\title{
Obese Zucker Rats as a Reverse Translational Model of Human Left Ventricular Hypertrophy
}

\author{
Mackenzie Shelby Newman \\ msnewman@mix.wvu.edu
}

Follow this and additional works at: https://researchrepository.wvu.edu/etd

Part of the Medical Biochemistry Commons, and the Medical Genetics Commons

\section{Recommended Citation}

Newman, Mackenzie Shelby, "Obese Zucker Rats as a Reverse Translational Model of Human Left Ventricular Hypertrophy" (2020). Graduate Theses, Dissertations, and Problem Reports. 7982.

https://researchrepository.wvu.edu/etd/7982

This Dissertation is protected by copyright and/or related rights. It has been brought to you by the The Research Repository @ WVU with permission from the rights-holder(s). You are free to use this Dissertation in any way that is permitted by the copyright and related rights legislation that applies to your use. For other uses you must obtain permission from the rights-holder(s) directly, unless additional rights are indicated by a Creative Commons license in the record and/ or on the work itself. This Dissertation has been accepted for inclusion in WVU Graduate Theses, Dissertations, and Problem Reports collection by an authorized administrator of The Research Repository @ WVU.

For more information, please contact researchrepository@mail.wvu.edu. 
Graduate Theses, Dissertations, and Problem Reports

2020

Obese Zucker Rats as a Reverse Translational Model of Human Left Ventricular Hypertrophy

Mackenzie Shelby Newman

Follow this and additional works at: https://researchrepository.wvu.edu/etd

Part of the Medical Biochemistry Commons, and the Medical Genetics Commons 


\title{
Obese Zucker Rats as a Reverse Translational Model of Human Left Ventricular Hypertrophy
}

\author{
Mackenzie Shelby Newman \\ Dissertation submitted to the School of Medicine at West Virginia University \\ in partial fulfillment of the requirements for the degree of \\ Doctor of Philosophy in \\ Cellular \& Integrative Physiology
}

\author{
Stan Hileman, Ph.D., Chair \\ Han-Gang Yu, Ph.D. \\ Timothy Nurkiewicz, Ph.D. \\ Karen Martin, Ph.D. \\ Kathleen Brundage, Ph.D. \\ Department of Physiology \& Pharmacology
}

Morgantown, West Virginia

2020

Keywords: left ventricular hypertrophy, transcriptome, obese Zucker rat, obesity, heart failure

Copyright 2020 Mackenzie Shelby Newman 


\title{
ABSTRACT \\ Obese Zucker Rats as a Reverse Translational Model of Human Left Ventricular Hypertrophy
}

\author{
Mackenzie Shelby Newman
}

Heart failure is a lifelong disability that is a comorbidity in nearly one out of eight deaths and leads to mortality within five years for over half of those affected. Left ventricular hypertrophy (LVH) is one of the most reliable independent predictors of heart failure. It has been observed to occur in nearly $20 \%$ of individuals in a large, representative US population study with sexspecific disparities. Obesity, cardiovascular diseases, and increased age are common comorbidities. Pathological LVH is irreversible in humans and early diagnosis is often missed due to lack of symptoms. Obese Zucker rats (OZR) are a rat strain that naturally develop phenotypical LVH without surgical intervention or a high fat diet. OZR develop obesity due to dysfunctional leptin signaling, which mimics the situation most often seen in obese humans. These animals also develop many conditions, such as hypertension and glucose intolerance, that mimic the human population at risk for LVH. These animal models are necessary for research as live human donor tissue is scarce. The central hypothesis is that genes and proteins that are differentially expressed during development of $\mathrm{LVH}$, with regard to sex and obesity status, may serve as clinical biomarkers or therapeutic targets for detection and prevention of heart failure. No previous studies have addressed these comparisons on an exome-wide basis. In the present research, I address these knowledge gaps with transcriptome analysis of rat and human left ventricle (LV) tissue in a sex- and obesity-specific manner. Specific genes that were identified and which are involved in cardiac development and function were then validated at the protein level to form a gene signature (NPPA, NPPB, HBB, MYL7, PDK4) that may be targetable as a future diagnostic or represent targets for intervention. Finally, I provide a novel method to reduce the expression of NPPA, the gene with the highest upregulation in both male and female obese humans and rats using targeted siRNA. In conclusion, this work defines novel genome-wide transcriptomes of LVH in male and female humans with or without regard to obesity, and male and female obese Zucker rats. Comparison of these datasets coupled to cross-species protein expression allows for confirmation of whether an individual gene or geneset is translationallyrelevant for further investigation in LVH. Future work should address transcriptomic and proteomic changes throughout the course of $\mathrm{LVH}$ development and whether intervention of specific gene targets can ameliorate onset of LVH. The work presented here provides a framework for the discovery of future LVH-related genes and proteins in order to improve the quality of life and burden on the healthcare system from LVH and, ultimately, heart failure. 


\section{ACKNOWLEDGEMENTS}

There's neither enough space nor time here to tell you all what you mean to me, so I'll try to make it brief.

To my committee-

Dr. Yu, thank you for these opportunities and everything else. You're one of the most intelligent people I've ever been lucky enough to learn from.

Drs. Hileman and Nurkiewicz, thanks for keeping this going.

Drs. Brundage and Martin, thank you for challenging my science at every corner.

To my friends here-

Emily, Brian, Timur, Drew, Quincy, Brad, Sion, Joe, Evan, Henry, Jesse, and everyone else, please don't ever lose touch.

To my family-

Brenda, Marc, Alex, Carrie, Daisy, Megan, et al, you've always been there and I don't tell you often enough what that means to me.

There are too many names to mention everyone here, but know that you're always on my mind.

"As we get close to the river, we see that everybody is already there, and I mean everyone."

This is dedicated to Franklin and Helen. 


\section{TABLE OF CONTENTS}

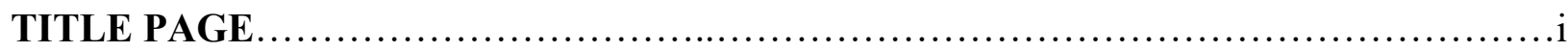

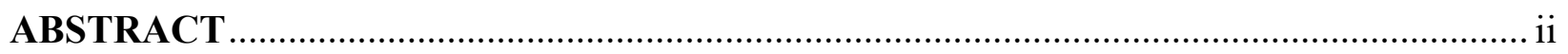

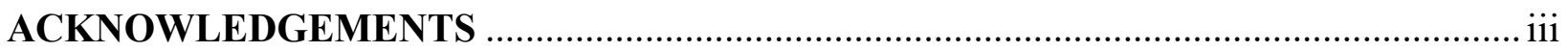

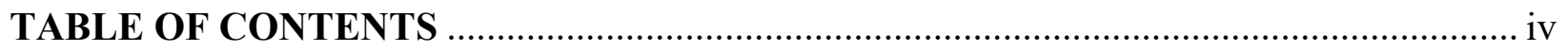

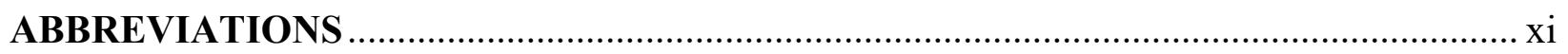

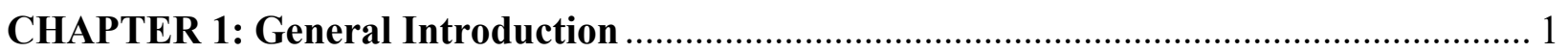

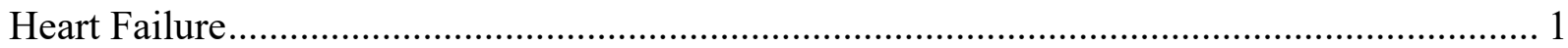

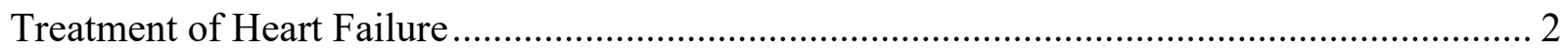

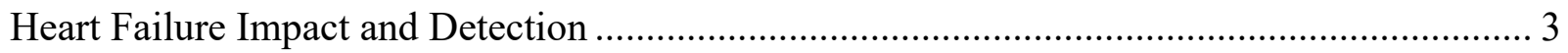

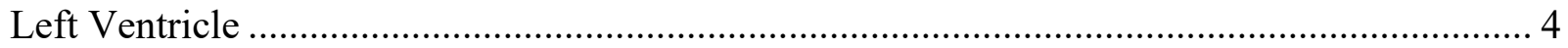

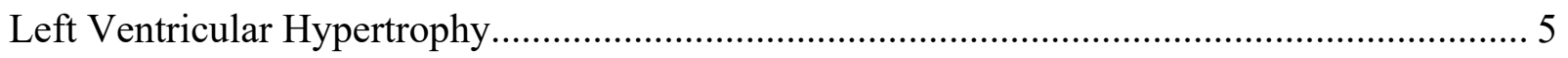

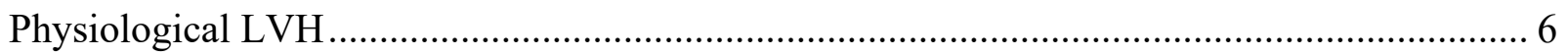

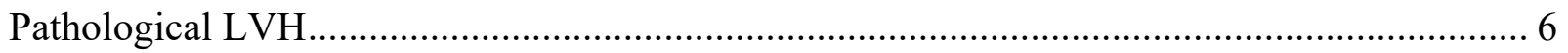

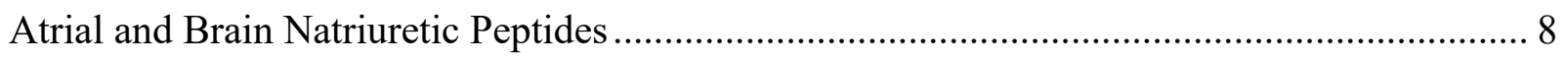

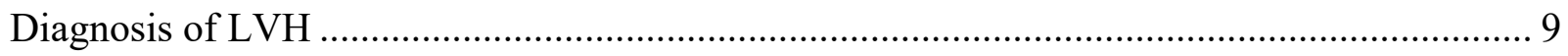

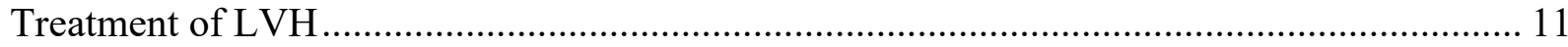

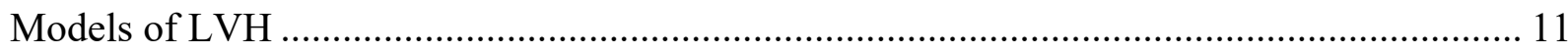

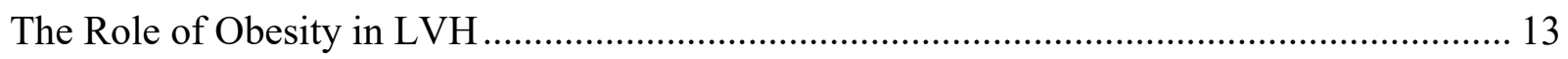

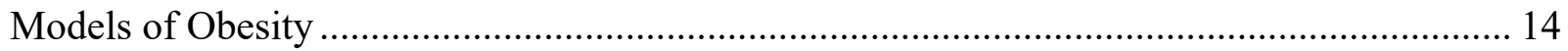

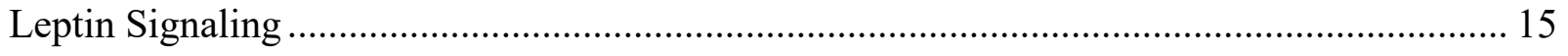

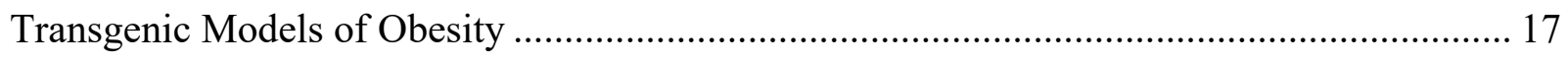

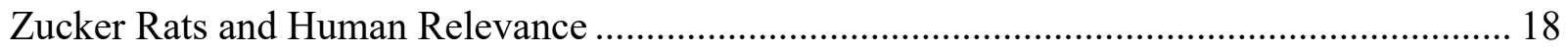

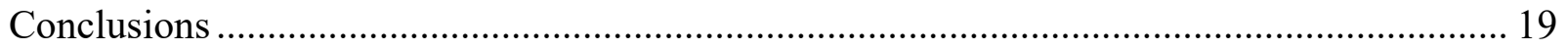

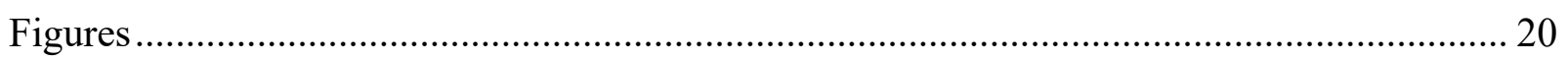

Figure 1. Representative pressure-volume loop of LVH and normal heart.......................... 20

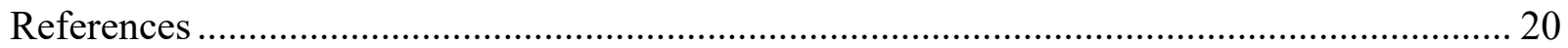

CHAPTER 2: Transcriptome profiling reveals novel BMI- and sex-specific gene expression

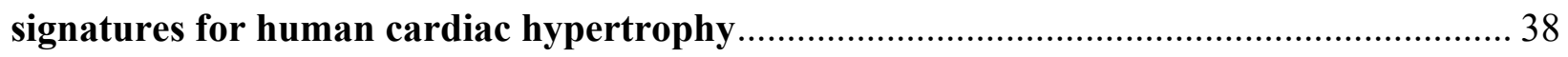

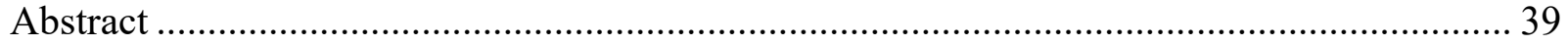

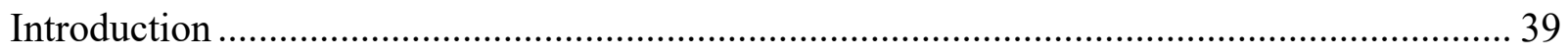




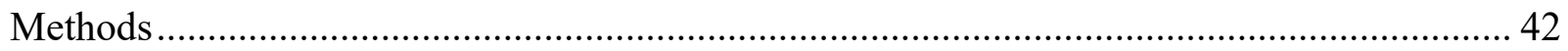

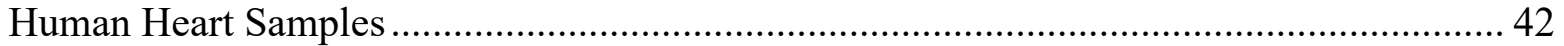

Total RNA Isolation, NGS (RNA-Seq), and Bioinformatics Analysis .................................. 43

Gene Ontology Enrichment and Pathway Analysis ............................................................ 44

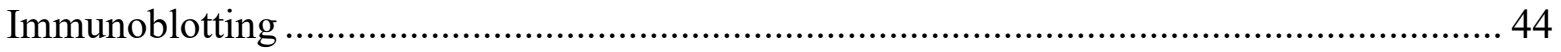

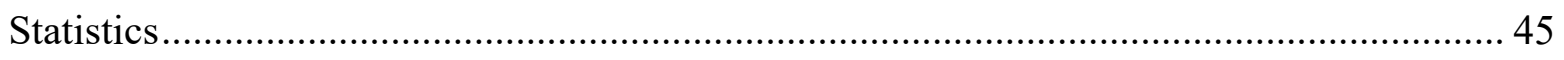

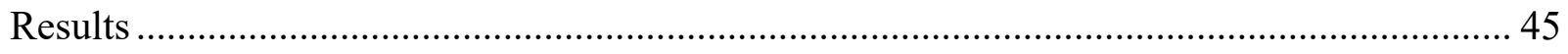

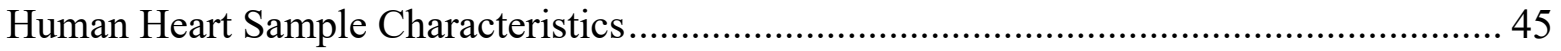

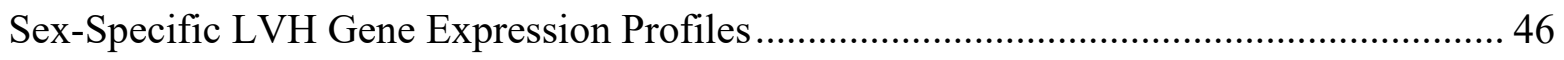

BMI- and Sex-Specific LVH Gene Expression Profiles ...................................................... 46

Distribution of Sex- and BMI-Specific Significant Cardiac Gene Expression ...................... 47

Sex- and BMI-Specific Significant Gene Expression Signature .......................................... 48

Significance of LVH DE Genes: Implication in Ischemic and/or Dilated Cardiomyopathy in

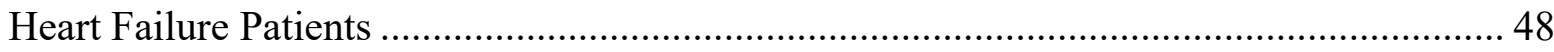

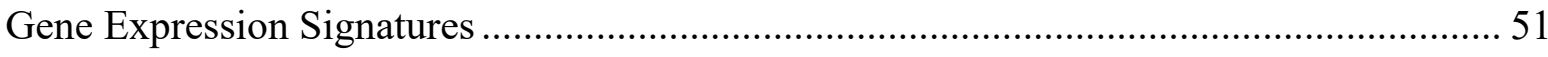

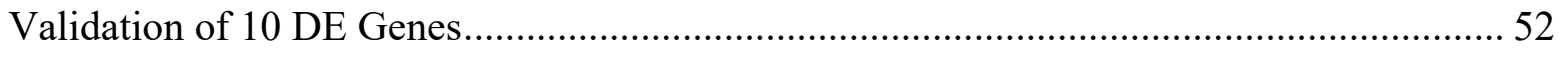

Expression of NPPA (ANP) and NPPB (BNP) in LVH, ISCH, and DCM......................... 52

Expression of HBA1 and HBB in LVH, ISCH, and DCM. ............................................. 53

Expression of GSTT1 and PLA2G2A in LVH, ISCH, and DCM....................................... 53

Expression of PDK4 and MYL7 in LVH, ISCH, and DCM.............................................. 54

Expression of HIST1H2AC in LVH, ISCH, and DCM. ................................................ 54

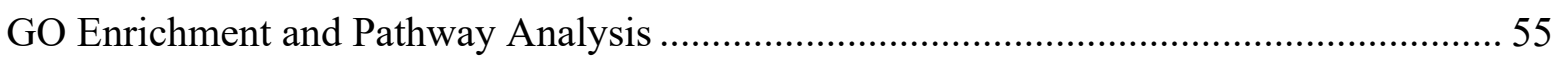

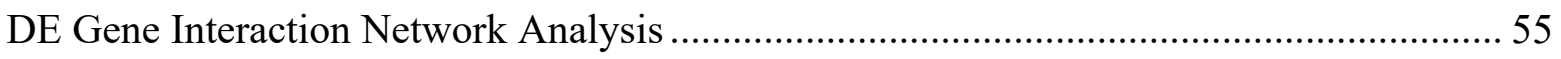

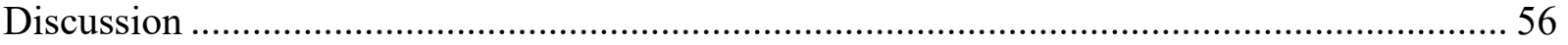

Roles of Nine DE Genes in Human Cardiac Hypertrophy and Failure................................. 57

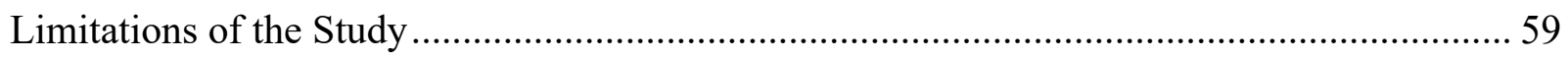

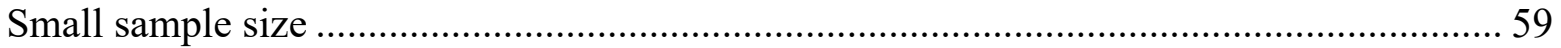

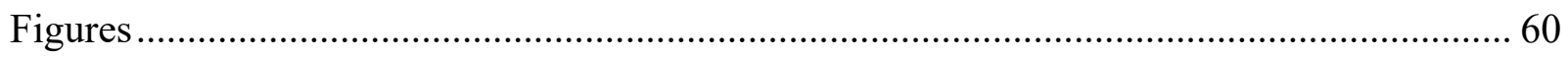

Figure 1: Sex-specific left ventricle hypertrophy (LVH) differential expression profiles (volcano) 
Figure 2: Body mass index (BMI)- and sex-specific LVH differential expression profiles (volcano)

Figure 3: Distribution of LVH differentially expressed genes under different conditions ... 63

Figure 4: Shared LVH differentially expressed (DE) genes in both sexes........................... 65

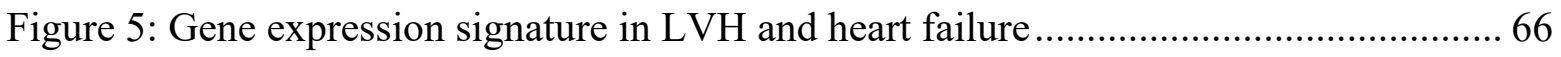

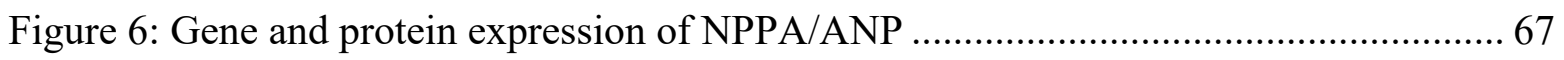

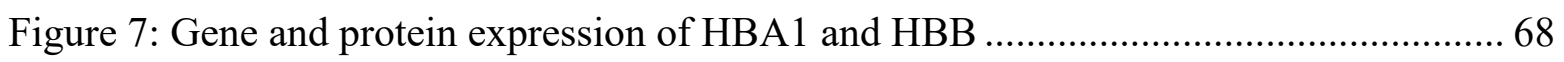

Figure 8: Gene and protein expression of GSTT1 and PLA2G2A...................................... 69

Figure 9: Gene and protein expression of PDK4 and MYL7 ……………...................... 70

Figure 10: Gene and protein expression of HIST1H2AC ................................................. 71

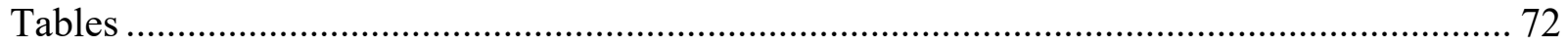

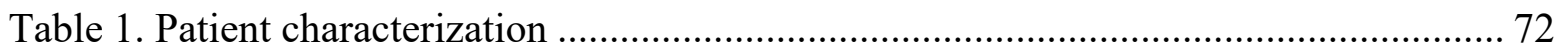

Table 2. Significant differentially expressed genes in LVH $[\operatorname{abs}(\log 2 \mathrm{FC})>2$, extreme small

p_adj, also found in heart failure data set]......................................................................... 73

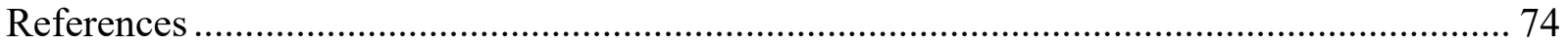

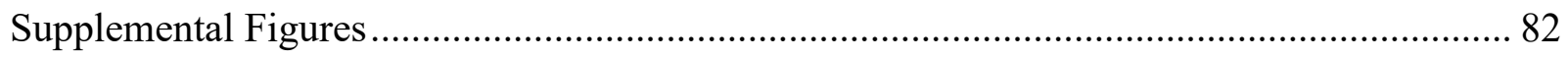

Supplemental Figure 1: Obese LVH DE genes found in heart failure patients..................... 82

Supplemental Figure 2: Sex-specific LVH DE genes found in heart failure patients ........... 84

Supplemental Figure 3: nine DE genes interaction network analysis by GeneMANIA ....... 87

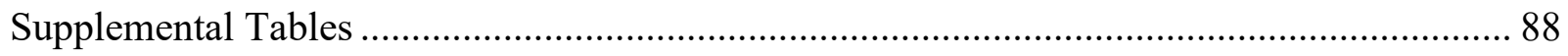

Supplemental Table 1. DEG from comparing men to women $\left(\mathrm{p} \_\right.$adj $\left.<0.05\right)$...................... 88

Supplemental Table 2. DEG in females when comparing NF to LVH (p_adj < 0.05) ......... 89

Supplemental Table 3. DEG in males when comparing NF to LVH (p_adj < 0.05) .......... 106

Supplemental Table 4. DEG in obese samples when comparing NF to LVH, $\left(p_{-}\right.$adj $\left.<0.05\right)$

Supplemental Table 5. DEG in all lean samples when comparing NF to LVH (p_adj < 0.05)

Supplemental Table 6. DEG in when comparing lean NF to obese LVH samples ( $0.05)$

Supplemental Table 7. DEG in when comparing lean NF to obese LVH samples, males only (p_adj $<0.05$ ) 128 
Supplemental Table 8. Sex- and BMI-specific DEG with (p_adj $<0.05$ ) and absolute value

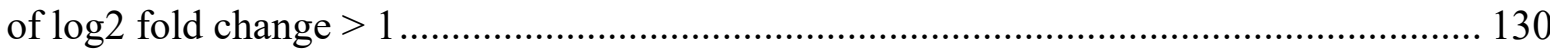
Supplemental Table 9. Sex- and BMI-specific DEG with $\left(p \_a d j<0.05\right)$ and absolute value of $\log 2$ fold change $>2$ 136

Supplemental Table 10. DEG shared between obesity-related LVH and ischemic heart datasets with (p_adj < 0.05).

Supplemental Table 11. DEG shared between obesity-related LVH and dilated

cardiomyopathy datasets with (p_adj < 0.05).

Supplemental Table 12. DEG shared between obesity-related LVH and both dilated ischemia and cardiomyopathy datasets with ( $p_{-}$adj $\left.<0.05\right)$

Supplemental Table 13. DEG shared between female obesity-related LVH and ischemia datasets with (p_adj < 0.05 ).

Supplemental Table 14. DEG shared between female obesity-related LVH and dilated cardiomyopathy datasets with ( $p_{-}$adj $\left.<0.05\right)$.

Supplemental Table 15. DEG shared between female obesity-related LVH and both dilated ischemia and cardiomyopathy datasets with $\left(p_{-}\right.$adj $\left.<0.05\right)$ 150

Supplemental Table 16. DEG shared between male obesity-related LVH and ischemia datasets with (p_adj < 0.05). 153

Supplemental Table 17. DEG shared between male obesity-related LVH and dilated cardiomyopathy datasets with ( $p_{-}$adj $<0.05$ ).

Supplemental Table 18. DEG shared between male obesity-related LVH and both dilated ischemia and cardiomyopathy datasets with $\left(p_{-}\right.$adj $\left.<0.05\right)$

Supplemental Table 19. Gene ontology results for "biological processes" from Ingenuity

Pathway Analysis 165

Supplemental Table 20. Gene ontology results for "cellular component" from Ingenuity

Pathway Analysis 175

Supplemental Table 21. Gene ontology results for "molecular function" from Ingenuity Pathway Analysis

CHAPTER 3: Obese Zucker Rats Share Similar Sex- and BMI-Specific Gene-Expression Signature with Human Left Ventricular Hypertrophy 179

Abstract 180 


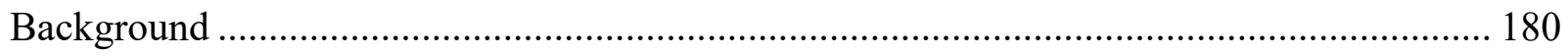

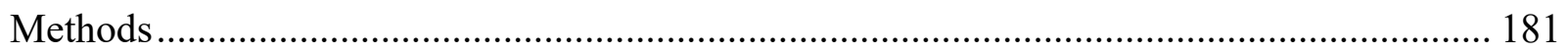

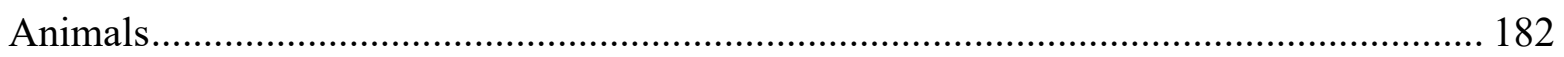

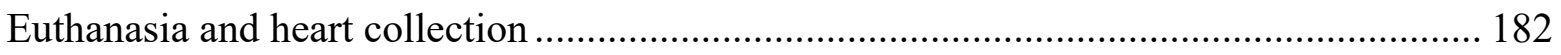

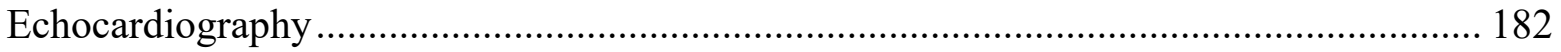

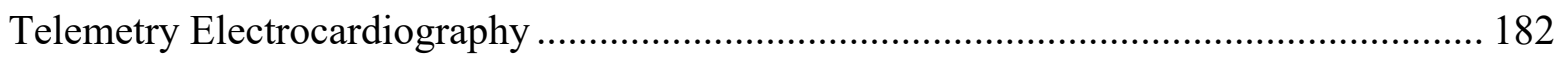

Transcriptome (RNA-Seq), bioinformatics analysis, and RT-qPCR ............................... 184

Gene expression levels were plotted in log2-fold (y-axis) from both transcriptome and

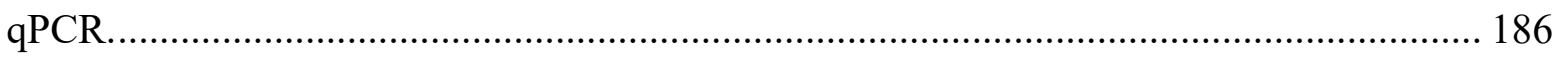

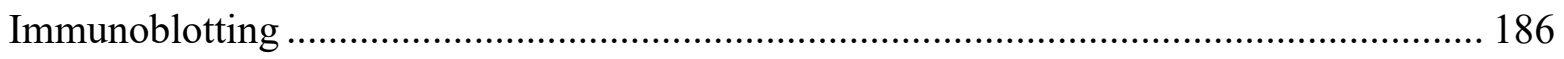

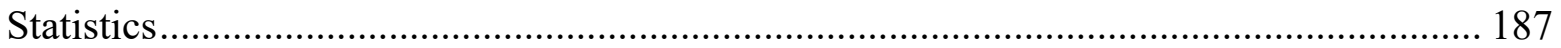

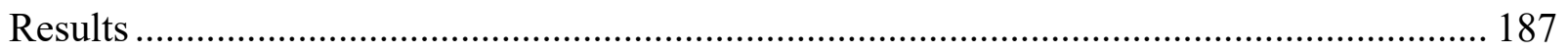

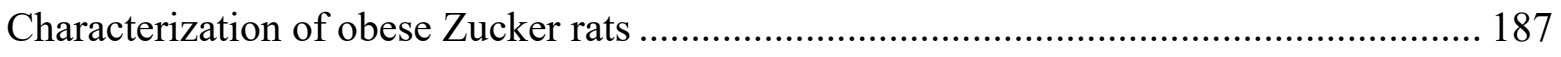

Altered heart rate, ST elevation, and heart rate variability in OZR from ECG recordings. 187

Identification of Differentially Expressed Genes (DEG) in OZR ..................................... 188

Shared Differentially Expressed Genes between rat and human obesity-related cardiac

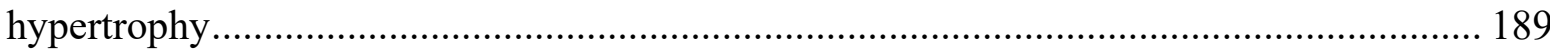

Validation of differentially expressed genes shared in OZR and obese human hypertrophied

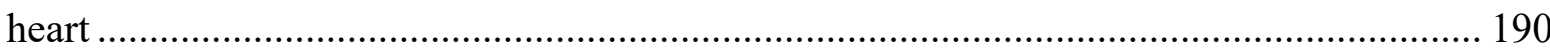

Sex- and obesity-dependent NPPA/NPPB expression .................................................... 192

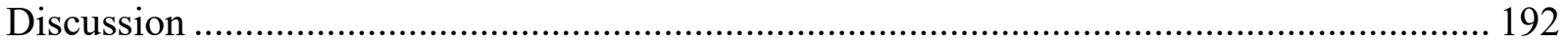

Echocardiography and electrophysiological characterizations of OZR ............................. 192

Differentially expressed genes associated with sex-specific obesity-related cardiac

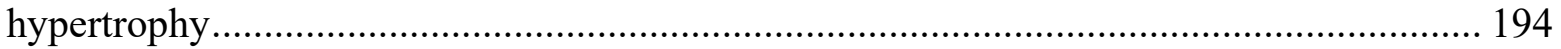

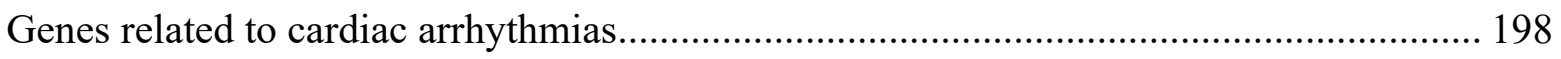

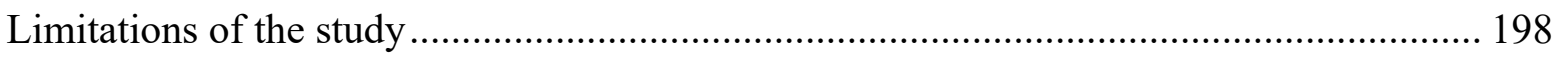

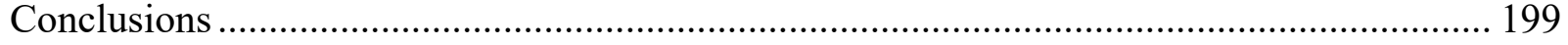

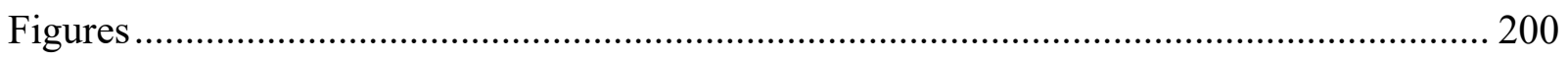

Figure 1: Representative ECG recordings of male (A) and female (B) Zucker rats ........... 200

Figure 2: Sex-specific distribution of significant genes in Zucker rat LVH ...................... 201

Figure 3: Sex-specific differentially expressed genes shared between human and rat ....... 202 
Figure 4. Comparison of seven obesity- and LVH-related differentially expressed genes

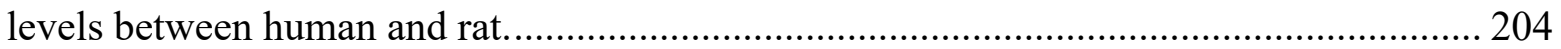

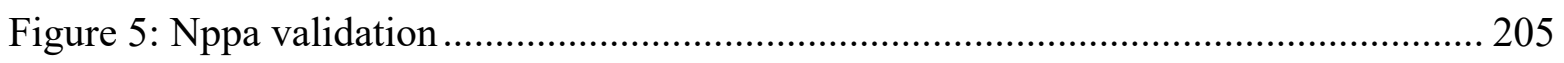

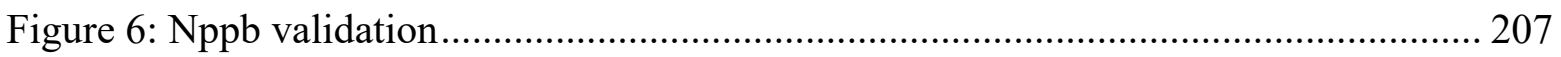

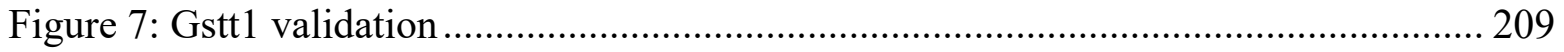

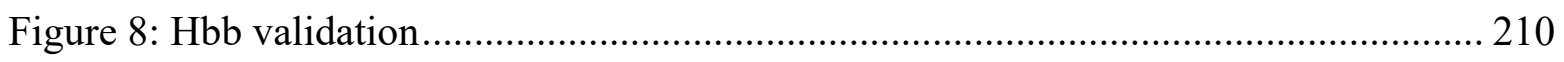

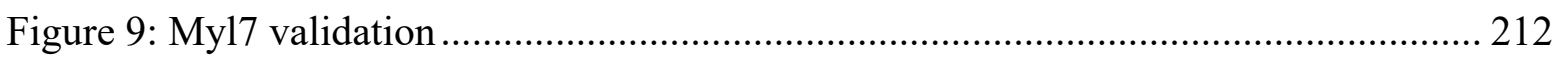

Figure 10: Pdk4 validation ................................................................................... 214

Figure 11: Roles of sex (A) and obesity (B) in NPPA and NPPB gene expression levels

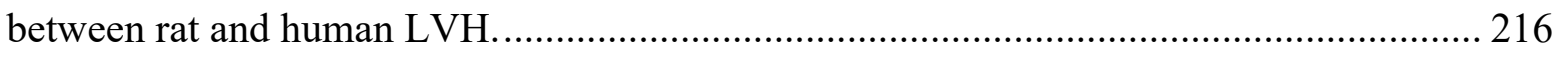

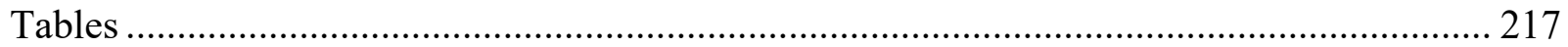

Table 1: Characteristics of OZR and echocardiogram results ...................................... 217

Table 2: Resting heart rate, ST elevation, and heart rate variability from ECG .............. 218

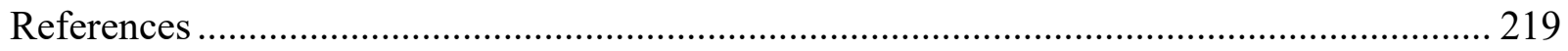

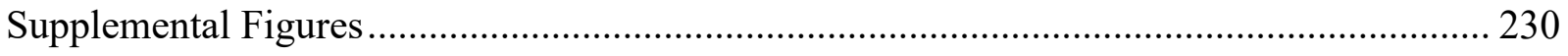

Figure S1: Principle component analysis (PCA) in female (left) and male rats showing that the groups of obese versus lean rats in both sexes are coherent................................... 230 Figure S2: Differential expression profiles between obese and lean animals (volcano plots) ..................................................... 232

Figure S3: Heatmaps of differentially expressed genes in OZR ................................. 233

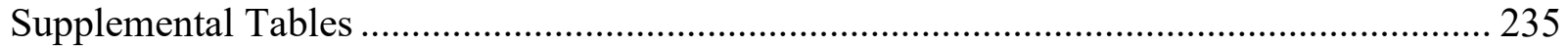

Supplemental Table 1. Primers used in qPCR. ............................................................ 235

Supplemental Table 2. Significant Differentially-Expressed Genes Per Sex ...................... 236

CHAPER 4: A Methodological Approach to Reducing Rat Nppa Using Targeted siRNA258

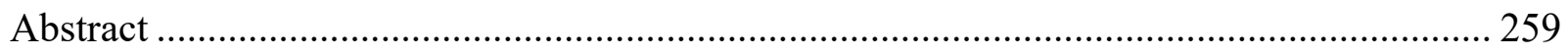

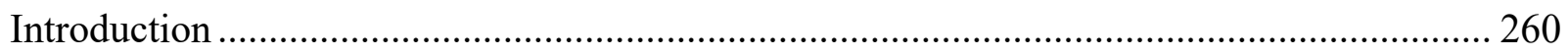

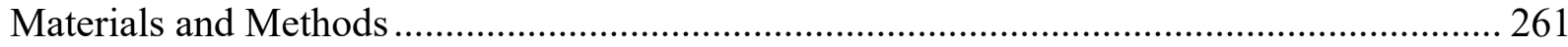

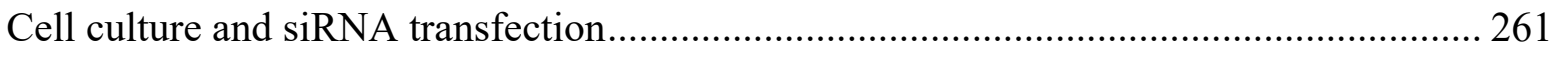

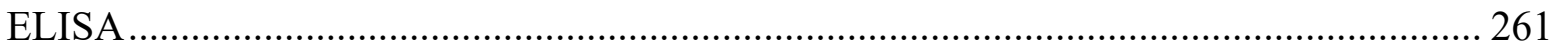

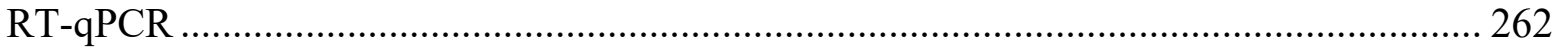

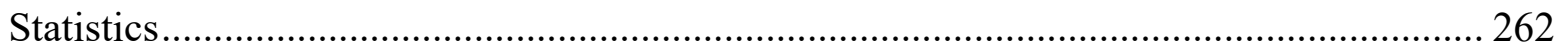




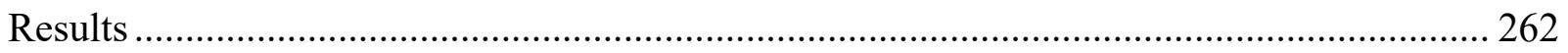

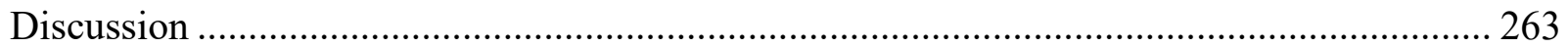

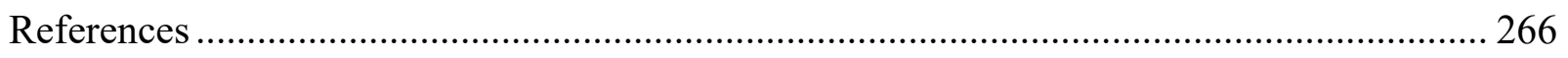

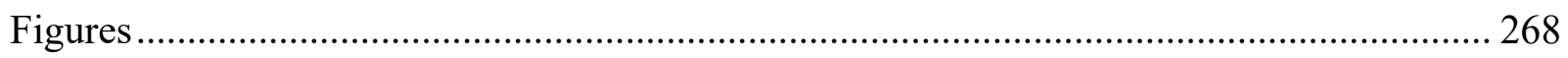

Figure 1: Time-course data from Anp ELISA from days 0 through 4.............................. 268

Figure 2: Day 0 quantification of Anp via ELISA …………………………………....... 269

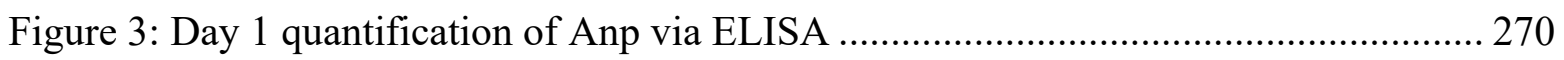

Figure 4: Day 2 quantification of Anp via ELISA …………………………………............ 271

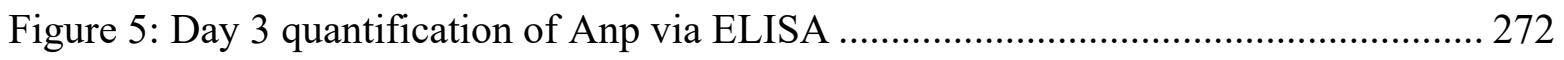

Figure 6: Representative RT-qPCR data for Nppa and Gapdh for day 2 ........................... 273

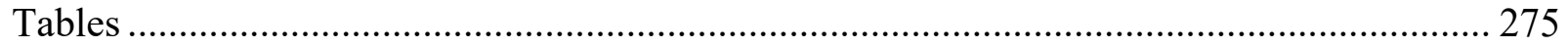

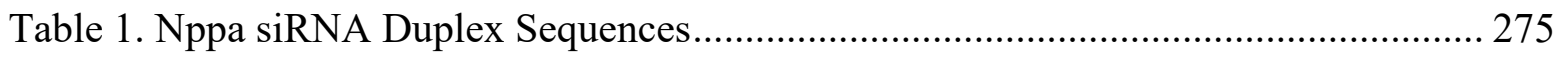

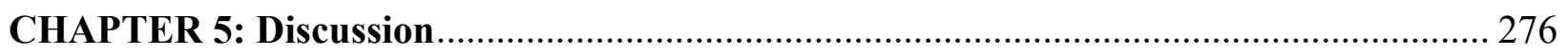

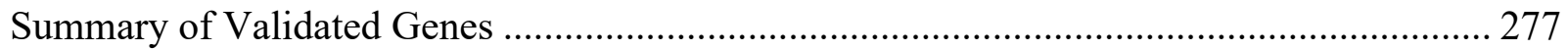

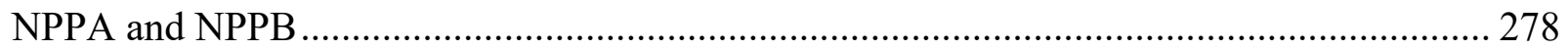

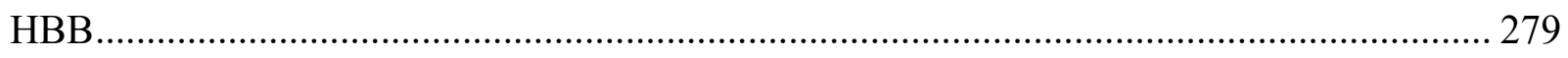

PDK4

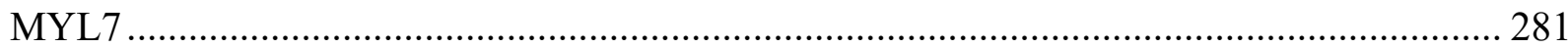

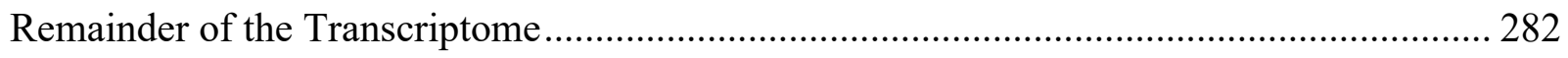

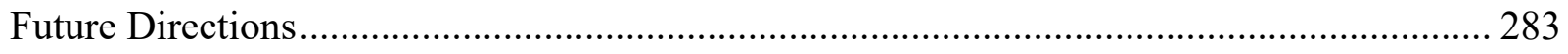

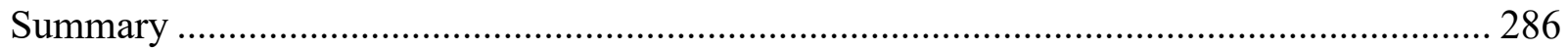

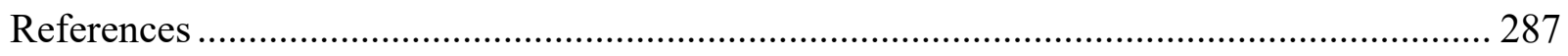




\begin{tabular}{|c|c|}
\hline ANP & atrial natriuretic peptide \\
\hline BMI & body mass index \\
\hline BMI25 & BMI less than 25 (lean) \\
\hline BMI30 & BMI greater than 30 (obese) \\
\hline BNP & brain natriuretic peptide \\
\hline DCM & dilated cardiomyopathy \\
\hline DE & differential expression \\
\hline DEG & differentially-expressed genes \\
\hline ECG & electrocardiography \\
\hline $\mathrm{ECHO}$ & echocardiography \\
\hline FPKM & fragments per kilobase of transcript per million mapped reads \\
\hline GSTT1 & glutathione S-transferase theta 1 \\
\hline HBA1 & hemoglobin subunit alpha \\
\hline HBB & hemoglobin subunit beta \\
\hline $\mathrm{HF}$ & heart failure \\
\hline HIST1H2AC & histone $\mathrm{H} 2 \mathrm{~A}$ type $1 \mathrm{C}$ \\
\hline ISCH & ischemic \\
\hline LEPR & leptin receptor (gene) \\
\hline LV & left ventricle \\
\hline LVH & left ventricular hypertrophy \\
\hline LZR & lean Zucker rat \\
\hline MAPK & mitogen-associated protein kinase \\
\hline MYL7 & myosin light chain 7 \\
\hline NF & non-failed heart \\
\hline NPPA & natriuretic peptide A (gene) \\
\hline NPPB & natriuretic peptide B (gene) \\
\hline NPRA & natriuretic peptide type A receptor \\
\hline NPRB & natriuretic peptide type B receptor \\
\hline OZR & obese Zucker rat \\
\hline p_adj & adjusted p-value \\
\hline PDK4 & pyruvate dehydrogenase kinase 4 \\
\hline PLA2G2A & phospholipase A2 group IIA \\
\hline $\mathrm{TAC}$ & trans-aortic constriction \\
\hline
\end{tabular}




\section{CHAPTER 1}

\section{General Introduction}

\section{Heart Failure (HF)}

HF occurs when the heart cannot sufficiently supply blood to the body. A lack of blood means a lack of oxygen and other nutrient delivery to tissues and organs, resulting in fatigue, peripheral edema, organ dysfunction, and fainting, among many other symptoms. It is an extremely detrimental disease because by the time it is diagnosed, significant irreversible damage is already present, thus explaining the high mortality rates. The most common comorbidities of HF, namely obesity, chronic kidney disease, hypertension, diabetes, and smoking, are present in $52 \%$ of HF patients $(13,38,52)$. HF can present acutely or chronically. In acute situations, frequently caused by underlying conditions such as arrhythmias or myocardial infarction, immediate treatment is pivotal because the heart has not had the same adaptive changes that accompany chronic HF. Although adaptive changes in chronic HF may be beneficial initially, they may ultimately lead to worse damage over time.

In HF, the Frank-Starling mechanism fails. In a healthy heart, this mechanism describes the positive correlation between simultaneous increases in cardiac output and right atrial pressure (reflective of blood returning to the heart from circulation). The hallmark of HF is decreased cardiac output, yet right atrial pressure is still increased. Cardiac output is defined as the product of stroke volume and heart rate. In HF, decreases in stroke volume are the result of diastolic and/or systolic dysfunction. In the former, ventricular filling is reduced (often due to stiffness or reduced lumen volume; ejection fraction is preserved), leading to less blood being pumped into circulation. In the latter, loss of myocyte contractility reduces the heart's capacity to pump. Changes in heart rate are most frequently the result of compensatory measures. 
Multiple systems facilitate adaptive changes for HF. Sympathetic input, endothelin, and angiotensin II are elevated in order to increase systemic vascular resistance and therefore both arterial and venous pressure. This causes vasoconstriction to compensate for reduced cardiac output. Sympathetic input, endothelin, and angiotensin II also increase contractility and heart rate to raise cardiac output in light of reduced stroke volume, but these can also promote arrythmias. Vasopressin and aldosterone are also elevated, in order to increase blood volume, but these can also cause peripheral edema. Atrial and brain natriuretic peptides (ANP and BNP, respectively) are released in HF to counterbalance the effects of these extremely elevated neurohumoral adaptations. Increases in blood volume (preload) and arterial vasoconstriction (afterload) can exacerbate HF by increasing the amount of total work output from the heart, as it is pumping a larger volume against a higher pressure gradient.

\section{Treatment of HF}

There is no treatment that can reverse HF, as cardiac tissue damage is irreparable (65). Despite this, numerous treatments have been shown to aid in patient survival, and survival rates have been increasing over the last few decades. Many medications have been shown to be effective post-HF; these have varying effects on the cardiovascular system that are principally related to hypertension and heart rate. Some of these classes of drugs include angiotensinconverting enzyme (ACE) inhibitors, angiotensin receptor blockers (ARBs), and angiotensin receptor neprilsyin inhibitors (ARNIs) (to reduce afterload and therefore reduce work required per stroke), If blockers and beta-blockers (to reduce heart rate/risk of arrhythmias in individuals with tachycardia), aldosterone antagonists and diuretics (to reduce blood volume), and digoxin (to increase contractility) (90). Heart transplant has been shown to be effective, but this is extremely costly and not recommended for elderly patients (72). The survival median is 
approximately 9.5 years after transplant, but the average is only five years. Transplantable hearts are in extreme demand, too, with less than $50 \%$ of patients desiring them actually receiving them from 1987-2012 (10). Heart transplant requires frequent post-operative attention because reduced parasympathetic cardiac innervation result in increased resting heart rate, reduced stroke volume, and increases in systolic and diastolic blood pressures $(3,104)$. Implantable devices, such as cardioverter-defibrillators, have been shown to be effective in patients with reduced ejection fraction. Left ventricular assist devices (LVAD), which are mechanical pumps that aid the LV to pump blood to the body, have been shown to be effective as well: from 2006-2014, the survival rate at one year was $80 \%$ and at two years was $70 \%$ in those who received implanted devices (58). Similarly, the in-hospital mortality rate dropped from $47 \%$ to $13 \%$ from $2005-2011$ (58). Though still costly, these devices are not as expensive as a transplant and are much more widely available. However, they are extremely invasive, too: the 30-day readmission rate after implantation was $44 \%$ in one meta-study (57). The most common complications were infection, stroke, and gastrointestinal bleeding, as well arrhythmias and device malfunction. Like pacemakers, LVAD require new batteries over time, and therefore another invasive surgery (72).

\section{HF Impact and Detection}

HF is a devastating condition that affects over $2 \%$ of the global population (48) and is a comorbidity in one out of eight deaths (22). Nearly \$40 billion USD in annual healthcare costs are attributed to HF (124), yet these are expected to rise to $\$ 69.8$ billion USD by 2030 (48). From 2013-2016, HF affected 6.2 million Americans aged 20 or greater, with projections of a $46 \%$ increase leading into 2030 and an overall $0.5 \%$ greater occurrence in the global population (48). Individuals aged 40 or greater are at a $20 \%$ risk of developing HF regardless of sex, but sex, race, age, and BMI are all factors in the development of HF (52). Males develop HF at rates 
that are $6-14 \%$ greater than females. In the $60-79$ age demographic, males and females develop $\mathrm{HF}$ at rates of $6.9 \%$ and $4.8 \%$, respectively, while above age 80 , these rates rise to $12.8 \%$ and $12 \%$, respectively (10). Lifetime risks are $30-42 \%$ in white males, $20-29 \%$ in black males, $32-$ $39 \%$ in white females, and $24-46 \%$ in black females (52). From $2005-2015$, there was a $27 \%$ increase in HF incidence $(10,48)$. The one-year mortality rate after HF for those aged less than 55 years was $17 \%$ in males and $14 \%$ in females, but for those aged 85 or greater, the rates sharply increased to $58 \%$ in men and $49 \%$ in women (10). The five-year mortality rate for all HF patients in a 1,282-patient study was $42 \%$, with no significant difference between sexes (68). Obesity (BMI $\left.>30 \mathrm{~kg} / \mathrm{m}^{2}\right)$ doubles the risk of HF over lean individuals $\left(\mathrm{BMI}<25 \mathrm{~kg} / \mathrm{m}^{2}\right)(52)$ with or without hypertension, a $100 \%$ increase in males and a $90 \%$ increase in females, according to the Framingham study $(56,63)$.

Common markers of cardiovascular damage, such as elevated circulating BNP, urinary albumin to creatine ratio, serum $\gamma$-glutamyl transferase, and hematocrit $(28,34,122)$, typically precede HF, but these may not be assessed in patients with no outward symptoms of cardiovascular damage. Left ventricular (LV) dysfunction is a hallmark feature of pending HF. Systolic LV dysfunction is present in $5 \%$ of patients, while diastolic dysfunction is present in $36 \%$ (60). In data from 2005-2010, lowered ejection fraction, a marker of LV function, was present in $50 \%$ of individuals (113) with HF.

\section{$\underline{\text { Left Ventricle }(\mathrm{LV})}$}

Proper function of the LV is critical to whole-body homeostasis as this chamber is responsible for pumping blood from the heart back into circulation. The LV is nearly $60 \%$ of overall heart volume (66) and is made up primarily of cardiomyocytes, a specialized type of muscle cell that contains a high density of mitochondria compared to skeletal muscle cells, and 
which carries cardiac action potentials that allow for rapid cycles of polarization and depolarization resulting in heart contraction. These cells have a low turnover rate with age; fewer than $50 \%$ are considered to be replaced under normal growth over a lifetime $(11,134)$. Fetal cardiomyocytes, instead of dividing, increase in size as the organism ages (134). There is a growing body of evidence for resident cardiac stem/progenitor cells, but their capacity to replace lost cells has not been fully elucidated $(11,134)$. The remaining minor cell populations in the LV are primarily fibroblasts, smooth muscle cells, and endothelial cells, which provide support and help maintain cardiomyocyte function (111).

\section{Left Ventricular Hypertrophy (LVH)}

The hallmark of $\mathrm{LVH}$, as the name implies, is an enlargement and thickening of the LV. There are two focal types: inward hypertrophic remodeling, wherein the chamber lumen volume is reduced, and outward hypertrophic remodeling, wherein the lumen volume increases. The former is associated with pathological cardiac hypertrophy and the latter is necessary in physiological hypertrophy. Numerous structural and functional changes underlie LVH, and although many have been investigated, none are completely understood. The most common feature of LVH is increased cardiomyocyte size (61). Electrical restructuring of the ventricle is secondary to cellular reorganization, as thickening of the ventricle wall leads to decreased conduction (18), which is usually accompanied by alterations in extracellular matrix composition and fibrosis (12). Compensatory mechanisms lead typically to increases in voltage within the QRS complex, as recorded by ECG (116), but changes have been witnessed in LVH such as increased R wave peak time, ST depression or elevation, and $\mathrm{T}$ wave inversion $(14,116)$. The QRS complex represents the period of ventricular depolarization (i.e. isovolumetric contraction) and the ST segment represents the period of ventricular repolarization (ejection). Changes in 
these parameters, such as lengthened time period or increased voltage, reflect thickened ventricle tissue, resulting in greater resistance to the propagation of electrical signal. However, these electrical changes do not occur in all cases $(4,5)$.

\section{Physiological LVH}

Importantly, not all LVH is detrimental. Physiological LVH occurs in normal circumstances: growth from childhood to adulthood, pregnancy, and exercise, i.e. situations where there is an increased tissue demand for oxygen and other nutrition in circulation (and therefore increased bloodflow) $(61,71,74)$. The focal biochemical pathways involved are often growth factor-based, such as the insulin-like growth factor-1 (IGF1) pathway, which leads to activation of phosphoinositol-3 kinase, Akt, and downstream mTOR $(75,85,99)$. Although increased activity of this pathway is seen in some cancers (128), it is considered to have only necessary, transient effects with regard to physiological LVH (74). Cardiac fetal gene expression (e.g. calcineurin, nuclear factor of activated T cells (NFAT), atrial natriuretic peptide (ANP), BNP, skeletal $\alpha$-actin, and myosin heavy chain 7$)$ is not significantly upregulated $(70,129)$. Proteins involved in the sarcomere, such sarco/endoplasmic reticulum Ca2+-ATPase, actin, and alpha- and beta-myosin heavy chains are increased in physiological LVH to facilitate contraction. The activation and increased production of these cellular components are considered to be reversible, even in cases of chronic exercise training. Cardiac function remains normal or increases over the period of exercise acclimation (74).

\section{Pathological LVH}

Pathological LVH develops due to a chronic insult such as increased pressure load (e.g. hypertension), volume load (valvular disease), or an underlying cardiomyopathy. Similar to physiological LVH, sarcomere counts and myocyte volume are increased, but in contrast, 
fibrosis and cellular necrosis/apoptosis are common. Changes in cardiac function are essentially the same as those seen in chronic HF: cardiac output decreases without a decrease in right atrial pressure. Hypertension in particular causes increased peripheral resistance and therefore increased right atrial pressure. Typically, diastolic dysfunction precedes systolic dysfunction, i.e. decreased passive compliance (filling) precedes decreased contractility.

Adaptive changes are similar as well. As previously discussed, increases in sympathetic input and angiotensin II (which are associated with and promote hypertension), and endothelin increase heart rate and contractility in order to compensate for reduced stroke volume and therefore raise cardiac output. These hormones all act through $G$ protein-coupled receptor pathways that are mediated by Gaq, which have been directly implicated in the development of cardiac hypertrophy. Gaq activates phospholipase C (PLC), which causes protein kinase C to activate numerous mitogen-activated protein kinases (MAPKs) associated with cellular stress. Activation of these pathways is also known to cause pro-fibrotic signaling, therefore further decreasing cardiac compliance. These hormones also activate phospholipase $\mathrm{C}$, which causes downstream changes in calcium homeostasis and activation/nuclear translocation of NFAT, a transcription factor that causes expression of many cardiac-specific genes $(31,49,74,77)$. Expression of fetal genes such as NPPA and NPPB, which encode for ANP and BNP, respectively, is increased $(32,35,44,50,86)$, in order to counterbalance elevations in aldosterone seen in hypertensive situations. Chronic elevations in these hormones ultimately contribute to irreversible damage of the myocardium and raise the risk of HF without early intervention. 


\section{Atrial and Brain Natriuretic Peptides}

The primary function of ANP and BNP is to decrease fluid volume by acting on distal portions of the nephron through binding to their cognate receptors, NPRA (gene NPR1) and NPRB (gene NPR2), respectively. Receptor activation in the heart and vasculature counteracts angiotensin: ANP and BNP are anti-hypertrophic, anti-fibroproliferative, and anti-hypertensive at physiological concentrations. ANP preferentially binds NPR1 and BNP preferentially binds NPR2, likely due to sequence homology. A third receptor, NPRC (gene NPR3), internalizes circulating natriuretic peptides, leading to their degradation. All three receptors are widely expressed, but with some tissue-specific enrichment: NPR1 in breast, lung, adipose tissue, and kidney, with NPR2 in brain, muscle, and female tissues (endometrium, cervix, uterus), and NPR3 in lung, kidney and urinary bladder, muscle, and adipose and soft tissue (121). NPRA and NPRB are GTPases upon activation; ligand binding causes receptor dimerization and initiates the conversion of intracellular GTP to cGMP. Elevated cGMP then may activate protein kinase G (PKG) to cause downstream effects such as ion channel modulation via phosphorylation (98, $115)$.

N-terminal pro B-type natriuretic peptide (pro-NT BNP) is currently used as a clinical marker for LVH and HF $(88,91,92)$. NPPA/ANP (measured as NT-pro-ANP) has been shown to be associated with cardiovascular dysfunction and death (110). However, these findings have not resulted in approval for use of ANP as a biomarker of HF. Pro-NT BNP is preferred to ANP due to its longer circulating half-life (40). ANP and ANP mimetics have been explored pharmaceutically for kidney injury due to expression of NPRA in distal nephron to regulate sodium clearance (84) and have been shown to have benefits in HF patients $(83,130)$, with one being approved in Japan. However, none have made it to market in the United States. 
Elevated ANP and BNP are generally considered to be beneficial in LVH. Despite this, extreme, persistent increases may be detrimental. Elevated levels of ANP have been shown to modulate heart rate (HR), effective refractory period (ERP), and action potential duration (APD) in cardiac tissue and cell preparations in mice, rats, dogs, rabbits, guinea pigs, and humans with mixed effects across species $(8,9,55,80,112)$. ANP was shown to have no effect or decrease HR in dogs $(8,9)$, decrease HR in rats $(80)$, and have no effect or an increase on HR in humans (80). ERP was shown to be increased in dogs and decreased in humans (80). ANP decreased APD in human, dog, rabbit, and guinea pig cardiac preparations (80), but had no effect on guinea pigs or dogs in another set of studies $(55,112)$. Natriuretic peptide receptor activation in cardiomyocytes is a likely cause of cardiac disturbances due to the dependence of hyperpolarization-activated cyclic nucleotide-gated (HCN) channels on intracellular cGMP; these channels are pivotal in maintaining the "funny" current in pacemaker cells that regulates and maintains heart rate. One human NPPA mutation, a single base deletion which causes a frameshift resulting in an extra 12 amino acids at the C-terminus, has been identified in humans that causes elevated, persistent ANP levels in the blood. Both humans and mice expressing this mutant NPPA exhibit atrial fibrillation (41). Overall, these data suggest that chronically elevated ANP signaling may contribute to the presentation of abnormal cardiac functions. Conversely, there is evidence in mice that NPPA or NPRA ablation results in pronounced cardiac hypertrophy and hypertension, leading to early mortality $(81,93)$.

\section{Diagnosis of LVH}

$\mathrm{LVH}$ is frequently asymptomatic, particularly early in its progression or in those with no obvious comorbidities. Initial diagnosis is derived from echocardiography (the same measurements can be derived by using an implanted catheter, computer tomography, or magnetic 
resonance imaging). These data can be analyzed as a pressure-volume loop, in order to determine basic cardiac parameters such as stroke volume, cardiac output, and ejection fraction. These parameters can then be used to gauge the presence of LVH. A basic representation of the changes in a pressure-volume loop due to LVH is shown in Figure 1. In line with this analysis, direct measurements of the LV can be taken directly from imaging and interpreted. These are often used in the Devereux formula to calculate LV mass:

LV mass $=0.8\left(1.04\left[\left([\operatorname{LVEDd}+\mathrm{IVSd}+\mathrm{PWd}]^{3}-\operatorname{LVEDd}^{3}\right)\right]\right)+0.6$

LVEDd: LV end diastolic diameter

IVSd: intraventricular septal diameter at end diastole

PWd: posterior wall thickness at end diastole

(all units are centimeters)

The resultant value is then normalized to body weight (39). LVH is initially suspected in males with LV mass $>115 \mathrm{~g} / \mathrm{m}^{2}$ and in females with LV mass $>95 \mathrm{~g} / \mathrm{m}^{2}$ in females (47).

However, LVH diagnosis is not based on a single test and, beyond echocardiography, 12lead electrocardiography is required in order to confirm electrical disturbances in the heart. Changes in electrical conduction manifest inconsistently in LVH, so clinical criteria seek to address multiple parameters at once. QRS complex alterations are most commonly seen in LVH (116) as this mechanism is active at the end of diastole and initiates systole, therefore reflecting the beginning of the period when the ventricle is contracting. Using Sokolow-Lyon criteria, the sum of the $\mathrm{S}$ wave in the V1 lead is added to the larger R wave in leads V5 or V6. Values greater 
than or equal to $35 \mathrm{~mm}$ and an $\mathrm{R}$ wave greater than or equal to $11 \mathrm{~mm}$ in the aVL lead are indicative of LVH $(47,109)$. Using Cornell and Cornell Product criteria, where the sum of the S wave in V3 and the R wave in aVL leads is used, a value greater than $28 \mathrm{~mm}$ in men or $20 \mathrm{~mm}$ in females indicates LVH (21). Differences in accuracy between the criteria have been shown to be dependent on BMI status (101), age (108), underlying condition such as myocardial infarction (102) or hypertension (132) and the population studied $(94,114)$.

Cardiac MRI may also be used to diagnose LVH. This technique allows for higher accuracy and precision versus echocardiography, as well as the identification of subtypes of LVH. This technique is expensive, however, and not necessary for patients who have met LVH diagnostic criteria using other methods $(89,107)$.

\section{Treatment of LVH}

There is no cure for $\mathrm{LVH}$, given that it is considered to be irreversible, yet many treatments have been implicated. Lifestyle changes, such as diet, exercise, and cessation of smoking, may have the largest impact on overall cardiovascular health. Like with HF, pharmaceuticals that reduce blood pressure have been shown to be effective at alleviating comorbidities of LVH, particularly hypertension or other conditions that increase the mechanical load put on the heart. Some of these drug classes include angiotensin converting enzyme inhibitors, angiotensin receptors blockers, calcium channel blockers, beta-blockers, and diuretics (133).

\section{Models of LVH}

LVH is nearly impossible to study in primary human cardiac tissue due to the scarcity of live donor tissue. Despite this, there are currently no laboratory models of cardiac hypertrophy that faithfully mimic the human disease. $\mathrm{LVH}$ is a progressive pathological adaptation which 
develops over an extended period of time. Surgical methods of LVH induction are extremely rapid and thus may not follow the same transcriptional changes seen in humans. Genetic animal models of LVH have phenotypical changes that are seen in human LVH but are not directly translatable due to the overexpression or deletion of a gene not being relevant to the vast majority of the population.

Transverse aortic constriction (TAC), the most common model used in rodents and larger mammals, requires invasive surgery to place a tie around the aorta and a follow-up procedure to remove it. Due to invasiveness and human variability with tightness of the tie, there is a mortality risk and experimental inconsistency with aortic banding $(62,118,119)$. Improvements to the model have been made, such as applying the band during closed-chest surgery, but many of the previous issues with variability remain $(36,51)$. A recent study used o-rings rather than nylon sutures around the aorta in order to allow for more reproducibility between animals and found a markedly decreased mortality rate and greater reproducibility in results (76).

Transgenic models have also been investigated, but often lack direct translational applicability in humans because humans seldom have only a single gene entirely knocked out or overexpressed in isolation. In a rat model overexpressing Ren2 (the gene encoding for renin, an enzyme that helps regulate angiotensin levels and therefore blood pressure), LV weights were increased in tandem with blood pressure and markers of fibrosis (123). These rats do not develop obesity. Overexpression of $\alpha_{1}$ adrenergic receptors leads to LVH by a proposed mechanism involving Gaq, as described above $(74,78,78)$. Overexpression of Gaq itself, a G-protein linked to alpha-1 adrenergic receptors, angiotensin II receptors, and endostatin type A receptors, has been shown to have a role in general cardiac hypertrophic development $(31,74,77)$. Overexpression of PKC- $\beta 2$, a further downstream mediator of these same receptors, led to LVH 
and expression of pro-fibrotic genes, purportedly via phospholipase $\mathrm{C}$ activation $(74,125)$. In a study comparing mice overexpressing RAS versus wild-type mice exposed to TAC treatment, the degree of LVH was equal between the two and this was associated with significant increases in collagen deposition (a marker of fibrosis) as well as in ANP and BNP levels $(43,74)$.

The MAPK signaling pathway has been widely accepted to play an important role in cardiac hypertrophy and HF in both humans and rodents, but the specific mechanisms involved are debated $(74,136)$. Neonatal rat cardiomyocytes in vitro have been shown to exhibit hypertrophy when expressing lentiviral-mediated, constituently-active MAP2K3 (126) or via pharmacological activation of the direct downstream effector p38 $\alpha$ (27). In contrast, transgenic mice overexpressing constituently-active MAP2K3 do not produce cardiac hypertrophy (64), and mice possessing dominant-negative $\mathrm{p} 38 \alpha$ in vivo have exhibited significant increases in hypertrophy versus controls according to one group of investigators (17) and demonstrated no changes in LV thickness compared to wild-type mice from another group (135). These inconsistent results from rodents do not clarify the role of MAP2K3 in myocyte hypertrophy. MAP2K3 transcript levels have been shown to increase 4.7-fold in human HF (15) and increased MAP2K3 activity has been associated with hypertrophy in human embryonic stem cell-derived cardiomyocytes (37). In our preliminary dataset of 18 human hearts, we found increased protein expression of MAP2K3 and phosphorylation of p38 in LVH that was positively associated with BMI in male, but not female, hearts (87).

\section{The Role of Obesity in LVH}

Obesity is the most powerful independent predictor of LVH and it nearly doubles the risk of HF in both males and females. It is one of the simplest diagnostics to measure due to lack of cost and invasiveness and therefore is extremely viable in initial determination of $\mathrm{LVH}$ 
susceptibility. Generally speaking, body mass increases in obesity require increased cardiac output due to more tissue needing to be perfused compared to lean individuals. This leads to an increase in blood volume, thus increasing venous pressure and thus right atrial pressure. Under the Frank-Starling mechanism, this causes an increase in overall cardiac output, and therefore work done by the heart. Obesity is also frequently accompanied by hypertension (over $50 \%$ of cases (2)), further perpetuating workload on the heart by increasing afterload $(96,105)$. Chronically, this leads to diastolic dysfunction and systolic dysfunction via the mechanisms described for LVH and HF.

\section{Models of Obesity}

As previously stated, obesity is one of the most common risk factors associated with both LVH and HF. Many rodent models of obesity have been developed, but the majority of them fall under two categories: diet-induced obesity (DIO) or transgenically-induced obesity. Whereas most models under either category are hyperphagic, sedentary, and develop insulin resistance, the transgenic models tend to develop more pronounced hyperglycemia, another risk factor for HF, as compared to DIO animals (69). DIO animals do not develop HF (16, 73). The most common type of DIO, using a $45 \%$ or $60 \%$ high-fat diet, does not produce the degree of obesity observed in many transgenic models (69). While transgenic animals allow for focused research into the mechanisms induced by discrete genetic changes with regard to obesity, these specific genetic changes may not be relevant to the wider human population. By contrast, obesity in humans is nearly always diet-induced. Although the polygenic nature of outbred animals in these studies may better mimic the wider human population, the underlying mechanisms may not be as easily determinable as compared with inbred strains. In addition, in transgenic models, it can be 
difficult to discern whether the physiological differences are the result of genetic alteration per se or versus the obesity itself.

\section{Leptin Signaling}

The most well-known transgenic models of rodent obesity share one common feature: disruptions in leptin input. Leptin is a hormone principally produced and secreted by adipose tissue in levels positively associated with fat mass. It was originally discovered by genome sequencing in the $o b / o b$ mouse, a mouse that exhibits extreme obesity due to the fact that it lacks leptin. There are multiple isoforms of the leptin receptor (all encoded from a single LEPR gene that undergoes exon shuffling), denoted as $\mathrm{Ob}-\mathrm{Ra}, \mathrm{Ob}-\mathrm{Rb}, \mathrm{Ob}-\mathrm{Rc}, \mathrm{Ob}-\mathrm{Rd}, \mathrm{Ob}-\mathrm{Re}$, and $\mathrm{Ob}-\mathrm{Rf}$. All six isoforms contain an N-terminal domain that binds leptin and contains fibronectin III binding domains. The short (Ob-Ra, Ob-Rc, Ob-Rd, and $\mathrm{Ob}-\mathrm{Rf}$ ) and long (Ob-Rb) isoforms have a single transmembrane spanning domain, then a box 1 motif and a janus kinase (JAK) binding domain, but the short isoforms do little to any intracellular signaling. The long isoform, $\mathrm{Ob}-\mathrm{Rb}$, also has a suppressor of cytokine signaling (SOCS) domain, a box 2 domain, and a box 3 domain; these are necessary for full JAK-STAT pathway signaling. Ob-Re is a circulating, soluble receptor that serves to bind and transport leptin, though its role, if any, in obesity is unclear. The short isoforms are thought to perhaps play a role in leptin transport in areas such as the blood-brain barrier, but they do not contain all domains necessary for full JAK-STAT activation, and thus only $\mathrm{Ob}-\mathrm{Rb}$ is considered to be a fully active receptor (42). Not surprisingly, loss of Ob-Rb, such as occurs in the $d b / d b$ mouse, also leads to excessive obesity.

Leptin receptors on the cell surface (10-20\% of the overall receptors per cell) typically exist as dimers (127). Upon a dimerized receptor binding one leptin molecule, the dimers form a tetramer (103) and a conformational change initiates signaling. JAK2 is recruited to the box 1 
and box 2 domains and phosphorylates the receptor at Tyr985 and Tyr1138. Next, members of the STAT protein family (notably STAT3) bind Tyr1138, are phosphorylated, then dimerize and translocate to the nucleus to act as transcription factors. PI3K, the major intracellular signaling molecule activated by insulin, is also activated by leptin binding Ob-Rb. This leads to downstream Akt/mTOR, MAPK/ERK/JNK, and p38MAPK induction $(33,42)$. One notable gene that is upregulated by STAT3 activation is SOCS3, which serves to negatively regulate leptin receptor activation by feeding back in a manner that inhibits intracellular signaling induced by leptin $(33,42)$. Downstream of STAT3 activation, protein tyrosine phosphatase 1B (PTP1B) is also activated and reduces leptin signaling.

Leptin receptors are widely distributed throughout the body and serve to regulate many physiological processes, including cell growth and energy expenditure, and possess the ability to communicate with other cytokines and hormones. The central effect of leptin on body weight comes from its actions in the arcuate nucleus (ARC) of the hypothalamus, where it binds functional leptin receptors on two distinct populations of neurons. Pro-opiomelanocortin (POMC) neurons are activated by leptin and secrete alpha-melanocyte stimulating hormone $(\alpha \mathrm{MSH})$ amongst other POMC-derived peptides whereas leptin inhibits neurons coexpressing Agouti-related peptide and neuropeptide Y (AgRP/NPY). These first-order neurons then project to other areas of the hypothalamus, such as the paraventricular nucleus and lateral hypothalamus, as well as to areas of the brain stem to regulate food intake and energy expenditure. $\alpha \mathrm{MSH}$ and AgRP neurons, along with melanocortin 3 receptor (MC3R) and melanocortin 4 receptors (MC4R), form what is referred to as the melanocortin system. aMSH and AgRP compete for binding at these receptors and deletion of MC4R leads to an obese phenotype, suggesting that aMSH input typically dominates in this system. Reciprocal innervations also exist between 
POMC and AgRP/NPY neurons, suggesting a highly coordinated response to changes in leptin that occurs with starvation or obesity. Overall, the actions of leptin in the hypothalamus lead to increased satiety, anorexia, and energy expenditure. By contrast, these central actions of leptin are directly opposed by that of ghrelin, a hormone released from the stomach that stimulates hunger $(1,42,69,120)$. Importantly, a lack of leptin is only rarely an explanation for human obesity and, typically, obese individuals have very high circulating levels of leptin. These individuals are considered to be resistant to the anorexigenic effects of leptin, and the sites of this resistance may include decreased transport at the blood-brain barrier as well as deficits in neural responses to leptin.

\section{Transgenic Models of Obesity}

The more commonly used rodent models of obesity are those with mutations in LEP or LEPR. There are notable examples of rodents with mutations or deletions in downstream effectors, such as POMC knockout mice $(23,131)$, POMC and AgRP double knockout mice (30), MC3R knockout mice (19), MC4R knockout mice and rats $(53,82)$, MC3R and MC4R double knockout mice (25), and AgRP overexpressing mice (45). In a mouse model with designer receptors exclusively activated by designer drugs (DREADDs), activation of AgRP neurons led to feeding and eventual increased adiposity, while inhibition of these populations reduced feeding (59). Lesser-used models that have direct leptin or leptin receptor mutations or deletions include s/s mice (mutated Tyr1138 in Ob-Rb, which is critical for STAT3 binding) (6, 7) and Koletsky rats (LEPR mutation resulting in undetectable Ob-Rb mRNA levels) (117). Nonetheless, the most common rodent models of obesity are the $o b / o b$ mouse, $d b / d b$ mouse, and OZR. $o b / o b$ mice contain an early stop codon in the mRNA for leptin, leading to the ablation of leptin protein production and circulating hormone levels (137). The effects of leptin deficiency 
on food intake, metabolism, and neuroendocrine dysfunction in this mouse model are entirely ameliorated by leptin injection (79). As mentioned above, obese humans are rarely obese due to a lack of leptin, so while the $o b / o b$ mouse has been invaluable as a tool to examine how leptin influences body weight regulation, they may not be a good translational model. $d b / d b$ mice and OZR have separate missense mutations in the LEPR gene that result in either an absence of receptor $(d b / d b$ mice) or a significantly reduced ability of $\mathrm{Ob}-\mathrm{Rb}$ to signal (OZR). Both models have elevated circulating concentrations of leptin $(46,69,95)$. A hallmark of $d b / d b$ mice is their

development of diabetes type II-like symptoms, thus they are frequently used as a model of diabetes in addition to obesity $(46,69)$. A parallel model of diabetes and obesity has been outbred into OZR, and is known as the Zucker diabetic fatty rat (106).

\section{Zucker Rats and Human Relevance}

OZR are used as a model for a variety of human diseases, e.g. hypertension, metabolic syndrome, chronic kidney disease, hyperlipidemia, and mild diabetes, depending on the age of the animal. They were initially inbred from rats that spontaneously developed obesity when given free access to food, developing up to $50 \%$ of their body mass as fat (compared to around $20 \%$ in lean controls). They are hyperphagic at a young age (fewer than three weeks) and can become over twice the body weight of lean controls as early as six weeks of age (24). In research, they have been historically favored, as compared to $o b / o b$ and $d b / d b$ mice, because Zuckers do not develop hyperglycemia to an extreme extent, therefore making this model more suitable for studying the effect of obesity alone $(54,74)$.

Despite a persistent increase in body weight starting early in life, no changes in mean arterial pressure of OZR were noted at 8 weeks of age in one study (97) and at both 9 and 13 months in another (29). Regardless, LVH has been established to be present by week 12 (100). 
Cardiomyocyte hypertrophy has been seen in OZR that exhibited increased QT interval as young as 16 weeks of age (67). A study of young rats (aged 9-13 weeks) looking at mRNA levels for NPPA and NPPB, both of which are elevated in human LVH and HF, only found a significant increase in NPPB, and this was unexpectedly in the lean animals (20). Another study in 5-6 month old Zucker rats found increases in both ANP and BNP, the peptide products of NPPA and NPPB (26). The longer amount of time required for LVH development in these animals is more comparable to human LVH than other TAC models, and their shared cardiac hormone profile, make them a viable model for obesity-related LVH.

\section{Conclusions}

Pathological LVH is a poorly-understood disease with many negative prognostic outcomes, most notably HF. As a comorbidity with obesity and rising obesity rates throughout the world, research into the development of LVH is implicit for future diagnoses and treatment. Investigating LVH in humans directly is hindered by the lack of availability of live donor tissue, therefore the development of new animal models is critical for the future of LVH research. The purpose of this research is to validate genes and proteins known to play a role in human HF that are also present in $\mathrm{LVH}$ as potential biomarkers of heart damage, then develop a novel model of LVH to further study the roles of these genes and proteins. The central hypothesis is that genes and proteins that are differentially expressed in $\mathrm{HF}$ and $\mathrm{LVH}$, with regard to sex and obesity status, may serve as clinical biomarkers or therapeutic targets for detection and prevention of HF. This will be explored via two specifics aims:

1. To identify differentially expressed genes that are common between human HF and LVH compared to non-failed hearts

2. To develop novel models to characterize the differentially expressed genes in human LVH 


\section{$\underline{\text { Figures }}$}

Figure 1. Representative pressure-volume loop of LVH and normal heart

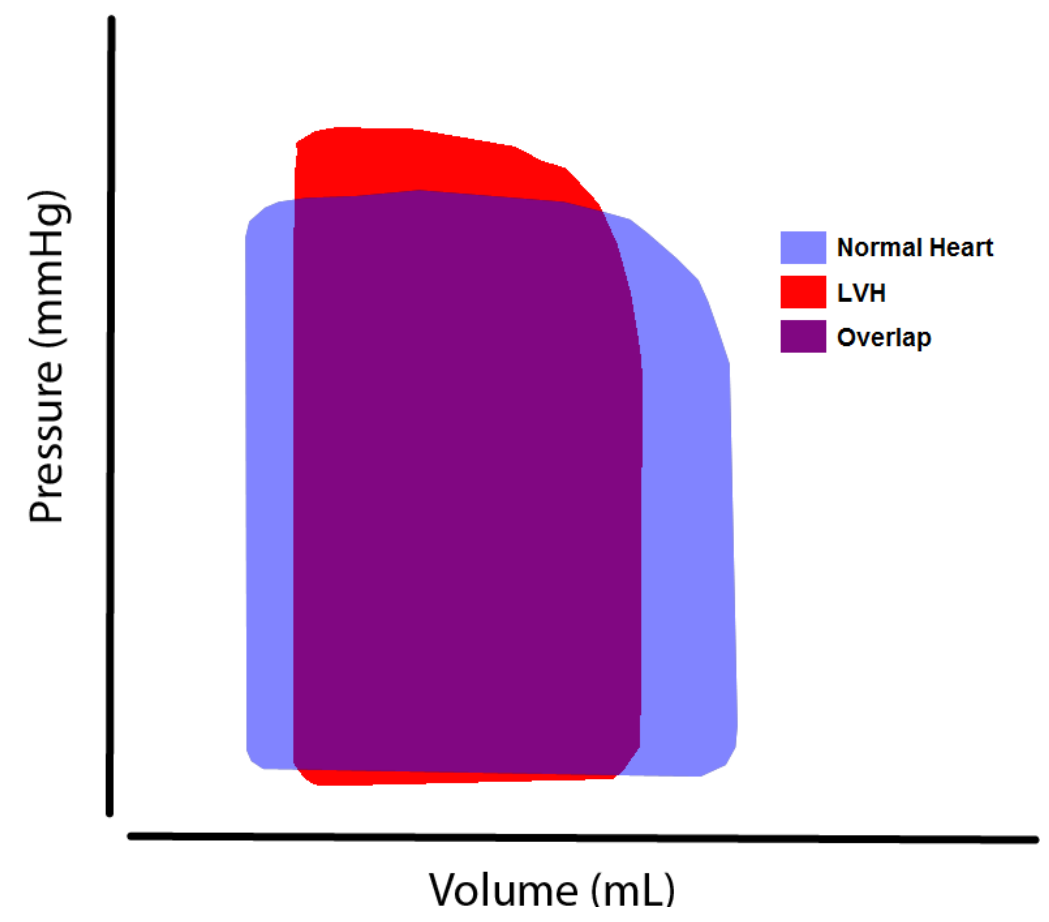

Figure 1. A basic representation of changes in a pressure-volume loop for a normal heart (blue) and an LVH heart (red). The narrowing of the LVH heart loop shows decreased total filling volume, reflecting reduced left ventricular lumen. The increased height of the loop represents increased pressure necessary for ejection, as seen in hypertension.

\section{$\underline{\text { References }}$}

(1) Andermann ML, Lowell BB. Toward a Wiring Diagram Understanding of Appetite Control. Neuron 2017 Aug 16;95(4):757-78.

(2) Aronow WS. Association of obesity with hypertension. Ann Transl Med 2017 Sep;5(17):350.

(3) Arrowood JA, Minisi AJ, Goudreau E, Davis AB, King AL. Absence of parasympathetic control of heart rate after human orthotopic cardiac transplantation. Circulation 1997 Nov $18 ; 96(10): 3492-8$. 
(4) Bacharova L. Left ventricular hypertrophy: disagreements between increased left ventricular mass and ECG-LVH criteria: the effect of impaired electrical properties of myocardium. J Electrocardiol 2014 Sep;47(5):625-9.

(5) Bacharova L, Estes EH. Left Ventricular Hypertrophy by the Surface ECG. J Electrocardiol 2017 Nov;50(6):906-8.

(6) Bates SH, Kulkarni RN, Seifert M, Myers MG, Jr. Roles for leptin receptor/STAT3dependent and -independent signals in the regulation of glucose homeostasis. Cell Metab 2005 Mar;1(3):169-78.

(7) Bates SH, Stearns WH, Dundon TA, Schubert M, Tso AW, Wang Y, et al. STAT3 signalling is required for leptin regulation of energy balance but not reproduction. Nature 2003 Feb 20;421(6925):856-9.

(8) Beaulieu P, Cardinal R, De LA, Lambert C. Direct chronotropic effects of atrial and C-type natriuretic peptides in anaesthetized dogs. Br J Pharmacol 1996 Aug;118(7):1790-6.

(9) Beaulieu P, Cardinal R, Page P, Francoeur F, Tremblay J, Lambert C. Positive chronotropic and inotropic effects of C-type natriuretic peptide in dogs. Am J Physiol 1997 Oct;273(4):H1933-H1940.

(10) Benjamin EJ, Muntner P, Alonso A, Bittencourt MS, Callaway CW, Carson AP, et al. Heart Disease and Stroke Statistics-2019 Update: A Report From the American Heart Association. Circulation 2019 Mar 5;139(10):e56-e528.

(11) Bergmann O, Bhardwaj RD, Bernard S, Zdunek S, Barnabe-Heider F, Walsh S, et al. Evidence for cardiomyocyte renewal in humans. Science 2009 Apr 3;324(5923):98-102.

(12) Berk BC, Fujiwara K, Lehoux S. ECM remodeling in hypertensive heart disease. J Clin Invest 2007 Mar;117(3):568-75. 
(13) Bibbins-Domingo K, Pletcher MJ, Lin F, Vittinghoff E, Gardin JM, Arynchyn A, et al. Racial differences in incident heart failure among young adults. N Engl J Med 2009 Mar 19;360(12):1179-90.

(14) Birnbaum Y, Alam M. LVH and the diagnosis of ST. J Electrocardiol 2014 Sep;47(5):65560.

(15) Boheler KR, Volkova M, Morrell C, Garg R, Zhu Y, Margulies K, et al. Sex- and agedependent human transcriptome variability: implications for chronic heart failure. Proc Natl Acad Sci U S A 2003 Mar 4;100(5):2754-9.

(16) Brainard RE, Watson LJ, Demartino AM, Brittian KR, Readnower RD, Boakye AA, et al. High fat feeding in mice is insufficient to induce cardiac dysfunction and does not exacerbate heart failure. PLoS One 2013;8(12):e83174.

(17) Braz JC, Bueno OF, Liang Q, Wilkins BJ, Dai YS, Parsons S, et al. Targeted inhibition of p38 MAPK promotes hypertrophic cardiomyopathy through upregulation of calcineurin-NFAT signaling. J Clin Invest 2003 May;111(10):1475-86.

(18) Burchfield JS, Xie M, Hill JA. Pathological ventricular remodeling: mechanisms: part 1 of 2. Circulation 2013 Jul 23;128(4):388-400.

(19) Butler AA, Kesterson RA, Khong K, Cullen MJ, Pelleymounter MA, Dekoning J, et al. A unique metabolic syndrome causes obesity in the melanocortin-3 receptor-deficient mouse. Endocrinology 2000 Sep;141(9):3518-21.

(20) Cabiati M, Raucci S, Liistro T, Belcastro E, Prescimone T, Caselli C, et al. Impact of obesity on the expression profile of natriuretic peptide system in a rat experimental model. PLoS One 2013;8(8):e72959. 
(21) Casale PN, Devereux RB, Alonso DR, Campo E, Kligfield P. Improved sex-specific criteria of left ventricular hypertrophy for clinical and computer interpretation of electrocardiograms: validation with autopsy findings. Circulation 1987 Mar;75(3):565-72.

(22) Centers for Disease Control and Prevention. Underlying Causes of Death, 1999-2017. 2020. National Center for Health Statistics.

(23) Challis BG, Coll AP, Yeo GS, Pinnock SB, Dickson SL, Thresher RR, et al. Mice lacking pro-opiomelanocortin are sensitive to high-fat feeding but respond normally to the acute anorectic effects of peptide-YY(3-36). Proc Natl Acad Sci U S A 2004 Mar 30;101(13):4695700.

(24) Charles River Laboratories. Zucker Rat Growth Chart. 2-1-2020. Charles River Laboratories.

(25) Chen AS, Marsh DJ, Trumbauer ME, Frazier EG, Guan XM, Yu H, et al. Inactivation of the mouse melanocortin-3 receptor results in increased fat mass and reduced lean body mass. Nat Genet 2000 Sep;26(1):97-102.

(26) Chen WK, Yeh YL, Lin YM, Lin JY, Tzang BS, Lin JA, et al. Cardiac hypertrophy-related pathways in obesity. Chin J Physiol 2014 Jun 30;57(3):111-20.

(27) Clerk A, Michael A, Sugden PH. Stimulation of the p38 mitogen-activated protein kinase pathway in neonatal rat ventricular myocytes by the $G$ protein-coupled receptor agonists, endothelin-1 and phenylephrine: a role in cardiac myocyte hypertrophy? J Cell Biol 1998 Jul 27;142(2):523-35.

(28) Coglianese EE, Qureshi MM, Vasan RS, Wang TJ, Moore LL. Usefulness of the blood hematocrit level to predict development of heart failure in a community. Am J Cardiol 2012 Jan 15;109(2):241-5. 
(29) Conti M, Renaud IM, Poirier B, Michel O, Belair MF, Mandet C, et al. High levels of myocardial antioxidant defense in aging nondiabetic normotensive Zucker obese rats. Am J Physiol Regul Integr Comp Physiol 2004 Apr;286(4):R793-R800.

(30) Corander MP, Rimmington D, Challis BG, O'Rahilly S, Coll AP. Loss of agouti-related peptide does not significantly impact the phenotype of murine POMC deficiency. Endocrinology 2011 May;152(5):1819-28.

(31) D'Angelo DD, Sakata Y, Lorenz JN, Boivin GP, Walsh RA, Liggett SB, et al. Transgenic Galphaq overexpression induces cardiac contractile failure in mice. Proc Natl Acad Sci U S A 1997 Jul 22;94(15):8121-6.

(32) Deschepper CF, Masciotra S, Zahabi A, Boutin-Ganache I, Picard S, Reudelhuber TL. Functional alterations of the Nppa promoter are linked to cardiac ventricular hypertrophy in WKY/WKHA rat crosses. Circ Res 2001 Feb 2;88(2):223-8.

(33) Devos R, Guisez Y, Van der Heyden J, White DW, Kalai M, Fountoulakis M, et al. Ligandindependent dimerization of the extracellular domain of the leptin receptor and determination of the stoichiometry of leptin binding. J Biol Chem 1997 Jul 18;272(29):18304-10.

(34) Dhingra R, Gona P, Wang TJ, Fox CS, D'Agostino RB, Sr., Vasan RS. Serum gammaglutamyl transferase and risk of heart failure in the community. Arterioscler Thromb Vasc Biol 2010 Sep;30(9):1855-60.

(35) Dries DL. Natriuretic peptides and the genomics of left-ventricular hypertrophy. Heart Fail Clin 2010 Jan;6(1):55-64.

(36) Eichhorn L, Weisheit CK, Gestrich C, Peukert K, Duerr GD, Ayub MA, et al. A Closedchest Model to Induce Transverse Aortic Constriction in Mice. J Vis Exp 2018 Apr 5;(134). 
(37) Foldes G, Mioulane M, Wright JS, Liu AQ, Novak P, Merkely B, et al. Modulation of human embryonic stem cell-derived cardiomyocyte growth: a testbed for studying human cardiac hypertrophy? J Mol Cell Cardiol 2011 Feb;50(2):367-76.

(38) Folsom AR, Shah AM, Lutsey PL, Roetker NS, Alonso A, Avery CL, et al. American Heart Association's Life's Simple 7: Avoiding Heart Failure and Preserving Cardiac Structure and Function. Am J Med 2015 Sep;128(9):970-6.

(39) Foppa M, Duncan BB, Rohde LE. Echocardiography-based left ventricular mass estimation. How should we define hypertrophy? Cardiovasc Ultrasound 2005 Jun 17;3:17.

(40) Fu S, Ping P, Wang F, Luo L. Synthesis, secretion, function, metabolism and application of natriuretic peptides in heart failure. J Biol Eng 2018;12:2.

(41) Galimberti ES, Kannankeril P, Kor K, Muhammad R, Blair M, Darbar D. Abstract 19074: NPPA Overexpression in Mice Increases Susceptibility to Atrial Fibrillation. Circulation 2012 Nov 20;126(Suppl 21):A19074.

(42) Gorska E, Popko K, Stelmaszczyk-Emmel A, Ciepiela O, Kucharska A, Wasik M. Leptin receptors. Eur J Med Res 2010 Nov 4;15 Suppl 2:50-4.

(43) Gottshall KR, Hunter JJ, Tanaka N, Dalton N, Becker KD, Ross J, Jr., et al. Ras-dependent pathways induce obstructive hypertrophy in echo-selected transgenic mice. Proc Natl Acad Sci U S A 1997 Apr 29;94(9):4710-5.

(44) Grabowski K, Riemenschneider M, Schulte L, Witten A, Schulz A, Stoll M, et al. Fetaladult cardiac transcriptome analysis in rats with contrasting left ventricular mass reveals new candidates for cardiac hypertrophy. PLoS One 2015;10(2):e0116807.

(45) Graham M, Shutter JR, Sarmiento U, Sarosi I, Stark KL. Overexpression of Agrt leads to obesity in transgenic mice. Nat Genet 1997 Nov;17(3):273-4. 
(46) Halaas JL, Gajiwala KS, Maffei M, Cohen SL, Chait BT, Rabinowitz D, et al. Weightreducing effects of the plasma protein encoded by the obese gene. Science $1995 \mathrm{Jul}$ 28;269(5223):543-6.

(47) Hashem MS, Kalashyan H, Choy J, Chiew SK, Shawki AH, Dawood AH, et al. Left ventricular relative wall thickness versus left ventricular mass index in non-cardioembolic stroke patients. Medicine (Baltimore) 2015 May;94(20):e872.

(48) Heidenreich PA, Albert NM, Allen LA, Bluemke DA, Butler J, Fonarow GC, et al. Forecasting the impact of heart failure in the United States: a policy statement from the American Heart Association. Circ Heart Fail 2013 May;6(3):606-19.

(49) Hein L, Stevens ME, Barsh GS, Pratt RE, Kobilka BK, Dzau VJ. Overexpression of angiotensin AT1 receptor transgene in the mouse myocardium produces a lethal phenotype associated with myocyte hyperplasia and heart block. Proc Natl Acad Sci U S A 1997 Jun 10;94(12):6391-6.

(50) Houweling AC, van Borren MM, Moorman AF, Christoffels VM. Expression and regulation of the atrial natriuretic factor encoding gene Nppa during development and disease. Cardiovasc Res 2005 Sep 1;67(4):583-93.

(51) Hu P, Zhang D, Swenson L, Chakrabarti G, Abel ED, Litwin SE. Minimally invasive aortic banding in mice: effects of altered cardiomyocyte insulin signaling during pressure overload. Am J Physiol Heart Circ Physiol 2003 Sep;285(3):H1261-H1269.

(52) Huffman MD, Berry JD, Ning H, Dyer AR, Garside DB, Cai X, et al. Lifetime risk for heart failure among white and black Americans: cardiovascular lifetime risk pooling project. J Am Coll Cardiol 2013 Apr 9;61(14):1510-7. 
(53) Huszar D, Lynch CA, Fairchild-Huntress V, Dunmore JH, Fang Q, Berkemeier LR, et al. Targeted disruption of the melanocortin-4 receptor results in obesity in mice. Cell 1997 Jan 10;88(1):131-41.

(54) Kava R, Greenwood MRC, Johnson PR. Zucker ( fa/fa ) Rat. ILAR Journal 32[3], 4-8. 1990.

(55) Kecskemeti V, Pacher P, Pankucsi C, Nanasi P. Comparative study of cardiac electrophysiological effects of atrial natriuretic peptide. Mol Cell Biochem 1996 Jul;160-161:539.

(56) Kenchaiah S, Evans JC, Levy D, Wilson PW, Benjamin EJ, Larson MG, et al. Obesity and the risk of heart failure. N Engl J Med 2002 Aug 1;347(5):305-13.

(57) Khazanie P, Hammill BG, Patel CB, Eapen ZJ, Peterson ED, Rogers JG, et al. Trends in the use and outcomes of ventricular assist devices among medicare beneficiaries, 2006 through 2011. J Am Coll Cardiol 2014 Apr 15;63(14):1395-404.

(58) Kirklin JK, Naftel DC, Pagani FD, Kormos RL, Stevenson LW, Blume ED, et al. Seventh INTERMACS annual report: 15,000 patients and counting. J Heart Lung Transplant 2015 Dec;34(12):1495-504.

(59) Krashes MJ, Koda S, Ye C, Rogan SC, Adams AC, Cusher DS, et al. Rapid, reversible activation of AgRP neurons drives feeding behavior in mice. J Clin Invest 2011 Apr;121(4):1424-8.

(60) Lam CS, Lyass A, Kraigher-Krainer E, Massaro JM, Lee DS, Ho JE, et al. Cardiac dysfunction and noncardiac dysfunction as precursors of heart failure with reduced and preserved ejection fraction in the community. Circulation $2011 \mathrm{Jul}$ 5;124(1):24-30. 
(61) Lazzeroni D, Rimoldi O, Camici PG. From Left Ventricular Hypertrophy to Dysfunction and Failure. Circ J 2016;80(3):555-64.

(62) Lei B, Chess DJ, Keung W, O'Shea KM, Lopaschuk GD, Stanley WC. Transient activation of p38 MAP kinase and up-regulation of Pim-1 kinase in cardiac hypertrophy despite no activation of AMPK. J Mol Cell Cardiol 2008 Sep;45(3):404-10.

(63) Levy D, Kenchaiah S, Larson MG, Benjamin EJ, Kupka MJ, Ho KK, et al. Long-term trends in the incidence of and survival with heart failure. $\mathrm{N}$ Engl $\mathrm{J}$ Med 2002 Oct 31;347(18):1397-402.

(64) Liao P, Georgakopoulos D, Kovacs A, Zheng M, Lerner D, Pu H, et al. The in vivo role of p38 MAP kinases in cardiac remodeling and restrictive cardiomyopathy. Proc Natl Acad Sci U S A 2001 Oct 9;98(21):12283-8.

(65) Lien CL, Harrison MR, Tuan TL, Starnes VA. Heart repair and regeneration: recent insights from zebrafish studies. Wound Repair Regen 2012 Sep;20(5):638-46.

(66) Lin FY, Devereux RB, Roman MJ, Meng J, Jow VM, Jacobs A, et al. Cardiac chamber volumes, function, and mass as determined by 64 -multidetector row computed tomography: mean values among healthy adults free of hypertension and obesity. JACC Cardiovasc Imaging 2008 Nov;1(6):782-6.

(67) Lin YC, Huang J, Kan H, Castranova V, Frisbee JC, Yu HG. Defective calcium inactivation causes long QT in obese insulin-resistant rat. Am J Physiol Heart Circ Physiol 2012 Feb 15;302(4):H1013-H1022.

(68) Loehr LR, Rosamond WD, Chang PP, Folsom AR, Chambless LE. Heart failure incidence and survival (from the Atherosclerosis Risk in Communities study). Am J Cardiol 2008 Apr 1;101(7):1016-22. 
(69) Lutz TA, Woods SC. Overview of animal models of obesity. Curr Protoc Pharmacol 2012 Sep;Chapter 5:Unit5.

(70) Maillet M, van Berlo JH, Molkentin JD. Molecular basis of physiological heart growth: fundamental concepts and new players. Nat Rev Mol Cell Biol 2013 Jan;14(1):38-48.

(71) Malhotra A, Sharma S. Hypertrophic Cardiomyopathy in Athletes. Eur Cardiol 2017 Dec;12(2):80-2.

(72) Marasco SF, Summerhayes R, Quayle M, McGiffin D, Luthe M. Cost comparison of heart transplant vs. left ventricular assist device therapy at one year. Clin Transplant 2016 May;30(5):598-605.

(73) Martins MA, Murucci CP, Bermond M, V, Dos SL, Monteiro de Assis ALE, Valentim NB, et al. Hypercaloric diet models do not develop heart failure, but the excess sucrose promotes contractility dysfunction. PLoS One 2020;15(2):e0228860.

(74) McMullen JR, Jennings GL. Differences between pathological and physiological cardiac hypertrophy: novel therapeutic strategies to treat heart failure. Clin Exp Pharmacol Physiol 2007 Apr;34(4):255-62.

(75) McMullen JR, Shioi T, Huang WY, Zhang L, Tarnavski O, Bisping E, et al. The insulin-like growth factor 1 receptor induces physiological heart growth via the phosphoinositide 3kinase(p110alpha) pathway. J Biol Chem 2004 Feb 6;279(6):4782-93.

(76) Melleby AO, Romaine A, Aronsen JM, Veras I, Zhang L, Sjaastad I, et al. A novel method for high precision aortic constriction that allows for generation of specific cardiac phenotypes in mice. Cardiovasc Res 2018 Oct 1;114(12):1680-90.

(77) Mende U, Kagen A, Cohen A, Aramburu J, Schoen FJ, Neer EJ. Transient cardiac expression of constitutively active Galphaq leads to hypertrophy and dilated cardiomyopathy by 
calcineurin-dependent and independent pathways. Proc Natl Acad Sci U S A 1998 Nov 10;95(23):13893-8.

(78) Milano CA, Dolber PC, Rockman HA, Bond RA, Venable ME, Allen LF, et al. Myocardial expression of a constitutively active alpha 1B-adrenergic receptor in transgenic mice induces cardiac hypertrophy. Proc Natl Acad Sci U S A 1994 Oct 11;91(21):10109-13.

(79) Mistry AM, Swick AG, Romsos DR. Leptin rapidly lowers food intake and elevates metabolic rates in lean and ob/ob mice. J Nutr 1997 Oct;127(10):2065-72.

(80) Moghtadaei M, Polina I, Rose RA. Electrophysiological effects of natriuretic peptides in the heart are mediated by multiple receptor subtypes. Prog Biophys Mol Biol 2016 Jan;120(1-3):3749.

(81) Mori T, Chen YF, Feng JA, Hayashi T, Oparil S, Perry GJ. Volume overload results in exaggerated cardiac hypertrophy in the atrial natriuretic peptide knockout mouse. Cardiovasc Res 2004 Mar 1;61(4):771-9.

(82) Mul JD, van BR, Bergen DJ, Brans MA, Brakkee JH, Toonen PW, et al. Melanocortin receptor 4 deficiency affects body weight regulation, grooming behavior, and substrate preference in the rat. Obesity (Silver Spring) 2012 Mar;20(3):612-21.

(83) Nagai T, Honda Y, Nakano H, Honda S, Iwakami N, Mizuno A, et al. Rationale and Design of Low-dose Administration of Carperitide for Acute Heart Failure (LASCAR-AHF). Cardiovasc Drugs Ther 2017 Dec;31(5-6):551-7.

(84) Naranjo V, Contreras A, Merino B, Plaza A, Lorenzo MP, Garcia-Caceres C, et al. Specific Deletion of the Astrocyte Leptin Receptor Induces Changes in Hippocampus Glutamate Metabolism, Synaptic Transmission and Plasticity. Neuroscience 2019 Nov 6. 
(85) Neri Serneri GG, Boddi M, Modesti PA, Cecioni I, Coppo M, Padeletti L, et al. Increased cardiac sympathetic activity and insulin-like growth factor-I formation are associated with physiological hypertrophy in athletes. Circ Res 2001 Nov 23;89(11):977-82.

(86) Newman MS, Nguyen T, Watson MJ, Hull RW, Yu HG. Transcriptome profiling reveals novel BMI- and sex-specific gene expression signatures for human cardiac hypertrophy. Physiol Genomics 2017 Jul 1;49(7):355-67.

(87) Newman M, Infanto AM, Watson MJ, Hull RW, Yu HG. Abstract 140: BMI- and Genderspecific Increase of MAP2K3/p38 Activity in Human Cardiac Hypertrophy. Circulation Research 2017 Feb 23;119(Suppl 1):A140.

(88) Ojji DB, Opie LH, Lecour S, Lacerda L, Adeyemi OM, Sliwa K. The proposed role of plasma NT pro-brain natriuretic peptide in assessing cardiac remodelling in hypertensive African subjects. Cardiovasc J Afr 2014 Sep;25(5):233-8.

(89) Oseni AO, Qureshi WT, Almahmoud MF, Bertoni AG, Bluemke DA, Hundley WG, et al. Left ventricular hypertrophy by ECG versus cardiac MRI as a predictor for heart failure. Heart 2017 Jan 1;103(1):49-54.

(90) Owens AT, Brozena SC, Jessup M. New Management Strategies in Heart Failure. Circ Res 2016 Feb 5;118(3):480-95.

(91) Paget V, Legedz L, Gaudebout N, Girerd N, Bricca G, Milon H, et al. N-terminal pro-brain natriuretic peptide: a powerful predictor of mortality in hypertension. Hypertension 2011 Apr;57(4):702-9.

(92) Palazzuoli A, Gallotta M, Quatrini I, Nuti R. Natriuretic peptides (BNP and NT-proBNP): measurement and relevance in heart failure. Vasc Health Risk Manag 2010 Jun 1;6:411-8. 
(93) Pandey KN. Genetic Ablation and Guanylyl Cyclase/Natriuretic Peptide Receptor-A: Impact on the Pathophysiology of Cardiovascular Dysfunction. Int J Mol Sci 2019 Aug $14 ; 20(16)$.

(94) Park JK, Shin JH, Kim SH, Lim YH, Kim KS, Kim SG, et al. A comparison of cornell and sokolow-lyon electrocardiographic criteria for left ventricular hypertrophy in korean patients. Korean Circ J 2012 Sep;42(9):606-13.

(95) Phillips MS, Liu Q, Hammond HA, Dugan V, Hey PJ, Caskey CJ, et al. Leptin receptor missense mutation in the fatty Zucker rat. Nat Genet 1996 May;13(1):18-9.

(96) Poirier P, Giles TD, Bray GA, Hong Y, Stern JS, Pi-Sunyer FX, et al. Obesity and cardiovascular disease: pathophysiology, evaluation, and effect of weight loss. Arterioscler Thromb Vasc Biol 2006 May;26(5):968-76.

(97) Porsti I, Kahonen M, Wu X, Arvola P, Ruskoaho H. Long-term physical exercise and atrial natriuretic peptide in obese Zucker rats. Pharmacol Toxicol 2002 Jul;91(1):8-12.

(98) Potter LR, Yoder AR, Flora DR, Antos LK, Dickey DM. Natriuretic peptides: their structures, receptors, physiologic functions and therapeutic applications. Handb Exp Pharmacol 2009;(191):341-66.

(99) Reiss K, Cheng W, Ferber A, Kajstura J, Li P, Li B, et al. Overexpression of insulin-like growth factor-1 in the heart is coupled with myocyte proliferation in transgenic mice. Proc Natl Acad Sci U S A 1996 Aug 6;93(16):8630-5.

(100) Ren J, Walsh MF, Jefferson L, Natavio M, Ilg KJ, Sowers JR, et al. Basal and ethanolinduced cardiac contractile response in lean and obese Zucker rat hearts. J Biomed Sci 2000 Sep;7(5):390-400. 
(101) Rider OJ, Ntusi N, Bull SC, Nethononda R, Ferreira V, Holloway CJ, et al. Improvements in ECG accuracy for diagnosis of left ventricular hypertrophy in obesity. Heart 2016 Oct 1;102(19):1566-72.

(102) Rodriguez-Padial L, Akerstrom F, Robles-Gamboa C, Andres J, Ruiz-Baena J. Diagnostic accuracy of left ventricular hypertrophy in patients with myocardial infarction by computerassisted electrocardiography (ELECTROPRES). Ann Noninvasive Electrocardiol 2013 Mar;18(2):170-80.

(103) Roujeau C, Jockers R, Dam J. New pharmacological perspectives for the leptin receptor in the treatment of obesity. Front Endocrinol (Lausanne) 2014;5:167.

(104) Sadowsk HS. Cardiac Translplantation: A Review. Physical Therapy 1996 May 1;76(5):498-515.

(105) Sardu C, De LC, Wallner M, Santulli G. Diabetes Mellitus and Its Cardiovascular Complications: New Insights into an Old Disease. J Diabetes Res 2019;2019:1905194.

(106) Shiota M, Printz RL. Diabetes in Zucker diabetic fatty rat. Methods Mol Biol 2012;933:103-23.

(107) Sipola P, Magga J, Husso M, Jaaskelainen P, Peuhkurinen K, Kuusisto J. Cardiac MRI assessed left ventricular hypertrophy in differentiating hypertensive heart disease from hypertrophic cardiomyopathy attributable to a sarcomeric gene mutation. Eur Radiol 2011 Jul;21(7):1383-9.

(108) Sohaib SM, Payne JR, Shukla R, World M, Pennell DJ, Montgomery HE. Electrocardiographic (ECG) criteria for determining left ventricular mass in young healthy men; data from the LARGE Heart study. J Cardiovasc Magn Reson 2009 Jan 16;11:2. 
(109) Sokolow M, Lyon TP. The ventricular complex in left ventricular hypertrophy as obtained by unipolar precordial and limb leads. 1949. Ann Noninvasive Electrocardiol 2001 Oct;6(4):34368.

(110) Song W, Wang H, Wu Q. Atrial natriuretic peptide in cardiovascular biology and disease (NPPA). Gene 2015 Sep 10;569(1):1-6.

(111) Souders CA, Bowers SL, Baudino TA. Cardiac fibroblast: the renaissance cell. Circ Res 2009 Dec 4;105(12):1164-76.

(112) Stambler BS, Guo GB. Atrial natriuretic peptide has dose-dependent, autonomically mediated effects on atrial refractoriness and repolarization in anesthetized dogs. J Cardiovasc Electrophysiol 2005 Dec;16(12):1341-7.

(113) Steinberg BA, Zhao X, Heidenreich PA, Peterson ED, Bhatt DL, Cannon CP, et al. Trends in patients hospitalized with heart failure and preserved left ventricular ejection fraction: prevalence, therapies, and outcomes. Circulation $2012 \mathrm{Jul}$ 3;126(1):65-75.

(114) Su FY, Li YH, Lin YP, Lee CJ, Wang CH, Meng FC, et al. A comparison of Cornell and Sokolow-Lyon electrocardiographic criteria for left ventricular hypertrophy in a military male population in Taiwan: the Cardiorespiratory fitness and Hospltalization Events in armed Forces study. Cardiovasc Diagn Ther 2017 Jun;7(3):244-51.

(115) Suga S, Nakao K, Hosoda K, Mukoyama M, Ogawa Y, Shirakami G, et al. Receptor selectivity of natriuretic peptide family, atrial natriuretic peptide, brain natriuretic peptide, and C-type natriuretic peptide. Endocrinology 1992 Jan;130(1):229-39.

(116) Szewieczek J, Gasior Z, Dulawa J, Francuz T, Legierska K, Batko-Szwaczka A, et al. ECG low QRS voltage and wide QRS complex predictive of centenarian 360-day mortality. Age (Dordr ) 2016 Apr;38(2):44. 
(117) Takaya K, Ogawa Y, Hiraoka J, Hosoda K, Yamori Y, Nakao K, et al. Nonsense mutation of leptin receptor in the obese spontaneously hypertensive Koletsky rat. Nat Genet 1996 Oct;14(2):130-1.

(118) Takimoto E, Champion HC, Li M, Belardi D, Ren S, Rodriguez ER, et al. Chronic inhibition of cyclic GMP phosphodiesterase 5A prevents and reverses cardiac hypertrophy. Nat Med 2005 Feb;11(2):214-22.

(119) Tarnavski O, McMullen JR, Schinke M, Nie Q, Kong S, Izumo S. Mouse cardiac surgery: comprehensive techniques for the generation of mouse models of human diseases and their application for genomic studies. Physiol Genomics 2004 Feb 13;16(3):349-60.

(120) Toda C, Santoro A, Kim JD, Diano S. POMC Neurons: From Birth to Death. Annu Rev Physiol 2017 Feb 10;79:209-36.

(121) Uhlen M, Fagerberg L, Hallstrom BM, Lindskog C, Oksvold P, Mardinoglu A, et al. Proteomics. Tissue-based map of the human proteome. Science 2015 Jan 23;347(6220):1260419. (122) Velagaleti RS, Gona P, Larson MG, Wang TJ, Levy D, Benjamin EJ, et al. Multimarker approach for the prediction of heart failure incidence in the community. Circulation 2010 Oct $26 ; 122(17): 1700-6$.

(123) Villarreal FJ, MacKenna DA, Omens JH, Dillmann WH. Myocardial remodeling in hypertensive Ren-2 transgenic rats. Hypertension 1995 Jan;25(1):98-104.

(124) Voigt J, Sasha JM, Taylor A, Krucoff M, Reynolds MR, Michael GC. A reevaluation of the costs of heart failure and its implications for allocation of health resources in the United States. Clin Cardiol 2014 May;37(5):312-21. 
(125) Wakasaki H, Koya D, Schoen FJ, Jirousek MR, Ways DK, Hoit BD, et al. Targeted overexpression of protein kinase $\mathrm{C}$ beta2 isoform in myocardium causes cardiomyopathy. Proc Natl Acad Sci U S A 1997 Aug 19;94(17):9320-5.

(126) Wang Y, Huang S, Sah VP, Ross J, Jr., Brown JH, Han J, et al. Cardiac muscle cell hypertrophy and apoptosis induced by distinct members of the p38 mitogen-activated protein kinase family. J Biol Chem 1998 Jan 23;273(4):2161-8.

(127) Wauman J, Zabeau L, Tavernier J. The Leptin Receptor Complex: Heavier Than Expected? Front Endocrinol (Lausanne) 2017;8:30.

(128) Weroha SJ, Haluska P. The insulin-like growth factor system in cancer. Endocrinol Metab Clin North Am 2012 Jun;41(2):335-50, vi.

(129) Wilkins BJ, Dai YS, Bueno OF, Parsons SA, Xu J, Plank DM, et al. Calcineurin/NFAT coupling participates in pathological, but not physiological, cardiac hypertrophy. Circ Res 2004 Jan 9;94(1):110-8.

(130) Yandrapalli S, Jolly G, Biswas M, Rochlani Y, Harikrishnan P, Aronow WS, et al. Newer hormonal pharmacotherapies for heart failure. Expert Rev Endocrinol Metab 2018 Jan;13(1):3549.

(131) Yaswen L, Diehl N, Brennan MB, Hochgeschwender U. Obesity in the mouse model of pro-opiomelanocortin deficiency responds to peripheral melanocortin. Nat Med 1999 Sep;5(9):1066-70.

(132) Yi S, Wang F, Wan M, Yi X, Zhang Y, Sun S. Prediction of stroke with electrocardiographic left ventricular hypertrophy in hypertensive patients: A meta-analysis. J Electrocardiol 2020 Apr 28;61:27-31. 
(133) Yildiz M, Oktay AA, Stewart MH, Milani RV, Ventura HO, Lavie CJ. Left ventricular hypertrophy and hypertension. Prog Cardiovasc Dis 2019 Nov 21.

(134) Yutzey KE. Cardiomyocyte Proliferation: Teaching an Old Dogma New Tricks. Circ Res 2017 Feb 17;120(4):627-9.

(135) Zhang S, Weinheimer C, Courtois M, Kovacs A, Zhang CE, Cheng AM, et al. The role of the Grb2-p38 MAPK signaling pathway in cardiac hypertrophy and fibrosis. J Clin Invest 2003 Mar;111(6):833-41.

(136) Zhang W, Elimban V, Nijjar MS, Gupta SK, Dhalla NS. Role of mitogen-activated protein kinase in cardiac hypertrophy and heart failure. Exp Clin Cardiol 2003;8(4):173-83.

(137) Zhang Y, Proenca R, Maffei M, Barone M, Leopold L, Friedman JM. Positional cloning of the mouse obese gene and its human homologue. Nature 1994 Dec 1;372(6505):425-32. 


\title{
CHAPTER 2
}

Transcriptome profiling reveals novel BMI- and sex-specific gene expression signatures for human cardiac hypertrophy

\author{
Mackenzie S. Newman ${ }^{1}$, Tina Nguyen ${ }^{1}$, Michael J. Watson ${ }^{2}$, Robert W. Hull ${ }^{3}$, and Han-Gang \\ $\mathrm{Yu}^{1}$
}

${ }^{1}$ Physiology and Pharmacology, West Virginia University, Morgantown, West Virginia;

${ }^{2}$ Department of Surgery, Duke University, Durham, North Carolina; and

${ }^{3}$ Department of Cardiology, West Virginia University, Morgantown, West Virginia

Original publication: Physiol Genomics. 2017 Jul 1;49(7):355-367. doi: 10.1152/physiolgenomics.00122.2016. Epub 2017 May 12. 


\section{$\underline{\text { Abstract }}$}

How obesity or sex may affect the gene expression profiles of human cardiac hypertrophy is unknown. We hypothesized that body-mass index (BMI) and sex can affect gene expression profiles of cardiac hypertrophy. Human heart tissues were grouped according to sex (male, female), BMI (lean $<25 \mathrm{~kg} / \mathrm{m}^{2}$, obese $>30 \mathrm{~kg} / \mathrm{m}^{2}$ ), or LVH and non-LVH nonfailed controls (NF). We identified 24 differentially expressed (DE) genes comparing female with male samples. In obese subgroup, there were $236 \mathrm{DE}$ genes comparing LVH with NF; in lean subgroup, there were seven DE genes comparing LVH with NF. In female subgroup, we identified 1,320 significant genes comparing LVH with NF; in male subgroup, there were 1,383 significant genes comparing LVH with NF. There were seven significant genes comparing obese LVH with lean NF; comparing male obese LVH with male lean NF samples we found 106 significant genes; comparing female obese LVH with male lean NF, we found no significant genes. Using absolute value of $\log _{2}$ fold-change $>2$ or extremely small $P$ value $\left(10^{-20}\right)$ as a criterion, we identified nine significant genes (HBA1, HBB, HIST1H2AC, GSTT1, MYL7, NPPA, NPPB, PDK4, PLA2G2A) in LVH, also found in published data set for ischemic and dilated cardiomyopathy in HF. We identified a potential gene expression signature that distinguishes between patients with high BMI or between men and women with cardiac hypertrophy. Expression of established biomarkers natriuretic peptide A (NPPA) and B (NPPB) were already significantly increased in hypertrophy compared with controls.

\section{Introduction}

In a recent global body mass index (BMI) mortality collaboration study from data collected from 3.9 million adults, the risk of dying before $70 \mathrm{yr}$ of age was $19 \%$ for men and $11 \%$ for women of normal weight (12). For obese men and women, that risk increased to $30 \%$ 
for men and $15 \%$ for women; thus, obesity caused an absolute increased risk of $11 \%$ for men and $4 \%$ for women (12). While this large-scale study confirmed the obesity-mortality causal link, it did not address the question, "why does obesity cause nearly three times more premature death in men than in women?"

While HF is frequently the final state of cardiovascular disease, cardiac hypertrophy is a major independent predictor of progressive heart disease and increased mortality (11). Cardiac hypertrophy is also one of the most common independent features in obesity, even in the absence of hypertension or diabetes mellitus $(1,3,20,34,45,48)$. Cardiomyocyte hypertrophy has been found to be the most common cause of sudden cardiac death in morbid obese patients (14). Advances in studies of signaling pathways in both physiological and pathological hypertrophies have led to a recent proposal that aims to treat cardiac hypertrophy as a new therapeutic target $(6$, 17).

Numerous studies from animal models, mostly rodents, have yielded at least 26 "key signaling molecules or processes" critical in hypertrophy and HF and thus are potential targets for new treatment of HF (38). However, clinical trials for new drugs have seldom been successful $(22,38)$. While finding new therapeutic targets in HF remains important, understanding genetic and molecular mechanisms of cardiac hypertrophy has recently gained increasing interest due to early-stage presentation during the time course of HF development (6, 17).

Studies in molecular signaling pathways have revealed different responses of several key signaling proteins to physiological and pathological hypertrophic stimuli (4). Notably, the expression levels of ANP and beta-myosin heavy chain ( $\beta$-MHC) protein increased only by 
receiving aortic banding compared with sham in an experimental mouse cardiac hypertrophy model (4).

Adding to the complexity of understanding the underlying mechanisms of cardiac hypertrophy is the potential contribution of obesity and sex. Obesity caused higher rates of cardiac hypertrophy, reduced quality of life, and shorter life expectancies compared with agematched lean individuals $(29,35)$. A recent study in 2.3 million adolescents from 1967 to 2010 found that overweight and obese individuals (measured by BMI) were strongly associated with increased cardiovascular mortality in adults (43). A high rate of sudden cardiac death in individuals with morbid obesity has been recognized for centuries (9). A high prevalence of sudden cardiac death has also been found in young obese people (5). For every $1 \mathrm{~kg} / \mathrm{m} 2$ increase in BMI, HF risk increases by $5 \%$ in men and $7 \%$ in women (23). In ventricular biopsy samples from obese patients, the number of adipocytes increases as the ejection fraction decreases (30). Comparatively, sex differences in cardiovascular physiology are well known, but sex-specific manifestations in human cardiovascular disease have only been recently recognized $(19,21,32)$. In the meantime, most mechanistic studies of cardiac hypertrophy have only been conducted in male animal models.

Methodologically, previous studies used Northern blotting, real-time PCR, and microarray cDNA for cardiac gene expression profiling under various hypertrophic conditions (26). Recent advances in next-generation sequencing (NGS) such as RNA-Seq (or transcriptome analysis) offer a unique opportunity to provide an overall snapshot of mRNA expression of all cardiac genes with high accuracy. Advantages of RNA-Seq over other sequencing methods such as cDNA microarrays are a combination of high-throughput sequencing, single-base resolution, 
low background noise, and a wide dynamic range for quantification of gene expression levels (46).

In this work, we used RNA-Seq to investigate the potential effects of BMI and sex on gene expression profiles of human heart with LVH.

\section{$\underline{\text { Methods }}$}

\section{Human Heart Samples}

Acquisition of human heart samples was approved by the Institutional Review Board (IRB) for the protection of human subjects at both West Virginia University and Duke University. Deidentified frozen human heart samples with pathological characterization were provided by the Department of Surgery at Duke University School of Medicine. Whole heart tissue was snap-frozen in liquid nitrogen immediately after collection from surgical procedures. $\mathrm{LV}$ were dissected and stored in $-80^{\circ} \mathrm{C}$ freezer until use. Patient characterizations of the samples are provided in Table 1. Average age of patients is $47.21 \pm 2.65 \mathrm{yr}$ (ranging from 19 to $67 \mathrm{yr}$ ). The control group, in which hearts had no hypertrophy or failure, is designated the nonfailed (NF) group, with a mean age of $46.00 \pm 3.69 \mathrm{yr}(\mathrm{n}=12)$. In the hypertrophy group, the mean age is $48.42 \pm 2.65 \mathrm{yr}(\mathrm{n}=12)$. $\mathrm{LVH}(\mathrm{n}=12,6$ women, 6 men $)$ samples were verified by echocardiograph (echo) measurement and interpreted by a cardiologist. NF hearts ( $\mathrm{n}=12,6$ female, 6 male) with echo data showing the absence of LVH were used as controls. Information of patients' age, sex, and BMI was obtained from pathological reports. BMI $<25$ was considered "lean" and BMI > 30 was considered "obese."

Ethics Approval and Consent to Participate

Use of human heart samples in this research was approved by the West Virginia University IRB and the Duke University IRB. 


\section{Total RNA Isolation, NGS (RNA-Seq), and Bioinformatics Analysis}

Total RNA was isolated using an RNA Fibrous Tissue Miniprep Kit (Qiagen). Quality of RNA was verified with an Agilent 2100 Bioanalyzer and RNA 6000 Pico Kit. Only samples that had RNA integrity number $>7.0$ were submitted for sequencing. The samples were then subjected to polyA enrichment followed by fragmentation, first- and second-strand synthesis, adenylation of 3'-ends, adapter ligation, DNA fragment enrichment, and real-time PCR quantification.

Sequencing was performed using NextSeq 500 (Illumina). Bcl sequencing data were converted to FastQ using onboard instrument software. Reads were mapped to human reference genome (hg38) using Spliced Transcripts Alignment to a Reference (STAR) (13).

Differential expression analysis was performed with NOISeq (v.2.14.1) (41) using RStudio version 0.99.879 (37). NOISeq is a newly developed tool for differential expression analysis. Compared with the commonly used DeSeq (2), NOISeq offered a set of tools for better quality control to avoid false positive discoveries (41). Gene annotation information was obtained from the Ensembl Biomart database, release 85 (50). Gene expression levels are indicated by FPKM (fragments per kilobase of transcript per million mapped reads) (42). FPKM was then normalized for batch effect using the ARSyNseq module included with the NOISeq package. Data were analyzed by the noiseqbio method under default conditions. The CPM filtering method was used for differential analyses where at least one group contained five or fewer replicates; otherwise, the Wilcoxon test was used for filtering.

The HF data set was extracted from a recent publication by Liu et al. (28). We used this HF data set against our LVH data set to explore the potential significance of newly identified 
differentially expressed (DE) genes as "a gene expression signature" for prediction during the course of HF.

\section{Gene Ontology Enrichment and Pathway Analysis}

Gene Ontology (GO) enrichment analysis was carried out by using a comprehensive gene set enrichment tool, Enrichr $(10,25)$. This web-based tool contains 180,184 annotated gene sets from 102 gene set libraries (25). It calculates four parameters: $\mathrm{P}$ value, $\mathrm{q}$ value or adjusted $\mathrm{P}$ value, $\mathrm{z}$ score, and a combinational score; higher indicates larger significance. Interaction Network Analysis of Differentially Expressed Genes (GeneMANIA) (47) was used for coexpression and association of significant DE genes.

\section{Immunoblotting}

Tissues sections were submerged in minimal lysis buffer [fresh protease and phosphatase inhibitors (Sigma), $20 \mathrm{mM}$ Tris, $150 \mathrm{mM} \mathrm{NaCl}, 10 \mathrm{mM}$ EGTA, and $10 \mathrm{mM}$ EDTA at $\mathrm{pH}$ 7.4] on ice and homogenized briefly at high speed. Samples were then centrifuged for 15 min increments at $10,000 \mathrm{~g}$ to pellet debris. Supernatants were placed into new tubes, and protein concentration was recorded with Bradford's method on an Eppendorf Biophotometer.

For Western blotting procedures, protein concentrations were normalized between samples to 10-30 $\mu \mathrm{g}$ and mixed with Non-Reducing Lane Marker (Thermo Scientific) with 5\% $\beta$-mercaptoethanol. After being heated in a water bath to $95^{\circ} \mathrm{C}$ for $5 \mathrm{~min}$, samples were cooled to $4^{\circ} \mathrm{C}$ and then loaded into a $4-12 \%$ bis-Tris gel (Invitrogen). Electrophoresis was carried out at 80 $\mathrm{V}$ for $30 \mathrm{~min}$ and then $140 \mathrm{~V}$ for the remainder.

Proteins were transferred to 0.45 micron nitrocellulose membranes (Thermo Fisher) at 30 V for $1 \mathrm{~h}$. Blots were blocked with 3\% BSA-V in Tris-buffered saline plus Tween 20 (TBS-T) for $1 \mathrm{~h}$ before primary antibody (1:1,000 dilution; Cell Signaling) was added on a shaker at $4{ }^{\circ} \mathrm{C}$ 
overnight. Primary antibody solution was replaced with fresh $3 \%$ BSA-V in TBS-T containing secondary antibodies at 1:10,000 dilution for $1 \mathrm{~h}$ at room temperature on a shaker. After five washes with TBS-T, blots were developed with a standard ECL kit (Life Technologies) or ECL Prime (Amersham) on X-ray film or using a G:BOX digital imaging system (Syngene).

\section{Statistics}

For Western blots, data are shown as means $\pm \mathrm{SE}$; Student's t-test was used for statistical analysis with $\mathrm{P}<0.05$ being considered as statistically significant, marked with the symbol *. For gene expression, gene size adjusted $\mathrm{P}$ value (false discovery rate) $<0.05$ was used ( $\mathrm{p}_{\text {adj }}<$ $0.05)$ to identify significant genes.

We used DE gene data (FPKM) and statistics in ischemic cardiomyopathy (ISCH) and dilated cardiomyopathy from a recent publication (28). It is possible to obtain the test statistic based on a single pair of objects (one disease, one nondisease control) due to the availability of multiple reads per subject in RNA-Seq methodology.

\section{$\underline{\text { Results }}$}

\section{Human Heart Sample Characteristics}

Table 1 summarizes the characteristics of human hearts used in the study. The average age of the patients is $47.21 \pm 2.65 \mathrm{yr}$ (ranging from 19 to $67 \mathrm{yr}, \mathrm{n}=24$ ), $48.42 \pm 3.93 \mathrm{yr}$ for the LVH group $(\mathrm{n}=12)$, and $46.00 \pm 3.69 \mathrm{yr}$ for the NF group $(\mathrm{n}=12)$. LVH $(\mathrm{n}=12,6$ women, 6 men) samples were diagnosed by echo measurement and interpreted by a cardiologist. Nonfailed without LVH (NF) hearts $(\mathrm{n}=12,6$ female, 6 male) confirmed with echo were used as controls for LVH. Information on patients' age, sex, and BMI was obtained from pathological reports. 


\section{Sex-Specific LVH Gene Expression Profiles}

Comparing LVH with NF samples ( $\mathrm{n}=9$ for each group), we found only one significant gene, NPPA (Fig. 1A). NPPA was increased by 11.6-fold in LVH (p_adj=0.004). This result contradicts previous gene expression reports on human cardiac hypertrophy, which have identified at least 76 significant genes by using conventional techniques such as PCR, Southern blotting, and Northern blotting (26). We wondered whether sex might play a role in this unexpected result.

When we compared gene expression profiles of women vs. men, independent of LVH and BMI, we found 24 significantly DE genes (Fig. 1B, Supplemental Table S1 in Appendix A). A heat map generated from these $24 \mathrm{DE}$ genes shows different patterns of cardiac gene expression between women and men (Fig. 1C). Furthermore, female and male samples can be clearly separated using the 24 DE genes, illustrated by principal component analysis (Fig. 1D).

Informed by the sex influence on gene expression, we next examined the effect of LVH on gene expression profiles in female and male groups separately. When comparing female LVH $(n=4)$ with female NF $(n=3)$ samples, we found 1,320 DE genes (Fig. 1E, Supplemental Table S2 in Appendix A). In the male LVH over NF comparison, we identified 1,383 DE genes (Fig. 1F, Supplemental Table S3 in Appendix A).

\section{BMI- and Sex-Specific LVH Gene Expression Profiles}

To investigate potential effects of obesity on cardiac gene expression, we compared obese (BMI30) with lean (BMI25) groups ( $\mathrm{n}=9$ for each group) and found no significant DE genes. However, in the obese group, we found 236 significant genes in LVH compared with NF samples ( $\mathrm{n}=4$ for each group) (Fig. 2A, Supplemental Table S4 in Appendix A). In the lean 
group, we found seven significant genes in LVH compared with NF samples $(\mathrm{n}=3$ for each group) (Fig. 2B, Supplemental Table S5 in Appendix A).

Next, when we compared obese LVH with lean NF samples ( $\mathrm{n}=4$ for each group), we found seven significant genes (Fig. 2C, also see Supplemental Table S6 in Appendix A). Considering the factor of sex, we compared male obese LVH with male lean NF samples $(n=3$ for each group) and found 106 significant genes (Fig. 2D, Supplemental Table S7 in Appendix A). However, comparing female obese LVH with female lean NF samples ( $\mathrm{n}=3$ for each group)

yielded no significant DE genes. One possibility is that LVH and obesity can independently alter the expression levels of DE genes but in opposite directions.

\section{Distribution of Sex- and BMI-Specific Significant Cardiac Gene Expression}

Figure 3 summarizes the distribution of DE genes under different conditions. We identified a total of 23,521 genes in human hearts. No significant DE genes were found in obesity over lean samples and in female lean NF compared with female obese LVH. One gene was found to be upregulated in LVH compared with NF. Seven DE genes were identified in lean LVH (six upregulated and one downregulated). Seven DE genes were found in lean NF vs. obese LVH (five upregulated and two downregulated). Among the $24 \mathrm{DE}$ genes found in women vs. men, five were upregulated, and 19 downregulated. Among $106 \mathrm{DE}$ genes found in male lean NF compared with male obese LVH, 61 were upregulated, and 45 were downregulated. Among the $236 \mathrm{DE}$ genes found in the obese group, 38 were upregulated, and 198 were downregulated. Among 1,320 DE genes found in female LVH, 330 were upregulated and 990 downregulated. Among 1,383 DE genes found in male LVH, 137 were upregulated, and 1,246 were downregulated. 


\section{Sex- and BMI-Specific Significant Gene Expression Signature}

To identify sex-specific LVH DE genes, we compared male LVH and female LVH DE genes. Figure 4A shows a scatterplot of DE genes shared by LVH-M and LVH-F. Quadrant I displays 80 genes that are upregulated in female LVH but downregulated in male LVH; quadrant II displays six genes that are upregulated in both female and male LVH; quadrant III displays 141 genes that are downregulated in both female and male LVH; quadrant IV displays 15 genes that are downregulated in female LVH but upregulated in male LVH. Figure 4B shows a heat map generated from the $80 \mathrm{DE}$ genes from quadrant I of Fig. 4A, demonstrating the sex-specific modulation of gene expression in LVH.

Using $\operatorname{abs}(\log 2 \mathrm{FC})>1$ as a criterion, we identified 213 sex- and BMI-specific significant genes (Supplemental Table S8 in Appendix A). Using $\operatorname{abs}(\log 2 \mathrm{FC})>2$ as a criterion, we identified 27 sex- and BMI-specific significant genes (Supplemental Table S9 in Appendix A).

Significance of LVH DE Genes: Implication in Ischemic and/or Dilated Cardiomyopathy in

\section{HF Patients}

To explore the potential significance of these findings, we compared LVH DE genes with those recently identified in ischemic cardiomyopathy (ISCH) and dilated cardiomyopathy (DCM) (28). Supplemental Figure S1A shows a scatterplot of DE genes found in obese LVH and in the previously published ISCH data set (28). There are $37 \mathrm{LVH}$ DE genes found in ISCH (Supplemental Table S10 in Appendix A). Quadrant I displays 16 genes that are upregulated in obese LVH but downregulated in ISCH; quadrant II displays four gene that is upregulated in both obese LVH and ISCH; quadrant III displays 14 genes that are downregulated in both obese LVH and ISCH; quadrant IV displays three genes that are downregulated in obese LVH and upregulated in ISCH. Supplemental Figure S1B shows a scatterplot of DE genes found in obese 
LVH and in the published DCM data set (28). There are $58 \mathrm{LVH}$ DE genes found in DCM (see Supplemental Table S11 in Appendix A). Quadrant I displays 27 genes that are upregulated in obese LVH but downregulated in DCM; quadrant II displays three genes that are upregulated in both obese LVH and DCM; quadrant III displays 17 genes that are downregulated in both obese LVH and DCM; quadrant IV displays 11 genes that are downregulated in obese LVH and upregulated in DCM. Supplemental Figure S1C shows a scatterplot of DE genes found in obese $\mathrm{LVH}$ and in the published ISCH/DCM data set (28). There are $31 \mathrm{LVH}$ DE genes found in ISCH/DCM data set (see Supplemental Table S12 in Appendix A). Quadrant I displays 16 genes that are upregulated in obese LVH but downregulated in ISCH/DCM; quadrant II displays three genes that are upregulated in both obese LVH and ISCH/DCM; quadrant III displays eight genes that are downregulated in both obese LVH and ISCH/DCM; quadrant IV displays four genes that are downregulated in obese LVH and upregulated in ISCH/DCM.

Supplemental Fig. S2A shows a scatterplot of DE genes found in female LVH and in the published ISCH data set (28). There are 111 female LVH DE genes found in ISCH (Supplemental Table S13 in Appendix A). Quadrant I displays 41 genes that are upregulated in obese LVH but downregulated in ISCH; quadrant II displays 14 genes that are upregulated in both obese LVH and ISCH; quadrant III displays 46 genes that are downregulated in both obese LVH and ISCH; quadrant IV displays 10 genes that are downregulated in obese LVH and upregulated in ISCH. Supplemental Fig. S2B shows a scatterplot of DE genes found in female LVH and in the published DCM data set (28). There are 181 female LVH DE genes found in DCM (Supplemental Table S14 in Appendix A). Quadrant I displays 63 genes that are upregulated in obese LVH but downregulated in DCM; quadrant II displays 20 genes that are upregulated in both obese LVH and DCM; quadrant III displays 76 genes that are downregulated 
in both obese LVH and DCM; quadrant IV displays 22 genes that are downregulated in obese LVH and upregulated in DCM.

Supplemental Figure S2C shows a scatterplot of DE genes found in female LVH and in the published ISCH/DCM data set (28). There are $98 \mathrm{LVH}$ DE genes found in ISCH/DCM (Supplemental Table S15 in Appendix A). Quadrant I displays 56 genes that are upregulated in obese $\mathrm{LVH}$ but downregulated in ISCH/DCM; quadrant II displays six genes that are upregulated in both obese LVH and ISCH/DCM; quadrant III displays 26 genes that are downregulated in both obese LVH and ISCH/DCM; quadrant IV displays 10 genes that are downregulated in obese LVH and upregulated in ISCH/DCM. Supplemental Figure S2D shows a scatterplot of DE genes found in male LVH and in published ISCH data set (28). There are 121 LVH DE genes found in ISCH (Supplemental Table S16 in Appendix A). Quadrant I displays 46 genes that are upregulated in obese LVH but downregulated in ISCH; quadrant II displays 16 genes that are upregulated in both obese LVH and ISCH; quadrant III displays 44 genes that are downregulated in both obese $\mathrm{LVH}$ and ISCH; quadrant IV displays 15 genes that are downregulated in obese LVH and upregulated in ISCH. Supplemental Figure S2E shows a scatterplot of DE genes found in male LVH and in the published DCM data set (28). There are 196 LVH DE genes found in DCM (Supplemental Table S17 in Appendix A). Quadrant I displays 98 genes that are upregulated in obese LVH but downregulated in DCM; quadrant II displays 17 genes that are upregulated in both obese LVH and DCM; quadrant III displays 46 genes that are downregulated in both obese LVH and DCM; quadrant IV displays 35 genes that are downregulated in obese LVH and upregulated in DCM. Supplemental Figure S2F shows a scatterplot of DE genes found in male LVH and in the published ISCH/DCM data set (28). There are $80 \mathrm{LVH}$ DE genes found in ISCH/DCM (see Supplemental Table S18 in Appendix A). 
Quadrant I displays 33 genes that are upregulated in obese LVH but downregulated in ISCH/DCM; quadrant II displays 15 genes that are upregulated in both obese LVH and ISCH/DCM; quadrant III displays 21 genes that are downregulated in both obese LVH and ISCH/DCM; quadrant IV displays 11 genes that are downregulated in obese LVH and upregulated in ISCH/DCM.

\section{Gene Expression Signatures}

To explore potential implications of LVH DE genes for future development of HF, we selected $10 \mathrm{DE}$ genes according to three criteria: $\operatorname{abs}(\log 2 \mathrm{FC})>2$, extremely small $\mathrm{p} \_$adj value (10-20), and whether they have been found in the published ISCH and DCM HF data set (28) (Table 2). Figure 5A shows the heat map of male LVH (LVH-M) and female LVH (LVH-F) compared with their respective NF controls. In male LVH, among expression levels of $10 \mathrm{DE}$ genes, four (HBB, PLA2G2A, HBA1, PLXDC2) are changed by less than one standard deviation, five (NPPA, NPPB, PDK4, HIST1H2AC, GSTT1) are increased, and one (MYL7) is decreased. In female LVH, among expression levels of 10 DE genes, eight (HBB, NPPA, NPPB, PDK4, PLA2G2A, HBA1, HIST1H2AC, PLXDC2) are increased, and two (MYL7, GSTT1) are decreased. Figure 5B shows the heat map of ISCH and DCM compared with NF using the published data (28). Among the expression levels of these 10 genes, seven (HBB, NPPA, NPPB, MYL7, PDK4, HIST1H2AC, PLXDC2) are increased in ISCH, seven (HBA1, HBB, NPPA, NPPB, MYL7, PDK4, HIST1H2AC) are increased in DCM, and two (PLA2G2A, GSTT1) are decreased, compared with controls. 


\section{Validation of 10 DE Genes}

\section{Expression of NPPA (ANP) and NPPB (BNP) in LVH, ISCH, and DCM.}

ANP and brain-type natriuretic peptide (BNP) are biomarkers for HF with left ventricular dysfunction $(8,16)$. We found that the transcripts of NPPA (gene that encodes ANP) were increased in LVH by 24-fold in men (LVH_M, 697 FPKM; NF_M, 29 FPKM; p_adj=0.01825), 7.4-fold in women (LVH_F, 148 FPKM; NF_F, 20 FPKM; p_adj=0.0324), 13.3-fold in BMI25 (LVH_BMI25, 601 FPKM; NF_BMI25, 45 FPKM; p_adj=7×10-15), and 16.4-fold in the BMI30 (LVH_BMI30, 327 FPKM, NF_BMI30, 23 FPKM; p_adj=1.4×10-14) subgroup, respectively (Fig. 6A, top). Its expression was also increased by 19-fold in ISCH (ISCH 234, 876 FPKM; NF-ISCH, 46 FPKM; p_adj=0) and 5.4-fold in DCM (DCM 333, 251 FPKM; NFDCM, 46 FPKM; p_adj=4.4×10-7) (Fig. 6A, top) (28). Immunoblotting experiments confirmed that ANP protein expression was increased by $90 \%$ in LVH compared with NF after being normalized to $\alpha$-actin (LVH: $1.10 \pm 0.15, \mathrm{n}=8$; NF: $0.58 \pm 0.05, \mathrm{n}=5$ ) (Fig. 6A, middle and bottom).

Similarly, we found that the transcripts of NPPB (gene that encodes BNP) were increased in LVH by 7.5-fold in male (LVH_M, 121 FPKM; NF_M, 16 FPKM; $p \_$adj=0.0136), fivefold in female (LVH_F, 130 FPKM; NF_F, 28 FPKM; p_adj=0.0146), and 3.4-fold in BMI30 (LVH_BMI30, 121 FPKM; NF_BMI30, 36 FPKM; p_adj=0.04) subgroups, respectively (Fig. $6 \mathrm{~B}$, top). Its expression was also increased by 11 -fold in ISCH (ISCH 234, 1,772 FPKM; NFISCH, 159 FPKM; p_adj=1.79×10-13) and fourfold in DCM (DCM 333, 617 FPKM; NFDCM, 159 FPKM; p_adj=8.96×10-7) (28) (Fig. 6B, top). BNP protein expression was increased by $151 \%$ in LVH compared with NF control (NF) after being normalized to $\alpha$-actin (LVH: $1.31 \pm 0.52, \mathrm{n}=9$; NF: $0.52 \pm 0.11, \mathrm{n}=9$ ) (Fig. 6B, middle and bottom). 


\section{Expression of HBA1 and HBB in LVH, ISCH, and DCM.}

Figure 7 shows the protein expression of HBA1 (Fig. 7A) and HBB (Fig. 7D). Both were increased in female LVH over NF (HBA1: LVH_F $=0.46 \pm 0.029, \mathrm{NF}_{-} \mathrm{F}=0.26 \pm 0.04, \mathrm{n}=3, \mathrm{P}$ $<0.05$; HBB: LVH_F $\left.=0.36 \pm 0.42, \mathrm{NF}_{-} \mathrm{F}=0.23 \pm 0.02, \mathrm{n}=3, \mathrm{P}<0.05\right)$ but were not changed in male LVH compared with NF (HBA1: $L V H \_M=0.17 \pm 0.07, \mathrm{NF} \_\mathrm{M}=0.17 \pm 0.06, \mathrm{n}=6, \mathrm{P}$ $>0.05 ;$ HBB: LVH_M $\left.=0.98 \pm 0.14, \mathrm{NF}_{-} \mathrm{M}=0.94 \pm 0.13, \mathrm{n}=6, \mathrm{P}>0.05\right)$ (Fig. 7, $\mathrm{B}$ and E). These female LVH-specific increases in protein expression of HBA1 and HBB are consistent with the corresponding increase in transcripts in female LVH ( 11-fold increase of HBA1, Fig. 7C; 44-fold increase of HBB, Fig. 7F). HBA1 transcripts were reported to increase by 3.8-fold in ischemic and 6.9-fold in dilated cardiomyopathy, respectively (Fig. 7C) (28); similarly, HBB transcripts were reported to increase by sixfold in ISCH and 11.6-fold in DCM, respectively (Fig. 7F) (28).

\section{Expression of GSTT1 and PLA2G2A in LVH, ISCH, and DCM.}

Figure 8 shows the protein expression of GSTT1 and PLA2G2A (Fig. 8A, men; Fig. 8B, women). GSTT1 levels were increased by 10 -fold in male LVH (LVH_M $=0.70 \pm 0.22$, NF_M $=0.07 \pm 0.06, \mathrm{n}=3, \mathrm{P}<0.05)$ but no significant changes were detected in female LVH $\left(\mathrm{LVH} \_\mathrm{F}\right.$ $=0.94 \pm 0.57$, NF_F $\left._{-}=0.97 \pm 0.61, \mathrm{n}=3, \mathrm{P}>0.05\right)($ Fig. 8C). PLA2G2A levels were increased in female LVH by fivefold $\left(\mathrm{LVH}_{-} \mathrm{F}=0.25 \pm 0.20, \mathrm{NF}_{-} \mathrm{F}=0.05 \pm 0.01, \mathrm{n}=3, \mathrm{P}<0.05\right)$ and in male LVH by sevenfold (LVH_M $=0.07 \pm 0.02, N F \_M=0.01 \pm 0.005, \mathrm{n}=3, \mathrm{P}<0.05$ ) (Fig. 8D). PLA2G2A levels are noticeably lower in male (Fig. 10A, middle lane) than in female (Fig. 8B, middle lane) LV.

Changes in protein expression levels of GSTT1 are consistent with those in transcripts, a 13.7fold increase in male LVH for GSTT1 (Fig. 8E). However, for PLA2G2A, there is a higher 
increase in transcripts in female LVH (6.4-fold) than in male LVH (1.8-fold) (Fig. 8F). In ISCH and DCM, GSTT1 transcripts were reported to increase by 40 and $260 \%$, respectively (28), whereas PLA2G2A transcripts were increased by 4.2-fold and 5.6-fold, respectively (28).

\section{Expression of PDK4 and MYL7 in LVH, ISCH, and DCM.}

Figure 9 shows the protein expression of PDK4 and MYL7 (Fig. 9A, women; Fig. 9B, men). PDK4 levels were increased by 2.2 -fold in male LVH over NF ( $L V H \_M=1.335 \pm 0.307$, $\left.\mathrm{NF} \_\mathrm{M}=0.514 \pm 0.107, \mathrm{n}=3, \mathrm{P}<0.05\right)$ but not significantly altered in female LVH compared with NF (LVH_M $\left.=1.282 \pm 0.3594, N F \_M=0.5801 \pm 0.08030, n=3, P>0.05\right)$ (Fig. 9C). On the other hand, MYL7 levels were decreased by $27 \%$ in female LVH over NF (LVH_F $=0.8958$ $\left.\pm 0.1007, \mathrm{NF}_{-} \mathrm{F}=1.225 \pm 0.04868, \mathrm{n}=3, \mathrm{P}<0.05\right)$ but not significantly changed in male LVH compared with NF (LVH_M $=1.687 \pm 0.2885, \mathrm{NF}_{-} \mathrm{M}=1.646 \pm 0.2225, \mathrm{n}=3, \mathrm{P}>0.05$ ) (Fig. 9D).

PDK4 transcript levels were increased in male LVH by 2.3 -fold and in female LVH by 1.4-fold (Fig. 9E). PDK4 transcripts levels were reported to increase (by 2.2-fold) only in ischemic HF (28) (Fig. 9E).

MYL7 transcript levels were increased in male LVH by $74 \%$ and in female LVH by $60 \%$ (Fig. 9F). MYL7 transcripts levels were reported to increase by 3.2-fold in both ISCH and DCM (28) (Fig. 9F).

\section{Expression of HIST1H2AC in LVH, ISCH, and DCM.}

Figure 10 shows the protein expression of HISTH2AC (Fig. 10A). The expression levels were increased by $154 \%$ in female LVH over NF $\left(\mathrm{LVH}_{-} \mathrm{F}=1.55 \pm 0.29, \mathrm{NF} \_\mathrm{F}=0.61 \pm 0.09, \mathrm{n}\right.$ $=3, \mathrm{P}<0.05)$ and by $254 \%$ in male $\mathrm{LVH}$ over NF $\left(\mathrm{LVH} \_\mathrm{M}=2.49 \pm 0.41, \mathrm{NF} \_\mathrm{M}=0.98 \pm 0.37\right.$, $\mathrm{n}=6, \mathrm{P}<0.05$ ), respectively (Fig. 10B), consistently with the increased transcripts in female 
LVH ( $\sim$-fold) and male-LVH ( 2-fold) (Fig. 10C). HIST1H2AC transcripts were reported to increase by 2.9-fold in ISCH and 5.4-fold in DCM, respectively (Fig. 10C) (28).

Finally, protein expression and re-examination of gene expression for PLXDC2 showed insignificant changes in LVH over NF. Thus, it was removed from DE genes for further data analysis.

\section{GO Enrichment and Pathway Analysis}

We performed GO enrichment analysis for nine DE genes (NPPA, NPPB, HBB, HBA1, PDK4, MYL7, HIST1H2AC, GSTT1, PLA2G2A). In "biological process," the top two most significant processes are receptor guanylyl cyclase signaling pathway $(\mathrm{q}$ value $=0.0004)$ (involving NPPA, NPPB) and oxygen transport $(\mathrm{q}$ value $=0.0005)($ involving HBB, HBA1) (Supplemental Table 19). In "cellular component," the top two most significant components are endocytic vesicle lumen and hemoglobin complex (q value $=0.0004)$, both involving HBB and HBA1 (Supplemental Table 20). In "molecular function," HBB and HBA1 are involved in the top five most significant functions, including oxygen transporter activity (q value $=0.0003$ ), oxygen binding $(\mathrm{q}$ value $=0.0008)$, peroxidase activity $(\mathrm{q}$ value $=0.0008)$, oxidoreductase activity $(\mathrm{q}$ value $=0.0008)$, and antioxidant activity $(\mathrm{q}$ value $=0.002)($ Supplemental Table 21$)$.

\section{DE Gene Interaction Network Analysis}

To further explore potential interactions among nine DE genes, we performed gene interaction network analysis using GeneMANIA (47). Network interaction in terms of predicted

physical interaction and coexpression was analyzed. Among our nine DE genes, we found three clusters: NPPA, NPPB, HBB, HBA1, HIST1H2AC, and MYL7 form the largest cluster; PDK4 and PLA2G2A are coexpressed together; and GSTT1 is not associated with other eight DE genes (Supplemental Fig. S3). Within the largest cluster, NPPA, NPPB, and MYL7 are coexpressed. 
HBB is coexpressed and associated with HBA1 and HIST1H2AC. NPPB also is associated with HBB and HIST1H2AC.

\section{$\underline{\text { Discussion }}$}

In the present work, we used transcriptome sequencing to explore the potential effects of BMI and sex on gene expression profiles of human hearts with and without LVH. We found both BMI and sex can unmask a large set of genes whose expression levels are significantly affected by LVH.

We explored the implications of BMI- and sex-specific LVH DE genes in HF. Previously, sex-specific differences in gene expression profiles of HF were investigated using cDNA microarray. In new-onset HF, 35 upregulated and 16 downregulated transcripts were identified in men vs. women (21). At end-stage DCM, there were 55 and 31 differentially regulated genes in female and male, respectively (19). Nineteen DE genes were shared by both males and females (19). Most recently, RNA-Seq was used on six patients to identify 983 DE genes in ISCH vs. NF [union of three pairs, see Table 1 in (28)], 1,109 DE genes in DCM vs. NF [union of six pairs, Table 1 in (28)], and 825 DE genes [union of two pairs, Table 1 in (28)] in which 476 genes were overexpressed in ISCH and 349 genes were overexpressed in DCM. Sex and obesity status were not specified in the article. We performed correlation studies of our LVH data and the published data in both types of HF, ISCH and DCM (28). Our scatterplots display numerous downregulated (quadrant II) and upregulated (quadrant III) DE genes in obese LVH, women, and male LVH, which are also found in ISCH and DCM.

We selected nine DE genes that were significantly changed in multiple LVH vs. NF

analyses within our data set and were shared by LVH, ISCH, and DCM, to provide a gene expression signature. There were five overexpressed genes (HBB, NPPA, NPPB, PDK4, 
HIST1H2AC); one downregulated gene, GSTT1; and three regulated in opposite directions (MYL7 decreased in LVH, increased in cardiomyopathy; PLA2G2A increased in female LVH, but decreased in cardiomyopathy; HBA1 increased in female LVH and DCM, but decreased in ISCH). For male LVH, the expression levels of three genes (HBB, PLA2G2A, HBA1) were not changed by more than one standard deviation.

\section{Roles of Nine DE Genes in Human Cardiac Hypertrophy and Failure}

Using GeneChip and TaqMan PCR, the first gene expression fingerprint of HF revealed 103 genes in 10 functional groups between NF and HF samples (40). ANP and BNP were two upregulated genes in HF. Using quantitative PCR, we found NPPA transcripts to be increased in dilated HF patients of both sexes (7). "Expression profiling-based biomarkers" was proposed in a transcriptome analysis of endomyocardial biopsies from $48 \mathrm{HF}$ patients, which used $96 \mathrm{DE}$ genes to predict cardiomyopathy etiology accurately (ischemic vs. nonischemic) (24). A clinical study in 2008 on 3,580 patients found that women had new-onset acute HF more frequently and less DCM compared with men (33).

In both male and female samples from end-stage DCM, microarrays identified upregulated NPPA and downregulated PLA2G2A expression, respectively (19). NPPA and NPPB overexpression was also detected, by microarrays, in new-onset HF patients (both male and female) with DCM (21). Most recently, RNA-Seq was used to show overexpressed NPPA and NPPB as well as decreased expression of PLA2G2A in both DCM and ischemic cardiomyopathy (Fig. 7) using a much smaller sample size (one disease vs. one control) (28).

ANP is mainly produced in the atria, whereas BNP is primarily produced in the ventricles, in response to myocardial stress. Both peptides have been reported as valuable diagnostic markers in HF (16). NH2-terminal pro-BNP (NT-proBNP) and midregional pro-ANP 
(MR-proANP) are inactive precursors of BNP and ANP, respectively, with long half-lives. These peptides are both current standards of care for patients with acute or chronic HF $(31,49)$. Increased NT-proBNP has also been found to be closely associated with HF patients with cachexia (BMI <20) (18). NT-proBNP has recently been confirmed as a reliable risk biomarker for fatal cardiovascular events in Type 2 diabetes patients (44).

Increased transcripts and protein expression of ANP and BNP were readily detected in LVH compared with NF in our study (Fig. 6). However, we found much higher NPPA expression than NPPB in male LVH (NPPA, 697 FPKM; NPPB, 121 FPKM) but not in female LVH (NPPA, 148 FPKM; NPPB, 130 FPKM). NPPA expression was increased more in male (24-fold) than in female (7.4-fold) LVH. In addition, the strongest association of LVH with increased NPPA expression was found between lean and obese groups, and such an association was not found for NPPA. In previously published HF gene expression data (28) we found both NPPA and NPPB expression levels are higher in ISCH (NPPA, 876 FPKM; NPPB, 1,772) than in DCM (NPPA, 251 FPKM; NPPB, 617).

In a 2014 report that examined mRNA expression of LV with sudden cardiac death, the expression of HBA1 and HBB mRNA was found to be increased, while PDK4 levels were downregulated (39). PDK4 is one of the key regulators of metabolism (36). Its expression levels in human hearts are decreased during development and tend to be reduced in HF (36). We found the gene expression levels for HBA1 and HBB were upregulated only in female LVH, while PDK4 levels were increased only in male LVH.

MYL7, one of the myosin light chains that plays a key role in cardiogenesis (15), is increased in human hypertrophic cardiomyopathy (26). We found its gene expression levels were increased only in female LVH. 
The roles of GSTT1, PLA2G2A, and HIST1H2AC in human cardiac hypertrophy or failure are unknown. We found that GSTT1 gene expression levels were upregulated in male, but not in female, LVH. Gene expression levels for PLA2G2A and HIST1H2AC were upregulated in LVH of both sexes with a greater increase in female LVH.

\section{Limitations of the Study}

\section{Small sample size}

We used a total of 18 samples. For subgroups such as obese female LVH, there were three samples for each group. Although it fulfills the minimal requirement for statistical analysis, it may lead to underestimates of the amount of significant genes in each subgroup, as well as large variations in expression levels. However, small samples sizes have demonstrated impact of the identified significant genes, particularly with very small p_adj values, such as PDK4 (p_adj < 10-6). In a recent study, the gene signatures identified by RNA-Seq from only six HF patients were used to accurately classify a large set of 313 patients with microarray data (28). It illustrated the use of RNA-Seq as an effective approach to discover novel gene expression signature based on an extremely small sample size.

Sample variations.

Intrinsic individual differences exist at genetic levels. Clinical diagnoses of LVH were based on echo. As shown in patient sample characteristics (Table 1), there are some samples with borderline LVH that were deemed as NF in pathological reports.

Despite these limitations our data provide an argument for using BMI- and sex-specific gene expression signatures containing multiple significant genes, rather than a single gene, to offer a more accurate prediction for future progression to HF. Conclusions 
We identified nine differentially expressed genes in LVH that are BMI- and sexsensitive. Validation of these nine genes (forming a "gene expression signature") in a large cardiac hypertrophy population may have the potential to help in the early diagnosis of HF, which occurs at a significantly high rate in the obese population.

\section{$\underline{\text { Figures }}$}

Figure 1: Sex-specific left ventricle hypertrophy (LVH) differential expression profiles (volcano)
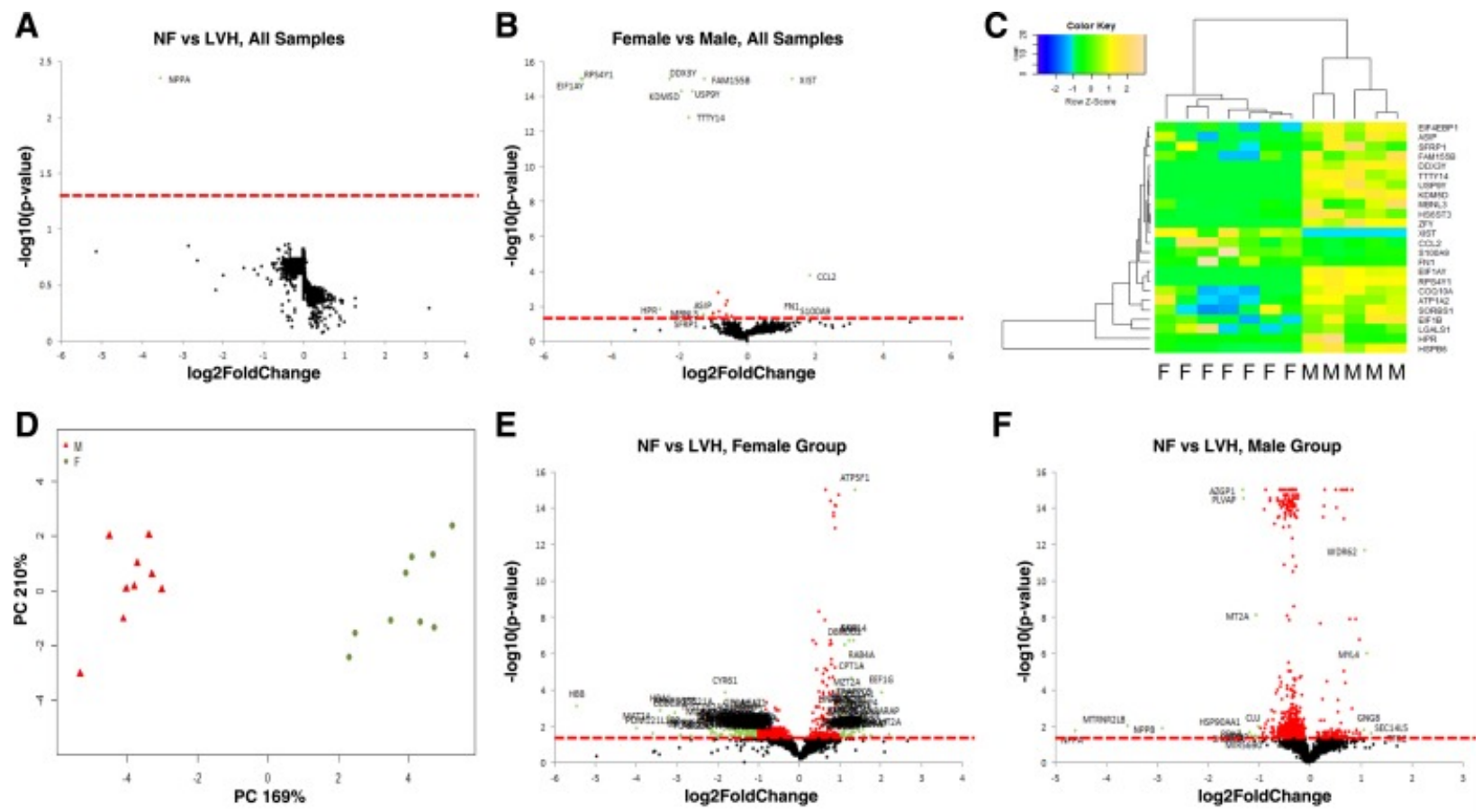

Figure 1. A: NF vs. LVH ( $\mathrm{n}=9$ in each group). B: female $(\mathrm{F})$ vs. male $(\mathrm{M})$ samples $(\mathrm{n}=9$ in each group). C: heat map generated from 24 differentially expressed genes comparing females with males (B) using unsupervised k-means clustering. D: principal component analysis plot of female and male samples from these 24 differentially expressed genes (B). E: differential expression profile of female NF $(n=4)$ vs. female LVH $(n=3)$. F: differential expression profile of male NF vs. male LVH ( $n=3$ in each group). Red dashed lines mark $p \_a d j=0.05$. Dark dots indicate genes without significant changes in expression levels comparing 2 individual groups 
(p_adj $>0.05)$. Red dots: significant genes $\left(p_{-}\right.$adj $\left.<0.05\right)$ with abs $(\log 2 \mathrm{FC}<1)$; green dots with labels: significant genes with $\mathrm{p} \__{-}$adj $<0.05$ and $\operatorname{abs}(\log 2 \mathrm{FC})>1$. NF, nonfailed controls. 
Figure 2: Body mass index (BMI)- and sex-specific LVH differential expression profiles (volcano)
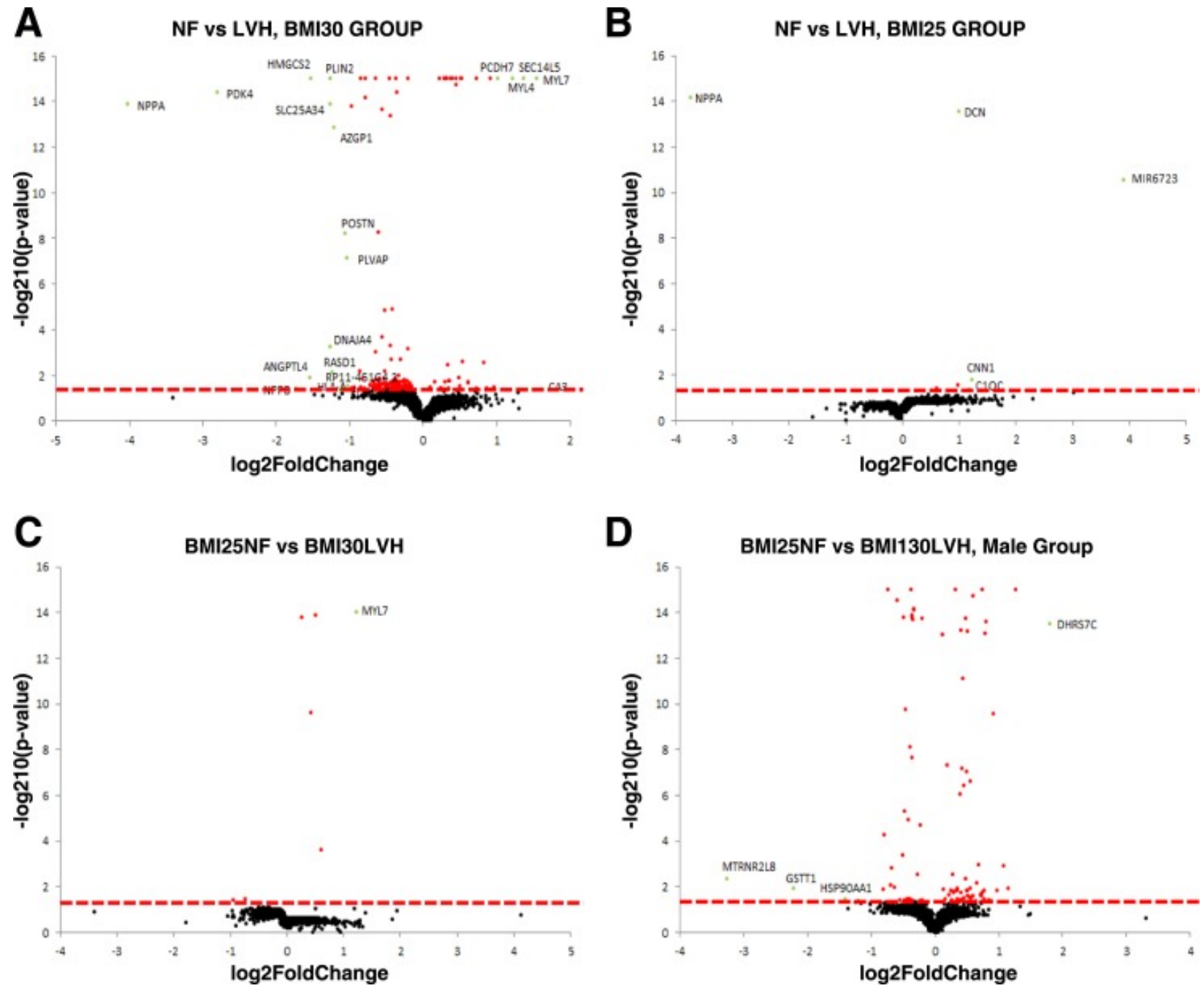

Figure 2. A: lean vs. obese samples ( $n=9$ in each group). B: obese NF vs. obese LVH ( $n=4$ in each group). C: lean NF vs. lean LVH ( $n=3$ in each group). D: lean NF vs. obese LVH $(n=3$ in each group). Red dashed lines mark $\mathrm{p} \_$adj $=0.05$. Dark dots indicate genes without significant changes in expression levels comparing 2 individual groups ( $p_{-}$adj $\left.>0.05\right)$. Red dots: significant genes ( $\mathrm{p} \_$adj $\left.<0.05\right)$ with abs $(\log 2 \mathrm{FC}<1)$; green dots with labels: significant genes with $\mathrm{p} \_$adj $<$ 0.05 and $\operatorname{abs}(\log 2 \mathrm{FC})>1$. 
Figure 3: Distribution of LVH differentially expressed genes under different conditions

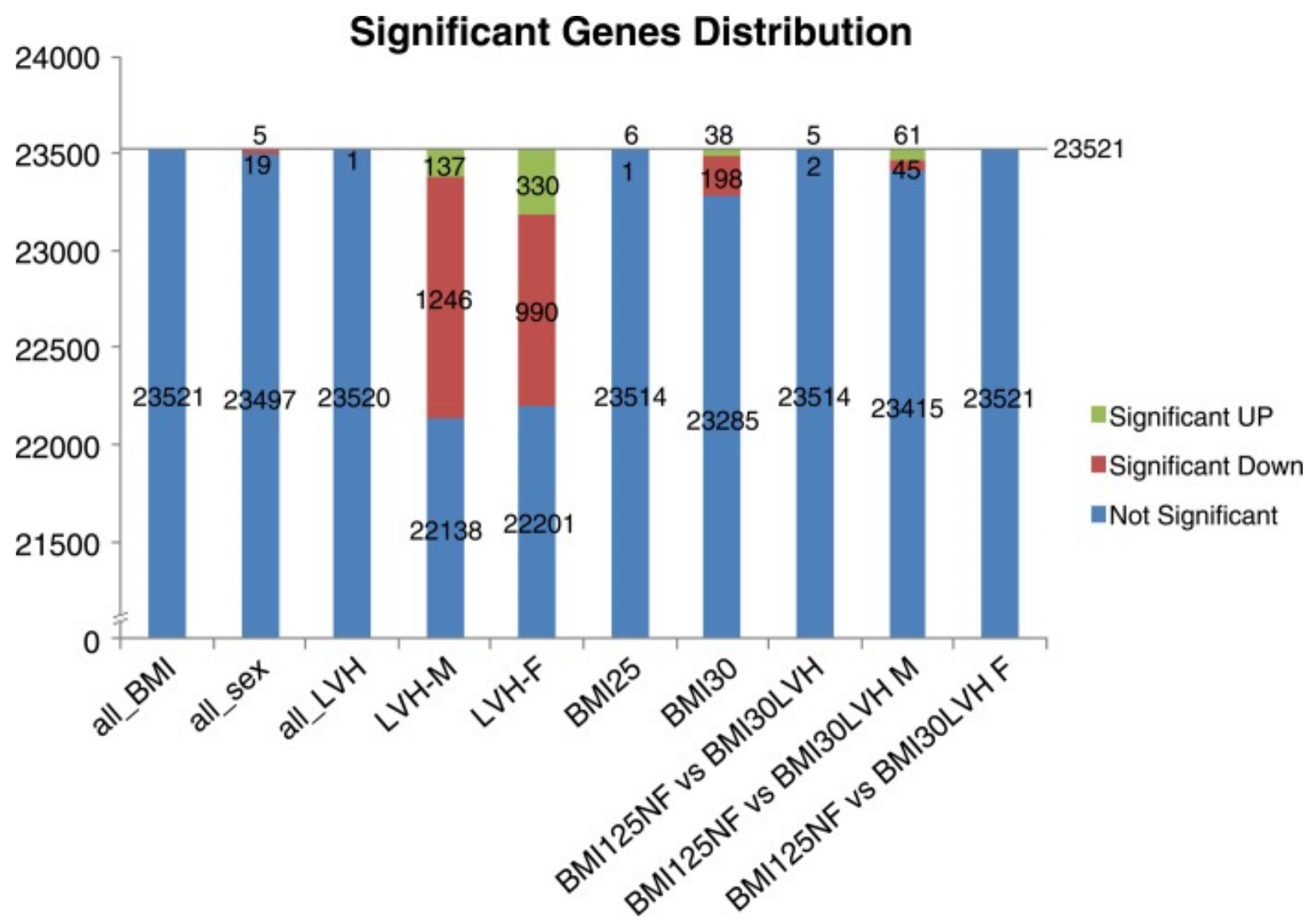

Figure 3. The number of significantly upregulated, downregulated, or non-significant genes when analyzed by different groupings (all_BMI = all samples, analyzed as lean versus obese; all_sex = all samples, analyzed as male versus female; all_LVH = all samples, analyzed as LVH versus non-failed control; LVH-M = male samples, analyzed as LVH versus non-failed control; LVH-F $=$ female samples, analyzed as LVH versus non-failed control; BMI25 = samples with $\mathrm{BMI}<25$, analyzed as LVH versus non-failed control; BMI30 = samples with BMI $>30$, analyzed as $\mathrm{LVH}$ versus non-failed control; BMI25NF vs BMI30LVH = non-failed control samples with $\mathrm{BMI}<25$ versus $\mathrm{LVH}$ samples with $\mathrm{BMI}>30$; $\mathrm{BMI} 25 \mathrm{NF}$ vs $\mathrm{BMI} 30 \mathrm{LVH} \mathrm{M}=$ non-failed control samples with $\mathrm{BMI}<25$ versus $\mathrm{LVH}$ samples with $\mathrm{BMI}>30$, males only; BMI25NF vs 
BMI30LVH F $=$ non-failed control samples with $\mathrm{BMI}<25$ versus $\mathrm{LVH}$ samples with $\mathrm{BMI}>30$, females only). Green indicates upregulation, red indicates downregulation, and blue indicates no significant change. 
Figure 4: Shared LVH differentially expressed (DE) genes in both sexes
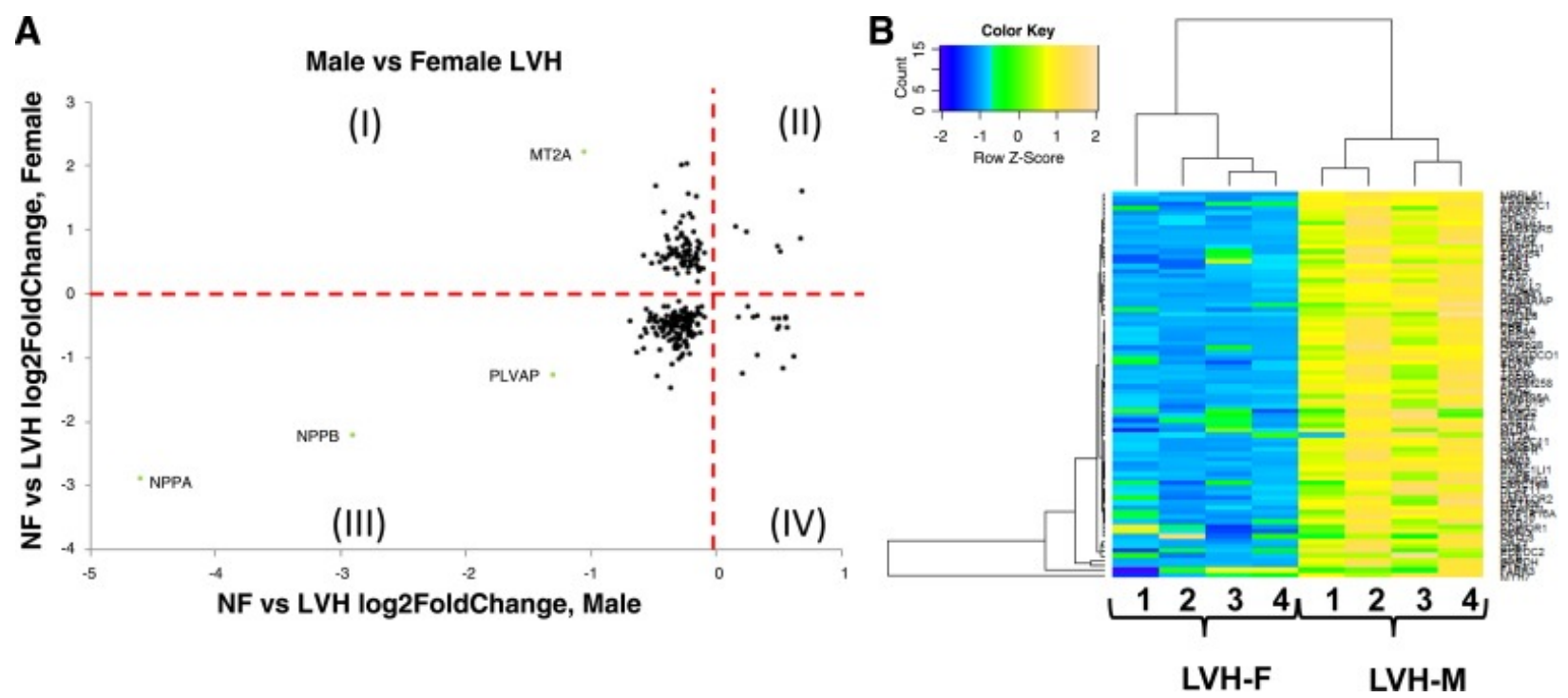

Figure 4. A: scatterplot of male vs. female LVH DE genes. Quadrant I contains DE genes upregulated in male LVH but downregulated in female LVH. Quadrant II contains DE genes downregulated in both male and female LVH. Quadrant III contains DE genes upregulated in both male and female LVH. Quadrant IV contains DE genes upregulated in female LVH but downregulated in male LVH. B: heat map from the $80 \mathrm{DE}$ genes from quadrant I of male vs. female DE gene scatterplot. 
Figure 5: Gene expression signature in LVH and HF

A
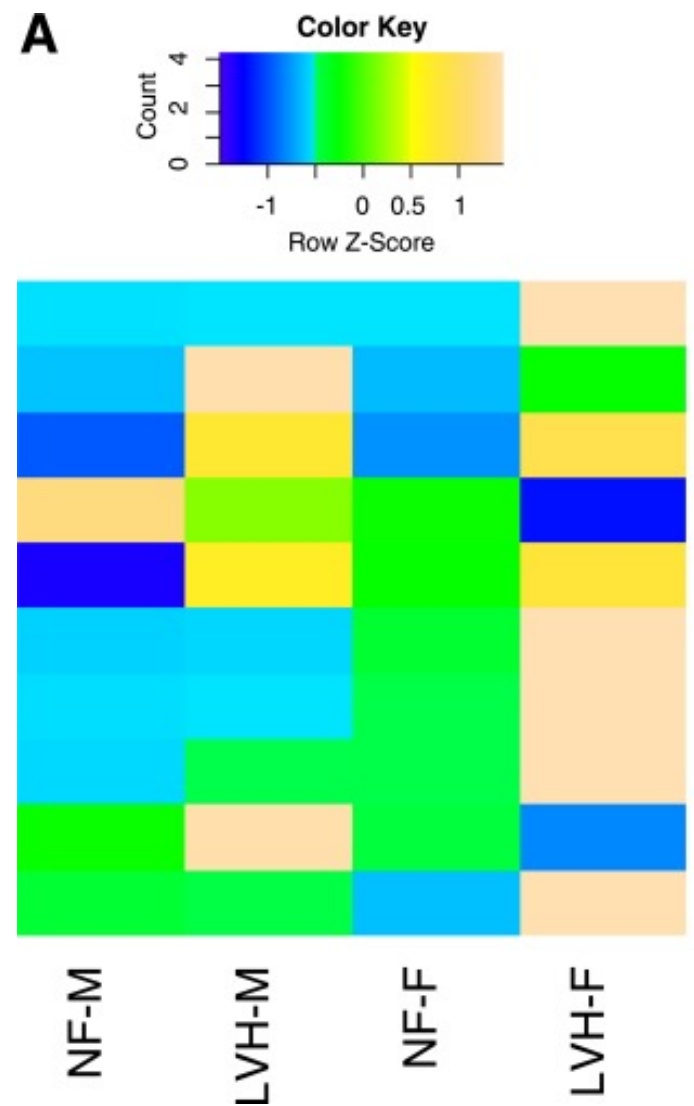

B

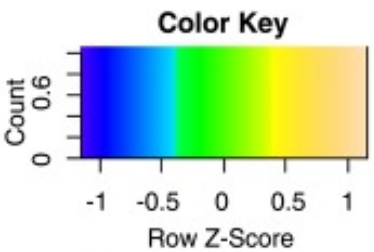

HBB

NPPA

NPPB

MYL7

PDK4

PLA2G2A

HBA1

HIST1H2AC

GSTT1

PLXDC2
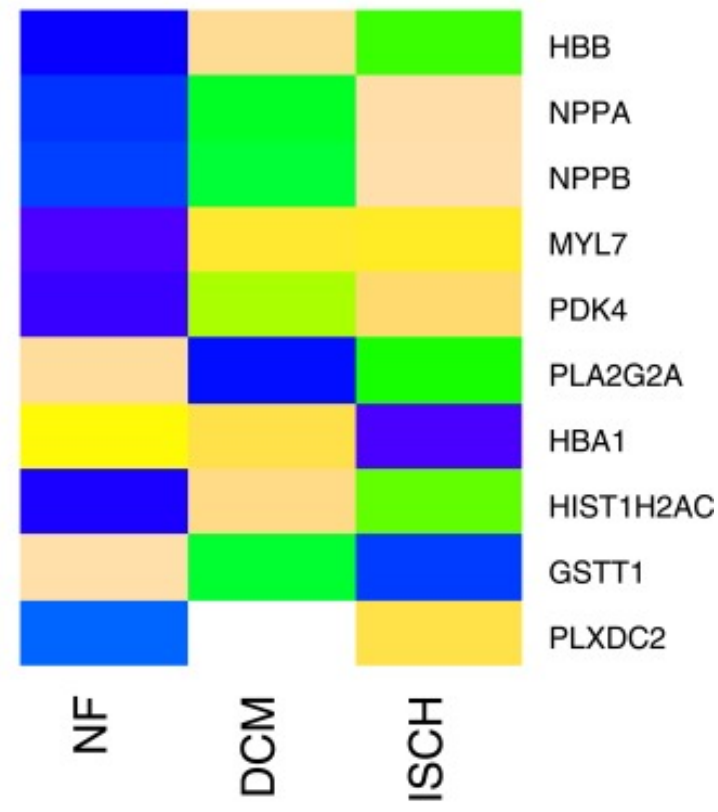

Figure 5. A: heat map of $10 \mathrm{DE}$ genes in male and female LVH; B: heat map of $10 \mathrm{DE}$ genes in ischemic cardiomyopathy (ISCH) and dilated cardiomyopathy (DCM). 
Figure 6: Gene and protein expression of NPPA/ANP
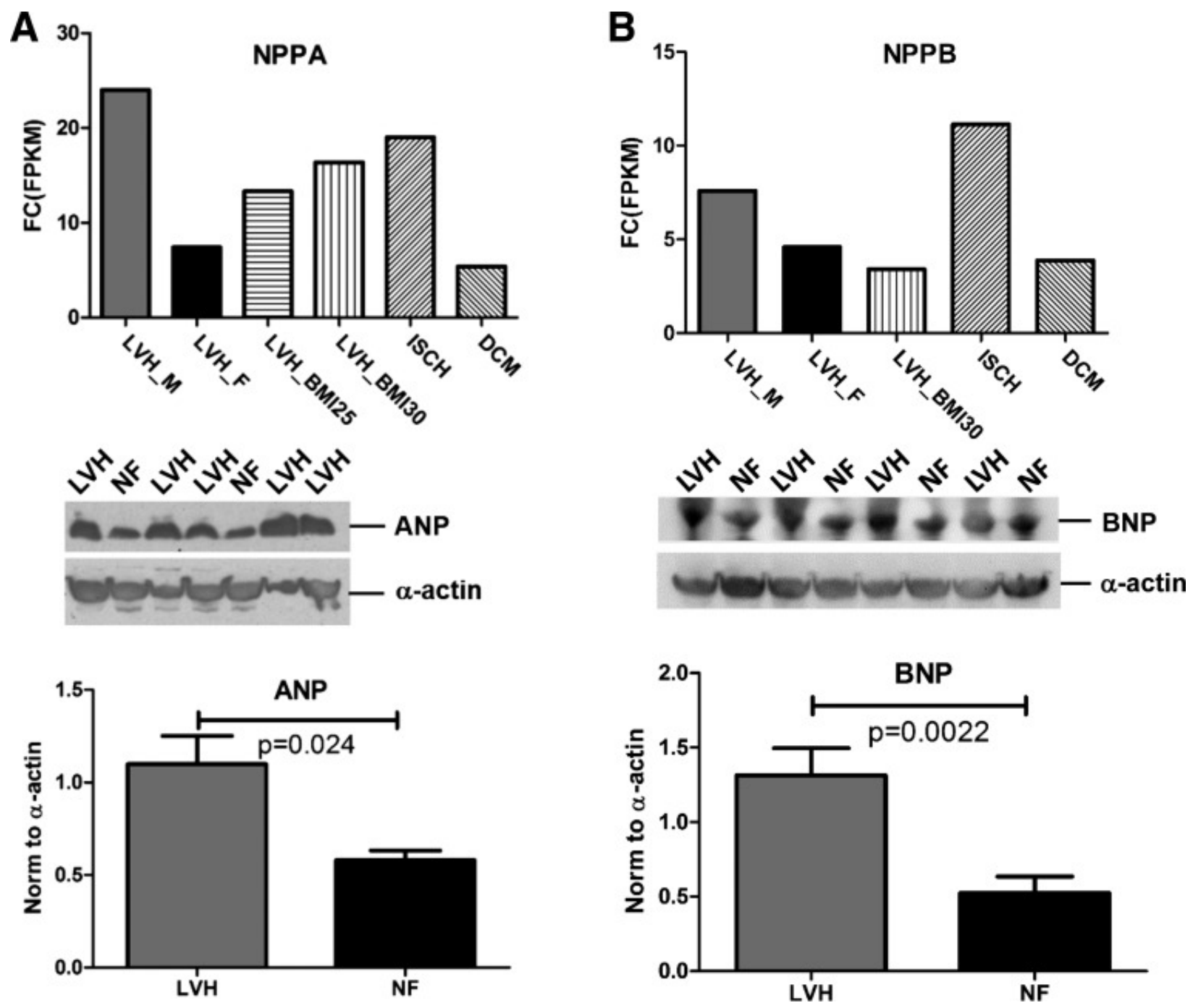

Figure 6. (A) and NPPB/BNP (B). Top: transcript expression of NPPA (A) or NPPB (B) in female and male LVH, ISCH, and DCM. Middle: ANP (A) or BNP (B) immunoblots in LVH and NF, $\alpha$-actin was used as a loading control. Bottom: ANP (A) or BNP (B) protein expression normalized to $\alpha$-actin in LVH and NF. ANP, NPPA, atrial natriuretic peptide A; BNP, NPPB, atrial natriuretic peptide $\mathrm{B}$. 
Figure 7: Gene and protein expression of HBA1 and HBB
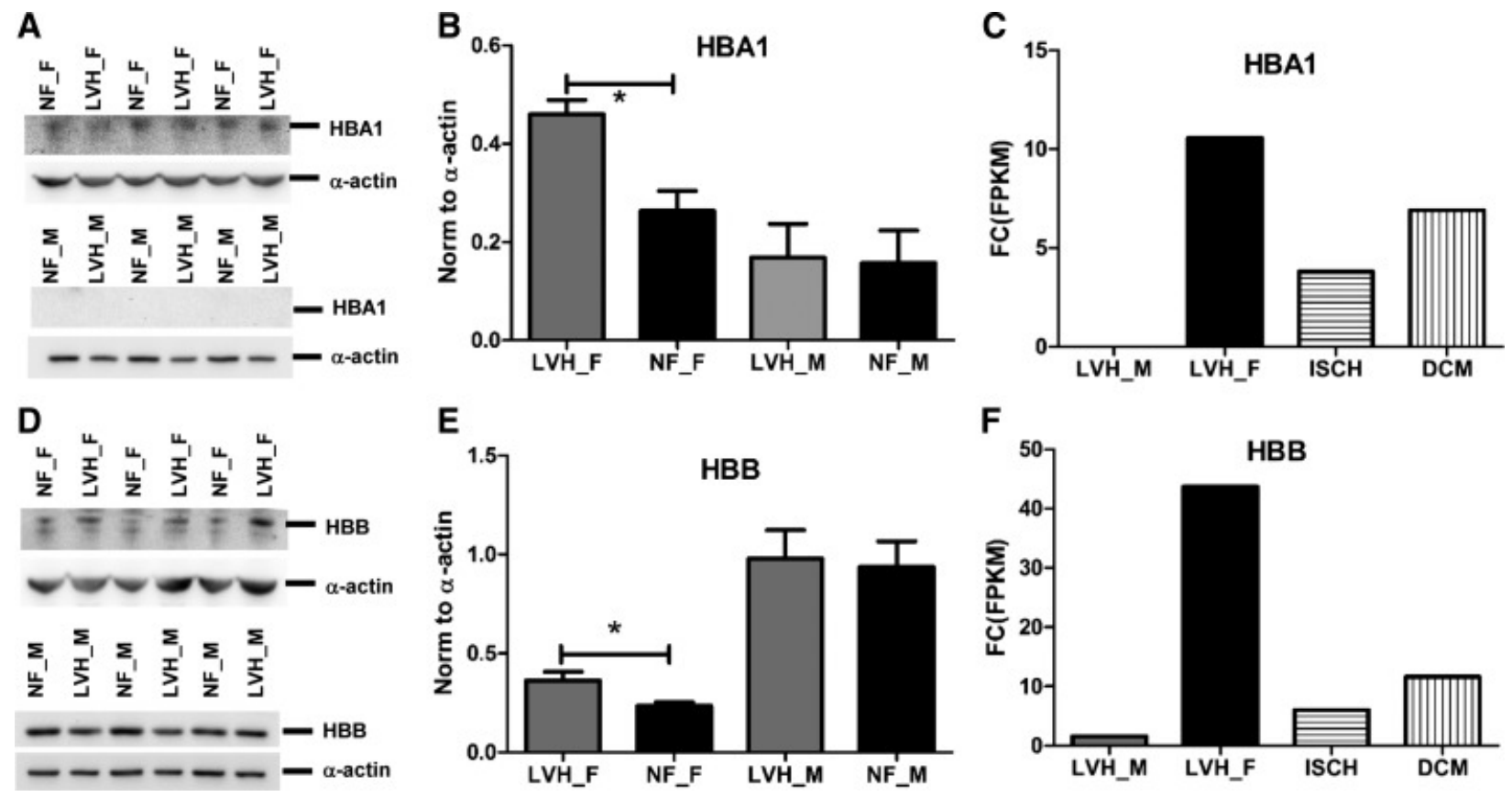

Figure 7. A: HBA1 immunoblots in female (top) and male (bottom) samples. $\alpha$-Actin was used as a loading control. B: HBA1 protein expression normalized to $\alpha$-actin in LVH and NF. C: HBA1 transcript changes in LVH over NF. D: HBB immunoblots in female (top) and male (bottom) samples. $\alpha$-Actin was used as a loading control. E: HBB protein expression normalized to $\alpha$-actin in LVH and NF. F: HBB transcript changes in LVH over NF. * Statistically significant difference between the 2 groups $(\mathrm{P}<0.05)$. FC, fold change; FPKM, fragments per kilobase of transcript per million mapped reads. 
Figure 8: Gene and protein expression of GSTT1 and PLA2G2A
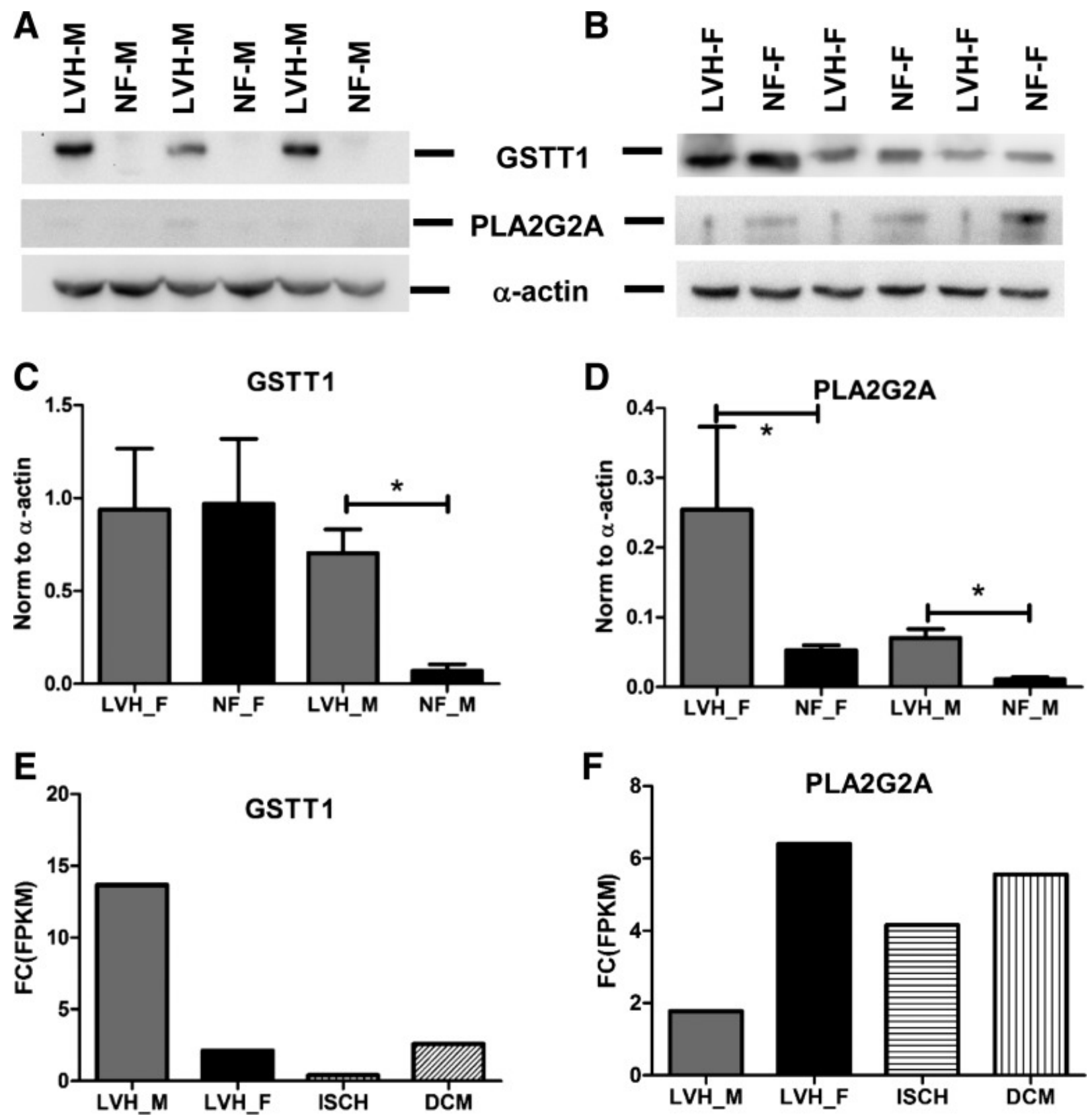

Figure 8. GSTT1 and PLA2G2A immunoblots in male (A) and female (B) samples. $\alpha$-Actin was used as a loading control. C: GSTT1 protein expression normalized to $\alpha$-actin in LVH and NF. D: PLA2G2A protein expression normalized to $\alpha$-actin in LVH and NF. E: GSTT1 transcript changes in LVH over NF. F: PLA2G2A transcript changes in LVH over NF. *Statistically significant difference between the 2 groups $(\mathrm{P}<0.05)$. FC, fold change. 
Figure 9: Gene and protein expression of PDK4 and MYL7

A

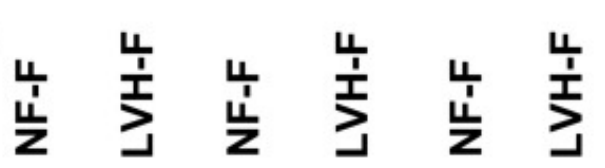

B

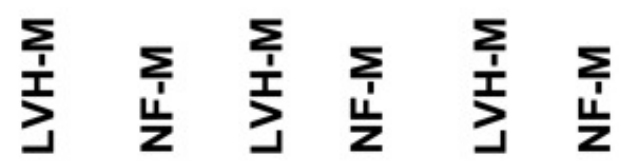

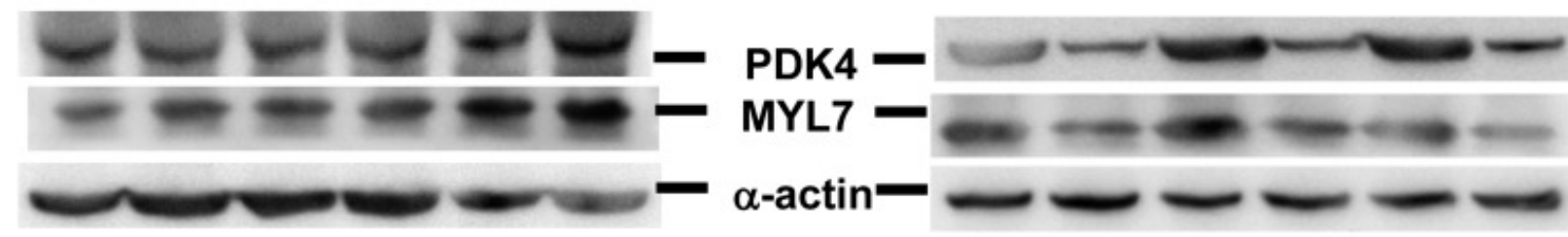

C
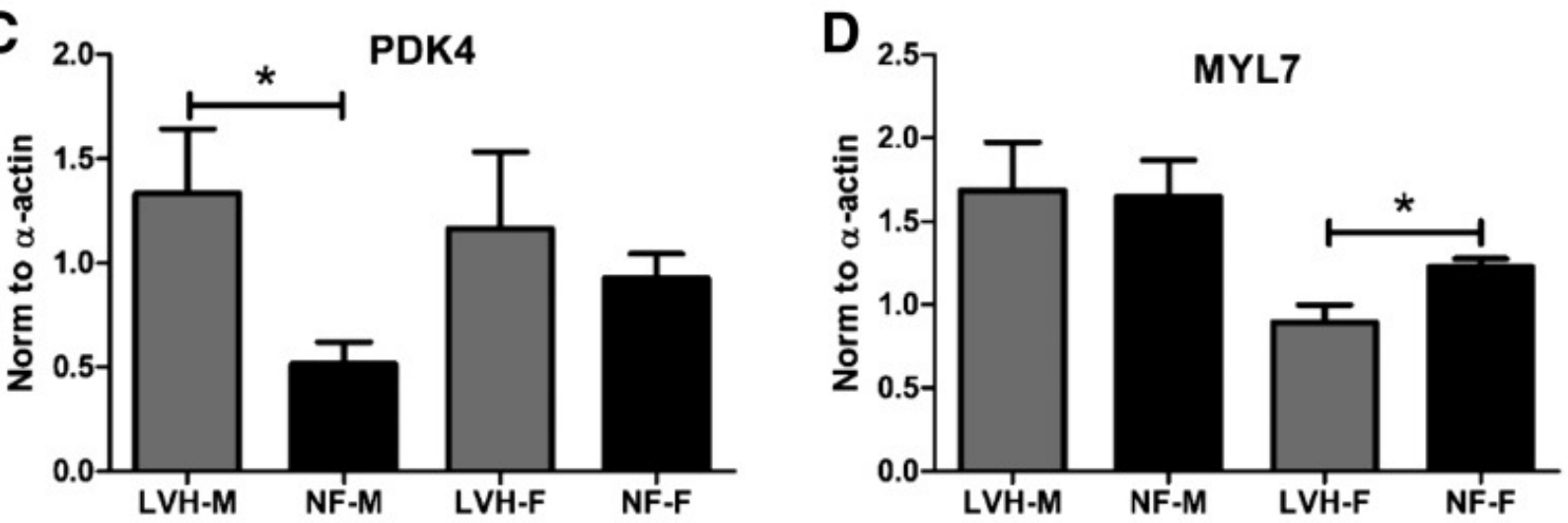

E

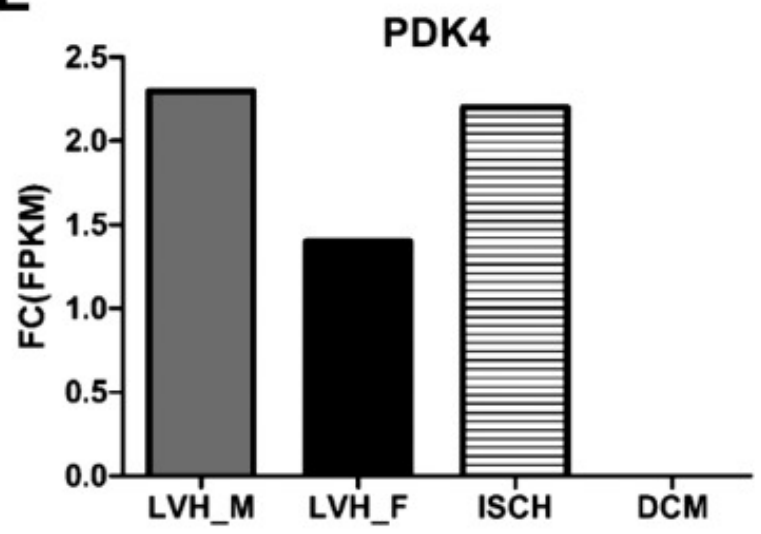

$\mathbf{F}$

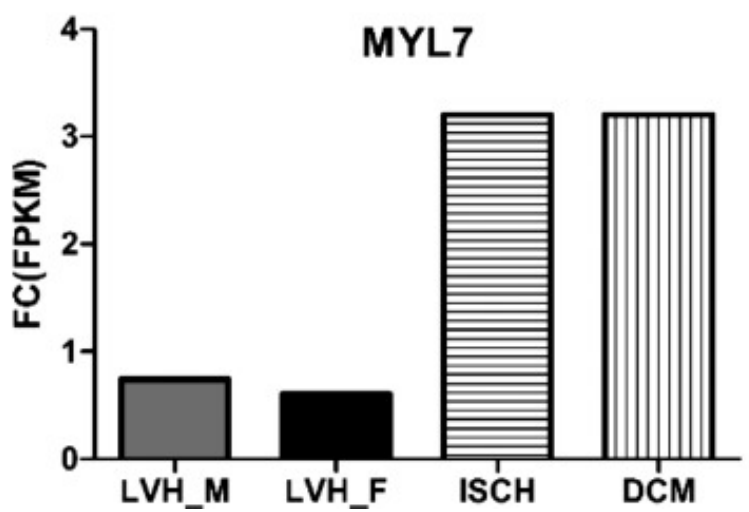

Figure 9. PDK4 and MYL7 immunoblots in female (A) and male (B) samples. $\alpha$-Actin was used as a loading control. C: PDK4 protein expression normalized to $\alpha$-actin in LVH and NF. D: MYL7 protein expression normalized to $\alpha$-actin in LVH and NF. E: PDK4 transcript changes in LVH over NF. F: MYL7 transcript changes in LVH over NF. *Statistically significant difference between the 2 groups $(\mathrm{P}<0.05)$. FC, fold change. 
Figure 10: Gene and protein expression of HIST1H2AC

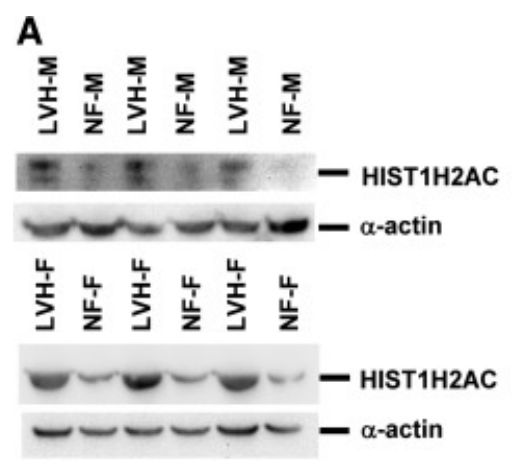

B

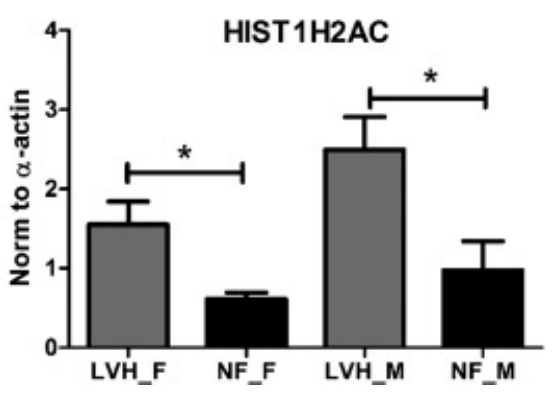

C

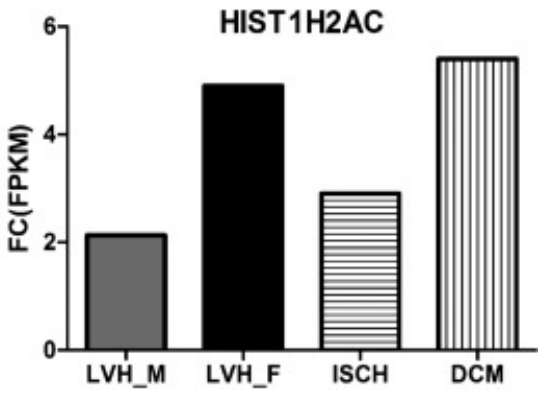

Figure 10. A: immunoblots in male (top) and female (bottom) samples. $\alpha$-Actin was used as a loading control. B: HIST1H2AC protein expression normalized to $\alpha$-actin in LVH and NF. C: HIST1H2AC transcript changes in LVH over NF. *Statistically significant difference between the 2 groups $(\mathrm{P}<0.05)$. FC, fold change. 
$\underline{\text { Tables }}$

Table 1. Patient characterization

\begin{tabular}{|l|l|l|l|l|l|}
\hline $\begin{array}{l}\text { Human } \\
\text { Sample }\end{array}$ & Sex & BMI & Age, yr & $\begin{array}{l}\text { LV Mass, } \\
\text { g }\end{array}$ & Path Report (LVH) \\
\hline NF1 & M & 29.3 & 57 & 126 & no \\
\hline NF2 & M & 17.2 & 54 & 175 & borderline \\
\hline NF3 & M & 30.1 & 41 & 194 & none to mild \\
\hline NF4 & M & 22.5 & 67 & 97 & no \\
\hline NF5 & M & 34.9 & 38 & & none-mild \\
\hline NF6 & M & 21.6 & 36 & 97 & no \\
\hline NF7 & F & 21.1 & 66 & & no \\
\hline NF8 & F & 23 & 33 & & no \\
\hline NF9 & F & 29.7 & 54 & 104 & no \\
\hline NF10 & F & 22.6 & 43 & 103 & no \\
\hline NF11 & F & 34.3 & 64 & & no \\
\hline NF12 & F & 38.1 & 28 & 162 & no \\
\hline LVH1 & M & 23 & 53 & 378 & moderate-severe \\
\hline LVH2 & M & 51.1 & 54 & 300 & moderate \\
\hline LVH3 & M & 33.2 & 51 & 248 & mild \\
\hline LVH4 & M & 19 & 53 & 347 & severe \\
\hline LVH5 & M & 39.1 & 59 & 276 & mild \\
\hline LVH6 & M & 21.1 & 19 & 348 & severe \\
\hline LVH7 & F & 42.9 & 25 & & mild-moderate \\
\hline LVH8 & F & 16.8 & 38 & 176 & mild \\
\hline LVH9 & F & 22.5 & 42 & 176 & mild \\
\hline LVH10 & F & 31.5 & 59 & 141 & moderate \\
\hline LVH11 & F & 33.5 & 49 & & severe \\
\hline LVH12 & F & 19.5 & 50 & & moderate \\
\hline
\end{tabular}

BMI, body mass index; M, male; F, female; LVH, left ventricular hypertrophy; NF: non-LVH nonfailed left ventricular tissue. 
Table 2. Significant differentially expressed genes in LVH $[\operatorname{abs}(\log 2 \mathrm{FC})>2$, extreme small p_adj, also found in HF data set]

\begin{tabular}{|l|l|l|l|l|l|}
\hline Gene & $\mathrm{N} / 25$ & $\mathrm{Y} / 30$ & $\log 2 \mathrm{FC}$ & $\mathrm{p} \_$adj & Protein \\
\hline HBB & 5.385036 & 236.2845 & -5.45543 & 0.000788 & hemoglobin subunit beta \\
\hline NPPA & 28.79361 & 697.1588 & -4.59767 & 0.018249 & natriuretic peptides A \\
\hline HBA1 & 2.670161 & 28.20004 & -3.4007 & 0.001351 & hemoglobin subunit alpha \\
\hline NPPB & 16.17904 & 120.645 & -2.89857 & 0.013588 & natriuretic peptides B \\
\hline PDK4 & 66.72739 & 465.2543 & -2.80167 & $4.00 \mathrm{E}-15$ & $\begin{array}{l}\text { pyruvate dehydrogenase kinase } \\
\text { isozyme 4 }\end{array}$ \\
\hline PLA2G2A & 22.78948 & 142.7502 & -2.64705 & 0.045658 & phospholipase A2 \\
\hline HIST1H2AC & 4.108 & 20.4747 & -2.31733 & 0.003876 & histone H2A type 1-C \\
\hline GSTT1 & 2.927 & 13.489 & -2.204 & 0.011703 & glutathione S-transferase theta-1 \\
\hline PLXDC2 & 4.073109 & 16.66727 & -2.03282 & 0.011265 & plexin domain- containing protein \\
& & & & & myosin light chain 7 \\
\hline MYL7 & 493.851 & 205.222 & 1.267 & $<10 \mathrm{E}-20$ & myin \\
\hline
\end{tabular}

$\mathrm{N}$, non-LVH nonfailed left ventricular tissue; 25, BMI 25; Y, LVH; 30, BMI 30; FC, foldchange. 


\section{$\underline{\text { References }}$}

(1) Abel ED, Litwin SE, Sweeney G. Cardiac remodeling in obesity. Physiol Rev 88: 389-419, 2008. doi:10.1152/physrev.00017.2007.

(2) Anders S, Huber W. Differential expression analysis for sequence count data. Genome Biol 11: R106, 2010. doi:10.1186/gb-2010-11-10-r106.

(3) Avelar E, Cloward TV, Walker JM, Farney RJ, Strong M, Pendleton RC, Segerson N, Adams TD, Gress RE, Hunt SC, Litwin SE. Left ventricular hypertrophy in severe obesity: interactions among blood pressure, nocturnal hypoxemia, and body mass. Hypertension 49: 34-39, 2007. doi:10.1161/01.HYP.0000251711.92482.14.

(4) Bernardo BC, Weeks KL, Pretorius L, McMullen JR. Molecular distinction between physiological and pathological cardiac hypertrophy: experimental findings and therapeutic strategies. Pharmacol Ther 128: 191-227, 2010. doi:10.1016/j.pharmthera.2010.04.005.

(5) Bharati S, Lev M. Cardiac conduction system involvement in sudden death of obese young people. Am Heart J 129: 273-281, 1995. doi:10.1016/0002-8703(95)90008-X.

(6) Bisping E, Wakula P, Poteser M, Heinzel FR. Targeting cardiac hypertrophy: toward a causal heart failure therapy. J Cardiovasc Pharmacol 64: 293-305, 2014. doi:10.1097/FJC.0000000000000126.

(7) Boheler KR, Volkova M, Morrell C, Garg R, Zhu Y, Margulies K, Seymour AM, Lakatta EG. Sex- and age-dependent human transcriptome variability: implications for chronic heart failure. Proc Natl Acad Sci USA 100: 2754-2759, 2003. doi:10.1073/pnas.0436564100.

(8) Bozkurt B, Mann DL. Use of biomarkers in the management of heart failure: are we there yet? Circulation 107: 1231-1233, 2003. doi:10.1161/01.CIR.0000057608.97285.20. 
(9) Chadwick J, Mann W. Medical Works of Hippocrates. Oxford: Blackwell, 1950. [Google Scholar]

(10) Chen EY, Tan CM, Kou Y, Duan Q, Wang Z, Meirelles GV, Clark NR, Ma'ayan A. Enrichr: interactive and collaborative HTML5 gene list enrichment analysis tool. BMC Bioinformatics 14: 128, 2013. doi:10.1186/1471-2105-14-128.

(11) Cooper RS, Simmons BE, Castaner A, Santhanam V, Ghali J, Mar M. Left ventricular hypertrophy is associated with worse survival independent of ventricular function and number of coronary arteries severely narrowed. Am J Cardiol 65: 441-445, 1990. doi:10.1016/00029149(90)90807-D.

(12) Di Angelantonio E, Bhupathiraju ShN, Wormser D, Gao P, Kaptoge S, Berrington de Gonzalez A, Cairns BJ, Huxley R, Jackson ChL, Joshy G, Lewington S, Manson JE, Murphy N, Patel AV, Samet JM, Woodward M, Zheng W, Zhou M, Bansal N, Barricarte A, Carter B, Cerhan JR, Smith GD, Fang X, Franco OH, Green J, Halsey J, Hildebrand JS, Jung KJ, Korda RJ, McLerran DF, Moore SC, O'Keeffe LM, Paige E, Ramond A, Reeves GK, Rolland B, Sacerdote C, Sattar N, Sofianopoulou E, Stevens J, Thun M, Ueshima H, Yang L, Yun YD, Willeit P, Banks E, Beral V, Chen Zh, Gapstur SM, Gunter MJ, Hartge P, Jee SH, Lam TH, Peto R, Potter JD, Willett WC, Thompson SG, Danesh J, Hu FB; Global BMI Mortality Collaboration . Body-mass index and all-cause mortality: individual-participant-data meta-analysis of 239 prospective studies in four continents. Lancet 388: 776-786, 2016. doi:10.1016/S01406736(16)30175-1.

(13) Dobin A, Davis CA, Schlesinger F, Drenkow J, Zaleski C, Jha S, Batut P, Chaisson M, Gingeras TR. STAR: ultrafast universal RNA-seq aligner. Bioinformatics 29: 15-21, 2013. doi:10.1093/bioinformatics/bts635. 
(14) Duflou J, Virmani R, Rabin I, Burke A, Farb A, Smialek J. Sudden death as a result of heart disease in morbid obesity. Am Heart J 130: 306-313, 1995. doi:10.1016/0002-8703(95)90445-X. (15) England J, Loughna S. Heavy and light roles: myosin in the morphogenesis of the heart. Cell Mol Life Sci 70: 1221-1239, 2013. doi:10.1007/s00018-012-1131-1.

(16) Falcão LM, Pinto F, Ravara L, van Zwieten PA. BNP and ANP as diagnostic and predictive markers in heart failure with left ventricular systolic dysfunction. J Renin Angiotensin Aldosterone Syst 5: 121-129, 2004. doi:10.3317/jraas.2004.028.

(17) Frey N, Katus HA, Olson EN, Hill JA. Hypertrophy of the heart: a new therapeutic target? Circulation 109: 1580-1589, 2004. doi:10.1161/01.CIR.0000120390.68287.BB.

(18) Gaggin HK, Belcher AM, Gandhi PU, Ibrahim NE, Januzzi JL Jr. Serial echocardiographic characteristics, novel biomarkers and cachexia development in patients with stable chronic heart failure. J Cardiovasc Transl Res 9: 429-431, 2016. doi:10.1007/s12265-016-9710-4.

(19) Haddad GE, Saunders LJ, Crosby SD, Carles M, del Monte F, King K, Bristow MR, Spinale FG, Macgillivray TE, Semigran MJ, Dec GW, Williams SA, Hajjar RJ, Gwathmey JK. Human cardiac-specific cDNA array for idiopathic dilated cardiomyopathy: sex-related differences. Physiol Genomics 33: 267-277, 2008. doi:10.1152/physiolgenomics.00265.2007.

(20) Heckbert SR, Post W, Pearson GD, Arnett DK, Gomes AS, Jerosch-Herold M, Hundley WG, Lima JA, Bluemke DA. Traditional cardiovascular risk factors in relation to left ventricular mass, volume, and systolic function by cardiac magnetic resonance imaging: the Multiethnic Study of Atherosclerosis. J Am Coll Cardiol 48: 2285-2292, 2006. doi:10.1016/j.jacc.2006.03.072.

(21) Heidecker B, Lamirault G, Kasper EK, Wittstein IS, Champion HC, Breton E, Russell SD, Hall J, Kittleson MM, Baughman KL, Hare JM. The gene expression profile of patients with 
new-onset heart failure reveals important gender-specific differences. Eur Heart J 31: 11881196, 2010. doi:10.1093/eurheartj/ehp549.

(22) Hulot JS, Ishikawa K, Hajjar RJ. Gene therapy for the treatment of heart failure: promise postponed. Eur Heart J 37: 1651-1658, 2016. doi:10.1093/eurheartj/ehw019.

(23) Kenchaiah S, Evans JC, Levy D, Wilson PWF, Benjamin EJ, Larson MG, Kannel WB, Vasan RS. Obesity and the risk of heart failure. N Engl J Med 347: 305-313, 2002. doi:10.1056/NEJMoa020245.

(24) Kittleson MM, Ye SQ, Irizarry RA, Minhas KM, Edness G, Conte JV, Parmigiani G, Miller LW, Chen Y, Hall JL, Garcia JG, Hare JM. Identification of a gene expression profile that differentiates between ischemic and nonischemic cardiomyopathy. Circulation 110: 3444-3451, 2004. doi:10.1161/01.CIR.0000148178.19465.11.

(25) Kuleshov MV, Jones MR, Rouillard AD, Fernandez NF, Duan Q, Wang Z, Koplev S, Jenkins SL, Jagodnik KM, Lachmann A, McDermott MG, Monteiro CD, Gundersen GW, Ma'ayan A. Enrichr: a comprehensive gene set enrichment analysis web server 2016 update. Nucleic Acids Res 44, W1: W90-W97, 2016. doi:10.1093/nar/gkw377.

(26) Lim D-S, Roberts R, Marian AJ. Expression profiling of cardiac genes in human hypertrophic cardiomyopathy: insight into the pathogenesis of phenotypes. J Am Coll Cardiol 38: 1175-1180, 2001. doi:10.1016/S0735-1097(01)01509-1.

(28) Liu Y, Morley M, Brandimarto J, Hannenhalli S, Hu Y, Ashley EA, Tang WH, Moravec CS, Margulies KB, Cappola TP, Li M; MAGNet Consortium . RNA-Seq identifies novel myocardial gene expression signatures of heart failure. Genomics 105: 83-89, 2015. doi:10.1016/j.ygeno.2014.12.002. 
(29) Mahgerefteh B, Vigue M, Freestone Z, Silver S, Nguyen Q. New drug therapies for the treatment of overweight and obese patients. Am Health Drug Benefits 6: 423-430, 2013.

(30) Marfella R, Di Filippo C, Portoghese M, Barbieri M, Ferraraccio F, Siniscalchi M, Cacciapuoti F, Rossi F, D’Amico M, Paolisso G. Myocardial lipid accumulation in patients with pressure-overloaded heart and metabolic syndrome. J Lipid Res 50: 2314-2323, 2009. doi:10.1194/jlr.P900032-JLR200.

(31) McMurray JJ, Adamopoulos S, Anker SD, Auricchio A, Böhm M, Dickstein K, Falk V, Filippatos G, Fonseca C, Gomez-Sanchez MA, Jaarsma T, Køber L, Lip GY, Maggioni AP, Parkhomenko A, Pieske BM, Popescu BA, Rønnevik PK, Rutten FH, Schwitter J, Seferovic P, Stepinska J, Trindade PT, Voors AA, Zannad F, Zeiher A, Bax JJ, Baumgartner H, Ceconi C, Dean V, Deaton C, Fagard R, Funck-Brentano C, Hasdai D, Hoes A, Kirchhof P, Knuuti J, Kolh P, McDonagh T, Moulin C, Popescu BA, Reiner Z, Sechtem U, Sirnes PA, Tendera M, Torbicki A, Vahanian A, Windecker S, McDonagh T, Sechtem U, Bonet LA, Avraamides P, Ben Lamin HA, Brignole M, Coca A, Cowburn P, Dargie H, Elliott P, Flachskampf FA, Guida GF, Hardman S, Iung B, Merkely B, Mueller C, Nanas JN, Nielsen OW, Orn S, Parissis JT, Ponikowski P; ESC Committee for Practice Guidelines . ESC Guidelines for the diagnosis and treatment of acute and chronic heart failure 2012: The Task Force for the Diagnosis and Treatment of Acute and Chronic Heart Failure 2012 of the European Society of Cardiology. Developed in collaboration with the Heart Failure Association (HFA) of the ESC. Eur Heart J 33: 1787-1847, 2012. doi:10.1093/eurheartj/ehs 104.

(32) Mosca L, Barrett-Connor E, Wenger NK. Sex/gender differences in cardiovascular disease prevention: what a difference a decade makes. Circulation 124: 2145-2154, 2011. doi:10.1161/CIRCULATIONAHA.110.968792. 
(33) Nieminen MS, Harjola VP, Hochadel M, Drexler H, Komajda M, Brutsaert D, Dickstein K, Ponikowski P, Tavazzi L, Follath F, Lopez-Sendon JL. Gender related differences in patients presenting with acute heart failure. Results from EuroHeart Failure Survey II. Eur J Heart Fail 10: 140-148, 2008. doi:10.1016/j.ejheart.2007.12.012.

(34) Perego L, Pizzocri P, Corradi D, Maisano F, Paganelli M, Fiorina P, Barbieri M, Morabito A, Paolisso G, Folli F, Pontiroli AE. Circulating leptin correlates with left ventricular mass in morbid (grade III) obesity before and after weight loss induced by bariatric surgery: a potential role for leptin in mediating human left ventricular hypertrophy. J Clin Endocrinol Metab 90: 4087-4093, 2005. doi:10.1210/jc.2004-1963.

(35) Preston SH, Stokes A. Contribution of obesity to international differences in life expectancy. Am J Public Health 101: 2137-2143, 2011. doi:10.2105/AJPH.2011.300219.

(36) Razeghi P, Young ME, Alcorn JL, Moravec CS, Frazier OH, Taegtmeyer H. Metabolic gene expression in fetal and failing human heart. Circulation 104: 2923-2931, 2001. doi:10.1161/hc4901.100526.

(37) RStudio-Team. RStudio: Integrated Development for R. Boston, MA: RStudio, Inc. 2015.

(38) Simpson PC. Where are the new drugs to treat heart failure? Introduction to the special issue on "key signaling molecules in hypertrophy and heart failure". J Mol Cell Cardiol 51: 435-437, 2011. doi:10.1016/j.yjmcc.2011.08.005.

(39) Son GH, Park SH, Kim Y, Kim JY, Kim JW, Chung S, Kim Y-H, Kim H, Hwang J-J, Seo J-S. Postmortem mRNA expression patterns in left ventricular myocardial tissues and their implications for forensic diagnosis of sudden cardiac death. Mol Cells 37: 241-247, 2014. doi:10.14348/molcells.2014.2344. 
(40) Tan F-L, Moravec CS, Li J, Apperson-Hansen C, McCarthy PM, Young JB, Bond M. The gene expression fingerprint of human heart failure. Proc Natl Acad Sci USA 99: 11387-11392, 2002. doi:10.1073/pnas.162370099.

(41) Tarazona S, García-Alcalde F, Dopazo J, Ferrer A, Conesa A. Differential expression in RNA-seq: a matter of depth. Genome Res 21: 2213-2223, 2011. doi:10.1101/gr.124321.111.

(42) Trapnell C, Williams BA, Pertea G, Mortazavi A, Kwan G, van Baren MJ, Salzberg SL, Wold BJ, Pachter L. Transcript assembly and quantification by RNA-Seq reveals unannotated transcripts and isoform switching during cell differentiation. Nat Biotechnol 28: 511-515, 2010. doi:10.1038/nbt.1621.

(43) Twig G, Yaniv G, Levine H, Leiba A, Goldberger N, Derazne E, Ben-Ami Shor D, Tzur D, Afek A, Shamiss A, Haklai Z, Kark JD. Body-mass index in 2.3 million adolescents and cardiovascular death in adulthood. N Engl J Med 374: 2430-2440, 2016. doi:10.1056/NEJMoa1503840.

(44) van der Leeuw J, Beulens JWJ, van Dieren S, Schalkwijk CG, Glatz JFC, Hofker MH, Verschuren WMM, Boer JMA, van der Graaf Y, Visseren FLJ, Peelen LM, van der Schouw YT. Novel biomarkers to improve the prediction of cardiovascular event risk in type 2 diabetes mellitus. J Am Heart Assoc 5: e003048, 2016. doi:10.1161/JAHA.115.003048.

(45) Vest AR, Heneghan HM, Agarwal S, Schauer PR, Young JB. Bariatric surgery and cardiovascular outcomes: a systematic review. Heart 98: 1763-1777, 2012. doi:10.1136/heartjnl2012-301778.

(46) Wang Z, Gerstein M, Snyder M. RNA-Seq: a revolutionary tool for transcriptomics. Nat Rev Genet 10: 57-63, 2009. doi:10.1038/nrg2484. 
(47) Warde-Farley D, Donaldson SL, Comes O, Zuberi K, Badrawi R, Chao P, Franz M, Grouios C, Kazi F, Lopes CT, Maitland A, Mostafavi S, Montojo J, Shao Q, Wright G, Bader GD, Morris Q. The GeneMANIA prediction server: biological network integration for gene prioritization and predicting gene function. Nucleic Acids Res 38, suppl_2: W214-W220, 2010. doi:10.1093/nar/gkq537.

(48) Wong CY, O’Moore-Sullivan T, Leano R, Byrne N, Beller E, Marwick TH. Alterations of left ventricular myocardial characteristics associated with obesity. Circulation 110: 3081-3087, 2004. doi:10.1161/01.CIR.0000147184.13872.0F.

(49) Yancy CW, Jessup M, Bozkurt B, Butler J, Casey DE Jr, Drazner MH, Fonarow GC, Geraci SA, Horwich T, Januzzi JL, Johnson MR, Kasper EK, Levy WC, Masoudi FA, McBride PE, McMurray JJ, Mitchell JE, Peterson PN, Riegel B, Sam F, Stevenson LW, Tang WH, Tsai EJ, Wilkoff BL; American College of Cardiology Foundation/American Heart Association Task Force on Practice Guidelines . 2013 ACCF/AHA guideline for the management of heart failure: a report of the American College of Cardiology Foundation/American Heart Association Task Force on practice guidelines. Circulation 128: e240-e327, 2013. doi:10.1161/CIR.0b013e31829e8807.

(50) Yates A, Akanni W, Amode MR, Barrell D, Billis K, Carvalho-Silva D, Cummins C, Clapham P, Fitzgerald S, Gil L, Girón CG, Gordon L, Hourlier T, Hunt SE, Janacek SH, Johnson N, Juettemann T, Keenan S, Lavidas I, Martin FJ, Maurel T, McLaren W, Murphy DN, Nag R, Nuhn M, Parker A, Patricio M, Pignatelli M, Rahtz M, Riat HS, Sheppard D, Taylor K, Thormann A, Vullo A, Wilder SP, Zadissa A, Birney E, Harrow J, Muffato M, Perry E, Ruffier M, Spudich G, Trevanion SJ, Cunningham F, Aken BL, Zerbino DR, Flicek P. Ensembl 2016. Nucleic Acids Res 44, D1: D710-D716, 2016. doi:10.1093/nar/gkv1157. 


\section{$\underline{\text { Supplemental Figures }}$}

\section{Supplemental Figure 1: Obese LVH DE genes found in HF patients}

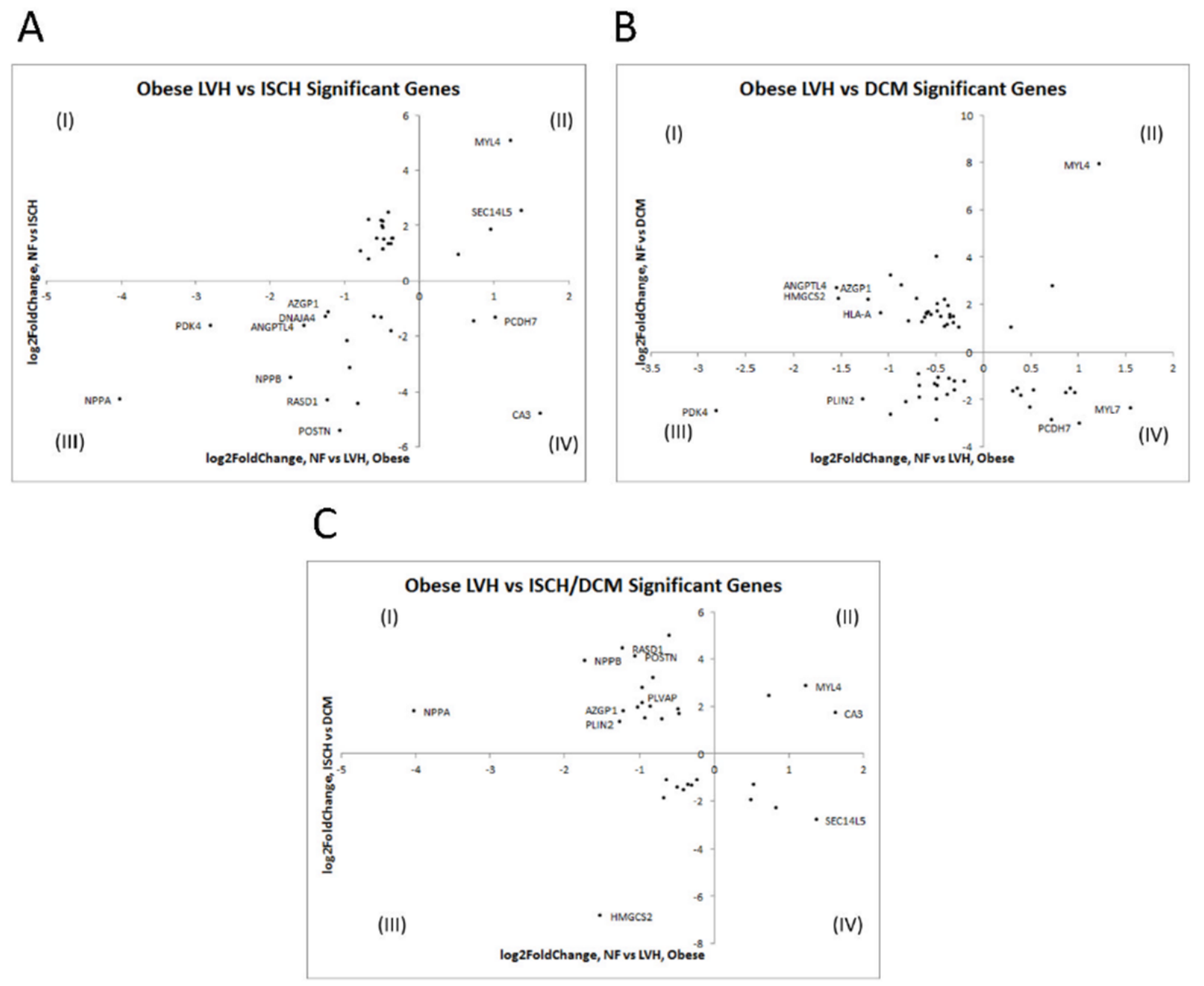

Supplementary Figure 1. A) Scatterplot of obese LVH and ischemia cardiomyopathy differentially expressed genes. Quadrant (I) contains DE genes upregulated in LVH but downregulated in ISCH. Quadrant (II) contains DE genes downregulated in both LVH and ISCH. Quadrant (III) contains DE genes upregulated in both LVH and ISCH. Quadrant (IV) contains DE genes upregulated in ISCH but downregulated in LVH. B) Scatterplot of obese LVH and dilated cardiomyopathy differentially expressed genes. Quadrant (I) contains DE genes upregulated in LVH but downregulated in DCM. Quadrant (II) contains DE genes 
downregulated in both LVH and DCM. Quadrant (III) contains DE genes upregulated in both LVH and DCM. Quadrant (IV) contains DE genes upregulated in DCM but downregulated in LVH. C) Scatterplot of obese LVH and ISCH/DCM differentially expressed genes. Quadrant (I) contains DE genes upregulated in LVH but downregulated in ISCH/DCM. Quadrant (II) contains DE genes downregulated in both LVH and ISCH/DCM. Quadrant (III) contains DE genes upregulated in both LVH and ISCH/DCM. Quadrant (IV) contains DE genes upregulated in ISCH/DCM but downregulated in LVH. 


\section{Supplemental Figure 2: Sex-specific LVH DE genes found in HF patients}

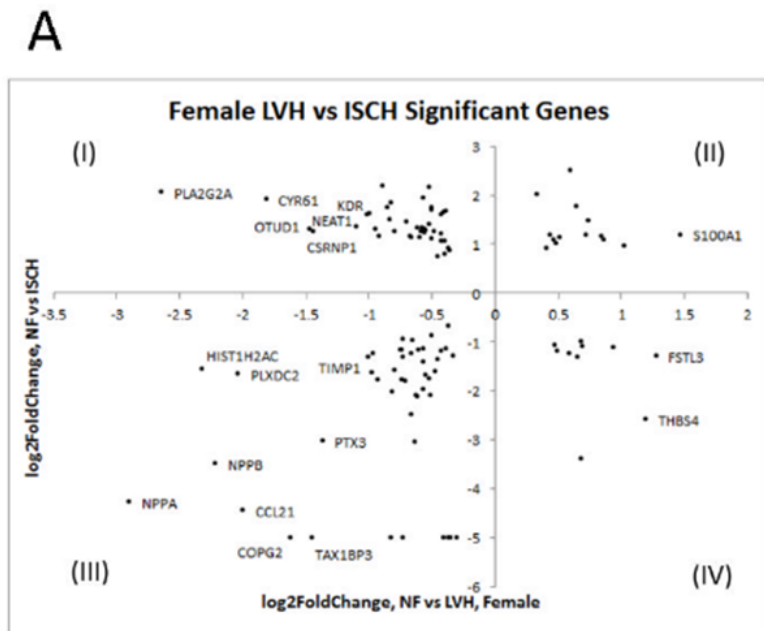

B

C
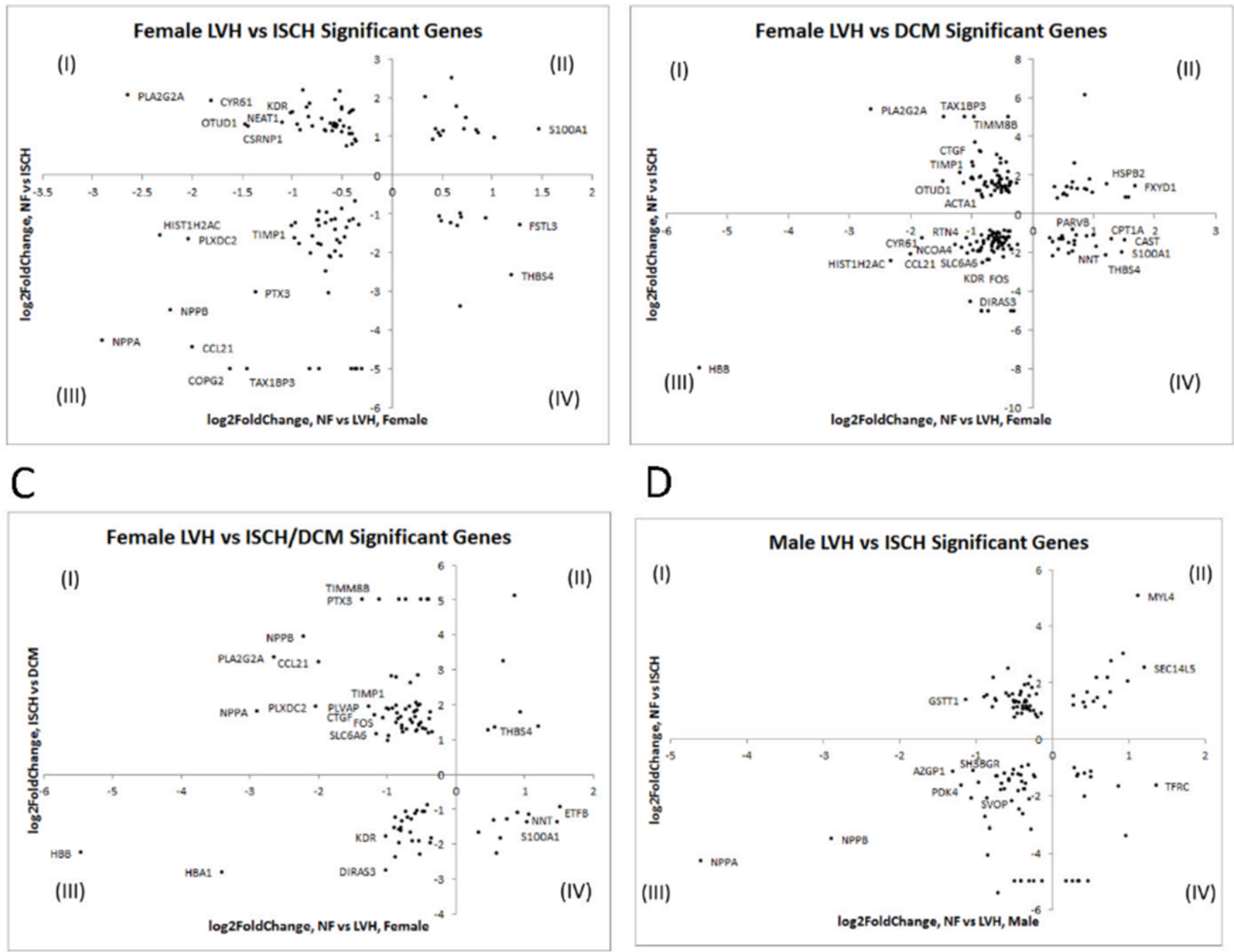

D
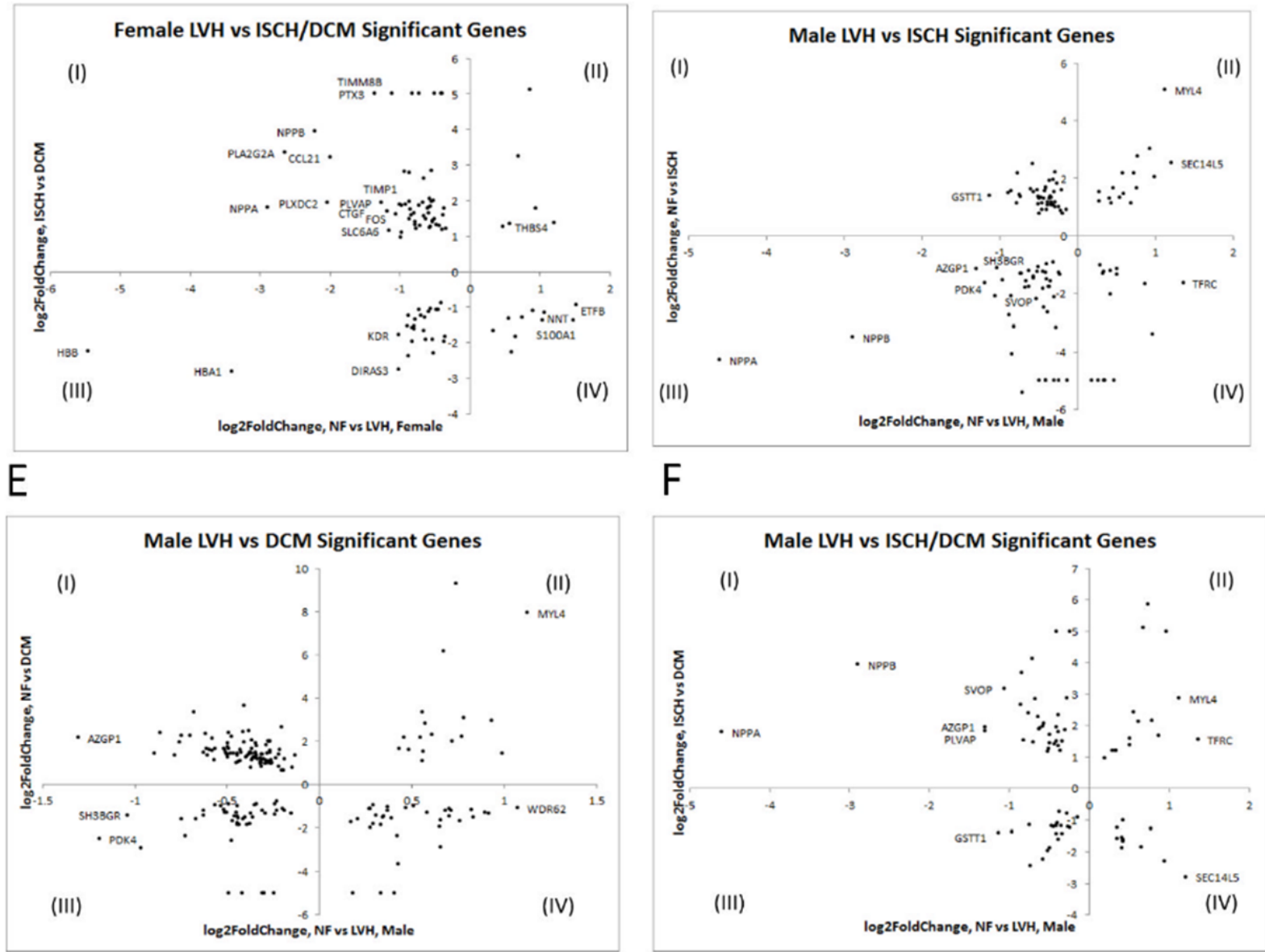

$\mathrm{F}$

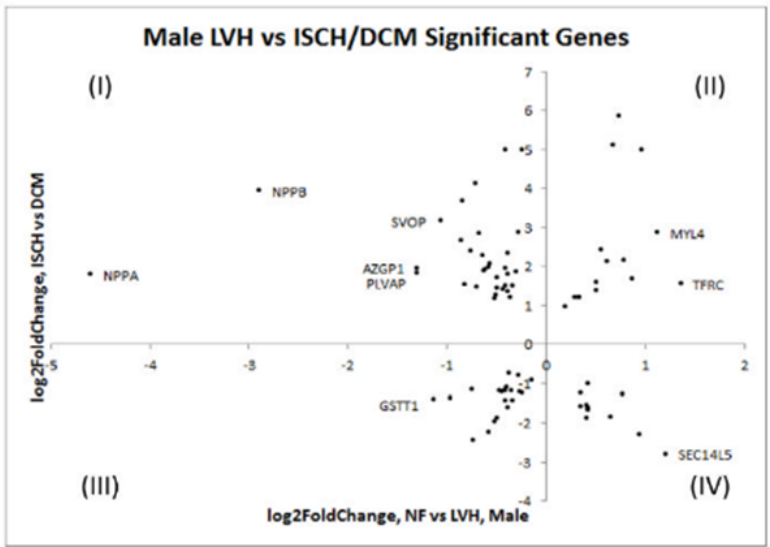

Supplementary Figure 2. A) Scatterplot of female LVH and ischemia cardiomyopathy differentially expressed genes. Quadrant (I) contains DE genes upregulated in LVH but 
downregulated in ISCH. Quadrant (II) contains DE genes downregulated in both LVH and ISCH. Quadrant (III) contains DE genes upregulated in both LVH and ISCH. Quadrant (IV) contains DE genes upregulated in ISCH but downregulated in LVH. B) Scatterplot of female LVH and dilated cardiomyopathy differentially expressed genes. Quadrant (I) contains DE genes upregulated in LVH but downregulated in DCM. Quadrant (II) contains DE genes downregulated in both LVH and DCM. Quadrant (III) contains DE genes upregulated in both LVH and DCM. Quadrant (IV) contains DE genes upregulated in DCM but downregulated in LVH. C) Scatterplot of female LVH and ISCH/DCM differentially expressed genes. Quadrant (I) contains DE genes upregulated in LVH but downregulated in ISCH/DCM. Quadrant (II) contains DE genes downregulated in both LVH and ISCH/DCM. Quadrant (III) contains DE genes upregulated in both LVH and ISCH/DCM. Quadrant (IV) contains DE genes upregulated in ISCH/DCM but downregulated in LVH. D) Scatterplot of male LVH and ischemia cardiomyopathy differentially expressed genes. Quadrant (I) contains DE genes upregulated in LVH but downregulated in ISCH. Quadrant (II) contains DE genes downregulated in both LVH and ISCH. Quadrant (III) contains DE genes upregulated in both LVH and ISCH. Quadrant (IV) contains DE genes upregulated in ISCH but downregulated in LVH. E) Scatterplot of male LVH and dilated cardiomyopathy differentially expressed genes. Quadrant (I) contains DE genes upregulated in LVH but downregulated in DCM. Quadrant (II) contains DE genes downregulated in both LVH and DCM. Quadrant (III) contains DE genes upregulated in both LVH and DCM. Quadrant (IV) contains DE genes upregulated in DCM but downregulated in LVH. F) Scatterplot of male LVH and ISCH/DCM differentially expressed genes. Quadrant (I) contains DE genes upregulated in LVH but downregulated in ISCH/DCM. Quadrant (II) contains DE genes downregulated in both LVH and ISCH/DCM. Quadrant (III) contains DE genes 
upregulated in both LVH and ISCH/DCM. Quadrant (IV) contains DE genes upregulated in ISCH/DCM but downregulated in LVH. 
Supplemental Figure 3: nine DE genes interaction network analysis by GeneMANIA
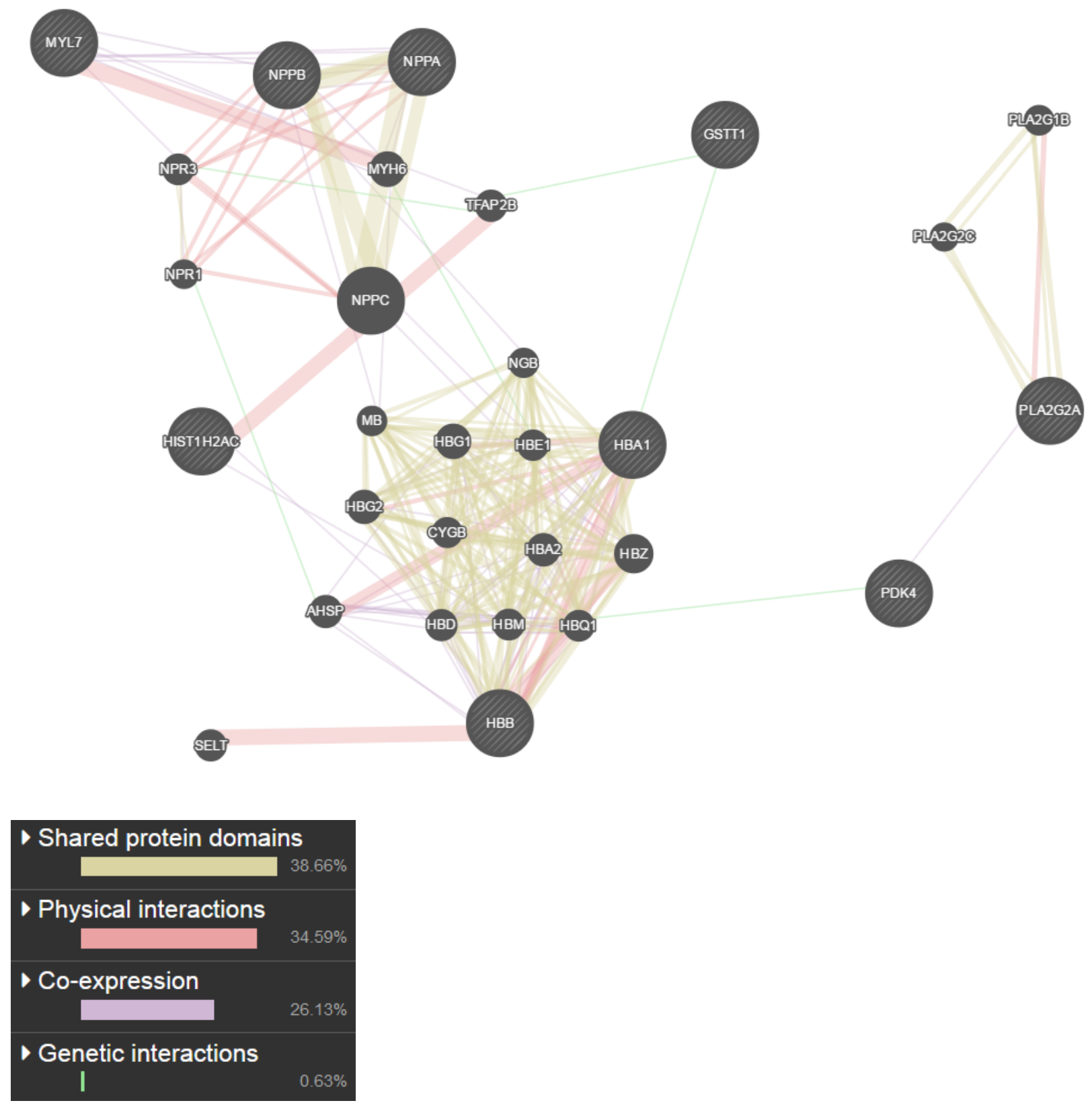

Supplementary Figure 3. Associations between the nine-gene panel with other genes estimated to have interactions were derived via GeneMANIA. Yellow indicates shared protein domains, red indicates physical interactions, purple indicates co-expression, and green indicates genetic interactions. 
Supplemental Tables

Supplemental Table 1. DEG from comparing men to women $\left(p_{-}\right.$adj $\left.<0.05\right)$

\begin{tabular}{|l|l|l|}
\hline GeneName & p_adj & log2FC \\
\hline XIST & 0 & 1.331247 \\
\hline DDX3Y & 0 & -2.28647 \\
\hline EIF1AY & 0 & -4.84517 \\
\hline RPS4Y1 & 0 & -4.89043 \\
\hline FAM155B & 0 & -1.25799 \\
\hline KDM5D & $5.00 \mathrm{E}-15$ & -1.59888 \\
\hline USP9Y & $5.00 \mathrm{E}-15$ & -1.93858 \\
\hline TTTY14 & $1.52 \mathrm{E}-13$ & -1.70475 \\
\hline CCL2 & 0.000175 & 1.84234 \\
\hline ZFY & 0.001617 & -0.83281 \\
\hline EIF4EBP1 & 0.004783 & -0.57846 \\
\hline COQ10A & 0.008654 & -0.61595 \\
\hline HPR & 0.015133 & -2.55647 \\
\hline HSPB6 & 0.020229 & -0.83095 \\
\hline HS6ST3 & 0.02362 & -0.98824 \\
\hline MBNL3 & 0.029631 & -1.27373 \\
\hline ASIP & 0.031702 & -1.01184 \\
\hline EIF1B & 0.03191 & -0.60913 \\
\hline SFRP1 & 0.03445 & -1.29097 \\
\hline SORBS1 & 0.036844 & -0.45915 \\
\hline S100A9 & 0.040507 & 1.311026 \\
\hline FN1 & 0.041244 & 1.560714 \\
\hline LGALS1 & 0.042249 & 0.379527 \\
\hline ATP1A2 & 0.042719 & -0.82381 \\
\hline
\end{tabular}


Supplemental Table 2. DEG in females

when comparing NF to LVH (p_adj <

0.05)

\begin{tabular}{|l|l|l|}
\hline GeneName & p_adj & log2FC \\
\hline ATP5F1 & 0 & 1.372359 \\
\hline COX5A & 0 & 0.655162 \\
\hline NDUFAB1 & $2.00 \mathrm{E}-15$ & 0.971683 \\
\hline UQCRC1 & $4.00 \mathrm{E}-15$ & 0.78536 \\
\hline DCAF6 & $6.99 \mathrm{E}-15$ & 0.894946 \\
\hline NDUFS2 & $7.99 \mathrm{E}-15$ & 0.903111 \\
\hline TPM1 & $1.80 \mathrm{E}-14$ & 0.852596 \\
\hline UQCRHL & $3.00 \mathrm{E}-14$ & 0.854599 \\
\hline ACADVL & $1.30 \mathrm{E}-13$ & 0.891492 \\
\hline CALM2 & $4.98 \mathrm{E}-09$ & 0.48869 \\
\hline PGK1 & $1.52 \mathrm{E}-08$ & 0.640531 \\
\hline RNF14 & $1.90 \mathrm{E}-07$ & 1.344973 \\
\hline ANXA6 & $1.92 \mathrm{E}-07$ & 0.346138 \\
\hline NFIX & $1.93 \mathrm{E}-07$ & 1.237286 \\
\hline AES & $1.98 \mathrm{E}-07$ & 0.773747 \\
\hline MYBPC3 & $3.07 \mathrm{E}-07$ & 0.794732 \\
\hline TNS1 & $3.08 \mathrm{E}-07$ & 0.423984 \\
\hline DBNDD2 & $3.19 \mathrm{E}-07$ & 1.116301 \\
\hline TNNI3 & $3.21 \mathrm{E}-07$ & 0.752848 \\
\hline TFG & $2.12 \mathrm{E}-06$ & 0.797028 \\
\hline SMIM4 & $4.14 \mathrm{E}-06$ & 0.795172 \\
\hline RAB4A & $5.53 \mathrm{E}-06$ & 1.515827 \\
\hline MLYCD & $6.48 \mathrm{E}-06$ & 0.893457 \\
\hline PTPRM & $7.06 \mathrm{E}-06$ & 0.629126 \\
\hline NDUFV1 & $7.71 \mathrm{E}-06$ & 0.442041 \\
\hline OAZ1 & $8.39 \mathrm{E}-06$ & 0.687205 \\
\hline CAPZB & $1.37 \mathrm{E}-05$ & 0.593617 \\
\hline CKMT2 & $1.95 \mathrm{E}-05$ & 0.826101 \\
\hline ANKRD9 & $2.22 \mathrm{E}-05$ & 0.733555 \\
\hline CPT1A & $2.31 \mathrm{E}-05$ & 1.291808 \\
\hline MRPL16 & $3.43 \mathrm{E}-05$ & 0.571236 \\
\hline COQ9 & $4.03 \mathrm{E}-05$ & 0.591581 \\
\hline MRPL33 & $5.81 \mathrm{E}-05$ & 0.685197 \\
\hline PDK2 & 0.000122 & 0.837148 \\
\hline TXLNB & 0.000129 & 0.492954 \\
\hline & & \\
\hline
\end{tabular}

\begin{tabular}{|l|l|l|} 
EEF1G & 0.000136 & 2.018869 \\
\hline DYNLL2 & 0.000138 & 0.593348 \\
\hline PTDSS1 & 0.00014 & 0.73936 \\
\hline CYR61 & 0.000146 & -1.8073 \\
\hline PPP1R12B & 0.000174 & 0.592238 \\
\hline MZT2A & 0.000177 & 1.152776 \\
\hline FTH1 & 0.000189 & 0.948478 \\
\hline GATA6 & 0.000195 & 0.756302 \\
\hline TIMM21 & 0.00028 & 0.528606 \\
\hline PDHB & 0.000373 & 0.973631 \\
\hline ATP6V1D & 0.000455 & 0.531776 \\
\hline PSMB1 & 0.000497 & -0.80433 \\
\hline TRAPPC5 & 0.000584 & 1.354619 \\
\hline DENND5A & 0.000663 & 0.90012 \\
\hline HBB & 0.000788 & -5.45543 \\
\hline ABCF3 & 0.000789 & 1.080317 \\
\hline S100A1 & 0.000813 & 1.472048 \\
\hline COX6C & 0.000854 & 0.434718 \\
\hline ASB8 & 0.000864 & 0.483151 \\
\hline SEPW1 & 0.000884 & 0.425352 \\
\hline C19orf43 & 0.001003 & -0.58583 \\
\hline CSDE1 & 0.001139 & 0.58907 \\
\hline GNL3 & 0.001164 & -0.81165 \\
\hline RPLP1 & 0.001217 & 0.862792 \\
\hline HBA1 & 0.001351 & -3.4007 \\
\hline SDHC & 0.001403 & 0.739299 \\
\hline SCARB2 & 0.00142 & -0.49507 \\
\hline MCL1 & 0.001421 & -0.55364 \\
\hline ADIPOR1 & 0.001502 & 1.190279 \\
\hline HNRNPA2B1 & 0.001525 & 1.093875 \\
\hline NDUFB1 & 0.001829 & 0.339606 \\
\hline ANKRD36B & 0.001861 & -3.04052 \\
\hline NNT & 0.002013 & 1.040709 \\
\hline TTC21A & 0.002043 & -2.47755 \\
\hline SLC8A1 & 0.002115 & 0.685781 \\
\hline ATP5A1 & 0.002269 & 0.754705 \\
\hline GPR4 & 0.002359 & -1.58775 \\
\hline ARL6IP4 & 0.002399 & 1.532074 \\
\hline NEAT1 & 0.002411 & -1.10139 \\
\hline ETFB & 0.002431 & 1.068367 \\
\hline TOM1L2 & 0.002475 & 0.570172 \\
\hline & & \\
\hline
\end{tabular}




\begin{tabular}{|l|l|l|} 
TFE3 & 0.002656 & -0.80107 \\
\hline CCDC39 & 0.002766 & -3.18253 \\
\hline SMARCA1 & 0.002783 & 0.990988 \\
\hline ETFA & 0.003112 & 0.602531 \\
\hline GOT2 & 0.003261 & 0.596243 \\
\hline NCOA4 & 0.003347 & -1.25799 \\
\hline TSFM & 0.003479 & 1.156395 \\
\hline CAST & 0.003565 & 1.515 \\
\hline TACC2 & 0.003703 & 0.563731 \\
\hline HIST1H2AC & 0.003876 & -2.31733 \\
\hline NDUFS6 & 0.004104 & 0.487713 \\
\hline PTGDS & 0.004501 & 0.646333 \\
\hline MRPL41 & 0.004511 & 0.420448 \\
\hline ILK & 0.004515 & 1.653967 \\
\hline FH & 0.004539 & 0.588753 \\
\hline GIMAP6 & 0.004831 & -0.94894 \\
\hline PFDN1 & 0.00486 & -1.49717 \\
\hline LTBP1 & 0.004871 & -1.15454 \\
\hline C1QTNF1 & 0.004932 & -0.867 \\
\hline BOD1 & 0.005182 & -0.51195 \\
\hline ATP5C1 & 0.005275 & 0.33629 \\
\hline NONO & 0.005596 & 1.256768 \\
\hline AK1 & 0.005699 & 0.897919 \\
\hline TADA3 & 0.005769 & -0.71523 \\
\hline MPC1 & 0.00577 & 0.985969 \\
\hline TRAP1 & 0.005888 & 0.768733 \\
\hline C15orf59 & 0.006123 & 0.693259 \\
\hline PARVB & 0.006333 & 1.004308 \\
\hline COX7B & 0.006368 & 1.091807 \\
\hline UCKL1 & 0.006433 & -0.91676 \\
\hline MAGEF1 & 0.006657 & -1.50688 \\
\hline ANXA2 & 0.006722 & -0.7149 \\
\hline GABARAP & 0.006902 & 2.005869 \\
\hline UBE2Q1 & 0.007324 & -0.77789 \\
\hline TIMMDC1 & 0.007489 & 0.460374 \\
\hline MOB4 & 0.007926 & -2.45158 \\
\hline GPRASP2 & 0.007945 & -1.95928 \\
\hline ACAA1 & 0.008231 & 0.976931 \\
\hline MDH2 & 0.008315 & 1.042324 \\
\hline FXYD1 & 0.009307 & 1.676 \\
\hline MYL3 & 0.00939 & 0.551532 \\
\hline
\end{tabular}

\begin{tabular}{|l|l|l|} 
HNRNPH2 & 0.009874 & -2.03727 \\
\hline VSTM5 & 0.010137 & -1.45653 \\
\hline MRPL37 & 0.010439 & 0.571961 \\
\hline GABARAPL1 & 0.011234 & -0.46525 \\
\hline PLXDC2 & 0.011265 & -2.03282 \\
\hline SRSF1 & 0.01148 & -0.72824 \\
\hline TIMM8B & 0.012035 & -1.1086 \\
\hline GNB1 & 0.012427 & -0.47342 \\
\hline TCEAL8 & 0.012464 & -0.48375 \\
\hline LMNA & 0.012465 & -0.36963 \\
\hline LINC00657 & 0.012465 & -0.4487 \\
\hline ERCC1 & 0.012481 & -0.63265 \\
\hline S100A8 & 0.012523 & -0.84811 \\
\hline FAM46A & 0.012548 & -0.6472 \\
\hline NEMF & 0.012557 & -0.70149 \\
\hline ATP5E & 0.01258 & -0.94383 \\
\hline MRPL9 & 0.012593 & -0.62379 \\
\hline RRAGA & 0.012651 & -0.42521 \\
\hline NTMT1 & 0.012669 & -1.15378 \\
\hline POLR2J & 0.012691 & -1.02605 \\
\hline NPTN-IT1 & 0.012711 & -1.49524 \\
\hline SDF4 & 0.012714 & -0.47181 \\
\hline MIR4458HG & 0.012752 & -0.92531 \\
\hline RDH14 & 0.012763 & -1.08698 \\
\hline AAMDC & 0.012768 & 0.720372 \\
\hline MAT2A & 0.012773 & -3.98597 \\
\hline CD93 & 0.012803 & -0.59718 \\
\hline FBLN5 & 0.012826 & -0.55134 \\
\hline PDIA3P1 & 0.01289 & -0.57568 \\
\hline SPARCL1 & 0.012917 & -0.59801 \\
\hline C12orf57 & 0.013003 & -0.54987 \\
\hline TMEM178B & 0.013058 & 0.987441 \\
\hline TMEM14B & 0.013071 & -0.8565 \\
\hline TRIM35 & 0.013302 & -1.52477 \\
\hline MB & 0.013673 & -0.36649 \\
\hline FAM162A & 0.013689 & 0.502189 \\
\hline TNPO1 & 0.013703 & -0.65171 \\
\hline ZNRF2P1 & 0.013769 & -0.88818 \\
\hline ANAPC11 & 0.013918 & 1.566538 \\
\hline SHC1 & 0.014001 & -0.77541 \\
\hline IDH3B & 0.014044 & 0.416677 \\
\hline
\end{tabular}




\begin{tabular}{|l|l|l|} 
TUSC1 & 0.014086 & -0.59556 \\
\hline MAGED2 & 0.014172 & -0.74103 \\
\hline COX17 & 0.014284 & -1.71685 \\
\hline GLRX5 & 0.014313 & 0.701564 \\
\hline SEPN1 & 0.014316 & -0.44066 \\
\hline DEXI & 0.01443 & 1.425424 \\
\hline SERTAD3 & 0.014546 & -0.90384 \\
\hline NPPB & 0.014572 & -2.21326 \\
\hline TINAGL1 & 0.01468 & 0.714199 \\
\hline ATP5J & 0.014702 & 0.284947 \\
\hline AKAP17A & 0.014985 & -1.03412 \\
\hline TGFBR3 & 0.014996 & -0.6507 \\
\hline GMEB1 & 0.015111 & -1.08091 \\
\hline FOXO3 & 0.015136 & -0.76924 \\
\hline EHD2 & 0.015141 & -0.48756 \\
\hline ANAPC2 & 0.015146 & -1.12143 \\
\hline RNF185 & 0.01517 & -0.83125 \\
\hline FAM217B & 0.015182 & -0.79726 \\
\hline NCBP2-AS2 & 0.015262 & -0.9356 \\
\hline IPO8 & 0.015302 & -0.77556 \\
\hline CCNG1 & 0.015352 & -1.33632 \\
\hline GBGT1 & 0.015357 & -1.08937 \\
\hline RFPL4AL1 & 0.015363 & -1.82569 \\
\hline KLHDC3 & 0.015367 & -0.74213 \\
\hline RAB12 & 0.015422 & -0.67548 \\
\hline FANCI & 0.015449 & -1.70713 \\
\hline AP1S2 & 0.015457 & -0.78901 \\
\hline PTGES3 & 0.015534 & -0.50628 \\
\hline CD2BP2 & 0.015536 & -0.58723 \\
\hline PSMB2 & 0.01563 & -0.88634 \\
\hline RFTN1 & 0.015701 & -0.77574 \\
\hline HMGN4 & 0.015742 & -0.54851 \\
\hline A2M & 0.015751 & -0.41594 \\
\hline NES & 0.015887 & -0.88792 \\
\hline GNAT1 & 0.015907 & -1.57915 \\
\hline SPRY1 & 0.01593 & -0.99367 \\
\hline SOCS5 & 0.015948 & -1.25689 \\
\hline ERLEC1 & 0.016148 & -0.75281 \\
\hline PTBP3 & 0.016169 & -0.95234 \\
\hline SLIRP & 0.016174 & 0.781915 \\
\hline ZNF22 & 0.016207 & -0.71381 \\
\hline
\end{tabular}

\begin{tabular}{|l|l|l|} 
ACSM1 & 0.016208 & -2.13365 \\
\hline SMPX & 0.016208 & -0.5787 \\
\hline LARP1B & 0.016209 & -1.10904 \\
\hline ZSWIM1 & 0.01621 & -1.14273 \\
\hline PBDC1 & 0.016215 & -0.60672 \\
\hline SPRY4 & 0.016248 & -1.08951 \\
\hline NDUFB8 & 0.016282 & -0.7828 \\
\hline IFT52 & 0.016284 & -0.80741 \\
\hline NEDD8 & 0.016298 & -0.83441 \\
\hline IMMP1L & 0.016304 & -1.26851 \\
\hline RPS24 & 0.016319 & -0.68556 \\
\hline SYNPO & 0.016324 & -0.61107 \\
\hline UQCC1 & 0.016391 & -1.03014 \\
\hline PDIA4 & 0.016406 & -0.53875 \\
\hline STAT1 & 0.016494 & -0.6334 \\
\hline ARHGAP29 & 0.016538 & -0.89775 \\
\hline MOGS & 0.016616 & -0.63599 \\
\hline MT1A & 0.016679 & -1.31961 \\
\hline PPIG & 0.016724 & -0.638 \\
\hline TRAM1 & 0.016725 & -0.37237 \\
\hline LMF2 & 0.016726 & -0.67529 \\
\hline OLFM1 & 0.016731 & -0.87629 \\
\hline DHRS4L2 & 0.01678 & -1.16709 \\
\hline A4GALT & 0.016789 & -0.51533 \\
\hline MEF2D & 0.016819 & -0.5289 \\
\hline FKBP8 & 0.016863 & -0.58714 \\
\hline MYLIP & 0.016882 & -0.77689 \\
\hline SLC6A6 & 0.016902 & -1.15071 \\
\hline HTR2B & 0.016906 & -1.63789 \\
\hline C14orf166 & 0.016924 & 0.698352 \\
\hline GAS7 & 0.016998 & -0.83087 \\
\hline HNRNPDL & 0.01702 & -0.43099 \\
\hline MCCC2 & 0.017026 & -0.90751 \\
\hline TXN & 0.017065 & -0.49235 \\
\hline PIN4P1 & 0.017103 & -0.79958 \\
\hline POMP & 0.017144 & -0.38022 \\
\hline NCLN & 0.017161 & -0.6726 \\
\hline IGF2 & 0.017209 & -1.4621 \\
\hline TPM3 & 0.017233 & -0.81961 \\
\hline ANP32B & 0.017281 & -0.50937 \\
\hline PMEPA1 & 0.017309 & -0.74767 \\
\hline
\end{tabular}




\begin{tabular}{|l|l|l|} 
FAM219A & 0.017339 & -0.54698 \\
\hline RNMTL1 & 0.017371 & -0.74583 \\
\hline TMEM14C & 0.017412 & -0.60308 \\
\hline PUM2 & 0.017423 & -0.45941 \\
\hline ZFP36 & 0.017465 & -0.99179 \\
\hline SF1 & 0.017507 & -1.01606 \\
\hline FAM160A2 & 0.017523 & -0.96128 \\
\hline SERPINB1 & 0.017524 & -0.63034 \\
\hline DENND2A & 0.017611 & -1.00146 \\
\hline PEF1 & 0.017654 & -0.93352 \\
\hline EIF3I & 0.017689 & -0.81232 \\
\hline PLVAP & 0.017747 & -1.26576 \\
\hline LAMP2 & 0.017906 & -0.9885 \\
\hline ZNF146 & 0.017951 & -0.67501 \\
\hline SYPL1 & 0.017992 & -0.31657 \\
\hline STK38 & 0.018017 & -0.66665 \\
\hline EIF2B5 & 0.018254 & -0.5767 \\
\hline CDC42EP1 & 0.018263 & -0.60669 \\
\hline RBMS1 & 0.018418 & -0.81146 \\
\hline MEA1 & 0.018451 & -0.71157 \\
\hline $\begin{array}{l}\text { MAPKAPK5- } \\
\text { AS1 }\end{array}$ & 0.018459 & -0.94441 \\
\hline SERINC1 & 0.018497 & -0.664 \\
\hline PTX3 & 0.018539 & -1.36678 \\
\hline BCAS2 & 0.018574 & -0.83626 \\
\hline COL6A2 & 0.018589 & -0.40003 \\
\hline OSBP & 0.018598 & -1.05728 \\
\hline ARHGDIA & 0.018617 & -0.83641 \\
\hline USP48 & 0.018636 & -0.94575 \\
\hline RASSF3 & 0.018653 & -0.75999 \\
\hline HTRA1 & 0.018696 & -0.60734 \\
\hline PPIL4 & 0.018708 & -0.9021 \\
\hline H6PD & 0.01873 & -0.69978 \\
\hline FUCA2 & 0.018749 & -1.24515 \\
\hline AAR2 & 0.018794 & -0.91337 \\
\hline PIP5K1C & 0.018903 & -0.63887 \\
\hline SAMHD1 & 0.018941 & -0.5876 \\
\hline CNTRL & 0.018993 & -1.53438 \\
\hline DDX6 & 0.019062 & -0.66268 \\
\hline PIP4K2A & 0.019064 & -0.86742 \\
\hline SLC45A3 & 0.019074 & -1.48551 \\
\hline
\end{tabular}

\begin{tabular}{|l|l|l|} 
SAP18 & 0.019095 & -0.46939 \\
\hline RFNG & 0.019152 & -0.98073 \\
\hline DCAF7 & 0.019219 & -0.87273 \\
\hline PARK7 & 0.019226 & -0.60096 \\
\hline CRKL & 0.019327 & -1.12111 \\
\hline CMYA5 & 0.019486 & 0.409973 \\
\hline EDN1 & 0.01956 & -0.97511 \\
\hline APMAP & 0.019589 & -0.5634 \\
\hline PSIP1 & 0.019633 & -0.76878 \\
\hline ATP1A1 & 0.01965 & -1.06399 \\
\hline ARL6IP5 & 0.019765 & -0.47494 \\
\hline GTPBP6 & 0.019788 & -1.53881 \\
\hline ST13 & 0.019796 & -0.40785 \\
\hline RN7SL2 & 0.019873 & 1.481054 \\
\hline FAM114A1 & 0.019887 & -0.71223 \\
\hline CACFD1 & 0.01989 & -0.89916 \\
\hline METTL7B & 0.019927 & -1.4323 \\
\hline NEK7 & 0.020017 & 1.095569 \\
\hline RARA & 0.020098 & -0.75894 \\
\hline FAM127B & 0.020183 & -0.71564 \\
\hline KLHDC8B & 0.020214 & -0.67781 \\
\hline EIF5 & 0.020246 & -0.58398 \\
\hline TEAD4 & 0.02039 & -0.83812 \\
\hline RD3L & 0.020528 & -0.84725 \\
\hline SLC25A5 & 0.020744 & -0.24487 \\
\hline DENND6A & 0.020744 & -0.74386 \\
\hline CTNND1 & 0.020838 & -0.49509 \\
\hline LMBRD1 & 0.020879 & -0.75413 \\
\hline CRTAP & 0.021056 & -0.60928 \\
\hline MTURN & 0.021131 & -1.28667 \\
\hline CD59 & 0.021177 & -0.57223 \\
\hline MTPN & 0.021198 & -2.09273 \\
\hline APOL1 & 0.021276 & -0.78233 \\
\hline WDR61 & 0.021294 & -1.02132 \\
\hline TMEM204 & 0.021325 & -0.6529 \\
\hline UFM1 & 0.021325 & -0.57161 \\
\hline ELOVL1 & 0.021326 & -1.17358 \\
\hline H3F3B & 0.02141 & -0.61227 \\
\hline MKNK2 & 0.021436 & -0.56249 \\
\hline PYURF & 0.021461 & 1.332057 \\
\hline CKAP5 & 0.021646 & 1.106586 \\
\hline
\end{tabular}




\begin{tabular}{|l|l|l|} 
NDUFB9 & 0.021655 & 0.376823 \\
\hline ABL1 & 0.021665 & -0.5416 \\
\hline RPS5 & 0.021779 & 0.882141 \\
\hline CSRP3 & 0.021814 & -1.03479 \\
\hline PPP1R3F & 0.021921 & -0.75372 \\
\hline JAG1 & 0.021947 & -0.64807 \\
\hline CBY1 & 0.021961 & -0.81745 \\
\hline ALS2 & 0.0221 & -0.83072 \\
\hline BTG2 & 0.022147 & -0.54751 \\
\hline FSTL3 & 0.022169 & 1.277475 \\
\hline MGST2 & 0.022194 & 0.697938 \\
\hline SNRPN & 0.022304 & -1.23539 \\
\hline SRSF7 & 0.02232 & -1.06689 \\
\hline SLC35D2 & 0.022325 & -0.74864 \\
\hline NOP56 & 0.02236 & -0.59664 \\
\hline CHST7 & 0.022374 & -0.85819 \\
\hline TIMP3 & 0.02239 & -0.39229 \\
\hline OSER1 & 0.022407 & -0.54049 \\
\hline SLC19A2 & 0.022419 & -0.7377 \\
\hline TLE1 & 0.022419 & -0.82577 \\
\hline F11R & 0.022427 & -0.96876 \\
\hline DRAM2 & 0.022444 & -0.72639 \\
\hline ASMTL & 0.022448 & -1.53306 \\
\hline ABCB7 & 0.022454 & -0.73559 \\
\hline RBBP4 & 0.022462 & -0.89412 \\
\hline MAGI & 0.022467 & -1.14043 \\
\hline SLC7A2 & 0.02247 & -0.88932 \\
\hline UGDH & 0.022474 & -0.74032 \\
\hline C10orf71 & 0.022517 & 0.497432 \\
\hline POPDC2 & 0.022602 & 0.851896 \\
\hline TRIM54 & 0.022801 & 0.39224 \\
\hline PHB & 0.022854 & 0.58039 \\
\hline LDHB & 0.023095 & 0.331912 \\
\hline TAX1BP3 & 0.023178 & -1.44566 \\
\hline HAGH & 0.023181 & 0.522402 \\
\hline BCKDHA & 0.023183 & 1.361748 \\
\hline DGCR6L & 0.023394 & -0.86207 \\
\hline RBM18 & 0.023495 & -0.88136 \\
\hline GNL2 & 0.023609 & -0.56566 \\
\hline SAT1 & 0.023627 & -0.40087 \\
\hline ZNF648 & 0.023648 & -0.99854 \\
\hline & & \\
\hline
\end{tabular}

\begin{tabular}{|l|l|l|} 
ELK3 & 0.023721 & -0.97643 \\
\hline HLA-DRB1 & 0.023762 & -2.0529 \\
\hline RNF11 & 0.023794 & -0.66954 \\
\hline ILF2 & 0.023796 & -0.58265 \\
\hline AMOTL1 & 0.023841 & -0.70949 \\
\hline POM121L10P & 0.023984 & -3.57981 \\
\hline HSPA14 & 0.024087 & -0.88951 \\
\hline CES2 & 0.024136 & 0.651625 \\
\hline WDR89 & 0.024138 & -1.19626 \\
\hline FAM134B & 0.024207 & -0.88409 \\
\hline CPXCR1 & 0.024216 & -1.39867 \\
\hline ARHGEF15 & 0.024341 & -0.67695 \\
\hline MFAP3 & 0.024431 & -0.78174 \\
\hline PELI1 & 0.024498 & -1.17581 \\
\hline PITPNA & 0.024619 & -0.55463 \\
\hline CNIH4 & 0.02465 & -0.61098 \\
\hline SDHD & 0.024683 & -0.56053 \\
\hline CHTF8 & 0.024691 & -0.48262 \\
\hline FABP3 & 0.024869 & 0.596267 \\
\hline TJP2 & 0.024913 & -0.66178 \\
\hline C19orf53 & 0.02494 & -0.83764 \\
\hline HSD17B11 & 0.02501 & -0.69315 \\
\hline CLIC2 & 0.025025 & -0.88174 \\
\hline OIP5-AS1 & 0.025046 & -0.37826 \\
\hline TBCK & 0.025073 & -0.90682 \\
\hline TAF10 & 0.025111 & 0.9115 \\
\hline MYO1B & 0.025112 & -0.60631 \\
\hline TRPC4AP & 0.025144 & -0.4905 \\
\hline MAPRE1 & 0.025203 & 1.119034 \\
\hline CREM & 0.025267 & -2.14778 \\
\hline ALDH2 & 0.025329 & 0.862575 \\
\hline CYBRD1 & 0.025364 & -0.90081 \\
\hline BRK1 & 0.025368 & -0.6395 \\
\hline RPL9 & 0.025397 & -1.02426 \\
\hline TOPORS & 0.025448 & -0.93365 \\
\hline SCNM1 & 0.025512 & -0.93321 \\
\hline SH3BP4 & 0.025523 & -0.70647 \\
\hline EDNRB & 0.025631 & -0.52333 \\
\hline ZBED1 & 0.025655 & -1.19893 \\
\hline HNRNPF & 0.025691 & -0.95398 \\
\hline KLHDC2 & 0.025808 & 0.823827 \\
\hline & &
\end{tabular}




\begin{tabular}{|l|l|l|} 
RMDN1 & 0.025816 & 0.62209 \\
\hline CERK & 0.025916 & -0.34257 \\
\hline BOP1 & 0.025938 & -0.50992 \\
\hline UBR4 & 0.02598 & -0.87303 \\
\hline ANGPTL4 & 0.026011 & -0.9783 \\
\hline RPN2 & 0.026012 & -0.32802 \\
\hline CNPY3 & 0.02602 & -1.06098 \\
\hline TMEM165 & 0.026022 & 1.050255 \\
\hline IL33 & 0.026093 & -0.79908 \\
\hline NUMB & 0.02612 & -0.70918 \\
\hline NUDT15 & 0.026191 & -0.84827 \\
\hline GTF3A & 0.02623 & 0.395008 \\
\hline NUP153 & 0.026253 & -0.59072 \\
\hline TMEM258 & 0.026373 & 0.779827 \\
\hline C1QBP & 0.026389 & 0.445172 \\
\hline ARID4A & 0.026396 & -0.623 \\
\hline BLOC1S1 & 0.026446 & -0.8507 \\
\hline TNFAIP1 & 0.026486 & -1.42484 \\
\hline FNBP1 & 0.026615 & -0.5942 \\
\hline RPS27A & 0.026615 & -0.50182 \\
\hline MAF1 & 0.026624 & -0.37243 \\
\hline PCBP1 & 0.026636 & 1.265688 \\
\hline MYOZ2 & 0.026657 & -0.55598 \\
\hline AKAP12 & 0.026665 & -0.73199 \\
\hline YJEFN3 & 0.026669 & -0.97526 \\
\hline TWF1 & 0.026748 & -0.78106 \\
\hline AFG3L2 & 0.02694 & 0.504829 \\
\hline SNRPD3 & 0.026984 & -0.90152 \\
\hline MGC16275 & 0.027125 & -1.16985 \\
\hline LAMTOR3 & 0.027132 & -0.73631 \\
\hline ATE1 & 0.02715 & -0.82397 \\
\hline NFKBIB & 0.027203 & -0.63057 \\
\hline TP53RK & 0.027302 & -0.98484 \\
\hline LAMTOR2 & 0.027347 & 1.016552 \\
\hline C17orf58 & 0.027375 & -0.97783 \\
\hline CC2D1A & 0.02742 & -0.61838 \\
\hline DCUN1D5 & 0.027445 & -0.74856 \\
\hline KDR & 0.027446 & -1.01649 \\
\hline TCHH & 0.027454 & -1.91902 \\
\hline ANKRD10 & 0.027476 & -0.70009 \\
\hline TRAPPC2 & 0.027511 & -0.90358 \\
\hline & & \\
\hline
\end{tabular}

\begin{tabular}{|l|l|l|} 
SNTA1 & 0.027513 & -0.50956 \\
\hline KCNH2 & 0.027536 & 0.701117 \\
\hline CHCHD3 & 0.027589 & -1.8757 \\
\hline WAC & 0.027652 & -0.54735 \\
\hline RAI2 & 0.027658 & -0.5362 \\
\hline ILF3 & 0.027679 & -0.62026 \\
\hline UXT & 0.02774 & -0.443 \\
\hline VEZF1 & 0.027757 & -0.62697 \\
\hline MAOA & 0.027791 & -0.67088 \\
\hline PRKACA & 0.027812 & 0.296659 \\
\hline PRPF4B & 0.027885 & -0.56489 \\
\hline PALM & 0.027912 & -0.62806 \\
\hline ATG9A & 0.028019 & 1.094103 \\
\hline CNN3 & 0.028099 & -0.67041 \\
\hline NAT6 & 0.028176 & -1.26624 \\
\hline SRF & 0.028178 & 0.885317 \\
\hline SGCA & 0.028242 & 0.496243 \\
\hline EDF1 & 0.028375 & -0.81059 \\
\hline PDLIM7 & 0.028378 & -0.57968 \\
\hline VBP1 & 0.028457 & 0.418934 \\
\hline CCDC3 & 0.028467 & -1.26406 \\
\hline BCAM & 0.028571 & -0.62411 \\
\hline LAMTOR5 & 0.028573 & 0.967197 \\
\hline SEMA3G & 0.028594 & -0.65991 \\
\hline IQGAP1 & 0.028623 & -0.86433 \\
\hline ACTG1 & 0.028634 & -0.80754 \\
\hline PSPH & 0.028652 & -0.83482 \\
\hline GIMAP7 & 0.028666 & -0.55439 \\
\hline RBM17 & 0.028738 & -0.4027 \\
\hline ITM2B & 0.028784 & 0.887048 \\
\hline RBP7 & 0.028813 & -0.8759 \\
\hline KCTD11 & 0.028872 & -1.32702 \\
\hline RETN & 0.02888 & -0.97095 \\
\hline TIMP1 & 0.028892 & -1.00851 \\
\hline VAMP7 & 0.028929 & -1.15256 \\
\hline DIRAS3 & 0.028944 & -1.01864 \\
\hline MT2A & 0.029011 & 2.208718 \\
\hline KCTD9 & 0.029115 & -0.65237 \\
\hline GAS5 & 0.029155 & -0.84549 \\
\hline CCNI & 0.029275 & -0.64834 \\
\hline MATR3 & 0.029278 & 1.100102 \\
\hline & &
\end{tabular}




\begin{tabular}{|l|l|l|} 
LRRFIP2 & 0.029348 & 0.529637 \\
\hline TMEM123 & 0.029487 & -0.88018 \\
\hline SUPT4H1 & 0.02952 & -0.44879 \\
\hline HNRNPA3 & 0.029563 & -0.54081 \\
\hline COX6A2 & 0.029613 & 0.439694 \\
\hline ARL4A & 0.029713 & -0.87392 \\
\hline GCSHP3 & 0.029797 & 1.258673 \\
\hline COL6A1 & 0.029819 & -0.56227 \\
\hline NPY6R & 0.030041 & -0.38687 \\
\hline EEF1B2 & 0.03009 & -0.36574 \\
\hline MON1B & 0.0301 & -0.59648 \\
\hline IPO7 & 0.030296 & -0.4767 \\
\hline CTGF & 0.030305 & -1.18947 \\
\hline TUBA8 & 0.030328 & 1.599595 \\
\hline CSRNP1 & 0.030347 & -1.44187 \\
\hline MIR3147 & 0.030514 & -1.29703 \\
\hline HBQ1 & 0.03057 & -1.68139 \\
\hline ALG2 & 0.030579 & -1.47132 \\
\hline SUMO1P3 & 0.030593 & -0.87541 \\
\hline EFHD2 & 0.030605 & -1.574 \\
\hline RAB28 & 0.030613 & -0.47433 \\
\hline UTP11L & 0.030623 & -0.75675 \\
\hline C17orf62 & 0.030703 & -0.72837 \\
\hline DCAF11 & 0.030771 & 0.664734 \\
\hline NDRG2 & 0.030812 & 0.411303 \\
\hline GSTK1 & 0.030825 & 0.410994 \\
\hline BOLA3 & 0.030894 & 0.76345 \\
\hline FUNDC2 & 0.030896 & 0.748066 \\
\hline COPG1 & 0.030907 & -0.35733 \\
\hline ATP13A1 & 0.030911 & -0.67598 \\
\hline MRPL4 & 0.030916 & 0.673332 \\
\hline PRELID1 & 0.030932 & -0.60757 \\
\hline SDCBP & 0.031003 & -0.79818 \\
\hline DDX54 & 0.031005 & -0.98504 \\
\hline MAPK14 & 0.031107 & -0.55155 \\
\hline NBL1 & 0.03112 & -0.79322 \\
\hline RBL1 & 0.031134 & -0.87852 \\
\hline PHAX & 0.031149 & -0.50805 \\
\hline RBMS2 & 0.031211 & -0.61912 \\
\hline EFNB2 & 0.031279 & -0.5343 \\
\hline MEIS3P1 & 0.031286 & -0.62686 \\
\hline & &
\end{tabular}

\begin{tabular}{|l|l|l|} 
ANXA9 & 0.031306 & -0.63019 \\
\hline C12orf45 & 0.031349 & -0.74622 \\
\hline HSPB2 & 0.031367 & 1.211078 \\
\hline UFL1 & 0.031372 & -0.64279 \\
\hline UBB & 0.03149 & -0.31754 \\
\hline PPP2CA & 0.03149 & -0.71545 \\
\hline OSMR & 0.031521 & -0.68846 \\
\hline TSR1 & 0.031523 & -0.67585 \\
\hline SDF2L1 & 0.031523 & -0.49901 \\
\hline ST3GAL4 & 0.031551 & -0.43762 \\
\hline 5-Sep & 0.031609 & -1.92603 \\
\hline ZFP62 & 0.031615 & -0.59505 \\
\hline ZNF436 & 0.031663 & -0.85345 \\
\hline AGPAT2 & 0.031696 & -0.86246 \\
\hline NDUFC1 & 0.031717 & 0.831897 \\
\hline SLC50A1 & 0.031719 & -0.62206 \\
\hline GPR157 & 0.031724 & -0.82673 \\
\hline PPP1R15A & 0.031771 & -0.60994 \\
\hline NCBP2 & 0.031874 & -0.49276 \\
\hline CLIC5 & 0.031906 & 0.419942 \\
\hline SRSF4 & 0.032 & -0.63244 \\
\hline ECSIT & 0.032002 & 0.285857 \\
\hline PIR & 0.032021 & -0.62167 \\
\hline AP3S2 & 0.032022 & -0.97934 \\
\hline SESTD1 & 0.032037 & -1.46671 \\
\hline POT1 & 0.032046 & -0.87773 \\
\hline MYL9 & 0.032117 & 0.544829 \\
\hline ZFAND5 & 0.032133 & -0.76114 \\
\hline FAM129B & 0.032265 & -0.71755 \\
\hline HOMER2 & 0.032295 & 0.967059 \\
\hline PSAP & 0.032314 & 0.423236 \\
\hline NPPA & 0.032393 & -2.89607 \\
\hline UTP14C & 0.032418 & -0.94049 \\
\hline ATF4 & 0.032427 & -0.7596 \\
\hline PGM3 & 0.032448 & -0.79205 \\
\hline SCN2B & 0.032449 & -0.81912 \\
\hline SLC39A1 & 0.03246 & -0.37595 \\
\hline MIR3164 & 0.032498 & -0.87443 \\
\hline HIAT1 & 0.032549 & -0.40538 \\
\hline FAM73B & 0.032558 & -0.49709 \\
\hline TRIP4 & 0.032559 & -0.47846 \\
\hline & & \\
\hline
\end{tabular}




\begin{tabular}{|l|l|l|} 
SOCS4 & 0.032568 & -0.632 \\
\hline NDUFB5 & 0.032568 & 0.694388 \\
\hline NRAS & 0.032582 & -0.45616 \\
\hline PUS7 & 0.032602 & -0.5256 \\
\hline BMI1 & 0.032605 & -0.66256 \\
\hline EIF5AL1 & 0.032612 & -0.47659 \\
\hline PTPDC1 & 0.032649 & -0.62867 \\
\hline SEC14L1 & 0.03269 & -0.42509 \\
\hline KLF8 & 0.032702 & -2.63556 \\
\hline RBKS & 0.032737 & -0.81206 \\
\hline 6-Sep & 0.032737 & -0.63976 \\
\hline CSNK1E & 0.032738 & -0.40218 \\
\hline ESPNP & 0.032743 & -2.91051 \\
\hline PDLIM3 & 0.032775 & -0.56979 \\
\hline RBCK1 & 0.032778 & -0.43379 \\
\hline GADD45B & 0.032794 & -0.53031 \\
\hline THOC2 & 0.032848 & -0.54631 \\
\hline CCDC71L & 0.032897 & -0.64345 \\
\hline MPHOSPH8 & 0.032906 & -0.49484 \\
\hline THAP10 & 0.032907 & -0.73962 \\
\hline METTL9 & 0.032916 & -0.4444 \\
\hline ARL5B & 0.03292 & -0.53217 \\
\hline CMC4 & 0.032923 & 1.087351 \\
\hline ALPK3 & 0.032942 & -0.44771 \\
\hline RPL5 & 0.032976 & -0.27665 \\
\hline GCA & 0.033035 & -0.53446 \\
\hline PRRC2B & 0.033054 & -0.64584 \\
\hline GDI2 & 0.033085 & -0.41097 \\
\hline GBAS & 0.033094 & 0.476362 \\
\hline YWHAH & 0.033102 & -0.32555 \\
\hline KIF5B & 0.033119 & -0.23947 \\
\hline KLHL11 & 0.033127 & -0.78492 \\
\hline USP9X & 0.033127 & 0.696856 \\
\hline MIR6766 & 0.033181 & -1.11136 \\
\hline MTHFR & 0.033204 & -0.68037 \\
\hline GPR173 & 0.033213 & -1.28738 \\
\hline REXO4 & 0.033222 & -0.51427 \\
\hline SMIM13 & 0.033326 & -0.85932 \\
\hline PTTG1IP & 0.033331 & -0.3002 \\
\hline UHMK1 & 0.033356 & -0.51687 \\
\hline VPS29 & 0.033375 & -0.31233 \\
\hline
\end{tabular}

\begin{tabular}{|l|l|l|} 
GCHFR & 0.033375 & -0.75276 \\
\hline ENG & 0.033416 & -0.57515 \\
\hline AZIN1 & 0.033503 & -0.45722 \\
\hline HTATIP2 & 0.033517 & -0.54413 \\
\hline DDI2 & 0.03353 & -0.90494 \\
\hline CLINT1 & 0.033752 & -0.69543 \\
\hline BZW2 & 0.033752 & 0.659553 \\
\hline SNORD116- & & \\
24 & 0.033788 & -0.91374 \\
\hline COQ10A & 0.033817 & 0.483454 \\
\hline PKD1 & 0.033835 & 0.806112 \\
\hline P2RY8 & 0.033891 & -0.76635 \\
\hline CDC73 & 0.033939 & -0.47904 \\
\hline LDB1 & 0.033968 & -0.37502 \\
\hline HIF1A-AS2 & 0.033996 & -1.13095 \\
\hline RTN3 & 0.034032 & -0.49811 \\
\hline MSANTD3 & 0.034098 & -0.78896 \\
\hline EZR & 0.034111 & -0.37383 \\
\hline TMEM184B & 0.034171 & -0.44936 \\
\hline GABARAPL2 & 0.034229 & -0.26784 \\
\hline MRPS10 & 0.034247 & -0.5015 \\
\hline B2M & 0.034341 & -0.4811 \\
\hline CTNNAL1 & 0.034383 & -0.66536 \\
\hline CCL21 & 0.034425 & -1.99891 \\
\hline RPS3 & 0.034478 & -0.40227 \\
\hline ARGLU1 & 0.034482 & -0.49591 \\
\hline CX3CL1 & 0.034502 & -0.86239 \\
\hline TRIM44 & 0.034555 & -0.36939 \\
\hline PIAS1 & 0.034565 & 0.893026 \\
\hline FBXO21 & 0.034565 & 0.614546 \\
\hline MRPS15 & 0.034569 & 0.417615 \\
\hline KRBA2 & 0.034582 & 0.801596 \\
\hline IWS1 & 0.034587 & -0.36654 \\
\hline PREX1 & 0.034587 & -0.99797 \\
\hline HAX1 & 0.034595 & 0.354228 \\
\hline MRPL28 & 0.034597 & 0.448777 \\
\hline TBCE & 0.034601 & -0.45664 \\
\hline WWP1 & 0.034621 & 0.461459 \\
\hline KRTCAP2 & 0.034655 & 0.910631 \\
\hline CD302 & 0.034665 & -0.72754 \\
\hline ELP3 & & -0.55687 \\
\hline
\end{tabular}




\begin{tabular}{|l|l|l|} 
SCP2 & 0.034692 & -0.63056 \\
\hline TBCA & 0.034697 & -0.48424 \\
\hline PSMB7 & 0.034751 & -0.55098 \\
\hline LUC7L & 0.03476 & -0.66488 \\
\hline NDUFA5 & 0.034772 & 0.423046 \\
\hline POLDIP2 & 0.034779 & -0.49773 \\
\hline MIR6723 & 0.034877 & 1.031906 \\
\hline MFGE8 & 0.034907 & 0.580005 \\
\hline MSANTD4 & 0.034921 & -0.88134 \\
\hline PTPN18 & 0.034949 & -0.67142 \\
\hline C10orf32 & 0.034966 & -0.73241 \\
\hline HDLBP & 0.034983 & 0.316667 \\
\hline MACROD1 & 0.035013 & 0.505591 \\
\hline GNAQ & 0.035014 & -0.81753 \\
\hline ALDH9A1 & 0.035017 & -0.30472 \\
\hline EEF2 & 0.035026 & -0.20504 \\
\hline SPEN & 0.035042 & -0.50484 \\
\hline ZNF516 & 0.035045 & -0.59417 \\
\hline SLC7A8 & 0.035062 & -0.97264 \\
\hline IFNGR1 & 0.035065 & -0.64936 \\
\hline HIST1H4H & 0.035099 & -1.18434 \\
\hline TES & 0.035128 & -0.61007 \\
\hline VAMP8 & 0.035165 & -0.56384 \\
\hline BTF3 & 0.035173 & -0.36123 \\
\hline CKB & 0.035218 & 0.524482 \\
\hline KLC1 & 0.035226 & 1.002591 \\
\hline ATG4D & 0.035229 & -0.43271 \\
\hline SEL1L & 0.035252 & -0.44253 \\
\hline HS2ST1 & 0.035255 & -0.88546 \\
\hline PMP22 & 0.035297 & 0.650094 \\
\hline STOML2 & 0.035338 & 0.283353 \\
\hline RAD21 & 0.035372 & 0.391218 \\
\hline MCTS1 & 0.035379 & -1.18667 \\
\hline IQCB1 & 0.035386 & -0.57608 \\
\hline FIGF & 0.035388 & -1.15941 \\
\hline PPP1R2P3 & 0.035424 & -0.80427 \\
\hline PGRMC2 & 0.035455 & -0.42042 \\
\hline ARPP19 & 0.035456 & -0.37512 \\
\hline EIF2S2 & 0.035465 & -0.51712 \\
\hline QSOX1 & 0.035483 & 0.570679 \\
\hline NOP14 & 0.035532 & -0.42275 \\
\hline & &
\end{tabular}

\begin{tabular}{|l|l|l|} 
HINT2 & 0.035535 & 0.617047 \\
\hline ZNF652 & 0.035536 & -0.6381 \\
\hline NOLC1 & 0.03558 & -0.51815 \\
\hline SSBP4 & 0.035593 & -0.55126 \\
\hline RAP1A & 0.035604 & -0.42128 \\
\hline MFN2 & 0.035641 & -0.7874 \\
\hline TREX1 & 0.035693 & -0.7033 \\
\hline LY75 & 0.035726 & -1.27058 \\
\hline STK39 & 0.035728 & -0.61687 \\
\hline CEBPB & 0.035736 & -0.4419 \\
\hline AFAP1 & 0.035746 & -0.60729 \\
\hline COPG2 & 0.035784 & -1.62398 \\
\hline VPS4A & 0.035788 & 0.72838 \\
\hline HSPA5 & 0.035795 & 1.758879 \\
\hline TGFB1 & 0.035802 & -0.4841 \\
\hline CUTC & 0.035822 & -0.55877 \\
\hline SLC25A29 & 0.035854 & -0.61363 \\
\hline ABCC2 & 0.035875 & -0.87705 \\
\hline FAM84B & 0.035885 & -0.91927 \\
\hline GAS2L1 & 0.035893 & -0.66278 \\
\hline NFYB & 0.035894 & -0.4614 \\
\hline TAF13 & 0.035928 & -0.51109 \\
\hline CMAHP & 0.035959 & -0.86088 \\
\hline UBA52 & 0.035992 & -0.33506 \\
\hline PTMS & 0.035995 & -0.42609 \\
\hline MCAM & 0.036015 & 0.68023 \\
\hline ARF1 & 0.036022 & -0.33003 \\
\hline PCBD1 & 0.03609 & -0.40595 \\
\hline FOSL2 & 0.036099 & -0.74138 \\
\hline HNRNPD & 0.036109 & -0.44658 \\
\hline CDV3 & 0.036113 & -0.49624 \\
\hline PRELP & 0.036116 & -0.5701 \\
\hline SRPX & 0.036118 & -0.38798 \\
\hline THBS3 & 0.036129 & -0.82807 \\
\hline STAT3 & 0.036138 & -0.97262 \\
\hline PA2G4P4 & 0.036143 & -0.6932 \\
\hline AGPAT9 & 0.036148 & -0.65693 \\
\hline DBN1 & 0.036149 & -0.64856 \\
\hline ADPRM & 0.036149 & -0.72515 \\
\hline SENP5 & 0.036158 & -1.4376 \\
\hline SRGN & 0.036167 & -0.36339 \\
\hline & &
\end{tabular}




\begin{tabular}{|l|l|l|} 
NDUFA7 & 0.036176 & 1.782766 \\
\hline PHKA2 & 0.036178 & 0.764127 \\
\hline RARG & 0.0362 & -0.61422 \\
\hline IL6ST & 0.036204 & -0.34621 \\
\hline CANX & 0.036208 & -0.61134 \\
\hline MRPS36 & 0.036218 & -0.4783 \\
\hline SNORD59A & 0.036337 & -0.74182 \\
\hline PFKP & 0.036359 & 0.496966 \\
\hline TRIB1 & 0.036371 & 0.87391 \\
\hline TMEM120A & 0.036375 & -0.19527 \\
\hline GPATCH1 & 0.036451 & -0.81312 \\
\hline SMC2 & 0.03646 & -0.61163 \\
\hline COMMD1 & 0.036537 & 0.628965 \\
\hline LIMS2 & 0.036658 & 0.460768 \\
\hline USF1 & 0.036674 & -0.78181 \\
\hline TAX1BP1 & 0.036848 & 0.279872 \\
\hline C17orf89 & 0.036896 & -0.36637 \\
\hline TMA7 & 0.036909 & 0.615817 \\
\hline CCT3 & 0.036911 & -0.20703 \\
\hline TRIM55 & 0.036942 & -0.56786 \\
\hline C19orf18 & 0.037 & -0.74323 \\
\hline LRPAP1 & 0.037026 & -0.34356 \\
\hline FAM219B & 0.037046 & 0.717804 \\
\hline CD151 & 0.037071 & 0.472685 \\
\hline SPTLC2 & 0.037092 & -0.48621 \\
\hline APOD & 0.037102 & -0.89267 \\
\hline ADAM19 & 0.037114 & -0.63014 \\
\hline FAM21A & 0.037179 & -0.91629 \\
\hline PIGU & 0.037222 & -0.48946 \\
\hline IL3RA & 0.037239 & -1.12638 \\
\hline GNG5 & 0.037263 & -0.2129 \\
\hline TRUB2 & 0.037263 & 0.703895 \\
\hline CFL1 & 0.037271 & 0.512821 \\
\hline DDX19A & 0.037332 & -0.66125 \\
\hline FAM133DP & 0.037353 & -0.73167 \\
\hline MTX2 & 0.03736 & -0.42275 \\
\hline GLIPR2 & 0.037362 & -0.67926 \\
\hline SCAND1 & 0.037369 & -0.34499 \\
\hline IDH3A & 0.037432 & 0.65059 \\
\hline SBK2 & 0.037448 & -0.71113 \\
\hline PEX6 & 0.037461 & -0.49797 \\
\hline & &
\end{tabular}

\begin{tabular}{|l|l|l|} 
RNU6-7 & 0.037551 & -1.09696 \\
\hline RSBN1L & 0.03758 & -0.60546 \\
\hline IL1R1 & 0.037637 & -0.51121 \\
\hline OTUD1 & 0.037677 & -1.47141 \\
\hline NAA10 & 0.03768 & 1.111668 \\
\hline PDLIM5 & 0.037684 & -0.75044 \\
\hline C8orf4 & 0.03769 & -0.88364 \\
\hline XRCC6 & 0.037731 & -0.38855 \\
\hline MAP7D1 & 0.037767 & 0.542422 \\
\hline CEACAM3 & 0.037795 & -0.83183 \\
\hline RGL1 & 0.037807 & -1.12518 \\
\hline GLB1 & 0.037873 & -0.55021 \\
\hline RWDD1 & 0.037879 & 0.742311 \\
\hline OAT & 0.037903 & -0.55549 \\
\hline IGSF8 & 0.037906 & -0.43315 \\
\hline UBE2G2 & 0.037969 & 0.673819 \\
\hline C7orf55- & & \\
LUC7L2 & 0.037975 & -0.94018 \\
\hline NGRN & 0.038009 & 0.494214 \\
\hline RNF145 & 0.038072 & -0.50542 \\
\hline NDUFA12 & 0.038093 & 0.614495 \\
\hline NME4 & 0.038097 & -0.48284 \\
\hline SUN1 & 0.038098 & -0.45082 \\
\hline MMGT1 & 0.038143 & -0.55294 \\
\hline MAP4 & 0.038209 & -0.67629 \\
\hline RHOU & 0.03821 & -0.64759 \\
\hline HAND2 & 0.038228 & 0.564561 \\
\hline CUL4A & 0.038228 & 0.372001 \\
\hline KLHL41 & 0.038234 & -0.70921 \\
\hline PCF11 & 0.038267 & -0.5163 \\
\hline ECI2 & 0.038296 & 0.474806 \\
\hline ZMAT3 & 0.038366 & -0.65019 \\
\hline AIMP1 & 0.038396 & -0.40934 \\
\hline SRPK2 & 0.038399 & -0.56518 \\
\hline UBXN4 & 0.038413 & -0.4632 \\
\hline TRA2A & 0.038457 & -0.55665 \\
\hline ENAH & 0.038479 & -0.95222 \\
\hline GAPDH & 0.03848 & 0.825841 \\
\hline SORT1 & 0.038485 & -0.68327 \\
\hline SPTSSA & 0.038521 & -0.72997 \\
\hline TLN1 & 0.038524 & -0.39651 \\
\hline & & \\
\hline
\end{tabular}




\begin{tabular}{|l|l|l|} 
UQCRB & 0.038548 & 0.451971 \\
\hline RPL38 & 0.038549 & -0.33469 \\
\hline VGLL2 & 0.038578 & -1.19911 \\
\hline CHURC1 & 0.038615 & -0.7249 \\
\hline C22orf46 & 0.038672 & -0.80934 \\
\hline TACC1 & 0.038676 & -0.37055 \\
\hline P2RY2 & 0.038731 & -0.64591 \\
\hline EGFL7 & 0.038757 & -0.82415 \\
\hline PEBP1 & 0.038836 & -0.40075 \\
\hline HMGN3 & 0.038909 & -0.59267 \\
\hline NFIA & 0.038917 & -0.44679 \\
\hline GRIN2D & 0.038948 & -0.83435 \\
\hline UBIAD1 & 0.038962 & -0.89191 \\
\hline SPCS1 & 0.038992 & 0.574248 \\
\hline SELE & 0.039042 & -0.7997 \\
\hline PLCB4 & 0.039118 & -0.76018 \\
\hline TNFSF12 & 0.039249 & -0.67128 \\
\hline GOLGA3 & 0.039346 & -0.58309 \\
\hline RTN4 & 0.039426 & -1.07648 \\
\hline ARFGAP3 & 0.039465 & -0.59693 \\
\hline GSTP1 & 0.039562 & -0.27793 \\
\hline TAF7 & 0.039646 & -0.23116 \\
\hline HIF1A & 0.039666 & -0.79076 \\
\hline ZMIZ1 & 0.039673 & -0.69877 \\
\hline GFM1 & 0.039675 & 0.593327 \\
\hline LINC00961 & 0.039683 & -0.63191 \\
\hline TMEM100 & 0.039693 & -0.62459 \\
\hline EMILIN1 & 0.039703 & -0.57114 \\
\hline CDC42 & 0.039715 & -0.44212 \\
\hline CNRIP1 & 0.039717 & -0.8034 \\
\hline CXCL1 & 0.039718 & -1.11979 \\
\hline RFC1 & 0.039781 & -0.48255 \\
\hline MFAP4 & 0.039794 & -0.52445 \\
\hline FAM168A & 0.039818 & -0.41783 \\
\hline ARL2BP & 0.039899 & 1.290096 \\
\hline NAALADL2- & & \\
\hline AS3 & 0.039902 & -0.92872 \\
\hline TEST4 & 0.039922 & -1.08126 \\
\hline ETS2 & 0.039962 & -0.44151 \\
\hline LRBA & 0.040005 & 0.83503 \\
\hline
\end{tabular}

\begin{tabular}{|l|l|l|} 
GPRASP1 & 0.040016 & -0.74586 \\
\hline STK25 & 0.040145 & -0.47793 \\
\hline VSTM2L & 0.04021 & -0.86326 \\
\hline EIF3K & 0.040228 & 0.330053 \\
\hline LLPH & 0.040231 & -0.65674 \\
\hline KIDINS220 & 0.040251 & -0.33558 \\
\hline SPOP & 0.040271 & -0.39701 \\
\hline MED18 & 0.040278 & -0.57297 \\
\hline ART4 & 0.04032 & -0.71393 \\
\hline GNA13 & 0.040322 & -0.90702 \\
\hline POU3F4 & 0.040361 & -0.92947 \\
\hline PSMB4 & 0.040406 & -0.2754 \\
\hline YIPF5 & 0.040414 & -0.54419 \\
\hline C19orf47 & 0.040417 & 0.457919 \\
\hline WIPI1 & 0.040437 & 0.488378 \\
\hline AP1M1 & 0.040449 & 0.584028 \\
\hline SPRYD3 & 0.040467 & -0.29592 \\
\hline SCO1 & 0.040469 & 0.476124 \\
\hline HIBCH & 0.040508 & 0.61161 \\
\hline LAMC2 & 0.040528 & -0.75161 \\
\hline ADNP & 0.040531 & -0.51295 \\
\hline C11orf68 & 0.040532 & -0.37281 \\
\hline BTN2A3P & 0.040561 & -0.8744 \\
\hline RAVER2 & 0.040586 & 0.4035 \\
\hline PPP1R16A & 0.040596 & 0.710287 \\
\hline DYNC1LI1 & 0.040613 & 0.455161 \\
\hline PSMC1 & 0.040634 & 0.78906 \\
\hline TP53BP2 & 0.040641 & -0.57646 \\
\hline UBTD2 & 0.040645 & -0.42758 \\
\hline FAM214B & 0.04066 & -0.55939 \\
\hline KLHL31 & 0.040682 & -0.96742 \\
\hline ATP5J2 & 0.040698 & 1.053234 \\
\hline RNF144B & 0.040792 & -0.53885 \\
\hline LPL & 0.040803 & -0.43864 \\
\hline PRMT2 & 0.040809 & -0.45257 \\
\hline CHCHD4 & 0.04081 & 0.979294 \\
\hline RPL18A & 0.040965 & 0.921168 \\
\hline CLNS1A & 0.040977 & 0.537933 \\
\hline HHATL & 0.041005 & 0.690944 \\
\hline MLLT1 & 0.041049 & -0.49458 \\
\hline ICA1L & 0.041076 & -0.64985 \\
\hline & & \\
\hline
\end{tabular}




\begin{tabular}{|l|l|l|} 
TMEM205 & 0.041111 & 0.656153 \\
\hline H2AFY & 0.041137 & 0.600628 \\
\hline SKI & 0.041146 & 0.527366 \\
\hline GRTP1 & 0.041158 & -0.73563 \\
\hline PRPF6 & 0.041213 & -0.3822 \\
\hline LYSMD1 & 0.041222 & -0.51025 \\
\hline GNAS & 0.041232 & 0.18082 \\
\hline ABCC4 & 0.041241 & -0.73659 \\
\hline NHP2L1 & 0.041246 & 0.325181 \\
\hline USF2 & 0.041262 & 0.461716 \\
\hline SMARCA5 & 0.041267 & -0.54153 \\
\hline AKAP8 & 0.041305 & 0.719672 \\
\hline ITGA6 & 0.041332 & -0.38656 \\
\hline CHMP2B & 0.041367 & -0.4139 \\
\hline PSMB6 & 0.041415 & 0.38315 \\
\hline RNF146 & 0.041492 & -0.37161 \\
\hline SZRD1 & 0.041493 & -0.30777 \\
\hline TMEM59 & 0.041521 & 0.442592 \\
\hline LZTS1 & 0.041571 & -0.84388 \\
\hline CLCC1 & 0.041678 & -0.7063 \\
\hline HADHB & 0.04173 & 0.454091 \\
\hline C1GALT1C1 & 0.041735 & -0.50868 \\
\hline FAM3C & 0.041765 & 0.732974 \\
\hline ANO5 & 0.04182 & 0.49683 \\
\hline CCL2 & 0.041833 & 0.861443 \\
\hline TRAM2 & 0.041837 & -0.55606 \\
\hline FXYD6 & 0.041837 & -0.61561 \\
\hline HSPA9 & 0.041839 & 0.33245 \\
\hline ATXN10 & 0.041858 & -0.31479 \\
\hline CTNNA1 & 0.041888 & 0.216737 \\
\hline SPRYD4 & 0.04193 & 0.677688 \\
\hline PKM & 0.041946 & 0.538092 \\
\hline LTV1 & 0.041958 & -0.48423 \\
\hline SDHA & 0.041978 & 0.649552 \\
\hline DCTN3 & 0.041992 & 0.483169 \\
\hline CMTM8 & 0.042006 & -0.53464 \\
\hline KDM5C & 0.042023 & -0.45856 \\
\hline PLS3 & 0.042088 & -0.59148 \\
\hline TCAP & 0.042106 & -0.13006 \\
\hline FXYD5 & 0.04211 & -0.74162 \\
\hline PTPN11 & 0.042125 & -0.40738 \\
\hline
\end{tabular}

\begin{tabular}{|l|l|l|} 
STX12 & 0.042183 & -1.23808 \\
\hline ZNF777 & 0.042188 & -0.65165 \\
\hline TEK & 0.042213 & -0.66509 \\
\hline HSPB6 & 0.04228 & 0.654175 \\
\hline KPNA6 & 0.042351 & 0.521901 \\
\hline ESF1 & 0.042366 & -0.4259 \\
\hline EID1 & 0.042432 & -0.4688 \\
\hline DENND5B- & & \\
AS1 & 0.042446 & -0.84422 \\
\hline CYB5R1 & 0.042453 & 0.622642 \\
\hline WASF2 & 0.042467 & -0.61661 \\
\hline RAMP3 & 0.04247 & -0.60506 \\
\hline ACTA1 & 0.042476 & -1.1199 \\
\hline DDX42 & 0.042477 & -0.42944 \\
\hline MYO18B & 0.042532 & -0.40501 \\
\hline ZCCHC24 & 0.04263 & -0.4355 \\
\hline HSPA6 & 0.042634 & -1.46953 \\
\hline GPR137B & 0.042676 & -0.41419 \\
\hline LPCAT3 & 0.042718 & 0.759429 \\
\hline PXYLP1 & 0.042773 & -0.70605 \\
\hline MBTPS1 & 0.042804 & 0.439015 \\
\hline ABHD14B & 0.042806 & -0.79084 \\
\hline LAMB1 & 0.042876 & -0.23987 \\
\hline PROS1 & 0.042894 & -0.925 \\
\hline RNF10 & 0.042923 & 0.322112 \\
\hline PPIA & 0.042948 & -0.39158 \\
\hline ULK1 & 0.042966 & 0.844633 \\
\hline WDR5 & 0.043029 & -0.35205 \\
\hline SGTA & 0.043111 & 0.405405 \\
\hline NR1D2 & 0.043119 & -0.36619 \\
\hline PSMB5 & 0.043129 & 0.264588 \\
\hline DNAJC11 & 0.043135 & 0.437261 \\
\hline SERPINH1 & 0.043145 & -0.564 \\
\hline RPA1 & 0.043152 & -0.34369 \\
\hline SPTAN1 & 0.043153 & -0.35344 \\
\hline ZBTB21 & 0.043154 & -0.573 \\
\hline S100P & 0.043155 & -0.62072 \\
\hline ANKRD11 & 0.043157 & -0.4622 \\
\hline MIR621 & 0.043158 & -0.65688 \\
\hline RPL28 & 0.04316 & -0.35673 \\
\hline MED4 & 0.043166 & -0.44131 \\
\hline & & \\
\hline
\end{tabular}




\begin{tabular}{|l|l|l|} 
CCNL2 & 0.043166 & -0.60767 \\
\hline BACE2 & 0.043168 & -0.47492 \\
\hline ST3GAL6 & 0.043173 & -0.37651 \\
\hline NDUFAF1 & 0.043183 & -0.445 \\
\hline LRRC2 & 0.043188 & -0.51305 \\
\hline ATP5SL & 0.04319 & 0.429372 \\
\hline TXNRD1 & 0.043213 & -0.39736 \\
\hline PAFAH2 & 0.04322 & -0.49065 \\
\hline HSPA8 & 0.043225 & -0.35936 \\
\hline ISCA1 & 0.043227 & -0.37738 \\
\hline CRIP2 & 0.043242 & 0.308317 \\
\hline SPOPL & 0.043249 & -0.48301 \\
\hline RHPN2 & 0.043263 & -0.6109 \\
\hline IRS1 & 0.043275 & -0.49894 \\
\hline CERS6 & 0.043281 & 0.829045 \\
\hline SLC35B3 & 0.0433 & -0.43175 \\
\hline PRICKLE3 & 0.043329 & -0.45225 \\
\hline TRIM65 & 0.043333 & -0.51273 \\
\hline WAC-AS1 & 0.04334 & -0.4838 \\
\hline B4GALT1 & 0.043353 & -0.63432 \\
\hline FKBP5 & 0.043381 & -0.51771 \\
\hline SLC35A2 & 0.043402 & -0.41879 \\
\hline RIMS1 & 0.043419 & -0.72291 \\
\hline ARV1 & 0.043446 & -0.4904 \\
\hline SNORD63 & 0.043456 & -0.60599 \\
\hline PANX1 & 0.043457 & -0.64611 \\
\hline MYH7 & 0.043476 & 0.725402 \\
\hline ADPRH & 0.043478 & -0.73527 \\
\hline HCFC1 & 0.043483 & -0.44703 \\
\hline GTPBP1 & 0.043497 & -0.39791 \\
\hline UBE2A & 0.043511 & 0.435335 \\
\hline UQCR11 & 0.043526 & 0.768241 \\
\hline TMEM70 & 0.043542 & -0.43259 \\
\hline CYC1 & 0.04356 & -0.51256 \\
\hline RHBDD1 & 0.043564 & -0.53431 \\
\hline POLE3 & 0.043578 & -0.30923 \\
\hline XPC & 0.043598 & -0.47842 \\
\hline LINC00998 & 0.043603 & 0.544187 \\
\hline ELK1 & 0.043625 & -0.39441 \\
\hline FOS & 0.043634 & -1.05858 \\
\hline NCKIPSD & 0.043648 & -0.40907 \\
\hline & &
\end{tabular}

\begin{tabular}{|l|l|l|} 
ZNHIT2 & 0.043652 & -0.96854 \\
\hline CASP3 & 0.043676 & -0.66391 \\
\hline DENND4C & 0.043721 & -0.4932 \\
\hline RBMXL1 & 0.043723 & -0.45167 \\
\hline RPS14 & 0.043761 & 0.724838 \\
\hline CALHM2 & 0.04377 & -0.4685 \\
\hline FAM210B & 0.043793 & -0.36254 \\
\hline ATP8B2 & 0.043809 & -0.42195 \\
\hline CTNNBL1 & 0.043831 & -0.42786 \\
\hline TGFB3 & 0.043859 & -0.45892 \\
\hline ROCK2 & 0.04386 & -0.38353 \\
\hline SMIM3 & 0.043864 & -0.3364 \\
\hline FAM8A1 & 0.043881 & -0.428 \\
\hline SCO2 & 0.043892 & -0.83004 \\
\hline DAAM2 & 0.043894 & -0.50534 \\
\hline FAM78B & 0.043911 & -0.62746 \\
\hline DNMBP & 0.043928 & -0.53882 \\
\hline MYO10 & 0.04393 & -0.53448 \\
\hline HDAC1 & 0.043974 & -0.57812 \\
\hline UPP1 & 0.043985 & -0.56422 \\
\hline LSM3 & 0.043996 & 0.678043 \\
\hline EPHA4 & 0.044019 & -0.5129 \\
\hline BCAR3 & 0.044029 & -0.79978 \\
\hline TRIP11 & 0.044051 & -0.38457 \\
\hline KLF7 & 0.044053 & -0.39001 \\
\hline MED6 & 0.044066 & -0.68885 \\
\hline CLUH & 0.044091 & 0.533426 \\
\hline DUSP27 & 0.044104 & -0.54408 \\
\hline TSC1 & 0.044115 & -0.53249 \\
\hline CDKN1A & 0.044115 & -0.42907 \\
\hline DGKE & 0.044135 & -0.55624 \\
\hline DYNLT3 & 0.044156 & -0.34186 \\
\hline NFIB & 0.044163 & -0.29183 \\
\hline STX5 & 0.044175 & -0.40774 \\
\hline PSMD11 & 0.044179 & 0.974021 \\
\hline CS & 0.044195 & 0.634064 \\
\hline CYP20A1 & 0.044198 & -0.60404 \\
\hline PPP1R17 & 0.044203 & -1.20029 \\
\hline FAM98B & 0.044205 & -0.46231 \\
\hline C1orf43 & 0.044205 & -0.23152 \\
\hline DPM1 & 0.044214 & -0.44745 \\
\hline & &
\end{tabular}




\begin{tabular}{|l|l|l|} 
INTS10 & 0.044233 & -0.43167 \\
\hline CRIPT & 0.04425 & -0.52125 \\
\hline SYT9 & 0.044262 & -0.71172 \\
\hline SPSB1 & 0.044285 & -0.41671 \\
\hline ODC1 & 0.044311 & -0.25745 \\
\hline SAYSD1 & 0.044315 & -0.49224 \\
\hline TECR & 0.044317 & 0.682883 \\
\hline CNBP & 0.044329 & -0.36659 \\
\hline CD68 & 0.044368 & 0.939055 \\
\hline GAR1 & 0.044412 & -0.42803 \\
\hline PPP1CC & 0.044417 & 0.660556 \\
\hline DANCR & 0.044424 & 0.485809 \\
\hline HSD3B7 & 0.044425 & -0.59878 \\
\hline DDR2 & 0.044432 & -0.52046 \\
\hline MYLK3 & 0.044447 & -0.4339 \\
\hline CREB1 & 0.04448 & -0.4684 \\
\hline PPP6R2 & 0.044507 & -0.32101 \\
\hline NSMF & 0.044562 & -0.43373 \\
\hline TRMT61A & 0.044654 & -0.44573 \\
\hline GATAD2A & 0.044678 & -0.36954 \\
\hline TMEM167A & 0.044683 & -0.51829 \\
\hline FBXL7 & 0.044687 & -0.37815 \\
\hline ARRDC1 & 0.044708 & -0.44123 \\
\hline RHAG & 0.044719 & -0.83399 \\
\hline S100A9 & 0.044725 & -0.93497 \\
\hline SFTPB & 0.044732 & -0.92639 \\
\hline THBS2 & 0.044745 & -0.66519 \\
\hline BLOC1S2 & 0.044748 & -0.37961 \\
\hline IKZF5 & 0.044775 & -0.51802 \\
\hline NUPL1 & 0.044795 & -0.41339 \\
\hline ZBTB44 & 0.044803 & -0.42604 \\
\hline MTIF3 & 0.044814 & -0.29802 \\
\hline CALM3 & 0.044816 & -0.26617 \\
\hline CFDP1 & 0.04486 & -0.3963 \\
\hline VPS28 & 0.044971 & 0.682919 \\
\hline NOP10 & 0.044991 & -0.32865 \\
\hline DMWD & 0.044996 & -0.36496 \\
\hline HYPK & 0.045092 & 0.872538 \\
\hline NR2F2 & 0.045107 & -0.51244 \\
\hline MSMP & 0.045127 & 0.807117 \\
\hline GPD2 & 045132 & -0.62732 \\
\hline
\end{tabular}

\begin{tabular}{|l|l|l|} 
KLHL38 & 0.045138 & 0.335281 \\
\hline ZNF324B & 0.045164 & -0.84753 \\
\hline NDUFS3 & 0.045181 & 0.280477 \\
\hline RAI14 & 0.045196 & -0.5097 \\
\hline GPKOW & 0.045223 & -0.35042 \\
\hline TRIM38 & 0.045223 & -0.5031 \\
\hline SBDS & 0.045268 & -0.21392 \\
\hline EMCN & 0.045297 & -0.39739 \\
\hline FTL & 0.045301 & -0.67871 \\
\hline AKT1S1 & 0.045308 & -0.3399 \\
\hline EFEMP1 & 0.04534 & -0.72991 \\
\hline TM9SF3 & 0.045344 & -0.294 \\
\hline RPS12 & 0.04535 & -0.36671 \\
\hline MINPP1 & 0.045369 & -0.5057 \\
\hline SGK223 & 0.04538 & -0.59039 \\
\hline BTBD10 & 0.045469 & -1.28796 \\
\hline THAP9-AS1 & 0.045504 & -0.54543 \\
\hline LPAR1 & 0.045525 & -0.46803 \\
\hline MAPK8IP3 & 0.045532 & -0.58917 \\
\hline XPO7 & 0.045555 & -0.35552 \\
\hline ARHGAP30 & 0.045572 & -0.69869 \\
\hline GPATCH11 & 0.045615 & -0.4265 \\
\hline LSM1 & 0.045632 & 0.470032 \\
\hline CXorf36 & 0.04564 & -0.52947 \\
\hline PLA2G2A & 0.045658 & -2.64705 \\
\hline DYRK2 & 0.045658 & -0.48669 \\
\hline CMAS & 0.04569 & -0.32507 \\
\hline ATXN3 & 0.04571 & -2.43236 \\
\hline AAK1 & 0.045716 & -1.78202 \\
\hline TUBA1B & 0.045721 & 0.712811 \\
\hline FAF1 & 0.045739 & 0.416979 \\
\hline TCEAL7 & 0.045743 & -0.55323 \\
\hline ENO2 & 0.045746 & -0.61311 \\
\hline NUDT16P1 & 0.045758 & -0.5736 \\
\hline CTTNBP2NL & 0.045785 & -0.53209 \\
\hline ZFYVE27 & 0.045792 & -0.45599 \\
\hline PRKCDBP & 0.045832 & -0.26048 \\
\hline DGAT1 & 0.045874 & -0.40077 \\
\hline LRRN4CL & 0.045889 & -0.74009 \\
\hline AC007392.3 & 0.045933 & -0.82613 \\
\hline DUSP1 & 0.045995 & -0.83021 \\
\hline
\end{tabular}




\begin{tabular}{|l|l|l|} 
ATN1 & 0.046005 & -0.36858 \\
\hline DNAJB1 & 0.046029 & -0.62176 \\
\hline CD81 & 0.046032 & 0.616659 \\
\hline PRDX2 & 0.046318 & 0.610194 \\
\hline RPL14 & 0.046364 & 0.820702 \\
\hline MIR568 & 0.046373 & -0.46123 \\
\hline SIK3 & 0.046385 & -0.53583 \\
\hline PBX4 & 0.046398 & -0.8201 \\
\hline CHMP7 & 0.04643 & -0.32032 \\
\hline PRKAA2 & 0.046432 & -0.42629 \\
\hline NEDD9 & 0.046449 & -0.52443 \\
\hline COPE & 0.046456 & 0.427074 \\
\hline DLGAP4 & 0.046479 & -0.46245 \\
\hline SLC11A2 & 0.046479 & -0.71511 \\
\hline PSMD8 & 0.046502 & -0.32555 \\
\hline DAPK2 & 0.046529 & 0.741447 \\
\hline SEC24A & 0.046569 & -0.50265 \\
\hline MYCT1 & 0.046578 & -0.54874 \\
\hline ZNF793 & 0.046632 & -0.78982 \\
\hline DNAJA2 & 0.046654 & -0.309 \\
\hline E2F6 & 0.0467 & -0.50392 \\
\hline RAB2A & 0.046706 & -0.35634 \\
\hline APP & 0.046741 & -0.19958 \\
\hline SLC25A37 & 0.046742 & -0.54723 \\
\hline INPP5J & 0.046762 & -0.45787 \\
\hline MRPL51 & 0.046781 & 0.399561 \\
\hline MAML1 & 0.046807 & -0.46279 \\
\hline LRRC14B & 0.046813 & 0.601274 \\
\hline FBXO7 & 0.04684 & -0.33304 \\
\hline RAB39B & 0.046864 & -0.6923 \\
\hline SNAPC2 & 0.046896 & -0.41126 \\
\hline ATP5H & 0.046897 & 1.01046 \\
\hline 7-Mar & 0.046901 & -0.49283 \\
\hline MAP2K7 & 0.046903 & -0.36906 \\
\hline PLAA & 0.046927 & -0.52849 \\
\hline CALCOCO1 & 0.047034 & 0.655995 \\
\hline ATP2B4 & 0.047037 & -0.47315 \\
\hline YAE1D1 & 0.047064 & -0.52899 \\
\hline SRL & 0.047072 & -0.49114 \\
\hline SLC29A3 & 0.047088 & -0.56336 \\
\hline PPP1R15B & 0.047125 & -0.66899 \\
\hline
\end{tabular}

\begin{tabular}{|l|l|l|} 
RPL41 & 0.047202 & -0.46263 \\
\hline CMC2 & 0.04727 & 0.928306 \\
\hline KANK2 & 0.047282 & 0.397004 \\
\hline KIAA0368 & 0.047288 & -0.21364 \\
\hline NDUFA13 & 0.047299 & 0.553808 \\
\hline DHRS3 & 0.047324 & -0.49842 \\
\hline COMMD10 & 0.047459 & -0.75317 \\
\hline ATP6AP2 & 0.047549 & -0.2996 \\
\hline MRPS6 & 0.047567 & 0.605533 \\
\hline TP53 & 0.04764 & -0.47344 \\
\hline INPP5A & 0.047664 & 0.353673 \\
\hline HIVEP1 & 0.047682 & -0.57953 \\
\hline GRHPR & 0.047712 & 0.471827 \\
\hline YDJC & 0.047741 & -0.4309 \\
\hline LRRC47 & 0.047796 & -0.28552 \\
\hline SPANXC & 0.047797 & -0.76043 \\
\hline DOCK1 & 0.047808 & -0.46818 \\
\hline NEXN & 0.04781 & -0.31415 \\
\hline MLIP & 0.047821 & 0.32587 \\
\hline PCYT1A & 0.047841 & 0.625988 \\
\hline KTN1-AS1 & 0.047945 & -0.62535 \\
\hline C1orf52 & 0.04795 & -0.45743 \\
\hline PCNXL2 & 0.047952 & -0.98865 \\
\hline CIAPIN1 & 0.047955 & -0.35584 \\
\hline SFXN1 & 0.047968 & -0.43105 \\
\hline THBS1 & 0.048008 & -0.37739 \\
\hline LTBP4 & 0.048019 & -0.40618 \\
\hline FAM195A & 0.048103 & 0.683693 \\
\hline CTNNB1 & 0.048125 & -0.36302 \\
\hline FLAD1 & 0.048152 & -0.44251 \\
\hline ATP6V0C & 0.048154 & 0.795255 \\
\hline UQCRC2 & 0.048197 & 0.327542 \\
\hline MAFG & 0.048208 & -0.65158 \\
\hline TPI1 & 0.048222 & 0.369575 \\
\hline ZNF768 & 0.048268 & -0.4432 \\
\hline FAM222B & 0.048367 & -0.55818 \\
\hline CRIM1 & 0.048377 & -0.4341 \\
\hline WDR26 & 0.048381 & 0.434243 \\
\hline TIMM50 & 0.048402 & 0.469425 \\
\hline NMRK2 & 0.048413 & 0.585886 \\
\hline DDB1 & 0.048418 & 0.390274 \\
\hline
\end{tabular}




\begin{tabular}{|l|l|l|} 
CWF19L2 & 0.048419 & -0.60657 \\
\hline GOLGA1 & 0.048422 & 0.732444 \\
\hline MORF4L2 & 0.048428 & -0.51525 \\
\hline JAK1 & 0.048446 & 0.507475 \\
\hline RPRD1B & 0.048459 & -0.50933 \\
\hline MAGOH & 0.048468 & -0.30745 \\
\hline SNORA33 & 0.048468 & -1.16312 \\
\hline NAAA & 0.048474 & -0.45585 \\
\hline CD1D & 0.04848 & -0.663 \\
\hline SOAT1 & 0.048506 & -0.50051 \\
\hline SPG7 & 0.048523 & 0.461449 \\
\hline THBS4 & 0.048544 & 1.196572 \\
\hline MTHFD1 & 0.048549 & 0.512123 \\
\hline JAZF1 & 0.048565 & -0.63113 \\
\hline NUCKS1 & 0.048583 & -0.33916 \\
\hline ZSCAN26 & 0.04859 & -0.48147 \\
\hline CLPTM1 & 0.048591 & 0.37098 \\
\hline MIR3936 & 0.048604 & -0.71114 \\
\hline DFFA & 0.048607 & -0.45858 \\
\hline TRIM24 & 0.048612 & 0.630467 \\
\hline PECAM1 & 0.048628 & -0.53376 \\
\hline HSBP1 & 0.048629 & 0.504809 \\
\hline SSH1 & 0.048667 & -0.62376 \\
\hline CLEC10A & 0.048724 & -0.78745 \\
\hline GOLGA5 & 0.048731 & -0.45546 \\
\hline TXNDC15 & 0.048733 & -0.45751 \\
\hline GRAMD4 & 0.048737 & -0.54644 \\
\hline CHTOP & 0.048751 & -0.42894 \\
\hline ASB1 & 0.048754 & -0.4207 \\
\hline C4orf27 & 0.048773 & -0.39327 \\
\hline CDH2 & 0.048778 & -0.28261 \\
\hline PARS2 & 0.048787 & -0.68577 \\
\hline TMEM181 & 0.048791 & -0.58895 \\
\hline SUSD1 & 0.048805 & -0.66238 \\
\hline RPE & 0.048814 & -0.62216 \\
\hline CAMK2D & 0.048827 & -0.51715 \\
\hline ZNF136 & 0.048863 & -0.7799 \\
\hline MRTO4 & 0.048896 & -0.51359 \\
\hline APLP2 & 0.048905 & -0.31988 \\
\hline XIRP1 & 0.048919 & -0.78224 \\
\hline MBD3 & 0.048919 & 0.640065 \\
\hline
\end{tabular}

\begin{tabular}{|l|l|l|} 
UBE2D3 & 0.048927 & 0.403792 \\
\hline HDHD2 & 0.048934 & 0.560806 \\
\hline ERRFI1 & 0.048981 & -0.49895 \\
\hline POLR2M & 0.049007 & -0.52703 \\
\hline TLR4 & 0.049015 & -0.51025 \\
\hline MTUS2 & 0.049071 & 0.370084 \\
\hline LARS & 0.049073 & -0.50774 \\
\hline TMSB4X & 0.049079 & -0.51899 \\
\hline CBLB & 0.049091 & -0.50192 \\
\hline POLI & 0.049111 & -0.65863 \\
\hline METRN & 0.049113 & 0.568665 \\
\hline JUND & 0.049129 & -0.30596 \\
\hline TNFRSF10B & 0.049134 & -0.50488 \\
\hline PORCN & 0.049136 & -0.64084 \\
\hline PWAR1 & 0.049174 & -1.02416 \\
\hline EGR1 & 0.049178 & -0.77867 \\
\hline TTL & 0.049195 & 0.703811 \\
\hline ASUN & 0.049196 & -0.39201 \\
\hline EIF4A3 & 0.04925 & -0.43556 \\
\hline PITPNM1 & 0.049337 & -0.57986 \\
\hline AVIL & 0.049382 & -0.61709 \\
\hline MPP1 & 0.049409 & 0.568199 \\
\hline NUDT4 & 0.049429 & -0.57452 \\
\hline ATL3 & 0.04943 & -0.54625 \\
\hline PNMAL1 & 0.049439 & -0.63464 \\
\hline ZDHHC3 & 0.049442 & -0.46304 \\
\hline FGD4 & 0.049444 & -0.70371 \\
\hline FBXO40 & 0.049521 & 0.559935 \\
\hline NCS1 & 0.049573 & -0.61313 \\
\hline DYNLL1 & 0.04959 & -0.74122 \\
\hline RPS15A & 0.049643 & 1.025891 \\
\hline RPL37A & 0.049679 & 0.515517 \\
\hline GJA1 & 0.049747 & -0.35022 \\
\hline GAS2 & 0.04977 & -0.53259 \\
\hline SYNM & 0.04979 & 0.325806 \\
\hline FASTKD1 & 0.049842 & 0.519079 \\
\hline ENPEP & 0.049861 & -0.57397 \\
\hline ZFAT & 0.049905 & -0.53663 \\
\hline CETN2 & 0.049919 & -0.28869 \\
\hline PDE4D & 0.04992 & -0.51969 \\
\hline TOPORS- & -0.55873 \\
\hline
\end{tabular}




\begin{tabular}{|l|l|l|} 
AS1 & & \\
\hline PGRMC1 & 0.049922 & -0.3399 \\
\hline RECK & 0.049922 & -0.42461 \\
\hline ANKIB1 & 0.049923 & -0.38159 \\
\hline RAB6A & 0.049923 & -0.1895 \\
\hline ANGPTL1 & 0.049924 & -0.41541 \\
\hline MGLL & 0.049925 & -0.37542 \\
\hline SLC25A16 & 0.049926 & -0.49931 \\
\hline ZNF438 & 0.049926 & -0.41161 \\
\hline ANKRD13C & 0.049934 & -0.42953 \\
\hline SLC31A1 & 0.049937 & -0.47657 \\
\hline ENOSF1 & 0.049938 & -0.51981 \\
\hline
\end{tabular}

\begin{tabular}{|l|l|l|} 
RBM12 & 0.049945 & -0.38949 \\
\hline ZNF302 & 0.049949 & -0.33246 \\
\hline DNAJC3 & 0.049951 & -0.37766 \\
\hline LIMA1 & 0.049959 & -0.57653 \\
\hline C14orf2 & 0.049972 & 0.367813 \\
\hline SLC25A38 & 0.049973 & -0.42679 \\
\hline ZNF841 & 0.049978 & -0.4047 \\
\hline ADAMTS9 & 0.049991 & -0.42334 \\
\hline
\end{tabular}


Supplemental Table 3. DEG in males

when comparing NF to LVH (p_adj <

0.05)

\begin{tabular}{|l|l|l|}
\hline GeneName & p_adj & log2FC \\
\hline AZGP1 & 0 & -1.30798 \\
\hline IGFBP2 & 0 & -0.86226 \\
\hline ACKR3 & 0 & 0.671192 \\
\hline HSPG2 & 0 & 0.733618 \\
\hline PPP1R12C & 0 & -0.42178 \\
\hline DYNLRB1 & 0 & -0.37811 \\
\hline IDH3A & 0 & 0.511248 \\
\hline FKBP8 & 0 & -0.42929 \\
\hline RAB11B & 0 & -0.48372 \\
\hline PSMA7 & 0 & -0.33594 \\
\hline SLC5A1 & 0 & 0.724038 \\
\hline SOD1 & 0 & -0.33697 \\
\hline GPNMB & 0 & 0.834657 \\
\hline TTN & 0 & 0.614038 \\
\hline NEXN & 0 & -0.52548 \\
\hline SRM & 0 & -0.35759 \\
\hline RHOC & 0 & -0.27958 \\
\hline C19orf53 & 0 & -0.31797 \\
\hline PNRC1 & 0 & -0.56502 \\
\hline HSD17B10 & 0 & -0.27013 \\
\hline RAMP1 & 0 & -0.59369 \\
\hline IDH2 & 0 & 0.289888 \\
\hline SPG21 & $2.00 \mathrm{E}-15$ & -0.50108 \\
\hline ARL2 & $2.00 \mathrm{E}-15$ & -0.47455 \\
\hline FAM50A & $2.00 \mathrm{E}-15$ & -0.42732 \\
\hline UBL7 & $2.00 \mathrm{E}-15$ & -0.28434 \\
\hline UBXN6 & $2.00 \mathrm{E}-15$ & -0.45076 \\
\hline PCBD1 & $2.00 \mathrm{E}-15$ & -0.44015 \\
\hline LDHD & $2.00 \mathrm{E}-15$ & -0.26791 \\
\hline PPAPDC3 & $2.00 \mathrm{E}-15$ & -0.22663 \\
\hline JUP & $2.00 \mathrm{E}-15$ & -0.35173 \\
\hline IGSF8 & $3.00 \mathrm{E}-15$ & -0.49134 \\
\hline PLVAP & $3.00 \mathrm{E}-15$ & -1.29896 \\
\hline CDIPT & $3.00 \mathrm{E}-15$ & -0.29615 \\
\hline C10orf10 & $3.00 \mathrm{E}-15$ & -0.77617 \\
\hline & & \\
\hline
\end{tabular}

\begin{tabular}{|l|l|l|} 
UBL5 & $3.00 \mathrm{E}-15$ & -0.28347 \\
\hline PPP2R1A & $3.00 \mathrm{E}-15$ & -0.27626 \\
\hline PACSIN3 & $3.00 \mathrm{E}-15$ & -0.34172 \\
\hline GPX4 & $3.00 \mathrm{E}-15$ & -0.22506 \\
\hline DUSP27 & $3.00 \mathrm{E}-15$ & -0.41684 \\
\hline TRIM63 & $3.00 \mathrm{E}-15$ & -0.64008 \\
\hline YPEL3 & $4.00 \mathrm{E}-15$ & -0.7847 \\
\hline HRSP12 & $4.00 \mathrm{E}-15$ & -0.4346 \\
\hline COPRS & $4.00 \mathrm{E}-15$ & -0.35458 \\
\hline MPHOSPH8 & $4.00 \mathrm{E}-15$ & -0.40987 \\
\hline DAPK3 & $4.00 \mathrm{E}-15$ & -0.57591 \\
\hline LRPAP1 & $4.00 \mathrm{E}-15$ & -0.39917 \\
\hline CD151 & $4.00 \mathrm{E}-15$ & -0.51077 \\
\hline PEBP1 & $4.00 \mathrm{E}-15$ & -0.34523 \\
\hline LMOD2 & $4.00 \mathrm{E}-15$ & -0.63874 \\
\hline CLPP & $5.00 \mathrm{E}-15$ & -0.34131 \\
\hline RBM42 & $5.00 \mathrm{E}-15$ & -0.35417 \\
\hline NUDC & $5.00 \mathrm{E}-15$ & -0.52612 \\
\hline SF3B5 & $5.00 \mathrm{E}-15$ & -0.40871 \\
\hline ATP6V0E1 & $5.00 \mathrm{E}-15$ & -0.36647 \\
\hline RRAS & $5.00 \mathrm{E}-15$ & -0.40779 \\
\hline PLEKHO1 & $6.00 \mathrm{E}-15$ & -0.59572 \\
\hline DYNLT1 & $6.00 \mathrm{E}-15$ & -0.44132 \\
\hline TRNP1 & $6.00 \mathrm{E}-15$ & -0.49843 \\
\hline EDF1 & $6.00 \mathrm{E}-15$ & -0.31412 \\
\hline RBPMS2 & $6.00 \mathrm{E}-15$ & -0.28934 \\
\hline DRG1 & $6.99 \mathrm{E}-15$ & -0.32335 \\
\hline ALDOC & $6.99 \mathrm{E}-15$ & -0.45447 \\
\hline GIPC1 & $7.99 \mathrm{E}-15$ & -0.3734 \\
\hline FDFT1 & $7.99 \mathrm{E}-15$ & -0.52811 \\
\hline DIAPH1 & $7.99 \mathrm{E}-15$ & -0.39074 \\
\hline PPP1R7 & $7.99 \mathrm{E}-15$ & -0.33032 \\
\hline SGCA & $7.99 \mathrm{E}-15$ & -0.45226 \\
\hline RBM3 & $7.99 \mathrm{E}-15$ & 0.274338 \\
\hline GABARAPL1 & $7.99 \mathrm{E}-15$ & -0.31891 \\
\hline TCAP & $7.99 \mathrm{E}-15$ & -0.29492 \\
\hline OS9 & $8.99 \mathrm{E}-15$ & -0.33479 \\
\hline PLA2G16 & $8.99 \mathrm{E}-15$ & -0.42933 \\
\hline ACTR1A & $8.99 \mathrm{E}-15$ & -0.28631 \\
\hline TBC1D8 & $9.99 \mathrm{E}-15$ & -0.5583 \\
\hline PWAR5 & $9.99 \mathrm{E}-15$ & 0.526286 \\
\hline & & \\
\hline
\end{tabular}




\begin{tabular}{|l|l|l|} 
DNASE2 & $1.10 \mathrm{E}-14$ & -0.45549 \\
\hline TMEM140 & $1.20 \mathrm{E}-14$ & -0.89372 \\
\hline TSPO & $1.20 \mathrm{E}-14$ & -0.44565 \\
\hline NDUFAF3 & $1.30 \mathrm{E}-14$ & -0.24658 \\
\hline SDF4 & $1.30 \mathrm{E}-14$ & -0.3565 \\
\hline TRIM54 & $1.30 \mathrm{E}-14$ & -0.40356 \\
\hline PSMD4 & $1.40 \mathrm{E}-14$ & -0.36071 \\
\hline SORT1 & $1.40 \mathrm{E}-14$ & -0.41997 \\
\hline FBXW5 & $1.60 \mathrm{E}-14$ & -0.39062 \\
\hline SYNGR2 & $1.80 \mathrm{E}-14$ & -0.48952 \\
\hline PNPLA2 & $1.80 \mathrm{E}-14$ & -0.4725 \\
\hline HSPB8 & $2.00 \mathrm{E}-14$ & -0.30308 \\
\hline NUCB1 & $2.10 \mathrm{E}-14$ & -0.31163 \\
\hline EIF5A & $2.40 \mathrm{E}-14$ & -0.38269 \\
\hline CTSD & $2.60 \mathrm{E}-14$ & -0.47497 \\
\hline TRIP10 & $2.80 \mathrm{E}-14$ & -0.59969 \\
\hline EPN1 & $2.90 \mathrm{E}-14$ & -0.36742 \\
\hline BRK1 & $2.90 \mathrm{E}-14$ & -0.28194 \\
\hline PDHX & $3.10 \mathrm{E}-14$ & 0.25964 \\
\hline AC018464.3 & $3.20 \mathrm{E}-14$ & -0.27882 \\
\hline CCDC85B & $3.60 \mathrm{E}-14$ & -0.62642 \\
\hline SNTA1 & $3.70 \mathrm{E}-14$ & -0.25705 \\
\hline KCNN2 & $3.90 \mathrm{E}-14$ & 0.669077 \\
\hline CCDC124 & $4.10 \mathrm{E}-14$ & -0.48253 \\
\hline CDK2AP2 & $7.21 \mathrm{E}-14$ & -0.69732 \\
\hline BSG & $1.07 \mathrm{E}-13$ & -0.40526 \\
\hline HIST2H2BE & $1.11 \mathrm{E}-13$ & -0.52488 \\
\hline HSPB7 & $4.91 \mathrm{E}-13$ & -0.3482 \\
\hline WDR62 & $2.15 \mathrm{E}-12$ & 1.07249 \\
\hline TALDO1 & $4.47 \mathrm{E}-12$ & -0.32684 \\
\hline RAB40B & $1.33 \mathrm{E}-11$ & -0.49743 \\
\hline RPL35 & $1.57 \mathrm{E}-11$ & -0.26922 \\
\hline EMD & $3.27 \mathrm{E}-11$ & -0.3251 \\
\hline BLVRA & $2.66 \mathrm{E}-09$ & -0.31132 \\
\hline MT2A & $7.38 \mathrm{E}-09$ & -1.04872 \\
\hline PEBP4 & $8.73 \mathrm{E}-09$ & -0.44419 \\
\hline S1PR3 & $1.29 \mathrm{E}-08$ & 0.779523 \\
\hline PTPRB & $1.29 \mathrm{E}-08$ & 0.897076 \\
\hline TANGO2 & $1.46 \mathrm{E}-08$ & -0.40924 \\
\hline NCOA4 & $2.30 \mathrm{E}-08$ & 0.20876 \\
\hline SCARA5 & $1.67 \mathrm{E}-07$ & 0.976601 \\
\hline & & \\
\hline
\end{tabular}

\begin{tabular}{|l|l|l|} 
MYL4 & $1.01 \mathrm{E}-06$ & 1.123475 \\
\hline ZBTB47 & $3.25 \mathrm{E}-06$ & -0.41997 \\
\hline CCL2 & $9.02 \mathrm{E}-06$ & 0.671466 \\
\hline CD81 & $9.22 \mathrm{E}-06$ & -0.36777 \\
\hline PLP2 & $9.72 \mathrm{E}-06$ & -0.41521 \\
\hline LAMTOR5 & $1.83 \mathrm{E}-05$ & -0.17459 \\
\hline RRAD & $1.96 \mathrm{E}-05$ & -0.58697 \\
\hline AURKAIP1 & $2.33 \mathrm{E}-05$ & -0.19937 \\
\hline SERF2 & $2.39 \mathrm{E}-05$ & -0.31704 \\
\hline MGAT4B & $2.62 \mathrm{E}-05$ & -0.29272 \\
\hline MYH14 & $3.04 \mathrm{E}-05$ & -0.29136 \\
\hline DLAT & $3.39 \mathrm{E}-05$ & 0.330348 \\
\hline KCTD17 & $3.80 \mathrm{E}-05$ & -0.56372 \\
\hline ITM2A & $4.45 \mathrm{E}-05$ & 0.703263 \\
\hline PRPF6 & $5.66 \mathrm{E}-05$ & -0.32279 \\
\hline MXI1 & $5.68 \mathrm{E}-05$ & -0.44513 \\
\hline LRRC14B & $6.69 \mathrm{E}-05$ & -0.58266 \\
\hline GTF3A & $9.33 \mathrm{E}-05$ & -0.37791 \\
\hline FAM89B & $9.57 \mathrm{E}-05$ & -0.44927 \\
\hline MASP1 & $9.84 \mathrm{E}-05$ & -0.6278 \\
\hline UXT & 0.000104 & -0.37965 \\
\hline MRPS23 & 0.000108 & -0.33421 \\
\hline HDAC5 & 0.000133 & -0.29006 \\
\hline ALDH2 & 0.000141 & -0.30653 \\
\hline LRRC23 & 0.000151 & -0.65684 \\
\hline ETFA & 0.00019 & -0.1549 \\
\hline SSB & 0.000191 & -0.3278 \\
\hline OSER1 & 0.00021 & -0.45161 \\
\hline RSL24D1 & 0.000229 & -0.32297 \\
\hline SLC6A8 & 0.000258 & -0.35093 \\
\hline DUSP26 & 0.000341 & -0.54462 \\
\hline SLC25A3 & 0.000341 & 0.25135 \\
\hline EMCN & 0.000356 & 0.55574 \\
\hline CDC34 & 0.000616 & -0.3254 \\
\hline B4GALT2 & 0.000621 & -0.35529 \\
\hline HSP90B1 & 0.000756 & -0.26208 \\
\hline RPL13A & 0.000944 & -0.18863 \\
\hline ZNF358 & 0.001 & -0.31875 \\
\hline KEAP1 & 0.001042 & -0.30105 \\
\hline INMT & 0.001049 & 0.824406 \\
\hline PIN1 & 0.001078 & -0.35181 \\
\hline
\end{tabular}




\begin{tabular}{|l|l|l|} 
WBP2 & 0.001109 & -0.377 \\
\hline LAMTOR2 & 0.001135 & -0.32041 \\
\hline C11orf68 & 0.001147 & -0.31843 \\
\hline SQSTM1 & 0.001194 & -0.39029 \\
\hline PIGT & 0.001263 & -0.25638 \\
\hline NRTN & 0.001353 & -0.74845 \\
\hline GYPC & 0.001467 & -0.25195 \\
\hline CKB & 0.001634 & -0.40248 \\
\hline ELN & 0.001639 & 0.558139 \\
\hline ST3GAL4 & 0.001644 & -0.68865 \\
\hline ANXA11 & 0.001677 & -0.20158 \\
\hline PARK7 & 0.001698 & -0.2137 \\
\hline ASIP & 0.001734 & -0.44131 \\
\hline SAT2 & 0.001785 & -0.27437 \\
\hline FXR2 & 0.001807 & -0.32765 \\
\hline ERP29 & 0.001823 & -0.36148 \\
\hline C11orf31 & 0.001837 & -0.51545 \\
\hline DAD1 & 0.001894 & -0.25769 \\
\hline HTRA1 & 0.001916 & -0.55285 \\
\hline MSRB1 & 0.001945 & -0.47805 \\
\hline BRE & 0.002095 & -0.31542 \\
\hline VPS4A & 0.002283 & -0.26511 \\
\hline TERF2IP & 0.002383 & -0.38456 \\
\hline JOSD2 & 0.002388 & -0.38476 \\
\hline TPRG1L & 0.0024 & -0.30609 \\
\hline CAMK2B & 0.002571 & -0.37192 \\
\hline SWI5 & 0.002661 & -0.40895 \\
\hline EIF4B & 0.002731 & -0.25102 \\
\hline TMEM256 & 0.002757 & -0.47367 \\
\hline LPL & 0.003074 & -0.52964 \\
\hline GSTP1 & 0.00341 & -0.26256 \\
\hline COBL & 0.003417 & -0.36775 \\
\hline HIST1H1C & 0.003543 & -0.41015 \\
\hline ZNF204P & 0.003762 & -0.5165 \\
\hline GPS1 & 0.003858 & -0.30609 \\
\hline SORBS1 & 0.00392 & -0.11485 \\
\hline PIGQ & 0.003927 & -0.54206 \\
\hline NECAB3 & 0.003934 & -0.24182 \\
\hline LSM10 & 0.003997 & -0.27941 \\
\hline HRC & 0.004002 & -0.29343 \\
\hline UROD & 0.004004 & -0.33644 \\
\hline & & \\
\hline
\end{tabular}

\begin{tabular}{|l|l|l|} 
JUND & 0.004008 & -0.34378 \\
\hline TMEM14C & 0.00412 & -0.20294 \\
\hline TNIP2 & 0.004136 & -0.43109 \\
\hline IK & 0.004208 & -0.28593 \\
\hline NKX2-5 & 0.004208 & -0.2459 \\
\hline SGSM3 & 0.004221 & -0.31954 \\
\hline DES & 0.004376 & -0.27321 \\
\hline NUPR1 & 0.004378 & -0.71661 \\
\hline UQCR10 & 0.004388 & -0.24074 \\
\hline ILVBL & 0.004582 & -0.26735 \\
\hline MIR4482 & 0.00467 & -0.81714 \\
\hline ATP6AP1 & 0.004765 & -0.30002 \\
\hline KARS & 0.004848 & -0.25315 \\
\hline GAPDH & 0.004859 & -0.14026 \\
\hline CYCS & 0.004879 & 0.279535 \\
\hline PFDN5 & 0.004886 & -0.22338 \\
\hline CHCHD2 & 0.00496 & -0.21494 \\
\hline EIF3I & 0.005083 & -0.28683 \\
\hline NPTN & 0.005114 & 0.209032 \\
\hline MYEOV2 & 0.005117 & -0.34901 \\
\hline PABPC1 & 0.005194 & -0.38188 \\
\hline CHPF & 0.005284 & -0.33398 \\
\hline SOD3 & 0.00539 & -0.40206 \\
\hline SLC25A39 & 0.005428 & -0.3899 \\
\hline LRP10 & 0.00543 & -0.36537 \\
\hline PRKAG1 & 0.005935 & -0.35304 \\
\hline MRPL28 & 0.005952 & -0.24936 \\
\hline CERK & 0.006085 & -0.48008 \\
\hline PPP1R16A & 0.006205 & -0.37085 \\
\hline CSTB & 0.00636 & -0.39138 \\
\hline EXOSC1 & 0.006372 & -0.39537 \\
\hline TMUB1 & 0.006399 & -0.47745 \\
\hline TADA3 & 0.00641 & -0.3553 \\
\hline MTA1 & 0.00647 & -0.28772 \\
\hline VIPAS39 & 0.006582 & -0.4012 \\
\hline CHMP2A & 0.006599 & -0.27158 \\
\hline LARP7 & 0.006649 & -0.34372 \\
\hline SPARC & 0.006824 & 0.610476 \\
\hline DDRGK1 & 0.006945 & -0.42941 \\
\hline POLDIP2 & 0.006972 & -0.14408 \\
\hline POLR2J & 0.007028 & -0.29525 \\
\hline & & \\
\hline
\end{tabular}




\begin{tabular}{|l|l|l|} 
HSPB1 & 0.00706 & -0.58607 \\
\hline INPP5 & 0.007167 & -0.44067 \\
\hline SNRPD2 & 0.007275 & -0.31241 \\
\hline ERI3 & 0.007414 & -0.29734 \\
\hline GTF3C6 & 0.007485 & -0.17151 \\
\hline TSPYL2 & 0.0075 & -0.8597 \\
\hline ENO2 & 0.007665 & -0.4759 \\
\hline SDC4 & 0.007675 & -0.3726 \\
\hline POMP & 0.007815 & -0.23334 \\
\hline CIR1 & 0.007818 & -0.37751 \\
\hline ANP32B & 0.008025 & -0.2556 \\
\hline SPR & 0.008157 & -0.57869 \\
\hline TRIM28 & 0.008165 & -0.31917 \\
\hline WDR83OS & 0.00831 & -0.36759 \\
\hline DYNLL1 & 0.008446 & -0.37751 \\
\hline PTGES3 & 0.008463 & -0.34736 \\
\hline SAP18 & 0.008474 & -0.1488 \\
\hline ALDH6A1 & 0.008789 & -0.45957 \\
\hline RRAS2 & 0.008991 & -0.4177 \\
\hline MXRA7 & 0.009181 & -0.53303 \\
\hline PABPC4 & 0.009273 & -0.26604 \\
\hline ATP6V0B & 0.00929 & -0.3209 \\
\hline GRHPR & 0.0093 & -0.20137 \\
\hline STX2 & 0.009303 & -0.38346 \\
\hline PLEKHM2 & 0.009372 & -0.27695 \\
\hline TMEM147 & 0.009384 & -0.27193 \\
\hline FAM134B & 0.009389 & -0.44629 \\
\hline MRPL15 & 0.009423 & -0.12168 \\
\hline IFT43 & 0.009442 & -0.50101 \\
\hline MTRNR2L8 & 0.009458 & -3.57542 \\
\hline RPS24 & 0.00946 & -0.32826 \\
\hline VAMP2 & 0.00948 & -0.16891 \\
\hline CCT3 & 0.00952 & -0.41748 \\
\hline OTUD1 & 0.009595 & -0.36497 \\
\hline LCMT1 & 0.009618 & -0.23454 \\
\hline UFC1 & 0.009768 & -0.23932 \\
\hline PSENEN & 0.009805 & -0.48937 \\
\hline C9orf78 & 0.009811 & -0.32238 \\
\hline KIAA2013 & 0.009924 & -0.26446 \\
\hline SPCS1 & 0.009987 & -0.2743 \\
\hline PACS1 & 0.01 & -0.26715 \\
\hline & & \\
\hline
\end{tabular}

\begin{tabular}{|l|l|l|} 
PSMB1 & 0.010017 & -0.23548 \\
\hline LSM4 & 0.010025 & -0.24831 \\
\hline NAP1L3 & 0.010078 & -0.49295 \\
\hline TSG101 & 0.010191 & -0.22809 \\
\hline CCT7 & 0.010264 & -0.31043 \\
\hline TBC1D17 & 0.01028 & -0.37313 \\
\hline SMARCC2 & 0.010316 & -0.30411 \\
\hline GSTM4 & 0.010332 & -0.26552 \\
\hline CCT4 & 0.01034 & -0.25732 \\
\hline TMEM164 & 0.010365 & -0.30622 \\
\hline ESF1 & 0.010457 & -0.41539 \\
\hline COPA & 0.010497 & -0.26554 \\
\hline SNRPC & 0.010573 & -0.33658 \\
\hline AP2S1 & 0.010589 & -0.31414 \\
\hline RNF181 & 0.01059 & -0.26769 \\
\hline STAMBP & 0.010618 & -0.36705 \\
\hline CLU & 0.010655 & -1.00447 \\
\hline GCDH & 0.010661 & -0.34981 \\
\hline PCOLCE2 & 0.010665 & -0.55002 \\
\hline POLRMT & 0.010672 & -0.42072 \\
\hline HHATL & 0.010686 & -0.32177 \\
\hline ZCRB1 & 0.010689 & -0.27024 \\
\hline DPM3 & 0.01069 & -0.37206 \\
\hline RNF20 & 0.010705 & -0.28119 \\
\hline VPS28 & 0.010734 & -0.21248 \\
\hline MLLT1 & 0.010817 & -0.41122 \\
\hline TMEM127 & 0.010858 & -0.19741 \\
\hline SLC25A5 & 0.010859 & -0.5597 \\
\hline PLOD3 & 0.010886 & -0.43705 \\
\hline GLUL & 0.010959 & -0.56715 \\
\hline GPC1 & 0.010965 & -0.33923 \\
\hline POLD4 & 0.01097 & -0.50326 \\
\hline GUK1 & 0.010998 & -0.19952 \\
\hline TPT1 & 0.011014 & -0.26429 \\
\hline FSTL3 & 0.011028 & -0.41328 \\
\hline POLR2E & 0.011031 & -0.22606 \\
\hline ZNF688 & 0.011044 & -0.42493 \\
\hline MYOF & 0.011076 & -0.39664 \\
\hline EI24 & 0.011102 & -0.21384 \\
\hline IER5 & 0.011172 & -0.51078 \\
\hline HOOK2 & 0.01119 & -0.33956 \\
\hline & & \\
\hline
\end{tabular}




\begin{tabular}{|l|l|l|} 
C11orf24 & 0.011191 & -0.33337 \\
\hline LAMTOR4 & 0.011205 & -0.34185 \\
\hline PKIG & 0.011212 & -0.19222 \\
\hline PRDX1 & 0.01138 & -0.42611 \\
\hline SDHAF1 & 0.011418 & -0.31634 \\
\hline EIF3B & 0.011534 & -0.26048 \\
\hline RTFDC1 & 0.011569 & -0.22657 \\
\hline C19orf43 & 0.011595 & -0.22252 \\
\hline COPS6 & 0.01161 & -0.16468 \\
\hline WBSCR16 & 0.01163 & -0.24281 \\
\hline TCEAL4 & 0.011643 & -0.23189 \\
\hline PCBP2 & 0.011658 & -0.19845 \\
\hline YBX3 & 0.011689 & -0.3657 \\
\hline PDAP1 & 0.01169 & -0.25235 \\
\hline GDI1 & 0.011732 & -0.28311 \\
\hline DKK3 & 0.011774 & -0.74641 \\
\hline EIF3G & 0.011847 & -0.24517 \\
\hline NELFB & 0.011884 & -0.40404 \\
\hline TSR3 & 0.011897 & -0.33253 \\
\hline C6orf1 & 0.011928 & -0.50915 \\
\hline DBI & 0.011937 & -0.28167 \\
\hline RAB1B & 0.011951 & -0.173 \\
\hline IFT22 & 0.011969 & -0.30612 \\
\hline PLEKHA6 & 0.011977 & -0.42819 \\
\hline TTC38 & 0.01202 & -0.61326 \\
\hline SLC27A6 & 0.012021 & -0.72589 \\
\hline C1orf122 & 0.012048 & -0.29349 \\
\hline SCYL1 & 0.012188 & -0.2842 \\
\hline TTLL12 & 0.012313 & -0.52602 \\
\hline TGM2 & 0.012428 & -0.49285 \\
\hline TCEA3 & 0.012457 & -0.33137 \\
\hline ARF5 & 0.012464 & -0.25538 \\
\hline UCHL1 & 0.012504 & -0.87673 \\
\hline CSNK2A2 & 0.012581 & -0.34957 \\
\hline ACOT11 & 0.012581 & -0.3613 \\
\hline PAIP2 & 0.012586 & -0.25219 \\
\hline CTSF & 0.012649 & -0.60939 \\
\hline COMTD1 & 0.01268 & -0.32352 \\
\hline MRPS21 & 0.012689 & -0.26227 \\
\hline RTN2 & 0.012714 & -0.31004 \\
\hline EFNA5 & 0.012737 & -0.32865 \\
\hline & & \\
\hline
\end{tabular}

\begin{tabular}{|l|l|l|} 
EXOSC4 & 0.012762 & -0.36018 \\
\hline ABTB1 & 0.012788 & -0.46099 \\
\hline POP5 & 0.012837 & -0.52448 \\
\hline RTCB & 0.012912 & -0.19915 \\
\hline ALDH4A1 & 0.012927 & -0.53248 \\
\hline MRFAP1 & 0.01297 & -0.12913 \\
\hline USP5 & 0.013017 & -0.34138 \\
\hline CNBP & 0.013039 & -0.08732 \\
\hline ZER1 & 0.013115 & -0.29479 \\
\hline EIF2S2 & 0.013166 & -0.30553 \\
\hline PHLDA1 & 0.013169 & -0.9679 \\
\hline GNAS & 0.013184 & -0.13999 \\
\hline COX14 & 0.013328 & -0.25528 \\
\hline ATP6V1F & 0.013414 & -0.3454 \\
\hline ROGDI & 0.01346 & -0.27556 \\
\hline EIF2S3 & 0.013473 & -0.27167 \\
\hline SCN1B & 0.013482 & -0.52847 \\
\hline RPS11 & 0.013484 & -0.14012 \\
\hline MFAP4 & 0.013485 & -0.36431 \\
\hline ULK1 & 0.013491 & -0.38666 \\
\hline CHP1 & 0.013576 & -0.30846 \\
\hline RALY & 0.013578 & -0.4703 \\
\hline NPPB & 0.013588 & -2.89857 \\
\hline RNF103 & 0.013768 & -0.31533 \\
\hline SCAND1 & 0.013792 & -0.35362 \\
\hline TST & 0.013797 & -0.72148 \\
\hline SYMPK & 0.013798 & -0.36044 \\
\hline PPCS & 0.013805 & -0.26008 \\
\hline ALDH1L1 & 0.013817 & -0.42288 \\
\hline PAF1 & 0.013831 & -0.23495 \\
\hline RABAC1 & 0.013844 & -0.34446 \\
\hline ECHS1 & 0.013861 & -0.25719 \\
\hline FAM58A & 0.013924 & -0.42815 \\
\hline SGTA & 0.013971 & -0.22424 \\
\hline RPS19BP1 & 0.013983 & -0.40534 \\
\hline AGPAT2 & 0.014057 & -0.30517 \\
\hline MRPL57 & 0.014129 & -0.29881 \\
\hline SAMD1 & 0.014196 & -0.44126 \\
\hline LSM7 & 0.014202 & -0.56597 \\
\hline DYNLL2 & 0.014215 & -0.20829 \\
\hline FASTK & 0.014245 & -0.27768 \\
\hline & & \\
\hline
\end{tabular}




\begin{tabular}{|l|l|l|} 
MAP3K3 & 0.014245 & -0.35698 \\
\hline FXYD1 & 0.014267 & -0.48232 \\
\hline EIF4A2 & 0.014294 & -0.39294 \\
\hline LSM3 & 0.014327 & -0.2158 \\
\hline EPDR1 & 0.014425 & 0.426137 \\
\hline HSF1 & 0.014428 & -0.24261 \\
\hline POLR2G & 0.014437 & -0.31933 \\
\hline LYNX1 & 0.014458 & -0.32746 \\
\hline C10orf76 & 0.014545 & -0.37873 \\
\hline DNTTIP2 & 0.014579 & -0.25529 \\
\hline NFE2L1 & 0.014752 & -0.27819 \\
\hline MTURN & 0.014763 & -0.47446 \\
\hline KIAA1191 & 0.014774 & -0.20948 \\
\hline PPP1R15A & 0.014806 & -0.32126 \\
\hline MB & 0.014812 & -0.22725 \\
\hline RPL36 & 0.014829 & -0.21354 \\
\hline MTMR14 & 0.014979 & -0.31443 \\
\hline NINJ1 & 0.014992 & -0.43303 \\
\hline BOLA1 & 0.015013 & -0.42337 \\
\hline CETN2 & 0.015106 & -0.33188 \\
\hline CYB5R1 & 0.015145 & -0.46927 \\
\hline ARF1 & 0.015199 & -0.12529 \\
\hline RNPS1 & 0.015199 & -0.29905 \\
\hline ST6GALNAC6 & 0.015234 & -0.41203 \\
\hline ZBED5-AS1 & 0.015267 & -0.6205 \\
\hline ST6GALNAC4 & 0.015328 & -0.441 \\
\hline ATP6V1E1 & 0.015334 & -0.24877 \\
\hline MRPL55 & 0.015397 & -0.34849 \\
\hline CDK5 & 0.015408 & -0.36628 \\
\hline EDNRB & 0.015444 & 0.572002 \\
\hline MLIP & 0.015451 & -0.22347 \\
\hline TMEM120A & 0.015642 & -0.3858 \\
\hline CRCP & 0.015655 & -0.25161 \\
\hline CYSTM1 & 0.015682 & -0.42572 \\
\hline FADS1 & 0.015691 & -0.32319 \\
\hline CRELD1 & 0.015765 & -0.57105 \\
\hline METRN & 0.015824 & -0.30801 \\
\hline UBXN1 & 0.015828 & -0.37412 \\
\hline KCTD2 & 0.015842 & -0.40662 \\
\hline LDHA & 0.016036 & -0.51813 \\
\hline CDKN2D & 0.016106 & -0.39297 \\
\hline & & \\
\hline
\end{tabular}

\begin{tabular}{|l|l|l|} 
MAF1 & 0.016223 & -0.28331 \\
\hline FGF12 & 0.016281 & 0.915237 \\
\hline MIRLET7D & 0.016287 & -0.56492 \\
\hline SLC14A1 & 0.016378 & 0.566344 \\
\hline DRAP1 & 0.016476 & -0.2622 \\
\hline GNG8 & 0.016495 & 1.058843 \\
\hline CAPN1 & 0.016533 & -0.22307 \\
\hline UBC & 0.016678 & -0.49693 \\
\hline POLR1D & 0.016683 & -0.34645 \\
\hline EMC2 & 0.016794 & -0.33008 \\
\hline ATRAID & 0.016827 & -0.33128 \\
\hline ELK1 & 0.016899 & -0.34555 \\
\hline DGCR6L & 0.016903 & -0.3252 \\
\hline C12orf10 & 0.016919 & -0.30345 \\
\hline HEPH & 0.016989 & -0.38567 \\
\hline TSSC4 & 0.017028 & -0.41571 \\
\hline KLF15 & 0.017053 & -0.70023 \\
\hline UBE2B & 0.017103 & -0.30749 \\
\hline DPP7 & 0.017114 & -0.26966 \\
\hline MAFK & 0.017121 & -0.41121 \\
\hline PRDX6 & 0.017153 & -0.41316 \\
\hline HINT2 & 0.017255 & -0.35427 \\
\hline MBD3 & 0.017335 & -0.31668 \\
\hline LIN52 & 0.017343 & -0.40293 \\
\hline CFL1 & 0.017353 & -0.2372 \\
\hline FBXW4 & 0.017386 & -0.27573 \\
\hline HSPE1 & 0.017401 & -0.62629 \\
\hline MAOA & 0.017421 & -0.29269 \\
\hline UBE2L3 & 0.017429 & -0.31198 \\
\hline ADRM1 & 0.017531 & -0.26227 \\
\hline SPTB & 0.017546 & -0.30281 \\
\hline HDGFRP2 & 0.017564 & -0.29361 \\
\hline MRPS33 & 0.017572 & -0.23304 \\
\hline CTNNBL1 & 0.017573 & -0.32895 \\
\hline ITPA & 0.017598 & -0.54934 \\
\hline DDAH1 & 0.017604 & -0.5688 \\
\hline RASL10B & 0.017747 & -0.60467 \\
\hline LYAR & 0.017756 & -0.50277 \\
\hline NEMF & 0.017773 & -0.28863 \\
\hline PPP1R1C & 0.017784 & 0.517569 \\
\hline NHP2 & 0.017786 & -0.26493 \\
\hline & & \\
\hline
\end{tabular}




\begin{tabular}{|l|l|l|} 
EIF1B & 0.017796 & -0.39273 \\
\hline RALBP1 & 0.017856 & -0.26441 \\
\hline TCEAL3 & 0.017945 & -0.35498 \\
\hline LRRC10 & 0.017955 & -0.47799 \\
\hline DCAF11 & 0.017956 & -0.23243 \\
\hline HMG20B & 0.018001 & -0.41925 \\
\hline ARL8A & 0.018211 & -0.16732 \\
\hline S100A13 & 0.018239 & -0.37145 \\
\hline CCDC59 & 0.018244 & -0.46895 \\
\hline NPPA & 0.018249 & -4.59767 \\
\hline ATG101 & 0.018275 & -0.35859 \\
\hline PTMS & 0.018308 & -0.20457 \\
\hline TUSC2 & 0.018326 & -0.24854 \\
\hline PCYT2 & 0.018328 & -0.44157 \\
\hline ZC3H15 & 0.018443 & -0.26553 \\
\hline ASNA1 & 0.018446 & -0.22283 \\
\hline HSP90AA1 & 0.018487 & -1.38355 \\
\hline DNAJB2 & 0.01849 & -0.41515 \\
\hline BTF3 & 0.018499 & -0.19986 \\
\hline RPLP2 & 0.018504 & -0.20578 \\
\hline SRI & 0.018627 & -0.2971 \\
\hline KDELR1 & 0.018636 & -0.36962 \\
\hline SLC25A38 & 0.018641 & -0.43073 \\
\hline EIF5B & 0.018641 & -0.22733 \\
\hline DPM2 & 0.018683 & -0.35311 \\
\hline TCEA2 & 0.018721 & -0.38428 \\
\hline CTDSP1 & 0.018866 & -0.26382 \\
\hline QSOX1 & 0.018983 & -0.3826 \\
\hline CDC37 & 0.018999 & -0.15495 \\
\hline RPS27A & 0.019056 & -0.32018 \\
\hline FNDC5 & 0.019081 & 0.420695 \\
\hline CALCRL & 0.019111 & 0.604543 \\
\hline FHL2 & 0.01912 & 0.565827 \\
\hline BAD & 0.019143 & -0.29838 \\
\hline SBDS & 0.019144 & -0.10968 \\
\hline ASCC2 & 0.019171 & -0.32559 \\
\hline ZSCAN18 & 0.019217 & -0.29695 \\
\hline AHSA1 & 0.019274 & -0.46542 \\
\hline RPL15 & 0.019279 & -0.25243 \\
\hline BAMBI & 0.019297 & -0.4425 \\
\hline STMN1 & 0.01937 & -0.58359 \\
\hline & & \\
\hline
\end{tabular}

\begin{tabular}{|l|l|l|} 
CES4A & 0.019489 & -0.70206 \\
\hline PPP1CA & 0.019498 & -0.34569 \\
\hline PSMB4 & 0.019554 & -0.27062 \\
\hline EMC10 & 0.019562 & -0.32654 \\
\hline DPH6-AS1 & 0.019774 & -0.6062 \\
\hline GSTM3 & 0.019815 & -0.53335 \\
\hline VWF & 0.019926 & 0.704071 \\
\hline FKBP4 & 0.020063 & -0.52346 \\
\hline ORMDL3 & 0.020082 & -0.28424 \\
\hline C5orf15 & 0.020115 & 0.35937 \\
\hline SMG5 & 0.020116 & -0.37037 \\
\hline DMWD & 0.020142 & -0.34524 \\
\hline C15orf65 & 0.020157 & -0.40466 \\
\hline SND1 & 0.020159 & -0.37093 \\
\hline NAP1L1 & 0.020236 & -0.3132 \\
\hline PSMC5 & 0.020254 & -0.27883 \\
\hline NT5M & 0.020328 & -0.38042 \\
\hline NPM1 & 0.020339 & -0.26878 \\
\hline CD300LG & 0.020405 & 0.770143 \\
\hline FBXO32 & 0.020543 & -0.51296 \\
\hline ZNHIT1 & 0.020717 & -0.31106 \\
\hline PLEKHA4 & 0.020773 & -0.49707 \\
\hline APLP1 & 0.020786 & -0.49427 \\
\hline DCBLD2 & 0.020852 & -0.2775 \\
\hline NDUFS1 & 0.020927 & 0.309691 \\
\hline CD59 & 0.020933 & -0.26825 \\
\hline OAZ1 & 0.021006 & -0.13158 \\
\hline RNPEPL1 & 0.021057 & -0.22856 \\
\hline ANAPC11 & 0.021095 & -0.22134 \\
\hline POR & 0.02111 & -0.51248 \\
\hline SMYD1 & 0.021132 & 0.293732 \\
\hline FAM195A & 0.021138 & -0.35168 \\
\hline HFE2 & 0.021178 & -0.41659 \\
\hline DBNL & 0.021185 & -0.43991 \\
\hline LINC01137 & 0.021186 & -0.48382 \\
\hline COL4A3BP & 0.021196 & -0.30889 \\
\hline RRAGA & 0.021263 & -0.18647 \\
\hline UBE2Z & 0.021294 & -0.22151 \\
\hline RBM10 & 0.021521 & -0.33803 \\
\hline SAE1 & 0.021552 & -0.31922 \\
\hline GDI2 & 0.021604 & -0.26871 \\
\hline & & \\
\hline
\end{tabular}




\begin{tabular}{|l|l|l|} 
B4GALNT3 & 0.021696 & -0.43816 \\
\hline SNRPA & 0.021733 & -0.32029 \\
\hline UNC45B & 0.021746 & -0.90511 \\
\hline PACRG & 0.021758 & -0.45468 \\
\hline SLU7 & 0.021769 & -0.29272 \\
\hline JAGN1 & 0.021776 & -0.32502 \\
\hline HIST1H2BD & 0.022086 & -0.44462 \\
\hline SET & 0.022102 & -0.25376 \\
\hline HAX1 & 0.022118 & -0.1331 \\
\hline MAPRE2 & 0.022163 & -0.31408 \\
\hline SMTN & 0.022189 & 0.264174 \\
\hline PRDX3 & 0.022218 & 0.14066 \\
\hline ITPKB & 0.022309 & 0.683888 \\
\hline CTNNAL1 & 0.022452 & -0.59542 \\
\hline PROS1 & 0.022481 & -0.63447 \\
\hline FAM3A & 0.022545 & -0.42221 \\
\hline PLK2 & 0.022569 & -0.74157 \\
\hline LSM1 & 0.022598 & -0.27895 \\
\hline SERPINB6 & 0.022689 & -0.2639 \\
\hline SOX12 & 0.022776 & -0.4053 \\
\hline PTDSS1 & 0.022819 & 0.495418 \\
\hline RPL18 & 0.022836 & -0.28869 \\
\hline RPL19 & 0.022951 & -0.16645 \\
\hline CWC15 & 0.02303 & -0.26389 \\
\hline IGFBP7 & 0.023042 & -0.36395 \\
\hline COL23A1 & 0.023043 & -0.75928 \\
\hline SURF1 & 0.023068 & -0.25652 \\
\hline SKP1 & 0.023151 & -0.16183 \\
\hline CYHR1 & 0.023158 & -0.37718 \\
\hline CNPPD1 & 0.023171 & -0.30237 \\
\hline SEC61G & 0.02318 & -0.29678 \\
\hline GTF2F1 & 0.02322 & -0.22978 \\
\hline TRAPPC1 & 0.023272 & -0.26542 \\
\hline UCK1 & 0.023313 & -0.38595 \\
\hline HMCES & 0.023375 & -0.21037 \\
\hline TP53I13 & 0.023406 & -0.36419 \\
\hline HSPBP1 & 0.02344 & -0.32118 \\
\hline PDK4 & 0.023484 & -1.19085 \\
\hline C110rf74 & 0.023496 & -0.33449 \\
\hline AMZ2 & 0.023585 & -0.35328 \\
\hline PYGM & 0.023586 & 0.297264 \\
\hline & & \\
\hline
\end{tabular}

\begin{tabular}{|l|l|l|} 
TUBB6 & 0.023596 & 0.430748 \\
\hline RPS4X & 0.023605 & -0.17722 \\
\hline HSP90AB1 & 0.02364 & -0.5772 \\
\hline CTSH & 0.02375 & 0.322121 \\
\hline COX6A1 & 0.023755 & -0.30993 \\
\hline HGS & 0.023923 & -0.45271 \\
\hline NENF & 0.023928 & -0.31267 \\
\hline PKN1 & 0.023964 & -0.29225 \\
\hline TAF7 & 0.023997 & -0.24279 \\
\hline COL15A1 & 0.024047 & 0.861246 \\
\hline MRPL14 & 0.024102 & -0.28668 \\
\hline ITFG3 & 0.024142 & -0.27646 \\
\hline PSMC3 & 0.024173 & -0.23743 \\
\hline EEF1B2 & 0.024322 & -0.25242 \\
\hline PLA2G15 & 0.024427 & -0.42828 \\
\hline RPL36AL & 0.024495 & -0.21821 \\
\hline ERH & 0.024512 & -0.27679 \\
\hline SHB & 0.024556 & -0.38377 \\
\hline AAMP & 0.024565 & -0.32935 \\
\hline TBX20 & 0.0246 & 0.935813 \\
\hline MFGE8 & 0.02466 & -0.42171 \\
\hline RP9 & 0.024743 & -0.31803 \\
\hline NOL7 & 0.024744 & -0.24137 \\
\hline DMPK & 0.024775 & -0.41788 \\
\hline C16orf13 & 0.024814 & -0.29099 \\
\hline GSTO1 & 0.024931 & -0.25031 \\
\hline FIS1 & 0.024969 & -0.28059 \\
\hline ZFAND5 & 0.024971 & -0.25691 \\
\hline C3 & 0.025013 & 0.990502 \\
\hline LRRC20 & 0.025057 & -0.2855 \\
\hline SF3B6 & 0.025115 & -0.34123 \\
\hline HINT1 & 0.025154 & -0.21309 \\
\hline SLC27A1 & 0.025159 & -0.45903 \\
\hline ZNF622 & 0.025306 & -0.34891 \\
\hline DSP & 0.025423 & 0.169735 \\
\hline FAM127B & 0.025447 & -0.29674 \\
\hline OST4 & 0.025479 & -0.14268 \\
\hline DTNBP1 & 0.025485 & -0.3453 \\
\hline C5orf46 & 0.025592 & -0.63812 \\
\hline GRN & 0.025626 & -0.33901 \\
\hline CLTB & 0.025627 & -0.31529 \\
\hline & & \\
\hline
\end{tabular}




\begin{tabular}{|l|l|l|} 
SSR4 & 0.02579 & -0.3286 \\
\hline LTBP3 & 0.025806 & -0.31484 \\
\hline SEC14L5 & 0.025826 & 1.203995 \\
\hline BCAT2 & 0.025828 & -0.39913 \\
\hline GAS6 & 0.025831 & -0.27298 \\
\hline SPRYD3 & 0.025875 & -0.23889 \\
\hline IQSEC1 & 0.025878 & 0.472077 \\
\hline MLYCD & 0.025974 & -0.39841 \\
\hline NSFL1C & 0.026126 & -0.27258 \\
\hline RRP12 & 0.026194 & -0.50065 \\
\hline EMC3 & 0.026352 & -0.39285 \\
\hline RPS12 & 0.026385 & 0.178465 \\
\hline NME3 & 0.026409 & -0.42944 \\
\hline XIRP2 & 0.026453 & 0.508832 \\
\hline RAB13 & 0.026459 & -0.35692 \\
\hline TNIP1 & 0.026482 & -0.34038 \\
\hline PA2G4 & 0.026486 & -0.26629 \\
\hline ERGIC3 & 0.026502 & -0.27067 \\
\hline MPST & 0.026537 & -0.49941 \\
\hline GPR137B & 0.026581 & -0.33007 \\
\hline CHST9 & 0.026745 & 0.537514 \\
\hline HIPK3 & 0.026941 & 0.347339 \\
\hline CTSA & 0.02735 & -0.22709 \\
\hline SLITRK4 & 0.027366 & -0.72626 \\
\hline WIPI2 & 0.027404 & -0.24828 \\
\hline SARS & 0.027439 & -0.21692 \\
\hline ST3GAL3 & 0.027532 & -0.29576 \\
\hline SYNJ2BP & 0.027682 & 0.275491 \\
\hline APLN & 0.027729 & 0.4261 \\
\hline INSR & 0.027943 & -0.25008 \\
\hline PMP22 & 0.027949 & -0.25453 \\
\hline RPL24 & 0.028027 & -0.20131 \\
\hline EIF2B4 & 0.028117 & -0.29439 \\
\hline GMPR & 0.028127 & -0.12988 \\
\hline HCFC1R1 & 0.028189 & -0.26988 \\
\hline POPDC2 & 0.028197 & -0.24067 \\
\hline GPR137 & 0.028202 & -0.33936 \\
\hline ISCU & 0.028242 & -0.13404 \\
\hline DNAJA1 & 0.028375 & -0.3996 \\
\hline BUD31 & 0.028401 & -0.3016 \\
\hline ZNF444 & 0.028468 & -0.32209 \\
\hline & & \\
\hline
\end{tabular}

\begin{tabular}{|l|l|l|} 
MAP1LC3A & 0.028534 & -0.23328 \\
\hline UQCR11 & 0.028558 & -0.20712 \\
\hline THBS1 & 0.028597 & -0.52502 \\
\hline NUTF2 & 0.028654 & -0.22124 \\
\hline KIF1B & 0.028657 & 0.307622 \\
\hline OSER1-AS1 & 0.028788 & -0.39574 \\
\hline RPS27 & 0.028815 & -0.3501 \\
\hline COMMD6 & 0.028834 & -0.28021 \\
\hline METTL5 & 0.028893 & -0.34246 \\
\hline CBX7 & 0.028918 & -0.1791 \\
\hline SUMO1 & 0.029073 & -0.2358 \\
\hline CBR1 & 0.029114 & -0.35687 \\
\hline MGLL & 0.029221 & 0.557831 \\
\hline TSPAN15 & 0.029257 & 0.632717 \\
\hline TJP2 & 0.029268 & -0.2175 \\
\hline NAA10 & 0.029307 & -0.28236 \\
\hline FAM180A & 0.029324 & -0.92246 \\
\hline COMMD7 & 0.029367 & -0.32804 \\
\hline RNF126 & 0.029394 & -0.40166 \\
\hline FBL & 0.029427 & -0.22109 \\
\hline TIMM17B & 0.029525 & -0.22288 \\
\hline YPEL2 & 0.029564 & -0.29997 \\
\hline MOB2 & 0.029577 & -0.35481 \\
\hline AGTRAP & 0.029608 & -0.43219 \\
\hline SURF2 & 0.029614 & -0.3161 \\
\hline SSNA1 & 0.029669 & -0.15168 \\
\hline BCL7C & 0.029712 & -0.26555 \\
\hline FABP3 & 0.029774 & -0.24525 \\
\hline AGPAT9 & 0.029837 & -0.47228 \\
\hline HSPB2 & 0.029863 & -0.26549 \\
\hline TEAD1 & 0.029968 & 0.289096 \\
\hline FIBP & 0.030052 & -0.27112 \\
\hline COA3 & 0.030084 & -0.24036 \\
\hline JTB & 0.030102 & -0.29281 \\
\hline TOM1 & 0.030337 & -0.40531 \\
\hline FAM228B & 0.030479 & -0.44692 \\
\hline SLC38A10 & 0.030654 & -0.31889 \\
\hline GSS & 0.030691 & -0.32203 \\
\hline CCDC167 & 0.030783 & -0.27559 \\
\hline WDR45 & 0.030806 & -0.38495 \\
\hline PRMT5 & 0.030842 & -0.30563 \\
\hline & & \\
\hline
\end{tabular}




\begin{tabular}{|l|l|l|} 
APEX1 & 0.030872 & -0.26722 \\
\hline UBA52 & 0.030973 & -0.18546 \\
\hline $\begin{array}{l}\text { ALDH1L1- } \\
\text { AS2 }\end{array}$ & 0.031011 & -0.56135 \\
\hline RUVBL1 & 0.031159 & -0.31242 \\
\hline PXDNL & 0.031173 & -0.58521 \\
\hline HIF3A & 0.031215 & -0.36726 \\
\hline LPAR5 & 0.031229 & 0.49944 \\
\hline MKRN1 & 0.03124 & -0.2462 \\
\hline SUGT1 & 0.031268 & -0.3227 \\
\hline CEBPB & 0.031278 & -0.46473 \\
\hline PSMD13 & 0.03144 & -0.16577 \\
\hline STX8 & 0.031652 & -0.26619 \\
\hline CLSTN1 & 0.031657 & -0.17756 \\
\hline SRP14 & 0.031665 & -0.16348 \\
\hline GATA6-AS1 & 0.031717 & -0.23575 \\
\hline MTCH1 & 0.031753 & -0.44486 \\
\hline ADO & 0.0318 & -0.25563 \\
\hline ACOT9 & 0.031855 & -0.18189 \\
\hline CYB5D2 & 0.031951 & -0.5814 \\
\hline RPL26 & 0.031971 & -0.14777 \\
\hline RUVBL2 & 0.032053 & -0.37922 \\
\hline NEAT1 & 0.032055 & -0.35596 \\
\hline SPATA24 & 0.032056 & -0.31896 \\
\hline CHN1 & 0.032057 & -0.34155 \\
\hline POLR2L & 0.032057 & -0.26405 \\
\hline KANK2 & 0.032067 & -0.32401 \\
\hline TUFM & 0.032069 & -0.14371 \\
\hline TMEM258 & 0.032069 & -0.24075 \\
\hline SSBP1 & 0.032073 & -0.28029 \\
\hline PSMA5 & 0.032107 & -0.22166 \\
\hline PERM1 & 0.03213 & -0.24044 \\
\hline WISP2 & 0.032136 & -0.61489 \\
\hline TYRP1 & 0.032138 & -0.2615 \\
\hline ABCF2 & 0.03214 & -0.29159 \\
\hline AP2A1 & 0.032148 & -0.27227 \\
\hline OARD1 & 0.032151 & -0.30922 \\
\hline CD55 & 0.032166 & -0.25869 \\
\hline COPE & 0.03217 & -0.26005 \\
\hline ABHD11 & 0.032183 & -0.29422 \\
\hline SLC25A29 & 0.032238 & -0.36921 \\
\hline & & \\
\hline
\end{tabular}

\begin{tabular}{|l|l|l|} 
TIMMDC1 & 0.032249 & -0.15166 \\
\hline RPL13 & 0.03225 & -0.25975 \\
\hline VPS45 & 0.032287 & -0.23252 \\
\hline ARHGAP24 & 0.032372 & -0.44656 \\
\hline PPP2R4 & 0.032413 & -0.24363 \\
\hline ITGA6 & 0.032435 & 0.499611 \\
\hline P4HB & 0.032444 & -0.27061 \\
\hline RPL34 & 0.032458 & -0.19978 \\
\hline TOMM40 & 0.032473 & -0.27085 \\
\hline CCNB1IP1 & 0.032583 & -0.20411 \\
\hline TOMM7 & 0.032642 & -0.18831 \\
\hline PHLDA3 & 0.032697 & -0.31311 \\
\hline SEMA5A & 0.0327 & -0.35377 \\
\hline IFI6 & 0.032703 & -0.34789 \\
\hline ISG15 & 0.032719 & -0.4353 \\
\hline DDX54 & 0.032735 & -0.27439 \\
\hline CDKN2AIPNL & 0.032748 & -0.25254 \\
\hline CIZ1 & 0.032753 & -0.25381 \\
\hline SYF2 & 0.032798 & -0.3305 \\
\hline TAF10 & 0.032805 & -0.27692 \\
\hline CNN2 & 0.032806 & -0.23453 \\
\hline PES1 & 0.032812 & -0.34474 \\
\hline APLP2 & 0.032846 & -0.21945 \\
\hline WDTC1 & 0.032879 & -0.24767 \\
\hline GPIHBP1 & 0.032929 & 0.716162 \\
\hline SLC2A4RG & 0.032933 & -0.31134 \\
\hline ADIPOR1 & 0.032961 & -0.19009 \\
\hline NSMCE2 & 0.033041 & -0.2607 \\
\hline ANTXR2 & 0.033088 & -0.23948 \\
\hline TXN & 0.033112 & -0.32177 \\
\hline HAGH & 0.033144 & -0.26948 \\
\hline CERS4 & 0.03322 & -0.42398 \\
\hline CCDC107 & 0.033226 & -0.28906 \\
\hline THYN1 & 0.033229 & -0.26346 \\
\hline RPL28 & 0.033283 & -0.21538 \\
\hline ZNF394 & 0.033332 & -0.30539 \\
\hline ARHGAP10 & 0.033369 & -0.2098 \\
\hline TUBA8 & 0.033375 & 0.687207 \\
\hline GSTT1 & 0.033376 & -1.14097 \\
\hline UBA1 & 0.033394 & -0.17864 \\
\hline MAP7D1 & 0.033484 & -0.17939 \\
\hline & & \\
\hline
\end{tabular}




\begin{tabular}{|l|l|l|} 
TMEM106C & 0.033492 & -0.33189 \\
\hline NSA2 & 0.033509 & -0.61507 \\
\hline PHB & 0.033533 & -0.1705 \\
\hline GABARAP & 0.033543 & -0.27524 \\
\hline RAI2 & 0.033563 & -0.27666 \\
\hline ZC3H13 & 0.03359 & -0.24509 \\
\hline IPO13 & 0.033605 & -0.19624 \\
\hline RHOBTB1 & 0.033645 & -0.34881 \\
\hline MORN4 & 0.033678 & -0.41256 \\
\hline ZRSR2 & 0.03368 & -0.30277 \\
\hline FKBP2 & 0.03378 & -0.28402 \\
\hline IMP4 & 0.033791 & -0.2654 \\
\hline CD320 & 0.033797 & -0.39382 \\
\hline CEP85 & 0.033799 & -0.35432 \\
\hline NDUFA2 & 0.033836 & -0.21085 \\
\hline EIF3D & 0.033886 & -0.21668 \\
\hline NUDT16P1 & 0.033931 & -0.35165 \\
\hline PNMA1 & 0.033934 & -0.2806 \\
\hline TAF1D & 0.033934 & -0.33269 \\
\hline NFIC & 0.033942 & -0.21991 \\
\hline BCAP31 & 0.03395 & -0.25814 \\
\hline ADCY5 & 0.033955 & -0.22636 \\
\hline ARMCX2 & 0.033972 & -0.35739 \\
\hline SF3A2 & 0.033972 & -0.25171 \\
\hline MT1E & 0.03398 & -0.37435 \\
\hline TIMM9 & 0.033989 & -0.24866 \\
\hline FLNC & 0.033991 & -0.29921 \\
\hline COG4 & 0.034012 & -0.28871 \\
\hline ZMAT2 & 0.034018 & -0.25549 \\
\hline TMEM219 & 0.034024 & -0.2405 \\
\hline MRPL40 & 0.034028 & -0.18299 \\
\hline FTSJ3 & 0.034082 & -0.32143 \\
\hline TNPO3 & 0.034111 & -0.24098 \\
\hline ARHGAP1 & 0.034159 & -0.2206 \\
\hline C1orf35 & 0.034189 & -0.29119 \\
\hline BRMS1 & 0.034189 & -0.35444 \\
\hline FAM177A1 & 0.03419 & -0.3486 \\
\hline TMEM109 & 0.034242 & -0.1514 \\
\hline TTF1 & 0.034247 & -0.28503 \\
\hline ARFRP1 & 0.034255 & -0.22561 \\
\hline CBY1 & 0.034258 & -0.33169 \\
\hline & & \\
\hline
\end{tabular}

\begin{tabular}{|l|l|l|} 
AAGAB & 0.034268 & -0.27342 \\
\hline EFTUD2 & 0.03428 & -0.28831 \\
\hline ENKD1 & 0.03433 & -0.45241 \\
\hline STK40 & 0.034331 & -0.38054 \\
\hline SVOP & 0.034337 & -1.06419 \\
\hline CREG1 & 0.034368 & -0.14789 \\
\hline TMEM44-AS1 & 0.034374 & -0.45809 \\
\hline DEDD2 & 0.034377 & -0.26013 \\
\hline IL11RA & 0.034381 & -0.34646 \\
\hline BOP1 & 0.034388 & -0.33889 \\
\hline NABP2 & 0.034394 & -0.30164 \\
\hline PSMB6 & 0.034398 & -0.10589 \\
\hline RPP25L & 0.034401 & -0.25943 \\
\hline PTRF & 0.03446 & -0.13256 \\
\hline MAP2K7 & 0.034484 & -0.2147 \\
\hline MYH7 & 0.034499 & -0.14756 \\
\hline PIK3IP1 & 0.034509 & -0.51557 \\
\hline MAGED2 & 0.034542 & -0.24398 \\
\hline GPAA1 & 0.034604 & -0.189 \\
\hline OXLD1 & 0.034606 & -0.33125 \\
\hline MARK4 & 0.034611 & -0.33795 \\
\hline CUEDC2 & 0.034613 & -0.16919 \\
\hline ATG14 & 0.034698 & -0.24888 \\
\hline SF3B2 & 0.034737 & -0.2289 \\
\hline COPG1 & 0.034749 & -0.16548 \\
\hline TBCA & 0.034805 & -0.26488 \\
\hline COMMD1 & 0.034815 & -0.15443 \\
\hline GADD45B & 0.034827 & -0.49784 \\
\hline SHISA4 & 0.034849 & -0.19029 \\
\hline CRNDE & 0.034849 & -0.52827 \\
\hline AHCY & 0.03485 & -0.3178 \\
\hline RBX1 & 0.034867 & -0.22541 \\
\hline RPL10A & 0.034873 & -0.17068 \\
\hline ODF2 & 0.034886 & -0.31579 \\
\hline NFKBIB & 0.034893 & -0.38029 \\
\hline HACL1 & 0.034896 & -0.35284 \\
\hline IFT46 & 0.034921 & -0.31926 \\
\hline ARID5B & 0.03493 & -0.36754 \\
\hline ANKH & 0.034957 & -0.25857 \\
\hline JMJD8 & 0.034976 & -0.31651 \\
\hline PGK1 & 0.03498 & -0.09618 \\
\hline & & \\
\hline
\end{tabular}




\begin{tabular}{|l|l|l|} 
RHEB & 0.034982 & -0.29912 \\
\hline BRSK1 & 0.034994 & -0.3673 \\
\hline THOC5 & 0.03501 & -0.39403 \\
\hline RBMS3-AS3 & 0.035034 & -0.51495 \\
\hline ZNF524 & 0.035041 & -0.33402 \\
\hline KPNB1 & 0.035045 & -0.20564 \\
\hline WDR18 & 0.035078 & -0.42631 \\
\hline ANKZF1 & 0.035082 & -0.27192 \\
\hline UBAC2 & 0.035092 & -0.2381 \\
\hline SUPV3L1 & 0.035109 & -0.2505 \\
\hline CALCOCO1 & 0.035111 & -0.21693 \\
\hline CTDNEP1 & 0.035115 & -0.17151 \\
\hline EMC9 & 0.035121 & -0.27157 \\
\hline RNF26 & 0.035136 & -0.31242 \\
\hline ATXN10 & 0.035143 & -0.19459 \\
\hline NDRG2 & 0.035152 & -0.14695 \\
\hline NCL & 0.035189 & -0.18666 \\
\hline SCCPDH & 0.03519 & -0.20731 \\
\hline GLTP & 0.035217 & -0.27896 \\
\hline NUBP2 & 0.035226 & -0.28845 \\
\hline ORAI3 & 0.035237 & -0.41031 \\
\hline NAB2 & 0.03527 & -0.35302 \\
\hline AIP & 0.035335 & -0.39889 \\
\hline TBL3 & 0.035351 & -0.32247 \\
\hline BABAM1 & 0.03536 & -0.21146 \\
\hline UBALD1 & 0.035361 & -0.30137 \\
\hline UBE2Q2 & 0.035361 & -0.21968 \\
\hline TWF2 & 0.035362 & -0.32439 \\
\hline GNB2 & 0.035362 & -0.28174 \\
\hline FLOT2 & 0.035363 & -0.24019 \\
\hline ANKRD1 & 0.035363 & -0.60096 \\
\hline CTIF & 0.035364 & -0.34031 \\
\hline CEBPZ & 0.03537 & -0.25298 \\
\hline CWC27 & 0.035398 & -0.25741 \\
\hline CD99L2 & 0.03543 & -0.21221 \\
\hline MPG & 0.035503 & -0.28327 \\
\hline RRP9 & 0.035529 & -0.36141 \\
\hline RORC & 0.035537 & -0.43576 \\
\hline UBE2T & 0.035552 & -0.44856 \\
\hline LRP3 & 0.03561 & -0.24281 \\
\hline RIOK3 & 0.035647 & -0.24306 \\
\hline & & \\
\hline
\end{tabular}

\begin{tabular}{|l|l|l|} 
ABAT & 0.035648 & -0.43632 \\
\hline RPL37 & 0.035649 & -0.15853 \\
\hline SDF2L1 & 0.035695 & -0.39462 \\
\hline USP11 & 0.035701 & -0.32699 \\
\hline LAMTOR1 & 0.035708 & -0.17128 \\
\hline C14orf1 & 0.035729 & -0.27531 \\
\hline CEBPD & 0.035732 & -0.49284 \\
\hline FAU & 0.035748 & -0.15049 \\
\hline RPL39 & 0.035752 & -0.28673 \\
\hline DYNC1LI1 & 0.035781 & -0.15102 \\
\hline RPL23A & 0.03584 & -0.22563 \\
\hline BTBD2 & 0.03585 & -0.38041 \\
\hline C11orf73 & 0.035856 & -0.24875 \\
\hline GTF2F2 & 0.035857 & -0.24101 \\
\hline COMMD9 & 0.035885 & -0.29688 \\
\hline RAD23A & 0.035914 & -0.15544 \\
\hline PRPF19 & 0.035933 & -0.16993 \\
\hline PLBD1 & 0.03597 & -0.24267 \\
\hline RPUSD3 & 0.035973 & -0.24711 \\
\hline DVL1 & 0.036015 & -0.20339 \\
\hline POLR3GL & 0.036017 & -0.2946 \\
\hline COPS3 & 0.036025 & -0.17417 \\
\hline NONO & 0.036063 & -0.20707 \\
\hline AP2M1 & 0.036118 & -0.21351 \\
\hline SNRPB2 & 0.036128 & -0.2526 \\
\hline TMEM203 & 0.036133 & -0.17728 \\
\hline H1FX & 0.036183 & -0.38973 \\
\hline TXNRD1 & 0.036189 & -0.49065 \\
\hline ZDHHC2 & 0.036191 & -0.26944 \\
\hline SCAF1 & 0.036192 & -0.17292 \\
\hline NOP16 & 0.036193 & -0.36398 \\
\hline C1orf226 & 0.036197 & -0.34229 \\
\hline GOLGA2 & 0.036199 & -0.19635 \\
\hline EDEM2 & 0.0362 & -0.33971 \\
\hline GLE1 & 0.036204 & -0.26479 \\
\hline HARS & 0.036207 & -0.25595 \\
\hline CTSB & 0.03621 & -0.22159 \\
\hline FBXL15 & 0.036216 & -0.30803 \\
\hline PKD1 & 0.036223 & -0.22321 \\
\hline ABHD8 & 0.036223 & -0.27618 \\
\hline NCBP2-AS2 & 0.03623 & -0.20476 \\
\hline & & \\
\hline
\end{tabular}




\begin{tabular}{|l|l|l|} 
SNCA & 0.036233 & -0.48798 \\
\hline PDK2 & 0.036234 & -0.23697 \\
\hline XIRP1 & 0.036235 & -0.31783 \\
\hline CDKN1B & 0.036237 & -0.28838 \\
\hline MMGT1 & 0.036237 & -0.2194 \\
\hline MAX & 0.036242 & -0.33015 \\
\hline FAM96B & 0.036243 & -0.2586 \\
\hline ELOF1 & 0.036243 & -0.2683 \\
\hline BANF1 & 0.036243 & -0.31316 \\
\hline TNK2 & 0.036253 & -0.31504 \\
\hline CALR & 0.036253 & -0.20634 \\
\hline TMUB2 & 0.036254 & -0.2077 \\
\hline ARHGAP5- & & \\
AS1 & 0.036256 & -0.36162 \\
\hline PRAF2 & 0.036294 & -0.33803 \\
\hline CDK4 & 0.036296 & -0.23838 \\
\hline TCP1 & 0.036297 & -0.2326 \\
\hline COMMD3 & 0.036309 & -0.26026 \\
\hline RNF167 & 0.036315 & -0.18438 \\
\hline DNAJC6 & 0.036332 & 0.473468 \\
\hline LAPTM4A & 0.036384 & -0.09958 \\
\hline CAMK2G & 0.036408 & -0.30398 \\
\hline MEAF6 & 0.036434 & -0.25225 \\
\hline ARPC4 & 0.036517 & -0.35209 \\
\hline PPID & 0.036546 & -0.3233 \\
\hline RFXANK & 0.036547 & -0.26278 \\
\hline COX7A2L & 0.036559 & -0.23144 \\
\hline AKR7A2 & 0.036639 & -0.26411 \\
\hline PDCD5 & 0.036652 & -0.18871 \\
\hline MKNK2 & 0.036684 & -0.56656 \\
\hline MIF4GD & 0.036688 & -0.34444 \\
\hline PREB & 0.036703 & -0.20891 \\
\hline SNAP47 & 0.036703 & -0.44979 \\
\hline SHMT2 & 0.036704 & -0.29907 \\
\hline PFDN2 & 0.036764 & -0.18553 \\
\hline HIGD2A & 0.036793 & -0.1969 \\
\hline A4GALT & 0.036802 & -0.21474 \\
\hline SYNPO & 0.036819 & -0.12529 \\
\hline PKDCC & 0.036829 & -0.50866 \\
\hline TP53TG1 & 0.036838 & -0.28175 \\
\hline EIF1 & 0.03684 & -0.22448 \\
\hline & & \\
\hline
\end{tabular}

\begin{tabular}{|l|l|l|} 
UGT2B4 & 0.036872 & 0.96781 \\
\hline AES & 0.036878 & -0.12894 \\
\hline TRAM1 & 0.036889 & 0.303004 \\
\hline NOL3 & 0.036899 & -0.28388 \\
\hline OGFR & 0.036914 & -0.28894 \\
\hline NCAM1 & 0.036984 & -0.17442 \\
\hline LYSMD1 & 0.036994 & -0.34905 \\
\hline TOX4 & 0.037001 & -0.21988 \\
\hline PPP2R5B & 0.037015 & -0.27286 \\
\hline NOC2L & 0.037018 & -0.2637 \\
\hline ABHD4 & 0.037021 & -0.32842 \\
\hline WBSCR22 & 0.037038 & -0.22916 \\
\hline CCND2 & 0.037071 & -0.33213 \\
\hline C19orf24 & 0.037155 & -0.44343 \\
\hline MARK2 & 0.037193 & -0.19623 \\
\hline NUDT16L1 & 0.037198 & -0.1828 \\
\hline LMAN2 & 0.037204 & -0.24025 \\
\hline HTATSF1 & 0.037221 & -0.17907 \\
\hline CDK5RAP3 & 0.037227 & -0.30484 \\
\hline CRIP2 & 0.037283 & -0.31158 \\
\hline ABHD12 & 0.037286 & -0.27832 \\
\hline PLIN4 & 0.037297 & -0.34226 \\
\hline MRFAP1L1 & 0.037329 & -0.19483 \\
\hline VPS51 & 0.037355 & -0.19631 \\
\hline FGFRL1 & 0.037363 & -0.32622 \\
\hline BCL7B & 0.037446 & -0.21312 \\
\hline TPD52L2 & 0.037452 & -0.23359 \\
\hline YPEL1 & 0.037534 & -0.31246 \\
\hline MYLK3 & 0.037545 & -0.29417 \\
\hline RBCK1 & 0.037584 & -0.32562 \\
\hline MLF2 & 0.037649 & -0.16014 \\
\hline ELP3 & 0.037705 & -0.21595 \\
\hline UBB & 0.037717 & -0.19496 \\
\hline NDUFB2 & 0.037727 & 0.167528 \\
\hline XAB2 & 0.037745 & -0.24261 \\
\hline NDUFA1 & 0.037752 & -0.23606 \\
\hline RAB20 & 0.037768 & -0.38825 \\
\hline ILKAP & 0.037808 & -0.28373 \\
\hline LRRC47 & 0.037826 & -0.14233 \\
\hline LDB3 & 0.037827 & -0.19309 \\
\hline MEA1 & 0.037845 & -0.29351 \\
\hline & & \\
\hline
\end{tabular}




\begin{tabular}{|l|l|l|} 
VPS16 & 0.037848 & -0.33445 \\
\hline KLHL21 & 0.03786 & -0.30959 \\
\hline FMOD & 0.03788 & -0.66788 \\
\hline HSPD1 & 0.037896 & -0.40253 \\
\hline ITPRIP & 0.037923 & -0.39424 \\
\hline ATOX1 & 0.037927 & -0.25732 \\
\hline CD2BP2 & 0.037935 & -0.21861 \\
\hline CTSL & 0.037947 & -0.16436 \\
\hline EPN2 & 0.037971 & -0.25437 \\
\hline CCDC53 & 0.037993 & -0.44427 \\
\hline TMEM160 & 0.037993 & -0.30858 \\
\hline GPX3 & 0.037995 & -0.28328 \\
\hline RSL1D1 & 0.038042 & -0.19426 \\
\hline GRINA & 0.03811 & -0.182 \\
\hline PRKCSH & 0.038133 & -0.19147 \\
\hline RPL11 & 0.038158 & -0.12005 \\
\hline GPR22 & 0.038161 & 0.72637 \\
\hline NKIRAS2 & 0.03819 & -0.21491 \\
\hline FAM98C & 0.038198 & -0.29988 \\
\hline LAD1 & 0.038212 & -0.70958 \\
\hline SLC25A34 & 0.038229 & -0.6356 \\
\hline RP11-451G4.2 & 0.038241 & -0.6335 \\
\hline VPS72 & 0.038241 & -0.28147 \\
\hline TSC22D4 & 0.038241 & -0.19438 \\
\hline ITGB1BP1 & 0.038246 & -0.3411 \\
\hline POLR2K & 0.038276 & -0.25759 \\
\hline ADAM9 & 0.038311 & 0.324659 \\
\hline SLCO2A1 & 0.038314 & -0.40719 \\
\hline PHC2 & 0.038315 & -0.25133 \\
\hline YIPF3 & 0.038328 & -0.23518 \\
\hline TCEB2 & 0.038371 & -0.26271 \\
\hline C1orf115 & 0.038375 & 0.560171 \\
\hline NUDT16 & 0.038384 & -0.31382 \\
\hline MIR126 & 0.038384 & 0.588636 \\
\hline PTOV1 & 0.038386 & -0.40275 \\
\hline CDC16 & 0.038389 & -0.20721 \\
\hline MAPKAPK5- & 0.038403 & -0.26467 \\
\hline AS1 & 0.03841 & 0.45269 \\
\hline LMBRD2 & 0.038423 & -0.36706 \\
\hline RPS19 & 0.038454 & -0.27768 \\
\hline FTH1 & & \\
\hline
\end{tabular}

\begin{tabular}{|l|l|l|} 
PDZD2 & 0.038455 & 0.357551 \\
\hline SART1 & 0.038459 & -0.25815 \\
\hline TMEM129 & 0.038468 & -0.20959 \\
\hline DCTN2 & 0.038477 & -0.18366 \\
\hline DGUOK & 0.038496 & -0.31123 \\
\hline CYB5R3 & 0.038505 & -0.22288 \\
\hline ARVCF & 0.038517 & -0.3541 \\
\hline UBALD2 & 0.038531 & -0.32891 \\
\hline GSK3A & 0.03855 & -0.23396 \\
\hline CDKN1A & 0.03855 & -0.3932 \\
\hline CCS & 0.038581 & -0.2738 \\
\hline TNS1 & 0.038613 & -0.19777 \\
\hline MAP2K3 & 0.038615 & -0.31403 \\
\hline ACOX2 & 0.038629 & -0.31187 \\
\hline RPL8 & 0.038643 & -0.1336 \\
\hline PMM1 & 0.038654 & -0.44972 \\
\hline EHD3 & 0.038657 & -0.41696 \\
\hline TIMM10 & 0.038672 & -0.32651 \\
\hline MIR5690 & 0.038673 & -1.01813 \\
\hline MRPL51 & 0.038675 & -0.1205 \\
\hline H2AFX & 0.038681 & -0.23258 \\
\hline CCDC9 & 0.038686 & -0.31105 \\
\hline AP3D1 & 0.038688 & -0.23903 \\
\hline EGLN3 & 0.038702 & -0.36243 \\
\hline SMPD1 & 0.038714 & -0.20845 \\
\hline GKAP1 & 0.038723 & -0.33471 \\
\hline IDH1-AS1 & 0.038727 & -0.43623 \\
\hline SERGEF & 0.03873 & -0.32063 \\
\hline SETD3 & 0.038731 & -0.20027 \\
\hline C1QTNF1 & 0.038739 & -0.5785 \\
\hline TMA7 & 0.038743 & -0.29814 \\
\hline MAD2L2 & 0.038743 & -0.41339 \\
\hline PLD3 & 0.038744 & -0.22871 \\
\hline DDOST & 0.038749 & -0.14211 \\
\hline RPF1 & 0.038758 & -0.20963 \\
\hline POLE4 & 0.038764 & -0.30762 \\
\hline C19orf70 & 0.038769 & -0.26739 \\
\hline CCM2 & 0.038772 & -0.27933 \\
\hline CTF1 & 0.038807 & -0.37463 \\
\hline NAT14 & 0.038835 & -0.45773 \\
\hline NDUFAB1 & 0.038846 & 0.249002 \\
\hline & & \\
\hline
\end{tabular}




\begin{tabular}{|l|l|l|} 
DCAKD & 0.038868 & -0.26436 \\
\hline DNAJC21 & 0.038868 & -0.26833 \\
\hline EIF3H & 0.038869 & -0.12197 \\
\hline RNF208 & 0.038875 & -0.21658 \\
\hline RPS27L & 0.038886 & -0.45338 \\
\hline RABGGTB & 0.038889 & -0.29911 \\
\hline CDK18 & 0.038997 & -0.20614 \\
\hline EEF1A1 & 0.039115 & -0.29709 \\
\hline ATP5J2 & 0.039351 & 0.152296 \\
\hline MTSS1 & 0.039649 & 0.405886 \\
\hline PFKFB2 & 0.03972 & -0.50091 \\
\hline CD248 & 0.039828 & 0.682005 \\
\hline COMP & 0.039889 & -0.84929 \\
\hline UTRN & 0.039902 & 0.391413 \\
\hline SYNE1 & 0.039974 & 0.420362 \\
\hline NDUFA11 & 0.040079 & -0.27112 \\
\hline XPR1 & 0.040079 & -0.40453 \\
\hline PKIA & 0.040133 & 0.190573 \\
\hline DUSP23 & 0.040206 & -0.3803 \\
\hline ARPC2 & 0.04023 & -0.18688 \\
\hline IVNS1ABP & 0.040303 & -0.50274 \\
\hline ZMYND19 & 0.040303 & -0.32232 \\
\hline AKT1S1 & 0.040327 & -0.30619 \\
\hline APOPT1 & 0.040328 & -0.31231 \\
\hline MANBAL & 0.040437 & -0.31077 \\
\hline HIST3H2A & 0.040552 & -0.39049 \\
\hline SNAP29 & 0.040615 & -0.26867 \\
\hline NCEH1 & 0.040649 & 0.47032 \\
\hline TIMP3 & 0.040691 & 0.458962 \\
\hline TUBB4B & 0.040782 & 0.721508 \\
\hline HMGN4 & 0.04093 & -0.21951 \\
\hline AQP7 & 0.040948 & -0.33909 \\
\hline TXN2 & 0.040982 & -0.2091 \\
\hline NR1H2 & 0.041104 & -0.33779 \\
\hline JAM3 & 0.0412 & -0.45255 \\
\hline TSR2 & 0.04123 & -0.23005 \\
\hline MAP7D3 & 0.041242 & -0.3786 \\
\hline P4HTM & 0.04128 & -0.23929 \\
\hline SH3BGR & 0.041381 & -1.03842 \\
\hline ETNPPL & 0.041395 & 0.520276 \\
\hline HMGA1 & 0.041419 & -0.19421 \\
\hline & & \\
\hline
\end{tabular}

\begin{tabular}{|l|l|l|} 
CHMP1A & 0.04147 & -0.3999 \\
\hline MIIP & 0.041477 & -0.58435 \\
\hline MT1X & 0.041477 & -0.82048 \\
\hline EML1 & 0.041581 & 0.398453 \\
\hline OPA1 & 0.041586 & 0.179787 \\
\hline TAOK1 & 0.041596 & 0.273087 \\
\hline TMEM261 & 0.04169 & -0.23682 \\
\hline KLHL31 & 0.041734 & 0.331983 \\
\hline EXOSC5 & 0.04175 & -0.33487 \\
\hline TFRC & 0.041854 & 1.359095 \\
\hline MAN2C1 & 0.042233 & -0.27072 \\
\hline ZNF41 & 0.042259 & 0.340503 \\
\hline ABCA8 & 0.042578 & 0.648874 \\
\hline FRA10AC1 & 0.042611 & -0.32812 \\
\hline RBPMS & 0.042622 & -0.19739 \\
\hline SPC24 & 0.042623 & -0.42661 \\
\hline CLNS1A & 0.042626 & -0.14629 \\
\hline PTGR2 & 0.042626 & -0.24052 \\
\hline GALNT18 & 0.042629 & -0.27594 \\
\hline KRI1 & 0.04263 & -0.3348 \\
\hline NAPA & 0.042632 & -0.1559 \\
\hline C18orf8 & 0.042648 & -0.32397 \\
\hline ARHGEF17 & 0.042658 & -0.2543 \\
\hline NUCB2 & 0.04266 & -0.22229 \\
\hline SNX17 & 0.042689 & -0.24286 \\
\hline VPS35 & 0.042693 & -0.16446 \\
\hline LINC00847 & 0.042694 & -0.30445 \\
\hline RTF1 & 0.042714 & -0.15155 \\
\hline GRK6 & 0.042721 & -0.27522 \\
\hline ASUN & 0.042725 & -0.21673 \\
\hline TMEM141 & 0.042731 & -0.18739 \\
\hline UBE2MP1 & 0.042739 & -0.20882 \\
\hline PFN1 & 0.042746 & -0.18697 \\
\hline BSDC1 & 0.042749 & -0.16824 \\
\hline SNHG8 & 0.042777 & -0.34416 \\
\hline R3HDM4 & 0.042781 & -0.20931 \\
\hline TSPAN17 & 0.042792 & -0.26126 \\
\hline PAFAH1B1 & 0.042805 & 0.142887 \\
\hline OSGIN1 & 0.042848 & -0.45382 \\
\hline KLHDC8B & 0.042867 & -0.18668 \\
\hline VEGFB & 0.042867 & -0.20507 \\
\hline & & \\
\hline
\end{tabular}




\begin{tabular}{|l|l|l|} 
KCND3 & 0.04292 & 0.403417 \\
\hline ITGB1 & 0.042933 & 0.292811 \\
\hline 4-Sep & 0.042957 & -0.21332 \\
\hline MIEN1 & 0.04298 & -0.21318 \\
\hline C7orf26 & 0.042984 & -0.21517 \\
\hline TM4SF1 & 0.04299 & 0.367067 \\
\hline EMILIN3 & 0.042993 & 0.743265 \\
\hline FAM127A & 0.043012 & -0.13189 \\
\hline GPR153 & 0.043028 & -0.68191 \\
\hline PTS & 0.043102 & -0.26375 \\
\hline LOH12CR1 & 0.043119 & -0.26707 \\
\hline CAST & 0.043171 & -0.1509 \\
\hline POSTN & 0.043196 & -0.71602 \\
\hline MAP3K10 & 0.043217 & -0.28638 \\
\hline NSRP1 & 0.043228 & -0.22539 \\
\hline ADAMTS15 & 0.043257 & 0.546947 \\
\hline TAF3 & 0.043265 & -0.23352 \\
\hline TCOF1 & 0.043275 & -0.24971 \\
\hline PPM1G & 0.043291 & -0.18035 \\
\hline STRA13 & 0.043304 & -0.34545 \\
\hline GARS & 0.043332 & -0.16787 \\
\hline DHX29 & 0.043332 & -0.19836 \\
\hline RBM24 & 0.043341 & -0.24186 \\
\hline EML2 & 0.043345 & -0.2887 \\
\hline IRX3 & 0.043472 & -0.40778 \\
\hline ZNF579 & 0.043485 & -0.24029 \\
\hline IRS2 & 0.043535 & -0.44685 \\
\hline PRDX5 & 0.043545 & -0.12374 \\
\hline ORC3 & 0.043559 & -0.17899 \\
\hline F3 & 0.043564 & -0.26721 \\
\hline LSAMP & 0.04362 & 0.662075 \\
\hline ANKRD12 & 0.043635 & -0.22516 \\
\hline MIR6748 & 0.043642 & 0.576951 \\
\hline BCCIP & 0.043651 & -0.23845 \\
\hline RXRG & 0.043663 & -0.43099 \\
\hline EXOSC7 & 0.04368 & -0.31322 \\
\hline OTUB1 & 0.043746 & -0.18332 \\
\hline YIF1B & 0.043766 & -0.26359 \\
\hline ADCK3 & 0.043788 & -0.32058 \\
\hline MRPS26 & 0.043817 & -0.16512 \\
\hline MRPS15 & 0.043821 & -0.13981 \\
\hline & & \\
\hline
\end{tabular}

\begin{tabular}{|l|l|l|} 
ZNF274 & 0.043824 & -0.22026 \\
\hline PPIG & 0.043832 & -0.1633 \\
\hline GLG1 & 0.043851 & -0.1679 \\
\hline FOXN3-AS1 & 0.043859 & -0.3671 \\
\hline TMOD1 & 0.043875 & -0.12936 \\
\hline PSME1 & 0.044005 & -0.18707 \\
\hline ATP5G1 & 0.044025 & 0.252811 \\
\hline CCT8 & 0.044075 & -0.23011 \\
\hline SSRP1 & 0.044078 & -0.22359 \\
\hline DNAJB1 & 0.044088 & -0.19553 \\
\hline POLR1E & 0.04409 & -0.2488 \\
\hline OGDH & 0.044109 & 0.164603 \\
\hline KIAA1462 & 0.044113 & 0.656201 \\
\hline C19orf54 & 0.044118 & -0.37015 \\
\hline VPS13D & 0.044122 & 0.260475 \\
\hline FIGF & 0.044151 & 0.53526 \\
\hline SHARPIN & 0.044177 & -0.1874 \\
\hline RPS13 & 0.044187 & -0.11095 \\
\hline PPP2R3C & 0.044233 & -0.25372 \\
\hline LPAR3 & 0.044328 & 0.474278 \\
\hline DDB1 & 0.044389 & -0.12906 \\
\hline IFITM1 & 0.044422 & -0.27235 \\
\hline SOBP & 0.044503 & -0.25994 \\
\hline AZIN1 & 0.044509 & -0.23306 \\
\hline MYPOP & 0.044571 & -0.19777 \\
\hline PPP1R3G & 0.044642 & -0.36934 \\
\hline IGBP1 & 0.04468 & -0.181 \\
\hline GOLGB1 & 0.044739 & -0.23189 \\
\hline LONP1 & 0.044766 & -0.2357 \\
\hline SLC12A4 & 0.044854 & -0.21426 \\
\hline PPP4C & 0.044869 & -0.26589 \\
\hline PDE6D & 0.044909 & -0.30053 \\
\hline ENO1 & 0.045018 & 0.368078 \\
\hline CBX3 & 0.045028 & -0.23295 \\
\hline CYGB & 0.045147 & 0.484722 \\
\hline PTTG1IP & 0.045153 & -0.14804 \\
\hline EEF1G & 0.045169 & -0.23034 \\
\hline C11orf96 & 0.045204 & -0.28625 \\
\hline DHPS & 0.045206 & -0.17092 \\
\hline PGLS & 0.045295 & -0.31026 \\
\hline SMAP1 & 0.045427 & -0.21296 \\
\hline & & \\
\hline
\end{tabular}




\begin{tabular}{|l|l|l|} 
TMEM245 & 0.045476 & 0.238885 \\
\hline CDPF1 & 0.045552 & -0.27819 \\
\hline TRAPPC6A & 0.04559 & -0.32744 \\
\hline TBC1D22A & 0.045678 & -0.22663 \\
\hline URM1 & 0.045749 & -0.23648 \\
\hline PPP1R35 & 0.04587 & -0.43023 \\
\hline DNAJA4 & 0.045952 & -0.73967 \\
\hline MFSD6 & 0.045963 & 0.455339 \\
\hline KAT2B & 0.045965 & 0.276942 \\
\hline YWHAB & 0.046168 & -0.09964 \\
\hline RAPGEF2 & 0.046195 & 0.348173 \\
\hline FGFBP2 & 0.046198 & 0.626356 \\
\hline ST3GAL6 & 0.046284 & -0.24462 \\
\hline TAF11 & 0.046295 & -0.24922 \\
\hline TRPC4AP & 0.046408 & -0.20512 \\
\hline TRDN & 0.046461 & -0.15193 \\
\hline TMEM71 & 0.046493 & -0.33857 \\
\hline TMED9 & 0.046493 & -0.23333 \\
\hline ALDH7A1 & 0.046532 & -0.28075 \\
\hline CSNK1E & 0.046613 & -0.19763 \\
\hline RPPH1 & 0.046663 & -0.44324 \\
\hline ARL3 & 0.046702 & -0.227 \\
\hline ATP6V1H & 0.046822 & -0.20221 \\
\hline RSG1 & 0.046887 & -0.35664 \\
\hline MAP3K7CL & 0.046917 & -0.50744 \\
\hline NT5C1A & 0.046935 & 0.656828 \\
\hline NAA20 & 0.046991 & -0.14086 \\
\hline STARD10 & 0.047049 & -0.29455 \\
\hline CRYAB & 0.047064 & -0.4513 \\
\hline C22orf39 & 0.047064 & -0.22494 \\
\hline ATP13A3 & 0.047239 & -0.30394 \\
\hline SCARNA7 & 0.047274 & -0.32296 \\
\hline INTS10 & 0.047293 & -0.23943 \\
\hline SAFB & 0.047321 & -0.19663 \\
\hline EEFSEC & 0.047366 & -0.33974 \\
\hline FBXW11 & 0.047424 & -0.19659 \\
\hline KLC2 & 0.047432 & -0.27713 \\
\hline CREB3L2 & 0.047452 & 0.320287 \\
\hline GNPAT & 0.047476 & -0.14615 \\
\hline SGCG & 0.047528 & -0.22029 \\
\hline BNIP3 & 0.047535 & -0.15576 \\
\hline & & \\
\hline
\end{tabular}

\begin{tabular}{|l|l|l|} 
ETV1 & 0.047575 & -0.34829 \\
\hline PAX8-AS1 & 0.047598 & 0.515569 \\
\hline CTNNA3 & 0.047603 & 0.420902 \\
\hline TLR4 & 0.047667 & 0.507093 \\
\hline C1orf43 & 0.047679 & -0.14213 \\
\hline EHBP1L1 & 0.047699 & -0.22378 \\
\hline UBE3A & 0.047775 & -0.14015 \\
\hline CDH5 & 0.047911 & 0.7136 \\
\hline SLC16A1 & 0.047959 & 0.342604 \\
\hline ZNF667-AS1 & 0.047976 & -0.3176 \\
\hline PRKAR2B & 0.048108 & -0.28691 \\
\hline ELK3 & 0.048157 & 0.621871 \\
\hline AIF1L & 0.04817 & 0.760357 \\
\hline MYCT1 & 0.048203 & 0.49137 \\
\hline APRT & 0.048238 & -0.26923 \\
\hline NEDD8 & 0.048326 & -0.23093 \\
\hline ZNF404 & 0.048442 & -0.30295 \\
\hline RPA1 & 0.048614 & -0.28105 \\
\hline APOOL & 0.048967 & 0.380361 \\
\hline NT5C & 0.049081 & -0.24269 \\
\hline LINC00957 & 0.049119 & -0.32978 \\
\hline HDDC2 & 0.04915 & -0.26761 \\
\hline HLA-B & 0.049228 & 0.583581 \\
\hline SHISA3 & 0.049266 & 0.929802 \\
\hline TNN1 & 0.049448 & 0.738987 \\
\hline MTHFD1 & 0.049485 & -0.20469 \\
\hline NDUFS5 & 0.049485 & -0.15074 \\
\hline GLTSCR2 & 0.049597 & -0.1596 \\
\hline NMT1 & 0.049633 & -0.13706 \\
\hline VPS4B & 0.049698 & 0.267645 \\
\hline QTRT1 & 0.049764 & -0.27948 \\
\hline ELOVL2 & 0.049783 & 0.395433 \\
\hline CAMK2A & 0.049788 & -0.25057 \\
\hline APP & 0.04983 & 0.252107 \\
\hline NEIL2 & 0.049882 & -0.37047 \\
\hline PPAP2B & 0.049882 & 0.270841 \\
\hline GSR & 0.049948 & -0.21135 \\
\hline IL6ST & 0.049957 & 0.330776 \\
\hline ALYREF & 0.049987 & -0.2162 \\
\hline & &
\end{tabular}


Supplemental Table 4. DEG in obese

samples when comparing NF to LVH,

(p_adj < 0.05)

\begin{tabular}{|l|l|l|}
\hline GeneName & p_adj & log2FC \\
\hline HMGCS2 & 0 & -1.52337 \\
\hline NUPR1 & 0 & -0.84992 \\
\hline MLYCD & 0 & -0.63952 \\
\hline SEC14L5 & 0 & 1.368913 \\
\hline PCDH7 & 0 & 1.017951 \\
\hline PLIN2 & 0 & -1.26669 \\
\hline TINAGL1 & 0 & -0.45178 \\
\hline PDHB & 0 & 0.283917 \\
\hline SUCLA2 & 0 & 0.381001 \\
\hline NDUFS1 & 0 & 0.45615 \\
\hline ACADM & 0 & 0.311292 \\
\hline DLD & 0 & 0.313703 \\
\hline CLIC5 & 0 & 0.403322 \\
\hline NDUFS2 & 0 & 0.220307 \\
\hline RGS5 & 0 & 0.517071 \\
\hline GPNMB & 0 & 0.917826 \\
\hline MYL4 & 0 & 1.223488 \\
\hline FAM129A & 0 & 0.530084 \\
\hline UBC & 0 & -0.78413 \\
\hline ACADVL & 0 & -0.20461 \\
\hline NEXN & 0 & -0.36827 \\
\hline IDH2 & 0 & 0.329235 \\
\hline RYR2 & 0 & 0.722121 \\
\hline MYL7 & 0 & 1.550622 \\
\hline AGT & $2.00 \mathrm{E}-15$ & 0.45158 \\
\hline PDK4 & $4.00 \mathrm{E}-15$ & -2.80167 \\
\hline TAF7 & $4.00 \mathrm{E}-15$ & -0.35599 \\
\hline CDKN1A & $6.99 \mathrm{E}-15$ & -0.78787 \\
\hline SLC25A34 & $1.40 \mathrm{E}-14$ & -1.2642 \\
\hline NPPA & $1.40 \mathrm{E}-14$ & -4.02274 \\
\hline GADD45G & $1.60 \mathrm{E}-14$ & -0.97254 \\
\hline TRIM63 & $2.20 \mathrm{E}-14$ & -0.55388 \\
\hline CTSD & $4.60 \mathrm{E}-14$ & -0.4451 \\
\hline AZGP1 & $1.46 \mathrm{E}-13$ & -1.21571 \\
\hline CEBPB & $5.28 \mathrm{E}-09$ & -0.61231 \\
\hline
\end{tabular}

\begin{tabular}{|l|l|l|} 
POSTN & $5.92 \mathrm{E}-09$ & -1.06062 \\
\hline PLVAP & $7.69 \mathrm{E}-08$ & -1.02931 \\
\hline FABP3 & $1.33 \mathrm{E}-05$ & -0.4155 \\
\hline TRIP10 & $1.47 \mathrm{E}-05$ & -0.51549 \\
\hline HSP90AB1 & 0.000225 & -0.55578 \\
\hline RPS24 & 0.000506 & -0.44797 \\
\hline DNAJA4 & 0.000586 & -1.25998 \\
\hline MRFAP1 & 0.000686 & -0.20271 \\
\hline SNHG5 & 0.000939 & -0.65023 \\
\hline PABPC1 & 0.002124 & -0.43332 \\
\hline ANXA11 & 0.002186 & -0.31079 \\
\hline ATP11A & 0.002511 & 0.533909 \\
\hline TBX20 & 0.002823 & 0.827152 \\
\hline PPIF & 0.003478 & 0.342752 \\
\hline ZNF622 & 0.007096 & -0.51671 \\
\hline FKBP5 & 0.007155 & -0.86411 \\
\hline RASD1 & 0.007468 & -1.23112 \\
\hline TSPO & 0.010087 & -0.32877 \\
\hline PDE3A & 0.012594 & 0.494364 \\
\hline ANGPTL4 & 0.012837 & -1.54119 \\
\hline DNAJB1 & 0.014125 & -0.59205 \\
\hline SS18L2 & 0.014448 & -0.53527 \\
\hline CCL21 & 0.014933 & -0.81735 \\
\hline RPS19BP1 & 0.015094 & -0.37503 \\
\hline SPSB1 & 0.015303 & -0.62767 \\
\hline ETFA & 0.016655 & -0.253 \\
\hline EIF4A2 & 0.017004 & -0.36697 \\
\hline TST & 0.017433 & -0.6759 \\
\hline PGAM2 & 0.017968 & 0.356582 \\
\hline SLC16A1 & 0.01809 & 0.359136 \\
\hline RPL10A & 0.018447 & -0.22134 \\
\hline PLA2G16 & 0.018502 & -0.4033 \\
\hline COX6A1 & 0.018897 & -0.27185 \\
\hline TSR3 & 0.019015 & -0.31082 \\
\hline SDHA & 0.019244 & 0.2945 \\
\hline THSD4 & 0.01937 & 0.612104 \\
\hline JTB & 0.019502 & -0.35856 \\
\hline TXNIP & 0.019841 & -0.67479 \\
\hline ATP5B & 0.020097 & 0.160013 \\
\hline SF3B5 & 0.020807 & -0.3282 \\
\hline C6orf1 & 0.021257 & -0.44422 \\
\hline & & \\
\hline
\end{tabular}




\begin{tabular}{|l|l|l|} 
C10orf10 & 0.02189 & -0.50913 \\
\hline GPR22 & 0.021962 & 0.6118 \\
\hline AMOTL2 & 0.022329 & -0.4103 \\
\hline EIF3D & 0.022381 & -0.25298 \\
\hline PNRC1 & 0.022442 & -0.32237 \\
\hline BTF3 & 0.022593 & -0.1762 \\
\hline SPR & 0.023243 & -0.54463 \\
\hline NSA2 & 0.023588 & -0.65499 \\
\hline CLPP & 0.023947 & -0.32218 \\
\hline HDDC2 & 0.024731 & -0.3461 \\
\hline PIM3 & 0.025119 & -0.48626 \\
\hline CHP1 & 0.025409 & -0.35118 \\
\hline CES2 & 0.025626 & -0.59991 \\
\hline ILF3-AS1 & 0.026093 & -0.60451 \\
\hline DDIT4 & 0.026101 & -0.5924 \\
\hline DNAJB2 & 0.026361 & -0.48136 \\
\hline KLF15 & 0.02653 & -0.70413 \\
\hline GLUL & 0.026805 & -0.57424 \\
\hline APOPT1 & 0.026905 & -0.40913 \\
\hline LAD1 & 0.026985 & -0.72085 \\
\hline HIST1H2BD & 0.026992 & -0.37751 \\
\hline HIGD2A & 0.027319 & -0.20007 \\
\hline MAP3K7CL & 0.027687 & -0.5808 \\
\hline P4HA2 & 0.027888 & -0.51364 \\
\hline ETFB & 0.028124 & -0.22952 \\
\hline FAM212B & 0.028241 & -0.58876 \\
\hline SCARB2 & 0.028244 & -0.19712 \\
\hline BCS1L & 0.028414 & -0.31361 \\
\hline AGTRAP & 0.028565 & -0.42563 \\
\hline FTL & 0.028623 & -0.35963 \\
\hline CERK & 0.028674 & -0.47531 \\
\hline SRSF3 & 0.028815 & -0.37009 \\
\hline TAF11 & 0.029432 & -0.32988 \\
\hline B3GALT2 & 0.029454 & 0.496169 \\
\hline LMAN2 & 0.029648 & -0.22971 \\
\hline ENO2 & 0.029835 & -0.40776 \\
\hline NDUFA1 & 0.030201 & -0.14984 \\
\hline SLC25A20 & 0.030399 & -0.63407 \\
\hline BRK1 & 0.030816 & -0.16216 \\
\hline DUSP1 & 0.031356 & -0.47569 \\
\hline FLOT2 & 0.031435 & -0.17131 \\
\hline & & \\
\hline
\end{tabular}

\begin{tabular}{|l|l|l|} 
SQSTM1 & 0.031502 & -0.29943 \\
\hline PTGES3 & 0.031626 & -0.26122 \\
\hline RP11- & & \\
451G4.2 & 0.031715 & -1.02284 \\
\hline RPS4X & 0.031805 & -0.17197 \\
\hline SNRNP70 & 0.032196 & -0.30914 \\
\hline GNL3 & 0.032279 & -0.386 \\
\hline MT1M & 0.032563 & -0.60151 \\
\hline PSME1 & 0.032774 & -0.20252 \\
\hline TXNRD1 & 0.032906 & -0.68006 \\
\hline CLU & 0.033301 & -0.8501 \\
\hline GSS & 0.033486 & -0.42971 \\
\hline CCDC85B & 0.033578 & -0.35189 \\
\hline HIF3A & 0.0337 & -0.48731 \\
\hline HSPA4 & 0.033707 & -0.24471 \\
\hline TMEM109 & 0.033713 & -0.20255 \\
\hline C11orf24 & 0.033819 & -0.37851 \\
\hline HLA-A & 0.033857 & -1.07959 \\
\hline CYSTM1 & 0.034106 & -0.47757 \\
\hline HS6ST1 & 0.034253 & -0.34455 \\
\hline CCT3 & 0.03428 & -0.41886 \\
\hline KEAP1 & 0.034344 & -0.22295 \\
\hline ATP6V1G1 & 0.034351 & -0.15847 \\
\hline PSMB4 & 0.03439 & -0.16743 \\
\hline MANBAL & 0.034404 & -0.32331 \\
\hline TSSC4 & 0.034527 & -0.33444 \\
\hline EEF1B2 & 0.034575 & -0.22103 \\
\hline HSPE1 & 0.034606 & -0.66784 \\
\hline CRYAB & 0.034918 & -0.64407 \\
\hline LRRC4 & 0.035271 & -0.49529 \\
\hline PLEKHO1 & 0.035305 & -0.59406 \\
\hline ADAM11 & 0.035425 & 0.963323 \\
\hline PEBP1 & 0.035486 & -0.18038 \\
\hline TNNC1 & 0.035576 & 0.099498 \\
\hline FDFT1 & 0.035763 & -0.35421 \\
\hline YBX3 & 0.035845 & -0.31249 \\
\hline MT1X & 0.036025 & -0.93198 \\
\hline IMPDH2 & 0.036094 & -0.39243 \\
\hline TRIM54 & 0.036123 & -0.31441 \\
\hline NGFR & 0.036183 & 0.731879 \\
\hline SLC25A3 & 0.036573 & 0.232832 \\
\hline & &
\end{tabular}




\begin{tabular}{|l|l|l|} 
SPRYD3 & 0.036704 & -0.26283 \\
\hline TUBA3E & 0.036916 & -0.48888 \\
\hline GGPS1 & 0.037146 & -0.23481 \\
\hline GGTA1P & 0.037154 & 0.872429 \\
\hline SND1 & 0.0372 & -0.3163 \\
\hline C10orf54 & 0.037269 & -0.31484 \\
\hline POLR3GL & 0.037398 & -0.38815 \\
\hline LSM7 & 0.037624 & -0.58806 \\
\hline GOT2 & 0.037858 & 0.255418 \\
\hline MRFAP1L1 & 0.037936 & -0.26112 \\
\hline DUSP23 & 0.038242 & -0.48478 \\
\hline ITGB5 & 0.038249 & -0.3956 \\
\hline MYO1C & 0.038317 & -0.21544 \\
\hline APOOL & 0.038404 & 0.351662 \\
\hline SCN1B & 0.038415 & -0.50331 \\
\hline KLHL36 & 0.038418 & -0.48906 \\
\hline RSL1D1 & 0.038745 & -0.21678 \\
\hline HADHA & 0.038791 & -0.34315 \\
\hline CHCHD2 & 0.038807 & -0.15763 \\
\hline TTLL12 & 0.039027 & -0.62207 \\
\hline ADCK3 & 0.039031 & -0.5208 \\
\hline SLC25A39 & 0.039036 & -0.32232 \\
\hline CCT7 & 0.039088 & -0.28018 \\
\hline ADO & 0.039112 & -0.29512 \\
\hline MIR5690 & 0.039171 & -0.61361 \\
\hline TERF2IP & 0.039215 & -0.26911 \\
\hline PRDX1 & 0.039357 & -0.41997 \\
\hline NPPB & 0.039417 & -1.72874 \\
\hline STIP1 & 0.039604 & -0.39623 \\
\hline H1FX & 0.039639 & -0.79503 \\
\hline FAM96B & 0.039689 & -0.25402 \\
\hline VSTM2L & 0.039785 & -0.97141 \\
\hline C110rf68 & 0.039816 & -0.24351 \\
\hline FIS1 & 0.040053 & -0.18151 \\
\hline DYNLRB1 & 0.040103 & -0.23809 \\
\hline OSER1 & 0.040119 & -0.40278 \\
\hline SAMD1 & 0.040196 & -0.42475 \\
\hline MAF1 & 0.040273 & -0.2453 \\
\hline MPST & 0.04035 & -0.39317 \\
\hline SRM & 0.040441 & -0.28869 \\
\hline & & \\
\hline
\end{tabular}

\begin{tabular}{|l|l|l|} 
PLOD1 & 0.04053 & -0.46537 \\
\hline KANK2 & 0.040589 & -0.29737 \\
\hline CHURC1 & 0.040622 & -0.34072 \\
\hline KLF10 & 0.040722 & -0.49371 \\
\hline CENPB & 0.040746 & -0.27682 \\
\hline IMP4 & 0.040824 & -0.30508 \\
\hline PHF5A & 0.040864 & -0.35502 \\
\hline HSPB2 & 0.041105 & -0.34623 \\
\hline SIAE & 0.041108 & -0.30127 \\
\hline SNHG8 & 0.041204 & -0.37071 \\
\hline UBXN6 & 0.041528 & -0.26849 \\
\hline CA3 & 0.041911 & 1.622109 \\
\hline POPDC2 & 0.042035 & -0.2717 \\
\hline LAMTOR4 & 0.042074 & -0.21479 \\
\hline ABCF2 & 0.042127 & -0.27423 \\
\hline HSPA2 & 0.042397 & -0.67426 \\
\hline PSMA7 & 0.042442 & -0.23883 \\
\hline RABAC1 & 0.043129 & -0.31624 \\
\hline DRAP1 & 0.043515 & -0.29077 \\
\hline PLEKHA4 & 0.043644 & -0.47818 \\
\hline GDI2 & 0.043684 & -0.21875 \\
\hline NABP2 & 0.043803 & -0.31278 \\
\hline MORN4 & 0.04407 & -0.48357 \\
\hline NGFRAP1 & 0.044122 & 0.294436 \\
\hline CMYA5 & 0.044239 & 0.34836 \\
\hline HMGA1 & 0.044499 & -0.27266 \\
\hline DBI & 0.045126 & -0.2712 \\
\hline FLJ42969 & 0.045495 & -1.10766 \\
\hline PLIN5 & 0.045642 & -0.36538 \\
\hline FAM3A & 0.04754 & -0.35208 \\
\hline SDPR & 0.047867 & -0.3549 \\
\hline CSNK2A2 & 0.04787 & -0.28847 \\
\hline ORAI3 & 0.047875 & -0.42071 \\
\hline ACSL1 & 0.047905 & -0.3018 \\
\hline NOP10 & 0.047961 & -0.24819 \\
\hline LARP7 & 0.048245 & -0.31615 \\
\hline TP53I3 & 0.048609 & -0.30731 \\
\hline TADA3 & 0.04919 & -0.31025 \\
\hline NANOS1 & 0.049464 & -0.94055 \\
\hline
\end{tabular}


Supplemental Table 5. DEG in all lean samples when comparing NF to LVH $\left(p_{-}\right.$adj $\left.<0.05\right)$

\begin{tabular}{|l|l|l|}
\hline GeneName & p_adj & log2FC \\
\hline NPPA & $6.99 \mathrm{E}-15$ & -3.73254 \\
\hline DCN & $2.90 \mathrm{E}-14$ & 1.003963 \\
\hline MIR6723 & $2.71 \mathrm{E}-11$ & 3.901165 \\
\hline CNN1 & 0.015874 & 1.230719 \\
\hline C1QA & 0.027665 & 0.985104 \\
\hline SPARCL1 & 0.038244 & 0.600471 \\
\hline C1QC & 0.043925 & 1.175501 \\
\hline
\end{tabular}


Supplemental Table 6. DEG in when comparing lean NF to obese LVH samples (p_adj < 0.05)

\begin{tabular}{|l|l|l|}
\hline GeneName & p_adj & $\log 2 \mathrm{FC}$ \\
\hline MYL7 & 0 & 1.231699 \\
\hline MYL9 & $1.30 \mathrm{E}-14$ & 0.501845 \\
\hline TPM1 & $1.60 \mathrm{E}-14$ & 0.26546 \\
\hline TPM2 & $2.49 \mathrm{E}-10$ & 0.422366 \\
\hline ACTC1 & 0.00024 & 0.611517 \\
\hline ECHDC3 & 0.035165 & -0.73491 \\
\hline RASD1 & 0.037077 & -0.94223 \\
\hline
\end{tabular}


Supplemental Table 7. DEG in when comparing lean NF to obese LVH samples, males only ( $p_{-}$adj $\left.<0.05\right)$

\begin{tabular}{|l|l|l|}
\hline GeneName & p_adj & log2FC \\
\hline SLC16A7 & 0 & 0.739186 \\
\hline DANCR & 0 & -0.371 \\
\hline IRX3 & 0 & -0.72525 \\
\hline MYL7 & 0 & 1.26689 \\
\hline PRKAR1A & 0 & 0.331577 \\
\hline SLC38A1 & $2.00 \mathrm{E}-15$ & 0.602805 \\
\hline FAM155B & $3.00 \mathrm{E}-15$ & -0.59298 \\
\hline HSPB3 & $6.99 \mathrm{E}-15$ & -0.32055 \\
\hline PPAPDC3 & $7.99 \mathrm{E}-15$ & -0.33143 \\
\hline FAM107A & $1.30 \mathrm{E}-14$ & -0.35431 \\
\hline NINJ1 & $1.60 \mathrm{E}-14$ & -0.48848 \\
\hline SAT2 & $1.70 \mathrm{E}-14$ & -0.35145 \\
\hline BSG & $1.90 \mathrm{E}-14$ & -0.20203 \\
\hline NRAP & $1.90 \mathrm{E}-14$ & 0.489586 \\
\hline LDHD & $2.10 \mathrm{E}-14$ & -0.33736 \\
\hline LGR4 & $2.60 \mathrm{E}-14$ & 0.811303 \\
\hline DHRS7C & $3.10 \mathrm{E}-14$ & 1.799902 \\
\hline NEBL & $5.90 \mathrm{E}-14$ & 0.406793 \\
\hline ACTN2 & $6.49 \mathrm{E}-14$ & 0.514332 \\
\hline ABCA8 & $8.50 \mathrm{E}-14$ & 0.783352 \\
\hline NDUFS5 & $9.10 \mathrm{E}-14$ & 0.130064 \\
\hline FAM129A & $7.66 \mathrm{E}-12$ & 0.445771 \\
\hline UBXN6 & $1.75 \mathrm{E}-10$ & -0.45321 \\
\hline NR1D2 & $2.66 \mathrm{E}-10$ & 0.916154 \\
\hline ECH1 & $7.66 \mathrm{E}-09$ & -0.3775 \\
\hline HYAL1 & $2.32 \mathrm{E}-08$ & -0.3605 \\
\hline MFN2 & $4.96 \mathrm{E}-08$ & 0.19523 \\
\hline MYL12A & $6.60 \mathrm{E}-08$ & 0.433722 \\
\hline RYR2 & $9.76 \mathrm{E}-08$ & 0.502612 \\
\hline EGLN1 & $2.47 \mathrm{E}-07$ & 0.555696 \\
\hline OIP5-AS1 & $3.69 \mathrm{E}-07$ & 0.45973 \\
\hline PDLIM5 & $8.57 \mathrm{E}-07$ & 0.395338 \\
\hline C17orf89 & $5.14 \mathrm{E}-06$ & -0.47029 \\
\hline C10orf54 & $1.24 \mathrm{E}-05$ & -0.41807 \\
\hline PEBP1 & $2.12 \mathrm{E}-05$ & -0.22826 \\
\hline & & \\
\hline
\end{tabular}

\begin{tabular}{|l|l|l|} 
VSTM2L & \multicolumn{1}{|l}{$5.47 \mathrm{E}-05$} & -0.7891 \\
\hline ID3 & 0.0004 & -0.49882 \\
\hline CPT1A & 0.001089 & 0.684059 \\
\hline AGPAT9 & 0.001219 & 1.075068 \\
\hline CIRBP & 0.001488 & -0.66858 \\
\hline GBAS & 0.002905 & 0.285877 \\
\hline SLC2A4 & 0.003014 & -0.26979 \\
\hline HNRNPH1 & 0.004275 & 0.48207 \\
\hline MTRNR2L8 & 0.004412 & -3.25627 \\
\hline PDE3A & 0.006769 & 0.664528 \\
\hline ASS1 & 0.008458 & -0.69383 \\
\hline NFIL3 & 0.010733 & -0.63558 \\
\hline GSTT1 & 0.011703 & -2.20421 \\
\hline HOPX & 0.011744 & 1.145151 \\
\hline RB1CC1 & 0.012092 & 0.556311 \\
\hline DECR1 & 0.012308 & 0.357415 \\
\hline SPR & 0.012736 & -0.80244 \\
\hline UQCR11 & 0.013373 & 0.134543 \\
\hline CFL2 & 0.015294 & 0.336491 \\
\hline PPAP2B & 0.015298 & 0.495698 \\
\hline ACE2 & 0.015324 & 0.775487 \\
\hline AREG & 0.015347 & 0.979501 \\
\hline LMOD3 & 0.015389 & 0.254639 \\
\hline ZNF844 & 0.015429 & 0.511165 \\
\hline MYOM2 & 0.015505 & 0.360828 \\
\hline MTUS1 & 0.017233 & 0.294426 \\
\hline TRAK2 & 0.017693 & 0.412516 \\
\hline C3 & 0.017999 & 0.745286 \\
\hline GOLGA4 & 0.018802 & 0.297001 \\
\hline RPS12 & 0.022689 & 0.22347 \\
\hline DPY19L2 & 0.022967 & 0.713779 \\
\hline ASB11 & 0.024143 & 0.518274 \\
\hline GATM & 0.024475 & 0.600525 \\
\hline NR1D1 & 0.024778 & 0.726608 \\
\hline PPARGC1A & 0.028884 & 0.53886 \\
\hline TNNT1 & 0.03236 & 0.454654 \\
\hline ADRB1 & 0.032499 & 0.578817 \\
\hline DHCR7 & 0.035029 & -0.42659 \\
\hline HINT2 & 0.035032 & -0.38789 \\
\hline NPY6R & 0.035286 & 0.161042 \\
\hline HSP90AA1 & 0.035951 & -1.39985 \\
\hline & &
\end{tabular}




\begin{tabular}{|l|l|l|} 
FAM124A & 0.036349 & 0.772011 \\
\hline HDDC2 & 0.03684 & -0.40888 \\
\hline PALMD & 0.036931 & 0.36049 \\
\hline PLOD1 & 0.037799 & -0.53847 \\
\hline FABP3 & 0.037937 & -0.19022 \\
\hline UBE2D1 & 0.038221 & 0.407133 \\
\hline TOMM22 & 0.038638 & -0.36846 \\
\hline PIK3IP1 & 0.039085 & 0.851293 \\
\hline SUCLG2 & 0.039289 & 0.429302 \\
\hline PDXK & 0.040214 & 0.395105 \\
\hline NACA & 0.040426 & 0.261319 \\
\hline HIST2H2BE & 0.04072 & -0.47358 \\
\hline MRPS26 & 0.041411 & -0.24566 \\
\hline SRM & 0.041446 & -0.33945 \\
\hline GJA1 & 0.042569 & 0.458062 \\
\hline KLHL24 & 0.043239 & 0.648831 \\
\hline F2RL3 & 0.04369 & -1.20011 \\
\hline
\end{tabular}

\begin{tabular}{|l|l|l|} 
C8orf4 & 0.04381 & -0.41677 \\
\hline GAPDH & 0.043989 & -0.21032 \\
\hline IFITM1 & 0.044485 & -0.32031 \\
\hline SFRP1 & 0.044853 & 0.897901 \\
\hline YBEY & 0.045711 & 0.543591 \\
\hline SNTA1 & 0.045962 & -0.21448 \\
\hline RXRG & 0.047003 & -0.75542 \\
\hline PFKFB3 & 0.047302 & 0.721138 \\
\hline PDE7B & 0.047321 & 0.646953 \\
\hline POPDC2 & 0.047669 & -0.43408 \\
\hline PIK3R3 & 0.048177 & -0.68814 \\
\hline HK2 & 0.049065 & -0.71184 \\
\hline AHCY & 0.049348 & -0.38926 \\
\hline
\end{tabular}


Supplemental Table 8. Sex- and BMI-specific DEG with (p_adj < 0.05$)$ and absolute value of $\log 2$ fold change $>1$

\begin{tabular}{|l|l|l|l|}
\hline GeneName & p_adj & $\log 2 \mathrm{FC}$ & Origin \\
\hline AAK1 & 0.045716 & -1.78202 & LVH-F \\
\hline ABCF3 & 0.000789 & 1.080317 & LVH-F \\
\hline ACSM1 & 0.016208 & -2.13365 & LVH-F \\
\hline ACTA1 & 0.042476 & -1.1199 & LVH-F \\
\hline ADIPOR1 & 0.001502 & 1.190279 & LVH-F \\
\hline & & & BMI25NF \\
AGPAT9 & 0.001219 & 1.075068 & BMI30LVH M \\
\hline AKAP17A & 0.014985 & -1.03412 & LVH-F \\
\hline ALG2 & 0.030579 & -1.47132 & LVH-F \\
\hline ANAPC11 & 0.013918 & 1.566538 & LVH-F \\
\hline ANAPC2 & 0.015146 & -1.12143 & LVH-F \\
\hline ANGPTL4 & 0.012837 & -1.54119 & BMI30 \\
\hline ANKRD36B & 0.001861 & -3.04052 & LVH-F \\
\hline ARL2BP & 0.039899 & 1.290096 & LVH-F \\
\hline ARL6IP4 & 0.002399 & 1.532074 & LVH-F \\
\hline ASMTL & 0.022448 & -1.53306 & LVH-F \\
\hline ATG9A & 0.028019 & 1.094103 & LVH-F \\
\hline ATP1A1 & 0.01965 & -1.06399 & LVH-F \\
\hline ATP5F1 & 0 & 1.372359 & LVH-F \\
\hline ATP5H & 0.046897 & 1.01046 & LVH-F \\
\hline ATP5J2 & 0.040698 & 1.053234 & LVH-F \\
\hline ATXN3 & 0.04571 & -2.43236 & LVH-F \\
\hline AZGP1 & $1.46 E-13$ & -1.21571 & BMI30 \\
\hline AZGP1 & 0 & -1.30798 & LVH-M \\
\hline BCKDHA & 0.023183 & 1.361748 & LVH-F \\
\hline BEST4 & 0.039922 & -1.08126 & LVH-F \\
\hline BTBD10 & 0.045469 & -1.28796 & LVH-F \\
\hline C1QC & 0.043925 & 1.175501 & BMI25 \\
\hline CA3 & 0.041911 & 1.622109 & BMI30 \\
\hline CAST & 0.003565 & 1.515 & LVH-F \\
\hline CCDC3 & 0.028467 & -1.26406 & LVH-F \\
\hline CCDC39 & 0.002766 & -3.18253 & LVH-F \\
\hline CCL21 & 0.034425 & -1.99891 & LVH-F \\
\hline CCNG1 & 0.015352 & -1.33632 & LVH-F \\
\hline CHCHD3 & 0.027589 & -1.8757 & LVH-F \\
\hline CKAP5 & 0.021646 & 1.106586 & LVH-F \\
\hline CLU & 0.010655 & -1.00447 & LVH-M \\
\hline & & & \\
\hline
\end{tabular}




\begin{tabular}{|l|l|l|l|} 
CMC4 & 0.032923 & 1.087351 & LVH-F \\
\hline CNN1 & 0.015874 & 1.230719 & BMI25 \\
\hline CNPY3 & 0.02602 & -1.06098 & LVH-F \\
\hline CNTRL & 0.018993 & -1.53438 & LVH-F \\
\hline COPG2 & 0.035784 & -1.62398 & LVH-F \\
\hline COX17 & 0.014284 & -1.71685 & LVH-F \\
\hline COX7B & 0.006368 & 1.091807 & LVH-F \\
\hline CPT1A & $2.31 E-05$ & 1.291808 & LVH-F \\
\hline CPXCR1 & 0.024216 & -1.39867 & LVH-F \\
\hline CREM & 0.025267 & -2.14778 & LVH-F \\
\hline CRKL & 0.019327 & -1.12111 & LVH-F \\
\hline CSRNP1 & 0.030347 & -1.44187 & LVH-F \\
\hline CSRP3 & 0.021814 & -1.03479 & LVH-F \\
\hline CTGF & 0.030305 & -1.18947 & LVH-F \\
\hline CXCL1 & 0.039718 & -1.11979 & LVH-F \\
\hline CYR61 & 0.000146 & -1.8073 & LVH-F \\
\hline DBNDD2 & $3.19 E-07$ & 1.116301 & LVH-F \\
\hline DCN & $2.90 \mathrm{E}-14$ & 1.003963 & BMI25 \\
\hline DENND2A & 0.017611 & -1.00146 & LVH-F \\
\hline DEXI & 0.01443 & 1.425424 & LVH-F \\
\hline DHRS4L2 & 0.01678 & -1.16709 & LVH-F \\
\hline DHRS7C & $3.10 E-14$ & 1.799902 & BMI30LVH M \\
\hline DIRAS3 & 0.028944 & -1.01864 & LVH-F \\
\hline DNAJA4 & 0.000586 & -1.25998 & BMI30 \\
\hline EEF1G & 0.000136 & 2.018869 & LVH-F \\
\hline EFHD2 & 0.030605 & -1.574 & LVH-F \\
\hline ELOVL1 & 0.021326 & -1.17358 & LVH-F \\
\hline ESPNP & 0.032743 & -2.91051 & LVH-F \\
\hline ETFB & 0.002431 & 1.068367 & LVH-F \\
\hline F2RL3 & 0.04369 & -1.20011 & BMI25NF \\
\hline FANCI & 0.015449 & -1.70713 & LVH-F \\
\hline FIGF & 0.035388 & -1.15941 & LVH-F \\
\hline FLJ42969 & 0.045495 & -1.10766 & BMI30 \\
\hline FOS & 0.043634 & -1.05858 & LVH-F \\
\hline FSTL3 & 0.022169 & 1.277475 & LVH-F \\
\hline FUCA2 & 0.018749 & -1.24515 & LVH-F \\
\hline FXYD1 & 0.009307 & 1.676 & LVH-F \\
\hline GABARAP & 0.006902 & 2.005869 & LVH-F \\
\hline GBGT1 & 0.015357 & -1.08937 & LVH-F \\
\hline GCSHP3 & 0.029797 & 1.258673 & LVH-F \\
\hline
\end{tabular}




\begin{tabular}{|c|c|c|c|}
\hline GMEB1 & 0.015111 & -1.08091 & LVH-F \\
\hline GNAT1 & \begin{tabular}{|l|}
0.015907 \\
\end{tabular} & -1.57915 & LVH-F \\
\hline GNG8 & 0.016495 & 1.058843 & LVH-M \\
\hline GPR173 & 0.033213 & -1.28738 & LVH-F \\
\hline GPR4 & 0.002359 & -1.58775 & LVH-F \\
\hline GPRASP2 & 0.007945 & -1.95928 & LVH-F \\
\hline GSTT1 & 0.011703 & -2.20421 & $\begin{array}{ll}\text { BMI25NF } & \text { vs } \\
\text { BMI30LVH M } & \\
\end{array}$ \\
\hline GSTT1 & 0.033376 & -1.14097 & LVH-M \\
\hline GTPBP6 & 0.019788 & -1.53881 & LVH-F \\
\hline HBA1 & 0.001351 & -3.4007 & LVH-F \\
\hline HBB & \begin{tabular}{|l|l|}
0.000788 \\
\end{tabular} & -5.45543 & LVH-F \\
\hline HBQ1 & 0.03057 & -1.68139 & LVH-F \\
\hline HIF1A-AS2 & \begin{tabular}{|l|}
0.033996 \\
\end{tabular} & -1.13095 & LVH-F \\
\hline HIST1H2AC & 0.003876 & -2.31733 & LVH-F \\
\hline HIST1H4H & 0.035099 & -1.18434 & LVH-F \\
\hline HLA-A & 0.033857 & -1.07959 & BMI30 \\
\hline HLA-DRB1 & 0.023762 & -2.0529 & LVH-F \\
\hline HMGCS2 & 0 & -1.52337 & BMI30 \\
\hline HNRNPA2B1 & \begin{tabular}{|l|l|}
0.001525 \\
\end{tabular} & 1.093875 & LVH-F \\
\hline HNRNPH2 & 0.009874 & -2.03727 & LVH-F \\
\hline HOPX & 0.011744 & 1.145151 & $\begin{array}{l}\text { BMI25NF } \\
\text { BMI30LVH M }\end{array}$ \\
\hline HSP90AA1 & 0.035951 & -1.39985 & $\begin{array}{ll}\text { BMI25NF } & \text { vs } \\
\text { BMI30LVH M }\end{array}$ \\
\hline HSP90AA1 & 0.018487 & -1.38355 & LVH-M \\
\hline HSPA5 & 0.035795 & 1.758879 & LVH-F \\
\hline HSPA6 & 0.042634 & -1.46953 & LVH-F \\
\hline HSPB2 & 0.031367 & 1.211078 & LVH-F \\
\hline HTR2B & 0.016906 & -1.63789 & LVH-F \\
\hline IGF2 & 0.017209 & -1.4621 & LVH-F \\
\hline IL3RA & 0.037239 & -1.12638 & LVH-F \\
\hline ILK & 0.004515 & 1.653967 & LVH-F \\
\hline IMMP1L & 0.016304 & -1.26851 & LVH-F \\
\hline KCTD11 & 0.028872 & -1.32702 & LVH-F \\
\hline KDR & 0.027446 & -1.01649 & LVH-F \\
\hline KLC1 & 0.035226 & 1.002591 & LVH-F \\
\hline KLF8 & 0.032702 & -2.63556 & LVH-F \\
\hline LAMTOR2 & 0.027347 & 1.016552 & LVH-F \\
\hline LARP1B & 0.016209 & -1.10904 & LVH-F \\
\hline LTBP1 & 0.004871 & -1.15454 & LVH-F \\
\hline LY75 & 0.035726 & -1.27058 & LVH-F \\
\hline
\end{tabular}




\begin{tabular}{|l|l|l|l|} 
MAGEF1 & 0.006657 & -1.50688 & LVH-F \\
\hline MAGI1 & 0.022467 & -1.14043 & LVH-F \\
\hline MAPRE1 & 0.025203 & 1.119034 & LVH-F \\
\hline MAT2A & 0.012773 & -3.98597 & LVH-F \\
\hline MATR3 & 0.029278 & 1.100102 & LVH-F \\
\hline MCTS1 & 0.035379 & -1.18667 & LVH-F \\
\hline MDH2 & 0.008315 & 1.042324 & LVH-F \\
\hline METTL7B & 0.019927 & -1.4323 & LVH-F \\
\hline MGC16275 & 0.027125 & -1.16985 & LVH-F \\
\hline MOB4 & 0.007926 & -2.45158 & LVH-F \\
\hline MT1A & 0.016679 & -1.31961 & LVH-F \\
\hline MT2A & 0.029011 & 2.208718 & LVH-F \\
\hline MT2A & $7.38 E-09$ & -1.04872 & LVH-M \\
\hline MTPN & 0.021198 & -2.09273 & LVH-F \\
\hline & & & BMI25NF \\
MTRNR2L8 & 0.004412 & -3.25627 & BMI30LVH M \\
\hline MTRNR2L8 & 0.009458 & -3.57542 & LVH-M \\
\hline MTURN & 0.021131 & -1.28667 & LVH-F \\
\hline MYL4 & 0 & 1.223488 & BMI30 \\
\hline MYL4 & $1.01 E-06$ & 1.123475 & LVH-M \\
\hline MYL7 & 0 & 1.231699 & BMI30LFH \\
\hline MYL7 & 0 & 1.26689 & BMI25NF \\
\hline MYL7 & 0 & 1.550622 & BMI30LVH M \\
\hline MZT2A & 0.000177 & 1.152776 & LVH-F \\
\hline NAA10 & 0.03768 & 1.111668 & LVH-F \\
\hline NAT6 & 0.028176 & -1.26624 & LVH-F \\
\hline NCOA4 & 0.003347 & -1.25799 & LVH-F \\
\hline NDUFA7 & 0.036176 & 1.782766 & LVH-F \\
\hline NEAT1 & 0.002411 & -1.10139 & LVH-F \\
\hline NEK7 & 0.020017 & 1.095569 & LVH-F \\
\hline NFIX & $1.93 E-07$ & 1.237286 & LVH-F \\
\hline NNT & 0.002013 & 1.040709 & LVH-F \\
\hline NONO & 0.005596 & 1.256768 & LVH-F \\
\hline NPPA & 0.004457 & -3.54329 & All_LVH \\
\hline NPPA & $6.99 E-15$ & -3.73254 & BMI25 \\
\hline NPPA & $1.40 E-14$ & -4.02274 & BMI30 \\
\hline NPPA & 0.032393 & -2.89607 & LVH-F \\
\hline NPPA & 0.018249 & -4.59767 & LVH-M \\
\hline NPPB & 0.039417 & -1.72874 & BMI30 \\
\hline NPPB & 0.014572 & -2.21326 & LVH-F \\
\hline
\end{tabular}




\begin{tabular}{|l|l|l|l|} 
NPPB & 0.013588 & -2.89857 & LVH-M \\
\hline NPTN-IT1 & 0.012711 & -1.49524 & LVH-F \\
\hline NTMT1 & 0.012669 & -1.15378 & LVH-F \\
\hline OSBP & 0.018598 & -1.05728 & LVH-F \\
\hline OTUD1 & 0.037677 & -1.47141 & LVH-F \\
\hline PARVB & 0.006333 & 1.004308 & LVH-F \\
\hline PCBP1 & 0.026636 & 1.265688 & LVH-F \\
\hline PCDH7 & 0 & 1.017951 & BMI30 \\
\hline PDK4 & $4.00 E-15$ & -2.80167 & BMI30 \\
\hline PDK4 & 0.023484 & -1.19085 & LVH-M \\
\hline PELI1 & 0.024498 & -1.17581 & LVH-F \\
\hline PFDN1 & 0.00486 & -1.49717 & LVH-F \\
\hline PLA2G2A & 0.045658 & -2.64705 & LVH-F \\
\hline PLIN2 & 0 & -1.26669 & BMI30 \\
\hline PLVAP & $7.69 E-08$ & -1.02931 & BMI30 \\
\hline PLVAP & 0.017747 & -1.26576 & LVH-F \\
\hline PLVAP & $3.00 E-15$ & -1.29896 & LVH-M \\
\hline PLXDC2 & 0.011265 & -2.03282 & LVH-F \\
\hline POLR2J & 0.012691 & -1.02605 & LVH-F \\
\hline POM121L10P & 0.023984 & -3.57981 & LVH-F \\
\hline POSTN & $5.92 \mathrm{E}-09$ & -1.06062 & BMI30 \\
\hline PPP1R17 & 0.044203 & -1.20029 & LVH-F \\
\hline PTX3 & 0.018539 & -1.36678 & LVH-F \\
\hline PWAR1 & 0.049174 & -1.02416 & LVH-F \\
\hline PYURF & 0.021461 & 1.332057 & LVH-F \\
\hline RAB4A & $5.53 E-06$ & 1.515827 & LVH-F \\
\hline RASD1 & 0.007468 & -1.23112 & BMI30 \\
\hline RDH14 & 0.012763 & -1.08698 & LVH-F \\
\hline RFPL4AL1 & 0.015363 & -1.82569 & LVH-F \\
\hline RGL1 & 0.037807 & -1.12518 & LVH-F \\
\hline RN7SL2 & 0.019873 & 1.481054 & LVH-F \\
\hline RNF14 & $1.90 E-07$ & 1.344973 & LVH-F \\
\hline RNU6-7 & 0.037551 & -1.09696 & LVH-F \\
\hline RP11- & & & \\
451G4.2 & 0.031715 & -1.02284 & BMI30 \\
\hline RPL9 & 0.025397 & -1.02426 & LVH-F \\
\hline RPS15A & 0.049643 & 1.025891 & LVH-F \\
\hline RTN4 & 0.039426 & -1.07648 & LVH-F \\
\hline S100A1 & 0.000813 & 1.472048 & LVH-F \\
\hline SEC14L5 & 0 & 1.368913 & BMI30 \\
\hline SEC14L5 & 0.025826 & 1.203995 & LVH-M \\
\hline
\end{tabular}




\begin{tabular}{|l|l|l|l|} 
SENP5 & 0.036158 & -1.4376 & LVH-F \\
\hline 5-Sep & 0.031609 & -1.92603 & LVH-F \\
\hline SESTD1 & 0.032037 & -1.46671 & LVH-F \\
\hline SF1 & 0.017507 & -1.01606 & LVH-F \\
\hline SH3BGR & 0.041381 & -1.03842 & LVH-M \\
\hline SLC25A34 & $1.40 \mathrm{E}-14$ & -1.2642 & BMI30 \\
\hline SLC45A3 & 0.019074 & -1.48551 & LVH-F \\
\hline SLC6A6 & 0.016902 & -1.15071 & LVH-F \\
\hline SNORA33 & 0.048468 & -1.16312 & LVH-F \\
\hline SNRPN & 0.022304 & -1.23539 & LVH-F \\
\hline SOCS5 & 0.015948 & -1.25689 & LVH-F \\
\hline SPRY4 & 0.016248 & -1.08951 & LVH-F \\
\hline SRSF7 & 0.02232 & -1.06689 & LVH-F \\
\hline STX12 & 0.042183 & -1.23808 & LVH-F \\
\hline SVOP & 0.034337 & -1.06419 & LVH-M \\
\hline TAX1BP3 & 0.023178 & -1.44566 & LVH-F \\
\hline TCHH & 0.027454 & -1.91902 & LVH-F \\
\hline TFRC & 0.041854 & 1.359095 & LVH-M \\
\hline THBS4 & 0.048544 & 1.196572 & LVH-F \\
\hline TIMM8B & 0.012035 & -1.1086 & LVH-F \\
\hline TIMP1 & 0.028892 & -1.00851 & LVH-F \\
\hline TMEM165 & 0.026022 & 1.050255 & LVH-F \\
\hline TNFAIP1 & 0.026486 & -1.42484 & LVH-F \\
\hline TRAPPC5 & 0.000584 & 1.354619 & LVH-F \\
\hline TRIM35 & 0.013302 & -1.52477 & LVH-F \\
\hline TSFM & 0.003479 & 1.156395 & LVH-F \\
\hline TTC21A & 0.002043 & -2.47755 & LVH-F \\
\hline TUBA8 & 0.030328 & 1.599595 & LVH-F \\
\hline UQCC1 & 0.016391 & -1.03014 & LVH-F \\
\hline VAMP7 & 0.028929 & -1.15256 & LVH-F \\
\hline VGLL2 & 0.038578 & -1.19911 & LVH-F \\
\hline VSTM5 & 0.010137 & -1.45653 & LVH-F \\
\hline WDR61 & 0.021294 & -1.02132 & LVH-F \\
\hline WDR62 & $2.15 E-12$ & 1.07249 & LVH-M \\
\hline WDR89 & 0.024138 & -1.19626 & LVH-F \\
\hline ZBED1 & 0.025655 & -1.19893 & LVH-F \\
\hline ZSWIM1 & 0.01621 & -1.14273 & LVH-F \\
\hline & & & \\
\hline & & \\
\hline
\end{tabular}


Supplemental Table 9. Sex- and BMI-specific DEG with $\left(p_{-} \_\right.$adj $\left.<0.05\right)$ and absolute value of $\log 2$ fold change $>2$

\begin{tabular}{|l|l|l|l|}
\hline GeneName & p_adj & log2FC & Origin \\
\hline HBB & 0.000788 & -5.45543 & LVH-F \\
\hline NPPA & 0.018249 & -4.59767 & LVH-M \\
\hline NPPA & $1.40 \mathrm{E}-14$ & -4.02274 & BMI30 \\
\hline MAT2A & 0.012773 & -3.98597 & LVH-F \\
\hline NPPA & $6.99 \mathrm{E}-15$ & -3.73254 & BMI25 \\
\hline POM121L10P & 0.023984 & -3.57981 & LVH-F \\
\hline MTRNR2L8 & 0.009458 & -3.57542 & LVH-M \\
\hline NPPA & 0.004457 & -3.54329 & All_LVH \\
\hline HBA1 & 0.001351 & -3.4007 & LVH-F \\
\hline & & & BMI25NF \\
MTRNR2L8 & 0.004412 & -3.25627 & BMI30LVH M \\
\hline CCDC39 & 0.002766 & -3.18253 & LVH-F \\
\hline ANKRD36B & 0.001861 & -3.04052 & LVH-F \\
\hline ESPNP & 0.032743 & -2.91051 & LVH-F \\
\hline NPPB & 0.013588 & -2.89857 & LVH-M \\
\hline NPPA & 0.032393 & -2.89607 & LVH-F \\
\hline PDK4 & $4.00 \mathrm{E}-15$ & -2.80167 & BMI30 \\
\hline PLA2G2A & 0.045658 & -2.64705 & LVH-F \\
\hline KLF8 & 0.032702 & -2.63556 & LVH-F \\
\hline TTC21A & 0.002043 & -2.47755 & LVH-F \\
\hline MOB4 & 0.007926 & -2.45158 & LVH-F \\
\hline ATXN3 & 0.04571 & -2.43236 & LVH-F \\
\hline HIST1H2AC & 0.003876 & -2.31733 & LVH-F \\
\hline NPPB & 0.014572 & -2.21326 & LVH-F \\
\hline MT2A & 0.029011 & 2.208718 & LVH-F \\
\hline & 0.011703 & -2.20421 & BMI25NF \\
\hline GSTT1 & 0.025267 & -2.14778 & LVH-F \\
\hline CREM & 0.016208 & -2.13365 & LVH-F \\
\hline ACSM1 & 0.021198 & -2.09273 & LVH-F \\
\hline MTPN & 0.023762 & -2.0529 & LVH-F \\
\hline HLA-DRB1 & 0.009874 & -2.03727 & LVH-F \\
\hline HNRNPH2 & 0.011265 & -2.03282 & LVH-F \\
\hline PLXDC2 & 0.000136 & 2.018869 & LVH-F \\
\hline EEF1G & 0.006902 & 2.005869 & LVH-F \\
\hline GABARAP & & & \\
\hline
\end{tabular}


Supplemental Table 10. DEG shared between obesity-related LVH and ischemic heart datasets with (p_adj < 0.05$)$

\begin{tabular}{|l|l|l|l|l|}
\hline Gene & $\begin{array}{l}\text { log2FC NF vs LVH, } \\
\text { BMI30 }\end{array}$ & p_adj & $\begin{array}{l}\text { log2FC NF vs } \\
\text { ISCH }\end{array}$ & p_adj \\
\hline ADAM11 & 0.963323248 & 0.035425 & 1.859819771 & $1.99 \mathrm{E}-05$ \\
\hline AMOTL2 & -0.410297052 & 0.022329 & 2.454941712 & $1.72 \mathrm{E}-09$ \\
\hline ANGPTL4 & -1.541190467 & 0.012837 & -1.62521166 & 0.046472 \\
\hline ATP11A & 0.533909197 & 0.002511 & 0.933140384 & 0.011395 \\
\hline AZGP1 & -1.215705163 & 0 & -1.138888768 & 0.040662 \\
\hline C10orf10 & -0.509127057 & 0.02189 & 2.175525819 & $1.54 \mathrm{E}-07$ \\
\hline CA3 & 1.622109468 & 0.041911 & -4.806058253 & $1.20 \mathrm{E}-09$ \\
\hline CCL21 & -0.817354828 & 0.014933 & -4.445963698 & $4.26 \mathrm{E}-13$ \\
\hline CDKN1A & -0.787871528 & 0 & 1.066409299 & 0.042891 \\
\hline CES2 & -0.599913795 & 0.025626 & -1.29788074 & 0.000568 \\
\hline DNAJA4 & -1.259979001 & 0.000586 & -1.318690893 & 0.005975 \\
\hline DUSP1 & -0.475690762 & 0.031356 & 1.50041234 & 0.002321 \\
\hline ENO2 & -0.407758301 & 0.029835 & 1.329284641 & 0.01159 \\
\hline GADD45G & -0.972541723 & 0 & -2.141900654 & $8.62 \mathrm{E}-06$ \\
\hline GLUL & -0.574244275 & 0.026805 & 1.511265015 & $4.26 \mathrm{E}-05$ \\
\hline HIF3A & -0.487311467 & 0.0337 & 1.90209774 & $2.88 \mathrm{E}-11$ \\
\hline HIST1H2BD & -0.377505615 & 0.026992 & -1.819527247 & 0.008223 \\
\hline KLF10 & -0.493714422 & 0.040722 & 1.981681166 & $2.83 \mathrm{E}-06$ \\
\hline KLHL36 & -0.489064934 & 0.038418 & 1.118522643 & 0.044766 \\
\hline MT1X & -0.931977954 & 0.036025 & -3.148047139 & $3.10 \mathrm{E}-06$ \\
\hline MYL4 & 1.223488273 & 0 & 5.061522714 & 0 \\
\hline NGFR & 0.731879187 & 0.036183 & -1.468816887 & 0.006195 \\
\hline NPPA & -4.022740194 & 0 & -4.275261149 & 0 \\
\hline NPPB & -1.728742349 & 0.039417 & -3.478505958 & $1.79 \mathrm{E}-13$ \\
\hline P4HA2 & -0.513644739 & 0.027888 & -1.327847131 & 0.000437 \\
\hline PCDH7 & 1.017950806 & 0 & -1.342352722 & 0.001533 \\
\hline PDK4 & -2.801668723 & 0 & -1.621761989 & 0.003644 \\
\hline PIM3 & -0.486255599 & 0.025119 & 1.148851509 & 0.031203 \\
\hline PLIN5 & -0.365375409 & 0.045642 & 1.522271323 & 0.000835 \\
\hline POSTN & -1.060620451 & $6.00 \mathrm{E}-09$ & -5.398096936 & 0 \\
\hline RASD1 & -1.231118925 & 0.007468 & -4.302868698 & 0 \\
\hline SDPR & -0.354902106 & 0.047867 & 1.540942135 & 0.001267 \\
\hline SEC14L5 & 1.368913328 & 0 & 2.532045159 & $4.07 \mathrm{E}-09$ \\
\hline SNHG8 & -0.370713939 & 0.041204 & 1.326354211 & 0.025361 \\
\hline TUBA3E & -0.488877251 & 0.036916 & 2.13283323 & $3.49 \mathrm{E}-07$ \\
\hline TXNIP & -0.67478941 & 0.019841 & 2.215508659 & $4.64 \mathrm{E}-06$ \\
\hline & & & & \\
\hline
\end{tabular}




\begin{tabular}{|l|l|l|l|l|} 
TXNRD1 & -0.6800562 & 0.032906 & 0.786257974 & 0.033516 \\
\hline
\end{tabular}


Supplemental Table 11. DEG shared between obesity-related LVH and dilated cardiomyopathy datasets with $\left(\mathbf{p} \_\right.$adj $\left.<0.05\right)$

\begin{tabular}{|c|c|c|c|c|}
\hline Gene & $\begin{array}{l}\log 2 \mathrm{FC} \text { NF vs LVH, } \\
\text { BMI30 }\end{array}$ & $\mathrm{p} \_$adj & $\begin{array}{l}\log 2 \mathrm{FC} \quad \mathrm{NF} \text { vs } \\
\mathrm{DCM}\end{array}$ & $\mathrm{p} \_$adj \\
\hline ACADM & 0.311292269 & 0 & -1.635131511 & 0.024369 \\
\hline ACSL1 & -0.301796691 & 0.047905 & -1.237893201 & 0.028694 \\
\hline ADAM11 & 0.963323248 & 0.035425 & -1.705143444 & 0.000396 \\
\hline ANGPTL4 & -1.541190467 & 0.012837 & 2.676261492 & $7.52 \mathrm{E}-07$ \\
\hline AZGP1 & -1.215705163 & 0 & 2.180581657 & $2.13 \mathrm{E}-06$ \\
\hline C10orf54 & -0.314841388 & 0.037269 & 1.198134446 & 0.039901 \\
\hline C11orf24 & -0.37851224 & 0.033819 & 1.146685791 & 0.045062 \\
\hline C6orf1 & -0.444222465 & 0.021257 & 1.462087575 & 0.00141 \\
\hline CCDC85B & -0.351885521 & 0.033578 & 1.451714992 & 0.003356 \\
\hline CCL21 & -0.817354828 & 0.014933 & -2.08871703 & 0.010486 \\
\hline CDKN1A & -0.787871528 & 0 & 1.293184109 & 0.041044 \\
\hline CEBPB & -0.612313557 & $5.00 \mathrm{E}-09$ & 1.446932885 & 0.038798 \\
\hline CES2 & -0.599913795 & 0.025626 & 1.624109893 & $3.83 \mathrm{E}-05$ \\
\hline CLIC5 & 0.403321879 & 0 & -1.829084965 & 0.024395 \\
\hline DUSP1 & -0.475690762 & 0.031356 & -1.092128327 & 0.041697 \\
\hline ENO2 & -0.407758301 & 0.029835 & 2.196136473 & 0.0023 \\
\hline FAM129A & 0.530083934 & 0 & -1.595061831 & 0.010247 \\
\hline FAM96B & -0.254020969 & 0.039689 & 1.007933098 & 0.042718 \\
\hline FKBP5 & -0.864105785 & 0.007155 & 2.79848211 & $1.39 \mathrm{E}-10$ \\
\hline GADD45G & -0.972541723 & 0 & -2.628999414 & $2.73 \mathrm{E}-09$ \\
\hline GGTA1P & 0.872428613 & 0.037154 & -1.706254443 & 0.012987 \\
\hline GLUL & -0.574244275 & 0.026805 & 1.664537577 & $5.94 \mathrm{E}-08$ \\
\hline GPNMB & 0.91782649 & 0 & -1.545983281 & 0.000353 \\
\hline HIF3A & -0.487311467 & 0.0337 & 2.0184829 & $6.70 \mathrm{E}-10$ \\
\hline HIST1H2BD & -0.377505615 & 0.026992 & -1.796075408 & 0.0122 \\
\hline HLA-A & -1.079594248 & 0.033857 & 1.632995353 & 0.003143 \\
\hline HMGCS2 & -1.523371969 & 0 & 2.238661292 & $2.33 \mathrm{E}-06$ \\
\hline HSPA2 & -0.6742577 & 0.042397 & -1.921073556 & 0.005923 \\
\hline HSPB2 & -0.346226709 & 0.041105 & 1.552376496 & 0.031126 \\
\hline HSPE1 & -0.66784361 & 0.034606 & -1.424482306 & 0.044167 \\
\hline KLF10 & -0.493714422 & 0.040722 & -2.844803667 & $7.04 \mathrm{E}-09$ \\
\hline KLF15 & -0.70413339 & 0.02653 & 2.244450656 & $6.56 \mathrm{E}-06$ \\
\hline KLHL36 & -0.489064934 & 0.038418 & -1.997317857 & 0.006246 \\
\hline MLYCD & -0.639518345 & 0 & 1.255474711 & 0.025587 \\
\hline MYL4 & 1.223488273 & 0 & 7.928132761 & 0 \\
\hline MYL7 & 1.550621715 & 0 & -2.355940042 & $1.32 \mathrm{E}-09$ \\
\hline
\end{tabular}




\begin{tabular}{|l|l|l|l|l|} 
NGFR & 0.731879187 & 0.036183 & 2.775612164 & $1.05 \mathrm{E}-07$ \\
\hline NGFRAP1 & 0.29443617 & 0.044122 & 1.03562481 & 0.043437 \\
\hline P4HA2 & -0.513644739 & 0.027888 & -1.327516966 & 0.022345 \\
\hline PCDH7 & 1.017950806 & 0 & -3.01148407 & $5.79 \mathrm{E}-13$ \\
\hline PDE3A & 0.494363508 & 0.012594 & -2.310334066 & $2.07 \mathrm{E}-05$ \\
\hline PDK4 & -2.801668723 & 0 & -2.495837398 & $3.53 \mathrm{E}-08$ \\
\hline PIM3 & -0.486255599 & 0.025119 & -1.433797174 & 0.004027 \\
\hline PLA2G16 & -0.403295041 & 0.018502 & 1.062296467 & 0.006247 \\
\hline PLEKHA4 & -0.478182032 & 0.043644 & 1.715005619 & 0.002446 \\
\hline PLEKHO1 & -0.594058511 & 0.035305 & 1.581454714 & 0.030276 \\
\hline PLIN2 & -1.26669077 & 0 & -1.979051483 & $5.95 \mathrm{E}-05$ \\
\hline PSME1 & -0.20251521 & 0.032774 & -1.220196145 & 0.026897 \\
\hline RABAC1 & -0.316244037 & 0.043129 & 1.466716007 & 0.048405 \\
\hline RYR2 & 0.722120711 & 0 & -2.846601211 & 0.001689 \\
\hline SDPR & -0.354902106 & 0.047867 & -1.109838725 & 0.036961 \\
\hline SIAE & -0.301274805 & 0.041108 & -1.596753323 & 0.029123 \\
\hline SLC16A1 & 0.359135811 & 0.01809 & -1.5319772 & 0.015032 \\
\hline SNHG8 & -0.370713939 & 0.041204 & 1.921250812 & 0.000585 \\
\hline SPR & -0.544625322 & 0.023243 & 1.53937965 & 0.003249 \\
\hline TUBA3E & -0.488877251 & 0.036916 & 4.00419486 & $1.18 \mathrm{E}-11$ \\
\hline TXNRD1 & -0.6800562 & 0.032906 & -0.917006565 & 0.005121 \\
\hline VSTM2L & -0.971412272 & 0.039785 & 3.223903018 & $5.23 \mathrm{E}-06$ \\
\hline
\end{tabular}


Supplemental Table 12. DEG shared between obesity-related LVH and both dilated ischemia and cardiomyopathy datasets with (p_adj $<0.05$ )

\begin{tabular}{|l|l|l|l|l|}
\hline Gene & $\begin{array}{l}\text { log2FC NF vs LVH, } \\
\text { BMI30 }\end{array}$ & p_adj & $\begin{array}{l}\text { log2FC ISCH vs } \\
\text { DCM }\end{array}$ & p_adj \\
\hline ACSL1 & -0.301796691 & 0.047905 & -1.36779967 & 0.040646 \\
\hline AMOTL2 & -0.410297052 & 0.022329 & -1.54606353 & 0.000364 \\
\hline ATP11A & 0.533909197 & 0.002511 & -1.31543383 & 0.000361 \\
\hline AZGP1 & -1.215705163 & 0 & 1.813024958 & 0.000192 \\
\hline CA3 & 1.622109468 & 0.041911 & 1.717173576 & 0.00744 \\
\hline CCL21 & -0.817354828 & 0.014933 & 3.211259363 & $1.73 \mathrm{E}-08$ \\
\hline ETFB & -0.229518502 & 0.028124 & -1.136020685 & 0.039885 \\
\hline FKBP5 & -0.864105785 & 0.007155 & 1.999049339 & $1.19 \mathrm{E}-05$ \\
\hline GADD45G & -0.972541723 & 0 & 2.127923456 & 0.000128 \\
\hline HMGCS2 & -1.523371969 & 0 & -6.83427681 & 0 \\
\hline KLF10 & -0.493714422 & 0.040722 & -1.429708621 & 0.00882 \\
\hline KLF15 & -0.70413339 & 0.02653 & 1.466101481 & 0.017679 \\
\hline MLYCD & -0.639518345 & 0 & -1.107128992 & 0.038156 \\
\hline MT1M & -0.601514184 & 0.032563 & 5 & 0.027723 \\
\hline MT1X & -0.931977954 & 0.036025 & 1.515877998 & 0.048194 \\
\hline MYL4 & 1.223488273 & 0 & 2.866610047 & $1.24 \mathrm{E}-05$ \\
\hline NGFR & 0.731879187 & 0.036183 & 2.460128189 & $3.52 \mathrm{E}-06$ \\
\hline NPPA & -4.022740194 & 0 & 1.804016681 & 0.002728 \\
\hline NPPB & -1.728742349 & 0.039417 & 3.95062945 & $1.28 \mathrm{E}-12$ \\
\hline PDE3A & 0.494363508 & 0.012594 & -1.958909858 & 0.000798 \\
\hline PLEKHA4 & -0.478182032 & 0.043644 & 1.693949792 & 0.000295 \\
\hline PLIN2 & -1.26669077 & 0 & 1.361068302 & 0.012164 \\
\hline PLVAP & -1.029311185 & $7.70 \mathrm{E}-08$ & 1.949632993 & 0.001075 \\
\hline POSTN & -1.060620451 & $6.00 \mathrm{E}-09$ & 4.131445065 & 0 \\
\hline RASD1 & -1.231118925 & 0.007468 & 4.447645806 & 0 \\
\hline SDPR & -0.354902106 & 0.047867 & -1.293987604 & 0.023136 \\
\hline SEC14L5 & 1.368913328 & 0 & -2.791126985 & $9.97 \mathrm{E}-09$ \\
\hline TBX20 & 0.827151711 & 0.002823 & -2.297082344 & $1.42 \mathrm{E}-06$ \\
\hline TUBA3E & -0.488877251 & 0.036916 & 1.87136163 & 0.00554 \\
\hline TXNIP & -0.67478941 & 0.019841 & -1.869992367 & 0.002275 \\
\hline VSTM2L & -0.971412272 & 0.039785 & 2.777014645 & $6.09 \mathrm{E}-05$ \\
\hline & & & & \\
\hline
\end{tabular}


Supplemental Table 13. DEG shared between female obesity-related LVH and ischemia datasets with (p_adj < 0.05$)$

\begin{tabular}{|l|l|l|l|l|}
\hline Gene & $\begin{array}{l}\text { log2FC NF vs } \\
\text { LVH_F }\end{array}$ & p_adj & $\begin{array}{l}\text { log2FC NF vs } \\
\text { ISCH }\end{array}$ & p_adj \\
\hline ADAM19 & -0.630142749 & 0.037114 & -3.048888723 & $1.74 \mathrm{E}-10$ \\
\hline AKAP12 & -0.731993228 & 0.026665 & -1.298370962 & 0.01369 \\
\hline AKAP8 & 0.719672413 & 0.041305 & 1.190883805 & 0.022442 \\
\hline AMOTL1 & -0.709487539 & 0.023841 & -1.806957882 & 0.00021 \\
\hline ANGPTL4 & -0.978298815 & 0.026011 & -1.62521166 & 0.046472 \\
\hline ANO5 & 0.496829866 & 0.04182 & -1.194968001 & 0.010258 \\
\hline APOD & -0.892669878 & 0.037102 & 2.188726773 & $4.39 \mathrm{E}-07$ \\
\hline ATE1 & -0.823974167 & 0.02715 & -5 & $2.25 \mathrm{E}-07$ \\
\hline ATP2B4 & -0.473150152 & 0.047037 & -1.590706441 & 0.000121 \\
\hline AZIN1 & -0.457215273 & 0.033503 & -1.355458125 & 0.00242 \\
\hline B2M & -0.481096356 & 0.034341 & 1.263669684 & 0.019571 \\
\hline BCAR3 & -0.799783333 & 0.044029 & 1.256167644 & 0.020878 \\
\hline BTG2 & -0.547514523 & 0.022147 & -1.680509038 & 0.000236 \\
\hline CBLB & -0.501922722 & 0.049091 & 1.736718946 & 0.000114 \\
\hline CCL21 & -1.998910656 & 0.034425 & -4.445963698 & $4.26 \mathrm{E}-13$ \\
\hline CD151 & 0.472684984 & 0.037071 & -1.076847186 & 0.003615 \\
\hline CD68 & 0.939054502 & 0.044368 & -1.102746724 & 0.045551 \\
\hline CDKN1A & -0.429068187 & 0.044115 & 1.066409299 & 0.042891 \\
\hline CES2 & 0.651625466 & 0.024136 & -1.29788074 & 0.000568 \\
\hline COPG2 & -1.623978704 & 0.035784 & -5 & $1.32 \mathrm{E}-05$ \\
\hline COQ10A & 0.483453731 & 0.033817 & 1.023565636 & 0.025646 \\
\hline COX6A2 & 0.439693907 & 0.029613 & 1.183127397 & 0.036892 \\
\hline CSRNP1 & -1.441867051 & 0.030347 & 1.25190798 & 0.017483 \\
\hline CYR61 & -1.807300387 & 0.000146 & 1.922649835 & $1.06 \mathrm{E}-05$ \\
\hline DAAM2 & -0.505335353 & 0.043894 & 1.699349677 & 0.000119 \\
\hline DAPK2 & 0.74144734 & 0.046529 & 1.472556324 & 0.003215 \\
\hline DRAM2 & -0.726386837 & 0.022444 & -5 & $2.46 \mathrm{E}-05$ \\
\hline DUSP1 & -0.830211926 & 0.045995 & 1.50041234 & 0.002321 \\
\hline DYNLL1 & -0.741219481 & 0.04959 & -1.775643022 & $3.34 \mathrm{E}-05$ \\
\hline EDNRB & -0.523326087 & 0.025631 & 2.168141571 & $1.62 \mathrm{E}-11$ \\
\hline EFEMP1 & -0.729911964 & 0.04534 & -0.95010613 & 0.030239 \\
\hline ELK1 & -0.394407987 & 0.043625 & 1.055086757 & 0.0086 \\
\hline EMILIN1 & -0.571141425 & 0.039703 & -1.138198488 & 0.04197 \\
\hline ENG & -0.575150638 & 0.033416 & 1.319210813 & 0.010059 \\
\hline ENO2 & -0.61311978 & 0.045746 & 1.329284641 & 0.01159 \\
\hline EPHA4 & -0.512902526 & 0.044019 & -2.090495025 & 0.000511 \\
\hline & & & & \\
\hline
\end{tabular}




\begin{tabular}{|c|c|c|c|c|}
\hline ETS2 & -0.554950903 & 0.039999 & $\mid 1.228682928$ & 0.014796 \\
\hline FBLN5 & -0.551338968 & 0.012826 & 1.290762964 & 0.006991 \\
\hline FSTL3 & 1.277475497 & 0.022169 & -1.278100975 & 0.030781 \\
\hline FXYD5 & -0.741622441 & 0.04211 & -1.168609909 & 0.049289 \\
\hline GIMAP6 & -0.948944237 & 0.004831 & 1.309671928 & 0.000231 \\
\hline GIMAP7 & -0.554386987 & 0.028666 & 1.30526632 & 0.021863 \\
\hline GPR157 & -0.826733847 & 0.031724 & 1.844800392 & 0.002285 \\
\hline HIST1H2AC & \begin{tabular}{|l|}
-2.317333894 \\
\end{tabular} & 0.003876 & -1.555247681 & 0.043713 \\
\hline IL33 & -0.799078418 & 0.026093 & -1.589206695 & 0.028392 \\
\hline IL6ST & -0.346209222 & 0.036204 & -5 & $1.55 \mathrm{E}-14$ \\
\hline ITGA6 & -0.386557844 & 0.041332 & -1.132163475 & 0.020601 \\
\hline KDR & -1.01649251 & 0.027446 & 1.609651748 & 0.000579 \\
\hline KIDINS220 & -0.335580105 & 0.040251 & -1.272954014 & 0.017108 \\
\hline KLHL31 & \begin{tabular}{|l}
-0.967419601 \\
\end{tabular} & 0.040682 & -1.242447658 & 0.022627 \\
\hline KLHL38 & 0.335280765 & 0.045138 & 2.014012569 & $2.44 \mathrm{E}-06$ \\
\hline LIMS2 & \begin{tabular}{|l|}
0.460768094 \\
\end{tabular} & 0.036658 & \begin{tabular}{|l|}
1.077500891 \\
\end{tabular} & 0.013085 \\
\hline LRRC14B & \begin{tabular}{|l|}
0.601273929 \\
\end{tabular} & 0.046813 & 2.497809238 & $2.29 \mathrm{E}-09$ \\
\hline MAGOH & -0.307452819 & 0.048468 & -5 & $2.79 \mathrm{E}-06$ \\
\hline MAOA & -0.670884366 & 0.027791 & 1.156827805 & 0.033939 \\
\hline MFAP4 & -0.524445582 & 0.039794 & -1.750721346 & 0.002055 \\
\hline MON1B & -0.59647824 & 0.0301 & 1.135406402 & 0.042159 \\
\hline MRPL33 & 0.685197212 & 5.81E-05 & -3.374524114 & 0 \\
\hline MYO1B & -0.606313032 & 0.025112 & -1.152455909 & 0.026489 \\
\hline NCKIPSD & -0.409067028 & 0.043648 & 1.647170331 & $5.65 \mathrm{E}-07$ \\
\hline NDRG2 & \begin{tabular}{|l|}
0.411302702 \\
\end{tabular} & 0.030812 & \begin{tabular}{|l|}
0.914021945 \\
\end{tabular} & $2.83 \mathrm{E}-06$ \\
\hline NEAT1 & \begin{tabular}{|l}
-1.101387739 \\
\end{tabular} & 0.002411 & 1.359837777 & 0.042058 \\
\hline NEDD9 & -0.524428571 & 0.046449 & 1.403249907 & 0.001187 \\
\hline NPPA & -2.896073668 & 0.032393 & -4.275261149 & 0 \\
\hline NPPB & -2.213258402 & 0.014572 & -3.478505958 & $1.79 \mathrm{E}-13$ \\
\hline NR1D2 & -0.366189625 & 0.043119 & -5 & $8.32 \mathrm{E}-11$ \\
\hline OTUD1 & -1.471407718 & 0.037677 & 1.307614166 & 0.008755 \\
\hline PLA2G2A & -2.647052214 & 0.045658 & 2.063800018 & $9.32 \mathrm{E}-07$ \\
\hline PLXDC2 & -2.032815142 & 0.011265 & -1.64696227 & 0.027561 \\
\hline PMEPA1 & \begin{tabular}{|l|}
-0.747667125 \\
\end{tabular} & 0.017309 & -1.156183207 & 0.005308 \\
\hline PPP1R12B & 0.592237795 & 0.000174 & -1.241293709 & 0.000151 \\
\hline PRELP & \begin{tabular}{|l|}
-0.570100677 \\
\end{tabular} & 0.036116 & 1.943073151 & $5.58 \mathrm{E}-07$ \\
\hline PREX1 & \begin{tabular}{|l}
-0.997966408 \\
\end{tabular} & 0.034587 & 1.63079897 & 0.000293 \\
\hline PRKAA2 & -0.426292519 & 0.046432 & -1.187609705 & 0.026793 \\
\hline PROS1 & -0.92499567 & 0.042894 & -1.777064031 & 0.000208 \\
\hline PTGDS & 0.646333156 & 0.004501 & 1.774781138 & 0.001074 \\
\hline PTMS & -0.426086888 & 0.035995 & 1.586696097 & 0.000379 \\
\hline
\end{tabular}




\begin{tabular}{|l|l|l|l|l|} 
PTX3 & -1.366782142 & 0.018539 & -3.020915462 & 0.000259 \\
\hline RNF146 & -0.371611904 & 0.041492 & -0.664744793 & 0.048359 \\
\hline RPL28 & -0.356732224 & 0.04316 & 0.86291538 & 0.047925 \\
\hline RPL37A & 0.515517464 & 0.049679 & 1.124108637 & 0.031403 \\
\hline RPLP1 & 0.862792317 & 0.001217 & 1.078124335 & 0.002645 \\
\hline RPS15A & 1.025890917 & 0.049643 & 0.955141541 & 0.018754 \\
\hline RTN3 & -0.498114556 & 0.034032 & -0.871600672 & 0.016901 \\
\hline S100A1 & 1.472047839 & 0.000813 & 1.194716866 & 0.038654 \\
\hline S100A8 & -0.848107813 & 0.012523 & 1.735255886 & 0.006747 \\
\hline SAMHD1 & -0.58759924 & 0.018941 & 1.250344989 & 0.014341 \\
\hline SCN2B & -0.819119857 & 0.032449 & -2.022686998 & $3.54 \mathrm{E}-05$ \\
\hline SEC14L1 & -0.425089014 & 0.03269 & 1.207579863 & 0.003966 \\
\hline SEMA3G & -0.659911145 & 0.028594 & 1.140983505 & 0.031186 \\
\hline SERPINH1 & -0.564001102 & 0.043145 & -1.417623133 & 0.004327 \\
\hline SLC8A1 & 0.685780929 & 0.002115 & -0.988155998 & 0.006057 \\
\hline SPEN & -0.504840729 & 0.035042 & 1.112858292 & 0.042159 \\
\hline STK38 & -0.666653177 & 0.018017 & -1.23246269 & 0.04036 \\
\hline STK39 & -0.616865536 & 0.035728 & -2.1051715 & 0.000835 \\
\hline SUN1 & -0.450823867 & 0.038098 & 0.750215245 & 0.022747 \\
\hline TACC1 & -0.370545039 & 0.038676 & 0.91919359 & 0.006701 \\
\hline TAX1BP3 & -1.445656879 & 0.023178 & -5 & $5.68 \mathrm{E}-07$ \\
\hline THBS2 & -0.665188472 & 0.044745 & -2.475548465 & $7.80 \mathrm{E}-08$ \\
\hline THBS4 & 1.196572038 & 0.048544 & -2.581450709 & $1.48 \mathrm{E}-09$ \\
\hline TIMP1 & -1.008514924 & 0.028892 & -1.301912191 & 0.012381 \\
\hline TIMP3 & -0.392285917 & 0.02239 & 1.659800666 & 0.004458 \\
\hline TMEM100 & -0.624594284 & 0.039693 & -2.083667615 & 0.01159 \\
\hline TNPO1 & -0.651711854 & 0.013703 & -0.97428252 & 0.044435 \\
\hline TXNRD1 & -0.397359953 & 0.043213 & 0.786257974 & 0.033516 \\
\hline UCKL1 & -0.916759905 & 0.006433 & 1.164593946 & 0.006452 \\
\hline ULK1 & 0.844633034 & 0.042966 & 1.158112048 & 0.027754 \\
\hline USP9X & 0.696855665 & 0.033127 & -1.085170923 & 0.041503 \\
\hline VAMP8 & -0.563839444 & 0.035165 & -1.974598288 & 0.008887 \\
\hline ZMIZ1 & -0.698773614 & 0.039673 & 1.461323404 & 0.004155 \\
\hline ZNF438 & -0.411605766 & 0.049926 & -5 & $2.62 \mathrm{E}-11$ \\
\hline & & & & \\
\hline
\end{tabular}


Supplemental Table 14. DEG shared between female obesity-related LVH and dilated cardiomyopathy datasets with $\left(\mathbf{p} \_\right.$adj $\left.<0.05\right)$

\begin{tabular}{|c|c|c|c|c|}
\hline Gene & $\begin{array}{lll}\text { log2FC } & \text { NF } & \text { vs } \\
\text { LVH F } & & \\
\end{array}$ & $\mathrm{p}$ adj & $\begin{array}{l}\log 2 \mathrm{FC} \quad \mathrm{NF} \text { vs } \\
\text { DCM }\end{array}$ & $p \_$adj \\
\hline ACAA1 & 0.976931371 & 0.008231 & 1.073799181 & 0.04415 \\
\hline ACTA1 & -1.119896192 & .042476 & 1.585652186 & .035032 \\
\hline AGF & .862461622 & 031696 & 1.173437054 & \\
\hline AGF & .656929279 & 036148 & -1.692568564 & 017186 \\
\hline AKAP12 & -0.731993228 & .026665 & -2.360153532 & $1.43 \mathrm{E}-05$ \\
\hline AKAP8 & 0.719672413 & 0.041305 & -1.108378062 & .027646 \\
\hline AMOTL1 & -0.709487539 & 0.023841 & -2.394648442 & 0.000287 \\
\hline ANAPC11 & 1.56653796 & 0.013918 & 0.824630217 & 0.002557 \\
\hline ANG & -0.978298815 & & 2.676261492 & \\
\hline ANX & -0.714896 & & -1.29 & \\
\hline $\mathrm{APC}$ & -0.8926 & & -1.9 & \\
\hline ARH & -0.6 & & 0.9 & \\
\hline $\mathrm{ARI}$ & 1.5 & & 0.8 & \\
\hline ATE1 & -0.8 & & -5 & \\
\hline ATP & -0.47 & & -2.249 & \\
\hline AZIN1 & -0.457215273 & 03 & -1.234736437 & .014779 \\
\hline $\mathrm{B} 2 \mathrm{M}$ & 096356 & 41 & -1.838140453 & .002514 \\
\hline $\mathrm{BC}$ & -0.7 & & -1.557 & \\
\hline BTC & -0.5475145 & & 1.5694 & \\
\hline & -0.36 & & 1.6 & \\
\hline & -0.8 & & 1.8 & \\
\hline $\mathrm{CA}$ & $\overline{-0.4}-4-3$ & 7 & -1.1 & 0.0 \\
\hline CALM2 & 0.48 & 09 & -1.133 & 0.0 \\
\hline CAMK2D & -0.517152358 & 0.048827 & -1.21846042 & 0.005703 \\
\hline CAST & 1.51500001 & 0.003565 & -1.367834595 & 0.000208 \\
\hline $\mathrm{CBL}$ & -0.501922722 & 0.049091 & -1.541442813 & 0.002264 \\
\hline $\mathrm{CCI}$ & 0.86144 & 33 & 6.143199949 & \\
\hline $\mathrm{CCl}$ & -199 & 25 & -2.08871703 & 0.010 \\
\hline $\mathrm{CD} 1$ & 0.472684984 & 71 & -1.317019331 & $2.63 \mathrm{E}-$ \\
\hline CD68 & 0.939 & 368 & 1.772692722 & 0.000442 \\
\hline CDKN1A & -0.429068187 & 0.044115 & 1.293184109 & 0.041044 \\
\hline CEBPB & -0.441897123 & 0.035736 & 1.446932885 & 0.038798 \\
\hline CES2 & 0.651625466 & 0.024136 & 1.624109893 & $3.83 \mathrm{E}-05$ \\
\hline CLIC & 0.419941576 & 0.031906 & \begin{tabular}{|l}
-1.829084965 \\
\end{tabular} & 0.024395 \\
\hline & 60542073 & 0.033752 & -0.858279003 & 0.046264 \\
\hline COL6A2 & -0.400028873 & 0.018589 & -1.204770251 & 0.036974 \\
\hline
\end{tabular}




\begin{tabular}{|c|c|c|c|c|}
\hline CPT1A & $\mid 1.291808397$ & 2.31E-05 & -1.279781009 & 0.007423 \\
\hline CRIM1 & -0.434102162 & 0.048377 & 1.256178203 & 0.006172 \\
\hline CTGF & -1.189465404 & 0.030305 & 2.120582413 & 0.001288 \\
\hline CXorf36 & -0.529466638 & 0.04564 & 1.723076035 & $7.56 \mathrm{E}-05$ \\
\hline CYBRD1 & -0.900806819 & 0.025364 & 1.184422202 & 0.02653 \\
\hline CYR61 & -1.807300387 & 0.000146 & -1.254650049 & 0.019218 \\
\hline DAAM2 & -0.505335353 & 0.043894 & 1.768775305 & $2.20 \mathrm{E}-05$ \\
\hline DAPK2 & 0.74144734 & \begin{tabular}{|l|}
0.046529 \\
\end{tabular} & 1.303940902 & 0.016556 \\
\hline DCAF11 & 0.664734382 & 0.030771 & -0.8027819 & 0.034408 \\
\hline DCAF6 & 0.894946081 & $6.99 \mathrm{E}-15$ & -1.170334921 & 0.002897 \\
\hline DIRAS3 & -1.018644618 & 0.028944 & -4.507392284 & $9.26 \mathrm{E}-13$ \\
\hline DNAJC3 & -0.377663049 & 0.049951 & -1.638522404 & 0.02877 \\
\hline DRAM2 & -0.726386837 & 0.022444 & -5 & 0.000183 \\
\hline DUSP1 & -0.830211 & 0.045995 & -1.092128 & \\
\hline DYRK2 & -0.486 & 5658 & -1.6 & 0.00945 \\
\hline EDF1 & -0.8 & 0.02 & & 0.040718 \\
\hline EDNRB & -0.5 & 0.025631 & 2.8 & $7.91 \mathrm{E}-13$ \\
\hline EFEMP1 & -0.72 & 0.04534 & 1.40 & 0.000575 \\
\hline EFNB2 & -0.534300677 & 0.031279 & -1.40383 & 0.019655 \\
\hline EHD2 & -0.487559748 & 0.015141 & 1.398161 & 0.004582 \\
\hline ELK1 & -0.394407987 & 0.043625 & 1.195326989 & 0.002194 \\
\hline EMILI & -0.571141425 & 0.039703 & 1.9582 & $5.42 \mathrm{E}-05$ \\
\hline ENG & -0.5751 & 0.033416 & 972805 & 0.026503 \\
\hline $\mathrm{ENC}$ & & 0.045746 & & \\
\hline ETS2 & & \begin{tabular}{|l|}
0.039999 \\
\end{tabular} & & \\
\hline FAM13 & -0.8 & \begin{tabular}{|l|}
0.024207 \\
\end{tabular} & -1.8 & 0.00021 \\
\hline FAM46A & -0.647202094 & 0.012548 & -1.451241958 & 0.04004 \\
\hline FBLN5 & -0.551338968 & 0.012826 & -1.514662511 & 0.003136 \\
\hline FBXL7 & \begin{tabular}{|l}
-0.378152289 \\
\end{tabular} & 0.044687 & -1.181297147 & 0.039006 \\
\hline FKBP5 & -0.517708568 & 0.043381 & 2.593668358 & $2.22 \mathrm{E}-11$ \\
\hline FOS & -1.058578228 & 0.043634 & -2.021564297 & 0.000552 \\
\hline FXYD1 & 1.676000427 & \begin{tabular}{|l|}
0.009307 \\
\end{tabular} & 1.416270491 & 0.002163 \\
\hline FXYD6 & & & & \\
\hline GADD45B & -0.53 & \begin{tabular}{|l|}
0.032794 \\
\end{tabular} & 1.914790 & 0.00068 \\
\hline GAS2L1 & -0.662780815 & 0.035893 & 1.569622643 & 0.009101 \\
\hline GAS5 & -0.845491655 & 0.029155 & -1.886659007 & 0.02464 \\
\hline GAS7 & -0.830867293 & 0.016998 & 0.970841425 & 0.003611 \\
\hline GFM1 & \begin{tabular}{|l|}
0.593327245 \\
\end{tabular} & 0.039675 & -1.50539242 & 0.044621 \\
\hline GIMAP6 & -0.948944237 & 0.004831 & 5 & $9.08 \mathrm{E}-12$ \\
\hline GIMAP & -0.554386987 & 0.028666 & -1.410024917 & 0.009221 \\
\hline GJA1 & -0.35022164 & 0.049747 & -1.825553014 & 0.00178 \\
\hline
\end{tabular}




\begin{tabular}{|c|c|c|c|c|}
\hline GPR157 & -0.826733847 & 0.031724 & -1.730715343 & 0.036692 \\
\hline HAGH & 0.522401715 & 0.023181 & 1.028491735 & 0.03694 \\
\hline HBB & -5.455425115 & \begin{tabular}{|l|}
0.000788 \\
\end{tabular} & \begin{tabular}{|l|}
-7.950773935 \\
\end{tabular} & 7.01E-06 \\
\hline HIST1H2AC & -2.317333894 & 0.003876 & -2.444267659 & 0.002105 \\
\hline HSPB2 & 1.21107834 & 0.031367 & 1.552376496 & 0.031126 \\
\hline HSPB6 & 0.654174777 & & -1.92991699 & 0.001118 \\
\hline IL6ST & \begin{tabular}{|l|}
-0.346209222 \\
\end{tabular} & 0.036204 & -5 & 1.49E-05 \\
\hline IQGAP1 & -0.864329128 & 0.028623 & -1.914008486 & 0.002439 \\
\hline JAG1 & -0.648073868 & 0.021947 & -1.500158672 & 0.004055 \\
\hline JAK1 & 0.507474682 & 0.048446 & -1.291085313 & 0.010722 \\
\hline KCTD9 & -0.652371069 & 0.029115 & -1.588741638 & 0.045577 \\
\hline KDR & -1.01649251 & 0.027446 & -1.618120478 & 0.001516 \\
\hline KIDINS & \begin{tabular}{|l|}
-0.335580105 \\
\end{tabular} & & & \\
\hline KIF5 & & & & \\
\hline $\mathrm{KLH}$ & & & -1.8 & \\
\hline KLHL38 & 0.33 & 0.045138 & -2.11 & 0.001188 \\
\hline MAFG & -0.6 & 0.048208 & -1.01 & 0.009541 \\
\hline MAGOH & -0.30 & 0.048468 & -5 & $3.86 \mathrm{E}-05$ \\
\hline METRN & 0568665031 & 0.049113 & 1.393 & 0.016556 \\
\hline MFAP4 & -0.52 & 0.039794 & 1.41250 & 0.002207 \\
\hline MGLL & -0.37 & & & 0.021445 \\
\hline MKNK & & & & \\
\hline MLYCD & & & & 5587 \\
\hline MON & -055 & 0.0301 & -1.3 & 0.016891 \\
\hline MRPL33 & 68 & $5.81 \mathrm{E}-05$ & 2.628185807 & 0.001411 \\
\hline MTUS2 & 0.37 & \begin{tabular}{|l|}
0.049071 \\
\end{tabular} & 1.366 & 0.005888 \\
\hline MYLK3 & -0.433901967 & 0.044447 & -1.349636972 & 0.037659 \\
\hline NBL1 & -0.793221485 & 0.03112 & -1.407958502 & 0.039406 \\
\hline NCKIPSD & -0.409067028 & 0.043648 & 1.807487423 & 0.000159 \\
\hline NCOA4 & & & & 04488 \\
\hline & & & & \\
\hline NDUF & & & & 0.031125 \\
\hline NNT & 1.040 & 0.002013 & -1.685801 & 0.004346 \\
\hline NUDT4 & -0.57452292 & 0.049429 & -1.175784242 & 0.014874 \\
\hline OTUD1 & -1.471407718 & 0.037677 & 1.656789936 & 0.002426 \\
\hline P2RY2 & -0.645910688 & 0.038731 & -0.949886077 & 0.044382 \\
\hline PALM & -0.628062223 & 0.027912 & -1.462084762 & 0.004055 \\
\hline PARVB & 1.004307731 & 0.006333 & -1.086567828 & 0.006525 \\
\hline PDLIM3 & 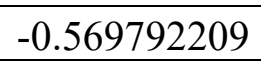 & 775 & -1.308620279 & 0.034935 \\
\hline PDLII & -0.579683763 & \begin{tabular}{|l|}
0.028378 \\
\end{tabular} & \begin{tabular}{|l|}
2.236962747 \\
\end{tabular} & 1.13E-06 \\
\hline PECAM1 & \begin{tabular}{|l|}
-0.533763511 \\
\end{tabular} & 0.048628 & \begin{tabular}{|l|}
1.602886378 \\
\end{tabular} & 0.0135 \\
\hline
\end{tabular}




\begin{tabular}{|c|c|c|c|c|}
\hline PEX6 & $\mid-0.497972807$ & 0.037461 & 1.100292214 & 0.041317 \\
\hline PLA2G2A & -2.647052214 & 0.045658 & 5.409285129 & 0 \\
\hline PNMAL1 & -0.634642632 & 0.049439 & -1.964423567 & 0.004 \\
\hline PPP1R12B & 0.592237795 & 0.000174 & -2.028461332 & 0.000287 \\
\hline PRELP & -0.570100677 & 0.036116 & 1.33153185 & 0.000945 \\
\hline PRKCDBP & -0.26048416 & 0.045832 & 1.595793765 & 0.02828 \\
\hline PTGDS & \begin{tabular}{|l|}
0.646333156 \\
\end{tabular} & 0.004501 & 1.322718047 & 0.028126 \\
\hline PTMS & -0.426086888 & 0.035995 & 2.640986855 & $3.74 \mathrm{E}-05$ \\
\hline PUM2 & -0.45940875 & 0.017423 & -1.520513073 & 0.025117 \\
\hline QSOX1 & 0.570678641 & 0.035483 & 1.402760158 & 0.000116 \\
\hline RAMP3 & -0.605059906 & 0.04247 & -1.03432831 & 0.046878 \\
\hline RFTN1 & -0.775743929 & 0.015701 & 1.628866348 & 0.000266 \\
\hline RNF146 & \begin{tabular}{|l|}
-0.371611904 \\
\end{tabular} & & & \\
\hline RNF & & & -5 & \\
\hline RPL & -0.3 & & 1.6 & \\
\hline RPLP1 & 0.86 & \begin{tabular}{|l|}
0.001217 \\
\end{tabular} & & 0.046657 \\
\hline RPS27A & -0.5 & 0.026615 & 1.22 & 0.024042 \\
\hline RTN4 & \begin{tabular}{|l|}
-1.07 \\
\end{tabular} & 0.039426 & -1.25 & 0.00121 \\
\hline S100A1 & 1.472 & 0.000813 & -2.005092261 & 0.000204 \\
\hline S100A8 & -0.84 & 0.012523 & 3.19243 & $1.63 \mathrm{E}-05$ \\
\hline $\mathrm{S} 100$ & -0.9 & 0.044725 & & $2.27 \mathrm{I}$ \\
\hline SAM & & & & \\
\hline $\mathrm{SCN}$ & & & & 05 \\
\hline SDC & -0.7 & 0.0 & -1.4 & 0.006884 \\
\hline SEC14L1 & 014 & 0.0 & 1.182 & 0.04 \\
\hline SEL1L & -0.44 & 0.035252 & 79115 & 0.017791 \\
\hline SEMA3G & -0.659911145 & 0.028594 & -1.095365967 & 0.02975 \\
\hline SERPINH1 & -0.564001102 & 0.043145 & -1.80990502 & 0.00023 \\
\hline SERTAD3 & -0.903840592 & 0.014546 & -1.43676246 & 0.013748 \\
\hline SGCA & 0.496242738 & & & \\
\hline SLC & & & & \\
\hline SLC6A6 & -1.1 & 0.0 & -1.74 & 6 \\
\hline SLC7A8 & -0.972640406 & 0.035062 & 2.456764225 & $1.18 \mathrm{E}-07$ \\
\hline SLC8A1 & \begin{tabular}{|l|}
0.685780929 \\
\end{tabular} & 0.002115 & -1.420048399 & 0.000244 \\
\hline SPARCL1 & -0.598007079 & 0.012917 & 1.47024442 & 0.003893 \\
\hline SPOP & -0.397007271 & 0.040271 & 5 & $9.57 \mathrm{E}-10$ \\
\hline SRGN & -0.363394399 & 0.036167 & 1.540982699 & 0.011232 \\
\hline SRPX & -0.3 & 0.036118 & 1.429851664 & 0.023931 \\
\hline STAT1 & -0.633400793 & 94 & -1.272018841 & 0.003775 \\
\hline STAT3 & -0.972618741 & 0.036138 & 1.755397946 & $4.58 \mathrm{~F}$ \\
\hline SUN1 & -0.450823867 & 0.038098 & -0.872885661 & 0.043073 \\
\hline
\end{tabular}




\begin{tabular}{|l|l|l|l|l|} 
SYNM & 0.325806265 & 0.04979 & -1.464381185 & 0.005351 \\
\hline TACC1 & -0.370545039 & 0.038676 & 1.464658566 & $1.73 \mathrm{E}-05$ \\
\hline TACC2 & 0.563731348 & 0.003703 & 0.951365548 & 0.043192 \\
\hline TAX1BP1 & 0.279872161 & 0.036848 & -1.262935914 & 0.024751 \\
\hline TAX1BP3 & -1.445656879 & 0.023178 & 5 & $7.34 \mathrm{E}-06$ \\
\hline TEAD4 & -0.838120353 & 0.02039 & 1.917580892 & 0.000583 \\
\hline TEK & -0.665089599 & 0.042213 & -1.904942937 & 0.000222 \\
\hline TGFB1 & -0.48409647 & 0.035802 & -1.545663311 & 0.005583 \\
\hline THBS4 & 1.196572038 & 0.048544 & -2.120653978 & 0.002596 \\
\hline TIMM8B & -1.108598614 & 0.012035 & 5 & $5.43 \mathrm{E}-10$ \\
\hline TIMP1 & -1.008514924 & 0.028892 & 1.840233596 & 0.006884 \\
\hline TIMP3 & -0.392285917 & 0.02239 & 2.182312486 & $4.85 \mathrm{E}-06$ \\
\hline TMEM204 & -0.652903815 & 0.021325 & 1.673222066 & 0.016904 \\
\hline TMSB4X & -0.518989998 & 0.049079 & -1.498036021 & 0.0037 \\
\hline TSC1 & -0.532485732 & 0.044115 & -0.986793547 & 0.020307 \\
\hline TXNRD1 & -0.397359953 & 0.043213 & -0.917006565 & 0.005121 \\
\hline USP9X & 0.696855665 & 0.033127 & -1.591714262 & 0.022522 \\
\hline VSTM2L & -0.863262469 & 0.04021 & 3.223903018 & $5.23 \mathrm{E}-06$ \\
\hline WDR26 & 0.434242526 & 0.048381 & -1.326486608 & 0.015158 \\
\hline XIRP1 & -0.78224014 & 0.048919 & 1.476941227 & 0.000878 \\
\hline ZFP36 & -0.991785116 & 0.017465 & 1.859309472 & 0.002371 \\
\hline ZMIZ1 & -0.698773614 & 0.039673 & -1.134288226 & 0.033385 \\
\hline
\end{tabular}


Supplemental Table 15. DEG shared between female obesity-related LVH and both dilated ischemia and cardiomyopathy datasets with $\left(p_{-}\right.$adj $\left.<0.05\right)$

\begin{tabular}{|c|c|c|c|c|}
\hline Gene & $\begin{array}{lll}\log 2 \mathrm{FC} & \text { NF } & \text { vs } \\
\text { LVH_F } & & \\
\end{array}$ & p_adj & $\begin{array}{l}\log 2 \mathrm{FC} \text { ISCH vs } \\
\text { DCM }\end{array}$ & $p$ adj \\
\hline ADAM19 & -0.630142749 & 0.037114 & 1.489280792 & 0.002551 \\
\hline AKAP12 & -0.731993228 & 0.026665 & -1.06178257 & 0.015975 \\
\hline AP1S2 & -0.789011417 & 0.015457 & 1.309426583 & 0.020855 \\
\hline APOD & -0.892669878 & 0.037102 & -1.524595823 & 0.000468 \\
\hline APOL1 & -0.782334647 & 0.021276 & 1.393911307 & 0.011075 \\
\hline ATE1 & -0.823974167 & 0.02715 & -1.582781094 & 0.001702 \\
\hline BCAR3 & -0.799783333 & 0.044029 & -1.610550008 & 0.004017 \\
\hline BTG2 & -0.547514523 & 0.022147 & 1.982556367 & 0.000194 \\
\hline C1QTNF1 & -0.866995909 & 0.004932 & 1.983690511 & 0.000181 \\
\hline C8orf4 & -0.883640814 & 0.03769 & -2.378198117 & $1.06 \mathrm{E}-06$ \\
\hline CASP3 & -0.66390716 & 0.043676 & 1.569891424 & 0.004874 \\
\hline CAST & 1.51500001 & 0.003565 & -0.923186229 & 0.005729 \\
\hline CCL2 & 0.861442892 & 0.041833 & 5.109077051 & 0.012536 \\
\hline CCL21 & -1.998910656 & 0.034425 & 3.211259363 & $1.73 \mathrm{E}-08$ \\
\hline CD151 & 0.472684984 & 0.037071 & 1.258355253 & 0.00377 \\
\hline CD68 & 0.939054502 & 0.044368 & 1.783816809 & 0.00035 \\
\hline CD93 & -0.59718142 & 0.012803 & 1.638558045 & 0.000696 \\
\hline COL6A2 & -0.400028873 & 0.018589 & 1.196810429 & 0.046365 \\
\hline CRIM1 & -0.434102162 & 0.048377 & 1.280530737 & 0.0142 \\
\hline CTGF & -1.189465404 & 0.030305 & 1.712090851 & 0.00187 \\
\hline CXorf36 & -0.529466638 & 0.04564 & 1.404338257 & 0.005522 \\
\hline DAPK2 & 0.74144734 & 0.046529 & -1.29432394 & 0.012646 \\
\hline DIRAS3 & -1.018644618 & 0.028944 & -2.755293509 & $1.15 \mathrm{E}-09$ \\
\hline DRAM2 & -0.726386837 & 0.022444 & 5 & $1.19 \mathrm{E}-05$ \\
\hline DYRK2 & -0.486689622 & 0.045658 & -1.060596127 & 0.047844 \\
\hline EFEMP1 & -0.729911964 & 0.04534 & 1.867728848 & $3.00 \mathrm{E}-06$ \\
\hline EFNB2 & -0.534300677 & 0.031279 & -1.914497416 & 0.000313 \\
\hline EGR1 & -0.778673334 & 0.049178 & -1.332175587 & 0.016758 \\
\hline EMILIN1 & -0.571141425 & 0.039703 & 2.041043672 & $8.13 \mathrm{E}-06$ \\
\hline ETFB & 1.068367319 & 0.002431 & -1.136020685 & 0.039885 \\
\hline FAM114A1 & -0.712234668 & 0.019887 & 1.298243506 & 0.023462 \\
\hline FAM134B & -0.884094691 & 0.024207 & -1.226478006 & 0.004017 \\
\hline FBXO40 & 0.559934599 & 0.049521 & -1.302725083 & 0.048941 \\
\hline FKBP5 & -0.517708568 & 0.043381 & 1.999049339 & $1.19 \mathrm{E}-05$ \\
\hline FOS & -1.058578228 & 0.043634 & 1.617142342 & 0.021996 \\
\hline GADD45B & -0.530310364 & 0.032794 & 1.423620172 & 0.03675 \\
\hline
\end{tabular}




\begin{tabular}{|c|c|c|c|c|}
\hline GJA1 & -0.35022164 & 0.049747 & -1.835417002 & $\mid 0.000968$ \\
\hline GLIPR2 & -0.67926169 & 0.037362 & 1.940228249 & 0.005741 \\
\hline GPR157 & -0.826733847 & 0.031724 & -1.956324683 & 0.003773 \\
\hline HBA1 & -3.400698784 & 0.001351 & -2.7929336 & $4.60 \mathrm{E}-12$ \\
\hline HBB & -5.455425115 & 0.000788 & -2.22489195 & $1.09 \mathrm{E}-07$ \\
\hline IL1R1 & -0.511212184 & 0.037637 & 1.483566641 & 0.024324 \\
\hline IL33 & -0.799078418 & 0.026093 & 1.634526823 & 0.021856 \\
\hline IL6ST & -0.346209222 & 0.036204 & \begin{tabular}{|l|}
1.207790986 \\
\end{tabular} & 0.000608 \\
\hline IQGAP1 & -0.864329128 & 0.028623 & 1.474355992 & 0.009234 \\
\hline ITGA6 & -0.386557844 & 0.041332 & 1.585582568 & 0.000365 \\
\hline JAG1 & -0.648073868 & 0.021947 & -1.288054053 & 0.02486 \\
\hline KDR & -1.01649251 & 0.027446 & -1.766277418 & 0.000681 \\
\hline KLHL38 & 0.335280765 & 0.045138 & -1.661614181 & 0.001099 \\
\hline LAMP2 & -0.988496109 & 0.017906 & \begin{tabular}{|l|}
0.967998379 \\
\end{tabular} & 0.031384 \\
\hline LRRC14B & 0.601273929 & 0.046813 & -2.25222654 & 2.04E-06 \\
\hline MAPK8IP3 & $\begin{array}{l}-0.589170794 \\
\end{array}$ & 0.045532 & \begin{tabular}{|l}
-1.122339641 \\
\end{tabular} & 0.000425 \\
\hline MLYCD & 0.893456574 & $6.48 \mathrm{E}-06$ & \begin{tabular}{|l}
-1.107128992 \\
\end{tabular} & 0.038156 \\
\hline MRPL33 & 0.685197212 & 5.81E-05 & 3.24989797 & 5.31E-05 \\
\hline MYO1B & -0.606313032 & 0.025112 & 1.770265741 & 0.000998 \\
\hline NCKIPSD & -0.409067028 & 0.043648 & -0.881265111 & 0.048721 \\
\hline NEDD9 & -0.524428571 & 0.046449 & -2.282312678 & 1.31E-07 \\
\hline NNT & 1.040709158 & 0.002013 & -1.370583183 & 0.001314 \\
\hline NPPA & -2.896073668 & 0.032393 & 1.804016681 & 0.002728 \\
\hline NPPB & -2.213258402 & 0.014572 & 3.95062945 & $1.28 \mathrm{E}-12$ \\
\hline NR1D2 & -0.366189625 & 0.043119 & \begin{tabular}{|l|}
1.769391525 \\
\end{tabular} & \begin{tabular}{|l}
0.000757 \\
\end{tabular} \\
\hline PDLIM3 & -0.569792209 & 0.032775 & \begin{tabular}{|l|}
-1.051610175 \\
\end{tabular} & 0.033209 \\
\hline PDLIM7 & -0.579683763 & 0.028378 & 1.237640222 & 0.003134 \\
\hline PLA2G2A & -2.647052214 & 0.045658 & 3.345485111 & $2.17 \mathrm{E}-08$ \\
\hline PLVAP & -1.265760002 & 0.017747 & 1.949632993 & 0.001075 \\
\hline PLXDC2 & -2.032815142 & 0.011265 & 1.934162391 & 0.005957 \\
\hline PNMAL1 & -0.634642632 & 0.049439 & -1.904599535 & 0.000101 \\
\hline PROS1 & -0.92499567 & 0.042894 & 1.897009877 & 9.67E-05 \\
\hline PTGDS & 0.646333156 & 0.004501 & \begin{tabular}{|l}
-1.841490956 \\
\end{tabular} & 0.001027 \\
\hline PTX3 & -1.366782142 & 0.018539 & 5 & $2.34 \mathrm{E}-05$ \\
\hline QSOX1 & 0.570678641 & 0.035483 & 1.356787899 & 0.000798 \\
\hline RFTN1 & -0.775743929 & 0.015701 & 1.201772963 & 0.036786 \\
\hline RNF145 & -0.505423328 & 0.038072 & 5 & $1.05 \mathrm{E}-10$ \\
\hline RNF185 & -0.83124553 & 0.01517 & 5 & 7.02E-08 \\
\hline S100A1 & 1.472047839 & 0.000813 & -1.356663715 & 0.0142 \\
\hline S100A9 & -0.934970606 & 0.044725 & 2.81213663 & 0.000516 \\
\hline SAMHD1 & -0.58759924 & 0.018941 & 1.816578453 & 0.002707 \\
\hline
\end{tabular}




\begin{tabular}{|l|l|l|l|l|} 
SDCBP & -0.798182227 & 0.031003 & -1.519540572 & $3.79 \mathrm{E}-05$ \\
\hline SERPINH1 & -0.564001102 & 0.043145 & 1.273626761 & 0.012686 \\
\hline SLC6A6 & -1.150709242 & 0.016902 & 1.147020506 & 0.034305 \\
\hline SLC7A8 & -0.972640406 & 0.035062 & 1.870262997 & 0.000332 \\
\hline SPOP & -0.397007271 & 0.040271 & 5 & $2.65 \mathrm{E}-11$ \\
\hline STAT3 & -0.972618741 & 0.036138 & 1.106890148 & 0.003681 \\
\hline SUN1 & -0.450823867 & 0.038098 & -1.068370923 & 0.000884 \\
\hline TCEAL7 & -0.553228932 & 0.045743 & 2.828337491 & 0.009234 \\
\hline TEAD4 & -0.838120353 & 0.02039 & 1.594275865 & 0.009234 \\
\hline TEK & -0.665089599 & 0.042213 & -1.674114374 & 0.001404 \\
\hline TGFB1 & -0.48409647 & 0.035802 & 1.319426652 & 0.027066 \\
\hline THBS1 & -0.377386586 & 0.048008 & -1.958430312 & $6.76 \mathrm{E}-05$ \\
\hline THBS2 & -0.665188472 & 0.044745 & 2.628736021 & $1.33 \mathrm{E}-08$ \\
\hline THBS4 & 1.196572038 & 0.048544 & 1.363856989 & 0.026136 \\
\hline TIMM8B & -1.108598614 & 0.012035 & 5 & $3.34 \mathrm{E}-11$ \\
\hline TIMP1 & -1.008514924 & 0.028892 & 1.891597357 & 0.000442 \\
\hline VAMP8 & -0.563839444 & 0.035165 & 1.823924832 & 0.024876 \\
\hline VSTM2L & -0.863262469 & 0.04021 & 2.777014645 & $6.09 \mathrm{E}-05$ \\
\hline ZMIZ1 & -0.698773614 & 0.039673 & -1.238874926 & 0.040865 \\
\hline ZNF436 & -0.853453662 & 0.031663 & 1.7512317 & 0.03428 \\
\hline ZNF438 & -0.411605766 & 0.049926 & 5 & $1.04 \mathrm{E}-11$ \\
\hline
\end{tabular}


Supplemental Table 16. DEG shared between male obesity-related LVH and ischemia datasets with (p_adj $<\mathbf{0 . 0 5})$

\begin{tabular}{|c|c|c|c|c|}
\hline Gene & $\begin{array}{lll}\log 2 \mathrm{FC} & \text { NF } & \text { Vs } \\
\text { LVH_M } & & \\
\end{array}$ & $p \_$adj & $\begin{array}{l}\log 2 \mathrm{FC} \quad \mathrm{NF} \text { vs } \\
\text { ISCH }\end{array}$ & $\mathrm{p} \_$adj \\
\hline ABTB1 & -0.460991597 & 0.012788 & 0.899951705 & 0.012014 \\
\hline ADAM9 & 0.324658867 & 0.038311 & -1.296514971 & 0.0169 \\
\hline ADAMTS15 & 0.546947449 & 0.043257 & 1.328317163 & 0.004927 \\
\hline AIF1L & 0.760356543 & 0.04817 & 1.666590241 & 0.000101 \\
\hline ALDH6A1 & -0.459566879 & 0.008789 & 1.123273643 & 0.036902 \\
\hline ANTXR2 & -0.239481735 & 0.033088 & 1.006537036 & 0.018857 \\
\hline APLN & 0.42609975 & 0.027729 & -2.034721693 & 0.001492 \\
\hline APLP1 & -0.494267388 & 0.020786 & -5 & $1.01 \mathrm{E}-09$ \\
\hline $\mathrm{AQP7}$ & -0.339087891 & 0.040948 & 1.111539965 & 0.040004 \\
\hline ATP13A3 & -0.303941208 & 0.047239 & -2.110450865 & $6.16 \mathrm{E}-06$ \\
\hline AZGP1 & -1.307980325 & 0 & -1.138888768 & 0.040662 \\
\hline AZIN1 & -0.233055614 & 0.044509 & -1.355458125 & 0.00242 \\
\hline B4GALNT3 & -0.438156567 & 0.021696 & 1.300729904 & 0.006208 \\
\hline C10orf10 & -0.776170461 & $3.00 \mathrm{E}-15$ & 2.175525819 & $1.54 \mathrm{E}-07$ \\
\hline C11orf96 & -0.286254062 & 0.045204 & 2.224645005 & $5.09 \mathrm{E}-06$ \\
\hline $\mathrm{C} 3$ & 0.99050202 & 0.025013 & 2.045972787 & $1.21 \mathrm{E}-05$ \\
\hline CD151 & -0.510771364 & $4.00 \mathrm{E}-15$ & -1.076847186 & 0.003615 \\
\hline CD300LG & 0.770142832 & 0.020405 & 2.773023736 & 0 \\
\hline CDKN1A & -0.393196018 & 0.03855 & 1.066409299 & 0.042891 \\
\hline CEP85 & -0.354319987 & 0.033799 & 1.377027078 & 0.004362 \\
\hline COL15A1 & 0.861245935 & 0.024047 & -1.666558498 & 0.000493 \\
\hline COL4A3BP & -0.308889968 & 0.021196 & -5 & $6.23 \mathrm{E}-08$ \\
\hline COMMD6 & -0.280205666 & 0.028834 & -3.151519642 & $3.22 \mathrm{E}-07$ \\
\hline COMP & -0.849294671 & 0.039889 & -4.065207514 & 2.18E-07 \\
\hline CRNDE & -0.528269658 & 0.034849 & -2.186615542 & 0.004521 \\
\hline CTIF & -0.340308488 & 0.035364 & 1.15914319 & 0.000568 \\
\hline DDAH1 & -0.568797795 & 0.017604 & -1.393084172 & 0.021863 \\
\hline DNAJA4 & -0.739665528 & 0.045952 & -1.318690893 & 0.005975 \\
\hline DYNLL1 & -0.377506314 & 0.008446 & -1.775643022 & $3.34 \mathrm{E}-05$ \\
\hline EDNRB & 0.572001611 & 0.015444 & 2.168141571 & $1.62 \mathrm{E}-11$ \\
\hline ELK1 & -0.345545015 & 0.016899 & 1.055086757 & 0.0086 \\
\hline ENO2 & -0.475902084 & 0.007665 & 1.329284641 & 0.01159 \\
\hline ETV1 & -0.348286938 & 0.047575 & -1.585575868 & 0.000271 \\
\hline FLNC & -0.299205249 & 0.033991 & 1.534788946 & 0.000252 \\
\hline FMOD & -0.667877526 & 0.03788 & -1.556832307 & 0.004055 \\
\hline FNDC5 & 0.420695209 & 0.019081 & -1.197431896 & 0.003018 \\
\hline
\end{tabular}




\begin{tabular}{|c|c|c|c|c|}
\hline FSTL3 & -0.413282056 & 0.011028 & -1.278100975 & 0.030781 \\
\hline GLUL & -0.567151422 & 0.010959 & 1.511265015 & $4.26 \mathrm{E}-05$ \\
\hline GPIHBP1 & 0.716162309 & 0.032929 & 2.175472642 & $1.92 \mathrm{E}-07$ \\
\hline GPR153 & -0.681905951 & 0.043028 & -1.810031993 & 0.001268 \\
\hline GSTT1 & -1.140967802 & 0.033376 & 1.379716623 & 0.004122 \\
\hline HCFC1R1 & -0.269876232 & 0.028189 & 1.813783392 & 4.68E-09 \\
\hline HDAC5 & -0.290056255 & 0.000133 & \begin{tabular}{|l|}
1.016822647 \\
\end{tabular} & 0.003741 \\
\hline $\mathrm{HEPH}$ & -0.385669396 & 0.016989 & -2.635587422 & $7.15 \mathrm{E}-08$ \\
\hline HFE2 & -0.416585779 & 0.021178 & -5 & $2.01 \mathrm{E}-15$ \\
\hline HIF3A & -0.36726417 & 0.031215 & 1.90209774 & $2.88 \mathrm{E}-11$ \\
\hline HIPK3 & 0.347338792 & 0.026941 & -5 & $1.85 \mathrm{E}-10$ \\
\hline HIST1H2BD & -0.444623519 & 0.022086 & -1.819527247 & 0.008223 \\
\hline HLA-B & 0.583581342 & 0.049228 & \begin{tabular}{|l|}
1.447469619 \\
\end{tabular} & 0.002137 \\
\hline IER5 & -0.5107848 & 0.011172 & 1.11415786 & 0.032784 \\
\hline IGFBP2 & -0.862259937 & 0 & -2.089911786 & 5.79E-06 \\
\hline IL6ST & 0.33077625 & 0.049957 & \begin{tabular}{|l|}
-0.96627322 \\
\end{tabular} & 0.007438 \\
\hline IRX3 & -0.407781337 & 0.043472 & 1.642289454 & 0.000158 \\
\hline ITGA6 & 0.49961087 & 0.032435 & -1.130173886 & 0.004787 \\
\hline ITGB1 & 0.292811102 & 0.042933 & -1.012564118 & 0.006673 \\
\hline ITPKB & 0.68388826 & 0.022309 & 1.136878349 & 0.031003 \\
\hline JUP & -0.351730759 & $2.00 \mathrm{E}-15$ & 1.340514078 & 0.000647 \\
\hline KCTD17 & -0.563715606 & $3.80 \mathrm{E}-05$ & -1.477646367 & 0.021895 \\
\hline KLHL21 & -0.309586543 & 0.03786 & 1.946075397 & $3.75 \mathrm{E}-05$ \\
\hline KLHL31 & 0.331983204 & 0.041734 & -1.242447658 & 0.022627 \\
\hline LDB3 & -0.193086896 & 0.037827 & \begin{tabular}{|l|}
0.780896708 \\
\end{tabular} & 0.021489 \\
\hline LDHA & -0.518131046 & 0.016036 & -1.229179915 & 0.019527 \\
\hline LRRC14B & -0.582658557 & $6.69 \mathrm{E}-05$ & 2.497809238 & $2.29 \mathrm{E}-09$ \\
\hline MAOA & -0.292685908 & 0.017421 & 1.156827805 & 0.033939 \\
\hline MASP1 & -0.627801182 & $9.84 \mathrm{E}-05$ & -1.201667492 & 0.015218 \\
\hline MFAP4 & -0.36430884 & 0.013485 & -1.750721346 & 0.002055 \\
\hline MT1X & -0.820480928 & 0.041477 & -3.148047139 & $3.10 \mathrm{E}-06$ \\
\hline MTMR14 & -0.314430647 & 0.014979 & 1.060913826 & 0.00318 \\
\hline MTSS1 & 0.405885732 & 0.039649 & 1.302231648 & 0.008775 \\
\hline MYL4 & 1.123474745 & 1.01E-06 & 5.061522714 & 0 \\
\hline NAP1L1 & -0.313200929 & 0.020236 & -0.910734866 & 0.027081 \\
\hline NCEH1 & 0.470319732 & 0.040649 & -5 & $1.70 \mathrm{E}-07$ \\
\hline NDRG2 & -0.146954763 & 0.035152 & \begin{tabular}{|l|}
0.914021945 \\
\end{tabular} & $2.83 \mathrm{E}-06$ \\
\hline NEAT1 & -0.355959133 & 0.032055 & 1.359837777 & 0.042058 \\
\hline NPPA & -4.597666596 & 0.018249 & -4.275261149 & 0 \\
\hline NPPB & -2.898570787 & 0.013588 & -3.478505958 & $1.79 \mathrm{E}-13$ \\
\hline NRTN & -0.748451624 & 0.001353 & \begin{tabular}{|l|}
1.414942419 \\
\end{tabular} & 0.045468 \\
\hline
\end{tabular}




\begin{tabular}{|c|c|c|c|c|}
\hline OPA1 & 0.179786546 & 0.041586 & -1.003990391 & 0.003612 \\
\hline OTUD1 & -0.364965273 & 0.009595 & 1.307614166 & 0.008755 \\
\hline PDK4 & -1.190849404 & 0.023484 & -1.621761989 & 0.003644 \\
\hline PHLDA1 & -0.967904287 & 0.013169 & -1.533891972 & 0.00837 \\
\hline PIK3IP1 & -0.515566884 & 0.034509 & 1.573888462 & 0.000162 \\
\hline PLEKHA6 & -0.428192332 & 0.011977 & 1.556511571 & 0.00051 \\
\hline PLK2 & -0.741565424 & 0.022569 & 1.372546472 & 0.009604 \\
\hline POR & \begin{tabular}{|l|}
-0.512484111 \\
\end{tabular} & 0.02111 & 1.351443234 & 0.004687 \\
\hline POSTN & -0.716023612 & 0.043196 & -5.398096936 & 0 \\
\hline PPAP2B & 0.270841062 & 0.049882 & 1.203371662 & 0.018584 \\
\hline PROS1 & -0.634473414 & 0.022481 & -1.777064031 & 0.000208 \\
\hline PTMS & -0.204572767 & 0.018308 & 1.586696097 & 0.000379 \\
\hline RASL10B & -0.604665337 & 0.017747 & 1.614289113 & 0.000481 \\
\hline RBM3 & 0.274338467 & 7.99E-15 & 1.527018038 & 0.000788 \\
\hline RPL28 & -0.215383175 & 0.033283 & 0.86291538 & 0.047925 \\
\hline RPS27L & \begin{tabular}{|l|}
-0.453376267 \\
\end{tabular} & 0.038886 & -1.237217046 & 0.026793 \\
\hline RRAS2 & \begin{tabular}{|l|}
-0.417699346 \\
\end{tabular} & 0.008991 & -5 & $9.89 \mathrm{E}-07$ \\
\hline RXRG & -0.430989662 & 0.043663 & -2.486881571 & 0.001101 \\
\hline SEC14L5 & 1.203995176 & 0.025826 & 2.532045159 & 4.07E-09 \\
\hline SH3BGR & -1.038419793 & 0.041381 & -1.126205863 & 0.042774 \\
\hline SHISA3 & 0.929801987 & 0.049266 & 3.030175389 & $1.33 \mathrm{E}-08$ \\
\hline SLC27A6 & -0.725885867 & 0.012021 & -1.314110357 & 0.029792 \\
\hline SLCO2A1 & \begin{tabular}{|l|}
-0.40718502 \\
\end{tabular} & 0.038314 & 1.689613686 & 0.000169 \\
\hline SNHG8 & -0.344159108 & 0.042777 & 1.326354211 & 0.025361 \\
\hline SOD3 & \begin{tabular}{|l|}
-0.402059082 \\
\end{tabular} & 0.00539 & -1.490774626 & 0.002217 \\
\hline SVOP & \begin{tabular}{|l|}
-1.064191722 \\
\end{tabular} & 0.034337 & -2.07588899 & 0.003099 \\
\hline TFRC & 1.359094592 & 0.041854 & -1.626296372 & 0.000835 \\
\hline TGM2 & -0.492854217 & 0.012428 & 1.284423864 & 0.028545 \\
\hline TIMM9 & -0.248657524 & 0.033989 & -5 & $1.94 \mathrm{E}-05$ \\
\hline TIMP3 & 0.458961699 & 0.040691 & 1.659800666 & 0.004458 \\
\hline TMEM140 & -0.89371551 & $1.20 \mathrm{E}-14$ & 1.502983443 & 0.001443 \\
\hline TOM1 & -0.405308093 & \begin{tabular}{|l|}
0.030337 \\
\end{tabular} & 0.901618286 & 0.045771 \\
\hline TSPYL2 & -0.85969565 & 0.0075 & 1.54357506 & 0.000704 \\
\hline TUBB6 & \begin{tabular}{|l|}
0.430747751 \\
\end{tabular} & 0.023596 & 1.122036783 & 0.036911 \\
\hline TXNRD1 & -0.490654157 & 0.036189 & 0.786257974 & 0.033516 \\
\hline UBAC2 & -0.238098048 & 0.035092 & -1.270103527 & 0.015028 \\
\hline UBE3A & -0.140145749 & 0.047775 & -5 & $2.61 \mathrm{E}-09$ \\
\hline UCHL1 & \begin{tabular}{|l}
-0.876726333 \\
\end{tabular} & 0.012504 & -2.745991144 & 0.002544 \\
\hline UGT2B4 & 0.967809667 & 0.036872 & -3.395510497 & $1.03 \mathrm{E}-05$ \\
\hline ULK1 & -0.386662051 & 0.013491 & 1.158112048 & 0.027754 \\
\hline VPS13D & 0.260474561 & 0.044122 & -5 & $6.36 \mathrm{E}-07$ \\
\hline
\end{tabular}




\begin{tabular}{|l|l|l|l|l|} 
XIRP2 & 0.508831734 & 0.026453 & -1.338335243 & 0.00205 \\
\hline XPR1 & -0.404531859 & 0.040079 & -0.992860816 & 0.035245 \\
\hline YPEL3 & -0.784698444 & $4.00 \mathrm{E}-15$ & 1.140709773 & 0.018303 \\
\hline
\end{tabular}


Supplemental Table 17. DEG shared between male obesity-related LVH and dilated cardiomyopathy datasets with $\left(\mathbf{p} \_\right.$adj $\left.<0.05\right)$

\begin{tabular}{|c|c|c|c|c|}
\hline Gene & $\begin{array}{lll}\log 2 \mathrm{FC} & \text { NF } & \text { vs } \\
\text { LVH_M } & & \\
\end{array}$ & $\mathrm{p} \_$adj & $\begin{array}{l}\log 2 \mathrm{FC} \text { NF vs } \\
\text { DCM }\end{array}$ & $p \_$adj \\
\hline ABAT & -0.436324244 & 0.035648 & -1.855169072 & 0.000245 \\
\hline ABCA8 & 0.648874255 & 0.042578 & -1.940300807 & 0.002435 \\
\hline ABHD11 & -0.294215215 & 0.032183 & 0.953401699 & 0.034878 \\
\hline ACOT11 & -0.361296457 & 0.012581 & -1.52973733 & 0.000207 \\
\hline ADAMTS15 & 0.546947449 & 0.043257 & 2.160891353 & $6.59 \mathrm{E}-06$ \\
\hline AGPAT2 & -0.305168768 & 0.014057 & 1.173437054 & 0.014742 \\
\hline AGPAT9 & -0.472276352 & 0.029837 & -1.692568564 & 0.017186 \\
\hline AIF1L & 0.760356543 & 0.04817 & -1.690830076 & 5.89E-05 \\
\hline ALDH1L1 & -0.422880657 & 0.013817 & 1.464875776 & 0.00475 \\
\hline ALDH6A1 & -0.459566879 & 0.008789 & -1.487913517 & 0.003356 \\
\hline ANAPC11 & -0.221336121 & 0.021095 & 0.824630217 & 0.002557 \\
\hline ANTXR2 & -0.239481735 & 0.033088 & -0.934274411 & 0.046 \\
\hline APLN & 0.42609975 & 0.027729 & -3.647631894 & $1.65 \mathrm{E}-07$ \\
\hline APLP1 & -0.494267388 & 0.020786 & -5 & $3.22 \mathrm{E}-07$ \\
\hline APRT & -0.2692331 & 0.048238 & 1.479862863 & 0.036549 \\
\hline AQP7 & -0.339087891 & 0.040948 & 1.320446161 & 0.012755 \\
\hline AZGP1 & -1.307980325 & 0 & 2.180581657 & $2.13 \mathrm{E}-06$ \\
\hline AZIN1 & -0.233055614 & 0.044509 & -1.234736437 & 0.014779 \\
\hline B4GALNT3 & -0.438156567 & 0.021696 & -1.571730374 & 0.002181 \\
\hline BAMBI & -0.442504318 & 0.019297 & 1.432540867 & 0.011909 \\
\hline C10orf76 & -0.378725282 & 0.014545 & -1.837503291 & 0.007708 \\
\hline C11 orf24 & -0.333366417 & 0.011191 & 1.146685791 & 0.045062 \\
\hline C11orf96 & -0.286254062 & 0.045204 & 1.838403629 & 0.010094 \\
\hline C1orf115 & 0.560171498 & 38375 & 1.522052774 & 0.004354 \\
\hline C1orf122 & -0.293493497 & 0.012048 & 1.113214233 & 0.036556 \\
\hline C1QTNF1 & -0.578503513 & 0.038739 & 2.113417594 & 0.001372 \\
\hline $\mathrm{C} 3$ & 0.99050202 & 0.025013 & 1.430493191 & 0.005785 \\
\hline C6orf1 & -0.509148869 & 0.011928 & 1.462087575 & 0.00141 \\
\hline CAMK2B & -0.371924966 & 0.002571 & 0.782785864 & 0.024498 \\
\hline CAST & -0.150895471 & 0.043171 & -1.367834595 & 0.000208 \\
\hline CCDC85B & -0.626416075 & $3.60 \mathrm{E}-14$ & 1.451714992 & 0.003356 \\
\hline CCL2 & 0.671466177 & $9.02 \mathrm{E}-06$ & 6.143199949 & 0.000908 \\
\hline CD151 & -0.510771364 & $4.00 \mathrm{E}-15$ & -1.317019331 & $2.63 \mathrm{E}-06$ \\
\hline CD300LG & 0.770142832 & 0.020405 & 2.221769286 & $1.13 \mathrm{E}-11$ \\
\hline $\mathrm{CDH} 5$ & 0.713600224 & 0.047911 & -1.238663795 & 0.005578 \\
\hline CDK18 & -0.206138497 & 0.038997 & 0.661435315 & 0.028167 \\
\hline
\end{tabular}




\begin{tabular}{|c|c|c|c|c|}
\hline CDKN1A & -0.393196018 & 0.03855 & 1.293184109 & 0.041044 \\
\hline CEBPB & -0.464728923 & 0.031278 & 1.446932885 & 0.038798 \\
\hline CEBPD & -0.492843424 & 0.035732 & 1.639611818 & 0.015646 \\
\hline CNN2 & -0.234532303 & 0.032806 & 1.205382083 & 0.042821 \\
\hline COL23A1 & -0.759281134 & 0.023043 & 1.965826273 & 0.00649 \\
\hline COL4A3BP & -0.308889968 & 0.021196 & -5 & $1.04 \mathrm{E}-06$ \\
\hline COMMD6 & -0.280205666 & 0.028834 & 1.986287051 & 0.003216 \\
\hline CRELD1 & -0.571053939 & 0.015765 & 1.119429701 & 0.003928 \\
\hline CTF1 & -0.374627485 & 0.038807 & 1.46343487 & 0.003932 \\
\hline CTIF & -0.340308488 & 0.035364 & -0.901524118 & 0.031209 \\
\hline CTNNA3 & 0.420902413 & 0.047603 & -2.358489586 & 0.000779 \\
\hline CTSF & -0.609388393 & 0.012649 & 1.636528981 & 0.001335 \\
\hline CYGB & 0.484721501 & 0.045147 & 1.603747399 & 0.000827 \\
\hline DCAF11 & -0.232426 & 0.017956 & -0.80 & \\
\hline DKK3 & -0.746405642 & 0.011774 & -1.591477235 & 5.19E-05 \\
\hline DPM3 & -0.372064209 & 0.01069 & -1.330865471 & 0.04489 \\
\hline DSP & 0.169735277 & 0.025423 & -1.738090398 & 0.024171 \\
\hline EDF1 & -0.314122494 & $6.00 \mathrm{E}-15$ & 0.831908111 & 0.040718 \\
\hline EDNRB & 0.572001611 & 0.015444 & 2.827625448 & $7.91 \mathrm{E}-13$ \\
\hline EGLN3 & -0.362429875 & 0.038702 & 1.213162938 & 0.017311 \\
\hline ELK1 & -0.345545015 & 0.016899 & 1.195326989 & 0.002194 \\
\hline ELN & 0.558139214 & 0.001639 & 3.353674038 & $7.56 \mathrm{E}-10$ \\
\hline ENO1 & 0.36807835 & 0.045018 & -1.04443571 & 0.039006 \\
\hline ENO2 & -0.475902084 & 0.007665 & 2.196136473 & 0.0023 \\
\hline EPN1 & -0.367415216 & $2.90 \mathrm{E}-14$ & 1.140407858 & 0.034801 \\
\hline FAM134B & -0.446289424 & 0.009389 & -1.884835279 & 0.00021 \\
\hline FAM58A & -0.428148244 & 0.013924 & 1.589540039 & 0.008686 \\
\hline FAM96B & -0.258604662 & 0.036243 & 1.007933098 & 0.042718 \\
\hline FAM98C & -0.299884112 & 0.038198 & -5 & 0.00225 \\
\hline FGF12 & 0.915237306 & 0.016281 & -1.357459243 & 0.041842 \\
\hline FMOD & & & & \\
\hline FXYD1 & -0.482324859 & 0.014267 & 1.416270491 & 0.002163 \\
\hline GADD45B & -0.497838091 & 0.034827 & 1.914790207 & 0.00068 \\
\hline GLUL & -0.567151422 & 0.010959 & 1.664537577 & $5.94 \mathrm{E}-08$ \\
\hline GPIHBP1 & 0.716162309 & 0.032929 & 1.997394735 & 0.000878 \\
\hline GPNMB & 0.834656999 & 0 & -1.545983281 & 0.000353 \\
\hline GPR153 & -0.681905951 & 0.043028 & 3.330351255 & $1.19 \mathrm{E}-10$ \\
\hline HAGH & -0.269484135 & 0.033144 & 1.028491735 & 0.03694 \\
\hline HCFC1R1 & -0.269876232 & 0.028189 & 2.009929055 & $7.11 \mathrm{E}-08$ \\
\hline HDAC5 & -0.290056255 & 0.000133 & 0.998631607 & 0.036506 \\
\hline HFE2 & -0.416585779 & 0.021178 & -5 & $9.12 \mathrm{E}-07$ \\
\hline
\end{tabular}




\begin{tabular}{|c|c|c|c|c|}
\hline HIF3A & -0.36726417 & 0.031215 & 2.0184829 & $6.70 \mathrm{E}-10$ \\
\hline HIST1H2BD & -0.444623519 & 0.022086 & -1.796075408 & 0.0122 \\
\hline HIST3H2A & -0.390486066 & 0.040552 & -1.888931371 & 0.048405 \\
\hline HLA-B & 0.583581342 & 0.049228 & -1.295070572 & 0.016126 \\
\hline HSPB2 & -0.265488233 & 0.029863 & 1.552376496 & 0.031126 \\
\hline HSPBP1 & -0.321175159 & 0.02344 & 1.023892449 & 0.014184 \\
\hline HSPE1 & -0.626289358 & 0.017401 & -1.424482306 & 0.044167 \\
\hline IER5 & -0.5107848 & 0.011172 & 1.61853436 & 0.023403 \\
\hline IFI6 & -0.347885446 & 0.032703 & 1.410291788 & 0.002942 \\
\hline IFT43 & -0.501012542 & 0.009442 & 1.37413865 & 0.020028 \\
\hline IL6ST & 0.33077625 & 0.049957 & -5 & $1.49 \mathrm{E}-05$ \\
\hline INMT & 0.824405867 & 0.001049 & -1.18429467 & 0.014254 \\
\hline IRX3 & -0.407781337 & 0.043472 & 1.929352226 & $2.35 \mathrm{E}-05$ \\
\hline ISCU & -0.134040 & 0.028242 & 1.3376 & \\
\hline ITGB1 & 0.292811102 & 0.042933 & -0.980421554 & 0.045357 \\
\hline ITM2A & 0.703262638 & $4.45 \mathrm{E}-05$ & -1.478001876 & 0.031951 \\
\hline ITPKB & 0.68388826 & 0.022309 & -1.228955592 & 0.023999 \\
\hline JOSD2 & -0.384763927 & 0.002388 & 2.475679235 & 0.001562 \\
\hline JUP & -0.351730759 & $2.00 \mathrm{E}-15$ & -0.947178548 & 0.021847 \\
\hline KAT2B & 0.27694211 & 0.045965 & -1.988769812 & 0.003654 \\
\hline KCND3 & 0.403416768 & 0.04292 & -5 & $1.18 \mathrm{E}-05$ \\
\hline KIAA146 & 0.656200891 & 0.044113 & -1.6 & 0.019311 \\
\hline KIF1B & 0.307621803 & 0.028657 & -1.420263708 & 0.020581 \\
\hline KLC2 & -0.277130347 & 0.047432 & 1.010816551 & 0.028992 \\
\hline KLF15 & -0.700234622 & 0.017053 & 2.244450656 & $6.56 \mathrm{E}-06$ \\
\hline KLHL21 & -0.309586543 & 0.03786 & -1.209988358 & 0.023398 \\
\hline KLHL31 & 0.331983204 & 0.041734 & -1.879304514 & 0.001189 \\
\hline LDB3 & -0.193086896 & 0.037827 & 0.661937621 & 0.036828 \\
\hline LDHA & -0.518131046 & 0.016036 & -1.193485774 & 0.036046 \\
\hline LRRC10 & -0.477986329 & 0.017955 & -2.556754092 & 9.14E-05 \\
\hline MAFK & & & & \\
\hline MASP1 & -0.627801182 & 9.84E-05 & -1.236931196 & 0.011745 \\
\hline METRN & -0.308008848 & 0.015824 & 1.393969653 & 0.016556 \\
\hline MFAP4 & -0.36430884 & 0.013485 & 1.412504009 & 0.002207 \\
\hline MGLL & 0.557831017 & 0.029221 & 1.082085931 & 0.021445 \\
\hline MKNK2 & -0.56656081 & 0.036684 & -0.940147819 & 0.03771 \\
\hline MLYCD & -0.398413389 & 0.025974 & 1.255474711 & 0.025587 \\
\hline MRPL55 & -0.348486272 & 0.015397 & 1.25082383 & 0.000798 \\
\hline MTMR14 & -0.314430647 & 0.014979 & 0.984248097 & 0.015083 \\
\hline MXI1 & -0.44513223 & $5.68 \mathrm{E}-05$ & -1.014610918 & 0.034023 \\
\hline MXRA7 & -0.533026994 & 0.009181 & -1.012060794 & 0.014742 \\
\hline
\end{tabular}




\begin{tabular}{|c|c|c|c|c|}
\hline MYL4 & 1.123474745 & $1.01 \mathrm{E}-06$ & 7.928132761 & 0 \\
\hline MYLK3 & -0.294168859 & 0.037545 & -1.349636972 & 0.037659 \\
\hline NCEH1 & 0.470319732 & 0.040649 & -1.070742336 & 0.035032 \\
\hline NCOA4 & 0.20875989 & $2.30 \mathrm{E}-08$ & -1.608733137 & 0.004488 \\
\hline NDRG2 & -0.146954763 & 0.035152 & 0.796941477 & 0.019726 \\
\hline NENF & -0.312674229 & 0.023928 & 1.113785717 & 0.041455 \\
\hline NOL3 & -0.283878573 & 0.036899 & 1.412768463 & 0.000199 \\
\hline NRTN & -0.748451624 & 0.001353 & 2.237726854 & 0.013565 \\
\hline NT5C1A & 0.656828147 & 0.046935 & -2.854945342 & 0.0023 \\
\hline OPA1 & 0.179786546 & 0.041586 & -5 & $4.03 \mathrm{E}-09$ \\
\hline ORMDL3 & -0.28424199 & 0.020082 & 1.083147377 & 0.040718 \\
\hline OTUD1 & -0.364965273 & 0.009595 & 1.656789936 & 0.002426 \\
\hline PACS1 & -0.267153098 & 0.01 & 1.081585456 & 0.020732 \\
\hline PDK4 & -1.190849404 & 0.023484 & -2.495837398 & $3.53 \mathrm{E}-08$ \\
\hline PEBP4 & -0.444188822 & $8.73 \mathrm{E}-09$ & 1.357320348 & 0.002526 \\
\hline PHLDA1 & $\begin{array}{l}-0.967904287 \\
\end{array}$ & 0.013169 & -2.900790044 & $9.89 \mathrm{E}-09$ \\
\hline PIK3IP1 & -0.515566884 & 0.034509 & 1.628750929 & 0.000141 \\
\hline PLA2G16 & -0.429325781 & $8.99 \mathrm{E}-15$ & 1.062296467 & 0.006247 \\
\hline PLEKHA4 & -0.4970689 & 0.020773 & 1.715005619 & 0.002446 \\
\hline PLEKHO1 & -0.595716203 & $6.00 \mathrm{E}-15$ & 1.581454714 & 0.030276 \\
\hline PLIN4 & -0.342258388 & 0.037297 & 1.770077196 & 0.005091 \\
\hline PLP2 & -0.41520749 & 9.72E-06 & 2.438307418 & 0.000171 \\
\hline PPAP2B & 0.270841062 & 0.049882 & -1.148180528 & 0.012479 \\
\hline PSME1 & -0.187065181 & 0.044005 & -1.220196145 & 0.026897 \\
\hline PTGR2 & -0.240516468 & 0.042626 & -1.384657793 & 0.018137 \\
\hline PTMS & -0.204572767 & 0.018308 & 2.640986855 & $3.74 \mathrm{E}-05$ \\
\hline PTPRB & 0.897075719 & $1.29 \mathrm{E}-08$ & -1.293901464 & 0.028365 \\
\hline QSOX1 & -0.382599933 & 0.018983 & 1.402760158 & 0.000116 \\
\hline RAB40B & -0.497432018 & $1.33 \mathrm{E}-11$ & 1.260992128 & 0.03673 \\
\hline RABAC1 & -0.344457803 & 0.013844 & 1.466716007 & 0.048405 \\
\hline RAPGEF2 & 0.348172878 & 0.046195 & -1.486548606 & 0.029188 \\
\hline RASL10B & & 0.017747 & 1.34844708 & 0.010022 \\
\hline RBM3 & 0.274338467 & $7.99 \mathrm{E}-15$ & -1.142839467 & 0.013191 \\
\hline RHOC & -0.279578803 & 0 & 1.024149951 & 0.003138 \\
\hline RPL13 & -0.259754186 & 0.03225 & 0.96882753 & 0.038432 \\
\hline RPL28 & -0.215383175 & 0.033283 & 1.619734681 & 0.00747 \\
\hline RPL34 & -0.19977586 & 0.032458 & -1.135965587 & 0.045212 \\
\hline RPPH1 & -0.443235403 & 0.046663 & -1.490980417 & 0.007622 \\
\hline RPS27A & -0.320176756 & 0.019056 & 1.226988387 & 0.024042 \\
\hline RRP12 & -0.500648788 & 0.026194 & -1.312136309 & 0.00381 \\
\hline S1PR3 & 0.77952287 & $1.29 \mathrm{E}-08$ & 3.072552131 & $9.00 \mathrm{E}-10$ \\
\hline
\end{tabular}




\begin{tabular}{|l|l|l|l|l|} 
SGCA & -0.452258165 & $7.99 \mathrm{E}-15$ & 0.975357587 & 0.00212 \\
\hline SGCG & -0.220288165 & 0.047528 & 1.390817319 & 0.00745 \\
\hline SH3BGR & -1.038419793 & 0.041381 & -1.456364189 & 0.004471 \\
\hline SHISA3 & 0.929801987 & 0.049266 & 2.945548655 & $2.73 \mathrm{E}-05$ \\
\hline SHISA4 & -0.190293722 & 0.034849 & 1.167445824 & 0.012045 \\
\hline SLC16A1 & 0.342603538 & 0.047959 & -1.5319772 & 0.015032 \\
\hline SLC25A29 & -0.369212061 & 0.032238 & 1.485004892 & 0.035932 \\
\hline SLC27A6 & -0.725885867 & 0.012021 & -2.371207698 & $5.90 \mathrm{E}-05$ \\
\hline SLCO2A1 & -0.40718502 & 0.038314 & 3.644338952 & $4.74 \mathrm{E}-08$ \\
\hline SNAP47 & -0.449792851 & 0.036703 & -1.291372522 & 0.025832 \\
\hline SNHG8 & -0.344159108 & 0.042777 & 1.921250812 & 0.000585 \\
\hline SPARC & 0.610476051 & 0.006824 & 2.314285316 & 0.00014 \\
\hline SPR & -0.578686275 & 0.008157 & 1.53937965 & 0.003249 \\
\hline ST6GALNAC4 & -0.440998042 & 0.015328 & 1.038108792 & 0.027679 \\
\hline TCEAL3 & -0.354979559 & 0.017945 & 1.259083776 & 0.040293 \\
\hline TCEB2 & -0.262708567 & 0.038371 & 1.00029373 & 0.029824 \\
\hline TEAD1 & 0.289096382 & 0.029968 & -1.8094642 & 0.003053 \\
\hline TGM2 & -0.492854217 & 0.012428 & 2.399523907 & 0.001434 \\
\hline TIMM9 & -0.248657524 & 0.033989 & -5 & 0.006 \\
\hline TIMP3 & 0.458961699 & 0.040691 & 2.182312486 & $4.85 \mathrm{E}-06$ \\
\hline TM4SF1 & 0.367067279 & 0.04299 & -1.212879539 & 0.037013 \\
\hline TMEM140 & -0.89371551 & $1.20 \mathrm{E}-14$ & 1.440124571 & 0.005012 \\
\hline TNK2 & -0.315040377 & 0.036253 & 1.394373146 & 0.042979 \\
\hline TNN11 & 0.738987233 & 0.049448 & 9.276823467 & 0 \\
\hline TRNP1 & -0.498430701 & $6.00 \mathrm{E}-15$ & 1.369548495 & 0.014466 \\
\hline TSPYL2 & -0.85969565 & 0.0075 & 2.390733186 & $2.13 \mathrm{E}-05$ \\
\hline TUBB6 & 0.430747751 & 0.023596 & 1.642746812 & 0.030168 \\
\hline TXNRD1 & -0.490654157 & 0.036189 & -0.917006565 & 0.005121 \\
\hline UTRN & 0.391412756 & 0.039902 & -1.589898147 & 0.013508 \\
\hline VWF & 0.704070796 & 0.019926 & -1.135546205 & 0.027555 \\
\hline WDR62 & 1.072490247 & $2.15 \mathrm{E}-12$ & -1.109653992 & 0.011913 \\
\hline WISP2 & -0.614891518 & 0.032136 & 2.328288849 & 0.002022 \\
\hline XIRP1 & -0.317827132 & 0.036235 & 0.949858802 & 0.011572 \\
\hline XIRP2 & 0.508831734 & 0.026453 & -1.017910018 & 0.001751 \\
\hline XPR1 & -0.404531859 & 0.040079 & -1.53208742 & 0.000285 \\
\hline YIF1B & -0.263588099 & 0.043766 & 1.10697907 & 0.029123 \\
\hline YPEL3 & -0.784698444 & $4.00 \mathrm{E}-15$ & 1.326294057 & 0.037659 \\
\hline & & & & \\
\hline & & & \\
\hline
\end{tabular}


Supplemental Table 18. DEG shared between male obesity-related LVH and both dilated ischemia and cardiomyopathy datasets with $\left(p_{-}\right.$adj $\left.<0.05\right)$

\begin{tabular}{|c|c|c|c|c|}
\hline Gene & \begin{tabular}{|l}
$\log 2 \mathrm{FC}$ \\
LVH_M
\end{tabular} & p a adj & $\begin{array}{l}\log 2 \mathrm{FC} \text { ISCH vs } \\
\text { DCM }\end{array}$ & $p$ adj \\
\hline ABAT & -0.436324244 & 0.035648 & 1.402124026 & 0.014941 \\
\hline ABCA8 & 0.648874255 & 0.042578 & -1.84507599 & 0.000274 \\
\hline ACOT11 & -0.361296457 & 0.012581 & 1.184750634 & 0.014629 \\
\hline ADAM9 & 0.324658867 & 0.038311 & 1.186817111 & 0.031098 \\
\hline APLN & 0.42609975 & 0.027729 & -1.612910202 & 0.002372 \\
\hline AQP7 & -0.339087891 & 0.040948 & -1.44003696 & 0.001261 \\
\hline ATP13A3 & -0.303941208 & 0.047239 & 1.838355362 & 0.000121 \\
\hline AZGP1 & -1.307980325 & 0 & 1.813024958 & 0.000192 \\
\hline C1QTNF1 & -0.578503513 & 0.038739 & 1.983690511 & 0.000181 \\
\hline C5orf46 & -0.638118011 & 0.025592 & 2.279946307 & 0.048626 \\
\hline CAMK2B & -0.371924966 & 0.002571 & -0.758761378 & 0.020855 \\
\hline CAST & -0.150895471 & 0.043171 & -0.923186229 & 0.005729 \\
\hline CCL2 & 0.671466177 & $9.02 \mathrm{E}-06$ & 5.109077051 & 0.012536 \\
\hline CD151 & -0.510771364 & $4.00 \mathrm{E}-15$ & 1.258355253 & 0.00377 \\
\hline CD300LG & 0.770142832 & 0.020405 & -1.275290819 & 0.007124 \\
\hline CHPF & -0.333975406 & 0.005284 & 1.502751997 & 0.018214 \\
\hline COL15A1 & 0.861245935 & 0.024047 & 1.680275526 & \begin{tabular}{|l|l|}
0.000418 \\
\end{tabular} \\
\hline COL23A1 & -0.759281134 & 0.023043 & 2.384872695 & $2.68 \mathrm{E}-05$ \\
\hline COMMD6 & -0.280205666 & 0.028834 & 2.877074001 & $3.04 \mathrm{E}-06$ \\
\hline COMP & -0.849294671 & 0.039889 & 3.672769871 & $1.37 \mathrm{E}-06$ \\
\hline CTNNA3 & 0.420902413 & 0.047603 & -1.665848899 & 0.004357 \\
\hline DKK3 & -0.746405642 & 0.011774 & -1.17219224 & 0.001464 \\
\hline ELN & 0.558139214 & 0.001639 & 2.405718414 & $2.62 \mathrm{E}-07$ \\
\hline FAM134B & -0.446289424 & 0.009389 & -1.226478006 & 0.004017 \\
\hline GADD45B & -0.497838091 & 0.034827 & 1.423620172 & \begin{tabular}{|l|}
0.03675 \\
\end{tabular} \\
\hline GPR153 & -0.681905951 & 0.043028 & 2.838701409 & 5.12E-07 \\
\hline GSTT1 & -1.140967802 & 0.033376 & -1.408853917 & 0.010118 \\
\hline HEPH & -0.385669396 & 0.016989 & 2.343603804 & 5.93E-07 \\
\hline HIPK3 & 0.347338792 & 0.026941 & -1.575941651 & $6.26 \mathrm{E}-05$ \\
\hline HIST3H2A & -0.390486066 & 0.040552 & -1.597503693 & 0.044378 \\
\hline IGFBP2 & -0.862259937 & 0 & 2.645422346 & $4.96 \mathrm{E}-07$ \\
\hline IL6ST & 0.33077625 & 0.049957 & 1.207790986 & 0.000608 \\
\hline ILVBL & -0.267348022 & 0.004582 & -1.210007211 & 0.012376 \\
\hline IRX3 & -0.407781337 & 0.043472 & -1.18400673 & 0.014833 \\
\hline ITGA6 & 0.49961087 & 0.032435 & 1.585582568 & 0.000365 \\
\hline ITGB1 & 0.292811102 & 0.042933 & 1.208608991 & 0.000586 \\
\hline
\end{tabular}




\begin{tabular}{|c|c|c|c|c|}
\hline JOSD2 & -0.384763927 & 0.002388 & 1.795954224 & 0.018766 \\
\hline KCND3 & 0.403416768 & 0.04292 & -1.876821251 & 0.001093 \\
\hline KCTD17 & -0.563715606 & $3.80 \mathrm{E}-05$ & 2.047177058 & 0.002859 \\
\hline KLF15 & -0.700234622 & 0.017053 & 1.466101481 & 0.017679 \\
\hline LDHA & -0.518131046 & 0.016036 & 1.163646348 & 0.034742 \\
\hline LRRC14B & -0.582658557 & $6.69 \mathrm{E}-05$ & -2.25222654 & $2.04 \mathrm{E}-06$ \\
\hline MAFK & -0.41121213 & 0.017121 & -1.418199767 & 0.005203 \\
\hline MLYCD & -0.398413389 & 0.025974 & -1.107128992 & 0.038156 \\
\hline MRPL55 & -0.348486272 & 0.015397 & -1.182167424 & 0.001452 \\
\hline MT1X & -0.820480928 & 0.041477 & 1.515877998 & 0.048194 \\
\hline MTSS1 & 0.405885732 & 0.039649 & -1.550740075 & 0.00301 \\
\hline MYL4 & 1.123474745 & $1.01 \mathrm{E}-06$ & 2.866610047 & $1.24 \mathrm{E}-05$ \\
\hline NOL3 & -0.283878573 & 0.036899 & -0.792023062 & 0.048467 \\
\hline NPPA & -4.597666596 & 0.018249 & 1.804016681 & 0.002728 \\
\hline NPPB & -2.898570787 & 0.013588 & 3.95062945 & $1.28 \mathrm{E}-12$ \\
\hline PFKFB2 & -0.500907657 & 0.03972 & -1.879265511 & $2.54 \mathrm{E}-08$ \\
\hline PHLDA1 & -0.967904287 & 0.013169 & -1.366898072 & 0.013516 \\
\hline PKIA & 0.190572744 & 0.040133 & 0.958191615 & 0.03428 \\
\hline PLEKHA4 & -0.4970689 & 0.020773 & 1.693949792 & 0.000295 \\
\hline PLK2 & -0.741565424 & 0.022569 & -2.454955955 & $5.88 \mathrm{E}-07$ \\
\hline PLP2 & -0.41520749 & $9.72 \mathrm{E}-06$ & 1.488051412 & 0.015975 \\
\hline PLVAP & -1.298964363 & $3.00 \mathrm{E}-15$ & 1.949632993 & 0.001075 \\
\hline PNPLA2 & -0.47250156 & $1.80 \mathrm{E}-14$ & -1.203871191 & 0.03428 \\
\hline POSTN & -0.716023612 & 0.043196 & 4.131445065 & 0 \\
\hline PROS1 & -0.634473414 & 0.022481 & 1.897009877 & $9.67 \mathrm{E}-05$ \\
\hline QSOX1 & -0.382599933 & 0.018983 & 1.356787899 & 0.000798 \\
\hline RAPGEF2 & 0.348172878 & 0.046195 & -1.258547528 & 0.029287 \\
\hline RBM24 & -0.241858165 & 0.043341 & -1.241857132 & 0.035328 \\
\hline RRAS2 & -0.417699346 & 0.008991 & 5 & $2.41 \mathrm{E}-05$ \\
\hline S1PR3 & 0.77952287 & $1.29 \mathrm{E}-08$ & 2.144826087 & 0.000239 \\
\hline SEC14L5 & 1.203995176 & 0.025826 & -2.791126985 & 9.97E-09 \\
\hline SLCO2A1 & -0.40718502 & 0.038314 & 1.954725266 & 0.004097 \\
\hline SPARC & 0.610476051 & 0.006824 & 2.112232792 & 0.000418 \\
\hline SVOP & -1.064191722 & 0.034337 & 3.167780548 & 4.70E-05 \\
\hline SYNE1 & 0.420361736 & 0.039974 & -1.017437725 & 0.025506 \\
\hline TBX20 & 0.935812719 & 0.0246 & -2.297082344 & $1.42 \mathrm{E}-06$ \\
\hline TFRC & 1.359094592 & 0.041854 & 1.549593619 & 0.001462 \\
\hline THBS1 & -0.525021016 & 0.028597 & -1.958430312 & $6.76 \mathrm{E}-05$ \\
\hline TIMM9 & -0.248657524 & 0.033989 & 5 & $1.32 \mathrm{E}-05$ \\
\hline TNNI1 & 0.738987233 & 0.049448 & 5.839519429 & 0 \\
\hline UGT2B4 & 0.967809667 & 0.036872 & 5 & $6.76 \mathrm{E}-05$ \\
\hline
\end{tabular}




\begin{tabular}{|l|l|l|l|l|} 
WDR18 & -0.426309848 & 0.035078 & -1.180729841 & 0.024547 \\
\hline WISP2 & -0.614891518 & 0.032136 & 1.907856911 & 0.005317 \\
\hline XIRP2 & 0.508831734 & 0.026453 & 1.365025666 & 0.000972 \\
\hline
\end{tabular}


Supplemental Table 19. Gene ontology results for "biological processes" from Ingenuity

Pathway Analysis

\begin{tabular}{|c|c|c|c|c|c|c|}
\hline Term & Overlap & P-value & $\begin{array}{l}\text { Adjusted P- } \\
\text { value }\end{array}$ & Z-score & $\begin{array}{l}\text { Combined } \\
\text { Score }\end{array}$ & Genes \\
\hline $\begin{array}{ll}\text { receptor guanylyl cyclase } \\
\text { signaling } & \text { pathway } \\
\text { (GO:0007168) } & \\
\end{array}$ & $2 / 11$ & $9.9 \mathrm{E}-06$ & 0.000403093 & 2.73352782 & 21.36619446 & $\begin{array}{l}\text { NPPB; } \\
\text { NPPA }\end{array}$ \\
\hline $\begin{array}{ll}\text { oxygen } & \text { transport } \\
(\mathrm{GO}: 0015671) & \end{array}$ & $2 / 16$ & $2.2 \mathrm{E}-05$ & 0.000531633 & - 2.64329808 & 19.92929626 & $\begin{array}{l}\text { HBB; } \\
\text { HBA1 }\end{array}$ \\
\hline $\begin{array}{l}\text { cGMP biosynthetic } \\
\text { process (GO:0006182) }\end{array}$ & $2 / 16$ & $2.2 \mathrm{E}-05$ & 0.000531633 & $\overline{-}-62154671$ & 19.76530058 & $\begin{array}{l}\text { NPPB; } \\
\text { NPPA }\end{array}$ \\
\hline $\begin{array}{lr}\begin{array}{l}\text { hydrogen } \\
\text { catabolic }\end{array} & \text { peroxide } \\
\text { (GO:0042744) } & \text { process } \\
\end{array}$ & $2 / 20$ & 3.4E-05 & 0.000631627 & $-\overline{2.61014597}$ & 19.22949714 & $\begin{array}{l}\text { HBB; } \\
\text { HBA1 }\end{array}$ \\
\hline $\begin{array}{ll}\text { gas } & \text { transport } \\
\text { (GO:0015669) } & \\
\end{array}$ & $2 / 20$ & 3.4E-05 & 0.000631627 & - & 18.81172467 & $\begin{array}{l}\text { HBB; } \\
\text { HBA1 }\end{array}$ \\
\hline $\begin{array}{l}\text { regulation of blood vessel } \\
\text { size (GO:0050880) }\end{array}$ & $3 / 63$ & $2.5 \mathrm{E}-06$ & 0.000264119 & $\overline{-} .26273855$ & 18.64295124 & $\begin{array}{l}\text { NPPB; } \\
\text { NPPA; } \\
\text { HBB }\end{array}$ \\
\hline $\begin{array}{l}\text { regulation of tube size } \\
\text { (GO:0035150) }\end{array}$ & $3 / 64$ & $2.6 \mathrm{E}-06$ & 0.000264119 & 2.24830864 & 18.52406162 & $\begin{array}{l}\text { NPPB; } \\
\text { NPPA; } \\
\text { HBB }\end{array}$ \\
\hline $\begin{array}{lr}\text { vascular process } & \text { in } \\
\text { circulatory } & \text { system } \\
(\text { GO:0003018) } & \\
\end{array}$ & $3 / 86$ & $6.3 \mathrm{E}-06$ & 0.000322775 & $\begin{array}{l}- \\
2.29516194\end{array}$ & 18.44978297 & $\begin{array}{l}\text { NPPB; } \\
\text { NPPA; } \\
\text { HBB }\end{array}$ \\
\hline $\begin{array}{ll}\text { reactive oxygen } & \text { species } \\
\text { metabolic } & \text { process } \\
(\text { GO:0072593) } & \\
\end{array}$ & $3 / 84$ & $5.9 \mathrm{E}-06$ & 0.000322775 & $\begin{array}{l}- \\
2.24768357\end{array}$ & 18.06812556 & $\begin{array}{l}\text { PDK4; } \\
\text { HBB; } \\
\text { HBA1 }\end{array}$ \\
\hline $\begin{array}{l}\text { cGMP metabolic process } \\
\text { (GO:0046068) }\end{array}$ & $2 / 24$ & $4.9 \mathrm{E}-05$ & 0.000840276 & $\overline{2}-45121922$ & 17.35899443 & $\begin{array}{l}\text { NPPB; } \\
\text { NPPA }\end{array}$ \\
\hline $\begin{array}{l}\text { circulatory } \quad \text { system } \\
\text { process (GO:0003013) }\end{array}$ & $3 / 130$ & $2.2 \mathrm{E}-05$ & 0.000531633 & -2.2966276 & 17.31555443 & $\begin{array}{l}\text { NPPB; } \\
\text { NPPA; } \\
\text { HBB }\end{array}$ \\
\hline $\begin{array}{l}\text { regulation of blood } \\
\text { pressure (GO:0008217) }\end{array}$ & $3 / 133$ & 2.3E-05 & 0.000531633 & -2.2953209 & 17.30570248 & $\begin{array}{l}\text { NPPB; } \\
\text { NPPA; } \\
\text { HBB }\end{array}$ \\
\hline $\begin{array}{lr}\text { cyclic purine } & \text { nucleotide } \\
\text { metabolic } & \text { process } \\
\text { (GO:0052652) } & \\
\end{array}$ & $2 / 33$ & 9.4E-05 & 0.001277978 & $\begin{array}{l}- \\
2.52856174\end{array}$ & 16.84648204 & $\begin{array}{l}\text { NPPB; } \\
\text { NPPA }\end{array}$ \\
\hline $\begin{array}{lr}\text { cyclic } & \text { nucleotide } \\
\text { biosynthetic } & \text { process } \\
(\text { GO:0009190) } & \end{array}$ & $2 / 34$ & 0.0001 & 0.001277978 & $\overline{-}-52746357$ & 16.83916551 & $\begin{array}{l}\text { NPPB; } \\
\text { NPPA }\end{array}$ \\
\hline $\begin{array}{lr}\text { hydrogen } & \text { peroxide } \\
\text { metabolic } & \text { process } \\
(\mathrm{GO}: 0042743) & \end{array}$ & $2 / 33$ & $9.4 \mathrm{E}-05$ & 0.001277978 & 2.45103358 & 16.3299525 & $\begin{array}{l}\text { HBB; } \\
\text { HBA1 }\end{array}$ \\
\hline $\begin{array}{l}\text { regulation of anatomical } \\
\text { structure } \\
\text { (GO:0090066) }\end{array}$ & $3 / 232$ & 0.00012 & 0.001475084 & $-\overline{2.41140828}$ & 15.72006712 & $\begin{array}{l}\text { NPPB; } \\
\text { NPPA; } \\
\text { HBB }\end{array}$ \\
\hline $\begin{array}{ll}\text { bicarbonate } & \text { transport } \\
(\text { GO:0015701) } & \end{array}$ & $2 / 31$ & $8.3 \mathrm{E}-05$ & 0.001277978 & $\overline{-}-32083616$ & 15.46251535 & $\begin{array}{l}\text { HBB; } \\
\text { HBA1 }\end{array}$ \\
\hline $\begin{array}{l}\text { organophosphate } \\
\text { biosynthetic process }\end{array}$ & $3 / 436$ & 0.00078 & 0.006685052 & - 2.38238235 & 11.93068786 & $\begin{array}{l}\text { NPPB; } \\
\text { PLA2G2A; }\end{array}$ \\
\hline
\end{tabular}




\begin{tabular}{|c|c|c|c|c|c|c|}
\hline (GO:0090407) & & & & & & NPPA \\
\hline $\begin{array}{lrr}\text { cellular } & \text { response } & \text { to } \\
\text { hydrogen } & \text { peroxide } \\
(\mathrm{GO}: 0070301) & \end{array}$ & $2 / 62$ & 0.00034 & 0.003603955 & $\overline{-} .09511044$ & 11.78651171 & $\begin{array}{l}\text { HBB; } \\
\text { HBA1 }\end{array}$ \\
\hline $\begin{array}{lr}\text { cyclic } & \text { nucleotide } \\
\text { metabolic } & \text { process } \\
(\text { GO:0009187) } & \end{array}$ & $2 / 58$ & 0.00029 & 0.003328491 & $\overline{-} .05442688$ & 11.72099042 & $\begin{array}{l}\text { NPPB; } \\
\text { NPPA }\end{array}$ \\
\hline $\begin{array}{l}\text { renal system process } \\
(\text { GO:0003014) }\end{array}$ & $2 / 71$ & 0.00044 & 0.004489781 & 2.16591455 & 11.70882865 & $\begin{array}{l}\text { NPPB; } \\
\text { HBB }\end{array}$ \\
\hline $\begin{array}{l}\text { protein } \\
\text { heterooligomerization } \\
\text { (GO:0051291) }\end{array}$ & $2 / 81$ & 0.00057 & 0.005562119 & $-\overline{2.18198987}$ & 11.32840277 & $\begin{array}{l}\text { HBB; } \\
\text { HBA1 }\end{array}$ \\
\hline $\begin{array}{l}\text { cellular response to } \\
\text { reactive oxygen species } \\
\text { (GO:0034614) }\end{array}$ & $2 / 89$ & 0.00069 & 0.00640506 & $-\overline{2.17389801}$ & 10.97963498 & $\begin{array}{l}\text { HBB; } \\
\text { HBA1 }\end{array}$ \\
\hline $\begin{array}{l}\text { response to hydrogen } \\
\text { peroxide (GO:0042542) }\end{array}$ & $2 / 95$ & 0.00079 & 0.006685052 & - 2.18694825 & 10.95197708 & $\begin{array}{l}\text { HBB; } \\
\text { HBA1 }\end{array}$ \\
\hline $\begin{array}{lr}\text { purine ribonucleotide } \\
\text { biosynthetic } & \text { process } \\
(\text { GO:0009152) } & \end{array}$ & $2 / 126$ & 0.00138 & 0.011237257 & $\overline{-} .18473896$ & 9.806245641 & $\begin{array}{l}\text { NPPB; } \\
\text { NPPA }\end{array}$ \\
\hline $\begin{array}{lr}\text { purine } & \text { nucleotide } \\
\text { biosynthetic } & \text { process } \\
(\text { GO:0006164) } & \\
\end{array}$ & $2 / 132$ & 0.00151 & 0.011846304 & $-\overline{2.20857659}$ & 9.796670106 & $\begin{array}{l}\text { NPPB; } \\
\text { NPPA }\end{array}$ \\
\hline $\begin{array}{lr}\text { response to } & \text { reactive } \\
\text { oxygen } & \text { species } \\
\text { (GO:0000302) } & \end{array}$ & $2 / 141$ & 0.00172 & 0.01238831 & $\overline{-} .22873446$ & 9.786377434 & $\begin{array}{l}\text { HBB; } \\
\text { HBA1 }\end{array}$ \\
\hline $\begin{array}{lr}\text { cellular response } & \text { to } \\
\text { oxidative } & \text { stress } \\
(\text { GO:0034599) } & \end{array}$ & $2 / 148$ & 0.00189 & 0.01238831 & $\overline{-} .22195018$ & 9.756587623 & $\begin{array}{l}\text { HBB; } \\
\text { HBA1 }\end{array}$ \\
\hline $\begin{array}{l}\text { purine-containing } \\
\text { compound biosynthetic } \\
\text { process (GO:0072522) }\end{array}$ & $2 / 146$ & 0.00184 & 0.01238831 & $-\overline{2} .20400803$ & 9.677803611 & $\begin{array}{l}\text { NPPB; } \\
\text { NPPA }\end{array}$ \\
\hline $\begin{array}{lr}\text { ribose } & \text { phosphate } \\
\text { biosynthetic } & \text { process } \\
(G O: 0046390) & \end{array}$ & $2 / 144$ & 0.00179 & 0.01238831 & $\begin{array}{l}- \\
2.19585593\end{array}$ & 9.642007714 & $\begin{array}{l}\text { NPPB; } \\
\text { NPPA }\end{array}$ \\
\hline $\begin{array}{l}\text { ribonucleotide } \\
\text { biosynthetic } \\
(\mathrm{GO} 0009260)\end{array}$ & $2 / 140$ & 0.0017 & 0.01238831 & $\overline{-} .19114785$ & 9.621334546 & $\begin{array}{l}\text { NPPB; } \\
\text { NPPA }\end{array}$ \\
\hline $\begin{array}{l}\text { negative regulation of cell } \\
\text { growth (GO:0030308) }\end{array}$ & $2 / 150$ & 0.00194 & 0.01238831 & 2.17058903 & 9.531060743 & $\begin{array}{l}\text { NPPB; } \\
\text { NPPA }\end{array}$ \\
\hline $\begin{array}{lrr}\text { cellular } & \text { response } & \text { to } \\
\text { external } & \text { stimulus } \\
(G O: 0071496) & \end{array}$ & $2 / 185$ & 0.00294 & 0.018147129 & $\overline{-} .27974751$ & 9.140061513 & $\begin{array}{l}\text { NPPA; } \\
\text { PDK4 }\end{array}$ \\
\hline $\begin{array}{l}\text { nucleotide biosynthetic } \\
\text { process (GO:0009165) }\end{array}$ & $2 / 208$ & 0.00369 & 0.02112531 & - 2.30417853 & 8.887869651 & $\begin{array}{l}\text { NPPB; } \\
\text { NPPA }\end{array}$ \\
\hline $\begin{array}{lr}\text { nucleoside } & \text { phosphate } \\
\text { biosynthetic } & \text { process } \\
(\text { GO:1901293) } & \end{array}$ & $2 / 209$ & 0.00373 & 0.02112531 & -2.3023159 & 8.880684986 & $\begin{array}{l}\text { NPPB; } \\
\text { NPPA }\end{array}$ \\
\hline $\begin{array}{l}\text { positive regulation of } \\
\text { renal sodium excretion } \\
(\mathrm{GO}: 0035815)\end{array}$ & $1 / 14$ & 0.00628 & 0.02563726 & $\overline{-} .36912468$ & 8.679782303 & NPPB \\
\hline $\begin{array}{l}\text { cellular modified amino } \\
\text { acid metabolic process } \\
(\mathrm{GO}: 0006575)\end{array}$ & $2 / 199$ & 0.00339 & 0.020321255 & $\overline{-} .18054286$ & 8.495586698 & $\begin{array}{l}\text { PLA2G2A; } \\
\text { GSTT1 }\end{array}$ \\
\hline
\end{tabular}




\begin{tabular}{|c|c|c|c|c|c|c|}
\hline $\begin{array}{l}\text { regulation of fatty acid } \\
\text { oxidation (GO:0046320) }\end{array}$ & $1 / 24$ & 0.01075 & 0.029401443 & $\overline{2} .39620447$ & 8.450721933 & PDK4 \\
\hline $\begin{array}{l}\text { response to } \\
(\mathrm{GO}: 0032868)\end{array}$ & $2 / 246$ & 0.00512 & 0.025330625 & $\overline{2}-28482003$ & 8.398406997 & $\begin{array}{l}\text { NPPA; } \\
\text { PDK4 }\end{array}$ \\
\hline $\begin{array}{l}\text { negative regulation of } \\
\text { growth (GO:0045926) }\end{array}$ & $2 / 221$ & 0.00416 & 0.022924024 & -2.2225645 & 8.391447455 & $\begin{array}{l}\text { NPPB; } \\
\text { NPPA }\end{array}$ \\
\hline $\begin{array}{l}\text { positive regulation of } \\
\text { urine } \\
\text { (GO:0035810) }\end{array}$ & $1 / 13$ & 0.00584 & 0.025330625 & $-\overline{2.26932608}$ & 8.341455256 & NPPB \\
\hline $\begin{array}{l}\text { regulation of homeostatic } \\
\text { process (GO:0032844) }\end{array}$ & $2 / 314$ & 0.00822 & 0.027502741 & $\overline{2.30668843}$ & 8.289014758 & $\begin{array}{l}\text { NPPB; } \\
\text { PDK4 }\end{array}$ \\
\hline $\begin{array}{lrr}\begin{array}{l}\text { regulation of renal } \\
\text { sodium }\end{array} & \begin{array}{r}\text { rexcretion } \\
(\text { GO:0035813) }\end{array} \\
\end{array}$ & $1 / 22$ & 0.00986 & 0.029401443 & $\overline{2.34779171}$ & 8.279984113 & NPPB \\
\hline $\begin{array}{lr}\text { regulation of } & \text { sulfur } \\
\text { metabolic } & \text { process } \\
(\text { GO:0042762) } & \\
\end{array}$ & $1 / 17$ & 0.00763 & 0.026820941 & $-\overline{2.28302805}$ & 8.261302138 & PDK4 \\
\hline $\begin{array}{ll}\text { renal absorption } \\
(\mathrm{GO} 00070293)\end{array}$ & $1 / 13$ & 0.00584 & 0.025330625 & $\begin{array}{l} \\
2.24317347\end{array}$ & 8.245325008 & HBB \\
\hline $\begin{array}{l}\text { regulation of urine } \\
\text { volume (GO:0035809) }\end{array}$ & $1 / 18$ & 0.00807 & 0.027446534 & $-\overline{2.29288751}$ & 8.244112312 & NPPB \\
\hline $\begin{array}{lr}\text { regulation of } & \text { coenzyme } \\
\text { metabolic } & \text { process } \\
(\mathrm{GO} 0051196) & \end{array}$ & $1 / 14$ & 0.00628 & 0.02563726 & $\begin{array}{l}- \\
2.24966527\end{array}$ & 8.242117842 & PDK4 \\
\hline $\begin{array}{l}\text { regulation of vascular } \\
\text { permeability } \\
\text { (GO:0043114) }\end{array}$ & $1 / 27$ & 0.01209 & 0.029401443 & $-\overline{2.32303767}$ & 8.192683755 & NPPB \\
\hline $\begin{array}{l}\text { response to oxidative } \\
\text { stress (GO:0006979) }\end{array}$ & $2 / 290$ & 0.00705 & 0.026820941 & $-\overline{2.25935012}$ & 8.175621808 & $\begin{array}{l}\text { HBB; } \\
\text { HBA1 }\end{array}$ \\
\hline $\begin{array}{ll}\text { regulation of } & \text { cofactor } \\
\text { metabolic } & \text { process } \\
\text { (GO:0051193) } & \\
\end{array}$ & $1 / 14$ & 0.00628 & 0.02563726 & $-\overline{2.22720499}$ & 8.159829903 & PDK4 \\
\hline $\begin{array}{l}\text { phosphatidylglycerol } \\
\text { acyl-chain remodeling } \\
\text { (GO:0036148) }\end{array}$ & $1 / 17$ & 0.00763 & 0.026820941 & - 2.24878469 & 8.137390053 & PLA2G2A \\
\hline $\begin{array}{l}\text { response to inorganic } \\
\text { substance (GO:0010035) }\end{array}$ & $2 / 370$ & 0.01128 & 0.029401443 & $\begin{array}{l}-29825494 \\
\end{array}$ & 8.105282199 & $\begin{array}{l}\text { HBB; } \\
\text { HBA1 }\end{array}$ \\
\hline $\begin{array}{l}\text { regulation of excretion } \\
\text { (GO:0044062) }\end{array}$ & $1 / 25$ & 0.0112 & 0.029401443 & $-\overline{2.28068583}$ & 8.043321033 & NPPB \\
\hline $\begin{array}{l}\text { negative regulation of } \\
\text { anoikis (GO:2000811) }\end{array}$ & $1 / 16$ & 0.00718 & 0.026820941 & - 2.21378836 & 8.010753305 & PDK4 \\
\hline $\begin{array}{lr}\text { regulation of } & \text { acyl-CoA } \\
\text { biosynthetic } & \text { process } \\
\text { (GO:0050812) } & \\
\end{array}$ & $1 / 13$ & 0.00584 & 0.025330625 & $\begin{array}{l}- \\
2.17524596\end{array}$ & 7.995641076 & PDK4 \\
\hline $\begin{array}{l}\text { response to peptide } \\
\text { hormone (GO:0043434) }\end{array}$ & $2 / 364$ & 0.01093 & 0.029401443 & $-\overline{2.25871606}$ & 7.96583997 & $\begin{array}{l}\text { NPPA; } \\
\text { PDK4 }\end{array}$ \\
\hline $\begin{array}{l}\text { negative regulation of } \\
\text { systemic arterial blood } \\
\text { pressure (GO:0003085) }\end{array}$ & $1 / 13$ & 0.00584 & 0.025330625 & $-\overline{2.15854699}$ & 7.934259986 & NPPA \\
\hline $\begin{array}{l}\text { response to peptide } \\
(G O: 1901652)\end{array}$ & $2 / 384$ & 0.01211 & 0.029401443 & $-\overline{2.23645603}$ & 7.887335279 & $\begin{array}{l}\text { NPPA; } \\
\text { PDK4 }\end{array}$ \\
\hline $\begin{array}{l}\text { regulation of anoikis } \\
(\mathrm{GO} 2000209)\end{array}$ & $1 / 20$ & 0.00897 & 0.028139038 & $\begin{array}{l} \\
2.20456099\end{array}$ & 7.871599745 & PDK4 \\
\hline $\begin{array}{l}\text { regulation of acetyl-CoA } \\
\text { biosynthetic process from }\end{array}$ & $1 / 12$ & 0.00539 & 0.025330625 & -2.1368309 & 7.85443726 & PDK4 \\
\hline
\end{tabular}




\begin{tabular}{|c|c|c|c|c|c|c|}
\hline e (GO:0010510) & & & & & & \\
\hline $\begin{array}{l}\text { regulation of cell growth } \\
\text { (GO:0001558) }\end{array}$ & $2 / 322$ & 0.00863 & 0.028139038 & $-\overline{2.19686961}$ & 7.844136932 & $\begin{array}{l}\text { NPPB; } \\
\text { NPPA }\end{array}$ \\
\hline $\begin{array}{l}\text { positive regulation of } \\
\text { macrophage derived foam } \\
\text { cell differentiation } \\
(\mathrm{GO}: 0010744)\end{array}$ & $1 / 15$ & 0.00673 & 0.026820941 & $\begin{array}{l}- \\
2.16462201\end{array}$ & 7.832841304 & PLA2G2A \\
\hline $\begin{array}{l}\text { phosphatidylcholine acyl- } \\
\text { chain remodeling } \\
\text { (GO:0036151) }\end{array}$ & $1 / 26$ & 0.01164 & 0.029401443 & $\overline{2} .21865045$ & 7.824540131 & PLA2G2A \\
\hline $\begin{array}{l}\text { organic anion transport } \\
(\mathrm{GO} 0015711)\end{array}$ & $2 / 328$ & 0.00895 & 0.028139038 & $-\overline{2.18397702}$ & 7.798102678 & $\begin{array}{l}\text { HBB; } \\
\text { HBA1 }\end{array}$ \\
\hline $\begin{array}{l}\text { phosphatidylinositol acyl- } \\
\text { chain remodeling } \\
\text { (GO:0036149) }\end{array}$ & $1 / 16$ & 0.00718 & 0.026820941 & $\overline{2} .15382746$ & 7.793780434 & PLA2G2A \\
\hline $\begin{array}{l}\text { phosphatidylserine acyl- } \\
\text { chain remodeling } \\
(\text { GO:0036150) }\end{array}$ & $1 / 17$ & 0.00763 & 0.026820941 & $\overline{2} .14615106$ & 7.766002824 & PLA2G2A \\
\hline $\begin{array}{l}\text { phosphatidylethanolamine } \\
\text { acyl-chain remodeling } \\
\text { (GO:0036152) }\end{array}$ & $1 / 23$ & 0.0103 & 0.029401443 & -2.1875258 & 7.714772466 & PLA2G2A \\
\hline $\begin{array}{lr}\text { carbohydrate } & \text { derivative } \\
\text { biosynthetic } & \text { process } \\
\text { (GO:1901137) } & \\
\end{array}$ & $2 / 377$ & 0.01169 & 0.029401443 & - & 7.619736157 & $\begin{array}{l}\text { NPPB; } \\
\text { NPPA }\end{array}$ \\
\hline $\begin{array}{l}\text { cellular response to fatty } \\
\text { acid (GO:0071398) }\end{array}$ & $1 / 27$ & 0.01209 & 0.029401443 & $\begin{array}{l} \\
2.15589578\end{array}$ & 7.603222498 & PDK4 \\
\hline $\begin{array}{l}\text { protein oligomerization } \\
\text { (GO:0051259) }\end{array}$ & $2 / 366$ & 0.01104 & 0.029401443 & -2.1468579 & 7.571348505 & $\begin{array}{l}\text { HBB; } \\
\text { HBA1 }\end{array}$ \\
\hline $\begin{array}{ll}\text { protein-lipid } & \text { complex } \\
\text { remodeling } & \\
\text { (GO:0034368) } & \\
\end{array}$ & $1 / 23$ & 0.0103 & 0.029401443 & $-\overline{2.14232935}$ & 7.55537763 & PLA2G2A \\
\hline $\begin{array}{l}\text { regulation of macrophage } \\
\text { derived foam cell } \\
\text { differentiation } \\
(\text { GO:0010743) }\end{array}$ & $1 / 28$ & 0.01253 & 0.029727456 & - & 7.541752258 & PLA2G2A \\
\hline $\begin{array}{l}\text { phosphatidylserine } \\
\text { metabolic process } \\
\text { (GO:0006658) }\end{array}$ & $1 / 27$ & 0.01209 & 0.029401443 & $\overline{2} .13577782$ & 7.532272253 & PLA2G2A \\
\hline $\begin{array}{l}\text { low-density } \\
\text { particle lipoprotein } \\
\text { (GO:0034374) }\end{array}$ & $1 / 11$ & 0.00494 & 0.025330625 & $\begin{array}{l}- \\
2.04798395\end{array}$ & 7.527858869 & PLA2G2A \\
\hline $\begin{array}{l}\text { macromolecular complex } \\
\text { remodeling } \\
\text { (GO:0034367) }\end{array}$ & $1 / 23$ & 0.0103 & 0.029401443 & - 2.13154487 & 7.517343872 & PLA2G2A \\
\hline $\begin{array}{l}\text { cardiac muscle adaptation } \\
\text { (GO:0014887) }\end{array}$ & $1 / 13$ & 0.00584 & 0.025330625 & -2.0425056 & 7.507721883 & NPPA \\
\hline $\begin{array}{ll}\text { plasma } & \text { lipoprotein } \\
\text { particle } & \text { remodeling } \\
(\text { GO:0034369) } & \end{array}$ & $1 / 23$ & 0.0103 & 0.029401443 & - & 7.485105269 & PLA2G2A \\
\hline $\begin{array}{l}\text { positive regulation of } \\
\text { nitric oxide biosynthetic } \\
\text { process (GO:0045429) }\end{array}$ & $1 / 32$ & 0.01431 & 0.03199631 & $\begin{array}{l}- \\
2.16432542\end{array}$ & 7.449899633 & HBB \\
\hline $\begin{array}{lr}\text { cellular amino } & \text { acid } \\
\text { metabolic } & \text { process } \\
(\text { GO:0006520) } & \end{array}$ & $2 / 421$ & 0.01443 & 0.03199631 & $\overline{-} .15253741$ & 7.409323703 & $\begin{array}{l}\text { PLA2G2A; } \\
\text { GSTT1 }\end{array}$ \\
\hline
\end{tabular}




\begin{tabular}{|c|c|c|c|c|c|c|}
\hline $\begin{array}{l}\text { muscle hypertrophy in } \\
\text { response to stress } \\
\text { (GO:0003299) }\end{array}$ & $1 / 13$ & 0.00584 & 0.025330625 & $-\overline{2.01519176}$ & 7.407323274 & NPPA \\
\hline $\begin{array}{ll}\text { cardiac } & \text { muscle } \\
\text { hypertrophy } & \\
\text { (GO:0003300) } & \\
\end{array}$ & $1 / 17$ & 0.00763 & 0.026820941 & $\overline{-} .03932258$ & 7.379436253 & NPPA \\
\hline $\begin{array}{l}\text { regulation of fatty acid } \\
\text { biosynthetic } \\
\text { (GO:0042304) }\end{array}$ & $1 / 29$ & 0.01298 & 0.03042917 & $\overline{-} .09889379$ & 7.330079239 & PDK4 \\
\hline $\begin{array}{l}\text { cardiac muscle } \\
\text { hypertrophy in response } \\
\text { to stress (GO:0014898) }\end{array}$ & $1 / 13$ & 0.00584 & 0.025330625 & $\overline{-} 1.96695067$ & 7.230001514 & NPPA \\
\hline $\begin{array}{l}\text { regulation of bone } \\
\text { resorption (GO:0045124) }\end{array}$ & $1 / 33$ & 0.01476 & 0.032022137 & $\begin{array}{l} \\
2.09602489\end{array}$ & 7.213108818 & PDK4 \\
\hline $\begin{array}{ll}\text { striated } & \text { muscle } \\
\text { hypertrophy } & \\
\text { (GO:0014897) } & \\
\end{array}$ & $1 / 18$ & 0.00807 & 0.027446534 & $\overline{-} .00283531$ & 7.201225173 & NPPA \\
\hline $\begin{array}{lr}\text { glutathione } & \text { derivative } \\
\text { biosynthetic } & \text { process } \\
\text { (GO:1901687) } & \end{array}$ & $1 / 27$ & 0.01209 & 0.029401443 & -2.0370459 & 7.184073249 & GSTT1 \\
\hline $\begin{array}{lr}\text { glutathione } & \text { derivative } \\
\text { metabolic } & \text { process } \\
\text { (GO:1901685) } & \\
\end{array}$ & $1 / 27$ & 0.01209 & 0.029401443 & $\overline{2} .03284516$ & 7.16925846 & GSTT1 \\
\hline $\begin{array}{l}\text { striated muscle adaptation } \\
\text { (GO:0014888) }\end{array}$ & $1 / 23$ & 0.0103 & 0.029401443 & $\overline{2}-03178992$ & 7.165536941 & NPPA \\
\hline $\begin{array}{l}\text { regulation of vasodilation } \\
\text { (GO:0042312) }\end{array}$ & $1 / 39$ & 0.01742 & 0.035889962 & $-\overline{2.14413362}$ & 7.13417069 & NPPB \\
\hline $\begin{array}{l}\text { muscle hypertrophy } \\
\text { (GO:0014896) }\end{array}$ & $1 / 20$ & 0.00897 & 0.028139038 & -1.9959265 & 7.126649966 & NPPA \\
\hline $\begin{array}{lr}\text { phosphatidic } & \text { acid } \\
\text { metabolic } & \text { process } \\
(\text { GO:0046473) } & \end{array}$ & $1 / 31$ & 0.01387 & 0.031430911 & $\begin{array}{l}- \\
2.05771477\end{array}$ & 7.119617883 & PLA2G2A \\
\hline $\begin{array}{lr}\text { phosphatidic } & \text { acid } \\
\text { biosynthetic } & \text { process } \\
\text { (GO:0006654) } & \\
\end{array}$ & $1 / 31$ & 0.01387 & 0.031430911 & - & 7.102906657 & PLA2G2A \\
\hline $\begin{array}{ll}\text { anion } & \text { transport } \\
(\mathrm{GO}: 0006820) & \\
\end{array}$ & $2 / 443$ & 0.0159 & 0.03343286 & -2.0855864 & 7.087273147 & $\begin{array}{l}\text { HBB; } \\
\text { HBA1 }\end{array}$ \\
\hline $\begin{array}{lr}\text { alditol } & \text { phosphate } \\
\text { metabolic } & \text { process } \\
(\text { GO:0052646) } & \\
\end{array}$ & $1 / 35$ & 0.01564 & 0.033242025 & $\overline{-} .06963168$ & 7.044902841 & PLA2G2A \\
\hline $\begin{array}{l}\begin{array}{l}\text { muscle } \\
(G O: 0043500)\end{array} \\
\text { adaptation }\end{array}$ & $1 / 28$ & 0.01253 & 0.029727456 & $-\overline{1.98762912}$ & 6.987876347 & NPPA \\
\hline $\begin{array}{l}\text { phosphatidylglycerol } \\
\text { metabolic process } \\
\text { (GO:0046471) }\end{array}$ & $1 / 34$ & 0.0152 & 0.032638693 & -2.0191409 & 6.910018702 & PLA2G2A \\
\hline $\begin{array}{ll}\text { plasma } & \text { lipoprotein } \\
\text { particle } & \text { organization } \\
\text { (GO:0071827) } & \end{array}$ & $1 / 31$ & 0.01387 & 0.031430911 & - & 6.909488048 & PLA2G2A \\
\hline $\begin{array}{l}\text { protein-lipid complex } \\
\text { subunit organization } \\
(\mathrm{GO}: 0071825)\end{array}$ & $1 / 33$ & 0.01476 & 0.032022137 & $\begin{array}{l}- \\
1.97523612\end{array}$ & 6.797435056 & PLA2G2A \\
\hline $\begin{array}{l}\text { negative regulation of } \\
\text { blood } \\
\text { (GO:0045776) }\end{array}$ & $1 / 39$ & 0.01742 & 0.035889962 & - & 6.784491452 & NPPA \\
\hline regulation of bone & $1 / 40$ & 0.01786 & 0.036074095 & - & 6.697027835 & PDK4 \\
\hline
\end{tabular}




\begin{tabular}{|c|c|c|c|c|c|c|}
\hline $\begin{array}{l}\text { remodeling } \\
\text { (GO:0046850) }\end{array}$ & & & & 2.01585324 & & \\
\hline \begin{tabular}{lr}
\multicolumn{2}{r}{ regulation of nitric oxide } \\
biosynthetic & process \\
(GO:0045428) &
\end{tabular} & $1 / 44$ & 0.01963 & 0.038879911 & $\begin{array}{l}- \\
1.97249456\end{array}$ & 6.405237357 & HBB \\
\hline $\begin{array}{l}\text { platelet aggregation } \\
\text { (GO:0070527) }\end{array}$ & $1 / 40$ & 0.01786 & 0.036074095 & $\begin{array}{l}- \\
1.89768215 \\
\end{array}$ & 6.304442187 & HBB \\
\hline $\begin{array}{ll}\text { somatic stem cell } \\
\text { maintenance } & \\
(\text { GO:0035019) } & \\
\end{array}$ & $1 / 44$ & 0.01963 & 0.038879911 & - & 6.113944817 & PLA2G2A \\
\hline $\begin{array}{l}\text { glutathione metabolic } \\
\text { process (GO:0006749) }\end{array}$ & $1 / 49$ & 0.02184 & 0.042838953 & $\begin{array}{l}- \\
1.87070624\end{array}$ & 5.893299843 & GSTT1 \\
\hline $\begin{array}{l}\begin{array}{l}\text { response to fatty acid } \\
(\mathrm{GO}: 0070542)\end{array} \\
\end{array}$ & $1 / 50$ & 0.02228 & 0.043288255 & $-\overline{1.85563355}$ & 5.826455398 & PDK4 \\
\hline $\begin{array}{l}\begin{array}{l}\text { regulation of tissue } \\
\text { remodeling } \\
\text { (GO:0034103) }\end{array} \\
\end{array}$ & $1 / 58$ & 0.0258 & 0.048294438 & - & 5.696046012 & PDK4 \\
\hline $\begin{array}{l}\begin{array}{l}\text { regulation of } \\
(\mathrm{GO}: 0006885)\end{array} \\
\end{array}$ & $1 / 52$ & 0.02316 & 0.044160653 & $\begin{array}{l}- \\
1.80164344 \\
\end{array}$ & 5.620985349 & PDK4 \\
\hline $\begin{array}{l}\text { regulation of fatty acid } \\
\text { metabolic } \\
(\text { GO:0019217) }\end{array}$ & $1 / 72$ & 0.03194 & 0.055696595 & -1.9297141 & 5.572698345 & PDK4 \\
\hline $\begin{array}{l}\text { defense response to } \\
\text { Gram-positive bacterium } \\
\text { (GO:0050830) }\end{array}$ & $1 / 52$ & 0.02316 & 0.044160653 & $\begin{array}{l}- \\
1.76201285\end{array}$ & 5.497341041 & PLA2G2A \\
\hline $\begin{array}{lcc}\begin{array}{l}\text { body fluid } \\
\text { (GO:0007589) }\end{array} & \text { secretion } \\
\end{array}$ & $1 / 71$ & 0.03151 & 0.055407562 & $-\overline{1.89751252}$ & 5.489578097 & NPPB \\
\hline $\begin{array}{l}\text { negative regulation of } \\
\text { angiogenesis } \\
\text { (GO:0016525) }\end{array}$ & $1 / 64$ & 0.02844 & 0.052267585 & $-\overline{1.83661158}$ & 5.420536652 & NPPB \\
\hline $\begin{array}{l}\text { negative regulation of } \\
\text { blood } \\
\text { morphogenesis } \\
(\text { vO:2000181) } \\
\end{array}$ & $1 / 66$ & 0.02932 & 0.053398367 & -1.8467018 & 5.410790326 & NPPB \\
\hline $\begin{array}{lr} & \text { cellular response to } \\
\text { mechanical } & \text { stimulus } \\
(\text { GO:0071260) } & \\
\end{array}$ & $1 / 67$ & 0.02976 & 0.053716999 & - & 5.407160611 & NPPA \\
\hline $\begin{array}{l}\text { homotypic cell-cell } \\
\text { adhesion (GO:0034109) }\end{array}$ & $1 / 57$ & 0.02536 & 0.047910801 & $-\overline{1.75912968}$ & 5.344964784 & HBB \\
\hline $\begin{array}{l}\text { negative regulation of } \\
\text { vasculature development } \\
\text { (GO:1901343) }\end{array}$ & $1 / 70$ & 0.03107 & 0.055407562 & $-\overline{1.84564916}$ & 5.339535356 & NPPB \\
\hline $\begin{array}{l}\text { cellular response to } \\
\text { starvation (GO:0009267) }\end{array}$ & $1 / 74$ & 0.03282 & 0.056735959 & $\begin{array}{l}- \\
1.85678295\end{array}$ & 5.32775474 & PDK4 \\
\hline $\begin{array}{l}\text { positive regulation of } \\
\text { inflammatory } \\
(\text { GO:0050729) }\end{array}$ & $1 / 88$ & 0.03892 & 0.065075659 & $\begin{array}{l}- \\
1.93289789\end{array}$ & 5.281072705 & PLA2G2A \\
\hline $\begin{array}{l}\text { phosphatidylcholine } \\
\text { metabolic process } \\
\text { (GO:0046470) }\end{array}$ & $1 / 62$ & 0.02756 & 0.051114936 & - & 5.190838994 & PLA2G2A \\
\hline $\begin{array}{lr}\begin{array}{l}\text { monovalent } \\
\text { cation }\end{array} & \text { inorganic } \\
(\mathrm{GO}: 0055067) & \end{array}$ & $1 / 82$ & 0.03631 & 0.061723153 & $-\overline{1.84987596}$ & 5.15208244 & PDK4 \\
\hline $\begin{array}{l}\text { pyruvate metabolic } \\
\text { process (GO:0006090) }\end{array}$ & $1 / 71$ & 0.03151 & 0.055407562 & $-\overline{1.77809292}$ & 5.144092529 & PDK4 \\
\hline
\end{tabular}




\begin{tabular}{|c|c|c|c|c|c|c|}
\hline $\begin{array}{l}\text { female pregnancy } \\
(\text { GO:0007565) }\end{array}$ & $1 / 85$ & 0.03761 & 0.063414586 & 1.85992222 & 5.129779653 & NPPA \\
\hline $\begin{array}{l}\text { cellular response } \\
\text { nutrient } \\
\text { (GO:0031669) }\end{array}$ & $1 / 99$ & 0.04369 & 0.071871484 & $\begin{array}{l}- \\
1.93686747\end{array}$ & 5.09953128 & PDK4 \\
\hline $\begin{array}{ll}\begin{array}{l}\text { regulation of } \\
\text { metabolic }\end{array} & \text { glucose } \\
\text { (GO:0010906) } & \\
\end{array}$ & $1 / 94$ & 0.04152 & 0.068865042 & $-\overline{1.90292583}$ & 5.091480909 & PDK4 \\
\hline $\begin{array}{l}\text { ethanolamine-containing } \\
\text { compound metabolic } \\
\text { process (GO:0042439) }\end{array}$ & $1 / 81$ & 0.03587 & 0.061495055 & $\begin{array}{l}- \\
1.79894728\end{array}$ & 5.016901485 & PLA2G2A \\
\hline $\begin{array}{ll}\begin{array}{l}\text { regulation of } \\
\text { biosynthetic } \\
(\text { GO:0046890) }\end{array} & \text { process } \\
\end{array}$ & $1 / 113$ & 0.04973 & 0.078635729 & $\begin{array}{l}- \\
1.95971673\end{array}$ & 4.98342072 & PDK4 \\
\hline $\begin{array}{lr}\text { negative regulation } & \text { of } \\
\text { epithelial } & \text { cell } \\
\text { proliferation } & \\
(\text { GO:0050680) } & \\
\end{array}$ & $1 / 110$ & 0.04843 & 0.077800036 & $\begin{array}{l}- \\
1.93886197\end{array}$ & 4.951103869 & PLA2G2A \\
\hline $\begin{array}{l}\text { response to starvation } \\
(\mathrm{GO} 0042594)\end{array}$ & $1 / 106$ & 0.04671 & 0.07623123 & $-\overline{1.91045262}$ & 4.917474601 & PDK4 \\
\hline \begin{tabular}{lr} 
cellular & \multicolumn{2}{c}{ response } & to \\
extracellular & stimulus \\
$($ GO:0031668) & \\
\end{tabular} & $1 / 119$ & 0.0523 & 0.082075886 & $\begin{array}{l}- \\
1.93929572\end{array}$ & 4.848454594 & PDK4 \\
\hline $\begin{array}{ll} & \text { positive regulation of } \\
\text { homeostatic } & \text { process } \\
(\text { GO:0032846) } & \end{array}$ & $1 / 130$ & 0.05701 & 0.08551943 & $-\overline{1.94446727}$ & 4.781467733 & NPPB \\
\hline $\begin{array}{l}\text { cellular response to acid } \\
\text { chemical (GO:0071229) }\end{array}$ & $1 / 147$ & 0.06425 & 0.091587408 & $-\overline{1.98818964}$ & 4.752690749 & PDK4 \\
\hline $\begin{array}{l}\text { phosphatidylinositol } \\
\text { metabolic process } \\
\text { (GO:0046488) }\end{array}$ & $1 / 125$ & 0.05487 & 0.083540694 & - & 4.752265974 & PLA2G2A \\
\hline $\begin{array}{l}\text { positive regulation of } \\
\text { response to wounding } \\
\text { (GO:1903036) }\end{array}$ & $1 / 130$ & 0.05701 & 0.08551943 & $\begin{array}{l}- \\
1.92665788\end{array}$ & 4.737674233 & PLA2G2A \\
\hline $\begin{array}{l}\text { peptide metabolic process } \\
\text { (GO:0006518) }\end{array}$ & $1 / 113$ & 0.04973 & 0.078635729 & $-\overline{1.85387676}$ & 4.714277173 & GSTT1 \\
\hline $\begin{array}{l}\text { stem cell maintenance } \\
\text { (GO:0019827) }\end{array}$ & $1 / 109$ & 0.048 & 0.077720098 & $\begin{array}{l}-84172747 \\
1.841\end{array}$ & 4.704953231 & PLA2G2A \\
\hline $\begin{array}{l}\text { cellular biogenic amine } \\
\text { metabolic } \\
\text { (GO:0006576) }\end{array}$ & $1 / 125$ & 0.05487 & 0.083540694 & $\begin{array}{l}- \\
1.88164422\end{array}$ & 4.671033906 & PLA2G2A \\
\hline $\begin{array}{l}\text { cellular amine metabolic } \\
\text { process (GO:0044106) }\end{array}$ & $1 / 125$ & 0.05487 & 0.083540694 & $-\overline{1.87913714}$ & 4.664810279 & PLA2G2A \\
\hline $\begin{array}{l}\text { insulin receptor signaling } \\
\text { pathway (GO:0008286) }\end{array}$ & $1 / 148$ & 0.06467 & 0.091587408 & $-\overline{1.94291262}$ & 4.644457778 & PDK4 \\
\hline $\begin{array}{l}\text { multi-multicellular } \\
\text { organism } \\
\text { (GO:0044706) }\end{array}$ & $1 / 121$ & 0.05316 & 0.082785256 & -1.8619101 & 4.638958887 & NPPA \\
\hline $\begin{array}{l}\text { regulation of cellular } \\
\text { carbohydrate metabolic } \\
\text { process (GO:0010675) }\end{array}$ & $1 / 137$ & 0.06 & 0.088694451 & $-\overline{1.90665765}$ & 4.618988666 & PDK4 \\
\hline $\begin{array}{l}\text { amine metabolic process } \\
\text { (GO:0009308) }\end{array}$ & $1 / 134$ & 0.05872 & 0.087437681 & $\begin{array}{l}- \\
1.88203617 \\
\end{array}$ & 4.586200233 & PLA2G2A \\
\hline $\begin{array}{lr}\text { regulation } & \text { of } \\
\text { carbohydrate } & \text { metabolic }\end{array}$ & $1 / 145$ & 0.0634 & 0.091084176 & $-\overline{1.91022418}$ & 4.576842093 & PDK4 \\
\hline
\end{tabular}




\begin{tabular}{|c|c|c|c|c|c|c|}
\hline process (GO:0006109) & & & & & & \\
\hline $\begin{array}{l}\text { carbohydrate homeostasis } \\
\text { (GO:0033500) }\end{array}$ & $1 / 143$ & 0.06255 & 0.090500943 & $-\overline{1.90358622}$ & 4.573166033 & PDK4 \\
\hline $\begin{array}{l}\text { glucose homeostasis } \\
\text { (GO:0042593) }\end{array}$ & $1 / 143$ & 0.06255 & 0.090500943 & $-\overline{1.90108069}$ & 4.567146763 & PDK4 \\
\hline $\begin{array}{l}\text { regulation of } \\
\text { homeostasis } \\
(G O: 2000021)\end{array}$ & $1 / 160$ & 0.06975 & 0.096798699 & $\begin{array}{l}- \\
1.95252547\end{array}$ & 4.559384651 & NPPB \\
\hline $\begin{array}{l}\text { glucose metabolic process } \\
\text { (GO:0006006) }\end{array}$ & $1 / 149$ & 0.0651 & 0.091587408 & $\begin{array}{l}- \\
1.89429783 \\
\end{array}$ & 4.528246002 & PDK4 \\
\hline $\begin{array}{l}\text { regulation of cellular } \\
\text { ketone metabolic process } \\
\text { (GO:0010565) }\end{array}$ & $1 / 178$ & 0.07732 & 0.103654301 & $-\overline{1.99449004}$ & 4.520898482 & PDK4 \\
\hline $\begin{array}{l}\text { multi-organism } \\
\text { reproductive } \\
\text { (GO:0044703) }\end{array}$ & $1 / 140$ & 0.06128 & 0.08993087 & $\begin{array}{l}- \\
1.87348024\end{array}$ & 4.512678108 & NPPA \\
\hline $\begin{array}{l}\text { xenobiotic metabolic } \\
\text { process (GO:0006805) }\end{array}$ & $1 / 156$ & 0.06806 & 0.095100819 & $-\overline{1.86636234}$ & 4.391210346 & GSTT1 \\
\hline $\begin{array}{l}\text { regulation of blood } \\
\text { circulation (GO:1903522) }\end{array}$ & $1 / 204$ & 0.08816 & 0.111704914 & $-\overline{1.99570569}$ & 4.374376481 & NPPB \\
\hline $\begin{array}{l}\text { positive regulation of } \\
\text { response to external } \\
\text { stimulus (GO:0032103) }\end{array}$ & $1 / 201$ & 0.08691 & 0.111345309 & $\begin{array}{l}- \\
1.98683895 \\
\end{array}$ & 4.361347956 & PLA2G2A \\
\hline $\begin{array}{l}\text { response to mechanical } \\
\text { stimulus (GO:0009612) }\end{array}$ & $1 / 176$ & 0.07648 & 0.103654301 & $\begin{array}{l} \\
\end{array}$ & 4.350262098 & NPPA \\
\hline $\begin{array}{lrr}\text { cellular } & \text { response } & \text { to } \\
\text { insulin } & \text { stimulus } \\
(G O: 0032869) & \end{array}$ & $1 / 195$ & 0.08442 & 0.110396313 & $-\overline{1.97115366}$ & 4.343789029 & PDK4 \\
\hline $\begin{array}{l}\text { regulation of angiogenesis } \\
\text { (GO:0045765) }\end{array}$ & $1 / 179$ & 0.07774 & 0.103654301 & $-\overline{1.91256909}$ & 4.335208772 & NPPB \\
\hline $\begin{array}{l}\text { cellular amide metabolic } \\
\text { process (GO:0043603) }\end{array}$ & $1 / 177$ & 0.0769 & 0.103654301 & $-\overline{1.90686125}$ & 4.322270856 & GSTT1 \\
\hline $\begin{array}{l}\text { hexose metabolic process } \\
\text { (GO:0019318) }\end{array}$ & $1 / 187$ & 0.08109 & 0.106719788 & $-\overline{1.92848107}$ & 4.315070283 & PDK4 \\
\hline $\begin{array}{l}\text { defense response to } \\
\text { bacterium (GO:0042742) }\end{array}$ & $1 / 179$ & 0.07774 & 0.103654301 & $\begin{array}{l}- \\
1.89719298 \\
\end{array}$ & 4.300355833 & PLA2G2A \\
\hline $\begin{array}{lr}\text { response to } \\
(G O: 0001666)\end{array}$ & $1 / 241$ & 0.10339 & 0.1240641 & $-\overline{2.03780663}$ & 4.252814632 & NPPA \\
\hline $\begin{array}{lr}\begin{array}{l}\text { response to } \\
\text { oxygen }\end{array} & \text { decreased } \\
\text { (GO:0036293) } & \\
\end{array}$ & $1 / 245$ & 0.10502 & 0.124557829 & $-\overline{2} .03765987$ & 4.244415298 & NPPA \\
\hline $\begin{array}{l}\text { regulation of vasculature } \\
\text { development } \\
\text { (GO:1901342) }\end{array}$ & $1 / 197$ & 0.08525 & 0.110774178 & $\begin{array}{l}- \\
1.91926706\end{array}$ & 4.222889581 & NPPB \\
\hline $\begin{array}{l}\text { response to oxygen levels } \\
\text { (GO:0070482) }\end{array}$ & $1 / 259$ & 0.11071 & 0.128326022 & -2.0533001 & 4.215797179 & NPPA \\
\hline $\begin{array}{l}\text { glycerophospholipid } \\
\text { biosynthetic process } \\
\text { (GO:0046474) }\end{array}$ & $1 / 178$ & 0.07732 & 0.103654301 & $\begin{array}{l}- \\
1.85850686\end{array}$ & 4.212666243 & PLA2G2A \\
\hline $\begin{array}{l}\text { response to bacterium } \\
\text { (GO:0009617) }\end{array}$ & $1 / 201$ & 0.08691 & 0.111345309 & -1.9173704 & 4.208856224 & PLA2G2A \\
\hline $\begin{array}{l}\text { transcription initiation } \\
\text { from RNA polymerase II } \\
\text { promoter (GO:0006367) }\end{array}$ & $1 / 187$ & 0.08109 & 0.106719788 & $-\overline{1.88035219}$ & 4.207379574 & NPPA \\
\hline
\end{tabular}




\begin{tabular}{|c|c|c|c|c|c|c|}
\hline $\begin{array}{lr}\text { cellular response to } \\
\text { abiotic } & \text { stimulus } \\
(\text { GO:0071214) } & \end{array}$ & $1 / 232$ & 0.0997 & 0.121793771 & $\begin{array}{l}- \\
1.97971767\end{array}$ & 4.168149185 & NPPA \\
\hline $\begin{array}{llr}\text { regulation of } & \text { lipid } \\
\text { metabolic } & \text { process } \\
(\mathrm{GO}: 0019216) & \end{array}$ & $1 / 245$ & 0.10502 & 0.124557829 & $\begin{array}{l}- \\
1.98657852\end{array}$ & 4.138013601 & PDK4 \\
\hline $\begin{array}{l}\text { regulation of epithelial } \\
\text { cell proliferation } \\
(\mathrm{GO}: 0050678)\end{array}$ & $1 / 258$ & 0.11031 & 0.128326022 & $\overline{2} .01373413$ & 4.134561082 & PLA2G2A \\
\hline $\begin{array}{lr}\begin{array}{l}\text { regulation } \\
\text { inflammatory } \\
(\text { GO:0050727) }\end{array} & \text { response } \\
\end{array}$ & $1 / 247$ & 0.10583 & 0.124799244 & $\begin{array}{l}- \\
1.98605804\end{array}$ & 4.133083846 & PLA2G2A \\
\hline $\begin{array}{l}\text { response to acid chemical } \\
\text { (GO:0001101) }\end{array}$ & $1 / 275$ & 0.11718 & 0.132070091 & $\begin{array}{l}- \\
2.02976969\end{array}$ & 4.109111444 & PDK4 \\
\hline $\begin{array}{l}\text { cellular response to } \\
\text { peptide hormone stimulus } \\
\text { (GO:0071375) }\end{array}$ & $1 / 261$ & 0.11152 & 0.128535376 & $\begin{array}{l}- \\
1.99780837\end{array}$ & 4.098605974 & PDK4 \\
\hline $\begin{array}{l}\text { monosaccharide } \\
\text { metabolic process } \\
(\mathrm{GO} 0005996)\end{array}$ & $1 / 221$ & 0.09518 & 0.118399598 & $\begin{array}{l}- \\
1.92031113\end{array}$ & 4.09734855 & PDK4 \\
\hline $\begin{array}{l}\text { glycerolipid biosynthetic } \\
\text { process (GO:0045017) }\end{array}$ & $1 / 202$ & 0.08733 & 0.111345309 & $\begin{array}{l}- \\
1.86097981\end{array}$ & 4.085072172 & PLA2G2A \\
\hline $\begin{array}{ll}\text { cellular response } & \text { to } \\
\text { peptide (GO:1901653) }\end{array}$ & $1 / 273$ & 0.11637 & 0.131890227 & $\begin{array}{l} \\
1.99892269\end{array}$ & 4.049388232 & PDK4 \\
\hline $\begin{array}{l}\text { DNA-templated } \\
\text { transcription, initiation } \\
\text { (GO:0006352) }\end{array}$ & $1 / 220$ & 0.09477 & 0.118399598 & $\begin{array}{l}- \\
1.89256968\end{array}$ & 4.038156913 & NPPA \\
\hline $\begin{array}{l}\text { phospholipid biosynthetic } \\
\text { process (GO:0008654) }\end{array}$ & $1 / 206$ & 0.08899 & 0.112059235 & $-\overline{1.84130636}$ & 4.030118151 & PLA2G2A \\
\hline $\begin{array}{lr}\text { positive regulation of } \\
\text { defense } & \text { response } \\
(\mathrm{GO}: 0031349) & \end{array}$ & $1 / 272$ & 0.11597 & 0.131890227 & $\begin{array}{l}- \\
1.98124152\end{array}$ & 4.013 & PLA2G2A \\
\hline $\begin{array}{l}\text { single organismal cell-cell } \\
\text { adhesion (GO:0016337) }\end{array}$ & $1 / 235$ & 0.10093 & 0.122561368 & - & 3.995899863 & HBB \\
\hline $\begin{array}{l}\text { muscle system process } \\
\text { (GO:0003012) }\end{array}$ & $1 / 237$ & 0.10175 & 0.122824304 & $\begin{array}{l}- \\
1.89958782 \\
\end{array}$ & 3.98343636 & NPPA \\
\hline $\begin{array}{l}\text { positive regulation of } \\
\text { secretion (GO:0051047) }\end{array}$ & $1 / 273$ & 0.11637 & 0.131890227 & $\begin{array}{l}- \\
1.96230497\end{array}$ & 3.975208583 & NPPB \\
\hline $\begin{array}{l}\text { glycerophospholipid } \\
\text { metabolic process } \\
\text { (GO:0006650) }\end{array}$ & $1 / 229$ & 0.09847 & 0.121748516 & $\begin{array}{l}- \\
1.88193914\end{array}$ & 3.962983126 & PLA2G2A \\
\hline $\begin{array}{l}\text { lipid catabolic process } \\
\text { (GO:0016042) }\end{array}$ & $1 / 232$ & 0.0997 & 0.121793771 & $\begin{array}{l} \\
1.87427142\end{array}$ & 3.946139893 & PLA2G2A \\
\hline $\begin{array}{l}\text { cellular response to lipid } \\
\text { (GO:0071396) }\end{array}$ & $1 / 315$ & 0.13317 & 0.145271214 & $\overline{2}-04511867$ & 3.945346484 & PDK4 \\
\hline $\begin{array}{l}\text { response to nutrient levels } \\
\text { (GO:0031667) }\end{array}$ & $1 / 291$ & 0.12361 & 0.137789387 & 1.98911959 & 3.942492597 & PDK4 \\
\hline $\begin{array}{l}\text { single organism cell } \\
\text { adhesion (GO:0098602) }\end{array}$ & $1 / 259$ & 0.11071 & 0.128326022 & $-\overline{1.89852701}$ & 3.898019985 & $\mathrm{HBB}$ \\
\hline $\begin{array}{l}\text { response to extracellular } \\
\text { stimulus (GO:0009991) }\end{array}$ & $1 / 313$ & 0.13237 & 0.145271214 & -1.9809919 & 3.821636155 & PDK4 \\
\hline $\begin{array}{l}\text { phospholipid metabolic } \\
\text { process (GO:0006644) }\end{array}$ & $1 / 288$ & 0.1224 & 0.137199649 & $-\overline{1.89627255}$ & 3.766600533 & PLA2G2A \\
\hline $\begin{array}{l}\text { regulation of response to } \\
\text { wounding (GO:1903034) }\end{array}$ & $1 / 347$ & 0.14577 & 0.156508623 & $\begin{array}{l}- \\
1.99853017\end{array}$ & 3.706562333 & PLA2G2A \\
\hline
\end{tabular}




\begin{tabular}{|c|c|c|c|c|c|c|}
\hline $\begin{array}{l}\text { glycerolipid metabolic } \\
\text { process (GO:0046486) }\end{array}$ & $1 / 296$ & 0.1256 & 0.139257235 & $\overline{1}-87625128$ & 3.69890266 & PLA2G2A \\
\hline $\begin{array}{lr}\text { sulfur } & \text { compound } \\
\text { metabolic } & \text { process } \\
(\text { GO:0006790) } & \\
\end{array}$ & $1 / 314$ & 0.13277 & 0.145271214 & $\begin{array}{l}- \\
1.87362498\end{array}$ & 3.614508959 & GSTT1 \\
\hline $\begin{array}{l}\text { defense response to other } \\
\text { organism (GO:0098542) }\end{array}$ & $1 / 328$ & 0.1383 & 0.150075488 & $\begin{array}{l}- \\
1.89956945\end{array}$ & 3.602755435 & PLA2G2A \\
\hline $\begin{array}{l}\text { regulation of system } \\
\text { process (GO:0044057) }\end{array}$ & $1 / 371$ & 0.15511 & 0.165669999 & $-\overline{1.99392402}$ & 3.584591709 & NPPB \\
\hline $\begin{array}{l}\text { cellular response to } \\
\text { organonitrogen } \\
\text { compound (GO:0071417) }\end{array}$ & $1 / 411$ & 0.17049 & 0.181140749 & $\overline{2} .06158381$ & 3.522176632 & PDK4 \\
\hline $\begin{array}{l}\text { alcohol metabolic process } \\
\text { (GO:0006066) }\end{array}$ & $1 / 340$ & 0.14303 & 0.154376223 & $\begin{array}{l} \\
1.87990284\end{array}$ & 3.512340243 & PLA2G2A \\
\hline $\begin{array}{l}\text { cellular response to } \\
\text { nitrogen } \\
(\text { GO:1901699) }\end{array}$ & $1 / 438$ & 0.18072 & 0.191021163 & $\begin{array}{l}- \\
2.05282397\end{array}$ & 3.398185388 & PDK4 \\
\hline $\begin{array}{lrr}\text { cellular } & \text { response } & \text { to } \\
\text { hormone } & \text { stimulus } \\
(G O: 0032870) & \\
\end{array}$ & $1 / 462$ & 0.18972 & 0.197615928 & $\begin{array}{l}- \\
2.00324322\end{array}$ & 3.248118432 & PDK4 \\
\hline $\begin{array}{l}\text { cation homeostasis } \\
\text { (GO:0055080) }\end{array}$ & $1 / 465$ & 0.19084 & 0.197615928 & - & 3.117220381 & PDK4 \\
\hline $\begin{array}{l}\text { response to other } \\
\text { organism (GO:0051707) }\end{array}$ & $1 / 462$ & 0.18972 & 0.197615928 & $\begin{array}{l}- \\
1.92119481 \\
\end{array}$ & 3.115082683 & PLA2G2A \\
\hline $\begin{array}{lc}\text { nitrogen } & \text { compound } \\
\text { transport (GO:0071705) }\end{array}$ & $1 / 464$ & 0.19047 & 0.197615928 & $\begin{array}{l}- \\
1.89214096\end{array}$ & 3.067973915 & HBB \\
\hline $\begin{array}{l}\text { blood coagulation } \\
\text { (GO:0007596) }\end{array}$ & $1 / 472$ & 0.19345 & 0.197615928 & $-\overline{1.88619266}$ & 3.058329153 & HBB \\
\hline $\begin{array}{l}\text { coagulation } \\
\text { (GO:0050817) }\end{array}$ & $1 / 472$ & 0.19345 & 0.197615928 & $\begin{array}{l}- \\
1.88360204 \\
\end{array}$ & 3.054128643 & $\mathrm{HBB}$ \\
\hline hemostasis (GO:0007599) & $1 / 478$ & 0.19568 & 0.197615928 & - 1.87614674 & 3.042040403 & HBB \\
\hline $\begin{array}{lr}\text { monocarboxylic } & \text { acid } \\
\text { metabolic } & \text { process } \\
\text { (GO:0032787) } & \end{array}$ & $1 / 473$ & 0.19382 & 0.197615928 & -1.8611017 & 3.017645924 & PDK4 \\
\hline $\begin{array}{l}\text { lipid biosynthetic process } \\
\text { (GO:0008610) }\end{array}$ & $1 / 491$ & 0.20049 & 0.201474791 & $\begin{array}{l}- \\
1.88047736 \\
\end{array}$ & 3.012695878 & PLA2G2A \\
\hline $\begin{array}{lr}\text { organic } & \text { hydroxy } \\
\text { compound } & \text { metabolic } \\
\text { process (GO:1901615) }\end{array}$ & $1 / 476$ & 0.19494 & 0.197615928 & $\begin{array}{l}- \\
1.85637011 \\
\end{array}$ & 3.009973985 & PLA2G2A \\
\hline $\begin{array}{l}\text { gene } \\
\text { (GO:0010467) }\end{array}$ & $1 / 672$ & 0.26483 & 0.264834524 & $\begin{array}{l}- \\
1.84167319\end{array}$ & 2.446939247 & NPPA \\
\hline
\end{tabular}


Supplemental Table 20. Gene ontology results for "cellular component" from Ingenuity

Pathway Analysis

\begin{tabular}{|c|c|c|c|c|c|c|}
\hline Term & Overlap & P-value & $\begin{array}{l}\text { Adjusted P- } \\
\text { value }\end{array}$ & Z-score & $\begin{array}{l}\text { Combined } \\
\text { Score }\end{array}$ & Genes \\
\hline $\begin{array}{l}\text { endocytic vesicle lumen } \\
\text { (GO:0071682) }\end{array}$ & $2 / 16$ & $2.2 \mathrm{E}-05$ & 0.000387551 & -2.4661 & 19.37315594 & HBB; HBA1 \\
\hline $\begin{array}{ll}\text { hemoglobin } & \text { complex } \\
\text { (GO:0005833) } & \end{array}$ & $2 / 12$ & $1.2 \mathrm{E}-05$ & 0.000387551 & -2.4402 & 19.16928532 & HBB; HBA1 \\
\hline $\begin{array}{ll}\text { extracellular } & \text { region } \\
\text { (GO:0005576) } & \end{array}$ & $5 / 1585$ & 0.0003 & 0.003581419 & -2.6121 & 14.71140055 & $\begin{array}{l}\text { NPPB; NPPA; } \\
\text { PLA2G2A; } \\
\text { HBB; HBA1 }\end{array}$ \\
\hline $\begin{array}{l}\text { cytoplasmic membrane- } \\
\text { bounded vesicle lumen } \\
\text { (GO:0060205) }\end{array}$ & $2 / 76$ & 0.0005 & 0.00363052 & -2.137 & 12.00654638 & HBB; HBA1 \\
\hline $\begin{array}{ll}\text { vesicle } & \text { lumen } \\
\text { (GO:0031983) } & \\
\end{array}$ & $2 / 76$ & 0.0005 & 0.00363052 & -2.1108 & 11.85949491 & HBB; HBA1 \\
\hline $\begin{array}{l}\text { blood microparticle } \\
\text { (GO:0072562) }\end{array}$ & $2 / 161$ & 0.00223 & 0.013404023 & -2.522 & 10.87518376 & HBB; HBA1 \\
\hline $\begin{array}{ll}\text { cytosolic } & \text { part } \\
\text { (GO:0044445) } & \\
\end{array}$ & $2 / 198$ & 0.00335 & 0.0161741 & -2.1824 & 9.000859897 & HBB; HBA1 \\
\hline $\begin{array}{l}\text { extracellular vesicular } \\
\text { exosome (GO:0070062) }\end{array}$ & $5 / 2717$ & 0.00361 & 0.0161741 & -2.1092 & 8.699115282 & $\begin{array}{l}\text { PLA2G2A; } \\
\text { HBB; GSTT1; } \\
\text { HBA1; } \\
\text { HIST1H2AC }\end{array}$ \\
\hline $\begin{array}{l}\text { cytoplasmic vesicle part } \\
\text { (GO:0044433) }\end{array}$ & $2 / 363$ & 0.01087 & 0.034211899 & -2.2201 & 7.493233854 & HBB; HBA1 \\
\hline $\begin{array}{ll}\text { extracellular } & \text { space } \\
(\text { GO:0005615) } & \\
\end{array}$ & $3 / 1120$ & 0.0114 & 0.034211899 & -2.0031 & 6.760721979 & $\begin{array}{l}\text { NPPB; NPPA; } \\
\text { PLA2G2A }\end{array}$ \\
\hline $\begin{array}{lcc}\text { mast cell } & \text { granule } \\
(\text { GO:0042629) } & \\
\end{array}$ & $1 / 18$ & 0.00807 & 0.029061036 & -1.8919 & 6.694189763 & NPPA \\
\hline $\begin{array}{l}\text { cytosolic small ribosomal } \\
\text { subunit (GO:0022627) }\end{array}$ & $1 / 39$ & 0.01742 & 0.048232212 & -1.8421 & 5.584864766 & HBA1 \\
\hline $\begin{array}{ll}\text { dendritic } & \text { spine } \\
(\mathrm{GO}: 0043197) & \\
\end{array}$ & $1 / 71$ & 0.03151 & 0.061430179 & -1.8738 & 5.227635379 & MYL7 \\
\hline $\begin{array}{ll}\text { neuron } & \text { spine } \\
(\mathrm{GO}: 0044309) & \\
\end{array}$ & $1 / 74$ & 0.03282 & 0.061430179 & -1.8378 & 5.127284847 & MYL7 \\
\hline $\begin{array}{ll}\text { myosin } & \text { complex } \\
\text { (GO:0016459) } & \end{array}$ & $1 / 64$ & 0.02844 & 0.061430179 & -1.8348 & 5.118816301 & MYL7 \\
\hline $\begin{array}{l}\text { small ribosomal subunit } \\
\text { (GO:0015935) }\end{array}$ & $1 / 62$ & 0.02756 & 0.061430179 & -1.7713 & 4.941658002 & HBA1 \\
\hline $\begin{array}{l}\text { nucleosome } \\
\text { (GO:0000786) }\end{array}$ & $1 / 71$ & 0.03151 & 0.061430179 & -1.7524 & 4.888981784 & HIST1H2AC \\
\hline $\begin{array}{l}\text { DNA packaging complex } \\
\text { (GO:0044815) }\end{array}$ & $1 / 77$ & 0.03413 & 0.061430179 & -1.7301 & 4.826699714 & HIST1H2AC \\
\hline $\begin{array}{l}\text { DNA bending complex } \\
\text { (GO:1990104) }\end{array}$ & $1 / 71$ & 0.03151 & 0.061430179 & -1.7266 & 4.816889438 & HIST1H2AC \\
\hline $\begin{array}{ll}\text { protein-DNA } & \text { complex } \\
\text { (GO:0032993) } & \\
\end{array}$ & $1 / 98$ & 0.04325 & 0.074149599 & -1.7808 & 4.633022425 & HIST1H2AC \\
\hline A band (GO:0031672) & $1 / 9$ & 0.00404 & 0.0161741 & -1.0513 & 4.336048765 & MYL7 \\
\hline
\end{tabular}




\begin{tabular}{|c|c|c|c|c|c|c|}
\hline $\begin{array}{l}\text { contractile fiber part } \\
(\mathrm{GO}: 0044449)\end{array}$ & $1 / 167$ & 0.0727 & 0.113794974 & -1.834 & 3.985955491 & MYL7 \\
\hline $\begin{array}{ll}\begin{array}{l}\text { secretory } \\
(G O: 0030141)\end{array} & \text { granule } \\
\end{array}$ & $1 / 176$ & 0.07648 & 0.114725126 & -1.8347 & 3.972485589 & PLA2G2A \\
\hline $\begin{array}{ll}\text { ribosomal } & \text { subunit } \\
(\mathrm{GO}: 0044391) & \\
\end{array}$ & $1 / 135$ & 0.05915 & 0.096785732 & -1.6955 & 3.959505965 & HBA1 \\
\hline $\begin{array}{ll}\text { mitochondrial } & \text { matrix } \\
(\text { GO:0005759) } & \end{array}$ & $1 / 208$ & 0.08982 & 0.129335749 & -1.8608 & 3.805919731 & PDK4 \\
\hline $\begin{array}{l}\text { mitochondrion } \\
\text { (GO:0005739) }\end{array}$ & $2 / 1269$ & 0.10762 & 0.138442768 & -1.8445 & 3.647181286 & $\begin{array}{l}\text { PLA2G2A; } \\
\text { PDK4 }\end{array}$ \\
\hline $\begin{array}{ll}\text { lytic } & \text { vacuole } \\
\text { (GO:0000323) } & \\
\end{array}$ & $1 / 261$ & 0.11152 & 0.138442768 & -1.799 & 3.557232948 & NPPA \\
\hline lysosome (GO:0005764) & $1 / 261$ & 0.11152 & 0.138442768 & -1.7797 & 3.519025579 & NPPA \\
\hline vacuole (GO:0005773) & $1 / 293$ & 0.12441 & 0.149286509 & -1.7819 & 3.388910633 & NPPA \\
\hline cytosol (GO:0005829) & $3 / 2529$ & 0.09443 & 0.130743337 & -1.6311 & 3.318418288 & $\begin{array}{ll}\text { HBB; } & \text { GSTT1 } \\
\text { HBA1 } & \\
\end{array}$ \\
\hline $\begin{array}{l}\text { mitochondrial inner } \\
\text { membrane (GO:0005743) }\end{array}$ & $1 / 341$ & 0.14342 & 0.166549167 & -1.7749 & 3.181532604 & PDK4 \\
\hline $\begin{array}{l}\text { organelle inner membrane } \\
\text { (GO:0019866) }\end{array}$ & $1 / 360$ & 0.15084 & 0.169696037 & -1.777 & 3.151907437 & PDK4 \\
\hline $\begin{array}{l}\text { perinuclear region of } \\
\text { cytoplasm (GO:0048471) }\end{array}$ & $1 / 411$ & 0.17049 & 0.185984084 & -1.7534 & 2.949390315 & NPPA \\
\hline $\begin{array}{l}\text { mitochondrial membrane } \\
\text { (GO:0031966) }\end{array}$ & $1 / 457$ & 0.18786 & 0.193223696 & -1.7167 & 2.822023032 & PDK4 \\
\hline $\begin{array}{lr}\text { cytoplasmic } & \text { membrane- } \\
\text { bounded } & \text { vesicle } \\
\text { (GO:0016023) } & \end{array}$ & $1 / 492$ & 0.20086 & 0.200856006 & -1.7176 & 2.757096016 & PLA2G2A \\
\hline $\begin{array}{l}\text { endoplasmic reticulum } \\
\text { membrane (GO:0005789) }\end{array}$ & $1 / 449$ & 0.18486 & 0.193223696 & -1.6636 & 2.734740612 & PLA2G2A \\
\hline
\end{tabular}


Supplemental Table 21. Gene ontology results for "molecular function" from Ingenuity

Pathway Analysis

\begin{tabular}{|c|c|c|c|c|c|c|}
\hline Term & Overlap & P-value & $\begin{array}{l}\text { Adjusted P- } \\
\text { value }\end{array}$ & Z-score & $\begin{array}{l}\text { Combined } \\
\text { Score }\end{array}$ & Genes \\
\hline $\begin{array}{l}\text { oxidoreductase } \\
\text { activity, acting on } \\
\text { peroxide as } \\
\text { acceptor } \\
\text { (GO:0016684) }\end{array}$ & $3 / 42$ & 7.17024E-07 & 8.60429E-06 & $\begin{array}{l}- \\
2.456896157\end{array}$ & 28.65539296 & $\begin{array}{l}\text { HBB; } \\
\text { GSTT1; } \\
\text { HBA1 }\end{array}$ \\
\hline $\begin{array}{l}\text { peroxidase } \\
\text { activity } \\
\text { (GO:0004601) }\end{array}$ & $3 / 42$ & $7.17024 \mathrm{E}-07$ & $8.60429 \mathrm{E}-06$ & -2.4349774 & 28.39974903 & $\begin{array}{l}\text { HBB; } \\
\text { GSTT1; } \\
\text { HBA1 }\end{array}$ \\
\hline $\begin{array}{l}\text { oxygen transporter } \\
\text { activity } \\
\text { (GO:0005344) }\end{array}$ & $2 / 14$ & $1.6335 \mathrm{E}-05$ & $9.801 \mathrm{E}-05$ & - 2.775041681 & 25.6148578 & HBB; HBA1 \\
\hline $\begin{array}{l}\text { antioxidant } \\
\text { activity } \\
\text { (GO:0016209) }\end{array}$ & $3 / 70$ & $3.39748 \mathrm{E}-06$ & $2.71798 \mathrm{E}-05$ & $-\overline{2.280737145}$ & 23.97747229 & $\begin{array}{l}\text { HBB; } \\
\text { GSTT1; } \\
\text { HBA1 }\end{array}$ \\
\hline $\begin{array}{l}\text { oxygen binding } \\
(\mathrm{GO}: 0019825)\end{array}$ & $2 / 37$ & 0.000118911 & 0.000570771 & $\begin{array}{l} \\
2.395657228\end{array}$ & 17.89202107 & HBB; HBA1 \\
\hline $\begin{array}{l}\text { hormone activity } \\
\text { (GO:0005179) }\end{array}$ & $2 / 122$ & 0.001291935 & 0.00516774 & $-\overline{2.332161538}$ & 12.27957659 & $\begin{array}{l}\text { NPPB; } \\
\text { NPPA }\end{array}$ \\
\hline $\begin{array}{l}\text { tetrapyrrole } \\
\text { binding } \\
\text { (GO:0046906) }\end{array}$ & $2 / 146$ & 0.001842388 & 0.005527164 & - 2.271718933 & 11.80857763 & HBB; HBA1 \\
\hline $\begin{array}{l}\text { heme binding } \\
\text { (GO:0020037) }\end{array}$ & $2 / 137$ & 0.001624924 & 0.005527164 & - 2.266033372 & 11.77902362 & HBB; HBA1 \\
\hline $\begin{array}{l}\text { iron ion binding } \\
\text { (GO:0005506) }\end{array}$ & $2 / 172$ & 0.00254417 & 0.006784455 & -2.29969838 & 11.48267312 & HBB; HBA1 \\
\hline $\begin{array}{l}\text { glutathione } \\
\text { peroxidase } \\
\text { activity } \\
(\mathrm{GO}: 0004602)\end{array}$ & $1 / 18$ & 0.00807251 & 0.01614502 & $\begin{array}{l}- \\
2.574585679\end{array}$ & 10.62311032 & GSTT1 \\
\hline $\begin{array}{l}\text { peptide hormone } \\
\text { receptor binding } \\
\text { (GO:0051428) }\end{array}$ & $1 / 17$ & 0.007625562 & 0.01614502 & $\begin{array}{l}- \\
2.364306114\end{array}$ & 9.755466632 & NPPA \\
\hline $\begin{array}{l}\text { glutathione } \\
\text { transferase } \\
\text { activity } \\
\text { (GO:0004364) }\end{array}$ & $1 / 25$ & 0.011196142 & 0.020669801 & -5.511179384 & 9.741069625 & GSTT1 \\
\hline $\begin{array}{l}\text { phospholipase } \mathrm{A} 2 \\
\text { activity } \\
\text { (GO:0004623) }\end{array}$ & $1 / 29$ & 0.012977146 & 0.022246536 & $-\overline{2.390870595}$ & 9.098622916 & PLA2G2A \\
\hline $\begin{array}{l}\text { calcium- } \\
\text { dependent } \\
\text { phospholipase A2 } \\
\text { activity } \\
\text { (GO:0047498) }\end{array}$ & $1 / 9$ & 0.004043525 & 0.00970446 & $-\overline{1.704169377}$ & 7.899114285 & PLA2G2A \\
\hline $\begin{array}{l}\text { transferase } \\
\text { activity, } \\
\text { transferring alkyl } \\
\text { or aryl (other than }\end{array}$ & $1 / 54$ & 0.024043964 & 0.038470342 & $-\overline{2.029011138}$ & 6.610249768 & GSTT1 \\
\hline
\end{tabular}




\begin{tabular}{|l|l|l|l|l|l|l|}
$\begin{array}{l}\text { methyl) groups } \\
\text { (GO:0016765) }\end{array}$ & & & & & \\
\hline $\begin{array}{l}\text { calcium ion } \\
\text { binding } \\
\text { (GO:0005509) }\end{array}$ & $2 / 698$ & 0.037207026 & 0.055810538 & 2.186146214 & 6.308764498 & $\begin{array}{l}\text { MYL7; } \\
\text { PLA2G2A }\end{array}$ \\
\hline $\begin{array}{l}\text { phospholipase } \\
\text { activity } \\
\text { (GO:0004620) }\end{array}$ & $1 / 90$ & 0.039786418 & 0.056169061 & 2.073175967 & 5.969480469 & PLA2G2A \\
\hline $\begin{array}{l}\text { lipase activity } \\
\text { (GO:0016298) }\end{array}$ & $1 / 105$ & 0.046278873 & 0.061705164 & -2.100801189 & 5.851545715 & PLA2G2A \\
\hline $\begin{array}{l}\text { carboxylic ester } \\
\text { hydrolase activity } \\
\text { (GO:0052689) }\end{array}$ & $1 / 111$ & 0.048864909 & 0.061724095 & 2.056264556 & 5.726863137 & PLA2G2A \\
\hline $\begin{array}{l}\text { hormone receptor } \\
\text { binding } \\
\text { (GO:0051427) }\end{array}$ & $1 / 160$ & 0.069752004 & 0.083702404 & 2.116892259 & 5.250924951 & NPPA \\
\hline $\begin{array}{l}\text { phospholipid } \\
\text { binding } \\
\text { (GO:0005543) }\end{array}$ & $1 / 296$ & 0.125604565 & 0.143548074 & 2.270665579 & 4.407555553 & PLA2G2A \\
\hline $\begin{array}{l}\text { protein } \\
\text { heterodimerization } \\
\text { activity } \\
\text { (GO:0046982) }\end{array}$ & $1 / 408$ & 0.169341136 & 0.184735784 & 2.209302288 & 3.73113304 & HIST1H2AC \\
\hline $\begin{array}{l}\text { protein } \\
\text { serine/threonine } \\
\text { kinase activity } \\
\text { (GO:0004674) }\end{array}$ & $1 / 449$ & 0.184858766 & 0.192896104 & 2.252252364 & 3.706314502 & PDK4 \\
\hline $\begin{array}{l}\text { ATP binding } \\
\text { (GO:0005524) }\end{array}$ & $1 / 1494$ & 0.5028586 & 0.5028586 & 2.111794514 & 1.451745246 & PDK4 \\
\hline
\end{tabular}




\title{
CHAPTER 3
}

Obese Zucker Rats Share Similar Sex- and BMI-Specific Gene-Expression Signature with Human Left Ventricular Hypertrophy

\author{
Mackenzie S. Newman ${ }^{1}$, Janelle Stricker ${ }^{1,2, \#, ~ H a n-G a n g ~ Y u^{1, *}}$
}

${ }^{1}$ Physiology and Pharmacology, West Virginia University, Morgantown, WV, USA

${ }^{2}$ Exercise Physiology, West Virginia University, Morgantown, WV, USA

\# present address: Pharmacology, California Institute for Biomedical Research, La Jolla, CA, USA 


\section{$\underline{\text { Abstract }}$}

Background: We recently identified in human cardiac hypertrophy a gene expression signature containing nine differentially-expressed genes (DEG) that are body-mass index (BMI) and sexdependent. To study mechanistic roles of these genes in hypertrophy, we asked whether these nine genes are differentially expressed in OZR known to develop cardiac hypertrophy.

Results: Rat LV were grouped according to sex (male, female) and obesity (obese, lean). OZR hypertrophy was characterized by increased left ventricular (LV) mass, LV inner diameter, interventricular septum, and LV wall thickness in a sex-specific manner. Altered electrophysiology in OZR was characterized by decreased resting heart rate, increased ST elevation, and suppressed sympathetic activity. 89 DEG were found when comparing all samples for obesity, but males alone had 2859 DEG and females had 826. Obese humans and rats shared 337 DEG. Five DEG in human LVH previously identified (Hbb, Hist1h2ac, Nppa, Nppb, Pdk4) were validated by qPCR and protein expression in OZR.

Conclusions: We identified a five-gene expression signature that is shared between rat and human LVH in an obesity and sex-specific manner. Expression of established biomarkers Anp/Nppa and Bnp/Nppb were already significantly increased in hypertrophy compared to

controls, with the absolute levels of Nppa significantly higher than Nppb. New genes (Hbb, Hist1h2ac, Pdk4) may provide potential new targets for early prognostic diagnosis in sexdependent obesity-related cardiac LVH.

\section{Background}

Obesity has been recognized as an independent risk for cardiovascular disease $(1,34)$. In fact, a high rate of sudden cardiac death in morbid obesity had been recognized in ancient times (13). High prevalence of sudden cardiac death also occurs in young obese people (7). For every 1 
$\mathrm{kg} / \mathrm{m}^{2}$ increase in body mass index (BMI), the HF risk is increased by $5 \%$ in men and $7 \%$ in women (37). In ventricular biopsy samples from obese patients, the number of adipocytes increases as the ejection fraction decreases (46). How these excessive adipocytes contribute to HF is unknown on the molecular level.

While HF is frequently the final state of cardiovascular disease, cardiac hypertrophy is a major independent predictor of progressive heart disease and increased mortality (15). Cardiac hypertrophy is also one of the most common independent features in obesity, even in the absence of hypertension or diabetes mellitus $(1,3,31,58,76,79)$. Myocyte hypertrophy has been found to be the most common cause of sudden cardiac death in morbid obese patients (19). Advances in studies of signaling pathways in both physiological and pathological hypertrophies have led to a recent proposal that aims to treat cardiac hypertrophy as a new therapeutic target $(10,25)$. Development of cardiac hypertrophy in obesity, especially in a sex-dependent manner, is an important question with significant clinical relevance.

Our previous study has found left ventricular myocyte hypertrophy in OZR, a common animal model for studies of obesity and metabolic syndrome (45). We have recently identified a sex-dependent gene expression signature for obesity-related human cardiac hypertrophy (56). To understand the genetic mechanisms that may mediate obesity-induced changes in cardiac electrophysiology and hypertrophy, we set out to identify an obese animal model associated with cardiac hypertrophy. We also noticed that almost all the previous studies used only male OZR. In this study, we examined the gene expression profile of OZR relative to the lean Zucker rats (LZR) in both sexes. We focused on genes that exhibited similar expression patterns from human heart, related to ventricular hypertrophy in obesity in a sex-dependent manner.

\section{Methods}




\section{Animals}

Lean and obese Zucker rats of both sexes (Charles River) were purchased at age of 8-10 weeks old. Experiments were performed when rats were at 14-17 weeks old. They were given access to food and water ad libitum. Proposed research using animals in this work was approved by West Virginia University Institutional Animal Care and Use Committee.

\section{Euthanasia and heart collection}

Animals were maintained for up to 10 days during the experiments. After echocardiography recordings, surgery was performed to insert the electrical leads. Recovery takes at least two days to warrant full recovery before electrocardiography recordings for up to 48 hours. Animals were sacrificed immediately following final electrocardiography data collection. Then, hearts were removed under deep anesthesia (3-5\% isoflurane), exsanguinated in Tyrode's solution, then flash-frozen and stored in liquid nitrogen until further RNA isolation and protein chemistry experiments.

\section{Echocardiography}

Echocardiography was performed using Vevo 2100 Micro-Ultrasound Imaging System (VisualSonics). An MS200 transducer (9-18 MHz) was used to obtain high-resolution heart images. Animals were anesthetized (2-3\% isoflurane) for 1-2 hours. Supplemental heat and close monitoring of temperature, pulse, and respiration were provided during study. LVH was identified as significantly increased left ventricular (LV) mass (animal body sized corrected, $\mathrm{M}=$ mode) (24). Other measured ventricular parameters included left ventricular internal diameter (diastole) $(L V I D, d)$, inter ventricular septum (diastole) $(I V S, d)$, and left ventricular posterior wall (diastole) $(L V P W, d)$.

\section{Telemetry Electrocardiography}




\section{Surgical procedure, Post-surgical recovery}

All survival surgeries were performed accordance with the WVU IACUC policy on Rodent Surgery and Post-Operative Care as well as the Pain and Distress Policy. Rats were weighed and anesthetized using an isoflurane (3-5\%) induction chamber. Sterile ophthalmic ointment was applied to the eyes of the animal to reduce corneal desiccation. Animals were shaved in relevant areas then transferred to the surgical area and externally heated with a temperature-controlled warming pad. Deep anesthesia was maintained with isoflurane. After disinfecting relevant areas with Betadine and $70 \%$ isopropanol, surgery was performed.

Animals were implanted with telemetry transmitters (Data Science Inc., St. Paul, MN) according to manufacturer's specifications. Briefly, a small midline incision was made below the xiphoid process to expose the abdominal muscles and a small pocket was made on the animals' left side, just slightly larger than the body of the transmitter. The pocket was then filled with sterile saline and the transmitter body inserted into the pocket. The ECG leads were then cut to allow for them to be placed subcutaneously while remaining flat along the muscle wall and with enough length to allow for normal animal movement. The red lead was then placed at the lowest rib and a 4-0 suture was used to secure the lead to the abdominal wall muscle. The clear lead was positioned on top of the right pectoral muscle. The leads were sutured into the subcutaneous muscle as described above. The skin was then sutured shut after gently cleaning up any excess blood and applying antibiotic ointment. Carprofen $(5 \mathrm{mg} / \mathrm{kg}$ subcutaneous) was administered parenterally to ensure pain relief without suppressing respiration. Animals were monitored for signs of pain twice daily post-surgery for five days and analgesics (stated above) were given during this period. Animals were then put on a 24-hour acquisition schedule to collect ECG data. 
Data acquisition and analysis were performed using the Ponemah 5.20 platform (Data Sciences International). A pair of lean and obese rats was studied simultaneously under identical conditions. Heart rate variability data analysis was performed according to procedures recommended directly by the manufacturer. ECG recordings of five minutes in length were isolated if they contained no signs of inconsistencies in the data or arrhythmias. Data were interpolated at $20 \mathrm{~Hz}$ and the Hanning method was applied for windowing. Default rate frequency bins were used, VLF (very low frequency): 0.05-0.25, LF (low frequency): 0.25-1, HF (high frequency): 1-3. LF and HF data were normalized to the sum of original LF and HF data. Graphs were produced by inserting raw R-wave interval (R-R) data from Ponemah into Kubios HRV Standard v. 3.0.0 $(72,73)$.

\section{Transcriptome (RNA-Seq), bioinformatics analysis, and RT-qPCR}

Total RNA was isolated using an RNA Fibrous tissue miniprep kit (Qiagen). Quality of RNA was tested and passed with (1) Nanodrop for purity (OD260/OD280), (2) Agarose gel electrophoresis for RNA degradation and potential contamination, and (3) an Agilent 2100 Bioanalyzer for RNA integrity. Only samples that had RNA Integrity Number (RIN)>7.0 were submitted for sequencing.

After the QC procedures, mRNA was enriched using oligo (dT) beads and then fragmented randomly in fragmentation buffer, followed by cDNA synthesis using random hexamers and reverse transcriptase. After first-strand synthesis, a custom second-strand synthesis buffer (Illumina) was added with dNTPs, RNase H and Escherichia coli polymerase I to generate the second strand by nick-translation. The final cDNA library was generated after purification, terminal repair, A-tailing, ligation of sequencing adapters, size selection, and PCR enrichment. Library concentration was first quantified using a Qubit 2.0 fluorometer (Life Technologies), and 
then diluted to $1 \mathrm{ng} / \mu \mathrm{l}$ before checking insert size on an Agilent 2100 and quantifying to greater accuracy by quantitative PCR (qPCR) (library activity $>2 \mathrm{nM}$ ).

Single-end sequencing with 20 million raw reads was performed using HiSeq ${ }^{\mathrm{TM}}$ PE150 (Illumina). The raw data were transformed to sequenced reads by base calling and converted to FastQ using onboard instrument software. Reads were mapped to human reference genome (hg38) using TopHat2 (39).

Differential expression analysis was performed with NOISeq (v.2.14.1) (71) using RStudio version 0.99.879 (65). NOISeq is a newly-developed tool for differential expression analysis. Compared to the commonly used DeSeq (2), NOISeq offered a set of tools for better quality control to avoid false positive discoveries (71). Gene annotation information was obtained from the Ensembl Biomart database, release 85 (80). Gene expression levels are indicated by FPKM (fragments Per Kilobase of transcript per Million mapped reads) (74). FPKM was then normalized for batch effect using the ARSyNseq module included with the NOISeq package. Data were analyzed using the noiseqbio method under default conditions. The CPM filtering method was used for differential analyses where at least one group contained five or fewer replicates; otherwise, the Wilcoxon test was used for filtering.

RT-qPCR was run on either a BioRad CFX96 Real Time System or Applied Biosystems 7500 using a Luna Universal One-Step RT-qPCR Kit (NEB E3005L) according to the manufacturer's instruction. Briefly, components were prepared for a $20 \mu \mathrm{L}$ reaction volume per well using 100-300ng template RNA and primers at $10 \mu \mathrm{M}$ concentration (primer sequences are listed in Supplemental Table 1). Each sample was prepared as two technical replicates, with three biological replicates total per group. GAPDH was used as a loading control on each plate. Reverse transcription was run at $55^{\circ} \mathrm{C}$ for 10 minutes, followed by one cycle of initial 
denaturation at $95^{\circ} \mathrm{C}$. This was followed by 40 cycles of $95^{\circ} \mathrm{C}$ denaturation $(10$ seconds) and $60^{\circ} \mathrm{C}$ extension ( 30 seconds). After the plate was read, melt curves were recorded using a $0.5^{\circ} \mathrm{C}$ step from $60-95^{\circ} \mathrm{C}$.

Gene expression levels were plotted in $\log 2$-fold (y-axis) from both transcriptome and qPCR.

\section{Immunoblotting}

Tissues sections were submerged in minimal lysis buffer (fresh protease and phosphatase inhibitors (Sigma), $20 \mathrm{mM}$ Tris, $150 \mathrm{mM} \mathrm{NaCl}, 10 \mathrm{mM}$ EGTA and 10mM EDTA at $\mathrm{pH} 7.4$ ) on ice and homogenized briefly at high speed. Samples were then centrifuged for 15 minute increments at $10,000 \mathrm{x}$ g to pellet debris. Supernatants were placed into new tubes and protein concentration was recorded using Bradford's method on an Eppendorf Biophotometer.

For Western blotting procedures, protein concentrations were normalized between samples to $10 \mu \mathrm{g}$ and mixed with Non-Reducing Lane Marker (Thermo Scientific) with $5 \% \beta$ mercaptoethanol. After heating in a water bath to $95^{\circ} \mathrm{C}$ for five minutes, samples were cooled to $4^{\circ} \mathrm{C}$ then loaded into a $4-12 \%$ bis-tris gel (Invitrogen). Electrophoresis was carried out at $80 \mathrm{~V}$ for 30 minutes then $140 \mathrm{~V}$ for the remainder.

Proteins were transferred to pre-wetted nitrocelluose membranes at $30 \mathrm{~V}$ for one hour. Blots were blocked with 3\% BSA-V in TBS-T for one hour before primary antibody (1:1000 dilution; Cell Signaling) was added on a shaker at $4^{\circ} \mathrm{C}$ overnight. Primary antibody solution was replaced with fresh $3 \%$ BSA-V in TBS-T containing secondary antibodies at 1:10,000 dilution for one hour at room temperature on a shaker. After five washes with TBS-T, blots were developed with a standard ECL kit (Life Technologies) on x-ray film or using a G:BOX digital imaging system (Syngene). 


\section{Statistics}

Data are presented as mean \pm SD. Student's $t$ test was used for statistical analysis on paired groups, with $p<0.05$ being considered as statistically significant, marked with the symbol *. One-way ANOVA with Tukey's post-hoc test was used for statistical analyses on more than two groups. Blinding and randomization was not performed during in vivo analysis due to the small number of animals in the study. For gene expression, gene size adjusted p-value (FDR false discovery rate) less than 0.05 was used (p_adj<0.05) to identify significant genes.

\section{$\underline{\text { Results }}$}

\section{Characterization of OZR}

Table 1 shows that in both sexes, body and heart weight in OZR are significantly increased compared to age-matched LZR. Echocardiogram (Echo) recordings revealed that although heart rate is decreased in OZR, it is statistically not significantly altered compared to lean controls of both sexes. On the other hand, left ventricular (LV) mass was significantly increased in OZR compared to LZR in both sexes: $32.7 \%$ increase in male and $59.1 \%$ increase in female, consistent with the enlarged heart. In comparison to LZR, the interventricular septum at diastole $(I V S ; d)$ was increased in OZR, but reached statistically significance only in female. Left ventricular internal diameter at diastole $(L V I D ; d)$ was increased by $24.2 \%$ in male OZR, but the increase in female OZR was not statistically significant compared to LZR. Left ventricular posterior wall thickness at diastole $(L V P W ; d)$ thickness was increased in OZR in OZR compared to their lean controls. We found no changes in OZR contractility since both ejection fraction (EF) and fraction shortening (FS) were not statistically significant.

\section{Altered heart rate, ST elevation, and heart rate variability in OZR from ECG recordings}


To investigate potential electrical abnormalities in OZR, we performed telemetry ECG recordings on paired OZR and LZR. A representative set of recordings is shown in Figure 1. In addition to apparent slowing in heart rate, the R-wave amplitude is larger in OZR than in LZR, especially in male OZR compared to male LZR (1A), which is a strong indicator of LVH (12, 17). However, averaged peaks of R-wave between obese and lean rats showed no statistical difference, although R-wave mean value in OZR-M is more than doubled than in LZR-M (OZRM: 0.862 , LZR-M: $0.367, p=0.065, n=4$. OZR-F: $0.761 \pm 0.416$, LZR-F: $0.696 \pm 0.158, p=0.813$, $\mathrm{n}=4)$

Table 2 summarized the most significant changes from ECG recordings. In comparison to age-matched male LZR controls, resting heart rate was significantly decreased, while ST elevation (STe) was significantly increased in male OZR. These changes were not observed in female OZR.

Heart rate variability also showed sex-dependent changes. To eliminate large intersubject variability in the total raw heart rate variability spectral power, we used normalized frequency power $(11)$. Normalized low-frequency $(\mathrm{nLF})$ power $(11,21)$ were decreased by $67 \%$ in male and by $40 \%$ in female obese rats compared to LZR controls, respectively. Normalized high-frequency (nHF) power was increased by $110 \%$ in male and by $57 \%$ in female obese rats. The ratio of LF/HF was decreased by $61 \%$ in female, but insignificantly decreased by $86 \%$ in male obese rats (possibly due to small number of animals).

\section{Identification of Differentially Expressed Genes (DEG) in OZR}

When comparing obese to lean left ventricular samples, 89 DEG (75 down, 14 up) associated with LVH were found (Figure 2). However, when female and male samples were separated, 826 female DEG (138 down, 688 up in obesity) and 2859 male DEG (1239 down, 
1620 up in obesity) were identified (Figure 2). There are 43 DEG that were upregulated in both sexes, whereas 253 DEG downregulated in both, and another 83 that were up in one sex and down in the other (Supplemental Table 2).

PCA plots to show cohesion of males and females are shown in Supplemental Figure 1. Detailed upregulation and downregulation of these DEG are shown in the volcano plots (Supplemental Figure 3) and heatmaps (Supplemental Figure 3).

Shared Differentially Expressed Genes between rat and human obesity-related cardiac hypertrophy

We have recently identified an RNA expression signature in LV for obesity-related human cardiac hypertrophy from 12 males and 12 females (Male BMI: 31.08 \pm 12.49 (LVH), $\mathrm{n}=6$; 25.93 \pm 6.57 (non-failure, NF, controls), $\mathrm{n}=6$. Female BMI: 27.78 $\pm 9.92(\mathrm{LVH}), \mathrm{n}=6 ; 28.13 \pm 7.02$ (NF controls), $\mathrm{n}=6$. Male age: 48.17 $\pm 14.54(\mathrm{LVH}), \mathrm{n}=6 ; 48.83 \pm 12.38$ (NF controls), $\mathrm{n}=6$. Female age: $43.83 \pm 11.72(\mathrm{LVH}), \mathrm{n}=6 ; 48.00 \pm 15.91$ ( $\mathrm{NF}$ controls), $\mathrm{n}=6)$ (56). The expression signature contains nine genes (HBA1, HBB, HIST1H2AC, GSTT1, MYL7, NPPA, NPPB, PDK4, PLA2G2A) with altered expression levels in a BMI- and sex-dependent manner (56). We compared expression profiles between rat and human heart samples and identified 337 shared DEG. In female hypertrophied samples (Figure 3A), 6 DEG are upregulated in both rat and human (lower left), 27 DEG are downregulated in both rat and human (upper right), 5 DEG are upregulated in rat but downregulated in human (lower right), and 55 DEG are downregulated in rat but upregulated in human (upper left). In male hypertrophied samples (Figure 3B), 28 DEG are upregulated in both rat and human (lower left), 22 DEG are downregulated in both rat and human (upper right), $8 \mathrm{DEG}$ are upregulated in rat but downregulated in human (lower right), and 193 DEG are downregulated in rat but upregulated in human (upper left). 
Among the nine-gene expression signature (HBA1, HBB, HIST1H2AC, GSTT1, MYL7, NPPA, NPPB, PDK4, PLA2G2A) identified in obesity-related human cardiac hypertrophy (56), seven DEG (Nppa, Nppb, Gstt1, Hbb, Hba1, Myl7, Pdk4) are potential DEG found in OZR compared to LZR in a sex-dependent manner. Figure 4 compares the expression levels of seven DEG in rat and human LVH. NPPA and NPPB expression levels were significantly increased in LVH of both rat and human of either sex. However, NPPA levels are notably higher than NPPB in rat and human of either sex. HBB gene expression was also drastically upregulated in rat and human LVH, but only in female. Upregulation of PDK4 is more significant in female than in male in both rat and human. MYL7 was upregulated in male but downregulated in female human LVH. In rats, Myl7 expression levels were significantly increased in females, inconsistent with that in human LVH. In human, GSTT1 and HBA1 levels were very low compared the other DEG.

Validation of differentially expressed genes shared in OZR and obese human hypertrophied heart

We set out to validate six DEG expression using $\mathrm{qPCR}$ and protein expression using western blotting. We did not include Hbal due to very low expression in human heart (Figure 4 bottom) (but dramatically high expression in rat heart (Figure 4 top)) and we could not detect protein expression in human heart. We included Gstt1 because it has a similar low expression pattern in both rat and human heart.

Figure 5A indicates a significant increase in Nppa transcript levels in OZR of both sexes from transcriptome experiment. Quantitative PCR experiments confirmed the statistically significant increase in male OZR, but not in female OZR (Figure 5B). At protein expression levels, Anp are remarkably higher in obese than in lean rats of both sexes (Figure 5C). The 
results are statistically significant (male: $\mathrm{p}=0.007, \mathrm{n}=3$; female: $\mathrm{p}=0.01, \mathrm{n}=3$ ) (Figure 5D), similar to human ANP protein expression patterns (56).

Figure 6A shows Nppb transcriptional level from transcriptome experiment, suggesting a higher expression in obese than in lean rats of both sexes, which was confirmed by quantitative PCR (Figure 6B). However, immunoblots (Figure 6C) did not reveal statistical difference in protein expression levels in either sex (Figure 6D) $(\mathrm{p}>0.05, \mathrm{n}=3)$.

Figure 7 shows Gstt1 transcript levels that are not significantly altered in OZR of both sexes (upper panel). Quantitative PCR also revealed insignificant changes of gene expression levels between OZR and LZR in both sexes (lower panel). We did not perform immunoblot experiments due to lack of justification.

Figure 8A shows an increase in Hbb transcriptional level only female OZR over LZR, which was confirmed by quantitative PCR (Figure 8B). However, immunoblots (Figure 8C) suggested that the protein expression levels are increased in OZR compared to LZR in both sexes (Figure $8 D$ ), which are statistically significant (male: $p=0.007, n=3$; female: $p=0.0003, n=3$ ).

Figure 9A shows a dramatic increase in Myl7 transcript levels in OZR compared to LZR in both sexes. However, quantitative PCR failed to validate increased gene expression, although very close in female OZR (Figure 9B) $(\mathrm{p}=0.0608)$. Immunoblots (Figure 9C) confirmed a significantly increased protein expression in female OZR compared to female LZR (Figure 9D) (OZR-F: 1.693, LZR-F: 0.769, $\mathrm{p}<0.05, \mathrm{n}=3$ ).

Figure 10A shows an increase in Pdk4 transcript levels in OZR compared to LZR in both sexes. Quantitative PCR validated these increases at gene expression levels (Figure 10B). Immunoblots (Figure 10C) confirmed an increased protein expression in female OZR, but not in male OZR, compared to LZR (Figure 10D) (male: $p=0.092, n=3$; female: $p<0.0001, n=3$ ). 


\section{Sex- and obesity-dependent NPPA/NPPB expression}

Figure 11A shows that NPPA gene expression levels are higher in obese female than in obese male rat and human with LVH. NPPB levels are higher only in obese female human compared to obese male human LVH; its levels are lower in obese female than in obese male rat. Figure 11B shows that in addition to LVH-mediated increase in expression of both NPPA and NPPB in human heart, obesity is associated with a significantly decreased expression of NPPB in both sexes, decreased expression of NPPA in male, but increased expression of NPPA in female human with LVH.

\section{$\underline{\text { Discussion }}$}

Obesity drastically increases the risk of sudden cardiac death and HF, with severity varying in a sex-specific manner. Cardiac hypertrophy is an independent feature from obesity but is the most common cause of sudden cardiac death in morbidly obese patients. Discovery of cross-species DEG validates an animal model to study how individual genes may contribute to sex-specific obesity-related cardiac hypertrophy in vivo. Novel biomarkers of cardiac hypertrophy should allow early intervention to delay or even prevent sudden cardiac death and HF.

In the present work, we investigated gene expression profiles of LV from OZR and LZR to test a hypothesis that OZR can serve as an animal model to study sex-specific obesity-related cardiac hypertrophy.

\section{Echocardiography and electrophysiological characterizations of OZR}

Enlargement of heart associated with obesity in Zucker rats can be detected at 12-weeks old and established in 14 weeks old of age (62). Our data on heart weight and LV mass in OZR of 14-17 weeks of age showed significant hypertrophy in both sexes, which is consistent with the 
cellular hypertrophy in OZR we previously reported (45). Although female hearts are smaller and lighter compared to males, the percent increase in LV mass is larger in female (59\%) than in male (33\%) (Table 1). The LVID is significantly increased in male OZR over LZR, but not in female OZR/LZR, although there is a tendency to increase. An increased IVS was found in female, but not in male, OZR compared to the respective LZR controls. OZR of both sexes showed an increased LVPW.

OZR has been known to have dysfunctional cardiac functions such as reduced contractility (81). However, we do not detect statistically significant changes in this study. Studies showing reduced contractility used old OZR (e.g. 20 weeks) (81), while younger OZR (e.g. 10-12 weeks old) do not exhibit altered contractility $(40,51)$. It is possible that while there is no significant difference in contractility between obese and lean rats at the tissue or wholeheart level, depressed contraction at the level of myocytes has already occurred (62).

ECG recordings showed that in comparison to LZR the resting heart rate was decreased in male but not in female OZR, although there is a statistically insignificant decrease in female OZR. ST elevation was also found to increase only in male OZR (Table 2). In OZR of both sexes LF (a combination of sympathetic and parasympathetic activities) was decreased, while HF (predominately vagal modulation) was increased, in agreement with reduced sympathetic baroreflex sensitivity in OZR due to leptin receptor dysfunction $(14,28,33,60)$. Increased HF component is more significant in male than in female OZR (Table 2). Reduced ratio of LF/HF further supported that the sympathetic tone in the obese heart was suppressed (21). It has been well established that reduced heart rate variability is a powerful independent risk factor for cardiovascular diseases such as hypertrophic cardiomyopathy and sudden cardiac death $(16,35$, 
$41,42,57)$, type 2 diabetes and metabolic disorders $(6,70)$, as well as in the general population $(63,75)$.

Differentially expressed genes associated with sex-specific obesity-related cardiac hypertrophy

It is not surprising for significantly increased Anp expression levels in both sexes of OZR compared to LZR, similar to what we found in obesity-related human cardiac hypertrophy (56). Consistent with human data, the Anp increase in LVH is significantly larger than Bnp, which has been clinically used as biomarker for diagnosis of $\operatorname{HF}(5,22,54,61)$.

ANP can directly modulate all voltage-dependent ion channels known to control heart rate and action potential duration $(52,59)$, yet, yields inconsistent results in experimental models and in humans. Nonetheless, ANP was found anti-arrhythmic, since a protein degradationresistant mutant can trigger atrial fibrillation (32). ANP can potentiate isoproterenol stimulation of the L-type voltage-gated calcium channel, while mutant ANP cannot (32).

While the mechanistic roles of ANP in cardiac electrophysiology and especially on ion channels remain to be clarified, the roles of ANP in hypertrophy and HF has been extensively studied and well defined $(38,61,69,77)$. ANP is predominantly released from atria, while it was later found also present in the ventricle (A:V ratio 40:1) (27). The synthesis of ANP is dramatically increased in the ventricle in response to hypertrophy, making it one of the surrogate markers (other than BNP) in advanced HF patients $(54,69)$. It was noted that the total amount of ANP in the failing ventricles is about 9-fold higher than BNP (ANP: $135 \pm 31 \mathrm{pmol} / \mathrm{g}$; BNP: 16.9 \pm 4.2$)$ (54). In Anp knockout mice, overload volume-induced cardiac hypertrophy was worsened, indicating that Anp protects the heart from hypertrophy development (53). Additionally, Anp protection is independent of blood pressure. Protective roles of ANP and BNP 
have recently been found in human studies. Increased LV mass and wall thickness were found in patients who carried allele $664 \mathrm{C}>\mathrm{G}$ in the ANP promoter, which results in lower levels of circulating ANP (66). On the other hand, patients with the rs5068 allele at the NPPA-NPPB locus, which results in higher circulating levels of ANP and BNP, had reduced LVH (36).

Consistent with our previous findings in obese human hypertrophied hearts (56), ANP expression levels are significantly increased in OZR of both sexes compared to LZR. Particular, ANP gene expression levels are higher in female than in male LZR and OZR, respectively (Figure 5), also in agreement with our previous findings in obese human hypertrophied heart (56). Unlike human hypertrophied heart, significant increases in BNP gene expression levels were found only in male OZR, which failed to be validated in protein expression (Figure 6). In both obese rat and human hypertrophied heart of both sexes, ANP expression levels are significantly higher than BNP. Thus, ANP not only can be used as an early biomarker in obesityrelated cardiac hypertrophy, but also a potential target to study how ANP affects cardiac electrophysiology during early development of hypertrophy under obesity condition.

Interestingly, we found several genes that were previously understudied or unknown with regard to their respective roles in cardiac hypertrophy and arrhythmias (Figure 4). In female (but not male) obese human hypertrophied heart, HBB expression was upregulated (56). In female OZR, Hbb gene expression was significantly upregulated compared to female LZR (Figure 8). Interestingly, immunoblots confirmed upregulated protein expression in both male and female OZR. The Hbb gene encodes $\beta$-globin, a subunit of hemoglobin. Its upregulation along with $\alpha$ globin (encoded by Hbal gene) may help explain the increased oxygen transport to peripheral tissues in obesity-related cardiac hypertrophy. 
Myosin light chain 7 (MYL7) was identified as a DEG in female (but not male) obese human hypertrophied heart (56). In OZR, Myl7 gene expression was significantly increased in both sexes, particularly in female (64-fold). Both quantitative PCR and immunoblots validated its upregulated expression in female OZR, in agreement with human data. MYL7 encodes myosin regulatory light chain protein, atrial isoform (MLC2a) in humans. Its expression is restricted to atria in healthy individuals, modulating cardiac development and contractility (23). In hypertrophic cardiomyopathy, MYL7 expression is readily detected in the ventricle (30). Its gene expression levels are very low in LZR of both sexes, but dramatically increased in OZR, consistent with its expression pattern in obese-related human cardiac hypertrophy (56).

We found PDK4 was significantly upregulated in male (but not female) obese human hypertrophied heart (56). In OZR, however, PDK4 was significantly upregulated in both male and male) heart (Figure 10). Its upregulation was validated by qPCR. PDK4 encodes a mitochondrial enzyme pyruvate dehydrogenase kinase 4. PDK4 downregulates glucose utilization and increases fat metabolism by decreasing glucose conversion to acetyl-CoA (43), resulting in a decreased production of ATP. Therefore, its upregulation may be resulted in increased demand for fat metabolism in obesity.

Currently, we do not know the biological roles of upregulated expression of Hbb, Myl7, and Pdk4, in association of obesity-related cardiac hypertrophy. Neither do we know whether these DEG might be used as potential new sex-dependent biomarkers for cardiac hypertrophy in obesity.

Unexpectedly, we did not detect the anticipated alterations of expression levels of genes that have been well documented in their contributions to development of cardiac hypertrophy and arrhythmia. Early study in mice identified that the expressions of 25 genes were altered in 
cardiac hypertrophy induced by angiotensin II and isoproterenol and that of 30 distinct genes were changed during regression of hypertrophy (26). Recently, using personalized and multiomics approaches in 100+ strains of genetically distance mice, 36 differentially expressed genes were identified (67). Microarray studies in a young hypertrophied heart rat model in the absence of hypertension, 65 genes altered their expression levels, and significantly more genes (390) changed their expression levels in the old hypertrophied heart (20). Notably, the Ras/mitogenactivated protein kinase (MAPK) signaling pathway and the tumor necrosis factor (TNF) receptor-mediated activation of nuclear factor- $\mathrm{B}(\mathrm{NF}-\kappa \mathrm{B})$ were found to play a crucial role in the development of hypertrophy (20). In human patients with cardiac hypertrophy, PCR screening found 35 (the article stated 36) genes were increased compared to normal human heart (44).

It is imperative to point out that hypertrophy has diverse phenotypes revealed by cardiac magnetic resonance imaging $(47-49,68)$. Pathologic hypertrophy caused by genetic mutations in sarcomere proteins are rare, representing $0.6 \%$ of prevalence in 3,600 unrelated subjects from the Framingham Heart Study and Jackson Heart Study cohorts $(8,68)$. The majority $(88.8 \%)$ of hypertrophic patients have benign mutations or variant of uncertain significance $(8,68)$. Cellular hypertrophy should occur earlier than changes in phenotype detected by echocardiography or cardiac magnetic imaging. Thus, altered gene expression patterns are important early markers during development of hypertrophy.

It is interesting to notice that obesity seems to inhibit gene expression of human NPPA and NPPB, except that in female with LVH obesity increased gene expression of NPPA (Figure 11B). Currently, we do not know the mechanism and how significant it may be in clinical settings. 


\section{Genes related to cardiac arrhythmias}

Electrical disturbance can be triggered by heart remodeling such as hypertrophy. Alterations of gene expression levels of membrane ion transporters including ion channels, pumps, and exchangers cause ionic imbalance which can trigger arrhythmias. Studies from experimental animal models demonstrated that ion channel remodeling occurs preceding clinical hypertrophy phenotype is diagnosed (9), since properties of ion channels can be altered when myocytes are enlarged (cellular hypertrophy).

We did not find changes in expression of genes that encode ion channels controlling the heart rate, such as hyperpolarization-activated, cyclic-nucleotide modulated (HCN) and voltagegated calcium channels (Cav1.2, Cav3.1) (64). Lack of heart rate-related ion channel gene expression changes supports the notion that the decreased heart rate in OZR is more likely caused by post-translational modulation under the hypertrophy conditions. Clinically, elevated ST segment is frequently associated with acute ischemic conditions such as acute coronary syndrome (29), however, it often occurs in healthy male individuals (78). In this study, STe was increased with statistical significance only in male OZR, but not in female OZR. ST elevation is associated with myocardial infarction (55). Recently, ST elevation is found to also occur in LVH (55) and in obesity $(4,18)$. The likely explanation for increased STe in male OZR is the LVHrelated cardiovascular dysfunction (50), female OZR may be more resistant to ischemic conditions at this age group (14-17 weeks).

\section{Limitations of the study}

The sample size is small (four animals per each group), which may have caused insignificant changes in expression levels for many genes related to cardiac hypertrophy and arrhythmias as well as in many ECG and Echo parameters. However, the small sample size also 
provided us an opportunity to focus on genes with significant alteration of expression levels. If confirmed in large samples of obese human cardiac hypertrophy, these genes (particularly the new genes) may represent novel targets for exploring early pharmacological treatment of sexspecific cardiac hypertrophy in obesity.

\section{Conclusions}

We identified five differentially expressed genes in LVH shared between OZR and human heart in obesity in a sex-dependent manner. ANP has the highest expression levels in LVH in both sexes. Future investigations of these five DEG may provide mechanistic insights in early development of hypertrophy in obesity in a sex-dependent manner. 


\section{Figures}

Figure 1: Representative ECG recordings of male (A) and female (B) Zucker rats
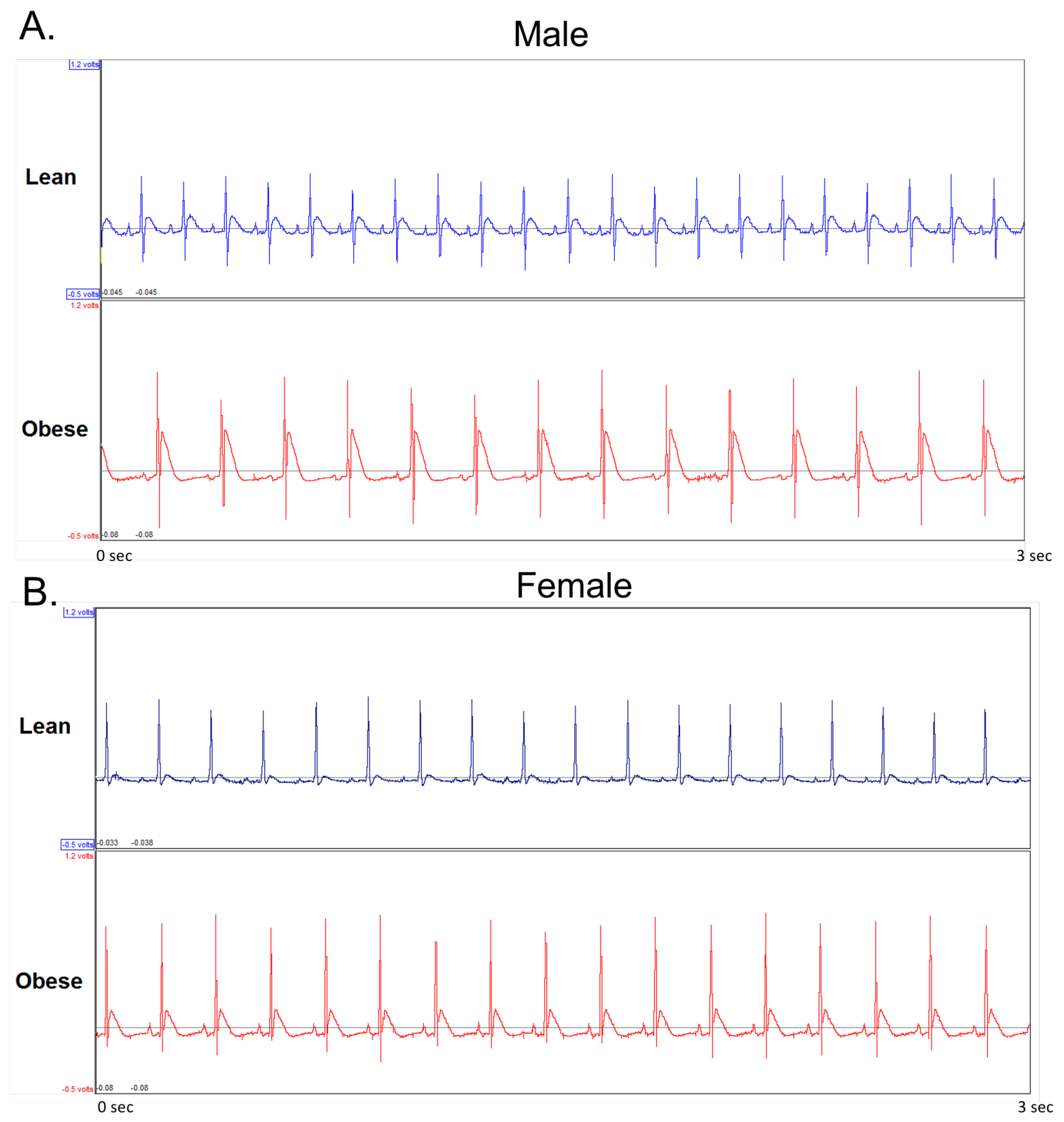

Figure 1A: Representative ECG data from males; B: Representative ECG data from females. Each $\mathrm{X}$-axis ranges from 0 to 3 seconds and each $\mathrm{Y}$-axis ranges from -0.5 to $1.2 \mathrm{~V}$. 
Figure 2: Sex-specific distribution of significant genes in Zucker rat LVH

\section{Significant Genes Distribution in LVH}

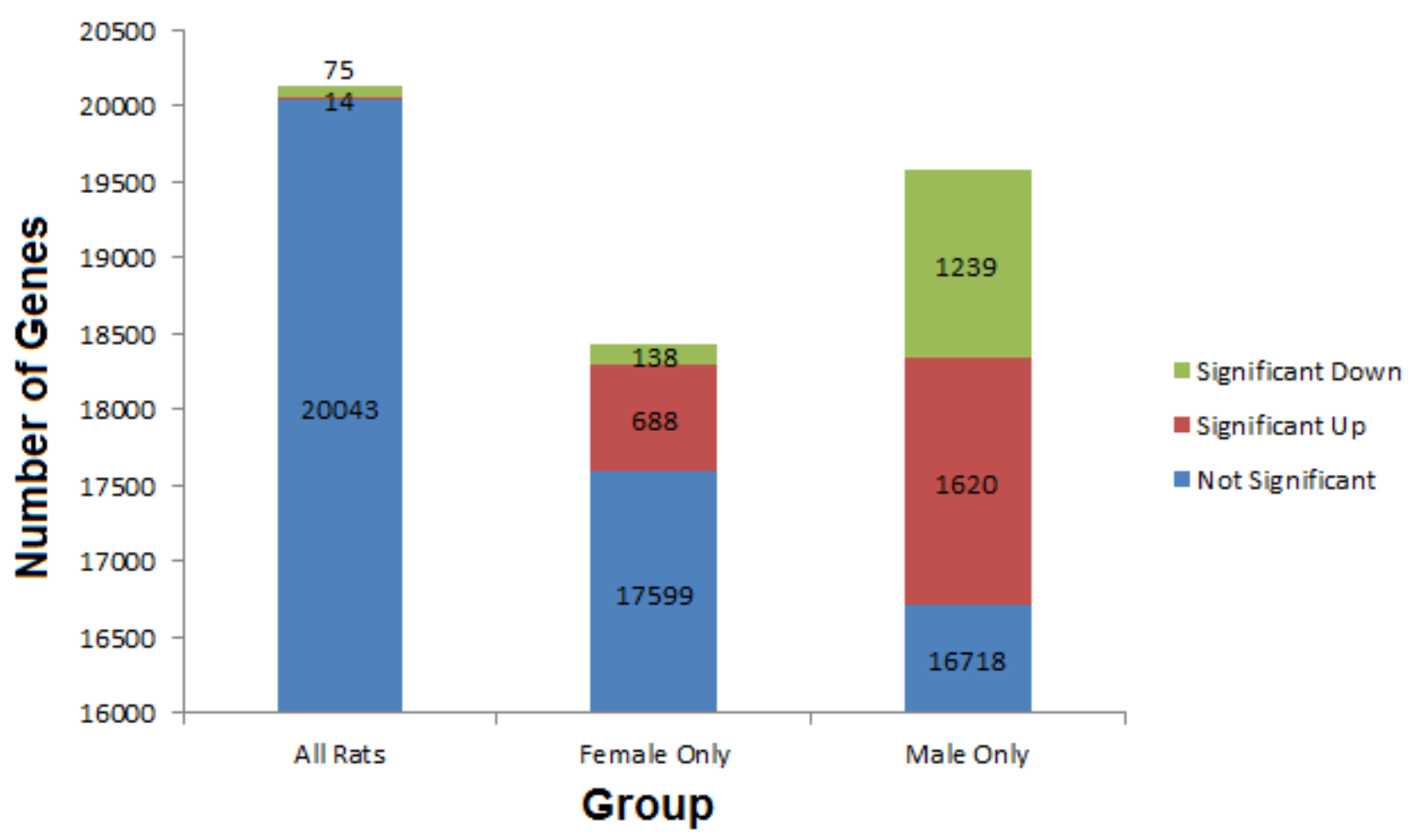

Figure 2. Significantly upregulated, downregulated, or not statistically significantly different total gene expression when comparing A) all rats; B) females only, and C) males only. The discrepancy in total number of genes between analyses is due to sex-specific expression: some genes are exclusively expressed in either males or females. If a gene had 0 expression in all samples, it was removed from analysis. 
Figure 3: Sex-specific differentially expressed genes shared between human and rat

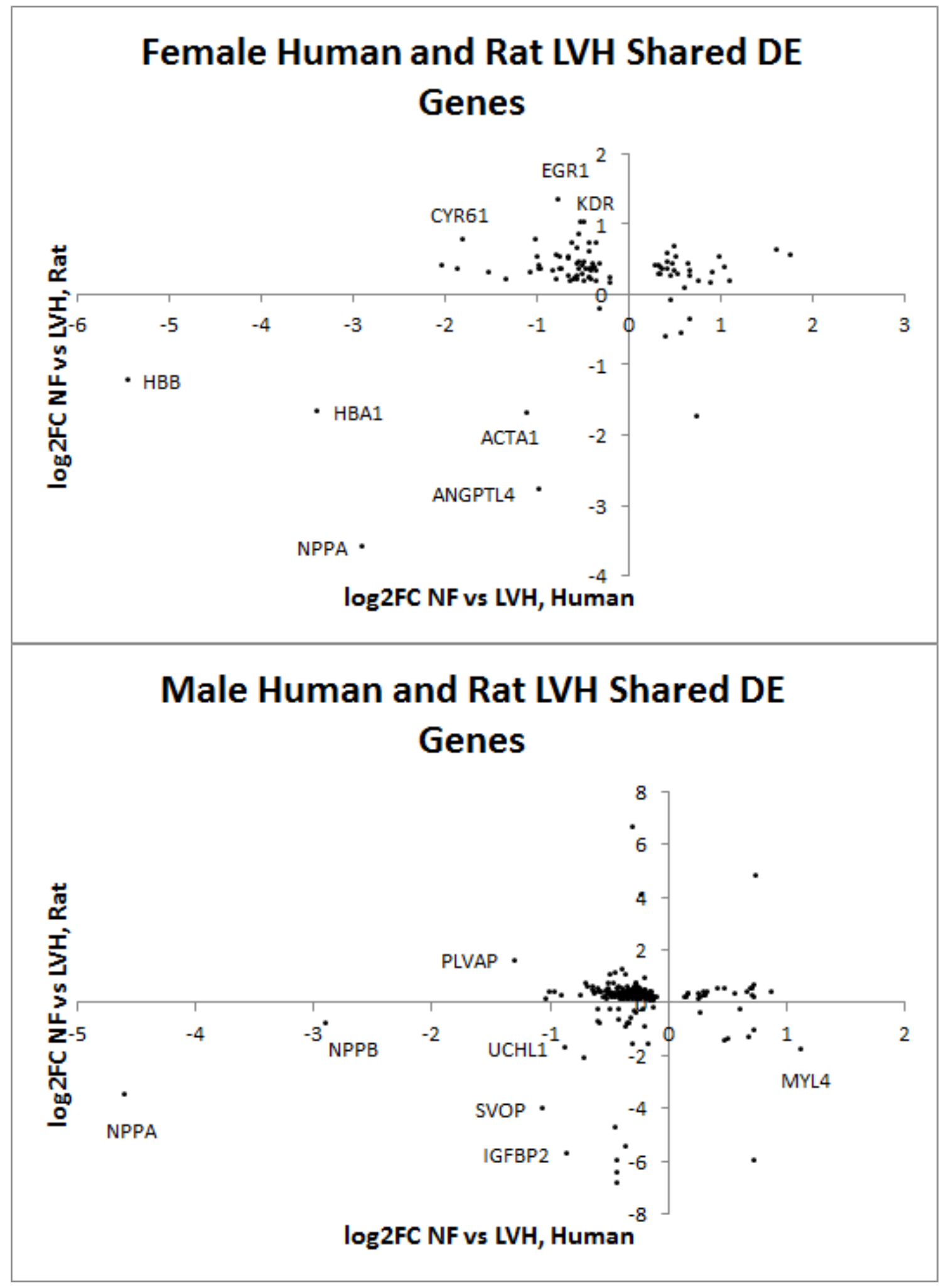


Figure 3. A: female, B: male. Bottom-left quadrants represent genes upregulated in human and rat LVH; upper-right quadrants represent genes downregulated in human and rat LVH; bottomright quadrants represent genes upregulated in rats but downregulated in humans; top-left quadrants represent genes upregulated in humans but downregulated in rats. 
Figure 4. Comparison of seven obesity- and LVH-related differentially expressed genes levels between human and rat.

\section{Rat 7 DEGs}
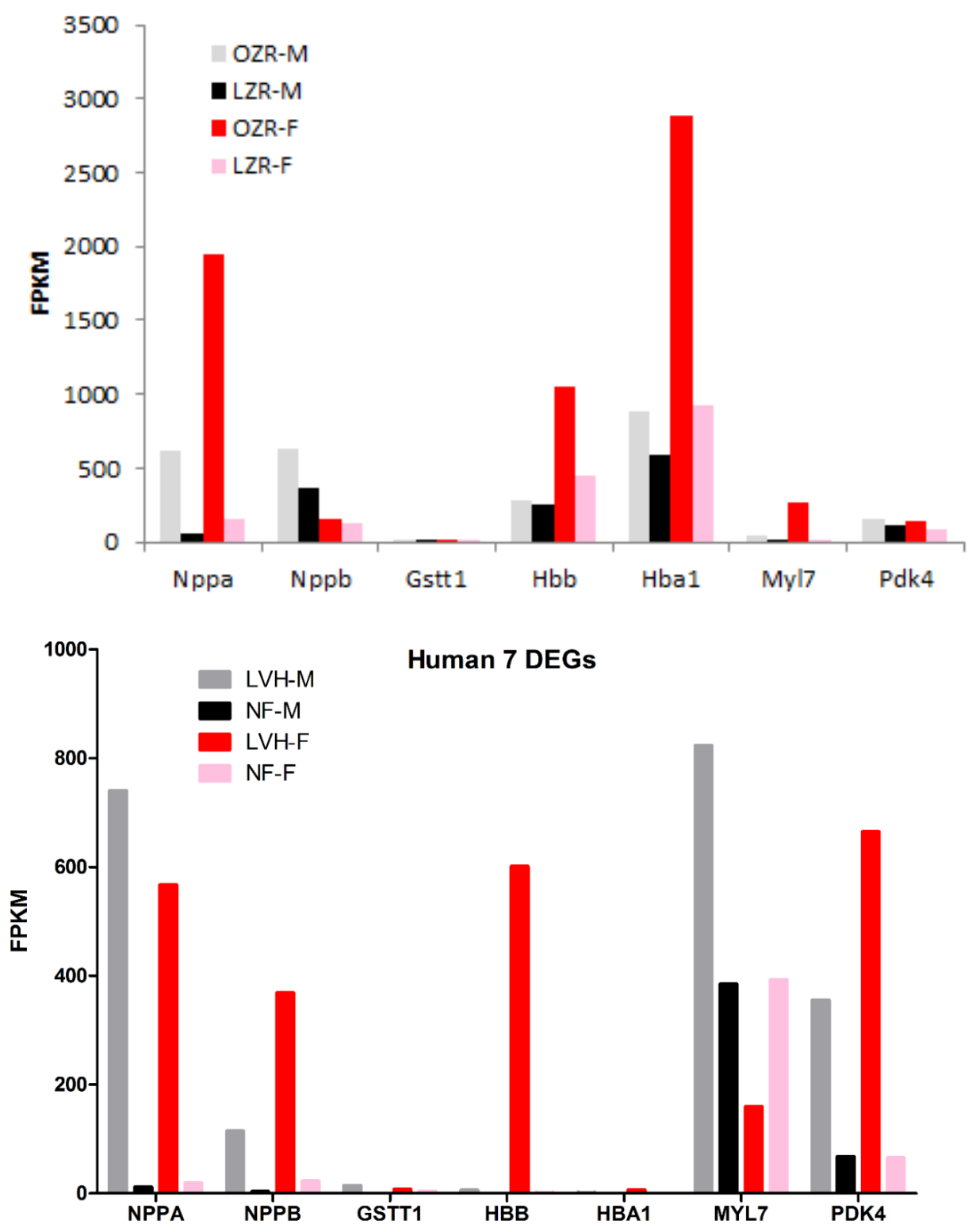

Figure 4. Gene panel transcript levels from transcriptome dataset in rats (top) and humans (bottom). The original nine-gene panel from humans was reduced to seven because two of the genes in humans were significant in neither male nor female Zucker rats. 
Figure 5: Nppa validation

A. Transcriptome

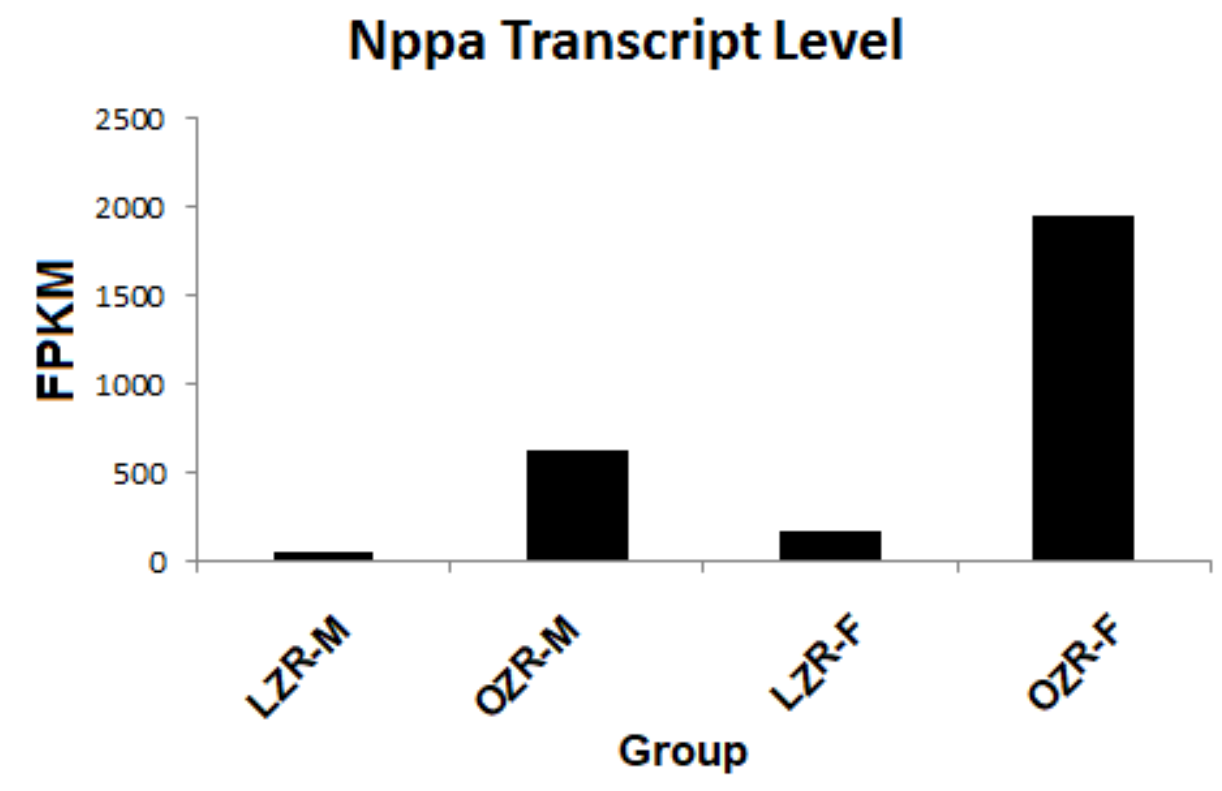

B. RT-qPCR

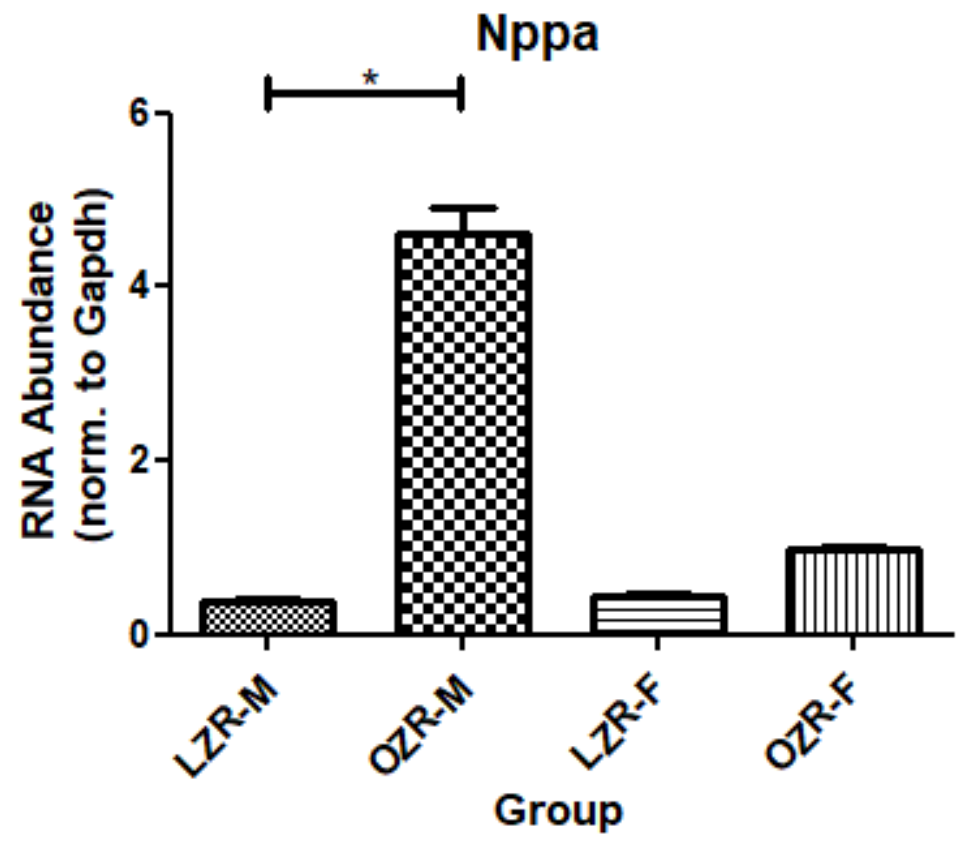


C. Western blots

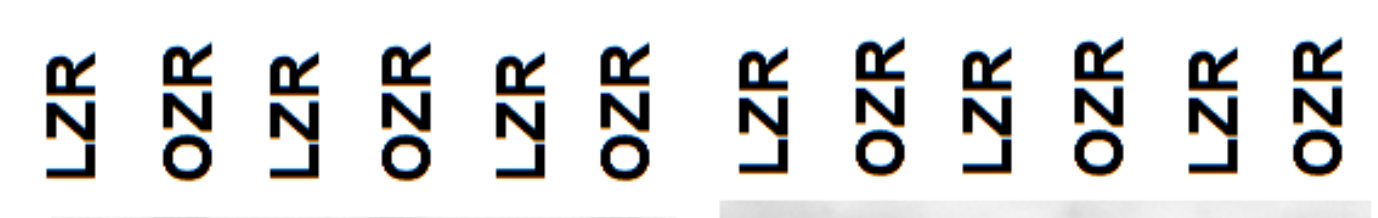

\section{Anp} Gapdh

\section{Male}

Female

D. Protein quantification

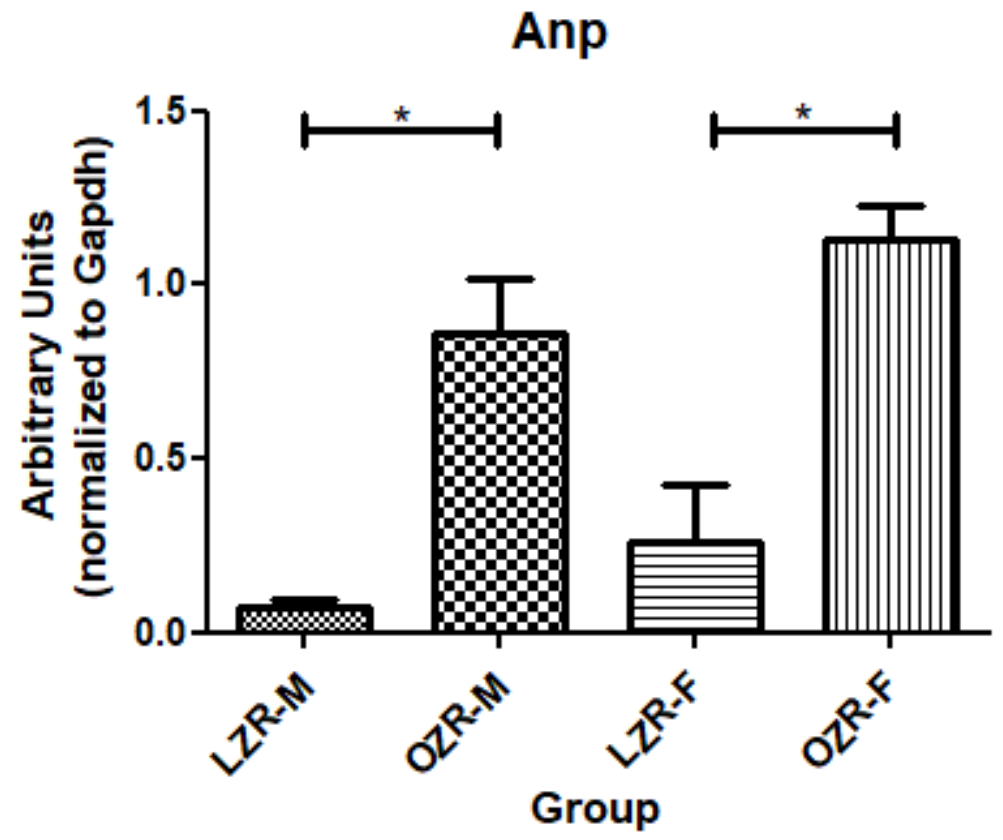

Figure 5. A: Gene expression from transcriptome. B: Gene expression from qPCR. C: Protein expression. D: Protein expression quantification. Protein level was normalized to $\alpha$-actin. * indicates statistically significant $(\mathrm{p}<0.05)$. 
Figure 6: Nppb validation

A. Transcriptome

Nppb Transcript Level

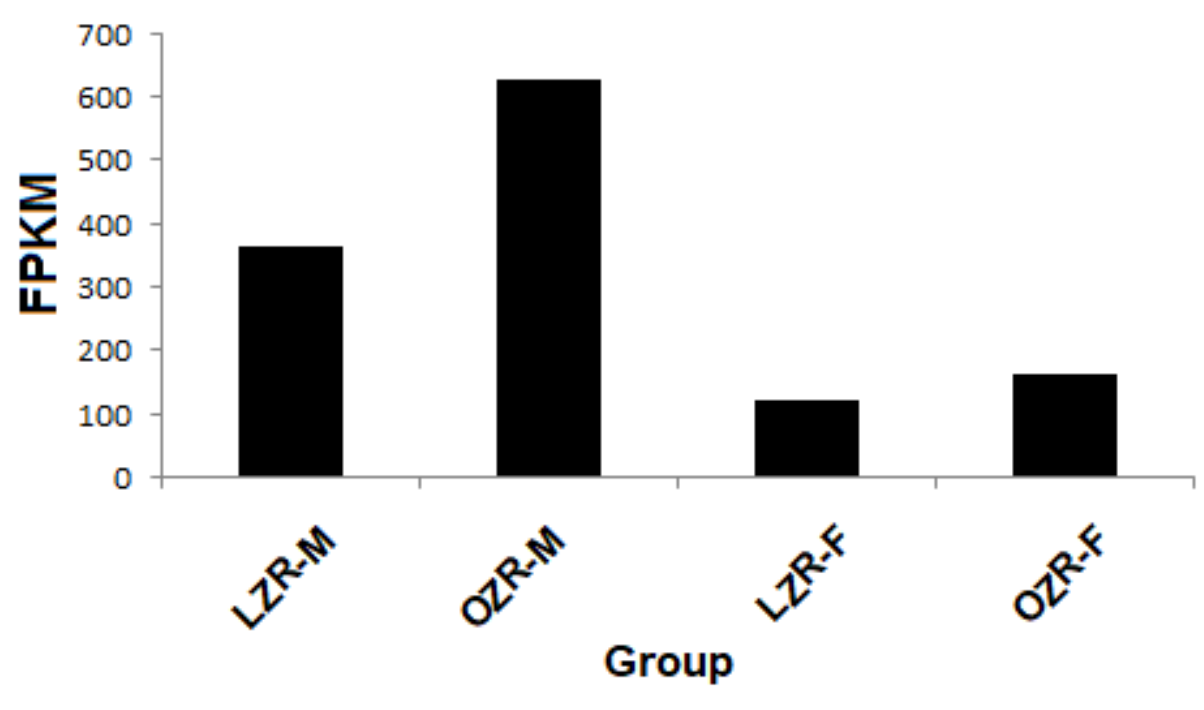

B. RT-qPCR

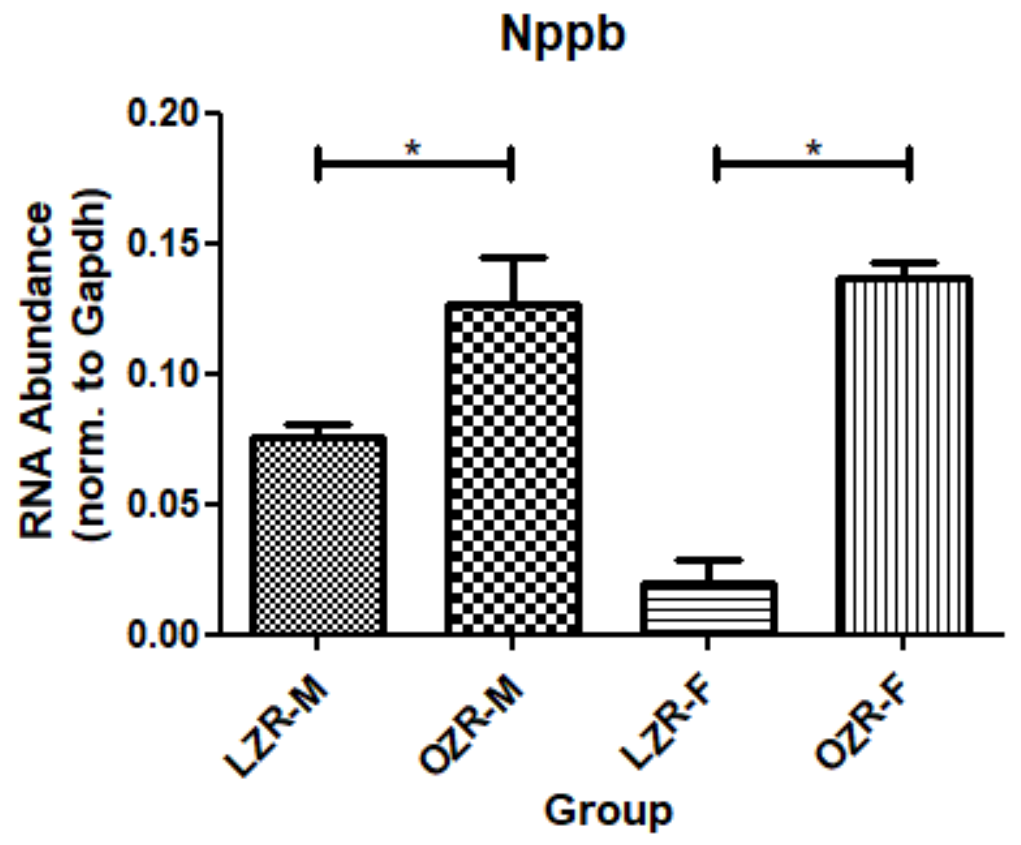


C. Western blots

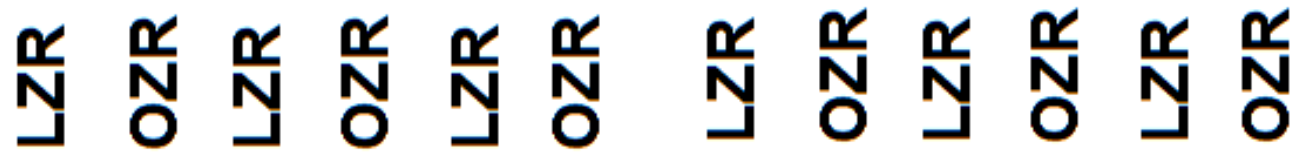

\section{Bnp} Gapdh

\section{Male}

\section{Female}

D. Protein quantification

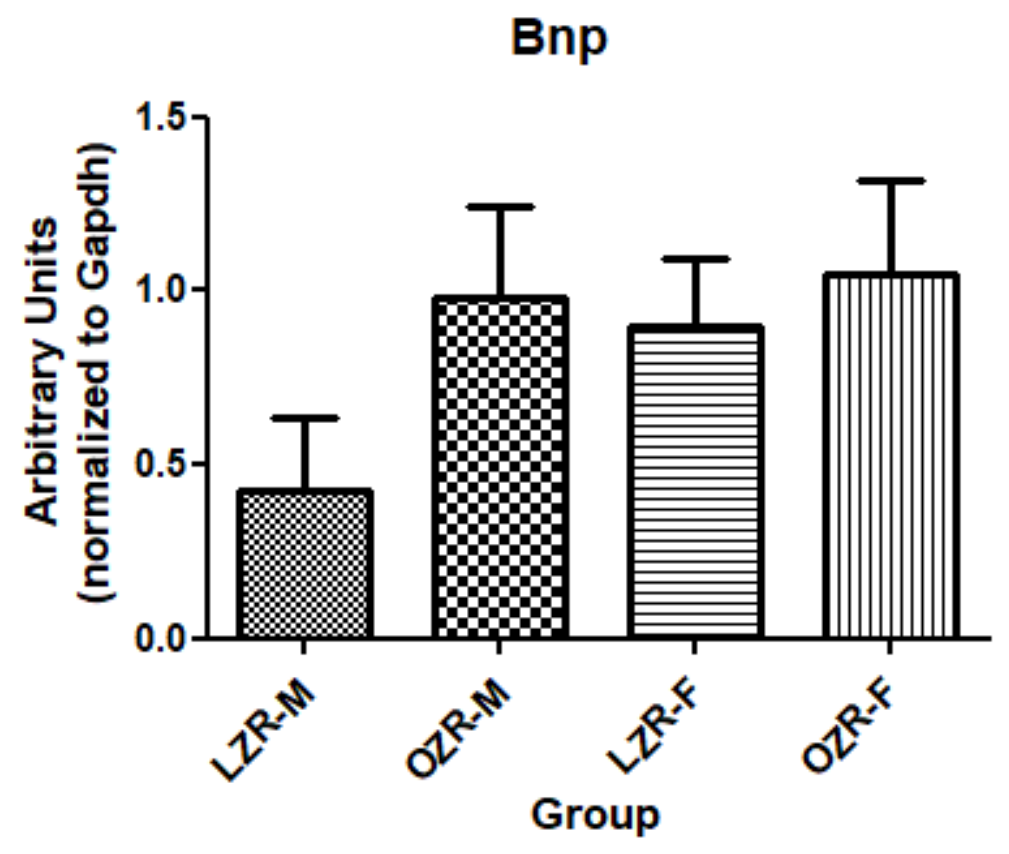

Figure 6. A: Gene expression from transcriptome. B: Gene expression from qPCR. C: Protein expression. D: Protein expression quantification. Protein level was normalized to $\alpha$-actin. * indicates statistically significant $(\mathrm{p}<0.05)$. 
Figure 7: Gstt1 validation

\section{Gstt1 Transcript Level}

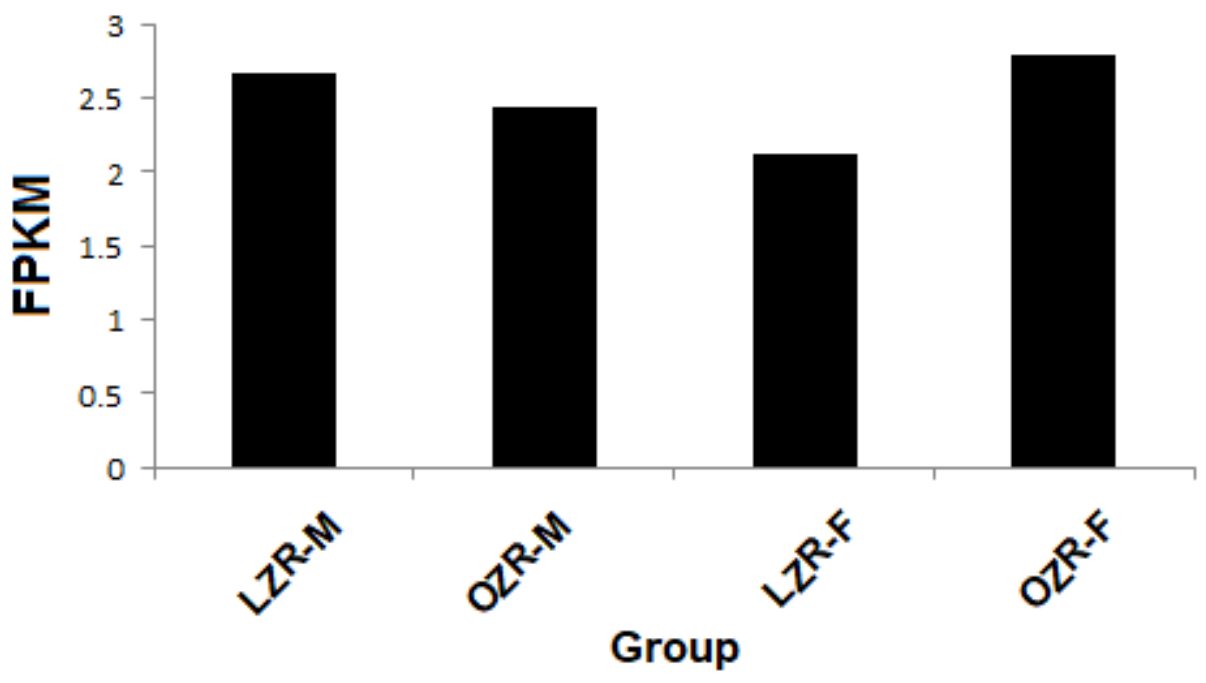

Gstt1

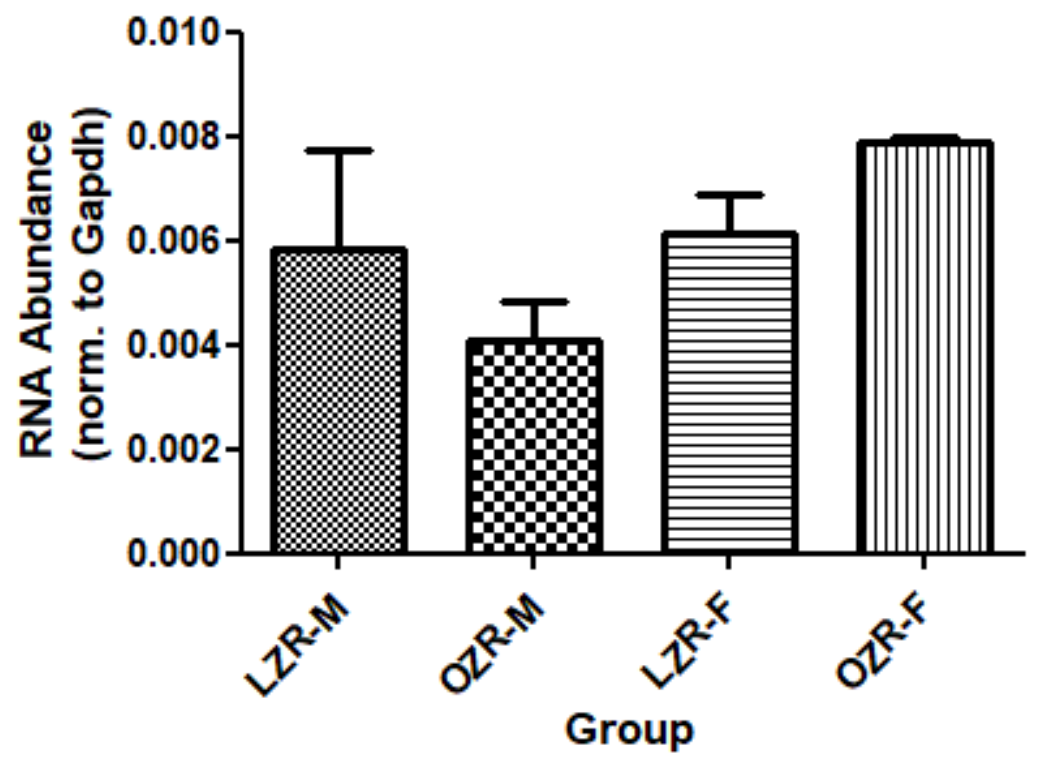

Figure 7. Upper: Gene expression from transcriptome, lower: Gene expression from qPCR. 
Figure 8: Hbb validation

A. Transcriptome

Hbb Transcript Level

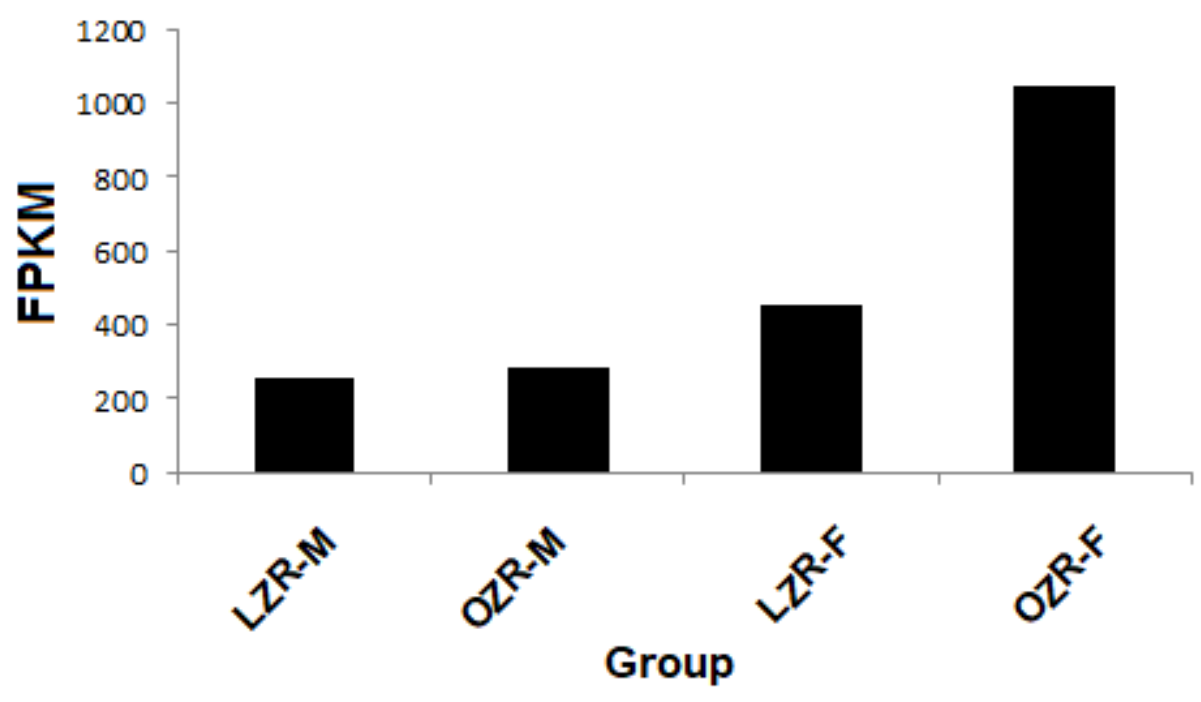

B. RT-qPCR

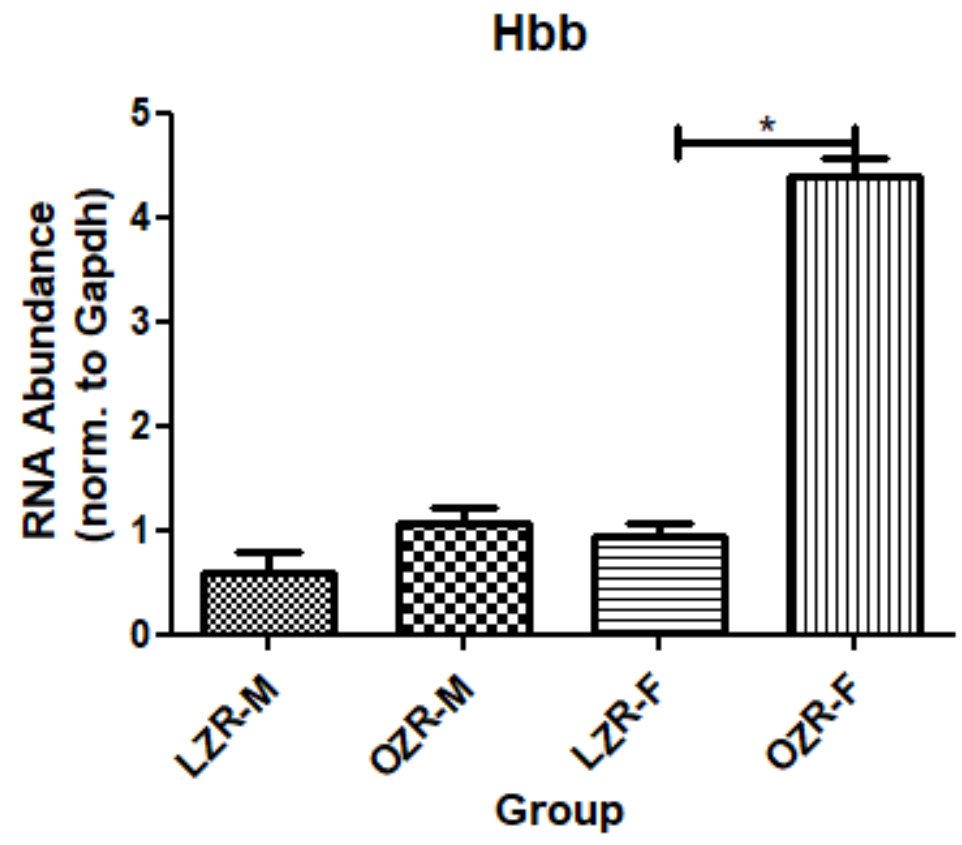




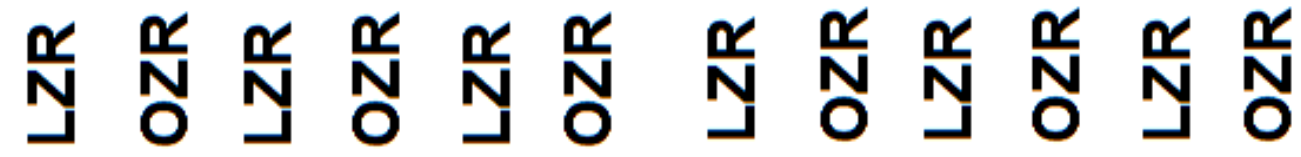

Hbb Gapdh

\section{Male}

Female

D. Protein quantification

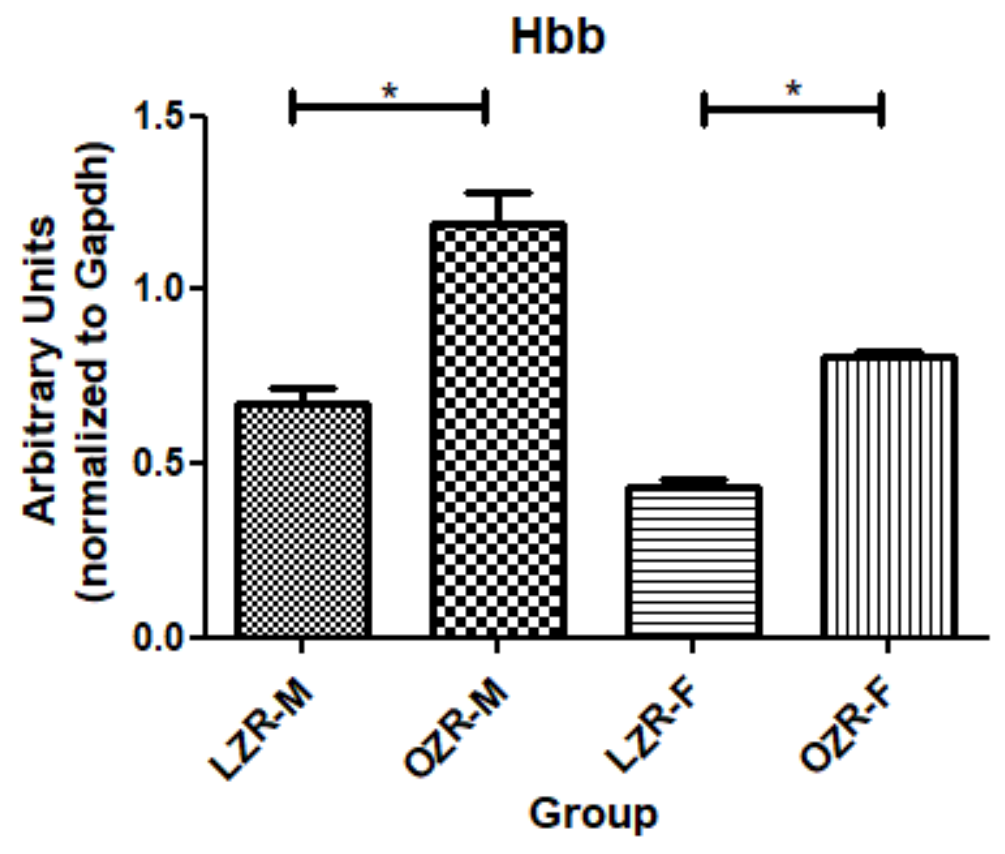

Figure 8. A: Gene expression from transcriptome. B: Gene expression from qPCR. C: Protein expression. D: Protein expression quantification. Protein level was normalized to $\alpha$-actin. * indicates statistically significant $(\mathrm{p}<0.05)$. 
Figure 9: Myl7 validation

A. Transcriptome

Myl7 Transcript Level

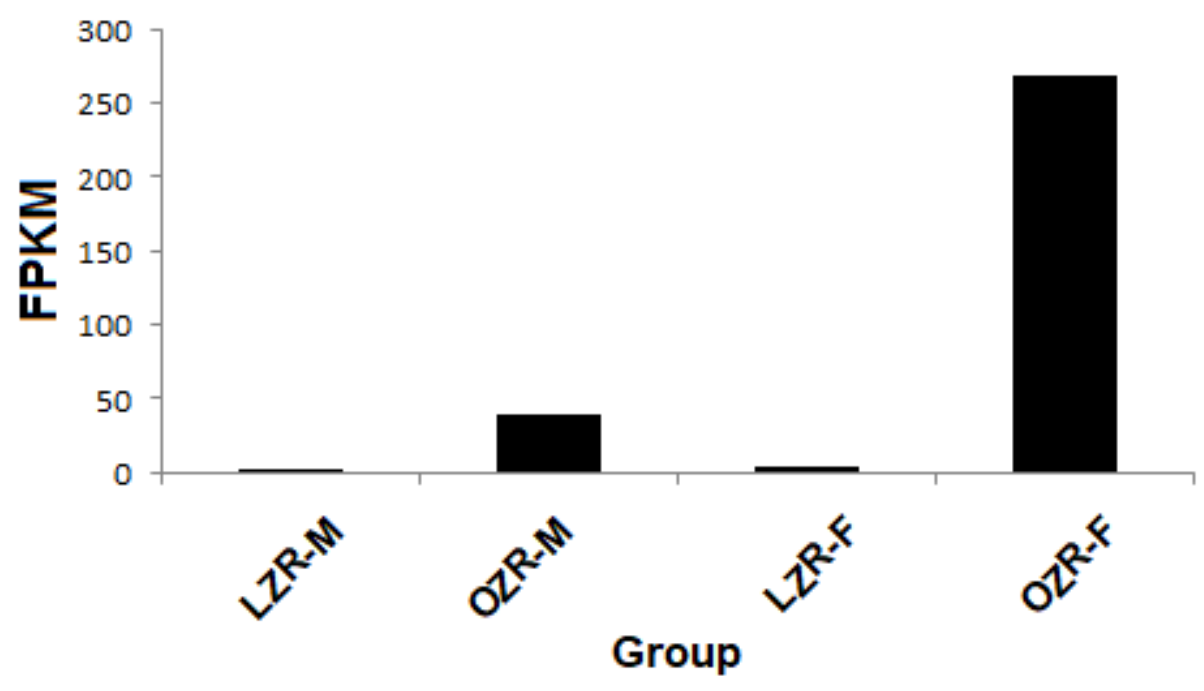

B. RT-qPCR

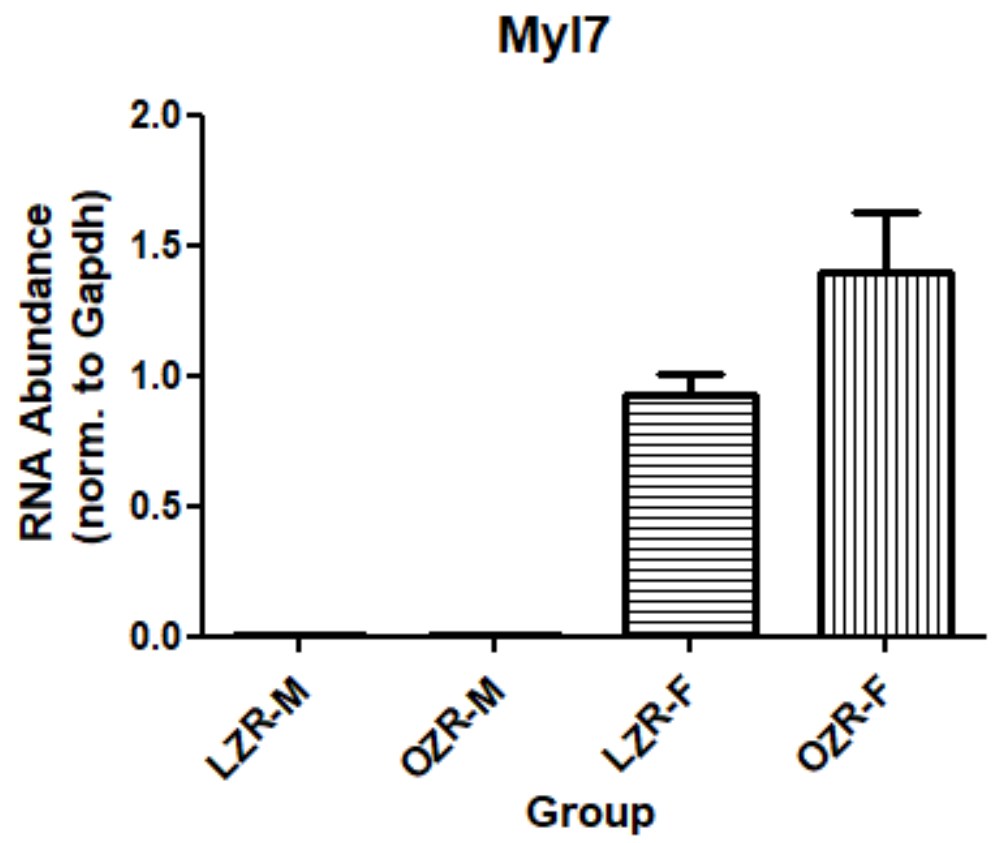


C. Western blots

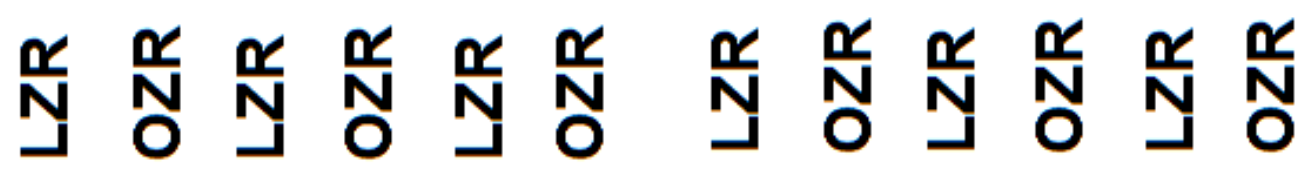

Myl7
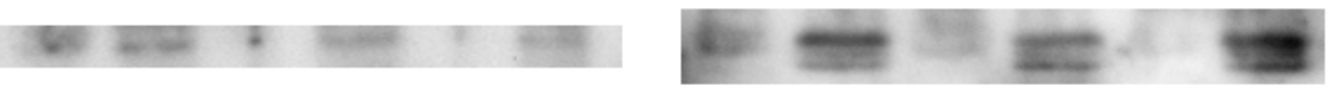

Gapdh

Male

Female

D. Protein quantification

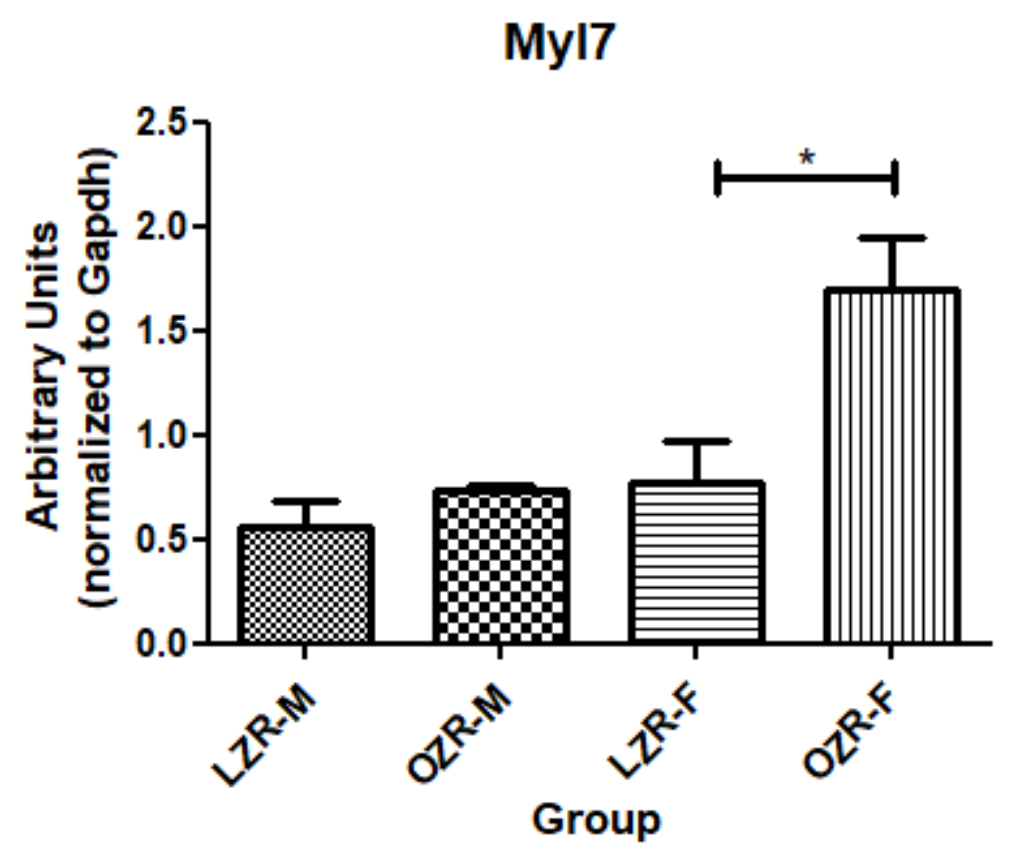

Figure 9. A: Gene expression from transcriptome. B: Gene expression from qPCR. C: Protein expression. D: Protein expression quantification. Protein level was normalized to $\alpha$-actin. * indicates statistically significant $(\mathrm{p}<0.05)$. 
Figure 10: Pdk4 validation

A. Transcriptome

Pdk4 Transcript Level

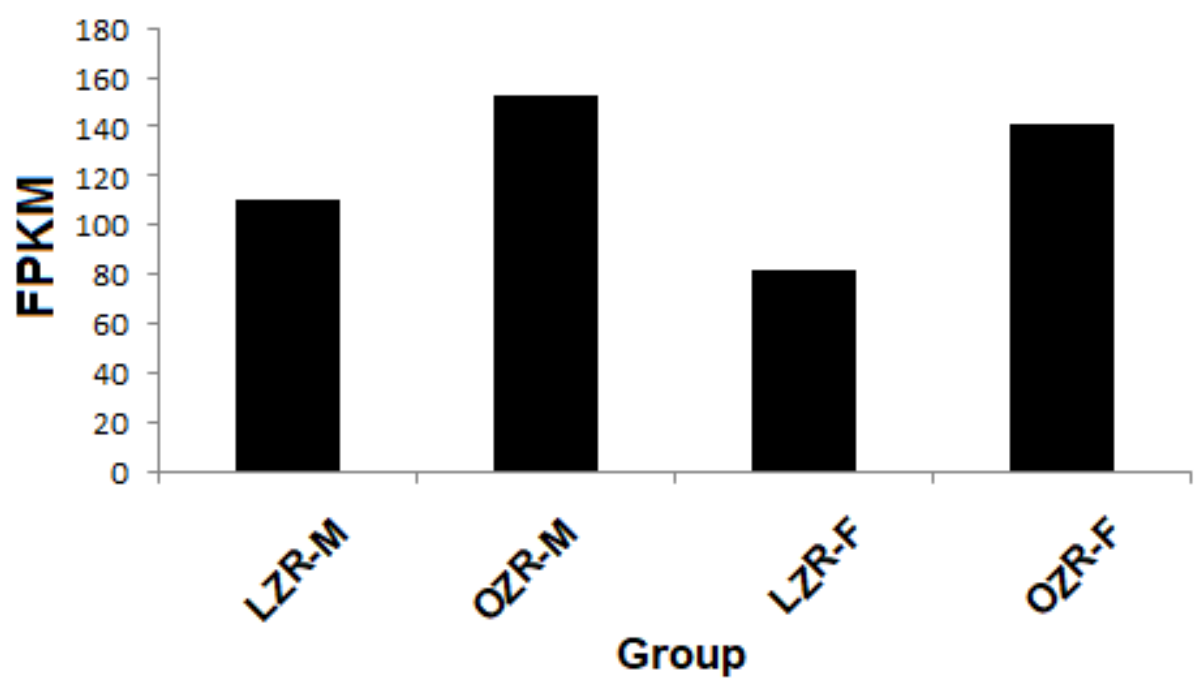

B. RT-qPCR

Pdk4

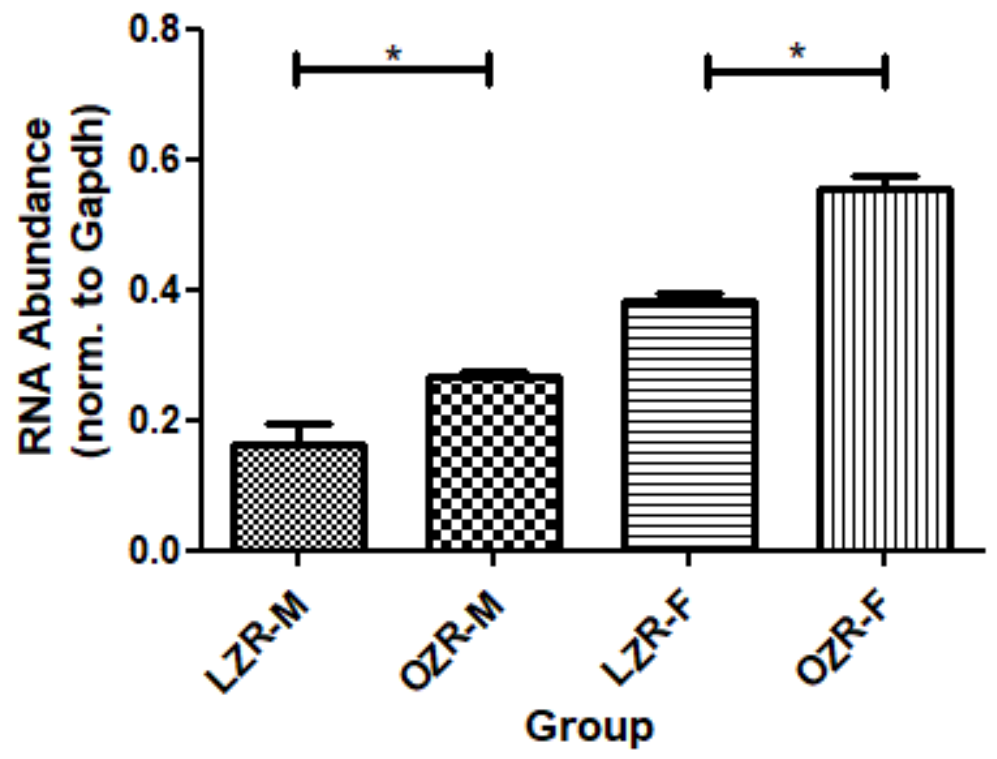


C. Western blots

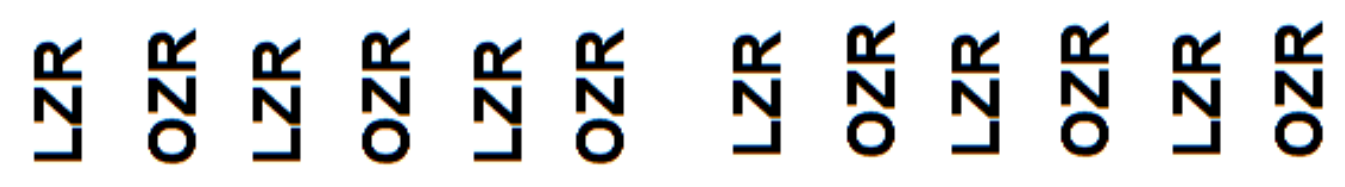

Pdk4

Gapdh

\section{Male}

Female

D. Protein quantification

Pdk4

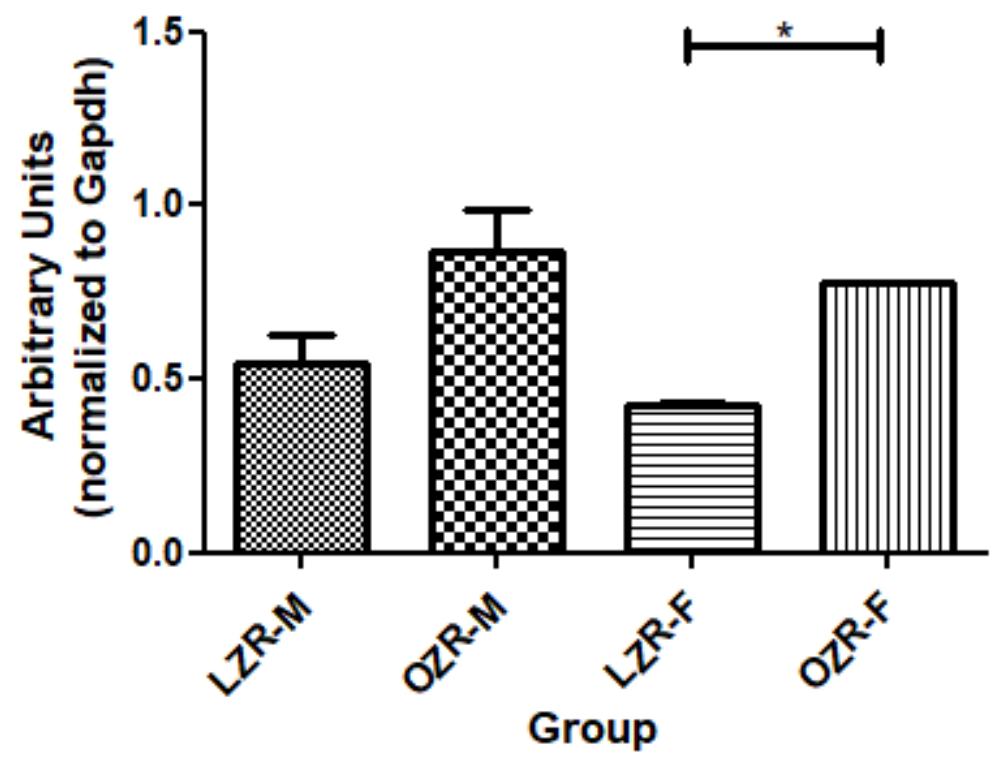

Figure 10. A: Gene expression from transcriptome. B: Gene expression from qPCR. C: Protein expression. D: Protein expression quantification. Protein level was normalized to $\alpha$-actin. * indicates statistically significant $(\mathrm{p}<0.05)$. 
Figure 11: Roles of sex (A) and obesity (B) in NPPA and NPPB gene expression levels between rat and human LVH.
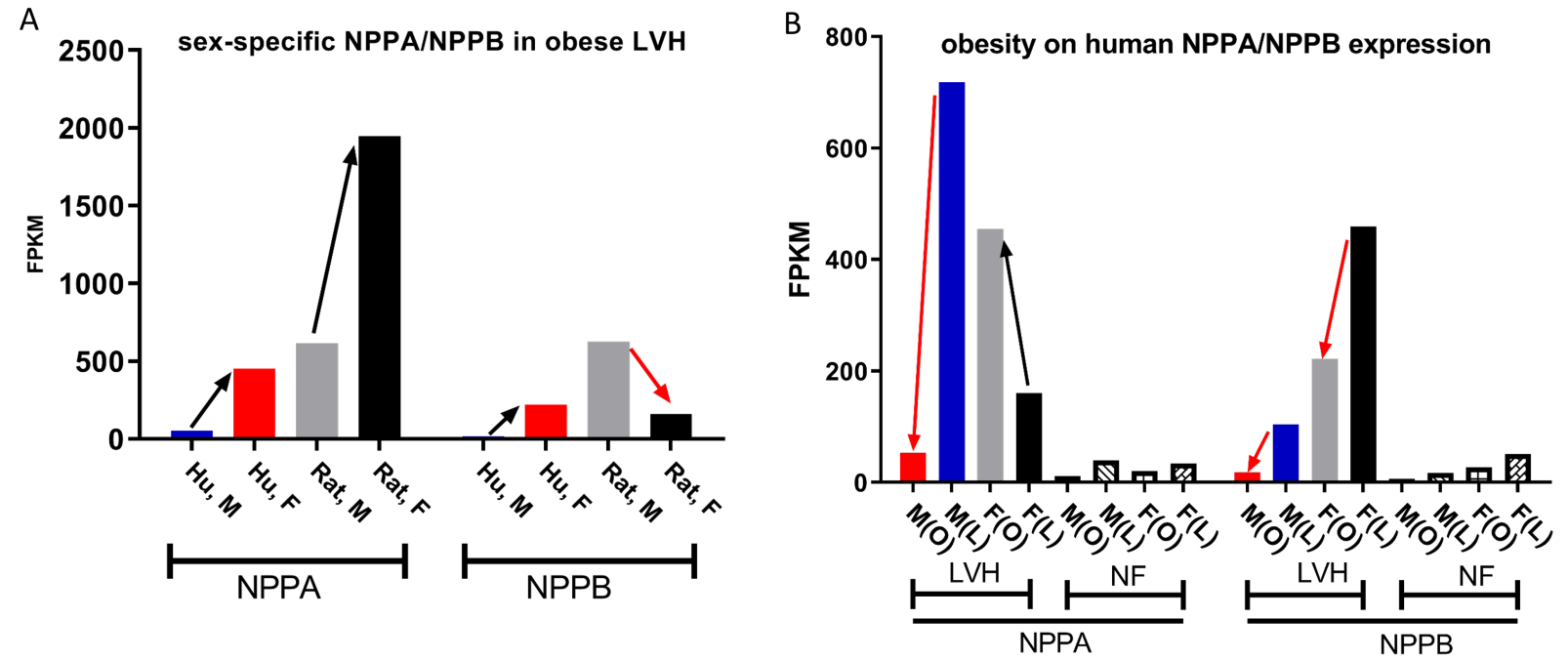

Figure 11. Black arrow: increase in paired group comparison. Red arrow: decrease in paired group comparison. NF: non-failed, non-LVH control. 


\section{$\underline{\text { Tables }}$}

\section{Table 1: Characteristics of OZR and echocardiogram results}

\begin{tabular}{|c|c|c|c|c|}
\hline & \multicolumn{2}{|c|}{ Male $(14-17 w k)(n=4)$} & \multicolumn{2}{|c|}{ Female $(14-17 w k)(n=4)$} \\
\hline & Lean & Obese & Lean & Obese \\
\hline Body weight(g) & $350 \pm 82$ & $607 \pm 57 *$ & $273 \pm 15$ & $575 \pm 24 *$ \\
\hline Heart weight(g) & $1.16 \pm 0.06$ & $1.75 \pm 0.15^{*}$ & $0.85 \pm 0.07$ & $1.55 \pm 0.06^{*}$ \\
\hline$H R(b p m)$ & $417 \pm 36$ & $371 \pm 11$ & $384 \pm 18$ & $374 \pm 6$ \\
\hline$L V$ mass $(m g)^{\&}$ & $786 \pm 39$ & $1043 \pm 96^{*}$ & $443 \pm 8$ & $705 \pm 33 *$ \\
\hline$I V S ; d(m m)$ & $1.79 \pm 0.21$ & $2.05 \pm 0.04$ & $1.72 \pm 0.04$ & $2.02 \pm 0.12 *$ \\
\hline LVID;d (mm) & $5.94 \pm 1.04$ & $7.84 \pm 0.1^{*}$ & $5.38 \pm 0.13$ & $6.95 \pm 0.15$ \\
\hline$L V P W ; d(m m) \#$ & $1.75 \pm 0.15$ & $2.31 \pm 0.35^{*}$ & $1.29 \pm 0.11$ & $1.76 \pm 0.11^{*}$ \\
\hline$E F(\%)$ & $83.16 \pm 7.25$ & $80.10 \pm 12.13$ & $89.92 \pm 6.90$ & $86.90 \pm 12.42$ \\
\hline$F S(\%)$ & $53.96 \pm 8.08$ & $51.67 \pm 12.25$ & $64.08 \pm 13.12$ & $60.81 \pm 15.08$ \\
\hline
\end{tabular}

*: $\mathrm{p}<0.05$ compared to sex-matched lean control group

\#: $n=6-8$ as more than one area was measured.

\&: body-size corrected. 
Table 2: Resting heart rate, ST elevation, and heart rate variability from ECG

\begin{tabular}{|l|l|l|l|l|}
\hline & \multicolumn{2}{|l|}{ Male } & Female \\
& Lean & Obese & Lean & Obese \\
\hline HR $(\mathrm{bpm})$ & $394 \pm 16$ & $310 \pm 17^{*}$ & $228 \pm 16$ & $198 \pm 19$ \\
\hline $\mathrm{STe}(\mathrm{mV})$ & $0.092 \pm 0.008$ & $0.140 \pm 0.025^{*}$ & $0.10 \pm 0.12$ & $0.079 \pm 0.057$ \\
\hline $\mathrm{nLF}$ & $0.621 \pm 0.169$ & $0.205 \pm 0.082^{*}$ & $0.589 \pm 0.009$ & $0.350 \pm 0.059^{*}$ \\
\hline $\mathrm{nHF}$ & $0.379 \pm 0.169$ & $0.795 \pm 0.082^{*}$ & $0.414 \pm 0.009$ & $0.650 \pm 0.059^{*}$ \\
\hline LF/HF & $1.951 \pm 1.062$ & $0.267 \pm 0.129 \#$ & $1.419 \pm 0.052$ & $0.545 \pm 0.136^{*}$ \\
\hline
\end{tabular}

*: $\mathrm{p}<0.05(\mathrm{n}=3)$ compared to sex-matched lean control group. $\#: \mathrm{p}=0.0526$ 


\section{$\underline{\text { References }}$}

(1) Abel ED, Litwin SE, and Sweeney G. Cardiac remodeling in obesity. Physiol Rev 88: 389419, 2008.

(2) Anders S, and Huber W. Differential expression analysis for sequence count data. Genome Biol 11: R106, 2010.

(3) Avelar E, Cloward TV, Walker JM, Farney RJ, Strong M, Pendleton RC, Segerson N, Adams TD, Gress RE, Hunt SC, and Litwin SE. Left ventricular hypertrophy in severe obesity: interactions among blood pressure, nocturnal hypoxemia, and body mass. Hypertension 49: 3439, 2007.

(4) Basoor A, Cotant J, Patel K, Randhawa G, Todorov M, Halabi A, Choksi N, Shafiq A, Stein P, and DeGregorio M. Obesity and ST Elevation Myocardial Infarction in Young. CHEST 140: 984A, 2011.

(5) Battistoni A, Rubattu S, and Volpe M. Circulating biomarkers with preventive, diagnostic and prognostic implications in cardiovascular diseases. Int J Cardiol 157: 160-168, 2012.

(6) Benichou T, Pereira B, Mermillod M, Tauveron I, Pfabigan D, Maqdasy S, and Dutheil F. Heart rate variability in type 2 diabetes mellitus: A systematic review and meta-analysis. PLoS One 13: e0195166, 2018.

(7) Bharati S, and Lev M. Cardiac conduction system involvement in sudden death of obese young people. Am Heart J 129: 273-281, 1995.

(8) Bick AG, Flannick J, Ito K, Cheng S, Vasan RS, Parfenov MG, Herman DS, DePalma SR, Gupta N, Gabriel SB, Funke BH, Rehm HL, Benjamin EJ, Aragam J, Taylor HA, Jr., Fox ER, Newton-Cheh C, Kathiresan S, O'Donnell CJ, Wilson JG, Altshuler DM, Hirschhorn JN, 
Seidman JG, and Seidman C. Burden of rare sarcomere gene variants in the Framingham and Jackson Heart Study cohorts. Am J Hum Genet 91: 513-519, 2012.

(9) Bignolais O, Quang KL, Naud P, El Harchi A, Briec F, Piron J, Bourge A, Leoni AL, Charpentier F, and Demolombe S. Early ion-channel remodeling and arrhythmias precede hypertrophy in a mouse model of complete atrioventricular block. J Mol Cell Cardiol 51: 713$721,2011$.

(10) Bisping E, Wakula P, Poteser M, and Heinzel FR. Targeting cardiac hypertrophy: toward a causal heart failure therapy. J Cardiovasc Pharmacol 64: 293-305, 2014.

(11) Burr RL. Interpretation of normalized spectral heart rate variability indices in sleep research: a critical review. Sleep 30: 913-919, 2007.

(12) Casale PN, Devereux RB, Kligfield P, Eisenberg RR, Miller DH, Chaudhary BS, and Phillips MC. Electrocardiographic detection of left ventricular hypertrophy: Development and prospective validation of improved criteria. 6: 572-580, 1985.

(13) Chadwick J, and Mann W. Medical Works of Hippocrates. Oxford: Black-well, 1950.

(14) Chua SC, Jr., White DW, Wu-Peng XS, Liu SM, Okada N, Kershaw EE, Chung WK, Power-Kehoe L, Chua M, Tartaglia LA, and Leibel RL. Phenotype of fatty due to Gln269Pro mutation in the leptin receptor (Lepr). Diabetes 45: 1141-1143, 1996.

(15) Cooper RS, Simmons BE, Castaner A, Santhanam V, Ghali J, and Mar M. Left ventricular hypertrophy is associated with worse survival independent of ventricular function and number of coronary arteries severely narrowed. Am J Cardiol 65: 441-445, 1990.

(16) Counihan PJ, Fei L, Bashir Y, Farrell TG, Haywood GA, and McKenna WJ. Assessment of heart rate variability in hypertrophic cardiomyopathy. Association with clinical and prognostic features. Circulation 88: 1682-1690, 1993. 
(17) Courand P-Y, Grandjean A, Charles P, Paget V, Khettab F, Bricca G, Boussel L, Lantelme P, and Harbaoui B. R Wave in aVL Lead Is a Robust Index of Left Ventricular Hypertrophy: A Cardiac MRI Study. American Journal of Hypertension 28: 1038-1048, 2015.

(18) Das SR, Alexander KP, Chen AY, Powell-Wiley TM, Diercks DB, Peterson ED, Roe MT, and de Lemos JA. Impact of Body Weight and Extreme Obesity on the Presentation, Treatment, and In-Hospital Outcomes of 50,149 Patients With ST-Segment Elevation Myocardial Infarction: Results From the NCDR (National Cardiovascular Data Registry). Journal of the American College of Cardiology 58: 2642-2650, 2011.

(19) Duflou J, Virmani R, Rabin I, Burke A, Farb A, and Smialek J. Sudden death as a result of heart disease in morbid obesity. Am Heart J 130: 306-313, 1995.

(20) Dwyer JP, Ritchie ME, Smyth GK, Harrap SB, Delbridge L, Domenighetti AA, and Di Nicolantonio R. Myocardial gene expression associated with genetic cardiac hypertrophy in the absence of hypertension. Hypertens Res 31: 941-955, 2008.

(21) Electrophysiology TFotESoCtNASoP. Heart Rate Variability. Standards of Measurement, Physiological Interpretation, and Clinical Use 93: 1043-1065, 1996.

(22) Emdin M, Passino C, Prontera C, Fontana M, Poletti R, Gabutti A, Mammini C, Giannoni A, Zyw L, Zucchelli G, and Clerico A. Comparison of brain natriuretic peptide (BNP) and amino-terminal ProBNP for early diagnosis of heart failure. Clin Chem 53: 1289-1297, 2007.

(23) England J, and Loughna S. Heavy and light roles: myosin in the morphogenesis of the heart. Cell Mol Life Sci 70: 1221-1239, 2013.

(24) Foppa M, Duncan BB, and Rohde LE. Echocardiography-based left ventricular mass estimation. How should we define hypertrophy? Cardiovasc Ultrasound 3: 17, 2005. 
(25) Frey N, Katus HA, Olson EN, and Hill JA. Hypertrophy of the heart: a new therapeutic target? Circulation 109: 1580-1589, 2004.

(26) Friddle CJ, Koga T, Rubin EM, and Bristow J. Expression profiling reveals distinct sets of genes altered during induction and regression of cardiac hypertrophy. Proc Natl Acad Sci US A 97: 6745-6750, 2000.

(27) Gardner DG. Natriuretic peptides: markers or modulators of cardiac hypertrophy? Trends Endocrinol Metab 14: 411-416, 2003.

(28) Guimaraes PS, Huber DA, Campagnole-Santos MJ, and Schreihofer AM. Development of attenuated baroreflexes in obese Zucker rats coincides with impaired activation of nucleus tractus solitarius. Am J Physiol Regul Integr Comp Physiol 306: R681-692, 2014.

(29) Gurm HS, and Topol EJ. The ECG in acute coronary syndromes: new tricks from an old dog. Heart 91: 851-853, 2005.

(30) Hailstones D, Barton P, Chan-Thomas P, Sasse S, Sutherland C, Hardeman E, and Gunning P. Differential regulation of the atrial isoforms of the myosin light chains during striated muscle development. J Biol Chem 267: 23295-23300, 1992.

(31) Heckbert SR, Post W, Pearson GD, Arnett DK, Gomes AS, Jerosch-Herold M, Hundley WG, Lima JA, and Bluemke DA. Traditional cardiovascular risk factors in relation to left ventricular mass, volume, and systolic function by cardiac magnetic resonance imaging: the Multiethnic Study of Atherosclerosis. J Am Coll Cardiol 48: 2285-2292, 2006.

(32) Hua R, MacLeod SL, Polina I, Moghtadaei M, Jansen HJ, Bogachev O, O’Blenes SB, Sapp JL, Legare J-F, and Rose RA. Effects of Wild-Type and Mutant Forms of Atrial Natriuretic Peptide on Atrial Electrophysiology and Arrhythmogenesis. Circ Arrhythm Electrophysiol 8: $1240-1254,2015$. 
(33) Huber DA, and Schreihofer AM. Attenuated baroreflex control of sympathetic nerve activity in obese Zucker rats by central mechanisms. J Physiol 588: 1515-1525, 2010.

(34) Hubert HB, Feinleib M, McNamara PM, and Castelli WP. Obesity as an independent risk factor for cardiovascular disease: a 26-year follow-up of participants in the Framingham Heart Study. Circulation 67: 968-977, 1983.

(35) Istenes I, Korei AE, Putz Z, Nemeth N, Martos T, Keresztes K, Kempler MS, Erzsebet VO, Vargha $\mathrm{P}$, and Kempler P. Heart rate variability is severely impaired among type 2 diabetic patients with hypertension. Diabetes Metab Res Rev 30: 305-312, 2014.

(36) Jujic A, Leosdottir M, Ostling G, Gudmundsson P, Nilsson PM, Melander O, and Magnusson M. A genetic variant of the atrial natriuretic peptide gene is associated with left ventricular hypertrophy in a non-diabetic population--the Malmo preventive project study. $B M C$ Med Genet 14: 64, 2013.

(37) Kenchaiah S, Evans JC, Levy D, Wilson PWF, Benjamin EJ, Larson MG, Kannel WB, and Vasan RS. Obesity and the Risk of Heart Failure. New England Journal of Medicine 347: 305$313,2002$.

(38) Kerkela R, Ulvila J, and Magga J. Natriuretic Peptides in the Regulation of Cardiovascular Physiology and Metabolic Events. J Am Heart Assoc 4: e002423, 2015.

(39) Kim D, Pertea G, Trapnell C, Pimentel H, Kelley R, and Salzberg SL. TopHat2: accurate alignment of transcriptomes in the presence of insertions, deletions and gene fusions. Genome Biol 14: R36, 2013.

(40) Kindel TL, Foster TOM, Harmann L, and Strande J. Sleeve Gastrectomy in Obese Zucker Rats Restores Cardiac Function and Geometry Toward a Lean Phenotype Independent of Weight Loss. J Card Fail 25: 372-379, 2019. 
(41) Kleiger RE, Miller JP, Bigger JT, Jr., and Moss AJ. Decreased heart rate variability and its association with increased mortality after acute myocardial infarction. Am J Cardiol 59: 256-262, 1987.

(42) La Rovere MT, Pinna GD, Maestri R, Mortara A, Capomolla S, Febo O, Ferrari R, Franchini M, Gnemmi M, Opasich C, Riccardi PG, Traversi E, and Cobelli F. Short-term heart rate variability strongly predicts sudden cardiac death in chronic heart failure patients. Circulation 107: 565-570, 2003.

(43) Lee I-K. The Role of Pyruvate Dehydrogenase Kinase in Diabetes and Obesity. Diabetes Metab J 38: 181-186, 2014.

(44) Lim DS, Roberts R, and Marian AJ. Expression profiling of cardiac genes in human hypertrophic cardiomyopathy: insight into the pathogenesis of phenotypes. J Am Coll Cardiol 38: 1175-1180, 2001.

(45) Lin Y-C, Huang J, Kan H, Castranova V, Frisbee JC, and Yu H-G. Defective calcium inactivation causes long QT in obese insulin-resistant rat. Am J Physiol Heart Circ Physiol 302: H1013-H1022, 2012.

(46) Marfella R, Di Filippo C, Portoghese M, Barbieri M, Ferraraccio F, Siniscalchi M, Cacciapuoti F, Rossi F, D'Amico M, and Paolisso G. Myocardial lipid accumulation in patients with pressure-overloaded heart and metabolic syndrome. Journal of Lipid Research 50: 2314$2323,2009$.

(47) Maron BJ, and Maron MS. Hypertrophic cardiomyopathy. Lancet 381: 242-255, 2013.

(48) Maron MS. Clinical utility of cardiovascular magnetic resonance in hypertrophic cardiomyopathy. J Cardiovasc Magn Reson 14: 13, 2012. 
(49) Maron MS, Lesser JR, and Maron BJ. Management implications of massive left ventricular hypertrophy in hypertrophic cardiomyopathy significantly underestimated by echocardiography but identified by cardiovascular magnetic resonance. Am J Cardiol 105: 1842-1843, 2010.

(50) Marsh SA, Powell PC, Agarwal A, Dell'Italia LJ, and Chatham JC. Cardiovascular dysfunction in Zucker obese and Zucker diabetic fatty rats: role of hydronephrosis. 293: H292H298, 2007.

(51) Marsh SA, Powell PC, Agarwal A, Dell'Italia LJ, and Chatham JC. Cardiovascular dysfunction in Zucker obese and Zucker diabetic fatty rats: role of hydronephrosis. Am J Physiol Heart Circ Physiol 293: H292-298, 2007.

(52) Moghtadaei M, Polina I, and Rose RA. Electrophysiological effects of natriuretic peptides in the heart are mediated by multiple receptor subtypes. Prog Biophys Mol Biol 120: 37-49, 2016.

(53) Mori T, Chen YF, Feng JA, Hayashi T, Oparil S, and Perry GJ. Volume overload results in exaggerated cardiac hypertrophy in the atrial natriuretic peptide knockout mouse. Cardiovasc Res 61: 771-779, 2004.

(54) Mukoyama M, Nakao K, Hosoda K, Suga S, Saito Y, Ogawa Y, Shirakami G, Jougasaki M, Obata $\mathrm{K}$, Yasue $\mathrm{H}$, and et al. Brain natriuretic peptide as a novel cardiac hormone in humans. Evidence for an exquisite dual natriuretic peptide system, atrial natriuretic peptide and brain natriuretic peptide. J Clin Invest 87: 1402-1412, 1991.

(55) Nepper-Christensen L, Lønborg J, Ahtarovski KA, Høfsten DE, Kyhl K, Ghotbi AA, Schoos MM, Göransson C, Bertelsen L, Køber L, Helqvist S, Pedersen F, Saünamaki K, Jørgensen E, Kelbæk H, Holmvang L, Vejlstrup N, and Engstrøm T. Left Ventricular Hypertrophy Is Associated With Increased Infarct Size and Decreased Myocardial Salvage in 
Patients With ST-Segment Elevation Myocardial Infarction Undergoing Primary Percutaneous Coronary Intervention. Journal of the American Heart Association 6: e004823, 2017.

(56) Newman MS, Nguyen T, Watson MJ, Hull RW, and Yu HG. Transcriptome profiling reveals novel BMI- and sex-specific gene expression signatures for human cardiac hypertrophy. Physiol Genomics 49: 355-367, 2017.

(57) Nolan J, Batin PD, Andrews R, Lindsay SJ, Brooksby P, Mullen M, Baig W, Flapan AD, Cowley A, Prescott RJ, Neilson JM, and Fox KA. Prospective study of heart rate variability and mortality in chronic heart failure: results of the United Kingdom heart failure evaluation and assessment of risk trial (UK-heart). Circulation 98: 1510-1516, 1998.

(58) Perego L, Pizzocri P, Corradi D, Maisano F, Paganelli M, Fiorina P, Barbieri M, Morabito A, Paolisso G, Folli F, and Pontiroli AE. Circulating leptin correlates with left ventricular mass in morbid (grade III) obesity before and after weight loss induced by bariatric surgery: a potential role for leptin in mediating human left ventricular hypertrophy. J Clin Endocrinol Metab 90: 4087-4093, 2005.

(59) Perrin MJ, and Gollob MH. The role of atrial natriuretic peptide in modulating cardiac electrophysiology. Heart Rhythm 9: 610-615, 2012.

(60) Phillips MS, Liu Q, Hammond HA, Dugan V, Hey PJ, Caskey CJ, and Hess JF. Leptin receptor missense mutation in the fatty Zucker rat. Nat Genet 13: 18-19, 1996.

(61) Potter LR, Yoder AR, Flora DR, Antos LK, and Dickey DM. Natriuretic peptides: their structures, receptors, physiologic functions and therapeutic applications. Handb Exp Pharmacol 341-366, 2009. 
(62) Ren J, Walsh MF, Jefferson L, Natavio M, Ilg KJ, Sowers JR, and Brown RA. Basal and ethanol-induced cardiac contractile response in lean and obese Zucker rat hearts. J Biomed Sci 7: 390-400, 2000.

(63) Rennie KL, Hemingway H, Kumari M, Brunner E, Malik M, and Marmot M. Effects of moderate and vigorous physical activity on heart rate variability in a British study of civil servants. Am J Epidemiol 158: 135-143, 2003.

(64) Roden DM, Balser JR, George AL, Jr., and Anderson ME. Cardiac ion channels. Annu Rev Physiol 64: 431-475, 2002.

(65) RStudio-Team. RStudio: Integrated Development for R. RStudio, Inc. Boston, MA: 2015.

(66) Rubattu S, Bigatti G, Evangelista A, Lanzani C, Stanzione R, Zagato L, Manunta P, Marchitti S, Venturelli V, Bianchi G, Volpe M, and Stella P. Association of atrial natriuretic peptide and type a natriuretic peptide receptor gene polymorphisms with left ventricular mass in human essential hypertension. J Am Coll Cardiol 48: 499-505, 2006.

(67) Santolini M, Romay MC, Yukhtman CL, Rau CD, Ren S, Saucerman JJ, Wang JJ, Weiss JN, Wang Y, Lusis AJ, and Karma A. A personalized, multiomics approach identifies genes involved in cardiac hypertrophy and heart failure. NPJ Syst Biol Appl 4: 12, 2018.

(68) Semsarian C, Ingles J, Maron MS, and Maron BJ. New perspectives on the prevalence of hypertrophic cardiomyopathy. J Am Coll Cardiol 65: 1249-1254, 2015.

(69) Sergeeva IA, and Christoffels VM. Regulation of expression of atrial and brain natriuretic peptide, biomarkers for heart development and disease. Biochim Biophys Acta 1832: 2403-2413, 2013. 
(70) Stuckey MI, Tulppo MP, Kiviniemi AM, and Petrella RJ. Heart rate variability and the metabolic syndrome: a systematic review of the literature. Diabetes Metab Res Rev 30: 784-793, 2014.

(71) Tarazona S, Garcia-Alcalde F, Dopazo J, Ferrer A, and Conesa A. Differential expression in RNA-seq: a matter of depth. Genome Res 21: 2213-2223, 2011.

(72) Tarvainen MP, Niskanen JP, Lipponen JA, Ranta-Aho PO, and Karjalainen PA. Kubios HRV--heart rate variability analysis software. Comput Methods Programs Biomed 113: 210-220, 2014.

(73) Tarvainen MP, Ranta-Aho PO, and Karjalainen PA. An advanced detrending method with application to HRV analysis. IEEE Trans Biomed Eng 49: 172-175, 2002.

(74) Trapnell C, Williams BA, Pertea G, Mortazavi A, Kwan G, van Baren MJ, Salzberg SL, Wold BJ, and Pachter L. Transcript assembly and quantification by RNA-Seq reveals unannotated transcripts and isoform switching during cell differentiation. Nat Biotech 28: 511$515,2010$.

(75) Tsuji H, Larson MG, Venditti FJ, Jr., Manders ES, Evans JC, Feldman CL, and Levy D. Impact of reduced heart rate variability on risk for cardiac events. The Framingham Heart Study. Circulation 94: 2850-2855, 1996.

(76) Vest AR, Heneghan HM, Agarwal S, Schauer PR, and Young JB. Bariatric surgery and cardiovascular outcomes: a systematic review. Heart 98: 1763-1777, 2012.

(77) Wang D, Gladysheva IP, Fan T-HM, Sullivan R, Houng AK, and Reed GL. Atrial natriuretic peptide affects cardiac remodeling, function, heart failure, and survival in a mouse model of dilated cardiomyopathy. Hypertension 63: 514-519, 2014. 
(78) Wang K, Asinger RW, and Marriott HJL. ST-Segment Elevation in Conditions Other Than Acute Myocardial Infarction. 349: 2128-2135, 2003.

(79) Wong CY, O'Moore-Sullivan T, Leano R, Byrne N, Beller E, and Marwick TH. Alterations of left ventricular myocardial characteristics associated with obesity. Circulation 110: 30813087, 2004.

(80) Yates A, Akanni W, Amode MR, Barrell D, Billis K, Carvalho-Silva D, Cummins C, Clapham P, Fitzgerald S, Gil L, Giron CG, Gordon L, Hourlier T, Hunt SE, Janacek SH, Johnson N, Juettemann T, Keenan S, Lavidas I, Martin FJ, Maurel T, McLaren W, Murphy DN, Nag R, Nuhn M, Parker A, Patricio M, Pignatelli M, Rahtz M, Riat HS, Sheppard D, Taylor K, Thormann A, Vullo A, Wilder SP, Zadissa A, Birney E, Harrow J, Muffato M, Perry E, Ruffier M, Spudich G, Trevanion SJ, Cunningham F, Aken BL, Zerbino DR, and Flicek P. Ensembl 2016. Nucleic Acids Res 44: D710-716, 2016.

(81) Zhou YT, Grayburn P, Karim A, Shimabukuro M, Higa M, Baetens D, Orci L, and Unger RH. Lipotoxic heart disease in obese rats: implications for human obesity. Proc Natl Acad Sci U $S A$ 97: 1784-1789, 2000. 


\section{$\underline{\text { Supplemental Figures }}$}

Figure S1: Principle component analysis (PCA) in female (left) and male rats showing that the groups of obese versus lean rats in both sexes are coherent.
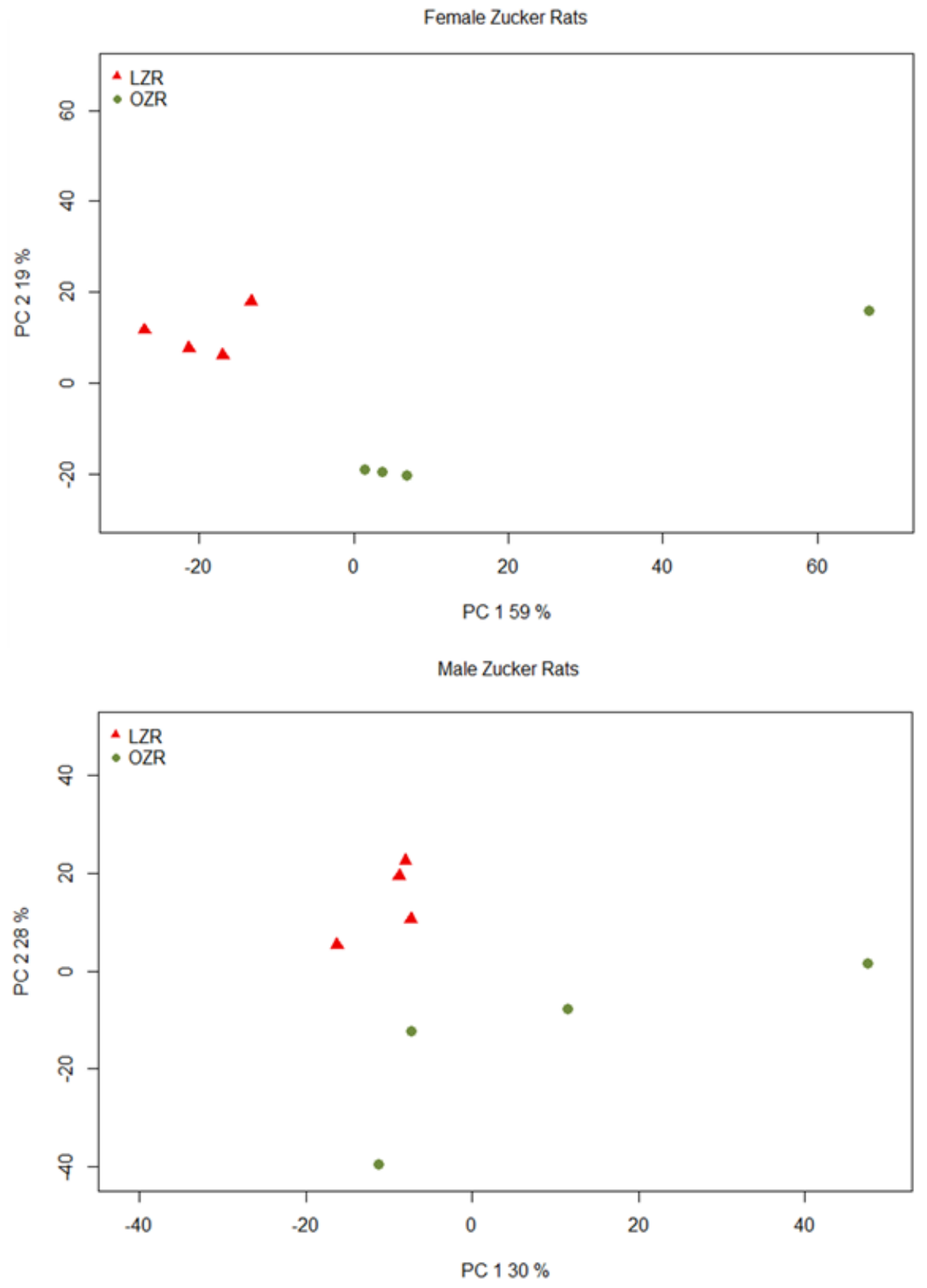
Figure S1. PCA plots displaying clustering of individual samples. Red triangles represent LZR and green circles are obese; females are displayed in the left graph and males are in the right graph. 
Figure S2: Differential expression profiles between obese and lean animals (volcano plots)
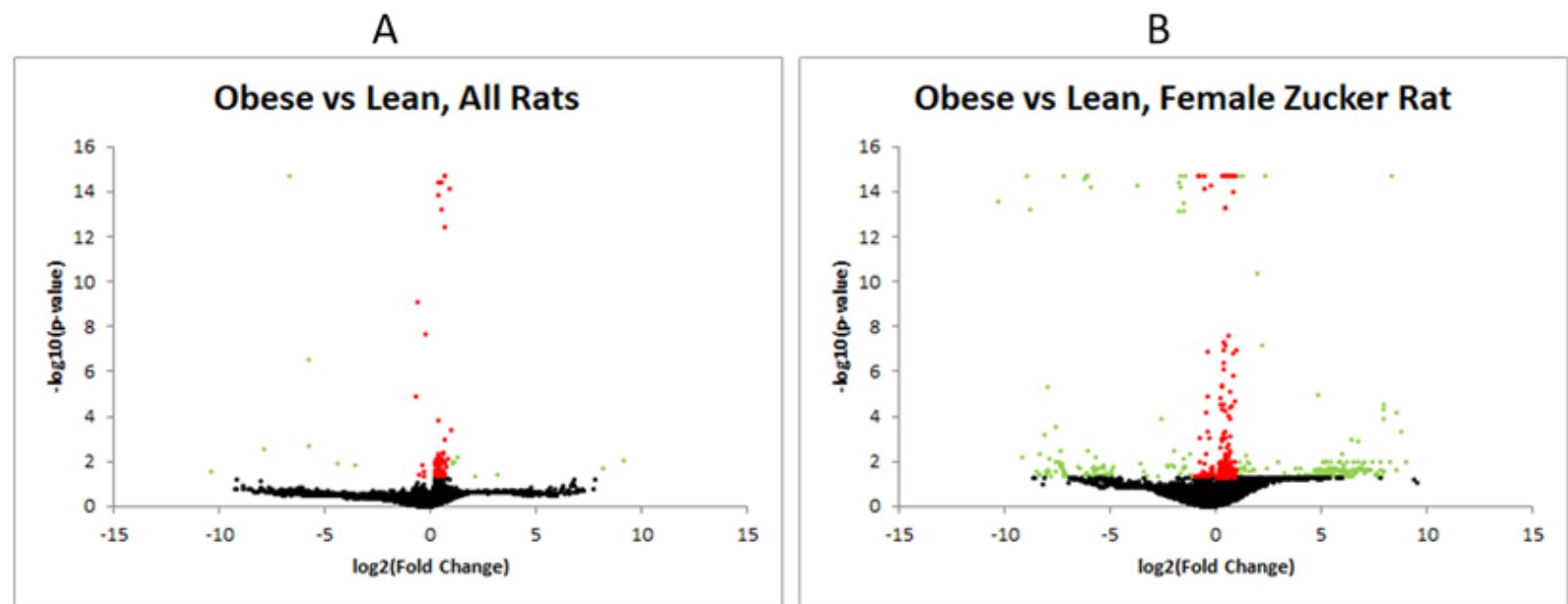

C

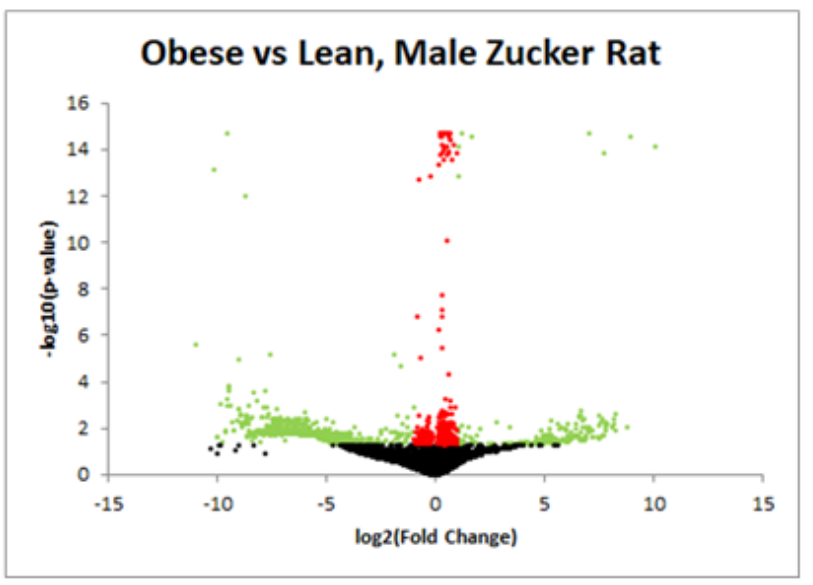

Figure S2. A: All samples analyzed without regard to sex. B: Obese versus lean females. C: Obese versus lean males. Black dots represent genes with $\mathrm{p}$-value $<0.05$. Red dots represent genes with $\mathrm{p}$-value $<0.05$ but less than a two-fold change in expression. Green dots represent genes with $p$-value $<0.05$ and expression level greater than two-fold. 
Figure S3: Heatmaps of differentially expressed genes in OZR

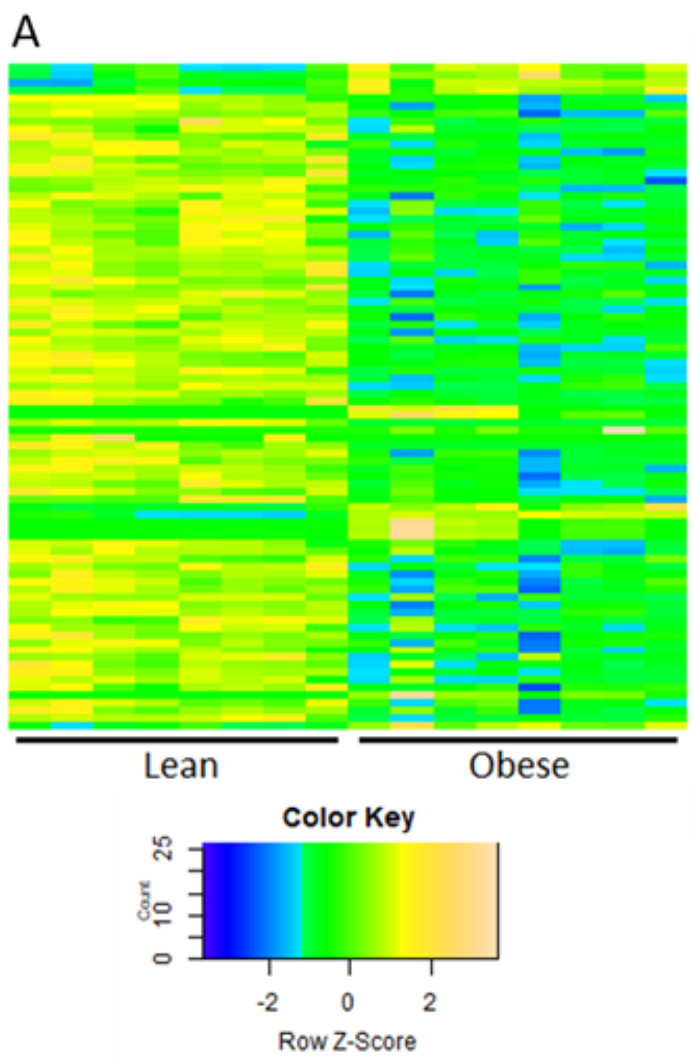

$89 \mathrm{DE}$ genes total

C

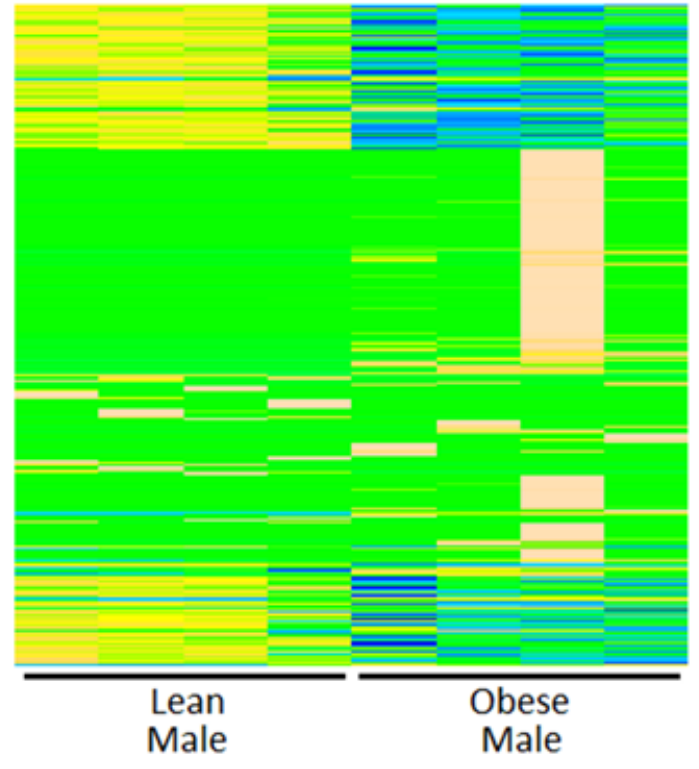

B
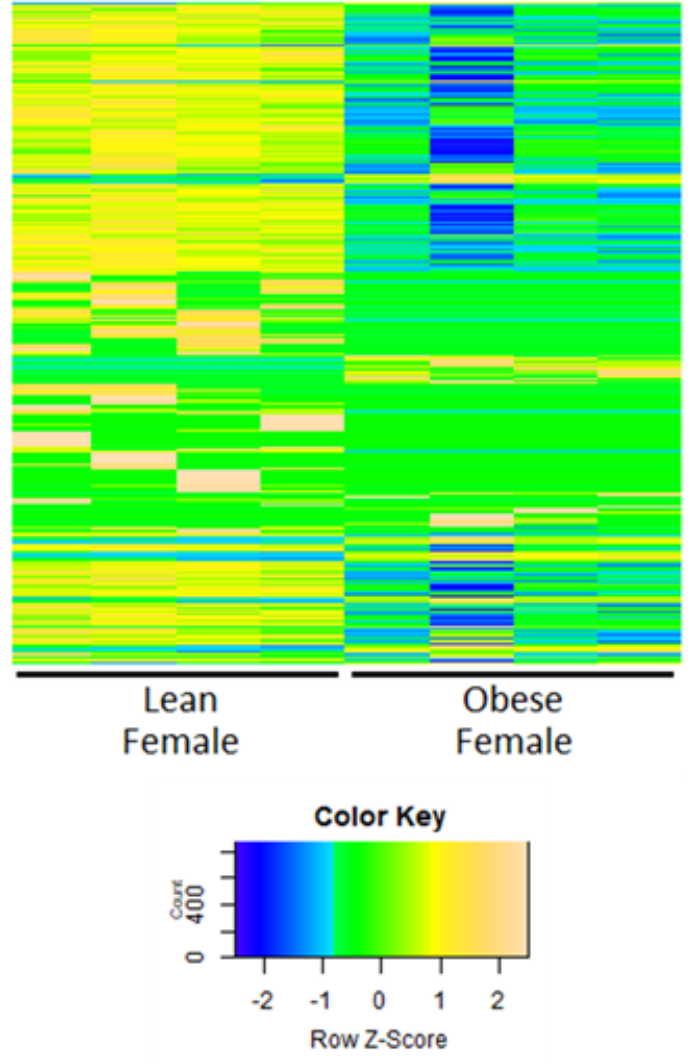

\section{- $\quad 826 \mathrm{DE}$ genes total}

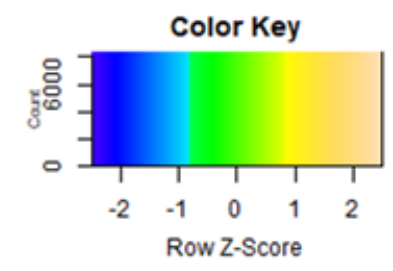

2859 DE genes total 
Figure S3. Each column represents one animal. Transcriptome data were analyzed for differential expression in obese versus lean animals for A: all samples (regardless of sex), B: females only, and C: males only. Heatmaps were generated using the gplot function in R. 


\section{$\underline{\text { Supplemental Tables }}$}

Supplemental Table 1. Primers used in qPCR.

\begin{tabular}{|l|l|l|}
\hline Gene & Strand & Sequence \\
\hline NPPA & F & GCAAACATCAGATCGTGCCC \\
\hline & R & GGTCTAGCAGGTTCTTGAAATCC \\
\hline NPPB & F & AGCTCTCAAAGGACCAAGGC \\
\hline & R & CGATCCGGTCTATCTTCTGCC \\
\hline HIST1H2AC & F & ACACCTTACCTTTTCACTTCC \\
\hline & R & GCGTCCAGACATCGTTATGC \\
\hline GSTT1 & F & TCCAGATGCATACTGTGGAGC \\
\hline & R & TGGCCACACTCTCACACAAGG \\
\hline PDK4 & F & TGGTGAAGAGCTGGTACATCC \\
\hline & R & ATCCCTTGTGCCATCGTAGG \\
\hline MYL7 & F & CTCAATGTTCGAGCAAGCCC \\
\hline & R & GACACTTACCCTCCCGAGC \\
\hline HBB & F & CTGGGCAGGCTGCTGG \\
\hline & R & TCAGATGAGCAAAGGTGCCC \\
\hline GAPDH & F & CAACTCCCTCAAGATTGTCAGCAA \\
\hline & R & GGCATGGACTGTGGTCATGA \\
\hline
\end{tabular}


Supplemental Table 2. Significant Differentially-Expressed Genes Per Sex

\begin{tabular}{|l|l|l|l|l|}
\hline GeneName & log2FC & p-value & log2FC & p-value \\
& Female & Female & Male & Male \\
\hline AABR06001092.3 & 5.730464004 & 0.006867631 & 5.587444214 & 0.017538338 \\
\hline & - & & - & \\
\hline AABR06002048.1 & 5.474802166 & 0.046768716 & 5.899857199 & 0.020329196 \\
\hline AABR06003762.1 & 5.64791736 & 0.021892925 & 6.049120464 & 0.020924976 \\
\hline AABR06008694.1 & 6.612430939 & 0.029549546 & 8.620401091 & 0.005975624 \\
\hline AABR06009495.2 & 6.641521781 & 0.026806303 & 6.739967734 & 0.032850468 \\
\hline AABR06027295.1 & 7.94417136 & 0.000132794 & 7.596415435 & 0.012709633 \\
\hline AABR06009537.6 & 7.593939062 & 0.030485885 & 7.054664058 & 0.014314151 \\
\hline & & & - & \\
\hline AABR06022532.1 & 5.425902591 & 0.04852144 & 4.676637294 & 0.044207941 \\
\hline AABR06024198.1 & 6.630507627 & 0.027811611 & 6.834896348 & 0.030537616 \\
\hline & & & - & \\
\hline & & & & - \\
\hline
\end{tabular}




\begin{tabular}{|c|c|c|c|c|}
\hline AABR06027333.1 & 4.885063042 & 0.041975951 & 7.725504514 & 0.018014809 \\
\hline AABR06028157.1 & 7.606891073 & 0.043823061 & $\begin{array}{l}- \\
8.620401091\end{array}$ & 0.005975624 \\
\hline AABR06028909.1 & 5.569160597 & 0.045447886 & $\begin{array}{l}- \\
6.724777073\end{array}$ & 0.016953624 \\
\hline AABR06030345.1 & 4.999134344 & 0.044856331 & \begin{tabular}{|l|}
-5.21384995 \\
\end{tabular} & \begin{tabular}{|l|}
0.026995547 \\
\end{tabular} \\
\hline AABR06030862.1 & 2.127493539 & 0.046006385 & 4.906853561 & 0.028547391 \\
\hline AABR06031450.1 & $\begin{array}{l}- \\
5.621704021\end{array}$ & 0.039238767 & $\begin{array}{l}- \\
6.242374048\end{array}$ & 0.016689447 \\
\hline AABR06031580.1 & 5.402081585 & 0.049376162 & $\begin{array}{l}- \\
4.845748232\end{array}$ & 0.03894374 \\
\hline AABR06031627.1 & 5.437967602 & 0.048072641 & $\begin{array}{l} \\
5.761342842\end{array}$ & 0.00989319 \\
\hline AABR06042542.1 & 6.268881516 & 0.041184128 & 6.473725938 & 0.021827543 \\
\hline AABR06043079.1 & 4.771726188 & 0.037246584 & $\mid-7.48026874$ & 0.006872807 \\
\hline AABR06045328.2 & $\begin{array}{l}- \\
9.157541849\end{array}$ & 0.006901706 & $\begin{array}{l}- \\
10.97271827\end{array}$ & $2.36148 \mathrm{E}-06$ \\
\hline AABR06045400.1 & $\begin{array}{l}- \\
5.694710338\end{array}$ & 0.025507481 & $\begin{array}{l}- \\
4.712676338\end{array}$ & 0.016914267 \\
\hline AABR06046610.1 & 5.404688557 & 0.049289009 & $\begin{array}{l}- \\
4.905580062\end{array}$ & 0.035350768 \\
\hline AABR06047190.1 & - & 0.024503013 & - & 0.01103747 \\
\hline
\end{tabular}




\begin{tabular}{|c|c|c|c|c|}
\hline & \begin{tabular}{|l}
7.221099856
\end{tabular} & & $\mid 7.590851117$ & \\
\hline AABR06047625.1 & 6.344460351 & 0.044357662 & $\begin{array}{l}- \\
6.724777073\end{array}$ & 0.016953624 \\
\hline AABR06050424.1 & 6.202888777 & 0.038097669 & $\begin{array}{l}- \\
8.160302291\end{array}$ & 0.012895458 \\
\hline AABR06052098.1 & 6.123638752 & 0.037293865 & $\begin{array}{l}- \\
9.756404167\end{array}$ & 0.04203545 \\
\hline AABR06052644.1 & 5.485454457 & 0.021961767 & 4.144056596 & 0.04674109 \\
\hline AABR06054455.1 & $\begin{array}{l}- \\
7.205285768\end{array}$ & 0.03794693 & $\begin{array}{l}- \\
6.604668142\end{array}$ & 0.015769722 \\
\hline AABR06055554.1 & 0.34234201 & 0.023637241 & $\mid 0.192215961$ & 0.044637305 \\
\hline AABR06055804.1 & 0.360817095 & 0.044907147 & 0.278251212 & 0.044665764 \\
\hline AABR06058815.1 & $\begin{array}{l}- \\
8.134764207\end{array}$ & 0.000605044 & $\begin{array}{l}- \\
7.691897758\end{array}$ & 0.002479804 \\
\hline AABR06059337.1 & $\begin{array}{l}- \\
7.100043994\end{array}$ & 0.043508355 & 6.183120784 & 0.028000384 \\
\hline AABR06059957.1 & 7.144611887 & 0.025697511 & $\begin{array}{l} \\
8.432012537\end{array}$ & 0.021791267 \\
\hline AABR06060284.1 & $\begin{array}{l}- \\
5.745410059\end{array}$ & 0.017610357 & $\begin{array}{l}- \\
6.747828475\end{array}$ & 0.018139359 \\
\hline AABR06062642.1 & 5.598698447 & 0.045957994 & $\begin{array}{l}- \\
8.547480448\end{array}$ & 0.012940666 \\
\hline AABR06065586.1 & 6.971913533 & 0.012116814 & - & 0.012945739 \\
\hline
\end{tabular}




\begin{tabular}{|c|c|c|c|c|}
\hline & & & 8.157589926 & \\
\hline AABR06065718.1 & 5.61563547 & 0.036360555 & $\begin{array}{l}- \\
5.040411106\end{array}$ & 0.027845096 \\
\hline AABR06066181.1 & 0.211521378 & 0.033099913 & 0.163550338 & 0.042769867 \\
\hline AABR06066677.1 & $\begin{array}{l}- \\
7.409937527\end{array}$ & 0.010771029 & 8.757888266 & 0.008370517 \\
\hline AABR06067381.1 & 5.417373268 & 0.048832775 & $\begin{array}{l} \\
6.466272791\end{array}$ & 0.014651542 \\
\hline AABR06068951.1 & 6.389230195 & 0.044904553 & $\begin{array}{l}- \\
7.812441519\end{array}$ & 0.01483145 \\
\hline AABR06072704.1 & 6.176198847 & 0.037337944 & 6.101534315 & 0.034619997 \\
\hline AABR06072709.2 & 7.613910522 & 0.033687553 & 7.790094159 & 0.005310569 \\
\hline AABR06074433.1 & 5.964886462 & 0.004543887 & $\begin{array}{l}- \\
7.079067219\end{array}$ & 0.021408484 \\
\hline AABR06080098.1 & 7.548375195 & 0.037056911 & -10.0337192 & 0.023031626 \\
\hline AABR06081787.1 & 7.157602788 & 0.023438023 & $\begin{array}{l}- \\
8.615733531\end{array}$ & 0.006343379 \\
\hline AABR06082987.1 & 6.071939834 & 0.022714779 & 6.210594527 & 0.025812336 \\
\hline AABR06086439.1 & 6.284162273 & 0.041952119 & $\begin{array}{l}- \\
9.569094396\end{array}$ & 0.001149496 \\
\hline AABR06086499.1 & 5.624067943 & 0.025697759 & $\begin{array}{l}- \\
6.880029517\end{array}$ & 0.016140955 \\
\hline AABR06090020.1 & 6.238806385 & 0.039655161 & - & 0.017698716 \\
\hline
\end{tabular}




\begin{tabular}{|c|c|c|c|c|}
\hline & & & 5.538778581 & \\
\hline & - & & - & \\
\hline AABR06091097.2 & 7.167437295 & 0.033067049 & 6.542939897 & 0.015052123 \\
\hline AABR06094518.1 & 6.257829499 & 0.040614306 & -6.37649517 & 0.014842829 \\
\hline AABR06095711.1 & 6.165015485 & 0.037148165 & 8.629781936 & 0.005308213 \\
\hline AABR06097617.1 & 7.54269473 & 0.036424528 & -7.02241795 & 0.015220933 \\
\hline AABR06098671.1 & 6.572853403 & 0.033570852 & $\begin{array}{l}- \\
7.792798317\end{array}$ & 0.013885729 \\
\hline AABR06099363.1 & 6.257829499 & 0.040614306 & 6.637953465 & 0.016207932 \\
\hline AABR06104230.1 & 5.410333607 & 0.049087063 & 5.108034597 & 0.014642567 \\
\hline AABR06108042.1 & 7.263823891 & 0.019223496 & 6.603650355 & 0.015756253 \\
\hline Abca12 & 3.459935696 & 0.045383266 & $\begin{array}{l}- \\
6.583349949\end{array}$ & 0.012305007 \\
\hline Acadl & $\begin{array}{l}- \\
0.607275514\end{array}$ & 0.045428879 & $\begin{array}{l}- \\
0.137793339\end{array}$ & 0.01684359 \\
\hline Acadsb & 0.890668799 & 0.010911172 & 0.610398798 & 0.011814675 \\
\hline Acot 2 & -0.58120987 & 6.99441E-15 & $\begin{array}{l}- \\
0.504184284\end{array}$ & 0.015381101 \\
\hline Acsf2 & 0.911905096 & 0 & 1.16347644 & 0 \\
\hline
\end{tabular}




\begin{tabular}{|c|c|c|c|c|}
\hline Acss 1 & 0.763344511 & 0 & 0.602589679 & $4.40688 \mathrm{E}-05$ \\
\hline Acy1 & 0.463633853 & 0.049980363 & 0.587055716 & 0.033199143 \\
\hline Adk & 0.255682302 & 0.029114696 & 0.219784555 & 0.04166912 \\
\hline Agxt2 & 2.900470908 & 0.049948297 & 5.904998099 & 0.0147047 \\
\hline Ahsal & 0.317805903 & 0.045691948 & 0.367810994 & 0.017583951 \\
\hline AI314180 & 0.322588664 & 0.045671081 & 0.262099643 & 0.041062529 \\
\hline Akt2 & 0.364876397 & 0.045299693 & 0.33098406 & 0.034807733 \\
\hline Aldh6a1 & 0.291433591 & 0.020504617 & 0.367517539 & 0.02018224 \\
\hline Alkbh6 & 5.021755859 & 0.037088315 & 4.378209885 & 0.049806205 \\
\hline Alkbh7 & 0.685059925 & 0.043948886 & 0.726260108 & 0.021886513 \\
\hline Angpt14 & $\begin{array}{l}- \\
2.762245514\end{array}$ & 0.044821936 & $\begin{array}{l}- \\
1.621592354\end{array}$ & 0.043044403 \\
\hline Ap1s2 & 0.575564747 & 0.04320077 & 0.421136111 & 0.022738184 \\
\hline Aqp1 & 0.84779176 & $9.99201 \mathrm{E}-15$ & 0.617937721 & 0.007820683 \\
\hline Araf & 0.444347287 & 0.046882239 & 0.488105854 & 0.015403714 \\
\hline Armc2 & 0.708916431 & 0.046359758 & 0.858175176 & 0.036384405 \\
\hline Art3 & 0.290284023 & 0.022083772 & 1.072787005 & $1.52989 \mathrm{E}-13$ \\
\hline Asb11 & 0.607055833 & $2.7531 \mathrm{E}-08$ & 0.629178896 & 0.020466789 \\
\hline Asb12 & 0.518211405 & 0.025290917 & 0.418729307 & 0.022169072 \\
\hline Asb15 & 1.084577028 & 0.036407893 & 0.683651026 & 0.045816184 \\
\hline Ascl1 & 4.352057597 & 0.046318649 & 7.054482108 & 0.004652836 \\
\hline
\end{tabular}




\begin{tabular}{|c|c|c|c|c|}
\hline Atad 1 & 0.540673758 & $\mid 0.010307023$ & 0.461574235 & 0.022269345 \\
\hline Atp1b4 & $\begin{array}{l}- \\
5.325617057\end{array}$ & 0.040634128 & -3.50164309 & 0.045642034 \\
\hline Atp2a2 & 0.430244727 & 5.44872E-05 & 0.578136476 & 0.020096166 \\
\hline Atp5a1 & 0.192687016 & 0.020807169 & 0.281540223 & 0 \\
\hline Atp5b & 0.226766549 & 0.02560679 & 0.233284958 & $1.9984 \mathrm{E}-15$ \\
\hline Atp5c1 & 0.305654859 & 0.025697696 & 0.322398406 & 0.024072964 \\
\hline Atp5g1 & 0.335923395 & 0.049960102 & 0.351669153 & 0.009577905 \\
\hline Atp5hl1 & 4.673080873 & 0.044926404 & 7.521594786 & 0.016802928 \\
\hline AY172581.2 & $\begin{array}{l}- \\
0.567404086\end{array}$ & 0 & $\begin{array}{l}- \\
0.862898681\end{array}$ & $1.47148 \mathrm{E}-07$ \\
\hline Bdh1 & 1.288249054 & 0 & 0.869124916 & 0.001274565 \\
\hline Bgn & $\begin{array}{l}- \\
0.443574479\end{array}$ & 6.35951E-05 & $\begin{array}{l}- \\
0.328716063\end{array}$ & 0.017853567 \\
\hline Btbd1 & 0.150384366 & 0.049941279 & 0.206918527 & 0.021789401 \\
\hline Car7 & $\begin{array}{l}- \\
5.079569134\end{array}$ & 0.03958806 & $\begin{array}{l}- \\
4.973620776\end{array}$ & 0.014694218 \\
\hline Ccdc47 & 0.373580226 & 0.025874844 & 0.273695476 & 0.038963574 \\
\hline Ccni & 0.196308539 & 0.021827199 & 0.161339802 & 0.043513846 \\
\hline Cct2 & 0.386163662 & 0.000938688 & 0.310511156 & 0.02105802 \\
\hline Cct7 & 0.357514942 & 0.044513607 & 0.234008547 & 0.048434217 \\
\hline Cct8 & 0.238013998 & 0.02248408 & 0.274560916 & 0.02695599 \\
\hline
\end{tabular}




\begin{tabular}{|c|c|c|c|c|}
\hline Cd40lg & 4.844293419 & 0.044485478 & 4.446372587 & 0.043451139 \\
\hline Cdh5 & 0.859440965 & 1.97549E-05 & 0.264170594 & 0.044545653 \\
\hline Cdkn1b & 0.527213781 & 0.019219126 & 0.346736296 & 0.047470007 \\
\hline Cdrt4 & 6.031707691 & 0.014068845 & 6.662422172 & 0.012687202 \\
\hline Chchd3 & 0.375916331 & 0.038491516 & 0.485882399 & 0.010444244 \\
\hline Cldn6 & 5.428940032 & 0.032789751 & $\begin{array}{l}- \\
7.080481776\end{array}$ & 0.004976843 \\
\hline $\mathrm{Cmbl}$ & 0.293924191 & 0.025443429 & 0.478210835 & 0.032304264 \\
\hline Cmya5 & 0.599965504 & 0.022969986 & 0.382578413 & 0.044684262 \\
\hline Cnpy1 & $\begin{array}{l}- \\
3.701346758\end{array}$ & $4.996 \mathrm{E}-15$ & $\begin{array}{l}- \\
2.085963564\end{array}$ & 0.043290677 \\
\hline $\operatorname{Cog} 6$ & 1.010510381 & 0.049082095 & 0.95711025 & 0.028081709 \\
\hline Coq10a & 0.700806087 & 0.039580092 & 0.6523307 & 0.000652482 \\
\hline Corin & 0.636234278 & 0.020801064 & 0.648546707 & 0.021778514 \\
\hline Cox 7c & 0.531007141 & 0.022827842 & 0.397048926 & 0.034978937 \\
\hline Cox $8 b$ & 0.646584078 & 0.044211433 & 0.49960678 & 0.033445553 \\
\hline Cpe & 0.199099236 & 0.021580116 & 0.431308743 & 0.006734814 \\
\hline Crip3 & 4.501527016 & 0.046122986 & -6.27141985 & 0.014287448 \\
\hline Ctnnall & 0.537455804 & 0.023560928 & 0.543498667 & 0.036115532 \\
\hline Ctsc & 0.287475747 & 0.03884358 & 0.554831424 & 0.002319039 \\
\hline Cxcr7 & 0.499272399 & 0.016356332 & 0.429882573 & 0.045035387 \\
\hline Cyb5a & 0.601692173 & 0.029027781 & 0.601826102 & 0.009020331 \\
\hline
\end{tabular}




\begin{tabular}{|c|c|c|c|c|}
\hline Dazap2 & $\mid 0.154060009$ & $\mid 0.022751422$ & 0.186642237 & 0.038636295 \\
\hline Dbt & 0.575277014 & 0.028058767 & 0.561446863 & $1.39888 \mathrm{E}-14$ \\
\hline Dcaf11 & 0.275451382 & 0.048440423 & 0.307994112 & $8.57317 \mathrm{E}-08$ \\
\hline Ddx17 & 0.501210902 & 0.043938017 & 0.323964102 & 0.036532778 \\
\hline Ddx5 & 0.268576837 & 0.04375523 & 0.249992452 & 0.02984819 \\
\hline Decr1 & $\begin{array}{l}- \\
0.652163543\end{array}$ & 0.027509875 & -0.5187071 & 0.014851021 \\
\hline Defb19 & 6.703738406 & 0.001312885 & 6.747663372 & 0.008733623 \\
\hline Dhrs7c & 0.515875476 & 0.048739116 & 0.587829231 & 0.031375558 \\
\hline Dmrtc1c1 & 5.388778039 & 0.020900257 & $\begin{array}{l}- \\
6.242374048\end{array}$ & 0.016689447 \\
\hline Dnajb9 & 0.635788109 & 0.015122747 & 0.571528072 & 0.005376241 \\
\hline Dnajc21 & 0.327115997 & 0.049947392 & 0.339162937 & 0.043396026 \\
\hline Eef2 & 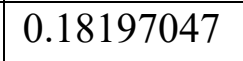 & 0.044097174 & 0.195351092 & 0.002458975 \\
\hline Eif2s3y & 0.258550834 & 0.025319813 & 0.152370094 & 0.048715015 \\
\hline Eif4a2 & 0.280357602 & $5.13946 \mathrm{E}-05$ & 0.228207161 & 0.026698777 \\
\hline Eif4g2 & 0.357566676 & 0.028476509 & 0.167969528 & 0.048776968 \\
\hline Errfi1 & 1.047374125 & 0.048916665 & 1.501277055 & 0.009129899 \\
\hline Esd & 0.34672104 & 0.024354071 & 0.444086828 & 0.009875889 \\
\hline Etfa & 0.088128009 & 0.04994981 & 0.093316917 & 0.009267487 \\
\hline Etfdh & 0.179875567 & 0.014792225 & 0.169547658 & 0.021716521 \\
\hline Fbp2 & 2.184454511 & 6.51504E-08 & 1.336645279 & 0.00848916 \\
\hline
\end{tabular}




\begin{tabular}{|c|c|c|c|c|}
\hline Fhl1 & 0.169828044 & 0.031326568 & 0.859618505 & 0.008266534 \\
\hline Fndc5 & 0.687941377 & 0.003705992 & 0.561655702 & 0.022023328 \\
\hline Fyco1 & 0.546744855 & 0.020474957 & 0.482205858 & 0.037543966 \\
\hline G0s2 & $\begin{array}{l}- \\
0.767032586\end{array}$ & 0.042781289 & $\begin{array}{l}- \\
0.334380674\end{array}$ & 0.042298642 \\
\hline Gbas & 0.443293473 & 0.000452828 & 0.359960788 & 0.008130815 \\
\hline Gbp1 & $\begin{array}{l}- \\
0.559215606\end{array}$ & 0.013353771 & \begin{tabular}{|l}
- \\
0.308920826
\end{tabular} & 0.04123228 \\
\hline Ghitm & 0.418202297 & 0 & 0.312865784 & 0.006634025 \\
\hline Ghr & 0.500716695 & 0.020634013 & 0.371526065 & 0.035498162 \\
\hline Gjal & 0.734775814 & 0 & 0.63683554 & $1.9984 \mathrm{E}-15$ \\
\hline Glud1 & 0.237804532 & 0.022793058 & 0.170494083 & 0.044330289 \\
\hline Gmps & 0.373609811 & 0.023583596 & 0.328009742 & 0.002981603 \\
\hline Gnb3 & 0.631796953 & 0.031813391 & 0.535669487 & 0.017021638 \\
\hline Gnpat & 0.405501911 & 0.048741215 & 0.408989621 & 0.022544858 \\
\hline Got1 & 0.193117007 & 0.046556087 & 0.213334232 & 0.021815551 \\
\hline Gpd1 & 0.693296476 & 0.045779873 & -0.60646849 & 0.018061497 \\
\hline Gramd3 & 0.386905724 & 0.015547556 & 0.528983479 & 0.009995589 \\
\hline Gstz1 & 0.904332157 & 0.031692461 & 0.973211633 & 0.012731107 \\
\hline H1 fo & 0.363319967 & 0.045481856 & 0.243498786 & 0.044077889 \\
\hline Hba1 & - & 5.9952E-15 & - & 0.026882394 \\
\hline
\end{tabular}




\begin{tabular}{|c|c|c|c|c|}
\hline & $\mid 1.647942938$ & & 0.600036835 & \\
\hline $\mathrm{Hba} 2$ & $\begin{array}{l}- \\
1.741094957\end{array}$ & 3.9968E-15 & $\begin{array}{l}- \\
0.643087765\end{array}$ & 0.021083096 \\
\hline Helt & 5.36513805 & 0.020991311 & $\begin{array}{l}- \\
7.449490192\end{array}$ & 0.017796224 \\
\hline Hibadh & 0.159283107 & 0.01226501 & 0.221075202 & 0.002311097 \\
\hline Higd1a & 0.624835392 & 0.02141784 & 0.487003321 & 0.031342491 \\
\hline Hist1h2an & 5.864474687 & 0.025573167 & 7.209439129 & 0.022926782 \\
\hline Hk2 & 1.33009822 & 0.023686687 & 0.92024078 & 0.00988869 \\
\hline Hnrnpa2b1 & 0.202225554 & 2.7491E-05 & 0.169472424 & 0.031424014 \\
\hline Hnrnph1 & 0.231889251 & 0.048935285 & 0.204406336 & 0.04417692 \\
\hline Hnrnpu & 0.197748288 & 0.021401164 & 0.229043924 & 0.045871693 \\
\hline Hsp90ab1 & 0.256396299 & 0.043298454 & 0.409652815 & 0.036188435 \\
\hline Hsp90b1 & 0.403250982 & 4.996E-14 & 0.646557788 & 0.008653939 \\
\hline Hspa5 & 0.575206625 & 0 & 0.672649519 & $3.9968 \mathrm{E}-15$ \\
\hline Hspa9 & 0.383406673 & 0.022723811 & 0.405433998 & 0.012355414 \\
\hline Hspd1 & 0.296398909 & 0.02366013 & 0.319764537 & 0.002528457 \\
\hline Hsph1 & 0.667322164 & 0.011084534 & 0.458800505 & 0.041292638 \\
\hline Hyou1 & 0.43884038 & 0.042917088 & 0.578139493 & 0.020104822 \\
\hline Ift81 & 0.496921735 & 0.046693081 & 0.611991751 & 0.021793987 \\
\hline Igfbp1 & 4.876591993 & 0.022645134 & 3.435201662 & 0.043325498 \\
\hline Igfbp3 & 0.488950937 & 0.02475388 & 1.221064305 & 0.007897261 \\
\hline Jak1 & \begin{tabular}{|l|l}
0.54779747 \\
\end{tabular} & 0.023598722 & 0.283372052 & 0.043344227 \\
\hline
\end{tabular}




\begin{tabular}{|c|c|c|c|c|}
\hline Jam2 & 0.519705325 & 0.024190673 & 0.330714399 & 0.048432169 \\
\hline Klhl13 & 0.876381078 & 0.022533084 & 0.737878985 & 0.04048652 \\
\hline Klhl23 & 0.653898816 & 0.021900883 & 0.560113954 & 0.044188559 \\
\hline Kpna2 & \begin{tabular}{|l|l|}
0.302032745 \\
\end{tabular} & $5.02835 \mathrm{E}-06$ & 0.496528075 & 0.012669943 \\
\hline Lamp2 & 0.370940598 & 0.028197874 & 0.321798796 & 0.02183601 \\
\hline Lbh & 0.448217973 & 0.031293342 & 0.357983796 & 0.036397665 \\
\hline Limd1 & 0.572987846 & 0.045310275 & 0.421221826 & 0.039165117 \\
\hline Lipa & $\begin{array}{l}- \\
0.747590807\end{array}$ & 0 & $\begin{array}{l}- \\
0.401158294\end{array}$ & 0.01976415 \\
\hline Lmod3 & 0.380277732 & 0.022340004 & 0.545633588 & 0.021276959 \\
\hline LOC100134871 & 1.527582317 & 3.39728E-14 & 0.719200354 & 0.044271973 \\
\hline LOC100361144 & 0.446578986 & 0.035059134 & 0.251863873 & 0.043349505 \\
\hline LOC100909678 & 4.285349624 & 0.045514903 & $\begin{array}{l}- \\
4.719098969\end{array}$ & 0.043554887 \\
\hline LOC100909892 & 0.343172566 & 0.037213383 & 0.263903409 & 0.040023567 \\
\hline LOC100910554 & $\begin{array}{l}- \\
6.487970228\end{array}$ & 0.02155601 & 5.999675928 & 0.03604115 \\
\hline LOC100911372 & 0.511294219 & 0.023801793 & 0.351489109 & 0.043467771 \\
\hline LOC 102551744 & 5.879498791 & 0.021013954 & 6.529870966 & 0.0149407 \\
\hline LOC257643 & 3.79915731 & 0.049956973 & 7.385770149 & 0.003845906 \\
\hline
\end{tabular}




\begin{tabular}{|l|l|l|l|l|}
\hline LOC680913 & 5.139484174 & 0.033896942 & -6.20223866 & 0.017623722 \\
\hline & & & - & \\
\hline & 7.922826547 & $4.84603 \mathrm{E}-05$ & 3.232189001 & 0.044311173 \\
\hline LOC688924 & 5.508452434 & 0.021488548 & 6.247875493 & 0.021049443 \\
\hline LOC690096 & 6.671551504 & 0.023709355 & 8.929140371 & 0.005623601 \\
\hline Lp1 & 0.621404397 & 0.012641857 & 0.449218712 & 0 \\
\hline Lppr4 & 4.840325657 & $1.12681 \mathrm{E}-05$ & 4.559431485 & 0.040330654 \\
\hline Lrrc2 & 0.421303682 & 0.006401647 & 0.306901233 & 0.04404651 \\
\hline Lsamp & 0.93932807 & $1.10439 \mathrm{E}-07$ & 0.395534115 & 0.044526818 \\
\hline Lsm14a & 0.278617135 & 0.022688379 & 0.188545533 & 0.048017797 \\
\hline Man1c1 & 0.58497033 & 0.037109006 & 0.769959253 & 0.020621537 \\
\hline Manf & 0.261364471 & 0.010788094 & 6.17403579 & 0.028786807 \\
\hline Mccc1 & 0.508168431 & 0.022870533 & 0.543207913 & 0.026367214 \\
\hline Mdh1 & 0.489089221 & 0.029125498 & 0.458541728 & 0.01263405 \\
\hline Mipep & 0.260865848 & 0.048808294 & 0.27247243 & 0 \\
\hline Mir106b & 0.43569083 & 0.045441366 & 0.351585974 & 0.049402502 \\
\hline Mir146b & 6.464125953 & 0.04273539 & 6.669207761 & 0.030672039 \\
\hline Mir185 & 6.60527377 & 0.030260155 & 7.501240133 & 0.026355749 \\
\hline Mir208b & & & & \\
\hline Mir215 & & & - & \\
\hline & & & & \\
\hline & & & & \\
\hline
\end{tabular}




\begin{tabular}{|c|c|c|c|c|}
\hline & & & 7.885770762 & \\
\hline Mir291a & 6.476929064 & 0.041980394 & 6.668336976 & 0.030633138 \\
\hline & & & - & \\
\hline Mir34b & 6.442459895 & 0.043778416 & 9.493619944 & 0.000152867 \\
\hline Mir3558 & 5.993562764 & 0.045828521 & 6.19714448 & 0.026840829 \\
\hline Mir3565 & 5.993562764 & 0.045828521 & 6.001284771 & 0.036077594 \\
\hline Mir3574 & 6.002450732 & 0.045012008 & 6.147908798 & 0.031084988 \\
\hline Mir6329 & 7.321269164 & 0.022741912 & $\begin{array}{l}- \\
6.801412021\end{array}$ & 0.016781534 \\
\hline Mirlet7c-1 & 7.11402254 & 0.026788678 & $\begin{array}{l}- \\
6.556130256\end{array}$ & 0.015181646 \\
\hline Mlf1 & 0.442539355 & 0.020875663 & 0.746257924 & 0.006799298 \\
\hline MORF4L1 & 0.269771631 & 0.001026021 & 0.360598336 & 0.021356446 \\
\hline Morf412 & 0.298507711 & 0.015985569 & 0.23363319 & 0.047139379 \\
\hline Mpc1 & 0.535685147 & 0 & 0.406339995 & 0.012515641 \\
\hline Mt-atp6 & $\begin{array}{l}- \\
0.322655684\end{array}$ & 0.000857489 & $\begin{array}{l}- \\
0.351029683\end{array}$ & 0.005498356 \\
\hline Mt-co3 & $\begin{array}{l}- \\
0.309782854\end{array}$ & 0.036781245 & $\begin{array}{l}- \\
0.376647377\end{array}$ & 0.009044942 \\
\hline Mut & 0.763244933 & 0.022715737 & 0.555791405 & 0.010471082 \\
\hline Mybphl & $\begin{array}{l}- \\
7.242669913\end{array}$ & 0 & $\begin{array}{l}- \\
5.283511114\end{array}$ & 0.017017428 \\
\hline Myh6 & 0.484722371 & 0 & 0.849185942 & 0.006508847 \\
\hline
\end{tabular}




\begin{tabular}{|c|c|c|c|c|}
\hline Myl1 & 2.066218174 & 0.01541496 & 1.714350843 & 0.02391371 \\
\hline Myl4 & $\begin{array}{l}- \\
6.180362206\end{array}$ & 0 & $\begin{array}{l}- \\
1.738837883\end{array}$ & 0.017899892 \\
\hline Myl7 & \begin{tabular}{|l} 
\\
5.894727421
\end{tabular} & 5.9952E-15 & $\begin{array}{l}- \\
5.062004985\end{array}$ & 0.034563751 \\
\hline Mylk3 & 0.364253423 & 0.040744886 & 0.61881681 & 0.013239686 \\
\hline Myoz2 & 0.477453054 & 0.032560618 & 0.389574779 & 0.00606133 \\
\hline Naa20 & 0.353671584 & 0.048926165 & 0.316937715 & 0.044198825 \\
\hline Nap114 & 0.276249662 & 0.033596126 & 0.167673247 & 0.039029575 \\
\hline ND3 & \begin{tabular}{|l}
- \\
0.541489246
\end{tabular} & 0.035012667 & $\begin{array}{l}- \\
0.333495057\end{array}$ & 0.014649944 \\
\hline Nde1 & 0.761744761 & $3.48818 \mathrm{E}-05$ & 0.529236216 & 0.021653924 \\
\hline Ndufa5 & 0.358119294 & 0.037786127 & 0.361412897 & 0.014493893 \\
\hline Ndufs2 & 0.320122194 & 0.036685242 & 0.158936416 & $6.10063 \mathrm{E}-07$ \\
\hline Nhp211 & 0.418574103 & 0.025257259 & 0.265651562 & 0.043265386 \\
\hline Nnt & 0.38728641 & 0.02776274 & 0.40994615 & 0.00059453 \\
\hline Nppa & $\begin{array}{l}- \\
3.587057807\end{array}$ & 0.012316634 & $\begin{array}{l}- \\
3.489060407\end{array}$ & 0.032429637 \\
\hline Nqo2 & 0.421595158 & 0.007587719 & 0.323755669 & 0.007605026 \\
\hline Nrd1 & 0.286643287 & 0.022615264 & 0.238406886 & 0.044404817 \\
\hline Nudt4 & 0.444316208 & $5.4956 \mathrm{E}-14$ & 0.514099741 & 0.0068294 \\
\hline Ogn & - & $7.50511 \mathrm{E}-14$ & 0.622915144 & 0.039415497 \\
\hline
\end{tabular}




\begin{tabular}{|l|l|l|l|l|} 
& 1.517044589 & & & \\
\hline & & & - & \\
\hline ORF1 & 6.947558389 & 0.023160009 & 7.066652498 & 0.014681191 \\
\hline Oxct1 & 0.859356796 & 0 & 0.522432963 & 0 \\
\hline Oxr1 & 0.338849137 & 0.037049793 & 0.343603787 & 0.020950045 \\
\hline & & & - & \\
\hline Oxt & 4.781612056 & 0.037103387 & 7.765527447 & 0.003316296 \\
\hline P4hb & 0.353801096 & $7.99805 \mathrm{E}-07$ & 0.435828318 & 0.018487266 \\
\hline Pabpc4 & 0.184400659 & 0.023130308 & 0.309675586 & 0.011851011 \\
\hline Paip2 & 0.147105462 & 0.046469447 & 0.279511843 & 0.031960318 \\
\hline Pcbp2 & 0.409162586 & 0.03835754 & 0.232195112 & 0.032978935 \\
\hline Pcca & 0.438856185 & 0.004480733 & 0.305408389 & 0.021879486 \\
\hline Pcmt1 & 0.374332624 & $5.16527 \mathrm{E}-08$ & 0.311904931 & 0.010215595 \\
\hline Pcmtd1 & 0.320113105 & 0.042688464 & 0.222062654 & 0.021684379 \\
\hline Pcnp & 0.651640312 & 0.038305947 & 0.430851599 & 0.034487043 \\
\hline Pdia3 & 0.311853604 & 0.042918382 & 0.250522661 & 0.032984969 \\
\hline Pdia6 & 0.270490103 & 0.001245801 & 0.382375449 & 0.010957247 \\
\hline Pdk1 & 0.796357319 & 0.047800947 & 0.42094529 & 0.021742818 \\
\hline Pdp2 & & 0.037086676 & - & 0.015561318 \\
\hline Perm1 & & & & \\
\hline & & & & \\
\hline & & & & \\
\hline
\end{tabular}




\begin{tabular}{|c|c|c|c|c|}
\hline & & & 8.066475888 & \\
\hline Pink1 & 0.303467083 & $1.9984 \mathrm{E}-15$ & 0.353786174 & 0 \\
\hline Pla2g12a & 0.429167334 & 0.046783241 & 0.497362911 & 0.019218194 \\
\hline Pla2g1b & 5.394230948 & 0.049637094 & 6.033111504 & 0.021139342 \\
\hline Pla2g5 & 0.531964139 & 0.012158376 & 0.418484755 & 0.00302196 \\
\hline Pln & 0.611885545 & 0 & 0.487171083 & 0 \\
\hline Ppap2b & 0.369534507 & 0.02452327 & 0.212369891 & 0.045522686 \\
\hline Ppm1k & 1.04256048 & 0.024098594 & 0.800913001 & 0.021906365 \\
\hline Prdx3 & 0.172331767 & 0.024719934 & 0.216779735 & 0.021343972 \\
\hline Prkab1 & 0.437144562 & 0.020731848 & 0.647356177 & 0.015332496 \\
\hline Prkag2 & $\begin{array}{l}- \\
0.741484668\end{array}$ & 0.038194487 & $\begin{array}{l}- \\
0.548375621\end{array}$ & 0.016227733 \\
\hline Prkar1a & 0.354324266 & 0.047191068 & 0.364608273 & 0.031162539 \\
\hline Prodh & 0.670490676 & 0.040112361 & 1.047710129 & $7.99361 \mathrm{E}-15$ \\
\hline Pygm & 0.177098891 & 0.04679001 & 0.307668088 & 0.027693398 \\
\hline Rab1 & 0.311944494 & 0.04335158 & 0.175881975 & 0.043402545 \\
\hline Rablb & 0.267434992 & 0.042236772 & 0.274824774 & 0.025815033 \\
\hline Rab2a & 0.193801686 & 0.020703123 & 0.296478933 & 0.012287851 \\
\hline Rabggtb & 0.374152168 & 0.024417697 & 0.301979266 & 0.047154418 \\
\hline Rangrf & 0.442322126 & 0.037094256 & 0.510070576 & 0.032934084 \\
\hline Rcan1 & -0.42039856 & 0.000489459 & 1.320637808 & 0.003622015 \\
\hline
\end{tabular}




\begin{tabular}{|c|c|c|c|c|}
\hline Rcan2 & 0.166987093 & 0.039926419 & 0.344161112 & 0.006382592 \\
\hline Reep5 & 0.363332992 & 0.023827933 & 0.319514914 & 0.020343535 \\
\hline RGD1311933 & 8.360321364 & 0 & 7.683618454 & $1.39888 \mathrm{E}-14$ \\
\hline RGD1359290 & 6.280055689 & 0.036709687 & -6.54281647 & 0.010808496 \\
\hline RGD1562055 & 5.388757506 & 0.021510049 & $\begin{array}{l}- \\
7.161374236\end{array}$ & 0.012051296 \\
\hline RGD1562977 & 6.39601092 & 0.04487618 & $\begin{array}{l}- \\
8.990813971\end{array}$ & 0.002255316 \\
\hline RGD1564827 & -2.29110917 & 0.018501671 & -3.70354597 & 0.008954433 \\
\hline Rgs5 & 0.815605773 & $1.61128 \mathrm{E}-07$ & 0.262803809 & 0.041313901 \\
\hline Rhox4g & 4.768200089 & 0.024449831 & 6.784021813 & 0.016881253 \\
\hline Rilpl1 & 0.293857942 & 0.023018377 & 0.607469596 & $2.9976 \mathrm{E}-15$ \\
\hline Rn50_1_1348.1 & 5.913640424 & 0.022710316 & $\begin{array}{l}- \\
5.542297373\end{array}$ & 0.01615152 \\
\hline Rn50_13_0855.2 & 5.476329943 & 0.046717937 & 8.751788286 & 0.004494618 \\
\hline Rn50_X_0554.2 & 7.028341431 & 0.011078713 & -7.02241795 & 0.015220933 \\
\hline Rn50_X_0648.2 & 5.573718404 & 0.045486901 & $\begin{array}{l}- \\
6.623984792\end{array}$ & 0.016026185 \\
\hline Rn50_X_0691.3 & 5.242856628 & 0.043068807 & 5.497035502 & 0.016992814 \\
\hline
\end{tabular}




\begin{tabular}{|c|c|c|c|c|}
\hline Rn50_X_0749.4 & | 3.791794496 & 0.049947288 & $\mid-5.70081855$ & 0.01923353 \\
\hline Rn5s & 6.002450732 & 0.045012008 & $\begin{array}{l}- \\
7.190719821\end{array}$ & 0.007305141 \\
\hline Rpn1 & 0.34064496 & 0.023902986 & 0.354985577 & 0.031364484 \\
\hline Rps25-ps2 & 7.418557907 & 0.010404298 & $\begin{array}{l}- \\
7.235210277\end{array}$ & 0.0085834 \\
\hline Rragd & \begin{tabular}{|l|}
0.579680961 \\
\end{tabular} & 0.025698149 & 0.395609451 & 0.048330715 \\
\hline RT1-O1 & 4.825195624 & 0.03770872 & $\begin{array}{l}- \\
4.264155894\end{array}$ & 0.048020153 \\
\hline Sat2 & 0.77246277 & 0.025685517 & 0.742306141 & 0.018788701 \\
\hline Sctr & 2.956339816 & 0.010797109 & 3.376015416 & 0.009691025 \\
\hline Sdha & 0.35019745 & 0.039134469 & 0.304294586 & 0.013006413 \\
\hline Sdhd & 0.221620844 & 0.040137032 & 0.230100248 & 0 \\
\hline Sdr39u1 & 0.421524854 & 0.045464425 & 0.467998642 & 0.034635779 \\
\hline Serinc1 & 0.27693989 & 0.026747424 & 0.244276175 & 0.007837025 \\
\hline Set & $\mid 0.353025993$ & 0.043537938 & 0.254170122 & 0.049427633 \\
\hline Sh3bp5 & 0.497247391 & 0.032909297 & 0.39812856 & 0.00753254 \\
\hline Sh3glb1 & 0.206964735 & 0.033614384 & 0.347115403 & 0.0055655973 \\
\hline Slc25a12 & 0.47972219 & 0.048245551 & 0.358647876 & 0.041323487 \\
\hline Slc25a48 & $\begin{array}{l}- \\
5.193900842\end{array}$ & 0.033309101 & $\begin{array}{l}- \\
6.692227933\end{array}$ & 0.018040929 \\
\hline Slc2a4 & 0.654282011 & 0.000134543 & 0.726193595 & 0.005782333 \\
\hline Slc41a3 & 0.630547009 & 0.024876889 & 0.518380514 & 0.04542402 \\
\hline
\end{tabular}




\begin{tabular}{|c|c|c|c|c|}
\hline Slc4alap & 0.519567558 & 0.041338813 & 0.483934951 & $\mid 0.049798653$ \\
\hline Slc4a3 & \begin{tabular}{|l|l|}
0.33876168 \\
\end{tabular} & 0.048698851 & 0.548709379 & 0.014402994 \\
\hline Slc6a8 & 0.626661284 & 0.024755298 & 0.364755932 & \begin{tabular}{|l|}
0.04957447 \\
\end{tabular} \\
\hline Sln & $\begin{array}{l}- \\
6.249252612\end{array}$ & $2.9976 \mathrm{E}-15$ & $\begin{array}{l}- \\
4.011773682\end{array}$ & 0.017158246 \\
\hline Spink8 & 0.386421654 & 0.025694247 & 0.265747102 & 0.043299275 \\
\hline Spop & \begin{tabular}{|l}
0.402770866 \\
\end{tabular} & $6.72427 \mathrm{E}-08$ & 0.238048871 & 0.034654498 \\
\hline Spp2 & 4.671133308 & 0.025519088 & $\begin{array}{l}- \\
5.862048085\end{array}$ & 0.019812403 \\
\hline Spryd7 & 0.184628386 & 0.029054706 & 0.594132823 & 0.016066519 \\
\hline Srsf5 & 0.267118927 & 0.048027235 & 0.3463088 & 0.024224051 \\
\hline Sspn & 0.439932453 & 0.045421261 & 0.42167133 & 0.043764684 \\
\hline Stip1 & 0.506683269 & 0.028194993 & 0.39469689 & 0.009583563 \\
\hline Stx4 & 0.439790665 & 0.028729016 & 0.422336005 & 0.013082737 \\
\hline Suclg2 & \begin{tabular}{|l}
0.255190729 \\
\end{tabular} & 0.023472475 & 0.272232127 & \begin{tabular}{|l|}
0.021658218 \\
\end{tabular} \\
\hline Sypl1 & 0.442189035 & 0.025134988 & 0.355162059 & 0.031693223 \\
\hline Tasp1 & \begin{tabular}{|l}
0.606585577 \\
\end{tabular} & 0.044612875 & 0.473013139 & \begin{tabular}{|l|l|}
0.034715117 \\
\end{tabular} \\
\hline Tcp1 & 0.29262856 & 0.016166257 & 0.33729851 & 0.02972758 \\
\hline Tcp1112 & 0.63296282 & $4.29345 \mathrm{E}-05$ & 0.554766865 & $1.29896 \mathrm{E}-14$ \\
\hline Tex13b & 4.933024755 & 0.047183382 & 4.599443036 & 0.018129662 \\
\hline Tex26 & $\begin{array}{l}- \\
6.079186686\end{array}$ & 0.035908667 & $\begin{array}{l}- \\
6.253715727\end{array}$ & 0.015393157 \\
\hline
\end{tabular}




\begin{tabular}{|c|c|c|c|c|}
\hline Tex37 & 4.20211932 & 0.048465795 & 5.955943617 & 0.021165841 \\
\hline Tfpi & 0.57414975 & 0.024658757 & 0.595962395 & 0.006691825 \\
\hline Tmed10 & 0.193621479 & 0.044902391 & 0.22526948 & \begin{tabular}{|l}
0.040122885 \\
\end{tabular} \\
\hline Tmed2 & 0.367457129 & 0 & 0.384159887 & 0.018175829 \\
\hline Tmem182 & 0.269216724 & 0.044261483 & 0.194705949 & 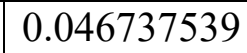 \\
\hline Tmem261 & 0.439418432 & 0.048301147 & 0.396610755 & \begin{tabular}{|l|l}
0.043369518 \\
\end{tabular} \\
\hline Tmem 262 & 4.897158934 & 0.041542947 & 4.857810664 & 0.049180656 \\
\hline Tmem38a & 0.404869712 & 0.029018463 & 0.429458054 & 0.008415459 \\
\hline Tmem59 & \begin{tabular}{|l}
0.257934708 \\
\end{tabular} & \begin{tabular}{|l|}
0.03835183 \\
\end{tabular} & 0.253998399 & 0.0437482 \\
\hline Tmod4 & 1.416557914 & 0.005591951 & 1.047646179 & 0.011330011 \\
\hline Tnni3k & 0.30914724 & 0.04115943 & 0.415083184 & 0.00253396 \\
\hline Tpp1 & 0.375004846 & 0.049396333 & 0.415871286 & 0.038192909 \\
\hline Trav7d-5 & 5.454847753 & 0.047454887 & 5.449836507 & 0.04968536 \\
\hline Trim35 & 0.322030152 & 0.048002951 & 0.339185352 & 0.049867665 \\
\hline Trim54 & $\begin{array}{l}- \\
0.585604296\end{array}$ & 0.047417392 & 0.346719167 & 0.045384247 \\
\hline Trim63 & 0.414119694 & 0.044891724 & 0.460606744 & 0.01331858 \\
\hline Trim72 & 0.218160248 & 0.045660042 & 0.292551644 & 0.03189256 \\
\hline Tspan3 & $\mid 0.279582296$ & 0.029474992 & 0.215675049 & 0.032321022 \\
\hline Ttc7b & 0.3999965663 & 0.046013492 & 0.303823738 & 0.047123805 \\
\hline Tuba8 & 0.651823455 & $8.23755 \mathrm{E}-06$ & 0.526405928 & 0.011618731 \\
\hline Txlnb & 0.334853654 & 0.024594464 & 0.425780453 & 0.002389614 \\
\hline
\end{tabular}




\begin{tabular}{|l|l|l|l|l|}
\hline Txn2 & 0.788925347 & $1.51281 \mathrm{E}-06$ & 0.936578764 & $1.4988 \mathrm{E}-14$ \\
\hline Ube2b & 0.235490055 & 0.012824273 & 0.241000663 & 0.021381362 \\
\hline Ucp2 & 0.559836801 & 0.023534574 & 0.622780018 & 0.010528044 \\
\hline Ugp2 & 0.229337073 & 0.01056207 & 0.586374642 & 0 \\
\hline Uqcrc2 & 0.301896919 & $3.99812 \mathrm{E}-06$ & 0.256027418 & 0.007314627 \\
\hline Uqcrfs1 & 0.205493555 & 0.041204366 & 0.119762276 & 0.044525471 \\
\hline Vdac2 & 0.180692074 & 0.023427809 & 0.294830726 & $2.9976 \mathrm{E}-15$ \\
\hline Vdac3 & 0.180565705 & 0.044786409 & 0.288191871 & $5.9952 \mathrm{E}-15$ \\
\hline Vegfa & 0.571307806 & 0.004749552 & 0.403157109 & 0.027571594 \\
\hline Vopp1 & 0.399416721 & 0.014177199 & 0.366496352 & 0.03517043 \\
\hline Wfdc1 & 0.604375385 & 0.001768803 & 0.788228807 & $5.9952 \mathrm{E}-15$ \\
\hline Ywhae & 0.105603012 & 0.047735897 & 0.151129557 & 0.022615446 \\
\hline Zdhhc11 & 3.916044848 & 0.041978058 & 3.989250623 & 0.042731048 \\
\hline
\end{tabular}




\section{CHAPTER 4}

A Methodological Approach to Reducing Rat Nppa Using Targeted siRNA

Mackenzie S. Newman

Department of Physiology and Pharmacology, West Virginia University, Morgantown, WV, USA 


\section{$\underline{\text { Abstract }}$}

Nppa is a gene that encodes for Anp, a hormone that causes diuresis and decreased blood volume. Despite the known beneficial roles in LVH and HF in reducing blood pressure and cardiac fibrosis, elevated levels of circulating Anp, have been shown to modulate electrical properties in cardiac tissue and cell preparations in many species, including dogs, rabbits, rodents, and humans, which may contribute to later development of arrhythmias and HF. Previous research has shown that complete knockout of Nppa or its primary receptor is embryonic lethal in rodents, but reducing the expression of the gene postnatally has not been explored. This study aims to validate whether rat Nppa can be knocked down using targeted siRNA duplexes in a rat cardiomyocyte cell line as a proof-of-concept for further application. The efficacy of three siRNA sequences targeted at Nppa were evaluated for their efficacy at reducing Anp secreted into cell culture media. All three Nppa siRNA significantly decreased Anp levels for three days post-treatment. siRNA sequence 1 decreased Anp levels to the greatest extent when comparing all three targeted siRNA, while siRNA 2 and 3 were not statistically different over multiple days. Day 2 Nppa levels were confirmed to be decreased in the siRNAtreated groups and positive control group via RT-qPCR. These results show that rat Nppa can be targeted using any of the three siRNA duplex sequences detailed herein. This study provides a tool for specific targeting of expression of this gene that can be used in further studies to determine the effects of reducing Anp in LVH and HF models. 


\section{Introduction}

Previously, Nppa gene expression has been demonstrated to be increased in both male and female OZR and obese humans. Its peptide product, Anp, followed the same pattern when analyzed via Western blot. Nppa is a gene expressed exclusively within cardiac tissue (15), thus the sole origin of Anp in circulation is the heart. As Nppa is part of the calcineurin-NFAT genetic program that is active primarily during fetal development with expression reduced over time $(9,16)$. In pathological $\mathrm{LVH}$, this genetic program becomes re-activated and Nppa levels rise in order to compensate for cardiac overload by decreasing blood volume by action at the kidney and therefore reducing blood pressure. Anp is secreted from cardiomyocytes in response to cardiac damage as a means of controlling fluid volume: it primarily acts to induce sodium secretion in the kidney. It is considered to be a physiological antagonist to the action of angiotensin $(3,4,6,7,12)$.

Despite the beneficial effects of Anp in many cardiovascular diseases, research has shown that exposure of cardiac tissues and cells to high concentrations of Anp can alter heart rate (HR), effective refractory period (ERP), and action potential duration (APD) from mice, rats, dogs, rabbits, guinea pigs, and humans $(1,2,8,10,14)$. Humans possessing a frameshift mutation that causes Anp to have an extended C-terminus and increased persistence in the blood exhibited atrial fibrillation, as well as mice with the gene knocked-in (5). Thus, the potential for elevated Anp to have a detrimental effect in situations of LVH cannot be ruled out. Deletion of Nppa or its cognate receptor gene, NPR1, has been shown to be cardiac-lethal in animal models $(5,11)$. To circumvent this issue, in this study, I sought to reduce, but not totally ablate, Nppa (and ultimately Anp), in an immortalized rat cardiomyocyte cell line via use of an siRNA approach. 


\section{Materials and Methods}

\section{Cell culture and siRNA transfection}

H9c2 cells, an immortalized rat cardiomyocyte cell line, were purchased from ATCC and routinely cultured in standard high glucose DMEM supplemented with 1X penicillin/streptomycin and 10\% fetal bovine serum. After cells reached $70-80 \%$ confluence in six-well plates, they were treated with 75 pmol siRNA for 1,2 , or 3 days. Control cells at day 0 were not treated with siRNA. Transfection was carried out using $7.5 \mu \mathrm{L}$ Lipofectamine 3000 . Rat Nppa siRNA duplexes were purchased from ABM (product i564970) and are listed in Table 1. Day 0 samples were taken from media that was already in the wells. Following this, all media was replaced and siRNA/Lipofectamine was added. Day 0 wells were used for day 1 samples. All wells were exposed to siRNA at the same time but no individual well was re-used beyond day 0 , e.g. samples for day 1 were not re-sampled for days 2 or 3. Anti-Gapdh siRNA in Lipofectamine (positive control, known for effectively knocking down a known gene but not sharing sequence homology with Nppa), a scrambled siRNA (also referred to as "Negative Control siRNA") construct, and Lipofectamine without siRNA were used as controls. Cell culture supernatant or cell pellets in RNALater were immediately frozen at $-80{ }^{\circ} \mathrm{C}$ for further use.

\section{ELISA}

ANP Competitive ELISA Kits (Thermo Fisher \#EIAANP) were used for all ELISA experiments following the manufacturer's instructions. Briefly, wells in pre-coated 96-well plates were incubated with standards or samples. Next, assay buffer, Anp conjugate, and Anp antibody were added sequentially (Anp antibody was not added to non-specific binding wells). Plates were then sealed and incubated with shaking for one hour at room temperature. Then, after four 
washes with wash buffer, TMB substrate was added to the wells and incubated for 30 minutes at room temperature. Stop solution was then added and readings were taken immediately at $450 \mathrm{~nm}$ on a plate reader. Data were fit to a four parameter logistic curve (by the manufacturer's suggestion) using Softmax Pro software. All ELISA samples consisted of three technical replicates and three biological replicates. Information about intra- and interassay coefficients of variation, detection limit, and cross-reactivity are available via the product manual.

\section{RT-qPCR}

RNA was extracted from cell pellets using an RNA Fibrous Tissue Miniprep Kit (Qiagen) according to the manufacturer's instructions and as previously described (13). RTqPCR for Nppa was only performed on Day 2 samples.

\section{Statistics}

ELISA data were analyzed using 1-way ANOVA with Tukey's test for multiple comparisons. RT-qPCR data were analyzed using the ddCt method.

\section{$\underline{\text { Results }}$}

In order to address whether expression of Nppa could be knocked down using siRNA, three different siRNA duplexes were designed against rat Nppa (Table 1). These sequences were run through the Thermo Fisher BLOCK-iT platform and verified via NIH blastn to be suitable for testing due to low homology with other known rat gene sequences. Using lipofectamine, a standard transfection vehicle, each sequence was tested in $\mathrm{H} 9 \mathrm{c} 2$ (rat immortalized cardiomyocyte cells) in comparison with a Gapdh siRNA (a positive control with known anti-proliferative effects on cells), scrambled Nppa siRNA (negative control), and vehicle with no siRNA. Media was sampled from wells after zero days (baseline), one, two, or three days. Because removing 
media from wells might have an effect on cell proliferation, each well was only sampled at one time point. For each individual treatment, three wells were sampled in triplicate.

Figure 1 displays the time-course trend for all of the data. Overall, levels of Anp in culture media decreased in the three siRNA-treated groups compared to all of the controls. The positive control Gapdh siRNA resulted in reduced levels of Anp compared to the two negative control groups, but this reduction was not to the same degree as the targeted siRNA. Figure 2 shows baseline data (i.e. no siRNA treatment). There was a statistically significant different between the "no treatment" and "Nppa siRNA 3" groups, but this may have been due to outliers. No other comparisons were significant. Figure 3 shows day one exposure data. All group comparisons resulted in significance $(\mathrm{p}<0.05)$ with the exception of the siRNA 2 versus siRNA 3. For days two and three (Figures 4 and 5), the same trends resulted: all comparisons were statistically significant $(\mathrm{p}<0.05)$ except for scrambled siRNA versus lipofectamine only (no treatment), siRNA 1 versus siRNA 2, and siRNA 2 versus siRNA 3 .

Figure 6 shows quantification of RT-qPCR for Nppa and Gapdh (normalized to $\beta$-actin) in cells from day 2 in order to verify the effect of these siRNA on transcript expression instead of Anp peptide production from ELISA. These data show that the anti-Nppa siRNA and Gapdh siRNA reduced Nppa transcript levels compared to controls (A, top panel) but only Gapdh siRNA reduced Gapdh transcript levels (A, bottom panel). Figure 6B confirms singular end products from RT-qPCR. Overall, these results show that the Nppa siRNA are effective at reducing Anp compared to the controls, but Nppa siRNA 1 has a slightly greater effect.

\section{Discussion}

In this study, the potential to reduce Anp secretion was investigated using siRNA constructs targeted toward Nppa. Given that Nppa and Anp are exclusively produced in the heart, 
the risk of these constructs producing side effects due to off-target binding in other tissues is minimal. Despite the potential for siRNA constructs to have off-target effects, none of them showed significant homology to other mRNA via BLAST (siRNA 1 and 2 had $66 \%$ homology to the next non-Nppa gene and siRNA 3 had 71\%). All three sequences did reduce Anp levels in culture media, but siRNA 1 appeared to have a slightly greater effect overall. Reducing circulating Anp levels via siRNA knockdown may have effects in other tissues in an animal model, primarily in the kidney, by increasing volume retention and decreasing diuresis, but this depends on the degree to which Anp is knocked down: if Anp receptors are super-saturated, reducing the amount of circulating hormone may not have a measurable effect.

As the ELISA assay only looks at secreted Anp and does not include intracellular stores, I attempted several western blots to verify a reduction in cellular Anp levels. Unfortunately, given the weak signal produced, these all required too much contrast adjustment above the background threshold to be usable for any kind of analysis. The weakness in signal is may be due to low concentrations of Anp in cells and is compounded by the lower sensitivity of Western blots compared to ELISA. In retrospect, the cells that I used for Western blots would have been much better used for RT-qPCR. In future studies, it might be advisable to perform and repeat more RTqPCR.

siRNA-based therapeutics are a newer technology with currently only limited use in approved pharmaceuticals. For laboratory research, their major use is for targeting individual genes without the need to modify the endogenous genome. Chemical modifications, such as replacing the phosphate backbone with thiophosphate or cholesterylation increase half-life and cellular uptake of an siRNA, respectively. Ideally, moving forward, I would like to package the 
siRNA 1 sequence into a modified siRNA construct and test it in OZR during the course of LVH development in order to gauge whether elevated Anp is necessary, beneficial, or detrimental.

In addition to using siRNAs, there are many other methods one could use to reduce the effect of circulating Anp. Small molecular agonists and antagonists of the Anp receptor, NPRA, have been researched in the past. However, due to the high homology between Anp and BNP, these drugs typically have affinities for both NPRA and NPRB, the cognate receptor for BNP. Another potential route to reduce the effect of Anp is direct administration of antibodies, but these have not been explored in obesity-related LVH models. An alternative way to modulate Nppa expression is through altering transcription of the gene Nppa antisense-1 (Nppa-AS1), an alternatively-spliced gene that runs concurrently with and beyond the length of Nppa. Its product is a long non-coding RNA whose role in disease is not understood. Induction of this gene, perhaps through siRNA or microRNA stabilization, is another avenue worth investigating.

Overall, the preliminary studies here show that knock-down of rat Nppa is achievable using the siRNA sequences presented. Future studies in whole animals are necessary to validate the safety and toxicity of reducing, while not completely ablating, Anp levels using the siRNA approach. Despite Anp being an important adaptive hormone against hypertrophic development, understanding the point in the development of LVH that circulating Anp levels are putting the individual at a higher risk of HF is critical. Application of the approach presented here in OZR would help address this significant issue. 


\section{$\underline{\text { References }}$}

(1) Beaulieu P, Cardinal R, De LA, Lambert C. Direct chronotropic effects of atrial and C-type natriuretic peptides in anaesthetized dogs. Br J Pharmacol 1996 Aug;118(7):1790-6.

(2) Beaulieu P, Cardinal R, Page P, Francoeur F, Tremblay J, Lambert C. Positive chronotropic and inotropic effects of C-type natriuretic peptide in dogs. Am J Physiol 1997 Oct;273(4):H1933-H1940.

(3) Deschepper CF, Masciotra S, Zahabi A, Boutin-Ganache I, Picard S, Reudelhuber TL. Functional alterations of the Nppa promoter are linked to cardiac ventricular hypertrophy in WKY/WKHA rat crosses. Circ Res 2001 Feb 2;88(2):223-8.

(4) Dries DL. Natriuretic peptides and the genomics of left-ventricular hypertrophy. Heart Fail Clin 2010 Jan;6(1):55-64.

(5) Galimberti ES, Kannankeril P, Kor K, Muhammad R, Blair M, Darbar D. Abstract 19074: NPPA Overexpression in Mice Increases Susceptibility to Atrial Fibrillation. Circulation 2012 Nov 20;126(Suppl 21):A19074.

(6) Grabowski K, Riemenschneider M, Schulte L, Witten A, Schulz A, Stoll M, et al. Fetal-adult cardiac transcriptome analysis in rats with contrasting left ventricular mass reveals new candidates for cardiac hypertrophy. PLoS One 2015;10(2):e0116807.

(7) Houweling AC, van Borren MM, Moorman AF, Christoffels VM. Expression and regulation of the atrial natriuretic factor encoding gene Nppa during development and disease. Cardiovasc Res 2005 Sep 1;67(4):583-93.

(8) Kecskemeti V, Pacher P, Pankucsi C, Nanasi P. Comparative study of cardiac electrophysiological effects of atrial natriuretic peptide. Mol Cell Biochem 1996 Jul;160-161:539. 
(9) Maillet M, van Berlo JH, Molkentin JD. Molecular basis of physiological heart growth: fundamental concepts and new players. Nat Rev Mol Cell Biol 2013 Jan;14(1):38-48.

(10) Moghtadaei M, Polina I, Rose RA. Electrophysiological effects of natriuretic peptides in the heart are mediated by multiple receptor subtypes. Prog Biophys Mol Biol 2016 Jan;120(1-3):3749.

(11) Mori T, Chen YF, Feng JA, Hayashi T, Oparil S, Perry GJ. Volume overload results in exaggerated cardiac hypertrophy in the atrial natriuretic peptide knockout mouse. Cardiovasc Res 2004 Mar 1;61(4):771-9.

(12) Newman MS, Nguyen T, Watson MJ, Hull RW, Yu HG. Transcriptome profiling reveals novel BMI- and sex-specific gene expression signatures for human cardiac hypertrophy. Physiol Genomics 2017 Jul 1;49(7):355-67.

(13) Newman M, Infanto AM, Watson MJ, Hull RW, Yu HG. Abstract 140: BMI- and Genderspecific Increase of MAP2K3/p38 Activity in Human Cardiac Hypertrophy. Circulation Research 2017 Feb 23;119(Suppl 1):A140.

(14) Stambler BS, Guo GB. Atrial natriuretic peptide has dose-dependent, autonomically mediated effects on atrial refractoriness and repolarization in anesthetized dogs. J Cardiovasc Electrophysiol 2005 Dec;16(12):1341-7.

(15) Uhlen M, Fagerberg L, Hallstrom BM, Lindskog C, Oksvold P, Mardinoglu A, et al. Proteomics. Tissue-based map of the human proteome. Science 2015 Jan 23;347(6220):1260419. (16) Wilkins BJ, Dai YS, Bueno OF, Parsons SA, Xu J, Plank DM, et al. Calcineurin/NFAT coupling participates in pathological, but not physiological, cardiac hypertrophy. Circ Res 2004 Jan 9;94(1):110-8. 


\section{Figures}

Figure 1: Time-course data from Anp ELISA from days 0 through 4.

\section{Effect of NPPA siRNA on Secreted ANP in H9c2 Cells}

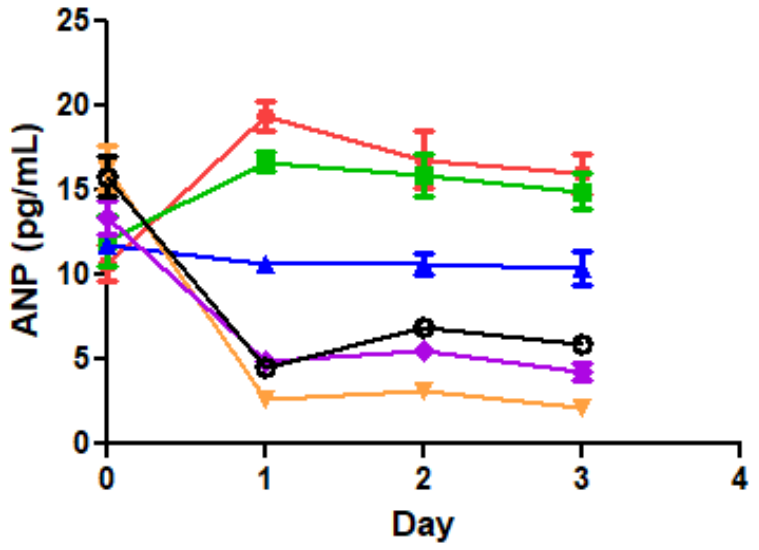

$$
\begin{aligned}
& * \text { No treatment } \\
& - \text { Scrambled siRNA } \\
& + \text { GAPDH siRNA } \\
& - \text { NPPA siRNA } 1 \\
& - \text { NPPA siRNA } 2 \\
& - \text { NPPA siRNA } 3
\end{aligned}
$$

Figure 1. Time-course data from Anp ELISA from days 0 through 4. Data points on each day are discrete (i.e. media was not sampled from the same wells on multiple days), but data points are connected in this plot to delineate the groups. Data are displayed as means $+/-$ standard deviation from three individual wells, with three technical replicates per well. 
Figure 2: Day 0 quantification of Anp via ELISA

\section{Day 0}

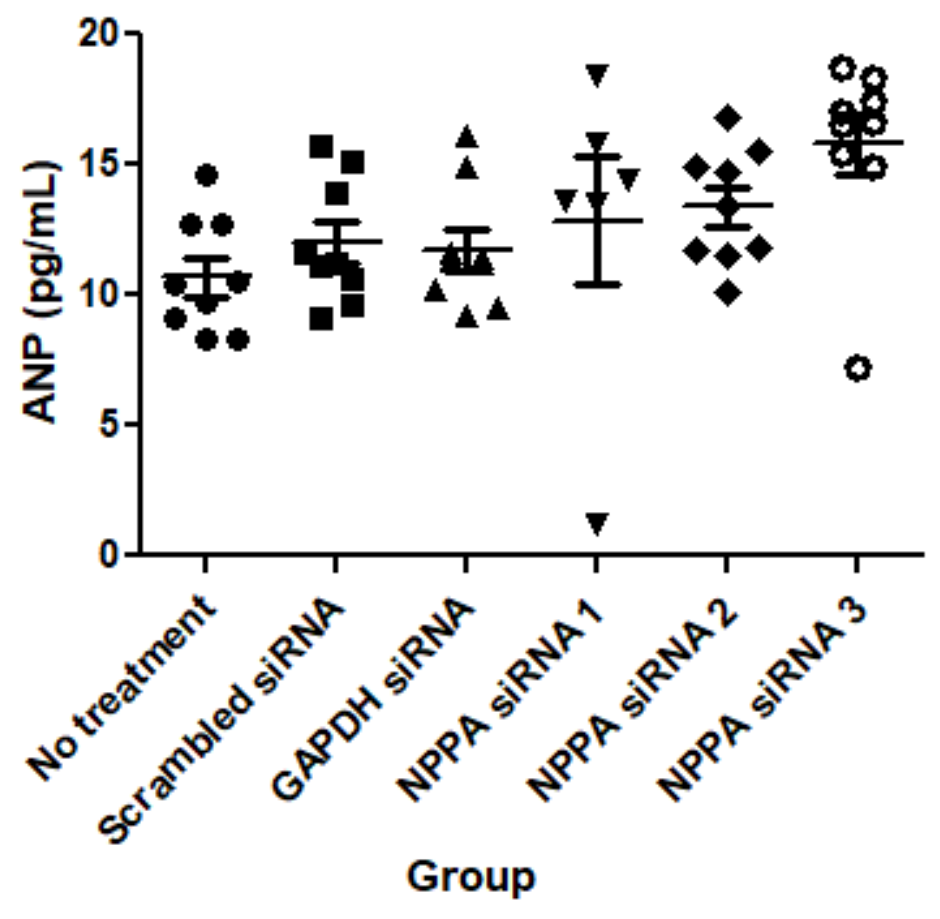

Figure 2. Day 0 quantification of Anp via ELISA. All pairwise comparisons were not statistically significant $(\mathrm{p}<0.05)$ except for siRNA 2 versus siRNA 3. Bars represent mean $+/$ - standard deviation for three separate wells, each with three technical replicates. Each symbol represents a signal replicate. 
Figure 3: Day 1 quantification of Anp via ELISA

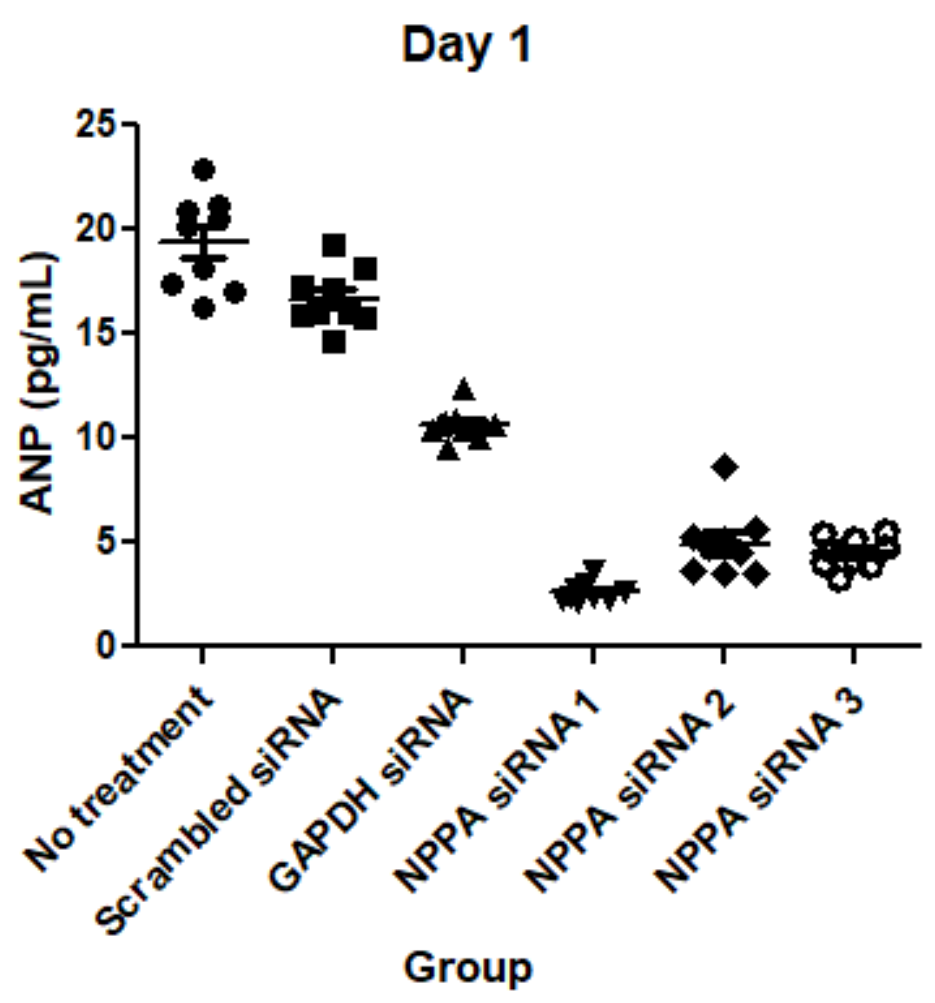

Figure 3. Day 1 quantification of Anp via ELISA. All pairwise comparisons were statistically significant $(\mathrm{p}<0.05)$ except for siRNA 2 versus siRNA 3. Bars represent mean $+/$ - standard deviation for three separate wells, each with three technical replicates. Each symbol represents a signal replicate. 
Figure 4: Day 2 quantification of Anp via ELISA

Day 2

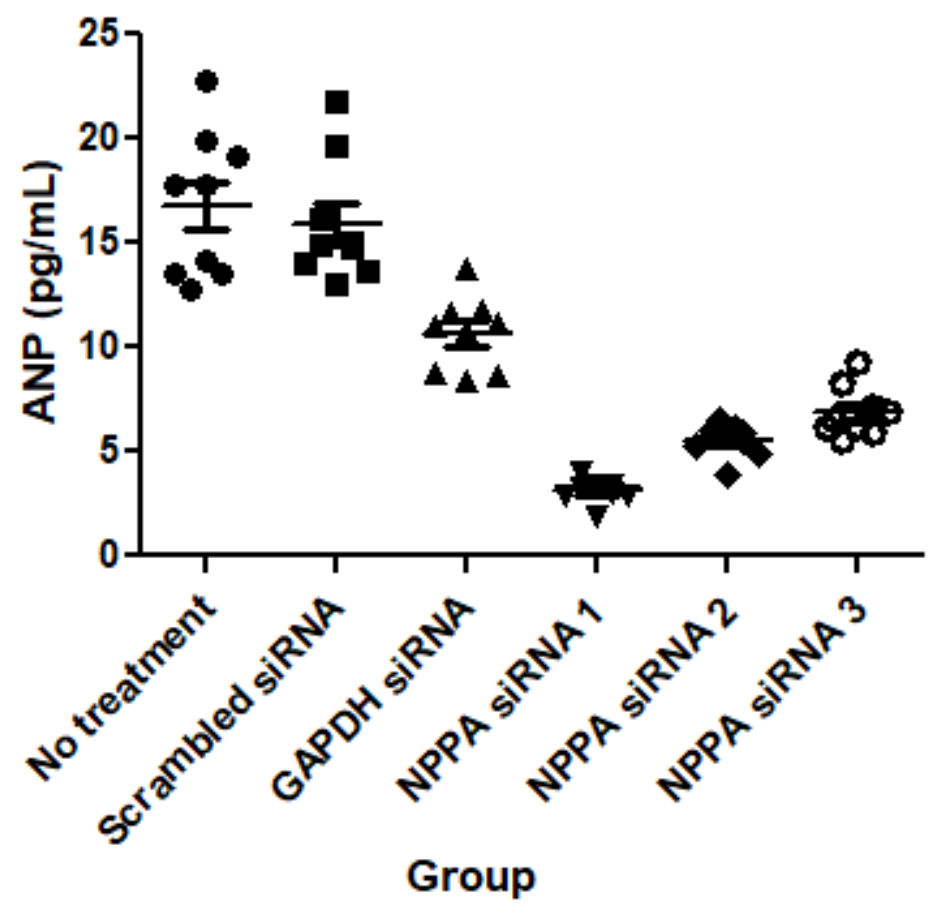

Figure 4. Day 2 quantification of Anp via ELISA. All pairwise comparisons were statistically significant $(\mathrm{p}<0.05)$ except for scrambled siRNA versus lipofectamine only (no treatment), siRNA 1 versus siRNA 2, and siRNA 2 versus siRNA 3. Bars represent mean $+/-$ standard deviation for three separate wells, each with three technical replicates. Each symbol represents a signal replicate. 
Figure 5: Day 3 quantification of Anp via ELISA

\section{Day 3}

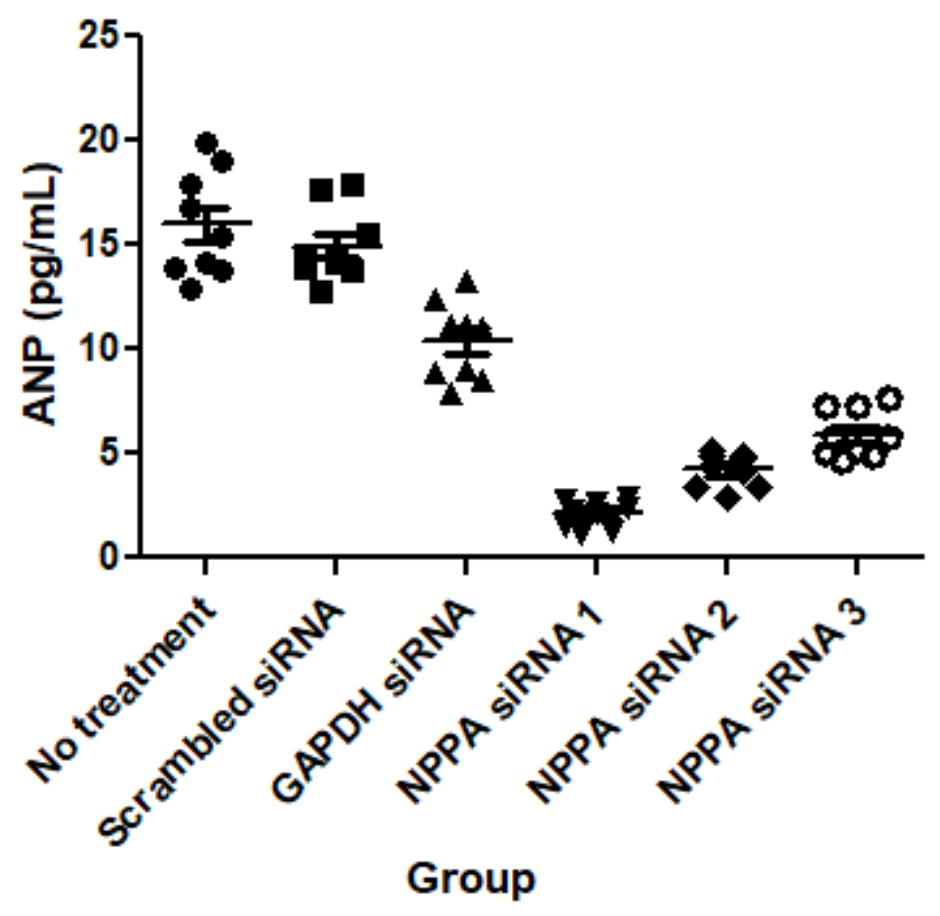

Figure 5. Day 3 quantification of Anp via ELISA. All pairwise comparisons were statistically significant $(\mathrm{p}<0.05)$ except for scrambled siRNA versus lipofectamine only (no treatment), SiRNA 1 versus siRNA 2, and siRNA 2 versus siRNA 3. Bars represent mean $+/-$ standard deviation for three separate wells, each with three technical replicates. Each symbol represents a signal replicate. 
Figure 6: Representative RT-qPCR data for Nppa and Gapdh for day 2.

A.
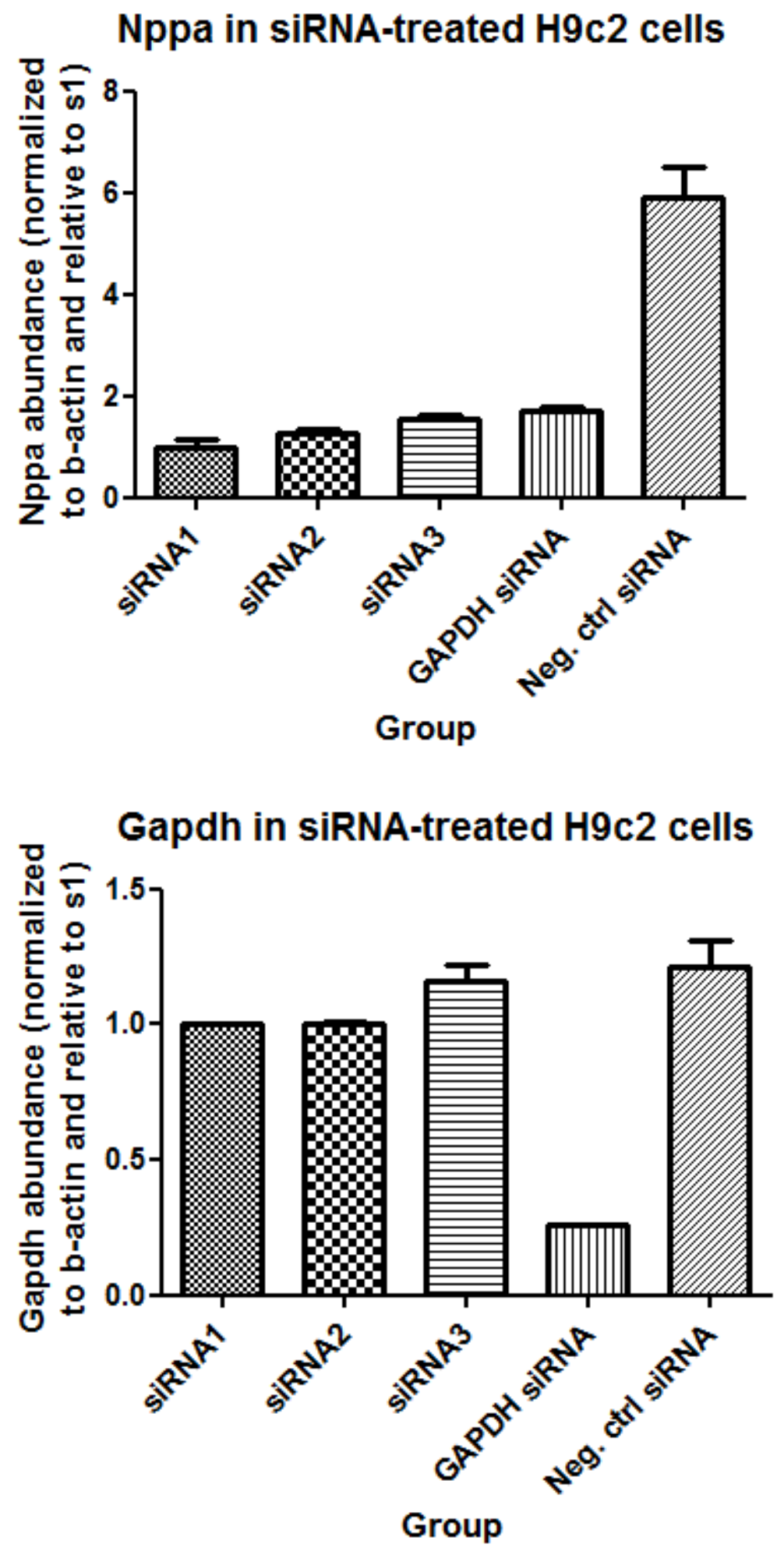
B.

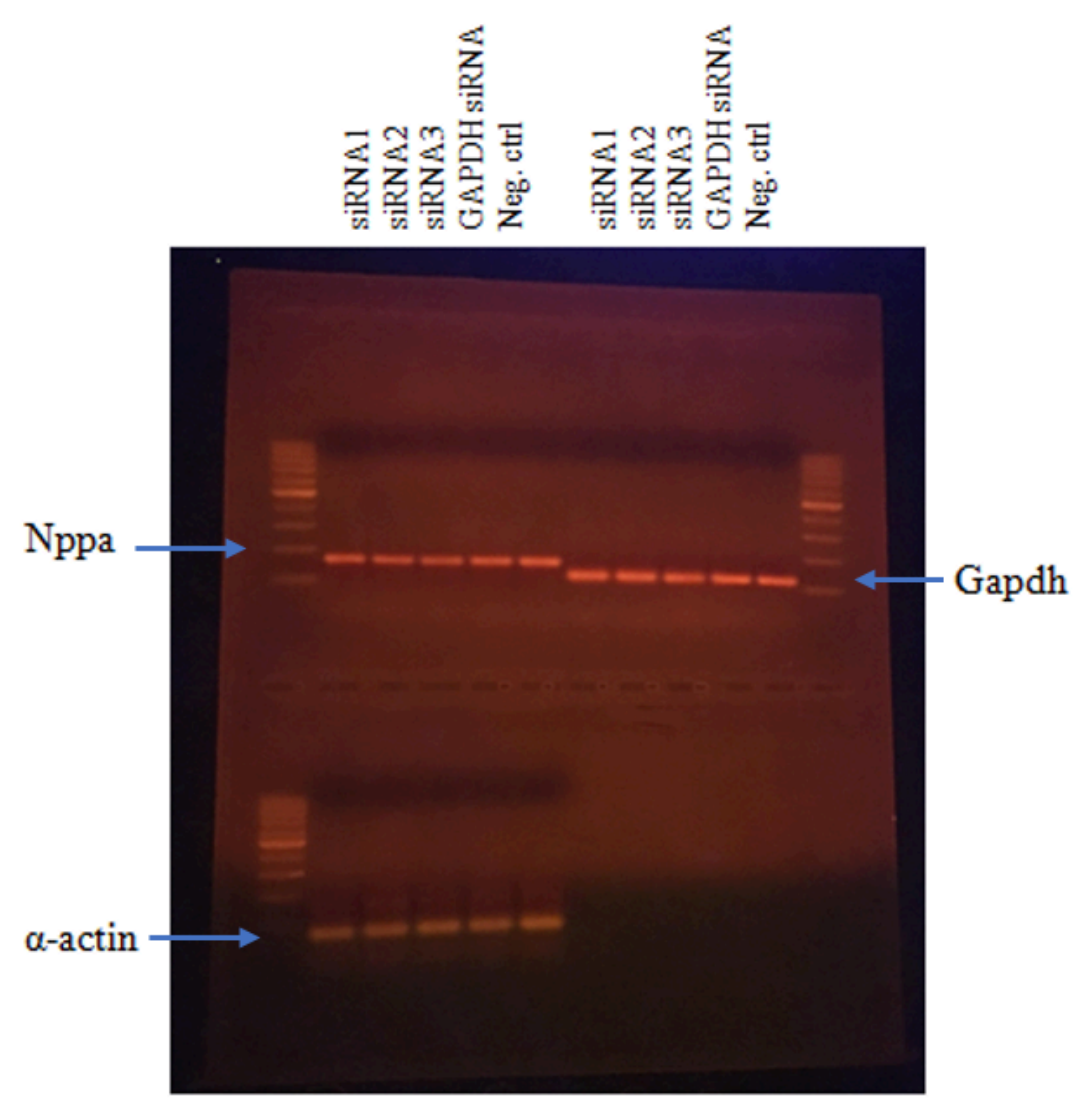

Figure 6. A: RT-qPCR quantification of Day 2 Nppa and Gapdh levels, normalized to $\beta$-actin and displayed as relative to siRNA 1. B: Gel displaying singular end products for RT-qPCR. 
$\underline{\text { Tables }}$

Table 1. Nppa siRNA Duplex Sequences

\begin{tabular}{|l|l|}
\hline & Sequence \\
\hline siRNA 1 & GGAGAAGAUGCCGGUAGAATT \\
& UUCUACCCGCAUCUUCUCCTT \\
\hline siRNA 2 & CCGAUAGAUCUGCCCUCUUTT \\
& AAGAGGGCAGAUCUAUCGGTT \\
\hline siRNA 3 & GACUAGGCUGCAACAGCUUTT \\
& AAGCUGUUGCAGCCUAGUCTT \\
\hline
\end{tabular}




\section{CHAPTER 5}

\section{Discussion}

In Chapters 1 and 2, I aimed to describe the effects of sex and obesity on the transcriptome of $\mathrm{LVH}$ in both humans and Zucker rats in order to investigate the use of Zucker rats as translational model. Given the difficulty of studying LVH in humans due to the scarcity of live tissue, particularly from healthy controls, there is a major need for research models of LVH. LVH is considered to be irreversible, but once it has progressed into HF, quality of life is significantly decreased and risk of death is majorly increased. Obesity is one of the most common comorbidities of both LVH and HF. Given these facts, early detection of LVH is pivotal in preventing the transition to HF. The results of these studies reveal a litany of potential gene targets that may serve as biomarkers or pharmaceutical targets for the detection or treatment, respectively, of LVH.

When comparing the overall transcriptomes of our human and rat samples, I identified a set of 45 genes that were differentially expressed during $\mathrm{LVH}$ in both species and in the same direction (i.e. upregulated in male or female humans and rats or downregulated in male or female humans and rats) and other genes with sex- or species-specific expression (Chapter 3, Supplemental Table 2). Because these genes are differentially expressed in a consistent direction for one or both sexes, they warrant examination into their potential roles in LVH. Data from my human studies provided a "gene signature" of nine genes, validated at both the RNA transcriptome and protein level, which may indicate the presence of LVH. Using that same signature as a starting point for my rat studies, I was able to validate five of those genes as potentially being translationally relevant. They are discussed below. 


\section{Summary of Validated Genes}

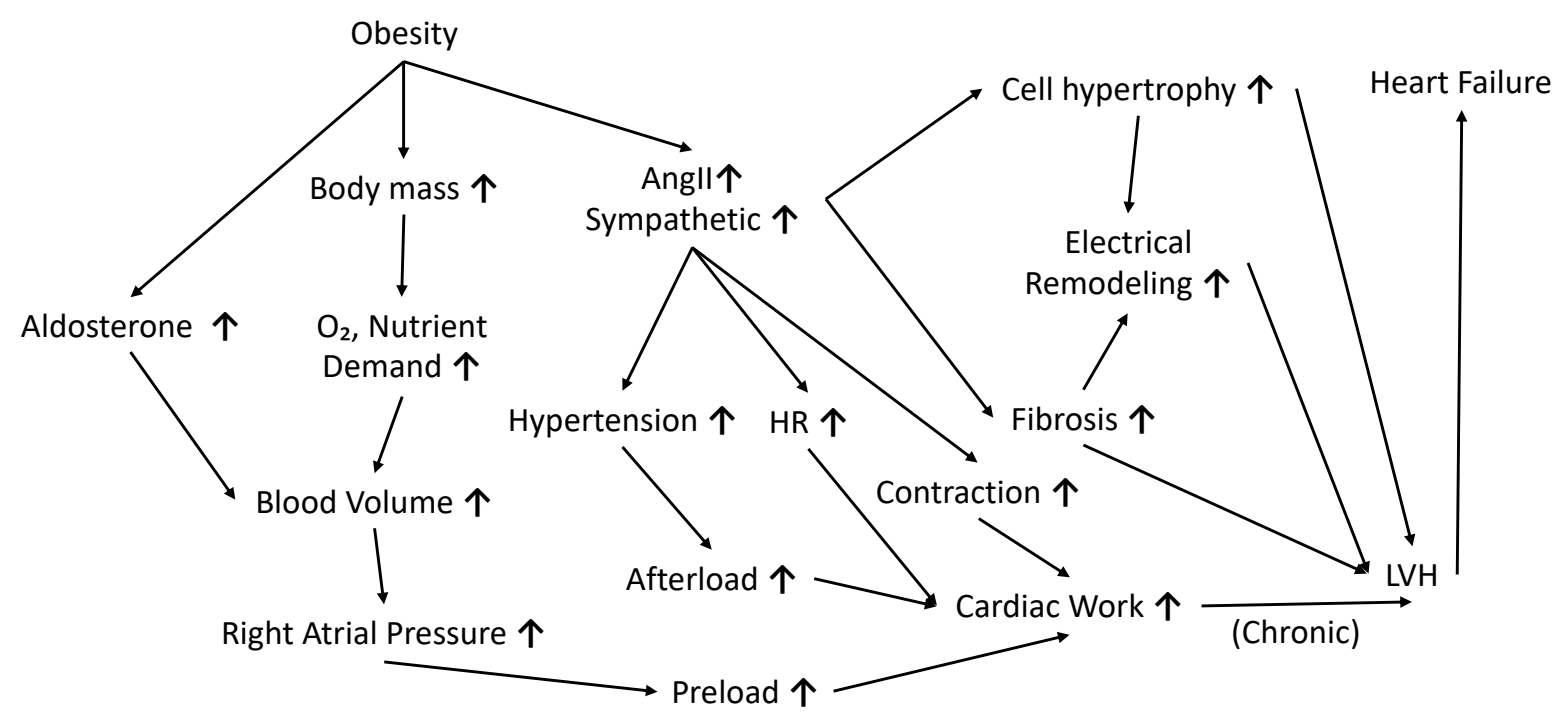

This model summarizes the main pathways that cause the development of LVH (and finally HF) as a result of obesity. Obesity, an increase in body mass, brings heightened demand for oxygen and nutrients to new tissue. Proteins such as HBB, an oxygen carrier, may play a role here. Obesity also means greater bioavailability of fat, therefore, a protein such as PDK4 may play a role in helping to utilize this energy source. A larger body mass means a larger blood volume is necessary. This is facilitated by a number of factors: 1, obesity directly causes increases in aldosterone which causes fluid retention; 2, obesity causes increases in circulating angiotensin II to increase, which causes further increases in aldosterone levels. The resultant elevation in blood volume raises right atrial pressure, which is reflected as a larger preload in the heart. Under normal conditions, under the Frank-Starling mechanism, this would cause increased cardiac output. Another branch of this mechanism is the rise in angiotensin II and catecholamines from obesity. This causes hypertension and therefore increased afterload, increases in contractility (facilitated by proteins such as MYL7), and increases in heart rate. 
These factors all contribute to increased cardiac work. Chronically, these factors cause the Frank-Starling mechanism to fail and cardiac output decreases despite right atrial pressure. Circulating angiotensin II and catecholamines also contribute directly to cellular hypertrophy and fibrosis, which together lead to electrical remodeling of the ventricle and independently to promote LVH. Hormones like ANP and BNP are released from the heart as "rescue" signals, to antagonize hypertension and reduce blood volume, but in the end, all of these compensatory mechanisms ultimately cause reductions in cardiac output that cannot be sustained, thus resulting in $\mathrm{HF}$.

\section{NPPA and NPPB}

I found NPPA to be the gene with the largest fold increase during LVH and obesity in both humans and OZR, with NPPB as the second-highest. Activity of NPPA in the ventricles is high during fetal development but later subsides under normal physiological conditions, while NPPB follows a similar trajectory but does not decrease to as large of an extent postnatally. ANP, the final peptide product encoded by NPPA, and BNP, the final peptide product encoded by NPPB, are circulating hormones that are primarily produced and secreted by the atria in adults, but are also produced and secreted from the ventricles in $\operatorname{LVH}(24,38)$. My findings are, thus, consistent with previous findings.

My research revealed several fold changes in NPPA and ANP expression to superphysiological levels in both sexes with the presence of obesity. As discussed in the introduction, elevated ANP has been shown to decrease heart rate in rats and dogs but increase it in humans $(1,2,26)$, increase the effective refractive period in dogs but decrease it in humans (26), and decrease action potential duration in human, dog, rabbit, and guinea pig cardiac preparations (26). In one family possessing a frameshift mutation in NPPA that produces an extended C- 
terminal, degradation-resistant ANP, all mutation carriers had atrial fibrillation (9). Although deletion of NPPA or its cognate receptor in mice produces early cardiac hypertrophy and ultimately death via HF $(28,32)$, elevated ANP may be a contributing factor in LVH as a precursor to HF.

In order to study the role of ANP further, a conditional knock-down, or an siRNAmediated knock-down of NPPA/ANP during the progression of LVH would be worth investigating. There is currently no research into the balance between NPR1 and NPR2 receptor saturation by ANP and activity of the clearance receptor NPR3; it is unknown whether ANP clearance is increased via NPR3 upon NPR1 and NPR2 saturation or if translation of the signaling receptors is upregulated instead. In a model such as OZR, where LVH presents before hypertension (unlike other transgenic obese rodent models), the role of supra-physiological ANP activity, specifically in cardiac disturbances, may be better elucidated.

\section{$\underline{\text { HBB }}$}

HBB is a gene that encodes for the protein precursor of hemoglobin subunit beta (HBB). It functions in coordination with hemoglobin subunit alpha, HBA1, to form an active hemoglobin complex (9). In my human studies, we found significant increases in HBA1 and HBB in both males and females via transcriptome analysis, but protein levels for each were increased only in females (30). In our rat studies, HBB was significantly increased in females only in transcriptome analysis, but both obese males and females showed increased protein levels. Measurable HBA1 protein levels were difficult to detect via Western blot but were not significantly different when quantified; this is likely due to fatty contamination of samples. HBB has not been previously implicated directly in population-wide hypertrophic conditions. Research into HBB with regard to cardiovascular disorders has largely focused on its role in 
sickle cell disease (16) and beta-thalassemia (3), but these diseases are associated with patientspecific gene polymorphisms and mutations $(3,16)$. One study of post-mortem hearts described increased levels of HBA1 and HBB mRNA associated with sudden cardiac death, but no direct mechanism was suggested (41). Increases in levels of HBA1 and HBB may occur in response to reactive oxygen species (19), which are elevated in the development of LVH and HF (37). The mechanism underlying HBB upregulation is not currently understood, though it may serve as an oxygen reserve or buffer to protect against hypoxic insult, and as a site to sequester reactive oxygen species. Unfortunately, HBB may not be a useful circulating biomarker as it is also always present in circulating erythrocytes, and elevated urinary poryphyrins (such as heme, produced by the breakdown of HBB) are common markers of liver dysfunction; however, LVH has been found in $12-30 \%$ of patients with cirrhosis $(7,31)$. Ventricular HBB levels may be indicative of underlying $\mathrm{LVH}$, but the risk of cardiac biopsy may outweigh its ultimate prognostic outcome compared to established biomarkers like pro-NT BNP.

\section{PDK4}

PDK4 is a gene that encodes for the protein precursor of pyruvate dehydrogenase kinase4 (PDK4). This mitochondrial enzyme is expressed in low-to-moderate amounts in many tissues, but cardiac and skeletal muscles have the greatest enrichment (45). Our studies found PDK4 to be significantly upregulated in LVH-presenting males and females via transcriptome analysis, but the protein was significantly increased only in males (30). In rats, expression was increased in both sexes in transcriptome analysis, but increases in protein were only significant in males. Despite these conflicting protein expression results, PDK4 has previously been positively associated with cardiac hypertrophy and $\mathrm{HF}(12,27,47)$, though some studies show a negative association $(33,39,43)$. Induction of PDK4 activity decreases glucose utilization and increases 
fat catabolism. PDK4 inhibits pyruvate dehydrogenase complexes, which are responsible for converting pyruvate (derived from glucose metabolism) to acetate (as acetyl-CoA) and carbon dioxide. Acetyl-CoA from this process is normally then used as a substrate for cellular respiration. Increased PDK4 activity decreases acetyl-CoA production from glucose which is counterbalanced by increases in fat metabolism to produce acetyl-CoA. Elevated concentrations of PDK4 in obesity may be simply due to the increased bioavailability of fats. Insulin insensitivity, a common comorbidity with obesity, has been linked to overexpression of PDK4 (44); this is likely due to decreased activation of PI3K, leading to reduced inhibition of the PDK4 promoter (18). PDK4 inhibitors have been explored with regard to treatment of cancers, diabetes, and acute cardiac ischemia (34), but have not been used to treat cardiac hypertrophy. Given the crucial role of PDK4 in regulating energy metabolism and the metabolic disturbances that are associated with obesity, and the widespread presence of PDK4 in skeletal muscle, inhibition of PDK4 may not be a suitable treatment for hypertrophy. PDK4 mRNA and protein enrichment in the heart may make it a useful local biomarker. Circulating PDK4 mRNA and protein levels have not been reported in the literature.

\section{MYL7}

MYL7 is a gene that encodes for the protein precursor of myosin light chain-7 (MYL7). Like NPPA, it is involved in cardiac development, but its expression is restricted to the atria in healthy adults $(8,40)$. Nonetheless, the presence of MYL7 in the ventricles has been reported in cardiac hypertrophy previously, but was not examined with regard to sex or obesity prior to my study $(17,21,30)$. The main function of MYL7 is to facilitate contractility; its upregulation in hypertrophy is attributed to the increased force demand from heart muscle (8). The functional differences between these and MYL7 is unknown. MYL7 may be a putative biomarker of 
hypertrophy due to being uniquely expressed within cardiac tissue, but whether it can be detected in blood is unknown. Due to its critical role in muscle contraction and sequence homology (36) with other, more ubiquitous myosin light chains (including MYL2, MYL5, MYL10, and MYL12), direct, specific targeting to increase the activity MYL7 by pharmaceutical intervention may be difficult. However, inhibition of myosin light chain kinase, which directly modulates the activity of MYL7 via reduced phosphorylation, has been explored as a treatment for cardiovascular conditions such as atherosclerosis (4) and heart ischemia/reperfusion injury (22) in rabbits and mice, respectively. These treatments have not been tested in humans.

\section{Remainder of the Transcriptome}

My studies uncovered a variety of ventricular genes that are shared in LVH by obese humans and Zucker rats. We validated a select group of them in humans and then examined these in our rat model. Not surprisingly, there are additional putative gene candidates within the dataset that may also be translationally relevant, but they are not within the scope of the present research. Both my human and rat data sets are limited by small sample size and age variability between samples. Beyond this, the human heart samples lacked full disclosure of pathology data, therefore we are not aware of any comorbidities or medications the patients were taking. Future studies would ideally rectify these issues (age-paired samples, number of samples, underlying conditions) and include functional human cardiac data for cross-comparison with the Zucker rat model. Given that LVH develops slowly over time, more research is warranted into transcriptomic and proteomic changes during the course of development, particularly with regard to specific isoforms of proteins that vary between atrial and ventricular expression. Our current transcriptome dataset does not differentiate between alternatively-spliced forms of genes. Alternate splicing is known to vary with age and can affect downstream protein structure and 
function (42). Other future studies might include identification of various species of RNA, such as long non-coding RNA (20) and microRNAs $(6,13,25,35,46)$, whose roles in the development of LVH are poorly understood. HF is not necessarily a death sentence but identifying and understanding what leads to it will save millions of lives in the future by allowing timely treatment.

\section{Future Directions}

Ideally, in order to continue to validate OZR as a model of human LVH, I would add more groups of animals: 1) LZR fed ad libitum, 2) OZR fed ad libitum, 3) LZR on a restricted diet, 4) OZR fed a restricted diet. Grouping the animals like this would allow for the results to be parsed better for the effect of obesity on LVH development. The LZR fed ad libitum and LZR fed a calorie-restricted diet should not show many transcriptomic changes and would serve as controls. The OZR with calorie restrictions will provide the most significant data in terms of overall LV expression: OZR with a restricted diet neither develop obesity nor LVH. Comparing these rats' transcriptomes to lean rats will reveal what genes are differentially-expressed as a product of deficient $\mathrm{Ob}-\mathrm{Rb}$ signaling in the obese animals. Comparing the calorie-restricted obese animals to the ones fed ad libitum will reveal which genes are differentially-expressed as a function of obesity. Leptin has effects on heart rate and parameters such as contractility but its direct role in LVH is poorly understood $(10,23)$. Adipose tissue, which directly secretes leptin, is known to be directly adjacent to cardiac tissue in obesity, therefore local levels of leptin are elevated above circulating concentrations. Comparing the calorie-restricted obese animals to the ones fed ad libitum will also help differentiate the effects of local leptin in the absence of Ob-Rb on the cardiac transcriptome in obesity-related LVH. If there are no differences between the OZR fed a calorie-restricted diet and lean controls, then the implications about what the presence 
of the OZR mutation does genomically and proteomically would be massive. However, the likelihood of there being zero differences between the two populations is minimal, as leptin signaling affects myriad second messenger and transcriptional pathways that have differential effects on the final proteome.

Similarly, with these groups, I would like to isolate LV RNA at different time points over the course of typical LVH development in the untreated obese group with free food access - i.e., before (e.g. week 8-10), during (e.g. week 11-14), and after pronounced LVH presentation (e.g. week 17-19). Genes and proteins involved in the development of LVH may not be present in the final presentation of $\mathrm{LVH}$, and these may serve as useful mechanistic or stage-specific markers of $\mathrm{LVH}$, whether for drug targeting or diagnosis. Adding groups such as Zucker diabetic fatty rats, a strain of OZR that develops diabetes, to these experiments would also allow for the effects of diabetes to be distinguished from obesity alone.

I would propose that the same experiments performed in Zucker rats herein should be performed in $o b / o b$ and $d b / d b$ mice. As discussed previously, these mice are similar to OZR with regard to dysfunctional leptin signaling at the level of the receptor $(d b / d b)$ and leptin itself $(o b / o b)$. These species develop many common pathological conditions to OZR despite not sharing the exact genetic mutation, but not all are the same or to the same degree, such as diabetes and hypertension. Moving into mice and comparing transcriptomic profiles would reveal differentially expressed genes that are shared between humans, rats, and mice, therefore further validating the translatability of the gene in the model and reducing the risk that a gene has different roles between species, e.g. a gene that is upregulated in mice but downregulated in humans and rats would be a bad target due to its role in LVH being inconsistent. 
Another potential avenue for continuing this research would be to induce the mutation found in OZR Ob-Rb protein, Gln269Pro, in another common rat strain, such as Wister-Kyoto rats. This would serve as a secondary confirmation for the role of the functional leptin receptor, $\mathrm{Ob}-\mathrm{Rb}$, as a contributor to obesity-related LVH. The same groups in the previous paragraph would be useful, again, to look at the developmental profile of LVH with or without obesity. The same mutation could be introduced to a common mouse strain, as well, such as BALB/c. Unlike $o b / o b$ and $d b / d b$ mice, having the exact mutation would allow for stricter control in cross-species analysis for the role of LepR in the development of LVH and obesity. Whereas OZR, $o b / o b$ mice, and $d b / d b$ mice, all are considered to have monogenically-derived obesity, Wister-Kyoto rats and TALLYHO mice have a polygenic background that better represents genetic variability like the general human population.

In order to further confirm the role of the leptin receptor in the development of $\mathrm{LVH}$, complete or partial ablation of the gene LepR within specific tissues or cell types might be considered. Numerous cell type-specific knockouts have been made, such as in endothelial cells (14), hepatocytes (15), neurons (5), astrocytes (29), and cardiomyocytes (11). Notably, the cardiomyocyte LepR knockout mice, which had a tamoxifen-inducible deletion, suffered from cardiac enlargement and eventual HF within ten days of induction (11). This evidence alone implies that LepR is critical in early development of cardiac tissue. Given that only one of the six LepR isoforms is considered dysfunctional in OZR (Ob-Rb) and they do not die of $\mathrm{HF}$ at such a young age, then the other isoforms might be playing a critical role in development. As discussed in Chapter 1, many leptin receptor isoforms have leptin binding capacity and therefore may serve to buffer circulating leptin levels or import leptin into cells, for example. The contributions of these other leptin receptors to cell signaling are considered to be minimal or absent, yet their 
roles have not been completely elucidated. Further mutating LepR to render the other isoforms inactive may be a useful way to look at their role in the development of LVH and ultimately HF.

\section{Summary}

In the studies presented here, I sought to validate novel biomarkers of LVH in humans as they pertained to HF and then define a model to further explore these genes and proteins. My human studies resulted in the generation of thousands of transcriptomic changes that have not been previously identified, particularly with respect to sex and obesity status. The genes and proteins that I validated for humans serve as a template signature that can be verified clinically, and the process described in those studies serves as a rapid pipeline to qualify genes for further investigation in humans. The goal of my rat studies was to show that there were genes and proteins shared across species in an obesity- and sex-specific manner. Genes that are differentially expressed in each species are viable tools for research into the mechanistic underpinnings of LVH. The role of sex, in particular, has not been explored frequently with regard to LVH, especially in model systems. It is well-known that estrogen has cardioprotective effects, and differentiating how LVH develops between males and females is critical in the development of appropriate biomarkers and suitable pharmaceutical interventions. Overall, there are myriad more directions that this research could be taken, but most importantly, the outcomes for human health are at the forefront. 


\section{$\underline{\text { References }}$}

(1) Beaulieu P, Cardinal R, De LA, Lambert C. Direct chronotropic effects of atrial and C-type natriuretic peptides in anaesthetized dogs. Br J Pharmacol 1996 Aug;118(7):1790-6.

(2) Beaulieu P, Cardinal R, Page P, Francoeur F, Tremblay J, Lambert C. Positive chronotropic and inotropic effects of C-type natriuretic peptide in dogs. Am J Physiol 1997 Oct;273(4):H1933-H1940.

(3) Cao A, Galanello R. Beta-thalassemia. Genet Med 2010 Feb;12(2):61-76.

(4) Cheng X, Wang X, Wan Y, Zhou Q, Zhu H, Wang Y. Myosin light chain kinase inhibitor ML7 improves vascular endothelial dysfunction via tight junction regulation in a rabbit model of atherosclerosis. Mol Med Rep 2015 Sep;12(3):4109-16.

(5) Cohen P, Zhao C, Cai X, Montez JM, Rohani SC, Feinstein P, et al. Selective deletion of leptin receptor in neurons leads to obesity. J Clin Invest 2001 Oct;108(8):1113-21.

(6) Curcio A, Torella D, Iaconetti C, Pasceri E, Sabatino J, Sorrentino S, et al. MicroRNA-1 downregulation increases connexin 43 displacement and induces ventricular tachyarrhythmias in rodent hypertrophic hearts. PLoS One 2013;8(7):e70158.

(7) De MM, Chinali M, Romano C, Benincasa M, D'Addeo G, D'Agostino L, et al. Increased left ventricular mass in pre-liver transplantation cirrhotic patients. J Cardiovasc Med (Hagerstown ) 2008 Feb;9(2):142-6.

(8) Doevendans PA, Bronsaer R, Lozano PR, Kubalak S, van BM. The murine atrial myosin light chain-2 gene: a member of an evolutionarily conserved family of contractile proteins. Cytogenet Cell Genet 2000;90(3-4):248-52. 
(9) Galimberti ES, Kannankeril P, Kor K, Muhammad R, Blair M, Darbar D. Abstract 19074: NPPA Overexpression in Mice Increases Susceptibility to Atrial Fibrillation. Circulation 2012 Nov 20;126(Suppl 21):A19074.

(10) Hall ME, Harmancey R, Stec DE. Lean heart: Role of leptin in cardiac hypertrophy and metabolism. World J Cardiol 2015 Sep 26;7(9):511-24.

(11) Hall ME, Smith G, Hall JE, Stec DE. Cardiomyocyte-specific deletion of leptin receptors causes lethal heart failure in Cre-recombinase-mediated cardiotoxicity. Am J Physiol Regul Integr Comp Physiol 2012 Dec 15;303(12):R1241-R1250.

(12) Horiuchi M, Kobayashi K, Masuda M, Terazono H, Saheki T. Pyruvate dehydrogenase kinase 4 mRNA is increased in the hypertrophied ventricles of carnitine-deficient juvenile visceral steatosis (JVS) mice. Biofactors 1999;10(2-3):301-9.

(13) Huang Y, Tang S, Huang C, Chen J, Li J, Cai A, et al. Circulating miRNA29 family expression levels in patients with essential hypertension as potential markers for left ventricular hypertrophy. Clin Exp Hypertens 2017;39(2):119-25.

(14) Hubert A, Bochenek ML, Schutz E, Gogiraju R, Munzel T, Schafer K. Selective Deletion of Leptin Signaling in Endothelial Cells Enhances Neointima Formation and Phenocopies the Vascular Effects of Diet-Induced Obesity in Mice. Arterioscler Thromb Vasc Biol 2017 Sep;37(9):1683-97.

(15) Huynh FK, Levi J, Denroche HC, Gray SL, Voshol PJ, Neumann UH, et al. Disruption of hepatic leptin signaling protects mice from age- and diet-related glucose intolerance. Diabetes 2010 Dec;59(12):3032-40.

(16) Kato GJ, Piel FB, Reid CD, Gaston MH, Ohene-Frempong K, Krishnamurti L, et al. Sickle cell disease. Nat Rev Dis Primers 2018 Mar 15;4:18010. 
(17) Kumar C, Saidapet C, Delaney P, Mendola C, Siddiqui MA. Expression of ventricular-type myosin light chain messenger RNA in spontaneously hypertensive rat atria. Circ Res 1988 Jun;62(6):1093-7.

(18) Kwon HS, Huang B, Unterman TG, Harris RA. Protein kinase B-alpha inhibits human pyruvate dehydrogenase kinase- 4 gene induction by dexamethasone through inactivation of FOXO transcription factors. Diabetes 2004 Apr;53(4):899-910.

(19) Li X, Wu Z, Wang Y, Mei Q, Fu X, Han W. Characterization of adult alpha- and betaglobin elevated by hydrogen peroxide in cervical cancer cells that play a cytoprotective role against oxidative insults. PLoS One 2013;8(1):e54342.

(20) Li Y, Liang Y, Zhu Y, Zhang Y, Bei Y. Noncoding RNAs in Cardiac Hypertrophy. J Cardiovasc Transl Res 2018 Dec;11(6):439-49.

(21) Lim DS, Roberts R, Marian AJ. Expression profiling of cardiac genes in human hypertrophic cardiomyopathy: insight into the pathogenesis of phenotypes. J Am Coll Cardiol 2001 Oct;38(4):1175-80.

(22) Lin HB, Cadete VJ, Sawicka J, Wozniak M, Sawicki G. Effect of the myosin light chain kinase inhibitor ML-7 on the proteome of hearts subjected to ischemia-reperfusion injury. J Proteomics 2012 Sep 18;75(17):5386-95.

(23) Lin YC, Huang J, Hileman S, Martin KH, Hull R, Davis M, et al. Leptin decreases heart rate associated with increased ventricular repolarization via its receptor. Am J Physiol Heart Circ Physiol 2015 Nov 15;309(10):H1731-H1739.

(24) Man J, Barnett P, Christoffels VM. Structure and function of the Nppa-Nppb cluster locus during heart development and disease. Cell Mol Life Sci 2018 Apr;75(8):1435-44. 
(25) Marketou ME, Parthenakis F, Vardas PE. Pathological Left Ventricular Hypertrophy and Stem Cells: Current Evidence and New Perspectives. Stem Cells Int 2016;2016:5720758.

(26) Moghtadaei M, Polina I, Rose RA. Electrophysiological effects of natriuretic peptides in the heart are mediated by multiple receptor subtypes. Prog Biophys Mol Biol 2016 Jan;120(1-3):3749.

(27) Mori J, Alrob OA, Wagg CS, Harris RA, Lopaschuk GD, Oudit GY. ANG II causes insulin resistance and induces cardiac metabolic switch and inefficiency: a critical role of PDK4. Am J Physiol Heart Circ Physiol 2013 Apr 15;304(8):H1103-H1113.

(28) Mori T, Chen YF, Feng JA, Hayashi T, Oparil S, Perry GJ. Volume overload results in exaggerated cardiac hypertrophy in the atrial natriuretic peptide knockout mouse. Cardiovasc Res 2004 Mar 1;61(4):771-9.

(29) Naranjo V, Contreras A, Merino B, Plaza A, Lorenzo MP, Garcia-Caceres C, et al. Specific Deletion of the Astrocyte Leptin Receptor Induces Changes in Hippocampus Glutamate Metabolism, Synaptic Transmission and Plasticity. Neuroscience 2019 Nov 6.

(30) Newman MS, Nguyen T, Watson MJ, Hull RW, Yu HG. Transcriptome profiling reveals novel BMI- and sex-specific gene expression signatures for human cardiac hypertrophy. Physiol Genomics 2017 Jul 1;49(7):355-67.

(31) Ortiz-Olvera NX, Castellanos-Pallares G, Gomez-Jimenez LM, Cabrera-Munoz ML, Mendez-Navarro J, Moran-Villota S, et al. Anatomical cardiac alterations in liver cirrhosis: an autopsy study. Ann Hepatol 2011 Jul;10(3):321-6.

(32) Pandey KN. Genetic Ablation and Guanylyl Cyclase/Natriuretic Peptide Receptor-A: Impact on the Pathophysiology of Cardiovascular Dysfunction. Int J Mol Sci 2019 Aug 14;20(16). 
(33) Razeghi P, Young ME, Ying J, Depre C, Uray IP, Kolesar J, et al. Downregulation of metabolic gene expression in failing human heart before and after mechanical unloading. Cardiology 2002;97(4):203-9.

(34) Roche TE, Hiromasa Y. Pyruvate dehydrogenase kinase regulatory mechanisms and inhibition in treating diabetes, heart ischemia, and cancer. Cell Mol Life Sci 2007 Apr;64(78):830-49.

(35) Sanchez-Ruderisch H, Queiros AM, Fliegner D, Eschen C, Kararigas G, Regitz-Zagrosek V. Sex-specific regulation of cardiac microRNAs targeting mitochondrial proteins in pressure overload. Biol Sex Differ 2019 Feb 6;10(1):8.

(36) Sayers EW, Agarwala R, Bolton EE, Brister JR, Canese K, Clark K, et al. Database resources of the National Center for Biotechnology Information. Nucleic Acids Res 2019 Jan 8;47(D1):D23-D28.

(37) Seddon M, Looi YH, Shah AM. Oxidative stress and redox signalling in cardiac hypertrophy and heart failure. Heart 2007 Aug;93(8):903-7.

(38) Sergeeva IA, Hooijkaas IB, Ruijter JM, van dM, I, de Groot NE, van de Werken HJ, et al. Identification of a regulatory domain controlling the Nppa-Nppb gene cluster during heart development and stress. Development 2016 Jun 15;143(12):2135-46.

(39) Sheeran FL, Angerosa J, Liaw NY, Cheung MM, Pepe S. Adaptations in Protein Expression and Regulated Activity of Pyruvate Dehydrogenase Multienzyme Complex in Human Systolic Heart Failure. Oxid Med Cell Longev 2019;2019:4532592.

(40) Small EM, Krieg PA. Molecular regulation of cardiac chamber-specific gene expression. Trends Cardiovasc Med 2004 Jan;14(1):13-8. 
(41) Son GH, Park SH, Kim Y, Kim JY, Kim JW, Chung S, et al. Postmortem mRNA expression patterns in left ventricular myocardial tissues and their implications for forensic diagnosis of sudden cardiac death. Mol Cells 2014 Mar;37(3):241-7.

(42) Stegeman R, Weake VM. Transcriptional Signatures of Aging. J Mol Biol 2017 Aug $4 ; 429(16): 2427-37$.

(43) Taegtmeyer H, Razeghi P, Young ME. Mitochondrial proteins in hypertrophy and atrophy: a transcript analysis in rat heart. Clin Exp Pharmacol Physiol 2002 Apr;29(4):346-50.

(44) Thoudam T, Ha CM, Leem J, Chanda D, Park JS, Kim HJ, et al. PDK4 Augments ERMitochondria Contact to Dampen Skeletal Muscle Insulin Signaling During Obesity. Diabetes 2019 Mar;68(3):571-86.

(45) Uhlen M, Fagerberg L, Hallstrom BM, Lindskog C, Oksvold P, Mardinoglu A, et al. Proteomics. Tissue-based map of the human proteome. Science 2015 Jan 23;347(6220):1260419. (46) Wang Y, Chen S, Gao Y, Zhang S. Serum MicroRNA-27b as a Screening Biomarker for Left Ventricular Hypertrophy. Tex Heart Inst J 2017 Dec;44(6):385-9.

(47) Zhao G, Jeoung NH, Burgess SC, Rosaaen-Stowe KA, Inagaki T, Latif S, et al. Overexpression of pyruvate dehydrogenase kinase 4 in heart perturbs metabolism and exacerbates calcineurin-induced cardiomyopathy. Am J Physiol Heart Circ Physiol 2008 Feb;294(2):H936-H943. 